\title{
Progress Report and Technical Evaluation of the ISCR Pilot Test Conducted at the Former CCC/USDA Grain Storage Facility in Centralia, Kansas
}

Environmental Science Division 
About Argonne National Laboratory

Argonne is a U.S. Department of Energy laboratory managed by UChicago Argonne, LLC under contract DE-AC02-06CH11357. The Laboratory's main facility is outside Chicago, at 9700 South Cass Avenue, Argonne, Illinois 60439. For information about Argonne and its pioneering science and technology programs, see www.anl.gov.

\section{Availability of This Report}

This report is available, at no cost, at http://www.osti.gov/bridge. It is also available on paper to the U.S. Department of Energy and its contractors, for a processing fee, from:

U.S. Department of Energy

Office of Scientific and Technical Information

P.O. Box 62

Oak Ridge, TN 37831-0062

phone (865) 576-8401

fax (865) 576-5728

reports@adonis.osti.gov

\section{Disclaimer}

This report was prepared as an account of work sponsored by an agency of the United States Government. Reference herein to any specific commercial product, process, or service by trade name, trademark, manufacturer, or otherwise, does not necessarily constitute or imply its endorsement, recommendation, or favoring by the United States Government or any agency thereof. The views and opinions of document authors expressed herein do not necessarily state or reflect those of the United States Government or any agency thereof, Argonne National Laboratory, or UChicago Argonne, LLC. 


\section{Progress Report and Technical Evaluation of the ISCR Pilot Test Conducted at the Former CCC/USDA Grain Storage Facility in Centralia, Kansas}

by

Applied Geosciences and Environmental Management Section

Environmental Science Division, Argonne National Laboratory

January 2009 


\section{Contents}

Notation.

1 Introduction and Background ................................................................................. 1-1

2 Pre-Treatment Baseline Characterization Studies ………………………………............... 2-1

2.1 Pre-Injection Baseline Characterization of Groundwater in the Pilot Test Area....... 2-1

2.1.1 Pre-Injection Groundwater Sampling and Analysis Methods ...................... 2-1

2.1.2 Results of the Pre-Injection Baseline Groundwater Analyses ..................... 2-3

2.2 Pre-Injection Baseline Characterization of Vadose Zone Soils in the Pilot Test Area

2.2.1 Soil Sampling and Analysis Methods ......................................................... 2-5

2.2.2 Pre-Injection Baseline Results for VOCs in Soil ....................................... 2-6

2.3 Summary of Pre-Injection Baseline Studies .......................................................... 2-6

3 Review of the ISCR Injection Process................................................................................. 3-1

3.1 Overview of the Field Injection Program ………................................................... 3-1

3.2 Evaluation of the Injection Process and Subsurface Fluid Distribution .................... 3-3

3.2.1 Leakage of the ISCR Injection Fluids to the Surface ................................ 3-3

3.2.2 Post-Injection Coring Studies ................................................................. $3-5$

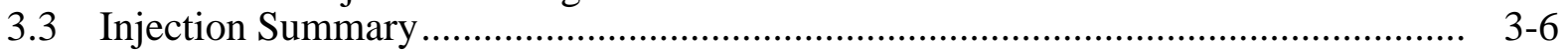

4 Preliminary Evaluation of the ISCR Pilot Test Results ........................................................ 4-1

4.1 Establishment of the Pilot Test Monitoring Well Network .................................... 4-1

4.2 Post-Injection Groundwater Data ..................................................................... 4-2

4.2.1 Post-Injection Groundwater Sampling and Analysis Methods....................... 4-2

4.2.2 Post-Injection Data for Bromide Tracer in Groundwater ............................ 4. 4-3

4.2.3 Post-Injection Data for Dissolved Oxygen and Oxidation-Reduction Potential in Groundwater Samples .............................................................. 4-5

4.2.4 Post-Injection Supplemental Geochemical Data for Groundwater............... 4 4-6

4.2.5 Post-Injection Data for VOCs in Groundwater........................................ 4-7

4.3 Post-Injection Data for VOCs in Vadose Zone Soil ............................................. 4-11

4.4 Estimated Costs of the Pilot ISCR Investigation .................................................. 4-12

5 Conclusions and Recommendations ………………................................................. $5-1$

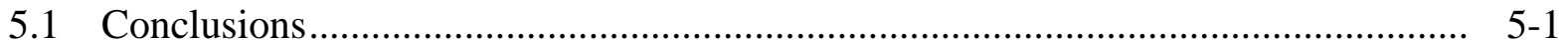

5.2 Recommendations............................................................................................... $5-3$

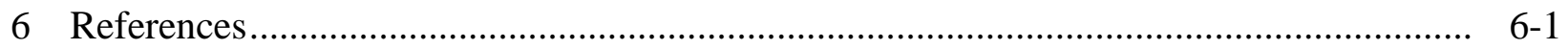

Appendix A: Sequence of Pre-Injection Baseline Sampling Activities and Post-Injection Monitoring Activities 
Appendix B: Photographs of Sediment Cores Collected in December 2007 at Post-Injection Investigation Points PSB10, PSB11, and PSB12

Appendix C: KDHE Completion Variance and Well Registration Forms.............................. C-1

Appendix D: Time Series Diagrams Presenting the Results of VOCs, DO, and ORP Analyses for Groundwater Monitoring Samples Collected during the ISCR Pilot Test

Supplement 1: Waste Handling, Characterization, and Disposal and Quality Control for Sample Collection, Handling, and Analysis

Supplement 2: Analytical Results for Groundwater and Soil Samples Collected during the Pre-Injection Baseline Sampling Activities

Supplement 3: Injection Program Summary Report for the Centralia ISCR Pilot Test, Prepared by Adventus Americas, Inc., March 4, 2008 on $\mathrm{CD}$

Supplement 4: Analytical Results for Groundwater and Soil Samples Collected during the Extended Post-Injection Monitoring Program on CD

Supplement 5: Outside Laboratory Data for Verification Organic Analyses. on $\mathrm{CD}$

\section{Figures}

1.1 Locations of the former CCC/USDA facility and the contaminated private well ........ 1-5

1.2 Analytical results for carbon tetrachloride in groundwater samples collected in September 2007, with interpreted plume boundaries in August 2004, September 2005, March and September 2006, and March and September 2007, and persistent contaminant hot spots identified at SB01, SB05, and MW02

2.1 Proposed pilot test area, with locations of soil boring SB12 and MW02 and nearby existing monitoring points

2.2 Proposed pilot test area, with locations of injection points and existing and new monitoring points

2.3 Carbon tetrachloride concentrations in groundwater samples collected at 50-55 ft BGL and at 55-60 ft BGL during the pre-injection baseline sampling event. 


\section{Figures (Cont.)}

2.4 Chloroform concentrations in groundwater samples collected at 50-55 ft BGL and at 55-60 ft BGL during the pre-injection baseline sampling event

2.5 Dissolved oxygen concentrations and oxidation-reduction potential levels in groundwater samples collected at 50-55 ft and 55-60 ft BGL during the pre-injection baseline sampling event.

2.6 Dissolved oxygen concentrations in groundwater samples collected at 50-55 ft and 55-60 ft BGL during the pre-injection baseline sampling event.

2.7 Oxidation-reduction potential levels in groundwater samples collected at $50-55 \mathrm{ft}$ and 55-60 ft BGL during the pre-injection baseline sampling event.

2.8 Vertical distribution of carbon tetrachloride identified in the vadose zone soils at pre-injection baseline sampling locations PSB1 and PSB2.

2.9 Vertical distribution of carbon tetrachloride identified in the vadose zone soils at pre-injection baseline sampling locations PSB3 and PSB4.

2.10 Vertical distribution of carbon tetrachloride identified in the vadose zone soils at 2002-2004 sampling location SB12.

3.1 Pilot test area, with the revised locations of injection points as implemented

3.2 Schematic representation of the locations where daylighting of EHC-A and EHC was observed in response to the injection of these amendments, with apparent migration directions

3.3 Daylighting of of EHC-A material outside the well casing at monitoring well MW02 and EHC slurry in open ground near injection point PT2

3.4 Locations of the pre-injection groundwater sampling points PSB1-PSB9, monitoring wells SB07R and MW02, injection points, and post-injection soil coring locations PSB10-PSB13

3.5 Appearance of EHC material along small, isolated fractures in the soil core recovered at $21 \mathrm{ft}$ BGL at post-injection soil coring and sampling location PSB13.

4.1 Locations of injection points and post-injection groundwater monitoring points 


\section{Figures (Cont.)}

4.2 Dissolved oxygen concentrations and oxidation-reduction potential levels in groundwater samples collected at permanent monitoring points during the initial post-injection sampling event

4.3 Dissolved oxygen concentrations in groundwater samples collected at permanent monitoring points during the initial post-injection sampling event....

4.4 Oxidation-reduction potential levels in groundwater samples collected at permanent monitoring points during the initial post-injection sampling event.

4.5 Dissolved oxygen concentrations and oxidation-reduction potential levels in groundwater samples collected at permanent monitoring points during post-injection sampling in February-September 2008

4.6 Dissolved oxygen concentrations in groundwater samples collected at permanent monitoring points during the September 2008 post-injection sampling event

4.7 Oxidation-reduction potential levels in groundwater samples collected at permanent monitoring points during the September 2008 post-injection sampling event

4.8 Appearance of groundwater collected from monitoring well MW02 during the September 2008 post-injection sampling event

4.9 Carbon tetrachloride concentrations in groundwater samples collected at permanent monitoring points during the initial post-injection sampling event, with pre-injection values for comparison.

4.10 Calculated fraction of carbon tetrachloride remaining, in comparison to pre-injection concentrations, in groundwater samples collected at permanent monitoring points during the initial post-injection sampling event

4.11 Carbon tetrachloride concentrations in groundwater samples collected at permanent monitoring points during the September 2008 post-injection sampling event, with calculated fraction of carbon tetrachloride remaining, in comparison to pre-injection concentrations

4.12 Chloroform concentrations in groundwater samples collected at permanent monitoring points during the September 2008 post-injection sampling event.....

4.13 Comparison of carbon tetrachloride concentrations in the vadose zone soils at pre-injection sampling point PSB1 and corresponding post-injection sampling point PSB13 


\section{Tables}

2.1 Results of organic analyses at the AGEM Laboratory for groundwater samples collected during the ISCR pilot study baseline sampling event

2.2 Results of bromide analyses for groundwater samples collected during the ISCR pilot study baseline sampling event

2.3 Results of purge-and-trap organic analyses at the AGEM Laboratory for soil samples collected during the ISCR pilot study baseline sampling event.

3.1 Summary of ISCR amendments injected at the Centralia pilot test site

4.1 Results of bromide analyses for groundwater samples collected during the ISCR pilot study post-injection sampling events.

4.2 Results of dissolved oxygen and oxidation-reduction potential analyses for groundwater samples collected during the ISCR pilot study post-injection sampling events

4.3 Results of organic analyses at the AGEM Laboratory for groundwater samples collected during the ISCR pilot study post-injection sampling events

4.4 Results of purge-and-trap organic analyses at the AGEM Laboratory for soil samples collected at PSB13 during the August 2008 post-injection sampling event. 


\section{Notation}

AGEM Applied Geosciences and Environmental Management

BER Bureau of Environmental Remediation (KDHE)

BGL below ground level

BOW Bureau of Water (KDHE)

$\mathrm{Br}^{-} \quad$ bromide

${ }^{\circ} \mathrm{C} \quad$ degree(s) Celsius

CCC Commodity Credit Corporation

CD compact disc

COC chain of custody

CPT cone penetrometer

DO dissolved oxygen

EDB ethylene dibromide

EPA U.S. Environmental Protection Agency

$\mathrm{ft} \quad$ foot (feet)

gal gallon(s)

IM Interim Measure

in. inch(es)

ISCR in situ chemical reduction

$\mathrm{KBr} \quad$ potassium bromide

KDHE Kansas Department of Health and Environment

$\mathrm{lb} \quad$ pound(s)

$\mu \mathrm{g} / \mathrm{kg} \quad$ microgram(s) per kilogram

$\mathrm{mg} / \mathrm{kg}$ milligram(s) per kilogram

$\mu \mathrm{g} / \mathrm{L} \quad$ microgram(s) per liter

$\mathrm{mg} / \mathrm{L} \quad$ milligram(s) per liter

MNA monitored natural attenuation

$\mathrm{mV} \quad$ millivolt(s)

ND not detected

ORP oxidation-reduction potential

psi pound(s) per square inch

PVC polyvinyl chloride

QA quality assurance

QC quality control

RBSL Risk-Based Screening Level 
Centralia Pilot Study Report

Version 00, 10/30/08

$\begin{array}{ll}\text { RWD } & \text { Rural Water District } \\ \mu \mathrm{S} / \mathrm{cm} & \text { microsiemen(s) per centimeter } \\ \text { TOC } & \text { total organic carbon } \\ \text { UIC } & \text { Underground Injection Control } \\ \text { USDA } & \text { U.S. Department of Agriculture } \\ \text { VOC } & \text { volatile organic compound }\end{array}$




\section{Progress Report and Technical Evaluation of the ISCR Pilot Test Conducted at the Former CCC/USDA Grain Storage Facility in Centralia, Kansas}

\section{Introduction and Background}

In October, 2007, the Commodity Credit Corporation of the U.S. Department of Agriculture (CCC/USDA) presented the document Interim Measure Conceptual Design (Argonne 2007a) to the Kansas Department of Health and Environment, Bureau of Environmental Remediation (KDHE/BER), for a proposed non-emergency Interim Measure (IM) at the site of the former CCC/USDA grain storage facility in Centralia, Kansas (Figure 1.1). The IM was recommended to mitigate existing levels of carbon tetrachloride contamination identified in the vadose zone soils beneath the former facility and in the groundwater beneath and in the vicinity of the former facility, as well as to moderate or decrease the potential future concentrations of carbon tetrachloride in the groundwater. The Interim Measure Conceptual Design (Argonne 2007a) was developed in accordance with the KDHE/BER Policy \#BERRS-029, Policy and Scope of Work: Interim Measures (KDHE 1996).

The hydrogeologic, geochemical, and contaminant distribution characteristics of the Centralia site, as identified by the CCC/USDA, factored into the development of the nonemergency IM proposal. These characteristics were summarized in the Interim Measure Conceptual Design (Argonne 2007a) and were discussed in detail in previous Argonne reports (Argonne 2002a, 2003, 2004, 2005a,b,c, 2006a,b, 2007b). The identified remedial goals of the proposed IM were as follows:

- To reduce the existing concentrations of carbon tetrachloride in groundwater in three "hot spot" areas identified at the site (at SB01, SB05, and SB12MW02; Figure 1.2) to levels acceptable to the KDHE.

- To reduce carbon tetrachloride concentrations in the soils near the location of former soil boring SB12 and existing monitoring well MW02 (Figure 1.2) to levels below the KDHE Tier 2 Risk-Based Screening Level (RBSL) of $200 \mu \mathrm{g} / \mathrm{kg}$ for this contaminant.

To address these goals, the potential application of an in situ chemical reduction (ISCR) treatment technology, employing the use of the EHC ${ }^{\circledR}$ treatment materials marketed by Adventus Americas, Inc. (Freeport, Illinois), was recommended. The EHC materials are proprietary 
mixtures of food-grade organic carbon and zero-valent iron that are injected into the subsurface as a slurry (EHC) or in dissolved form (EHC-A) and subsequently released slowly into the formation. The materials are designed to create highly reducing geochemical conditions in the vadose and saturated zones that foster both thermodynamic and biological reductive dechlorination of carbon tetrachloride.

As a precursor to implementation of the full IM, however, the CCC/USDA recommended an initial short-term, field-scale pilot test of the Adventus materials in one of the hot-spot areas, near monitoring well MW02 and former soil boring SB12 (Figure 1.2). The SB12-MW02 hot spot was chosen for testing because of the presence of elevated carbon tetrachloride concentrations in both the vadose and saturated zones at this location, coupled with subsurface lithologic conditions that appeared more favorable for the injection process than those underlying the remaining two groundwater-only hot-spot areas (at SB01 and SB05; Figure 1.2).

The technical and logistic objectives of the pilot test were as follows:

- To operationally test and critically evaluate the viability of ISCR, and specifically the Adventus materials, as a remedial approach for restoration of the subsurface soils and groundwater at Centralia, as well as potentially at other former CCC/USDA investigation sites in Kansas that might have similar hydrogeologic characteristics, geochemical features, and remedial requirements.

- To gain practical technical and logistic experience in implementation of the ISCR approach and thus facilitate the development of optimal methods and techniques for the potential application of this technology at Centralia and other CCC/USDA investigation sites where the approach might be applicable.

- To critically evaluate the ability of the ISCR technology to achieve the remedial goals outlined above in a cost- and time-effective manner.

The technical specifications for the proposed Centralia ISCR pilot test were jointly developed by the CCC/USDA and Argonne National Laboratory in consultation with Adventus representatives. These specifications and are summarized in Section 4 of the Interim Measure 
Conceptual Design (Argonne 2007a). The primary elements of the pilot testing program included the following:

- Pre-treatment soil and groundwater sampling in and near the targeted hot-spot area, to provide baseline data for evaluation of the ISCR process.

- Injection of the EHC materials on a grid pattern within the targeted treatment area.

- Initial post-injection soil coring to investigate the subsurface distribution of the materials resulting from the injection process, along with groundwater sampling and analyses to identify possible short-term ISCR effects on the groundwater geochemistry and contaminant levels.

- Extended groundwater sampling and analyses, with follow-up vadose zone soil sampling and analyses, to evaluate the longer-term effectiveness of the ISCR treatment.

The recommended testing activities were discussed by representatives of the KDHE/BER, CCC/USDA, and Argonne in a teleconference on November 8, 2007. On November 9, 2007, the KDHE/BER (KDHE 2007a) approved the pilot ISCR testing program as outlined in the Interim Measure Conceptual Design (Argonne 2007a), with minor revisions.

With the approval of the CCC/USDA and KDHE/BER project managers, the pilot testing program was implemented at Centralia as follows:

- Pre-treatment baseline sampling associated with the ISCR pilot test was conducted on November 12-16, 2007.

- Injection of the EHC materials was performed on November 26-December 5, 2007.

- Post-injection soil coring was initiated on December 5, 2007, and completed on December 17, 2007. 
- The installation of permanent groundwater monitoring points at locations approved by the KDHE and initial post-injection groundwater sampling were conducted on January 8-10, 2008.

- Extended monitoring of the groundwater in the pilot test area was initiated on January 24, 2008, and continued approximately monthly through September 2008.

- Vadose zone soil sampling for analyses of volatile organic compounds (VOCs) was conducted as part of the extended monitoring effort on August 21, 2008.

This report documents and provides a technical evaluation of the results of the Centralia ISCR pilot test that had been obtained as of September 2008. These results were discussed with representatives of the CCC/USDA and the KDHE/BER in a web-based conference conducted on September 25, 2008. This section provides a brief background and chronological history of the pilot testing program. Section 2 presents an overview of the planned injection program as originally approved by the CCC/USDA and KDHE/BER project managers (on November 9, 2007), along with the related pre-treatment baseline sampling studies. Section 3 summarizes modifications to the planned injection program that were made with the approval of the CCC/USDA and KDHE/BER project managers as a result of the pre-treatment characterization studies, and it also presents an overview of the injection field activities. In Section 4, the results of the initial post-injection characterization studies and the extended groundwater and vadose zone monitoring program are summarized. The conclusions drawn from the pilot testing studies to date and preliminary recommendations for further action at the Centralia site based on these results are presented in Section 5.

All of the soil and groundwater collection, sample analysis, and monitoring point/piezometer installation activities conducted in conjunction with the Centralia ISCR pilot test and described in this report were performed in accord with the detailed methodologies and procedures in the KDHE-approved Master Work Plan for environmental investigations in Kansas (Argonne 2002b). The site-specific quality control measures employed during sample collection, handling, and analysis and for the handling and disposal of investigation-derived wastes generated throughout the pilot testing program are summarized in Supplement 1, which is on the compact disc (CD) inside the back cover of this report. 


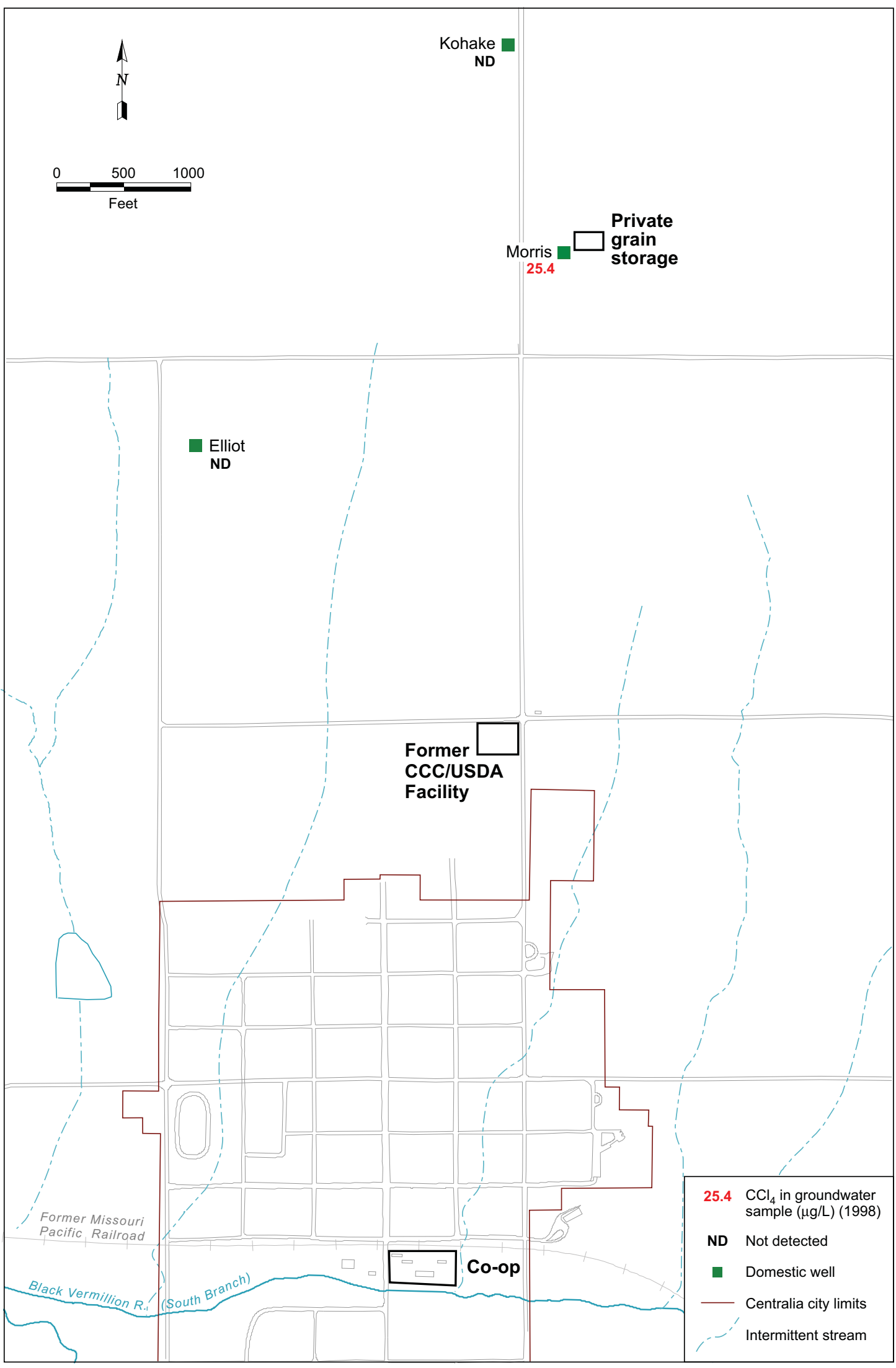

FIGURE 1.1 Locations of the former CCC/USDA facility and the contaminated private well. 


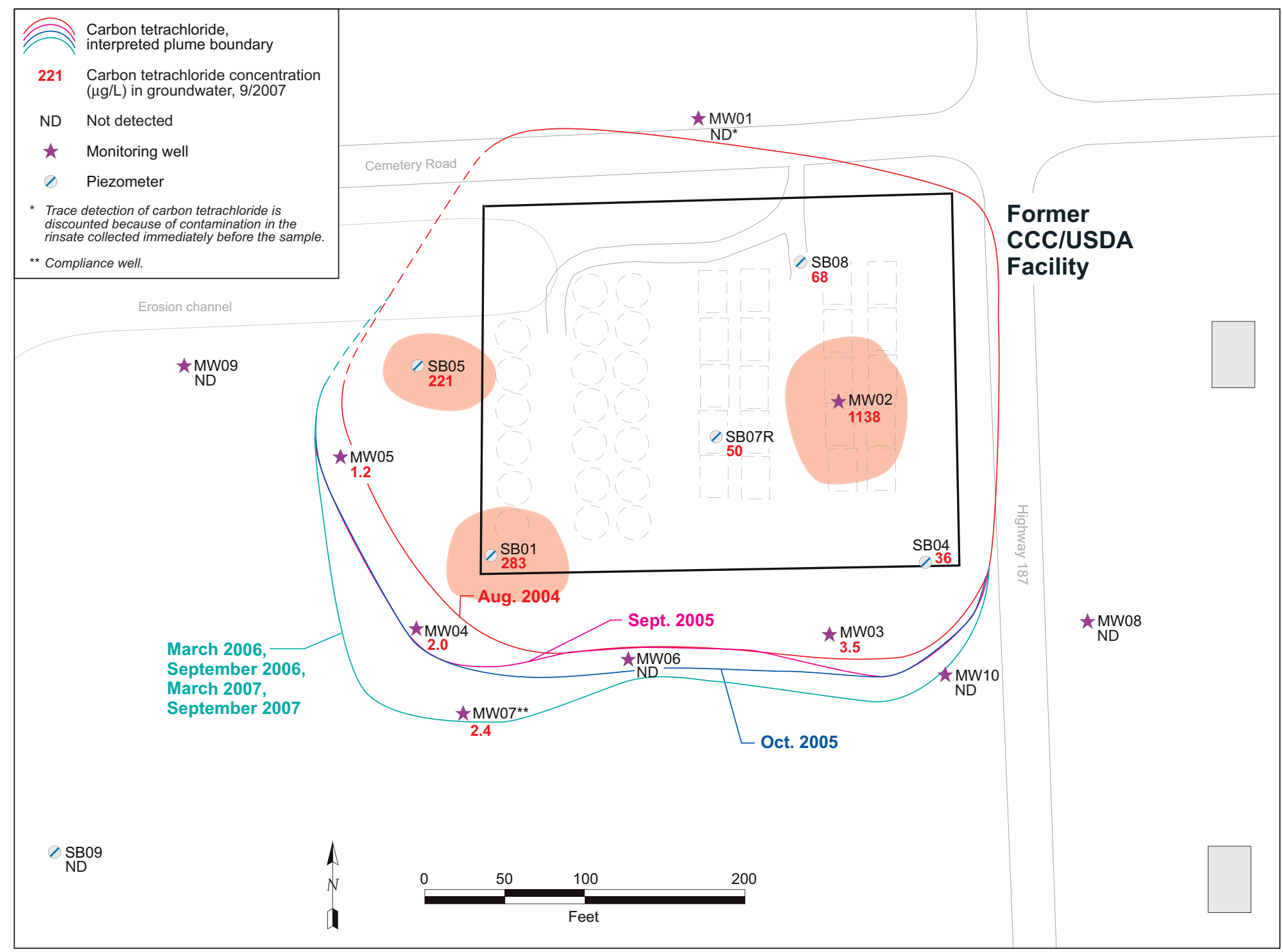

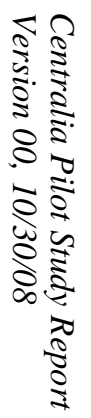

FIGURE 1.2 Analytical results for carbon tetrachloride in groundwater samples collected in September 2007, with interpreted plume boundaries in August 2004, September 2005, March and September 2006, and March and September 2007, and persistent contaminant hot spots identified at SB01, SB05, and MW02. 


\section{Pre-Treatment Baseline Characterization Studies}

Figure 2.1 illustrates the area targeted for ISCR treatment (using the Adventus EHC materials) in the Centralia pilot test, as approved by the CCC/USDA and KDHE/BER project managers in November 2007. The area, approximately $45 \mathrm{ft}$ wide by $75 \mathrm{ft}$ long, was selected to encompass the locations of existing monitoring well MW02 and former investigative boring SB12; these two borings have consistently exhibited the highest concentrations of carbon tetrachloride identified in both the vadose zone soils and groundwater at the Centralia investigation site (Argonne 2007a). In Figure 2.2, the proposed distribution of EHC injection points (PT1-PT15) is shown. As outlined in the approved Interim Measure Conceptual Design (Argonne 2007a), the ten injection points nearest former boring location SB12 were targeted for injection of the soluble EHC-A material in the vadose zone soils from $20 \mathrm{ft}$ to $40 \mathrm{ft}$ below ground level (BGL) and EHC slurry in the deeper soils and aquifer materials from $40 \mathrm{ft}$ to $60 \mathrm{ft}$ BGL. At the remaining five injection points, EHC slurry was to be used only over the depth interval at 40-60 ft BGL.

With the approval of the CCC/USDA and KDHE/BER project managers, 15 locations (Figure 2.2) were selected for pre-treatment sampling to provide baseline data on the detailed distribution of carbon tetrachloride in the vadose zone soils in the injection area (PSB1-PSB4) and on the carbon tetrachloride levels and inorganic geochemical characteristics of the groundwater in (PSB1-PSB4 and MW02) and near (PSB5-PSB9, SB04, SB07R, SB08, and MW03) the proposed injection field. The results of the baseline sampling studies are discussed below.

\subsection{Pre-Injection Baseline Characterization of Groundwater in the Pilot Test Area}

\subsubsection{Pre-Injection Groundwater Sampling and Analysis Methods}

Groundwater sampling for baseline analyses of VOCs and selected geochemical parameters was performed at 9 locations (PSB1-PSB9; Figure 2.2) by using the Argonne 20-ton track-mounted cone penetrometer (CPT) vehicle. At each location, groundwater samples were obtained at depths of approximately 50-55 ft BGL and 55-60 ft BGL within the previously identified aquifer interval (Argonne 2007a) by advancing a temporary, 0.5-in.-diameter polyvinyl chloride (PVC) screen and riser to the desired sampling depth through the CPT rods. 
Attempts to recover groundwater from shallower depths in the sediment profile at several locations (40-45 ft BGL and 45-50 ft BGL at PSB1; 45-50 ft BGL at PSB3; Figure 2.2) were unsuccessful.

Sample aliquots for laboratory analyses of VOCs, total organic carbon, and methane were collected by using a stainless steel bailer. Sample aliquots for laboratory analyses of inorganic geochemical parameters (described below) were recovered by using an inertial pumping assembly (disposable, small-diameter tubing and foot valve). The sequence of activities during the baseline sampling event is in Appendix A.

At the request of the KDHE/BER, a sample of the water to be used for mixing of the pilot test injection fluids (obtained from the Centralia municipal water system, which is supplied by Nemaha County Rural Water District 3 [RWD 3]) was also collected for geochemical analysis as part of the pre-injection sampling event.

With the approval of the CCC/USDA and KDHE/BER project managers, analytical parameter data for groundwater samples collected by Argonne in September 2007 at permanent monitoring points SB04, SB07R, SB08, MW02, and MW03 (Figure 2.2) are included in the baseline data set. The September 2007 analyses (Argonne 2008a) were obtained as part of ongoing sampling of the complete Centralia monitoring well network that is performed twice yearly at the request of the KDHE/BER. These monitoring points are screened over the full aquifer thickness (at approximately 50-60 ft BGL) at each location.

Measurements of temperature, $\mathrm{pH}$, conductivity, carbon dioxide, iron(II), dissolved oxygen (DO) and oxidation-reduction potential (ORP) were made for all samples by using calibrated field instruments and appropriate field test kits in accord with the manufacturers' instructions and the Master Work Plan (Argonne 2002b). Groundwater samples designated for VOCs analyses were collected in appropriate laboratory containers, labeled, packaged, and chilled to $4^{\circ} \mathrm{C}$ by placement in ice-filled coolers. The samples were shipped by overnight delivery service to the Applied Geosciences and Environmental Management (AGEM) Laboratory at Argonne for VOCs analyses with U.S. Environmental Protection Agency (EPA) Method 524.2 (EPA 1995). Aliquots of selected samples (chosen in the field) were also shipped to Envirosystems, Inc., Columbia, Maryland, for verification analyses. 
Samples designated for geochemical analyses were collected in appropriate laboratory containers, labeled, packaged, preserved (as required for the parameter of interest, in accord with the Master Work Plan [Argonne 2002b]), and shipped to TestAmerica Laboratories, Inc., in Burlington, Vermont, and Chicago, Illinois. The analyses included dissolved chloride, bromide, sulfate, nitrate, and phosphate concentrations by EPA Method 300; total alkalinity by EPA Method 310.1; nitrate-nitrite nitrogen by EPA Method 353.2; nitrite nitrogen by EPA Method 354.1; sulfide by EPA Method 376.2; total organic carbon by EPA Method 415.1; and dissolved metals (calcium, magnesium, manganese, potassium, silicon, and sodium) by EPA Method 6010 (EPA 1998). Analyses for methane were conducted with Method RSK-175 (Kampbell and Vandegrift 1998).

\subsubsection{Results of the Pre-Injection Baseline Groundwater Analyses}

The complete results of the baseline groundwater analyses are on CD, in Supplement 2.

\subsubsection{Baseline VOCs Analysis Results for Groundwater}

The results of the VOCs analyses of baseline groundwater samples are summarized in Table 2.1 and are illustrated in Figures 2.3-2.4.

Figure 2.3 demonstrates that the carbon tetrachloride distribution in groundwater in the pilot test investigation area was highly heterogeneous, with concentrations varying by as much as two orders of magnitude and more over relatively short vertical (approximately $5 \mathrm{ft}$ ) and lateral ( $\leq 50 \mathrm{ft}$ ) distances. Carbon tetrachloride levels in the upper portion of the aquifer interval (50-55 ft BGL) ranged from non-detectable (at an analytical method detection limit of $0.1 \mu \mathrm{g} / \mathrm{L}$ ) to $2,646 \mu \mathrm{g} / \mathrm{L}$. Carbon tetrachloride concentrations in the lower aquifer interval (55-60 ft BGL) ranged from $1.4 \mu \mathrm{g} / \mathrm{L}$ to $2,029 \mu \mathrm{g} / \mathrm{L}$. Concentrations in the lower aquifer interval were generally slightly lower than the corresponding values in the shallower aquifer interval.

The results of the baseline analyses confirmed the continuing presence of a hot spot in the groundwater carbon tetrachloride concentrations in the vicinity of monitoring well MW02, as previously identified (Argonne 2007a). The results indicated, however, that elevated contaminant levels (> $100 \mu \mathrm{g} / \mathrm{L}$ ) associated with the hot spot extended laterally to the northeast, south, and southwest, beyond the limits of the proposed ISCR injection field. The highest carbon 
tetrachloride concentrations in groundwater detected during the baseline characterization (2,029 $\mu \mathrm{g} / \mathrm{L}$ and 2,646 $\mu \mathrm{g} / \mathrm{L}$; Figure 2.3) were identified at sampling location PSB8, outside the southwestern margin of the proposed injection area.

Figure 2.4 shows the baseline distributions of chloroform in the aquifer intervals at 50-55 ft BGL and 55-60 ft BGL, respectively. Chloroform concentrations varied from $<1 \mu \mathrm{g} / \mathrm{L}$ to $103 \mu \mathrm{g} / \mathrm{L}$, with relatively greater values (> $10 \mu \mathrm{g} / \mathrm{L}$ ) identified in association with high levels of carbon tetrachloride (Figure 2.3). Trace levels of methylene chloride were also detected at several locations (PSB5, PSB7, and PSB8; Table 2.1) in conjunction with the higher carbon tetrachloride values. The observed detections of chloroform and methylene chloride in association with carbon tetrachloride are consistent with previous monitoring results (Argonne 2005, 2006a, 2006b, 2007b), which suggested that natural biodegradation of carbon tetrachloride might locally occur, to a limited degree, within the Centralia aquifer.

\subsubsection{Baseline Geochemical Analysis Results for Groundwater}

A compilation of the complete inorganic geochemical and field parameter data set obtained during the pre-injection groundwater sampling event is on CD, in Supplement 2. The results of selected geochemical parameter analyses of particular concern to the implementation and evaluation of the ISCR pilot testing program are discussed below.

As noted in Section 1, the Adventus EHC materials recommended for the pilot injection program at Centralia are formulated to generate a highly reducing (low ORP), oxygen-depleted environment within the subsurface that is amenable to both thermodynamic and anaerobic biological reductive dechlorination of carbon tetrachloride. Figures 2.5-2.7 summarize the preinjection DO and ORP values in groundwater that were determined as baseline data for the pilot test area. The DO levels ranged from approximately $2.9 \mathrm{mg} / \mathrm{L}$ to $8.3 \mathrm{mg} / \mathrm{L}$ (Figures 2.5 and 2.6), and ORP values generally (with only two exceptions) were more positive than $-70 \mathrm{mV}$ (Figures 2.5 and 2.7). The observed results indicate that the ambient groundwater conditions in the testing area are generally aerobic and therefore are not conducive to the widespread natural occurrence of reductive dechlorination. Values for ORP more negative than $-300 \mathrm{mV}$ were detected at two locations (Figure 2.7): PSB5 (-311 mV, at 55-60 ft BGL) and PSB7 (-343 mV, at 50-55 ft BGL); however, these results appear inconsistent with the DO levels observed at these points (3.9 mg/L and $3.7 \mathrm{mg} / \mathrm{L}$, respectively; Figure 2.5 ). 
At the request of the KDHE/BER (KDHE 2007a), bromide $\left(\mathrm{Br}^{-}\right)$was identified as a conservative species (under the anticipated conditions of the ISCR pilot test) for possible use as a geochemical tracer, to assist in tracking the subsurface distribution and movement of the injected fluids during the Centralia pilot test. To determine suitable $\mathrm{Br}^{-}$concentrations for potential use in the recommended tracer studies (see Section 3.1), ambient levels of $\mathrm{Br}^{-}$were determined for the baseline groundwater samples collected at points PSB1-PSB9 (Figure 2.2) and for a sample of the water (obtained from the Centralia municipal system and supplied by RWD 3) to be used in mixing of the EHC injection fluids. The results of the analyses are in Table 2.2. Bromide levels in the Centralia formation waters varied by approximately one order of magnitude, from $0.14 \mathrm{mg} / \mathrm{L}$ to $2.0 \mathrm{mg} / \mathrm{L}$, with the highest concentrations occurring at PSB5 (0.95-2.0 mg/L) and PSB8 (0.78-1.0 mg/L), to the northeast and southwest, respectively, of the proposed injection field (Figure 2.2). An average $\mathrm{Br}^{-}$concentration of $0.52 \mathrm{mg} / \mathrm{L}$ was calculated for all groundwater samples; an average of $0.30 \mathrm{mg} / \mathrm{L}$ was calculated for the sampling points (PSB1-PSB4) in the proposed injection field. The $\mathrm{Br}^{-}$concentration $(0.29 \mathrm{mg} / \mathrm{L})$ in the sample of water from the Centralia municipal system was comparable to the levels identified in groundwater in the pilot test area.

\subsection{Pre-Injection Baseline Characterization of Vadose Zone Soils in the Pilot Test Area}

\subsubsection{Soil Sampling and Analysis Methods}

At the request of the KDHE/BER, continuous soil cores were collected from ground surface to a depth of $40 \mathrm{ft}$ BGL at locations PSB1-PSB4 (Figure 2.2) by using the Argonne 20-ton track-mounted CPT vehicle, to facilitate vertical-profile soil sampling for VOCs analyses. Soil samples for VOCs analyses were recovered from the cores at 4-ft intervals, from $4 \mathrm{ft}$ to $40 \mathrm{ft}$ BGL. The samples were immediately placed in appropriate laboratory containers, labeled, preserved on dry ice, and shipped by an overnight delivery service to the AGEM Laboratory for sample preparation and VOCs analyses with modified EPA Methods 5030B and 8260B. The sequence of activities during the baseline soil sampling is in Appendix A. The complete baseline analytical results are on CD, in Supplement 2. 


\subsubsection{Pre-Injection Baseline Results for VOCs in Soil}

The results of the VOCs analyses on baseline soil samples are summarized in Table 2.3 and are illustrated in Figures 2.8 and 2.9. Carbon tetrachloride was detected (at an analytical method detection limit of $1.0 \mu \mathrm{g} / \mathrm{kg}$ ) in the vadose zone soils at all of the locations sampled, with quantifiable levels of the contaminant (at a method quantitation limit of $10 \mu \mathrm{g} / \mathrm{kg}$ ) most commonly occurring at depths of approximately $24 \mathrm{ft}$ BGL or greater. Carbon tetrachloride levels greater than $100 \mu \mathrm{g} / \mathrm{kg}$ were identified at borings PSB1 and PSB2 only (at depths ranging from $24 \mathrm{ft}$ to $36 \mathrm{ft}$ BGL; Figure 2.8), in the northern portion of the targeted injection area. The lowest carbon tetrachloride concentrations in soils (ranging from non-detectable to $20 \mu \mathrm{g} / \mathrm{kg}$ ) were identified at PSB3, in the southwestern portion of the test area (Figure 2.9).

Sampling point PSB1 was located to coincide with earlier Argonne investigative boring SB12 (Argonne 2004, 2007a). Soil samples collected at this location had previously exhibited carbon tetrachloride levels exceeding the KDHE Tier 2 RBSL (200 $\mu \mathrm{g} / \mathrm{kg}$ ) for this contaminant in soils (Figures 2.2 and 2.10). Maximum carbon tetrachloride concentrations identified were $219 \mu \mathrm{g} / \mathrm{kg}$ in boring PSB1 and $173 \mu \mathrm{g} / \mathrm{kg}$ at PSB2; these levels are comparable to the maximum carbon tetrachloride concentrations identified at SB12 (202-219 $\mathrm{g} / \mathrm{kg})$, confirming the continued presence of a localized contamination hot spot in the vadose zone soils near the SB12PSB1 location. The net extent and levels of carbon tetrachloride contamination in the vadose zone soils at PSB1 and PSB2 were somewhat less, however, than those previously detected at SB12 (Figures 2.8 and 2.10). The observed variations in contaminant levels might reflect natural mobilization and limited degradation of the carbon tetrachloride in the vadose zone soils over time (April 2003 to November 2007), as well as inherent localized heterogeneity in the contaminant distribution in soils.

\subsection{Summary of Pre-Injection Baseline Studies}

The key findings of the pre-injection baseline sampling studies are as follows:

- The distribution of carbon tetrachloride contamination in groundwater in the area targeted for the ISCR pilot test is heterogeneous, with identified levels varying by more than two orders of magnitude over relatively short vertical and lateral distances (see Figure 2.3). 
- The previously identified contaminant hot spot in the groundwater in the vicinity of monitoring well MW02 was confirmed; carbon tetrachloride concentrations up to $1,285 \mu \mathrm{g} / \mathrm{L}$ were detected at sampling points PSB1-PSB4 and MW02, within the proposed injection test area.

- Carbon tetrachloride concentrations in groundwater ranging from $114 \mu \mathrm{g} / \mathrm{L}$ to 2,646 $\mu \mathrm{g} / \mathrm{L}$ were also identified at sampling points (PSB5-PSB8) adjacent to the proposed injection area to the northeast, south, and southwest of MW02. The maximum pre-injection values $(2,029-2,646 \mu \mathrm{g} / \mathrm{L})$ were identified at PSB8, to the southwest of MW02. The concentrations detected at PSB8 represent the highest levels of carbon tetrachloride identified in groundwater at Centralia to date.

- $\quad$ Observed DO levels of approximately 2.9-8.3 mg/L and ORP values generally more positive than $-70 \mathrm{mV}$ indicated that the ambient groundwater in the proposed testing area is aerobic and that the aquifer conditions in the proposed test area are not conducive to the widespread natural occurrence of reductive dechlorination.

- The compound potassium bromide $(\mathrm{KBr})$ was identified for possible use as a tracer to monitor the movement of injected fluids during the ISCR pilot test. Relatively low ambient $\mathrm{Br}^{-}$levels $(0.14-2.0 \mathrm{mg} / \mathrm{L})$ were detected in the Centralia formation waters. Average $\mathrm{Br}^{-}$concentrations calculated were $0.52 \mathrm{mg} / \mathrm{L}$ for all groundwater samples and $0.30 \mathrm{mg} / \mathrm{L}$ for groundwater samples collected within the proposed injection field (PSB1-PSB4). The $\mathrm{Br}^{-}$ concentration in a sample of water from the Centralia municipal system $(0.29 \mathrm{mg} / \mathrm{L})$ was comparable to the levels identified in groundwater in the pilot test area.

- Baseline carbon tetrachloride concentrations exceeding $100 \mu \mathrm{g} / \mathrm{kg}$ were identified in the vadose zone soils at locations PSB1 and PSB2 in the northern portion of the proposed injection field, confirming the hot spot previously identified at former investigative boring SB12. 
- A carbon tetrachloride concentration (219 $\mu \mathrm{g} / \mathrm{kg})$ exceeding the KDHE/BER Tier 2 RBSL $(200 \mu \mathrm{g} / \mathrm{kg})$ for this contaminant in vadose zone soils was detected in one sample only, collected at $24 \mathrm{ft}$ BGL in PSB1. This identified maximum level was comparable to the maximum concentration in soils previously identified at investigative boring SB12. 
TABLE 2.1 Results of organic analyses at the AGEM Laboratory for groundwater samples collected during the ISCR pilot study baseline sampling event.

\begin{tabular}{|c|c|c|c|c|c|c|}
\hline \multirow[b]{2}{*}{ Location } & \multirow[b]{2}{*}{ Sample } & \multirow[b]{2}{*}{$\begin{array}{l}\text { Sample } \\
\text { Date }\end{array}$} & \multirow[b]{2}{*}{$\begin{array}{l}\text { Depth } \\
\text { (ft BGL) }\end{array}$} & \multicolumn{3}{|c|}{ Concentration $(\mu \mathrm{g} / \mathrm{L})$} \\
\hline & & & & $\begin{array}{c}\text { Carbon } \\
\text { Tetrachloride }\end{array}$ & Chloroform & $\begin{array}{c}\text { Methylene } \\
\text { Chloride }\end{array}$ \\
\hline PSB1 & CNPSB1-W-16245 & $11 / 12 / 07$ & $50-55$ & 782 & 27 & $N D^{a}$ \\
\hline PSB1 & CNPSB1-W-16246 & $11 / 13 / 07$ & $55-60$ & 309 & 11 & ND \\
\hline PSB2 & CNPSB2-W-16247 & $11 / 13 / 07$ & $50-55$ & 405 & 3.4 & ND \\
\hline PSB2 & CNPSB2-W-16248 & $11 / 13 / 07$ & $55-60$ & 16 & 2.2 & ND \\
\hline PSB3 & CNPSB3-W-16249 & $11 / 13 / 07$ & $50-55$ & 987 & 50 & ND \\
\hline PSB3 & CNPSB3-W-16250 & $11 / 14 / 07$ & $55-60$ & 1285 & 30 & ND \\
\hline PSB4 & CNPSB4-W-16251 & $11 / 14 / 07$ & $50-55$ & 908 & 17 & ND \\
\hline PSB4 & CNPSB4-W-16252 & $11 / 14 / 07$ & $55-60$ & 830 & 25 & ND \\
\hline PSB5 & CNPSB5-W-16255 & $11 / 14 / 07$ & $50-55$ & 770 & 16 & ND \\
\hline PSB5 & CNPSB5-W-16256 & $11 / 14 / 07$ & $55-60$ & 460 & 80 & $0.6 \mathrm{~J}^{\mathrm{b}}$ \\
\hline PSB6 & CNPSB6-W-16253 & $11 / 14 / 07$ & $50-55$ & 114 & 2.5 & ND \\
\hline PSB6 & CNPSB6-W-16254 & $11 / 14 / 07$ & $55-60$ & 116 & 5.2 & ND \\
\hline PSB7 & CNPSB7-W-16258 & $11 / 15 / 07$ & $50-55$ & 326 & 82 & $0.7 \mathrm{~J}$ \\
\hline PSB7 & CNPSB7-W-16257 & $11 / 15 / 07$ & $55-60$ & 148 & 6.6 & ND \\
\hline PSB8 & CNPSB8-W-26060 & $11 / 15 / 07$ & $50-55$ & 2646 & 103 & 2.5 \\
\hline PSB8 & CNPSB8-W-26061 & $11 / 15 / 07$ & $55-60$ & 2029 & 18 & ND \\
\hline PSB9 & CNPSB9-W-26063 & $11 / 15 / 07$ & $50-55$ & ND & $0.2 \mathrm{~J}$ & ND \\
\hline PSB9 & CNPSB9-W-26064 & $11 / 15 / 07$ & $55-60$ & 1.4 & $0.2 \mathrm{~J}$ & ND \\
\hline $\mathrm{MWO} 2^{\mathrm{C}}$ & CNMW02-W-16227 & $9 / 26 / 07$ & $49.5-59.5$ & 1138 & 18 & ND \\
\hline $\mathrm{MWO}^{\mathrm{C}}$ & CNMW03-W-16223 & $9 / 25 / 07$ & $50.5-60.5$ & 3.5 & ND & ND \\
\hline $\mathrm{SBO} 4^{\mathrm{C}}$ & CNSB04-W-16230 & $9 / 26 / 07$ & $51-61$ & 36 & $0.4 \mathrm{~J}$ & ND \\
\hline $\mathrm{SB} 07 \mathrm{R}^{\mathrm{C}}$ & CNSB07R-W-16225 & $9 / 25 / 07$ & $45-60$ & 50 & 2.4 & ND \\
\hline $\mathrm{SB08C}$ & CNSB08-W-16229 & 9/26/07 & $52-62$ & 68 & 1.8 & ND \\
\hline
\end{tabular}

a ND, not detected at an instrument detection limit of $0.1 \mu \mathrm{g} / \mathrm{L}$.

b Qualifier J indicates as estimated concentration below the purge-and-trap method quantitation limit of $1.0 \mu \mathrm{g} / \mathrm{L}$.

c Results from the September 2007 well sampling were accepted as baseline values. 
TABLE 2.2 Results of bromide analyses for groundwater samples collected during the ISCR pilot study baseline sampling event.

\begin{tabular}{lcccc}
\hline Location & Sample & $\begin{array}{c}\text { Sample } \\
\text { Date }\end{array}$ & $\begin{array}{c}\text { Depth } \\
\text { (ft BGL) }\end{array}$ & $\begin{array}{c}\text { Bromide } \\
(\mathrm{mg} / \mathrm{L})\end{array}$ \\
\hline PSB1 & CNPSB1-W-16245 & $11 / 12 / 07$ & $50-55$ & 0.24 \\
PSB1 & CNPSB1-W-16246 & $11 / 13 / 07$ & $55-60$ & 0.14 \\
& & & & \\
PSB2 & CNPSB2-W-16247 & $11 / 13 / 07$ & $50-55$ & 0.19 \\
PSB2 & CNPSB2-W-16248 & $11 / 13 / 07$ & $55-60$ & 0.40 \\
& & & & \\
PSB3 & CNPSB3-W-16249 & $11 / 13 / 07$ & $50-55$ & 0.49 \\
PSB3 & CNPSB3-W-16250 & $11 / 14 / 07$ & $55-60$ & 0.49 \\
& & & & \\
PSB4 & CNPSB4-W-16251 & $11 / 14 / 07$ & $50-55$ & 0.16 \\
PSB4 & CNPSB4-W-16252 & $11 / 14 / 07$ & $55-60$ & 0.31 \\
& & & & \\
PSB5 & CNPSB5-W-16255 & $11 / 14 / 07$ & $50-55$ & 0.95 \\
PSB5 & CNPSB5-W-16256 & $11 / 14 / 07$ & $55-60$ & 2.0 \\
& & & & \\
PSB6 & CNPSB6-W-16253 & $11 / 14 / 07$ & $50-55$ & 0.72 \\
PSB6 & CNPSB6-W-16254 & $11 / 14 / 07$ & $55-60$ & 0.26 \\
& & & & \\
PSB7 & CNPSB7-W-16258 & $11 / 15 / 07$ & $50-55$ & 0.49 \\
PSB7 & CNPSB7-W-16257 & $11 / 15 / 07$ & $55-60$ & 0.43 \\
PSB8 & CNPSB8-W-26060 & $11 / 15 / 07$ & $50-55$ & 1.0 \\
PSB8 & CNPSB8-W-26061 & $11 / 15 / 07$ & $55-60$ & 0.78 \\
PSB9 & CNPSB9-W-26063 & $11 / 15 / 07$ & $50-55$ & 0.15 \\
PSB9 & CNPSB9-W-26064 & $11 / 15 / 07$ & $55-60$ & 0.15 \\
& & & & \\
\hline
\end{tabular}


TABLE 2.3 Results of purge-and-trap organic analyses at the AGEM Laboratory for soil samples collected during the ISCR pilot study baseline sampling event.

\begin{tabular}{|c|c|c|c|c|c|c|}
\hline \multirow[b]{2}{*}{ Location } & \multirow[b]{2}{*}{ Sample } & \multirow[b]{2}{*}{$\begin{array}{l}\text { Sample } \\
\text { Date }\end{array}$} & \multirow[b]{2}{*}{$\begin{array}{l}\text { Depth } \\
\text { (ft BGL) }\end{array}$} & \multicolumn{3}{|c|}{ Concentration $(\mu \mathrm{g} / \mathrm{kg})$} \\
\hline & & & & $\begin{array}{c}\text { Carbon } \\
\text { Tetrachloride }\end{array}$ & Chloroform & $\begin{array}{l}\text { Methylene } \\
\text { Chloride }\end{array}$ \\
\hline \multirow[t]{10}{*}{ PSB1 } & CNPSB1-S-16338 & $11 / 12 / 07$ & 4 & $N D^{a}$ & ND & ND \\
\hline & CNPSB1-S-16339 & $11 / 12 / 07$ & 8 & $1.7 \mathrm{Jb}^{\mathrm{b}}$ & $1.2 \mathrm{~J}$ & ND \\
\hline & CNPSB1-S-16340 & $11 / 12 / 07$ & 12 & $4.4 \mathrm{~J}$ & $3 \mathrm{~J}$ & ND \\
\hline & CNPSB1-S-16341 & $11 / 12 / 07$ & 16 & 42 & $6.4 \mathrm{~J}$ & ND \\
\hline & CNPSB1-S-16342 & $11 / 12 / 07$ & 20 & 84 & 14 & ND \\
\hline & CNPSB1-S-16343 & $11 / 12 / 07$ & 24 & 219 & $3.7 \mathrm{~J}$ & ND \\
\hline & CNPSB1-S-16344 & $11 / 12 / 07$ & 28 & 112 & 11 & ND \\
\hline & CNPSB1-S-16345 & $11 / 12 / 07$ & 32 & 91 & $8.3 \mathrm{~J}$ & ND \\
\hline & CNPSB1-S-16346 & $11 / 12 / 07$ & 36 & 122 & $3 \mathrm{~J}$ & ND \\
\hline & CNPSB1-S-19946 & $11 / 12 / 07$ & 40 & 44 & $4.6 \mathrm{~J}$ & ND \\
\hline \multirow[t]{10}{*}{ PSB2 } & CNPSB2-S-19949 & $11 / 13 / 07$ & 3.8 & ND & ND & ND \\
\hline & CNPSB2-S-19950 & $11 / 13 / 07$ & 8 & ND & ND & ND \\
\hline & CNPSB2-S-19951 & $11 / 13 / 07$ & 12 & ND & ND & ND \\
\hline & CNPSB2-S-19952 & $11 / 13 / 07$ & 16 & ND & ND & ND \\
\hline & CNPSB2-S-19953 & $11 / 13 / 07$ & 20 & $9 \mathrm{~J}$ & $3.5 \mathrm{~J}$ & ND \\
\hline & CNPSB2-S-19954 & $11 / 13 / 07$ & 24 & 103 & $6.1 \mathrm{~J}$ & ND \\
\hline & CNPSB2-S-19955 & $11 / 13 / 07$ & 28 & 66 & $8.8 \mathrm{~J}$ & ND \\
\hline & CNPSB2-S-19956 & $11 / 13 / 07$ & 32 & 152 & $5.3 \mathrm{~J}$ & ND \\
\hline & CNPSB2-S-19957 & $11 / 13 / 07$ & 36 & 173 & $3.5 \mathrm{~J}$ & ND \\
\hline & CNPSB2-S-19958 & $11 / 13 / 07$ & 40 & 62 & $2.9 \mathrm{~J}$ & ND \\
\hline \multirow[t]{10}{*}{ PSB3 } & CNPSB3-S-25970 & $11 / 16 / 07$ & 4 & ND & ND & ND \\
\hline & CNPSB3-S-25961 & $11 / 16 / 07$ & 8 & ND & ND & ND \\
\hline & CNPSB3-S-25962 & $11 / 16 / 07$ & 12 & ND & ND & ND \\
\hline & CNPSB3-S-25963 & $11 / 16 / 07$ & 16 & ND & ND & ND \\
\hline & CNPSB3-S-25964 & $11 / 16 / 07$ & 20 & ND & ND & ND \\
\hline & CNPSB3-S-25965 & $11 / 16 / 07$ & 24 & $1.2 \mathrm{~J}$ & ND & ND \\
\hline & CNPSB3-S-25966 & $11 / 16 / 07$ & 28 & $2.7 \mathrm{~J}$ & $1.2 \mathrm{~J}$ & ND \\
\hline & CNPSB3-S-25967 & $11 / 16 / 07$ & 32 & 13 & $1.7 \mathrm{~J}$ & ND \\
\hline & CNPSB3-S-25968 & $11 / 16 / 07$ & 36 & 20 & $2.4 \mathrm{~J}$ & ND \\
\hline & CNPSB3-S-25969 & $11 / 16 / 07$ & 40 & 15 & $4.3 \mathrm{~J}$ & ND \\
\hline \multirow[t]{10}{*}{ PSB4 } & CNPSB4-S-19966 & $11 / 16 / 07$ & 4 & ND & ND & ND \\
\hline & CNPSB4-S-19967 & $11 / 16 / 07$ & 8 & ND & ND & ND \\
\hline & CNPSB4-S-19968 & $11 / 16 / 07$ & 12 & ND & ND & ND \\
\hline & CNPSB4-S-19970 & $11 / 16 / 07$ & 16 & ND & ND & ND \\
\hline & CNPSB4-S-19971 & $11 / 16 / 07$ & 20 & ND & ND & ND \\
\hline & CNPSB4-S-19972 & $11 / 16 / 07$ & 24 & $1.4 \mathrm{~J}$ & $1.4 \mathrm{~J}$ & ND \\
\hline & CNPSB4-S-19973 & $11 / 16 / 07$ & 28 & 24 & $4.7 \mathrm{~J}$ & ND \\
\hline & CNPSB4-S-19974 & $11 / 16 / 07$ & 32 & 89 & $7.8 \mathrm{~J}$ & ND \\
\hline & CNPSB4-S-19975 & $11 / 16 / 07$ & 36 & 58 & $6 \mathrm{~J}$ & ND \\
\hline & CNPSB4-S-25960 & $11 / 16 / 07$ & 40 & 57 & $9.7 \mathrm{~J}$ & ND \\
\hline
\end{tabular}

a ND, not detected at an instrument detection limit of $1.0 \mu \mathrm{g} / \mathrm{kg}$.

b Qualifier J indicates an estimated concentration below the purge-and-trap method quantitation limit of $10 \mu \mathrm{g} / \mathrm{kg}$. 


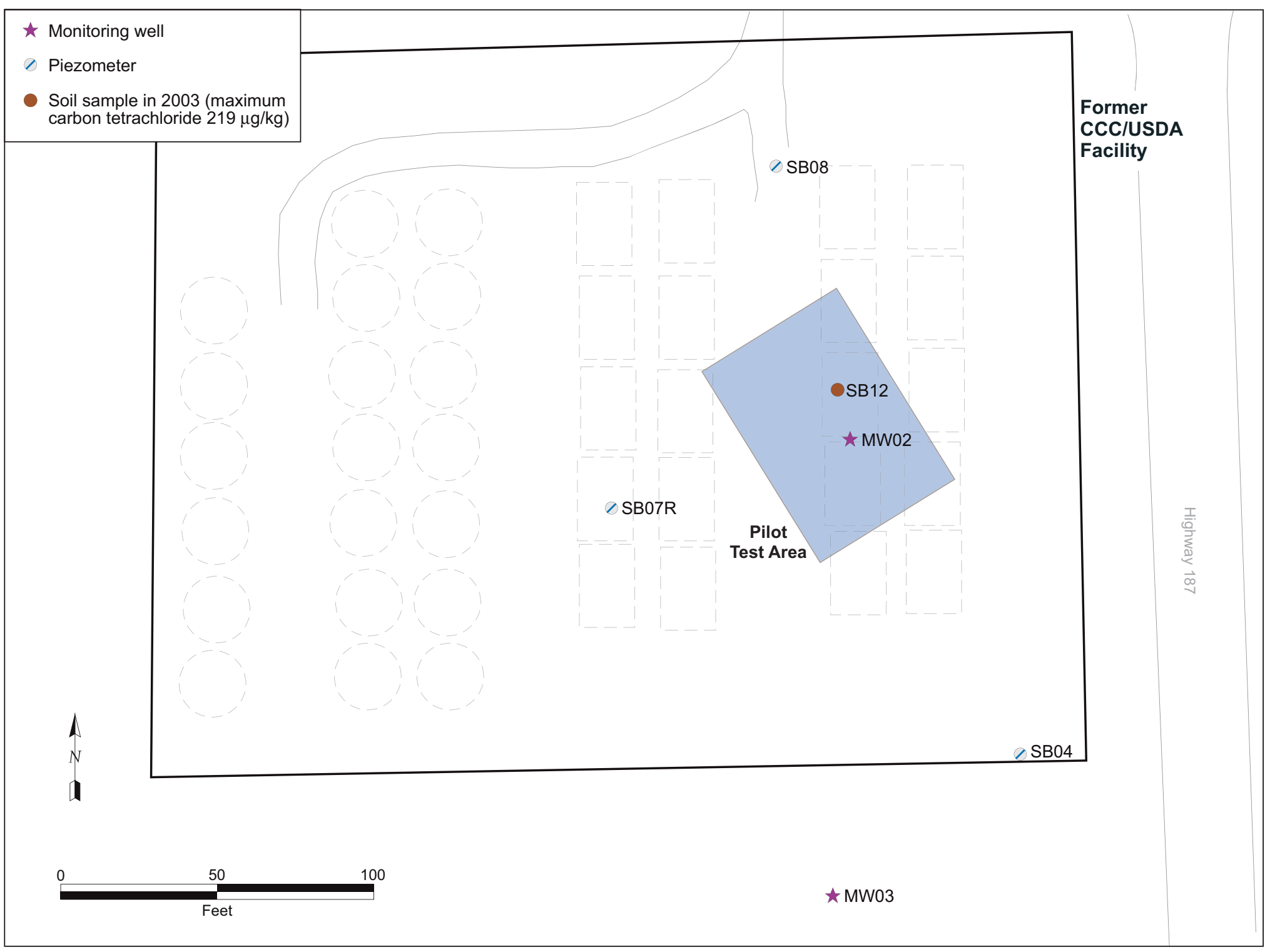

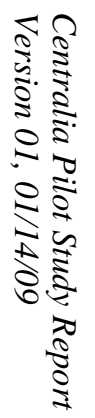

FIGURE 2.1 Proposed (November 2007) pilot test area, with locations of soil boring SB12 and MW02 (which define the hot spot area to be treated in the pilot test) and nearby existing monitoring points. 


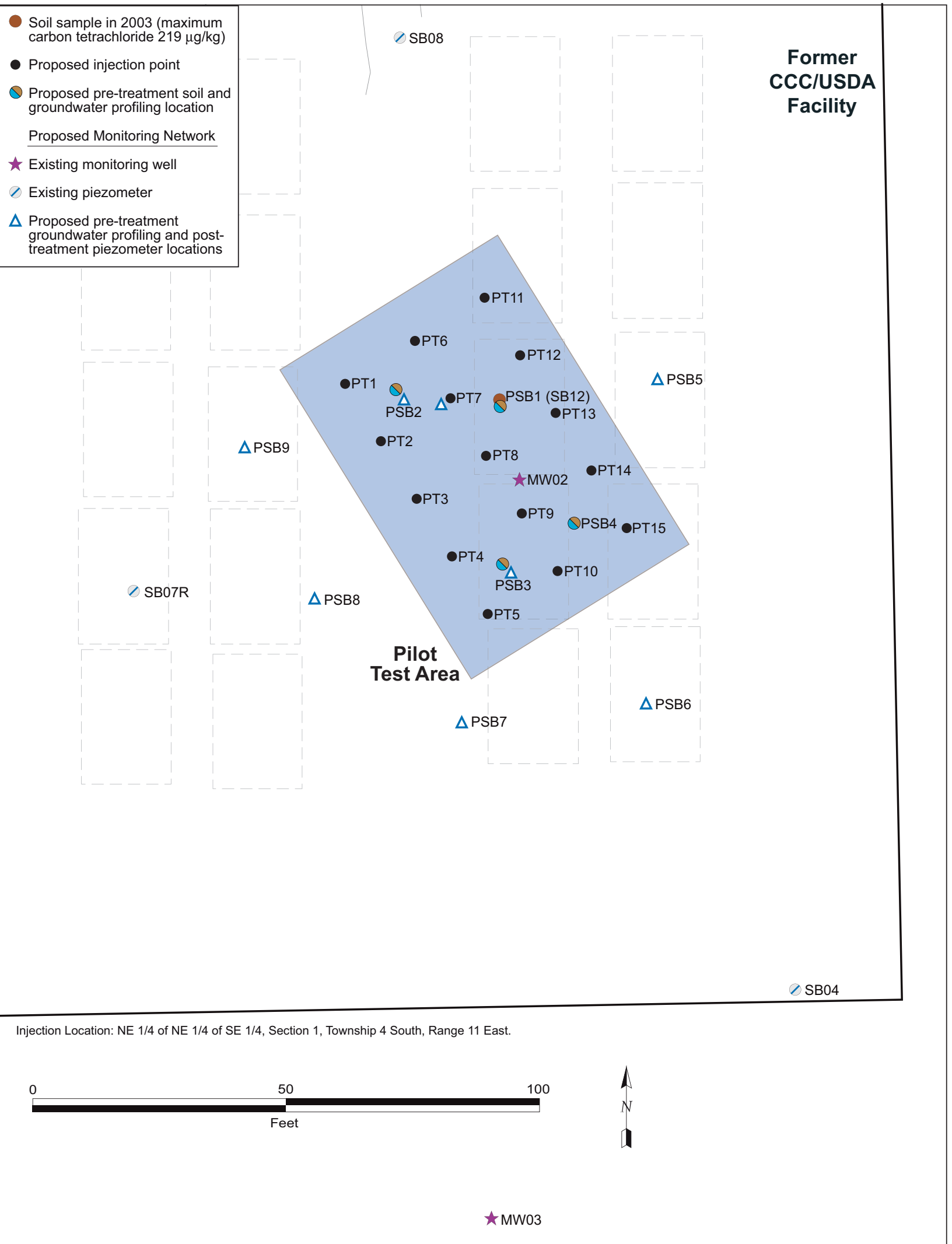

FIGURE 2.2 Proposed (November 2007) pilot test area, with locations of injection points and existing and new monitoring points. 


\section{Pre-Injection Sampling - Groundwater $50-55 \mathrm{ft}$ \\ Pre-Injection Sampling - Groundwater 55-60 ft}

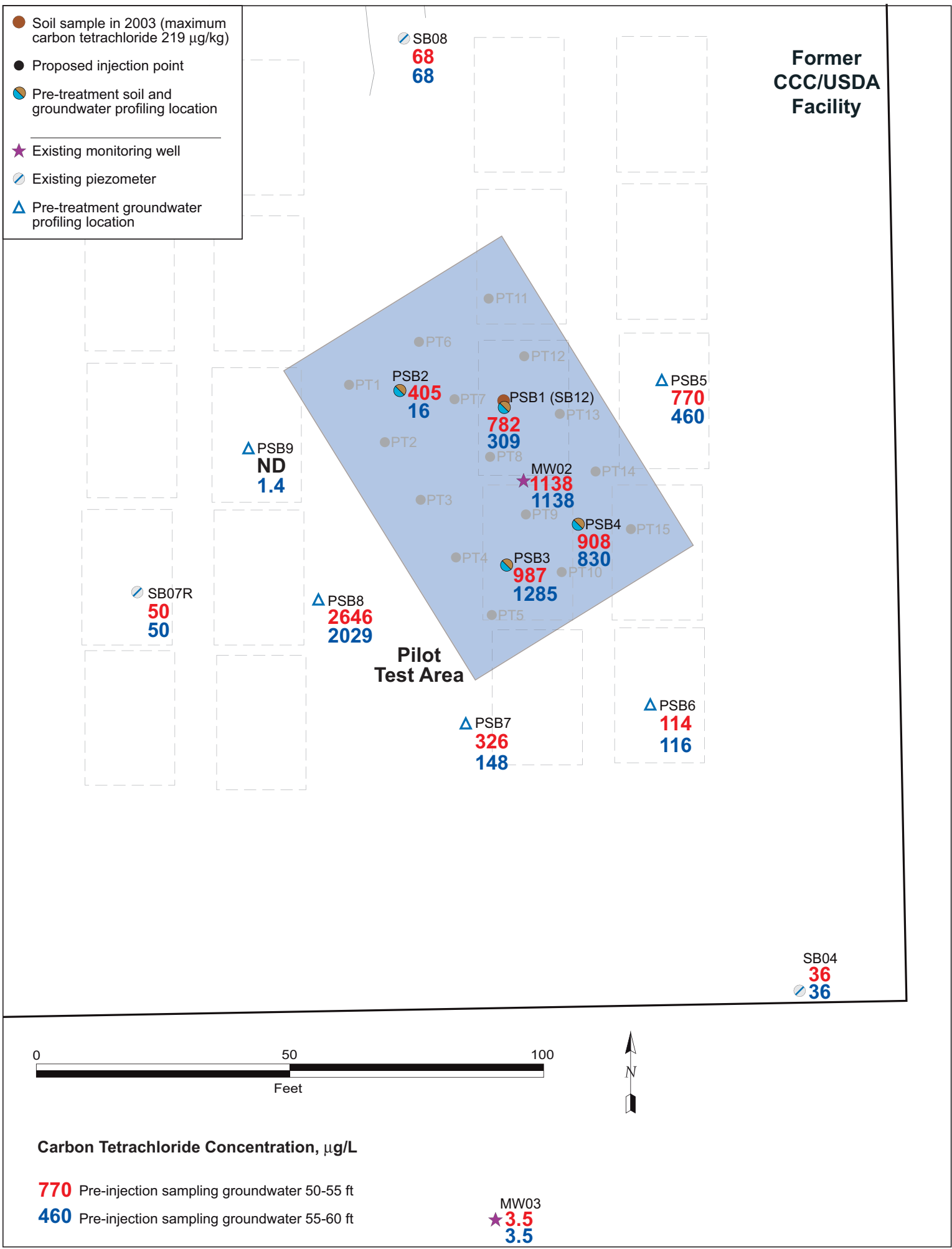

FIGURE 2.3 Carbon tetrachloride concentrations in groundwater samples collected at 50-55 $\mathrm{ft}$ BGL and at 55-60 ft BGL during the pre-injection baseline sampling event. 
Pre-Injection Sampling - Groundwater 55-60 ft

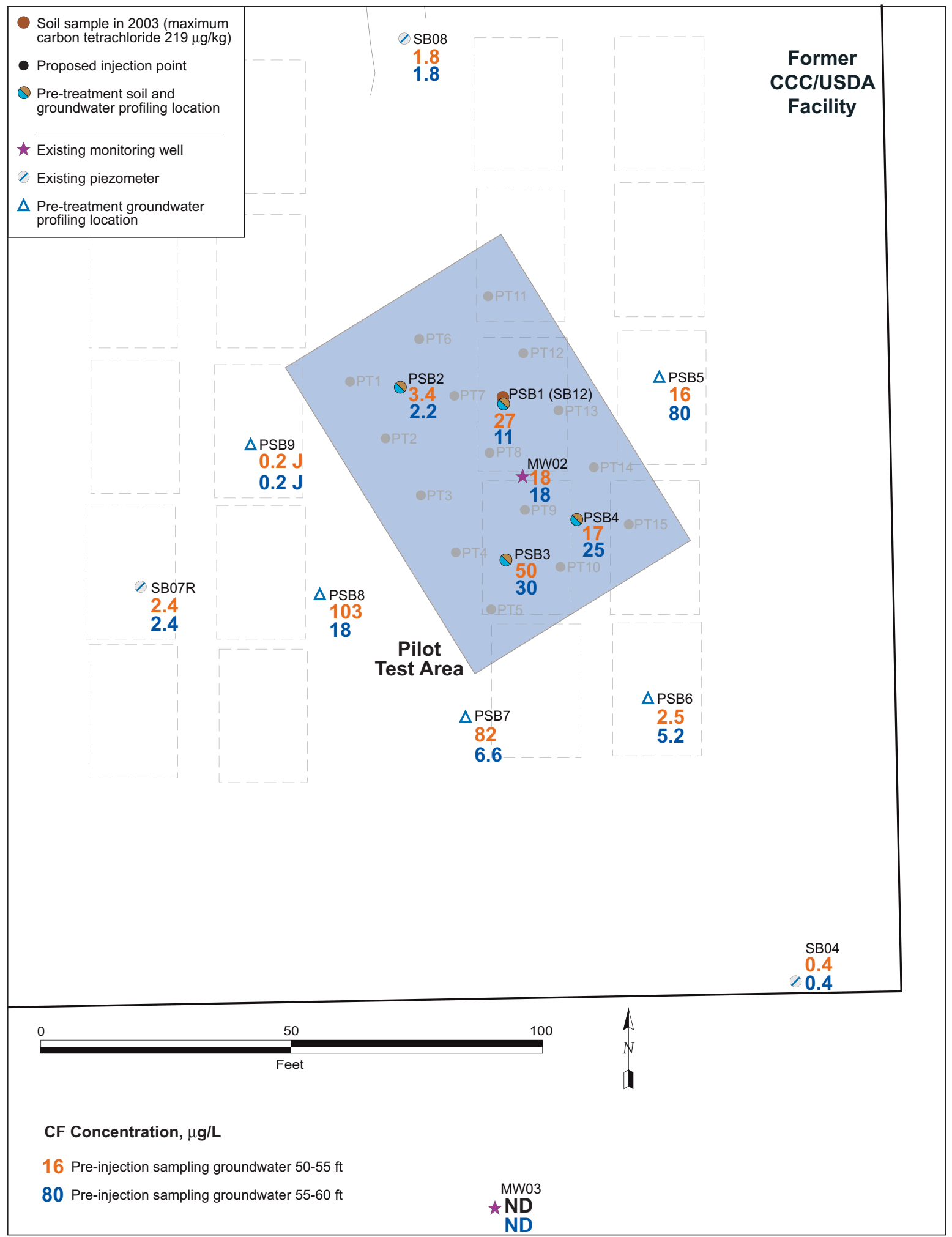

FIGURE 2.4 Chloroform concentrations in groundwater samples collected at 50-55 ft BGL and at 55-60 ft BGL during the pre-injection baseline sampling event. 


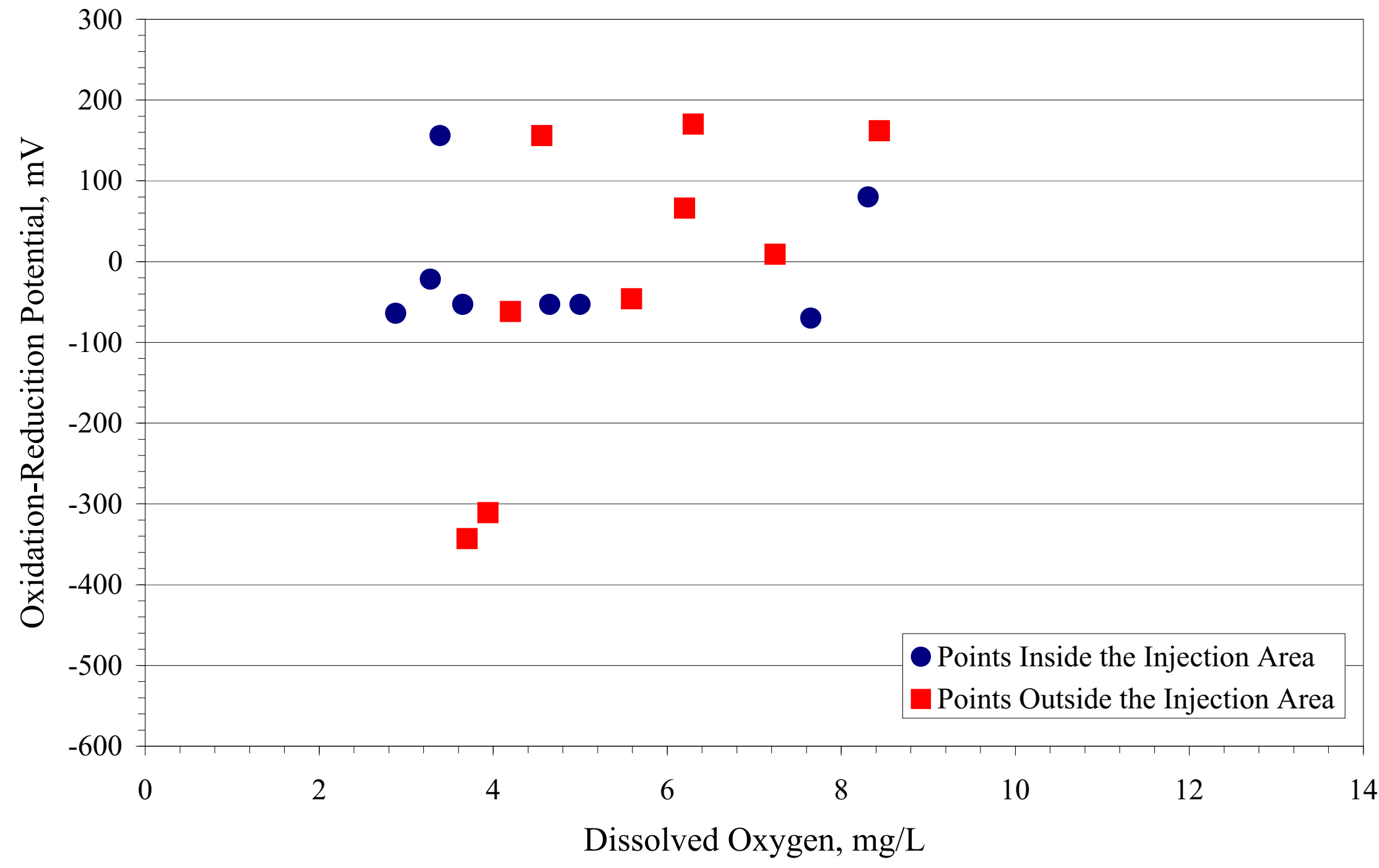

FIGURE 2.5 Dissolved oxygen concentrations and oxidation-reduction potential levels in groundwater samples collected at 50-55 ft and $55-60 \mathrm{ft}$ BGL during the pre-injection baseline sampling event. 


\section{Pre-Injection Sampling - Groundwater \\ $50-55 / 55-60 \mathrm{ft}$}

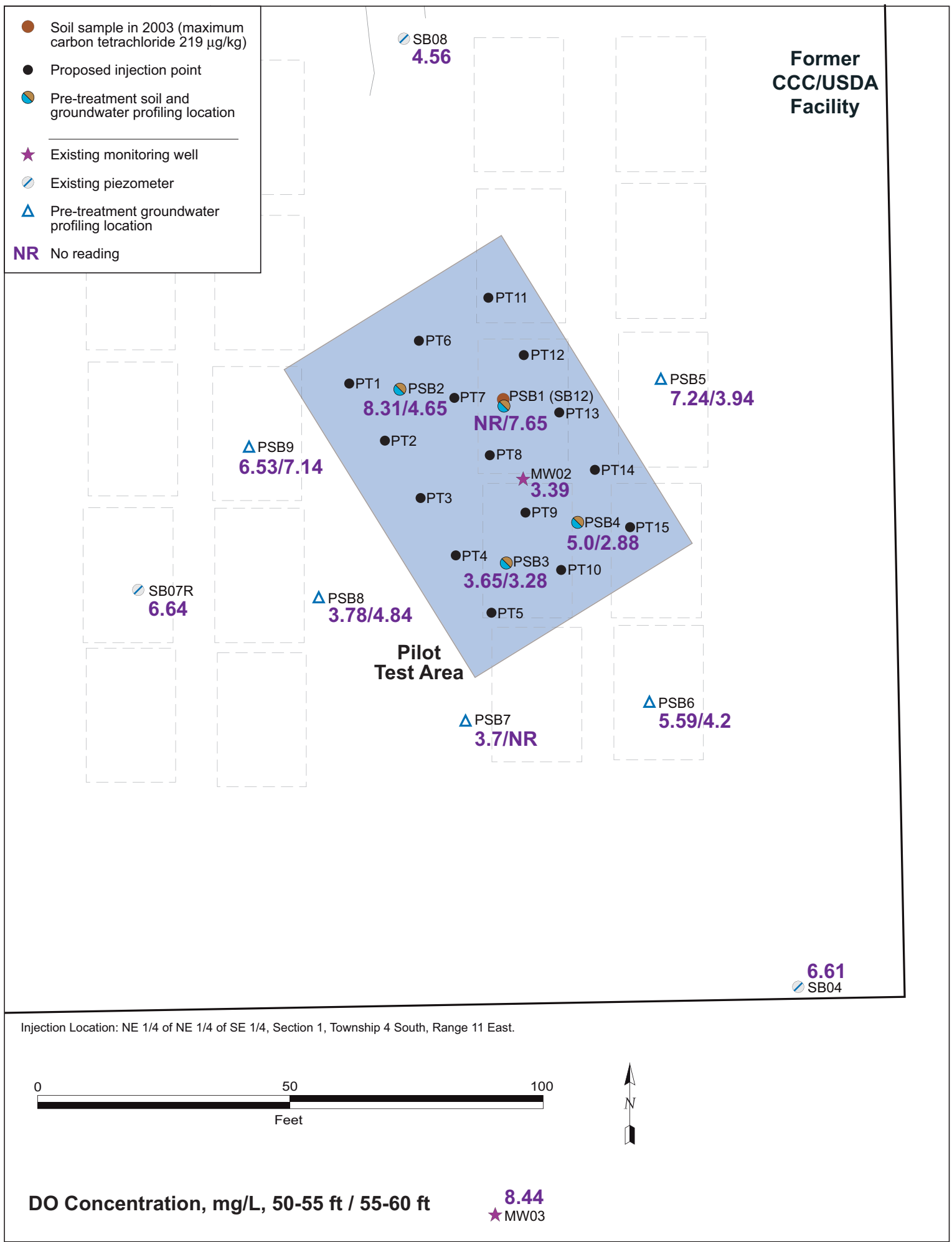

FIGURE 2.6 Dissolved oxygen concentrations in groundwater samples collected at $50-55 \mathrm{ft}$ and 55-60 ft BGL during the pre-injection baseline sampling event. 


\section{Pre-Injection Sampling - Groundwater \\ $50-55 / 55-60 \mathrm{ft}$}

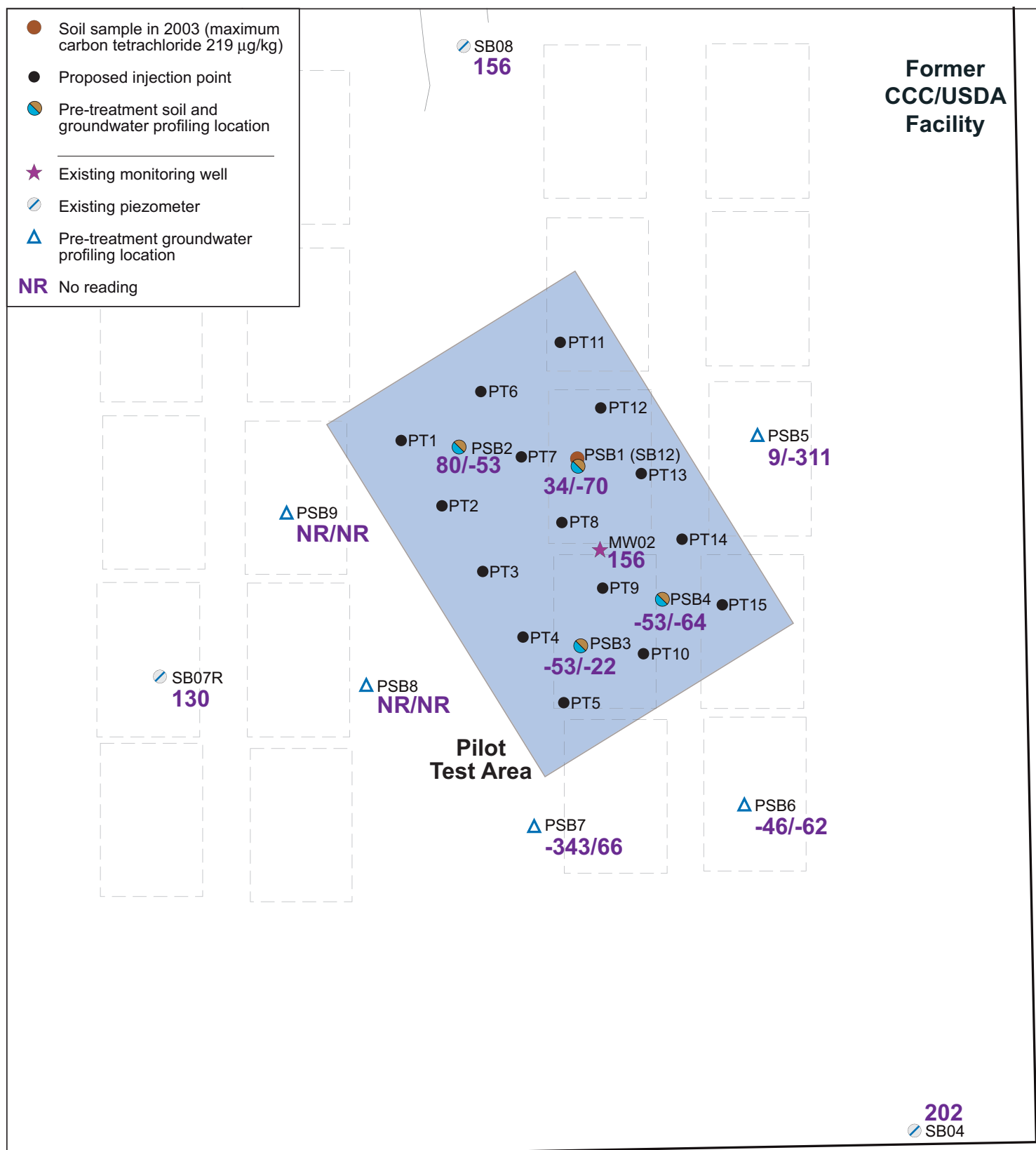

Injection Location: NE 1/4 of NE 1/4 of SE 1/4, Section 1, Township 4 South, Range 11 East.

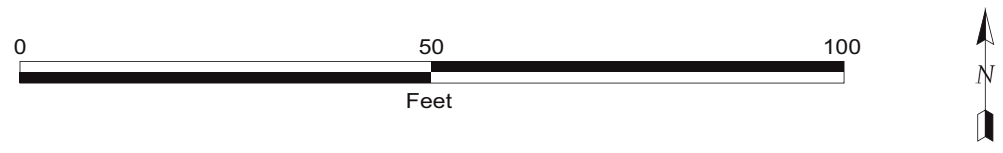

ORP, $\mathrm{mV}, 50-55 \mathrm{ft} / 55-60 \mathrm{ft}$

162

$\uparrow$ MW03

FIGURE 2.7 Oxidation-reduction potential levels in groundwater samples collected at $50-55 \mathrm{ft}$ and 55-60 ft BGL during the pre-injection baseline sampling event. 


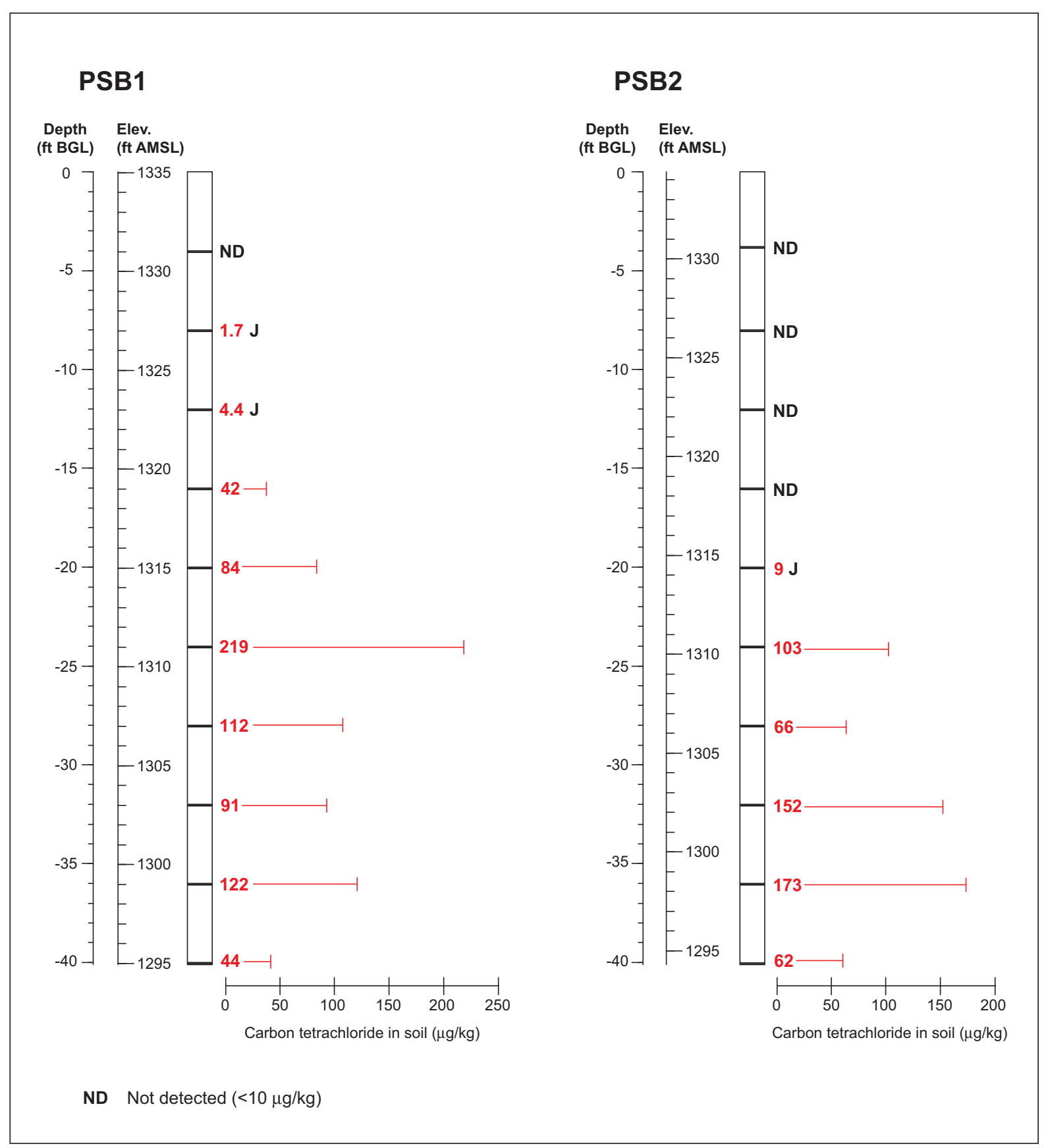

FIGURE 2.8 Vertical distribution of carbon tetrachloride identified in the vadose zone soils at preinjection baseline sampling locations PSB1 and PSB2. 


\section{PSB3}

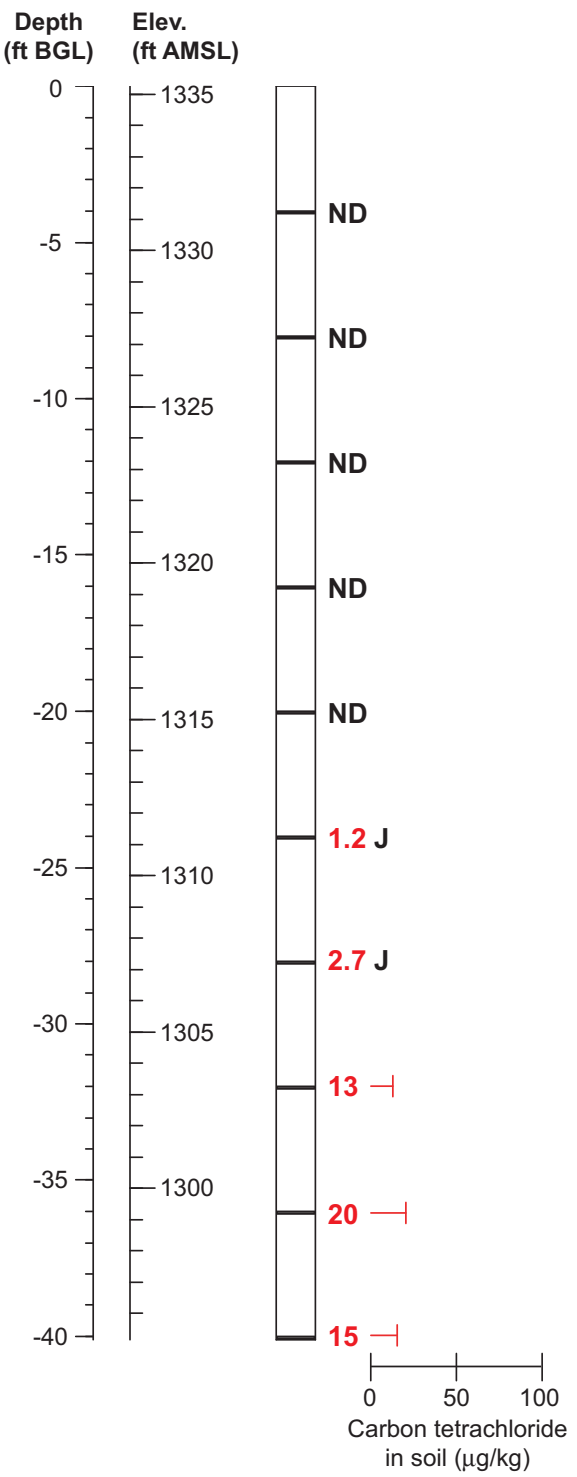

\section{PSB4}

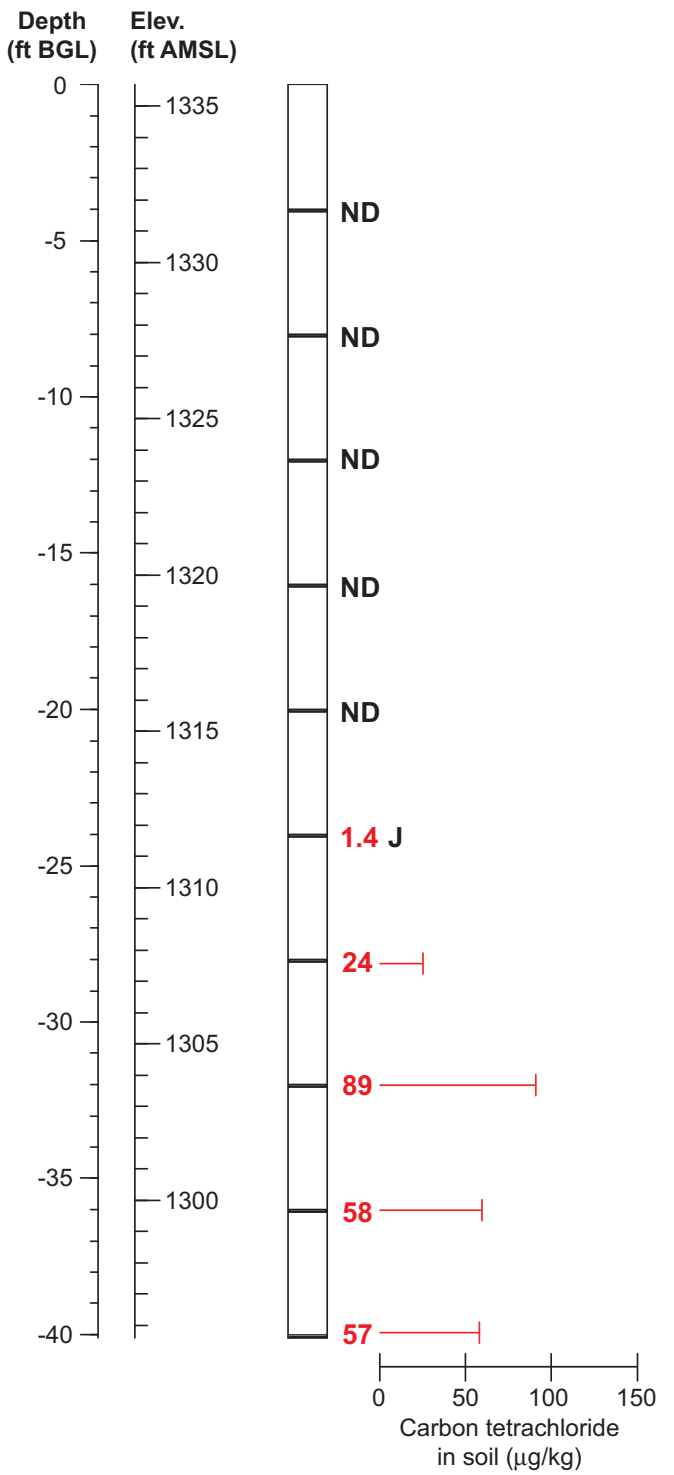

ND Not detected $(<10 \mu \mathrm{g} / \mathrm{kg})$

FIGURE 2.9 Vertical distribution of carbon tetrachloride identified in the vadose zone soils at preinjection baseline sampling locations PSB3 and PSB4. 


\section{SB12}
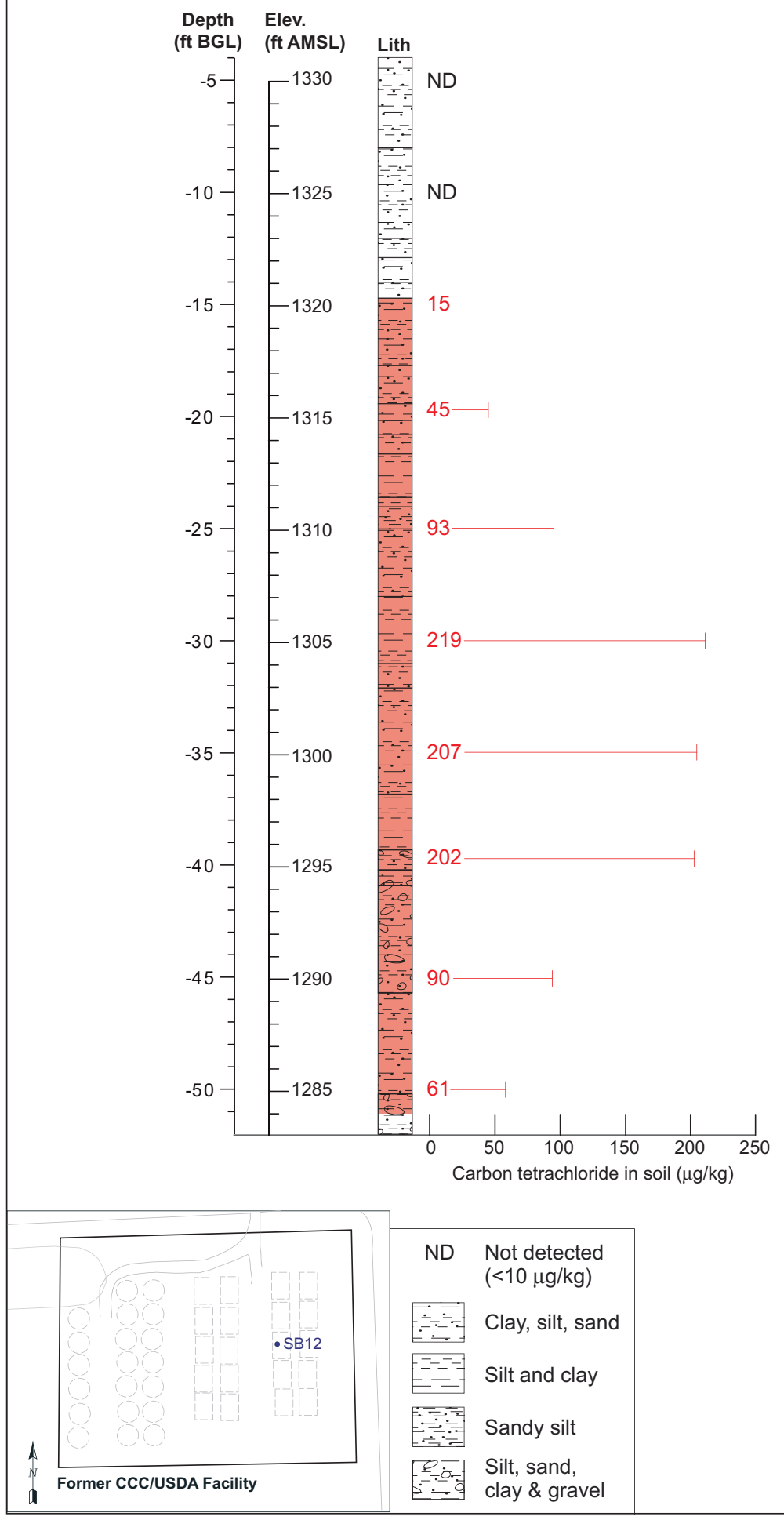

FIGURE 2.10 Vertical distribution of carbon tetrachloride identified in the vadose zone soils at 2002-2004 sampling location SB12. 


\section{Review of the ISCR Injection Process}

\subsection{Overview of the Field Injection Program}

The results of the pre-injection baseline characterization studies presented in Section 2 were discussed with the KDHE/BER and CCC/USDA project managers and with technical representatives from Adventus prior to field implementation of the injection program at Centralia, and continuing technical dialogue took place among these agencies as the injection activities progressed. On the basis of these discussions, and with the approval of the KDHE/BER and CCC/USDA project managers, several modifications were made to the original injection plan (Argonne 2007a) to more effectively address the distribution of carbon tetrachloride contamination identified at the pilot test site and the field conditions encountered during the injection efforts. The distribution of injection points as originally planned and the injection program as implemented are illustrated in Figures 2.2 and 3.1, respectively. The revisions made include the following:

- Relocation of three injection points (PT1, PT6, and PT11) planned along the northwestern edge of the injection field (Figure 2.2) to the vicinity of preinjection sampling point PSB8 (Figure 3.1) to address the high carbon tetrachloride concentrations identified in baseline groundwater samples from location PSB8 (Figure 2.3).

- Injection into both the vadose and saturated zones at 7 locations near preinjection sampling points PSB1 and PSB8, instead of at 10 locations near PSB1 only.

- Injection of EHC-A at 20-40 ft BGL and a thin slurry of EHC at 40-50 ft BGL into the vadose zone soils, in lieu of EHC-A only, to provide additional EHC mass near the top of the saturated zone.

- Injection of EHC into the saturated zone only at 7 locations, in lieu of the originally proposed 5 locations. 
- Elimination of injection point PT8 and relocation of injection point PT9 (Figure 2.2) to reduce the potential for invasion of injected fluid at monitoring well MW02 (see below).

- Use of reagent-grade $\mathrm{KBr}$ as a geochemical tracer compound, at the request of the KDHE/BER (KDHE 2007a). The KBr tracer was added at a rate of $84.3 \mathrm{~g}$ per 100 gal of water used for mixing of the EHC slurry and EHC-A fluids, to yield an initial dissolved $\mathrm{Br}^{-}$concentration of $150 \mathrm{mg} / \mathrm{L}$ in the tagged injection water.

The injections at Centralia were performed by Redox Tech, LLC (Cary, North Carolina), under the supervision of representatives from the KDHE/BER, the KDHE Bureau of Water (KDHE/BOW) Underground Injection Control (UIC) program, the CCC/USDA, Adventus, and Argonne. The program was conducted under authorization received from the KDHE/BOW (KDHE 2007b) by using a direct-push (GeoProbe) technique to inject the EHC-A and EHC amendments from the top down, at the request of the KDHE/BER (KDHE 2007a).

Documentation summarizing the injection field program, including discussion of the field methods employed; a chronology of the daily activities at the site during this phase of the pilot study; and data on the fluid types, quantities, injection depths, and injection pressures employed at each injection location, was provided by Adventus (2008a). The Adventus report was submitted to the KDHE/BOW in compliance with the UIC program authorization for the site (Argonne 2008b), and is on CD in the present report as Supplement 3.

The quantities of EHC and EHC-A employed at each injection location are summarized in Table 3.1. Totals of 3,650 lb of EHC-A and 1,300 lb of EHC were injected into the vadose zone soils (20-50 ft BGL) at locations PT1, PT2, PT6, PT7, and PT11-PT13 (Figure 3.1). In all, $5,300 \mathrm{lb}$ of EHC material was injected into the saturated zone at the 14 locations shown in Figure 3.1 (PT1-PT7 and PT9-PT15). Approximately 8,650 gal of (Br--tagged) water was used to make up the EHC and EHC-A injection fluids and for limited additional (subsurface) flushing of the downhole injection tool in conjunction with the placement of the EHC materials, as described in Supplement 3. Calculations provided by Adventus (2008a) (Tables 9 and 10 in Supplement 3) indicate that the combined volumes of flush water, EHC slurry, and EHC-A solution injected into the subsurface at Centralia represented approximately 3-6\% of the total estimated pore space in the saturated and vadose zones targeted for treatment in the pilot test injection area. 


\subsection{Evaluation of the Injection Process and Subsurface Fluid Distribution}

Field observations during the injection program and subsequent testing conducted with the approval of the KDHE/BER suggested that the heterogeneous subsurface conditions present at Centralia significantly affected both the injection process and the ultimate distribution of the ISCR amendments in the vadose zone and saturated zone soils, in a complex and unpredictable manner.

\subsubsection{Leakage of the ISCR Injection Fluids to the Surface}

Adventus indicated prior to the injection program that pre-existing boreholes within and near the injection field could provide possible conduits for the leakage of injected fluids to the surface. In light of this, monitoring well MW02 (within the injection field) was pre-loaded with uncontaminated (by carbon tetrachloride) water above the screened interval, and the casing headspace was slightly pressurized, to deter possible invasion of the casing by injection fluids. At the request of Adventus, all pre-injection investigative (CPT) borings were plugged with a proprietary, Bentonite-based material (Adventus HoleBlok+TM). Each injection point was also immediately plugged (with HoleBlok+) upon completion of the injections at that location, with methods approved by the KDHE/BOW (Adventus [2008a]; Supplement 3).

The field logs included in the Adventus (2008a) report (Supplement 3) document numerous instances of EHC-A and EHC fluids leaking to the ground surface (“daylighting”) as the injections occurred, indicating probable movement of the fluids along preferred vertical and lateral migration pathways within the subsurface. The occurrences of daylighting showed no consistent relationship to the identified surface or subsurface conditions at the site, however, or to the operational parameters or fluid types employed in the injection procedure. The measures used to control the loss of fluids at the daylighting locations were described by Adventus (2008a) (Supplement 3).

Figure 3.2 presents a schematic representation, based on determinations by Argonne staff, of the approximate lateral direction(s) and distance(s) at which daylighting occurred in response to the introduction of fluids at the various injection points. Daylighting was noted at distances ranging from less than $1 \mathrm{ft}$ to more than $25 \mathrm{ft}$ from the source injection points. Daylighting occurred at various depth intervals (one or more at each location), as follows: 
- During injection of EHC-A solution into the vadose zone at PT1, PT6, PT7, and PT11 (20-40 ft BGL)

- During injection of thin EHC slurry into the vadose zone at PT7, PT12, and PT13 (40-50 ft BGL)

- During injection of standard EHC slurry into the saturated zone at PT2, PT10, PT14, and PT15 (50-60 ft BGL)

The KDHE/BOW initially authorized the use of injection pressures (measured at the outlet of the injection pump) of up to 300 psi during the injection program (KDHE 2007b). Injection pressures exceeding $300 \mathrm{psi}$ were required, however, to achieve placement of the required EHC fluids at multiple locations and depths in the saturated zone sediments. These pressures were permitted upon notification of the KDHE/BOW as the injection program progressed (Adventus [2008a]; Supplement 3). Injection pressures associated with the instances of daylighting ranged from $<100$ psi to 300 psi during injection into vadose zone soils and from approximately 200 psi to 600 psi during injection into the saturated zone. The observed pressures are comparable to those recorded for corresponding subsurface intervals at other locations from which no daylighting occurred.

Figures 3.2 and 3.3 (top) illustrate that fluids from multiple injection points (PT7, PT13, PT14) daylighted outside the well casing at MW02. Daylighting outside the casing at monitoring well SB07R occurred in response to injection at point PT6 (approximately $27 \mathrm{ft}$ northwest of SB07R) (Figure 3.2). Daylighting was also observed (from injection points PT10 and PT14) along pathways roughly intersecting the locations of (plugged) pre-injection investigative borings PSB3 and PSB4 and at (plugged) injection point PT13 (from injection points PT7 and PT12), apparently confirming the influence of the pre-existing borings on the subsurface movement of the injected fluids. Daylighting occurred through previously undisturbed ground, however, in response to the injections at points PT2 (Figure 3.3, bottom) and PT15, suggesting that the surfacing of fluids at Centralia is not uniquely controlled by the presence of earlier borings. 


\subsubsection{Post-Injection Coring Studies}

To obtain further insight into the subsurface movement and distribution of the injected fluids, soil cores were collected at three radial distances from one of the injection points for visual identification of injection fluid, per Adventus recommendations (Argonne 2007a). Injection point PT7 was selected, in consultation with the KDHE/BER project manager, as the focus for this investigation, in light of the multiple daylighting events that occurred in association with injections at this location. Continuous soils cores were collected from $2 \mathrm{ft}$ to $60 \mathrm{ft}$ BGL by using the Argonne CPT in accord with procedures outlined in the Master Work Plan (Argonne 2002b), at distances of $11 \mathrm{ft}, 5 \mathrm{ft}$, and $3 \mathrm{ft}$ (PSB10-PSB12, respectively) from point PT7, along the approximate pathway of daylighting observed from this injection point toward pre-injection sampling point PSB1 and injection point PT13 (Figure 3.4). The coring at locations PSB10 and PSB11 (11-ft and 5-ft distances) was performed within two days of completion of the injection program. Because of weather and logistic limitations, the coring at PSB12 (3-ft distance) was completed approximately two weeks after the injection program ended. Photographs of the recovered cores are in Appendix B.

No indications of vertically or laterally pervasive invasion of the vadose zone or aquifer soils were observed in the soil cores collected near PT7. No clear evidence of the presence of injection fluid was identified in the vadose zone soils at the 11-ft distance (PSB10; see Appendix B). One possible thin (approximately 2-in.) horizon of the dark gray EHC material was noted near the top of the aquifer interval at PSB10, at approximately $52 \mathrm{ft}$ BGL; however, no saturation of the associated soils was apparent.

Isolated zones of increased soil moisture were identified at approximately $26 \mathrm{ft}, 34 \mathrm{ft}$, $42 \mathrm{ft}$, and $50 \mathrm{ft}$ BGL in the vadose zone soils recovered at the 5-ft distance (PSB11; see Appendix B), and slight residual pressures were detected in the CPT rods during the recovery of these cores. Sufficient saturated soil (approximately $1 \mathrm{ft}$ ) was retrieved from the coring barrel at the 26-ft depth interval at PSB11 to permit limited geochemical analyses of the associated fluid. Negative ORP levels $(<-140 \mathrm{mV})$ and detection of the $\mathrm{Br}^{-}$tracer in this sample at an elevated concentration (86 mg/L; Supplement 4 [on CD]) confirmed the presence of the EHC-A solution at this horizon. A small sample of free water was retrieved from the PSB11 core barrel at 54-58 ft BGL, within the previously identified aquifer zone. The $\mathrm{Br}^{-}$tracer was detected in this sample at $0.55 \mathrm{mg} / \mathrm{L}$ (approximately the ambient concentration for groundwater), and the ORP value was positive (+50 mV) (Supplement 4). 
In contrast to the 5-ft distance (PSB11) from PT7, at the 3-ft distance (PSB12) little evidence of the injected EHC-A fluid was again identified in the vadose zone soils (see Appendix B). Dark gray EHC material observed along a vertical fracture through the soil core recovered at 34-36 ft BGL, however, suggested migration of EHC materials injected deeper (> $40 \mathrm{ft}$ BGL) in the stratigraphic section into the overlying vadose zone soils at this location. A negative ORP measurement $(-71 \mathrm{mV})$ obtained for a small sample of free water also recovered from the aquifer core at 54-58 $\mathrm{ft}$ BGL at PSB12 suggested the possible influence of the EHC material. The $\mathrm{Br}^{-}$tracer was again identified, however, at an apparently ambient groundwater concentration $(0.15 \mathrm{mg} / \mathrm{L})$.

Additional soil coring was conducted from the ground surface to $40 \mathrm{ft}$ BGL at a fourth location (PSB13), approximately nine months after completion of the injection program, for the purposes of follow-up soil VOCs analyses, as discussed in Section 4.3. Boring PSB13 was located approximately $2 \mathrm{ft}$ southeast of pre-injection sampling point PSB1, roughly equidistant from injection points PT7, PT12, and PT13 (Figure 3.4). Examination of the PSB13 cores identified apparent traces of the dark gray EHC material along isolated, small vertical fractures in the soils at $21 \mathrm{ft}, 25 \mathrm{ft}$, and $27.5 \mathrm{ft}$ BGL (Figure 3.5).

\subsection{Injection Summary}

Detailed, three-dimensional characterization of the subsurface distribution of the ISCR amendments achieved by the injection program at Centralia is beyond both the defined scope and the intent of the current pilot study (Argonne 2007a). The information outlined in this section, however, supports the hypothesis that under the heterogeneous lithologic conditions present at this site, the pilot injection program resulted in a highly non-uniform distribution of the EHC and EHC-A materials. The daylighting observations and coring results together strongly suggest that movement of the injected fluids in both the vadose zone and aquifer soils occurred primarily along complex, preferred migration pathways, the locations and orientations of which cannot be predicted on the basis of available subsurface lithologic controls or the operational parameters of the injection procedures employed. Under these conditions, no effective "average" radius of influence in the stratigraphic section at Centralia can be estimated for use of the present ISCR injection technology (or for other potential treatment alternatives that might require the subsurface injection of materials). 
TABLE 3.1 Summary of ISCR amendments injected at the Centralia pilot test site. ${ }^{a}$

\begin{tabular}{|c|c|c|c|c|c|}
\hline \multirow[b]{3}{*}{ Location } & \multicolumn{5}{|c|}{ Weight (lb) } \\
\hline & \multicolumn{2}{|c|}{$\begin{array}{l}\text { Vadose } \\
\text { Zone }\end{array}$} & \multirow{2}{*}{$\begin{array}{c}\text { Saturated } \\
\text { Zone } \\
\text { EHC }\end{array}$} & \multicolumn{2}{|c|}{ Totals } \\
\hline & EHC-A & $\mathrm{EHC}$ & & EHC-A & $\mathrm{EHC}$ \\
\hline PT1 & 500 & 150 & 350 & 500 & 500 \\
\hline PT2 & 500 & 150 & 250 & 500 & 400 \\
\hline PT3 & - & - & 500 & - & 500 \\
\hline PT4 & - & - & 500 & - & 500 \\
\hline PT5 & - & - & 500 & - & 500 \\
\hline PT6 & 500 & 150 & 300 & 500 & 450 \\
\hline PT7 & 500 & 150 & 350 & 500 & 500 \\
\hline PT9 & - & - & 500 & - & 500 \\
\hline PT10 & - & - & 300 & - & 300 \\
\hline PT11 & 650 & 300 & 350 & 650 & 650 \\
\hline PT12 & 500 & 150 & 350 & 500 & 500 \\
\hline PT13 & 500 & 150 & 50 & 500 & 200 \\
\hline PT14 & - & 100 & 500 & - & 600 \\
\hline PT15 & - & - & 500 & - & 500 \\
\hline Totals & 3,650 & 1,300 & 5,300 & 3,650 & 6,600 \\
\hline
\end{tabular}

a Approximately $8,650 \mathrm{gal}$ of water was used for the injection program. 


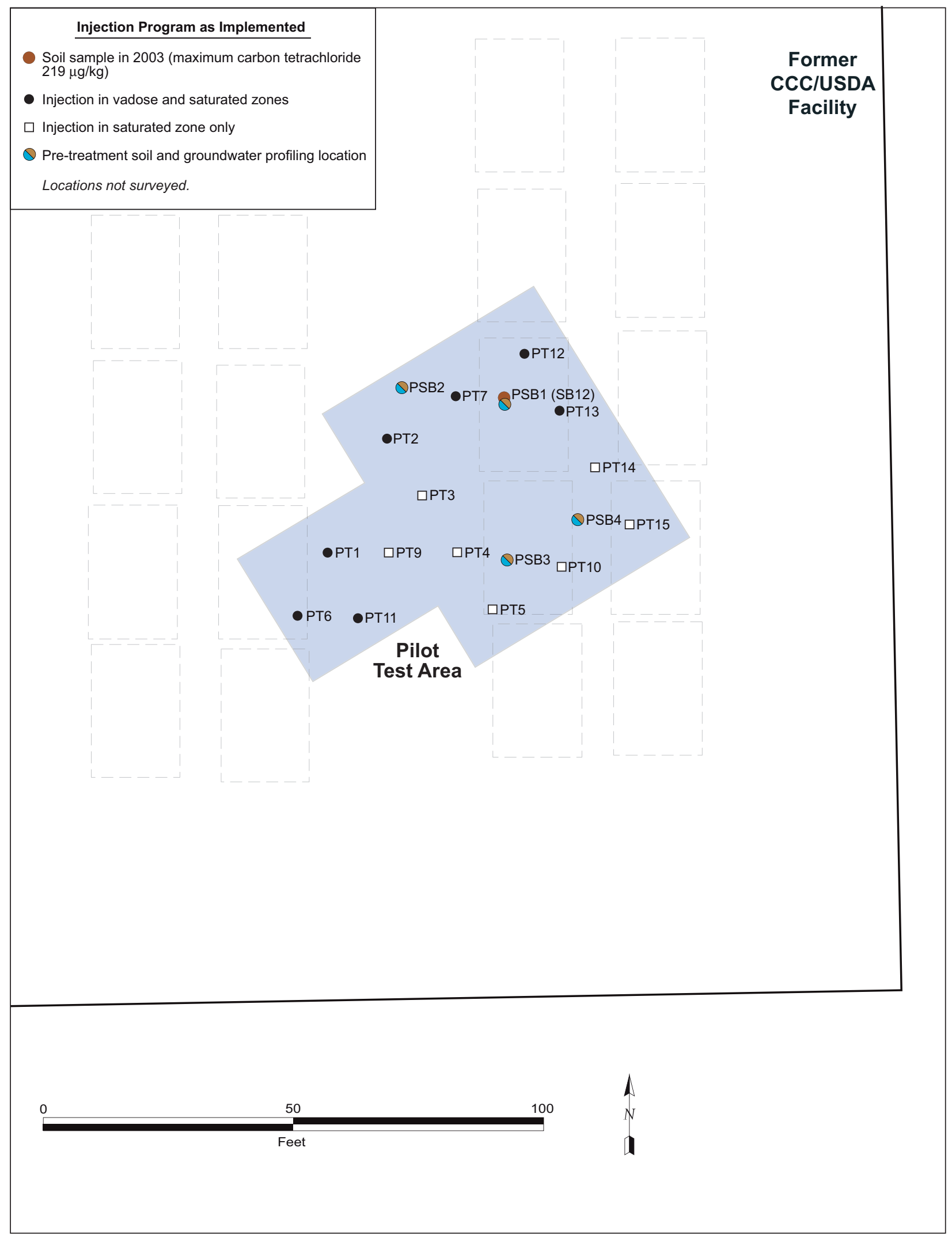

FIGURE 3.1 Pilot test area, with the revised locations of injection points as implemented. 


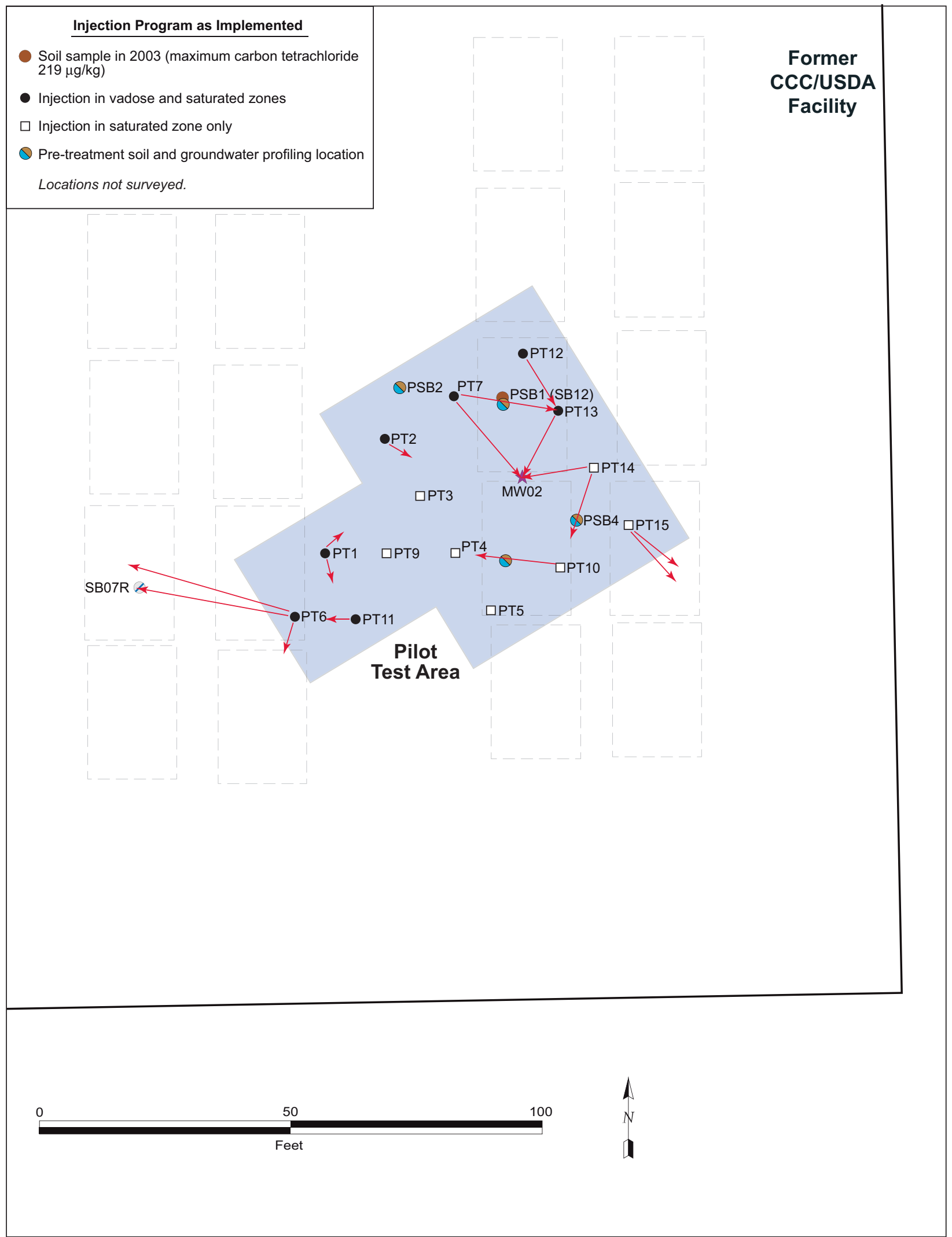

FIGURE 3.2 Schematic representation of the locations where daylighting of EHC-A and EHC was observed in response to the injection of these amendments, with apparent migration directions. 


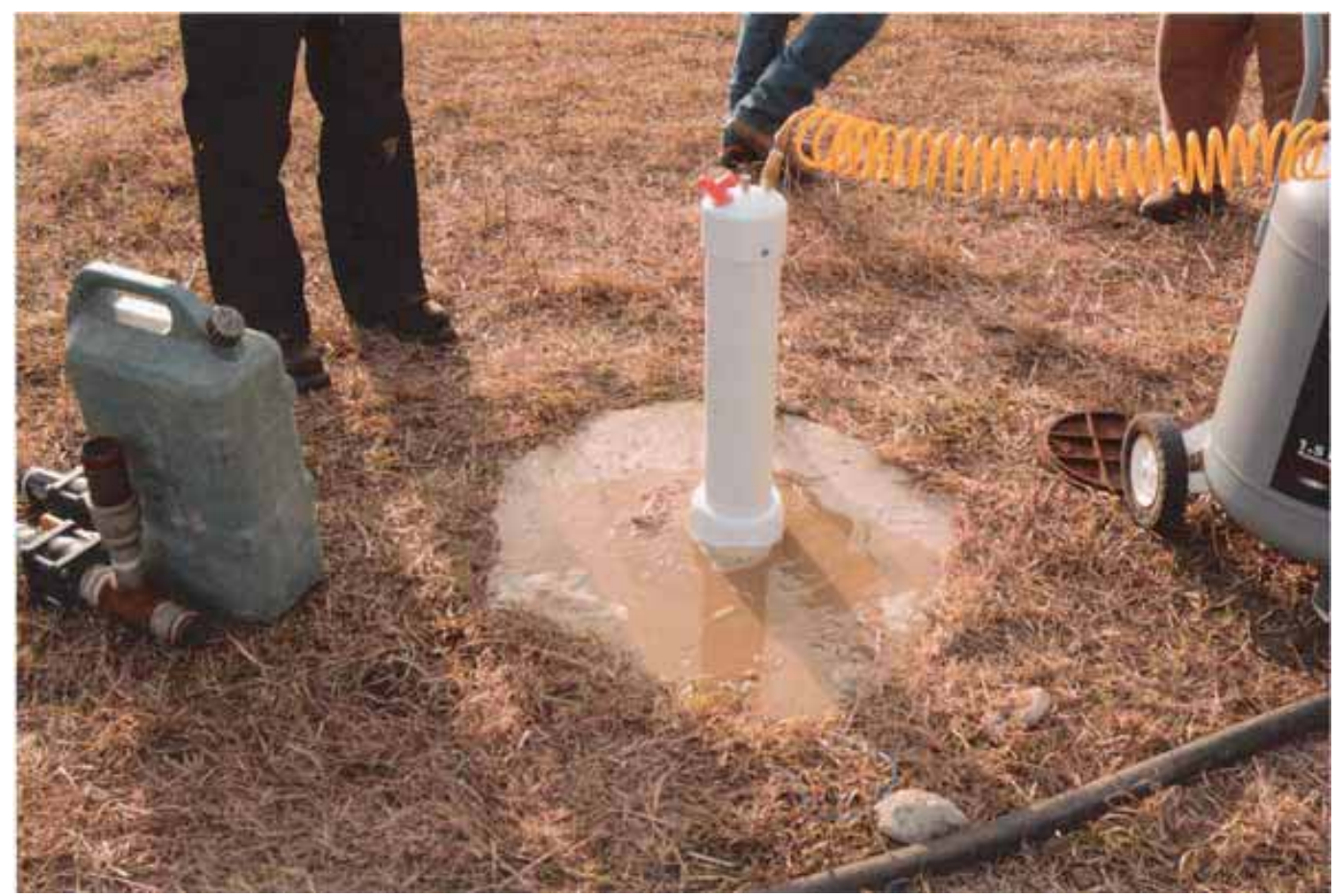

Daylighting of EHC-A solution observed at monitorng well MW02.

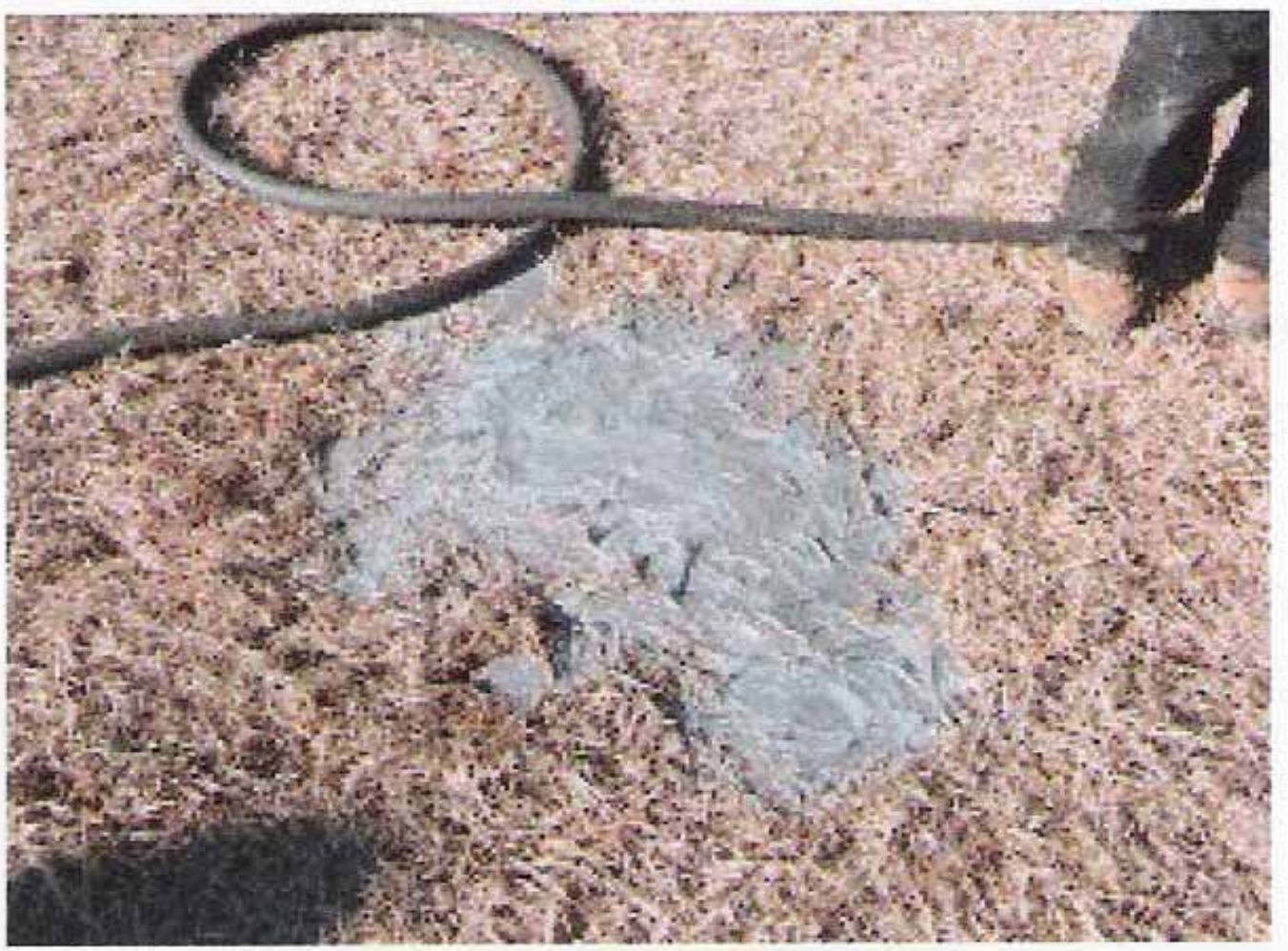

Daylighting of EHC slurry observed in open ground near injection point PT2.

FIGURE 3.3 Daylighting of (top) of EHC-A material outside the well casing at monitoring well MWO2 and (bottom) EHC slurry in open ground near injection point PT2. 


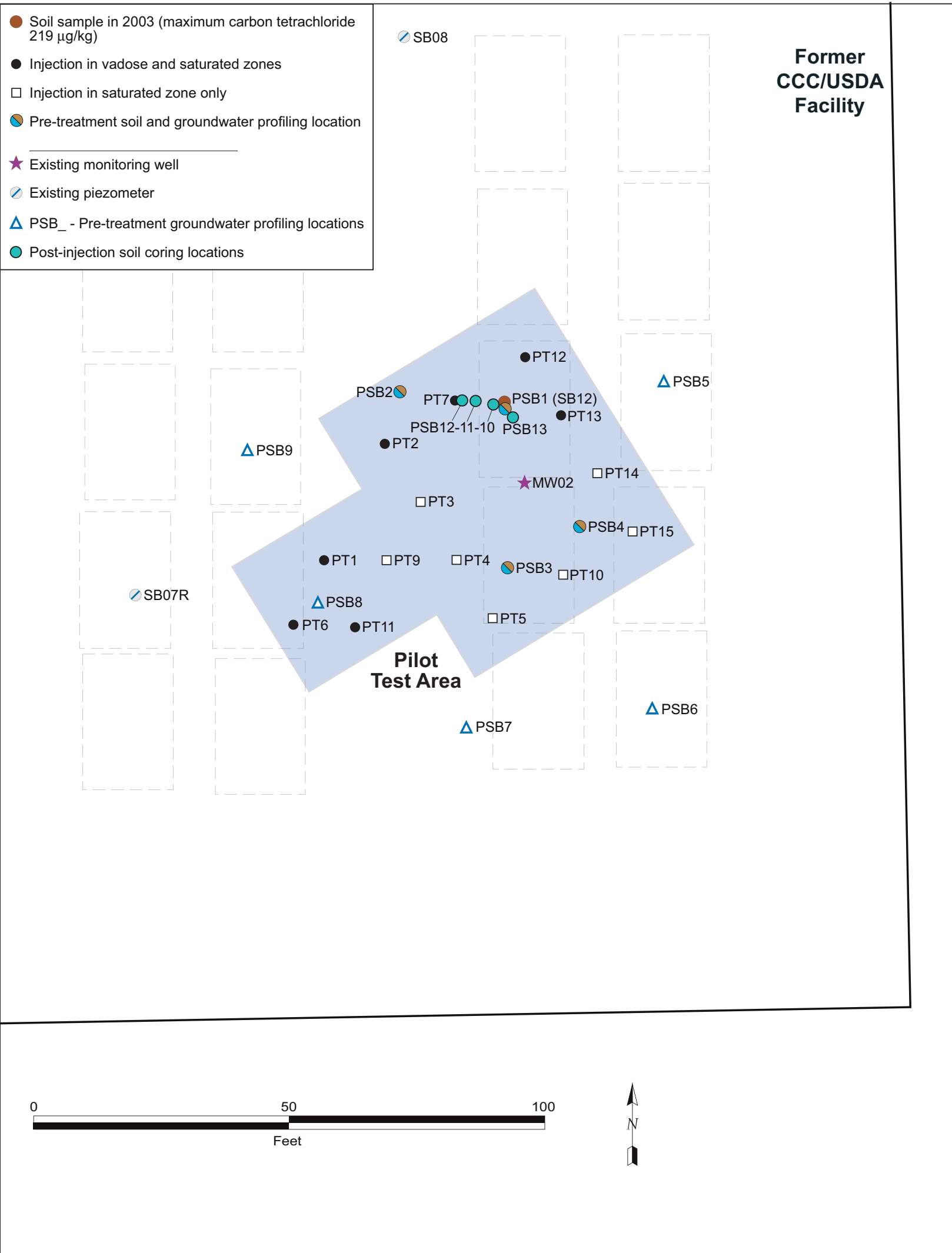

FIGURE 3.4 Locations of the pre-injection groundwater sampling points PSB1-PSB9, monitoring wells SB07R and MW02, injection points, and post-injection soil coring locations PSB10-PSB13. 


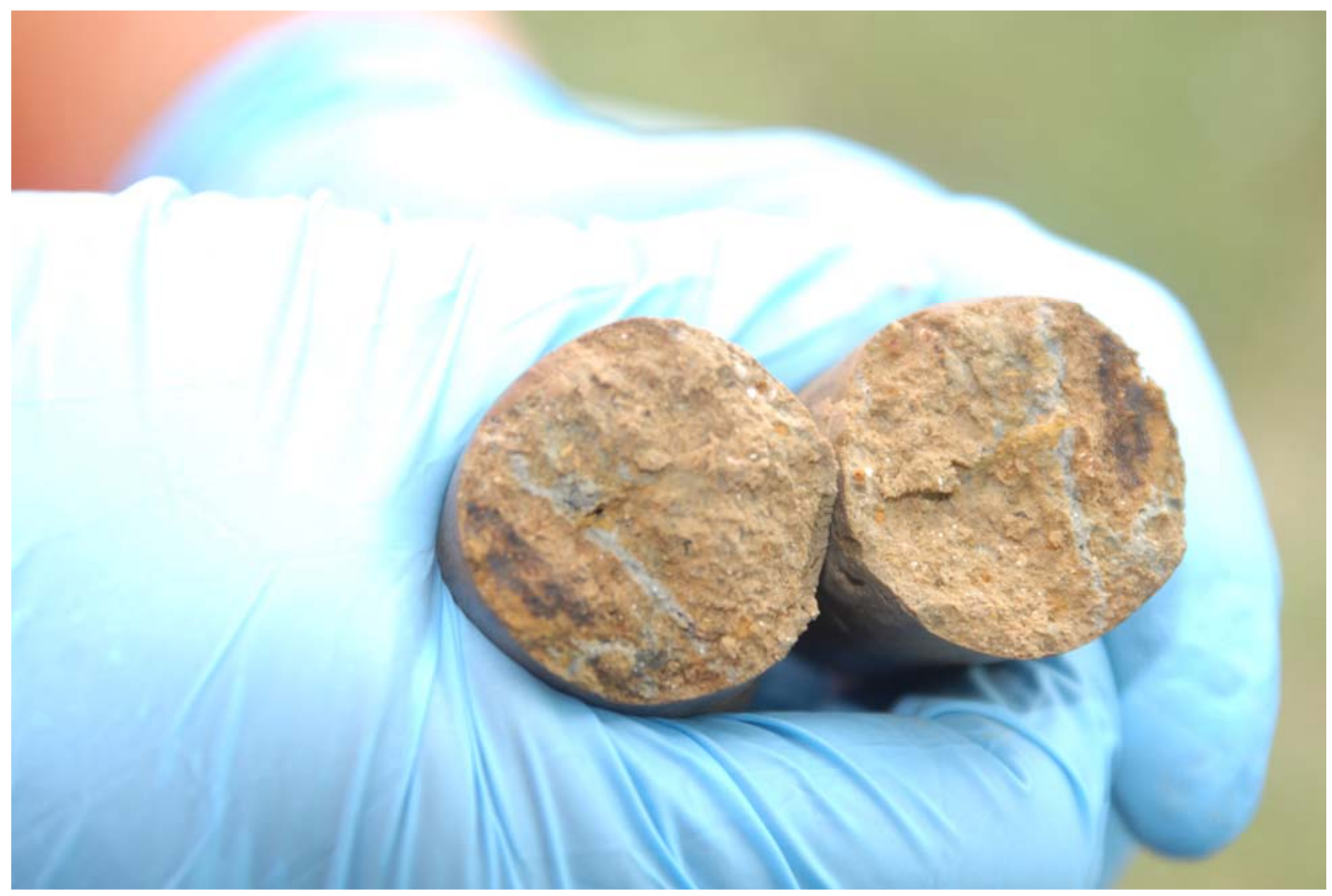

FIGURE 3.5 Appearance of EHC material along small, isolated fractures in the soil core recovered at $21 \mathrm{ft} \mathrm{BGL}$ at post-injection soil coring and sampling location PSB13. 


\section{Preliminary Evaluation of the ISCR Pilot Test Results}

With the approval of the CCC/USDA and KDHE/BER project managers, Argonne initiated an extended program of sampling and analyses at the Centralia pilot test site to monitor the potential impacts of the ISCR treatment technology on the VOCs contaminant levels and the associated geochemical characteristics of the groundwater and vadose zone soils in and near the injection area. The results of the monitoring program to date are presented and reviewed below, with emphasis on examining the parameters that appear most diagnostic of the effects of ISCR treatment technology.

\subsection{Establishment of the Pilot Test Monitoring Well Network}

To facilitate monitoring of the ISCR pilot test, nine new permanent monitoring wells (PMP1-PMP9) were installed to supplement the five pre-existing monitoring points (SB04, SB07R, SB08, MW02, MW03) in the testing area (Figure 4.1).

Wells PMP1-PMP9 were installed by using the direct-push capabilities of the Argonne CPT in accord with KDHE regulations and procedures in the Master Work Plan (Argonne 2002b). With the approval of the KDHE/BER project manager, wells PMP1-PMP9 were constructed with a single 10 -ft screen across the previously identified aquifer interval at each location (50-60 ft BGL at PMP1-PMP3 and PMP5-PMP9; 48.75-58.75 ft BGL at PMP4), in keeping with the construction of pre-existing monitoring points SB04, SB07R, SB08, MW02, and MW03. Wells PMP1-PMP9 were completed flush with the ground surface with the approval of the KDHE/BOW (KDHE 2008a); the surface completion variance and Kansas water well completion registrations (WWC-5 records) are in Appendix C.

As outlined in the approved Interim Measure Conceptual Design (Argonne 2007a), four new monitoring points (PMP1-PMP3 and PMP8, Figure 4.1) were installed within the pilot test injection field. Monitoring well PMP1 was constructed as a 3-ft offset from injection point PT7, and well PMP2 was installed as a 7.5-ft offset from injection point PT7, near the northern margin of the injection field. (PMP2 is also offset by $7.5 \mathrm{ft}$ from injection point PT2 and pre-injection sampling point PSB2.) Wells PMP3 and PMP8 were installed to coincide with pre-injection groundwater sampling points PSB3 and PSB8, respectively (Figures 2.2 and 4.1). 
Monitoring points PMP4-PMP7 and PMP9 were installed near the inferred upgradient and downgradient margins of the injection area. Wells PMP5-PMP7 and PMP9 were constructed at the locations of corresponding pre-injection sampling points PSB5-PSB7 and PSB9, respectively. Well PMP4 was installed, at the request of the KDHE/BER project manager, at a new location southwest of the injection field and the groundwater contamination hot spot identified at PSB8 (Figures 2.2 and 4.1).

\subsection{Post-Injection Groundwater Data}

\subsubsection{Post-Injection Groundwater Sampling and Analysis Methods}

The installation of monitoring wells PMP1-PMP9 and initial sampling of the complete pilot test monitoring network was delayed until January 8-10, 2008, approximately five weeks after the injection program was completed, because of weather-imposed limitations and logistic concerns at the site. A second groundwater sampling event was conducted approximately two weeks later, on January 24, 2008. Groundwater sampling was subsequently performed approximately monthly from February through September 2008 (in lieu of quarterly sampling after the initial six months, as originally proposed; Argonne 2007a). With the approval of the KDHE/BER, sampling in January 2008 was performed without purging to address concerns regarding potential remobilization of the injected ISCR fluids in the immediate vicinity of the monitoring wells if they were pumped. All subsequent sampling events were performed by using a low-flow purging and sampling technique, at the request of the KDHE/BER project manager, in accord with technical guidance recommended by the KDHE (Puls and Barcelona 1996). Groundwater samples were collected for VOCs analyses during each sampling event. Sample aliquots for analyses of the $\mathrm{Br}^{-}$tracer were collected during the initial post-injection monitoring event (January 8-10, 2008) and in February, April, and August 2008. Aliquots were also collected for selected inorganic geochemical analyses in the February and August 2008 sampling events. All sampling and analyses were performed in accord with the procedures outlined in Section 2.1.1.

The complete results of the post-injection groundwater analyses are on CD, in Supplement 4. The results of the VOCs, DO, and ORP analyses are illustrated over time for each sampling location in Appendix D. 


\subsubsection{Post-Injection Data for Bromide Tracer in Groundwater}

The tracer compound $\mathrm{KBr}$ was added to all water used for formulation of the injected EHC-A solution and EHC slurry, as well as for subsurface flushing of the GeoProbe injection tool, at a $\mathrm{Br}^{-}$concentration of $150 \mathrm{mg} / \mathrm{L}$. The tracer was employed to address two primary technical concerns identified prior to implementation of the injection program:

- To facilitate the detection of potential mixing, and hence dilution, of the groundwater sampled for VOCs and geochemical analyses subsequent to completion of the injection program, by a component of injected water.

- To permit tracking of the potential movement of the injected water into and through the Centralia aquifer zone over time.

As noted in Section 2.1.2.2, $\mathrm{Br}^{-}$levels in the untreated (pre-injection) groundwater in the pilot test area varied by approximately an order of magnitude, ranging from $0.14 \mathrm{mg} / \mathrm{L}$ to $2.0 \mathrm{mg} / \mathrm{L}$. The maximum concentrations $(0.78-1.0 \mathrm{mg} / \mathrm{L}$ and $0.95-2.0 \mathrm{mg} / \mathrm{L}$, respectively) were detected at sampling points PSB8 and PSB5. The $\mathrm{Br}^{-}$concentration in a sample of water from the Centralia municipal system used for mixing of the injection fluids was $0.29 \mathrm{mg} / \mathrm{L}$.

The results of $\mathrm{Br}^{-}$analyses for the groundwater samples collected from the monitoring wells in January, February, April, and August 2008 are summarized in Table 4.1. With the exception of one location (MW02), measured $\mathrm{Br}^{-}$values for the post-injection samples ranged from $<0.1 \mathrm{mg} / \mathrm{L}$ to $32 \mathrm{mg} / \mathrm{L}$ (at PMP3, during the January sampling event). Bromide concentrations exceeding $1.0 \mathrm{mg} / \mathrm{L}$ have thus far been detected only in groundwater samples collected within the injection field (MW02, PMP2, PMP3, and PMP8; Table 4.1). Bromide concentrations detected at well PMP3 have declined steadily during the monitoring period, from $32 \mathrm{mg} / \mathrm{L}$ in January to $1.1 \mathrm{mg} / \mathrm{L}$ in August 2008, while Br- levels at PMP8 have shown a net increase (from $4.7 \mathrm{mg} / \mathrm{L}$ to $9.7 \mathrm{mg} / \mathrm{L}$ ) over the same time period, suggesting possible localized redistribution of the injected fluids within the injection field in the vicinity of these monitoring wells. The observed $\mathrm{Br}^{-}$concentrations at PMP2 have shown no significant changes.

Table 4.1 indicates that erratic $\mathrm{Br}^{-}$results were obtained for groundwater samples recovered from monitoring well MW02 during the January, February, April, and August 2008 sampling events, as well as in additional samples collected for verification of the $\mathrm{Br}^{-}$analyses at 
this location in June and July 2008. Apparent $\mathrm{Br}^{-}$levels exceeding the maximum $\mathrm{Br}^{-}$ concentration $(150 \mathrm{mg} / \mathrm{L})$ originally present in the tagged injection water were reported by TestAmerica (Chicago, Illinois) for the samples collected in April, June, and August 2008, while no detectable $\mathrm{Br}^{-}$was reported in the sample from MW02 in February 2008. TestAmerica has indicated that the anomalous MW02 values indicated for $\mathrm{Br}^{-}$(and several other inorganic constituents; Supplement 4) were influenced by an unresolved interference in the response of the analytical instrument for these samples; hence, the reliability of these values for interpretive purposes is questionable.

The post-injection $\mathrm{Br}^{-}$results have been used to estimate the potential fraction of injection-derived water that might be represented in each of the groundwater samples collected during the January, February, April, and August 2008 sampling events, under the highly conservative assumption that the minimum $\mathrm{Br}^{-}$concentration identified in the pre-treatment groundwater at the site $(0.14 \mathrm{mg} / \mathrm{L}$; Table 2.3) is representative of the ambient groundwater conditions throughout the pilot study area. The results of these calculations, included in Table 4.1, suggest maximum estimated fractions of water derived from the injected fluids of approximately 18-21\% for groundwater samples collected at PMP3 (in the injection field) during the January and February 2008 sampling events and 6-7\% for groundwater samples collected at PMP8 (in the injection field) in April and August 2008. For all other samples, an estimated injection-derived component of $\leq 3 \%$ is indicated. The $\mathrm{Br}^{-}$tracer analyses therefore suggest that little dilution of the groundwater sampled for VOCs analysis at the Centralia pilot site has occurred as a result of direct mixing of the native groundwater with the (uncontaminated) injected water. This interpretation is qualitatively consistent with the relatively low volume of injected water (approximately 3-6\% of the estimated total pore volume targeted for treatment within the injection field; see Section 3.1) employed for the ISCR pilot investigation. ${\mathrm{The} \mathrm{Br}^{-}}^{-}$ tracer analyses also demonstrate that the injected fluids are not being preferentially extracted from the subsurface in response to the low-flow purging and sampling technique used for the pilot test monitoring program.

Bromide concentrations at all monitoring locations outside the injection field (PMP4PMP7, PMP9, SB04, SB07R, SB08, MW03) have been $\leq 0.6 \mathrm{mg} / \mathrm{L}$ throughout the period of record, values comparable to or less than the corresponding pre-treatment $\mathrm{Br}^{-}$levels at these locations. Calculations based on the conservative assumption outlined above yield estimated factors for dilution by uncontaminated injection water of $<1 \%$ for the groundwater samples from these monitoring points. The results, therefore, provide no evidence for migration of the $\left(\mathrm{Br}^{-}-\right.$ 
tagged) injected water beyond the limits of the injection field as of August 2008. This observation is qualitatively consistent with the relatively slow movement of groundwater (and carbon tetrachloride contamination) at Centralia documented previously (Argonne 2005b, 2005c, 2006a,b, 2007b, 2008a).

\subsubsection{Post-Injection Data for Dissolved Oxygen and Oxidation-Reduction Potential in Groundwater Samples}

As noted in Section 1, the Adventus EHC and EHC-A materials are formulated to generate a highly reducing, anaerobic subsurface environment that will foster both thermodynamic and enhanced biological reductive dechlorination of carbon tetrachloride and its primary degradation products (chloroform and methylene chloride). Generally aerobic, relatively oxidizing conditions were identified in the groundwater in the pilot test area prior to the injection of the ISCR fluids (see Section 2.1.2.2 and Figure 2.5).

The DO and ORP levels in groundwater identified during the post-injection monitoring events are summarized in Table 4.2 and Figures 4.2-4.6.

Figures 4.2-4.4 illustrate that significant decreases in both DO and ORP levels were detected in the initial (January 8-10, 2008) post-injection monitoring event at all monitoring locations in and near the pilot test injection field (with the exception of locations SB04, SB07R, SB08, and MW03), in apparent response to the injection of the ISCR amendments. DO concentrations of $0.08-3.11 \mathrm{mg} / \mathrm{L}$ and ORP levels of -189 to $-556 \mathrm{mV}$ were detected, with the lowest values for these parameters (DO $<1 \mathrm{mg} / \mathrm{L}$, ORP $<-500 \mathrm{mV}$ ) occurring exclusively within the injection field. The minimum DO and ORP values noted above are consistent with experimental and field results previously reported by Adventus in response to application of the EHC product (Adventus 2006, 2008b-f). These values are conducive to both abiotic and enhanced biological reductive dechlorination of carbon tetrachloride.

The results of continued monitoring of DO and ORP in the pilot test area are illustrated as time series data plots for the individual monitoring points in Appendix D; combined data for the February to September 2008 sampling events are summarized in Figure 4.5. The trends in DO and ORP levels observed at the monitoring points since the initial post-injection sampling event (January 8-10, 2008; Figure 4.2) have varied in detail among the individual wells; however, the results for all monitoring points (with the exception of locations SB04, SB07R, SB08, and 
MW03) show an increase in ORP from minimum levels generally achieved during the initial or second January sampling events. As shown in Figure 4.5, DO and ORP levels at monitoring points outside the injection field have returned to values similar to the pre-injection baseline levels (Figure 2.5). Maximum transient DO concentrations were recorded at most locations during the July or August 2008 sampling events. (See the time series plots in Appendix D.)

The ORP levels detected at wells SB04, SB07R, SB08, and MW03 have remained positive $(>50 \mathrm{mV}$ ) throughout the post-injection monitoring period, and no decrease in DO concentrations was detected at these locations during the initial post-injection monitoring event (January 8-10, 2008). The DO and ORP levels observed at these locations have fluctuated during the period of monitoring but have shown little net change, as of September 2008, relative to the corresponding pre-treatment baseline levels.

The data presented in Figures 4.6 and 4.7 for the September 2008 sampling event illustrate that measured DO and ORP levels at monitoring points in the injection field remain lower than both the pre-injection baseline levels at these locations and the contemporaneous concentrations at the surrounding monitoring wells outside the injection field. Since February 2008, the observed levels of DO (0.03-3.52 mg/L) and ORP (-192.6 mV to $44.7 \mathrm{mV})$ within the injection field have shown periodic fluctuations but have predominantly remained at levels that might locally support potential biological reductive dechlorination of carbon tetrachloride but are insufficient to promote the thermodynamic breakdown of this contaminant.

\subsubsection{Post-Injection Supplemental Geochemical Data for Groundwater}

Groundwater obtained from monitoring points within the injection field (particularly PMP3, PMP8, and MW02) under the reducing, oxygen-depleted conditions represented in Figures 4.6 and 4.7 is dark gray to black in color (Figure 4.8) and has a very strong, unpleasant, fetid odor. Groundwater samples obtained from the pilot test monitoring points outside the injection field (PMP4-PMP7, PMP9, SB04, SB07R, SB08, MW03) are generally clear and colorless to cloudy tan with some fine sediment, with no distinctive odor.

Analyses of selected anions (chloride, nitrate, and sulfate) were performed for the groundwater samples collected from the 14 pilot test monitoring wells in February 2008, and analyses of selected metals and anions were conducted for these wells in August 2008. 
Comparison of these results (Supplement 4) to equivalent data for groundwater samples collected in September 2007 from the full suite of permanent monitoring wells at Centralia (Argonne 2008a) and for samples collected at pre-injection points PSB1-PSB9 in November 2007 (Supplement 2) suggests that the ISCR treatment has not significantly affected the major ion chemistry of the groundwater in and near the injection field. The August 2008 analyses suggest, however, that within the injection field, the post-injection concentrations of magnesium, manganese, and iron at monitoring points PMP3 and PMP8 and the post-injection iron and manganese concentrations at PMP1 and PMP2 are slightly elevated relative to the levels of these elements detected in the remaining pilot test wells sampled in August 2008 and in the Centralia wells sampled in 2007. The dissolved iron concentrations identified at monitoring wells PMP1, PMP3, and PMP8 in August 2008 (0.42-7.6 mg/L) exceeded the level for this contaminant $(0.3 \mathrm{mg} / \mathrm{L})$ recommended under the National Secondary Drinking Water Regulations (EPA 2008). Iron was not detected above this regulatory level in groundwater collected at these locations in the pre-injection baseline sampling event (see Table S4.3).

\subsubsection{Post-Injection Data for VOCs in Groundwater}

The results of the post-injection VOCs analyses of groundwater samples are summarized in Table 4.3 and Figures 4.9-4.12. The concentrations of carbon tetrachloride, chloroform, and methylene chloride detected at the individual groundwater monitoring points are illustrated as time series plots in Appendix D. Permanent monitoring wells PMP1 and PMP2 were installed as offsets from injection point PT7, and well PMP4 (Figure 4.1) was installed at a new location requested by the KDHE/BER project manager upon completion of the baseline study. Wells PMP1, PMP2, and PMP4 do not directly correspond to locations sampled during the baseline study (see Section 4.1). Comparisons of pre- and post-injection contaminant levels for PMP1 and PMP2 noted in this section are therefore based on the average of the baseline contaminant concentrations determined for nearby pre-injection sampling points PSB1 and PSB2 (Figure 4.1). No baseline sampling analog is available for the PMP4 well location. Comparisons of pre- and post-injection contaminant levels at PMP3 and PMP5-PMP9 are based on the average contaminant concentrations measured at corresponding pre-injection sampling points PSB3 and PSB5-PSB9 (see Figure 4.1 and Table 2.1).

With the exception of two locations (PMP2 and PMP9), carbon tetrachloride concentrations determined at all of the monitoring points showed a decrease during the initial post-injection sampling event (January 8-10, 2008; Figure 4.9) relative to corresponding pre- 
injection baseline values (Figure 2.3). The decreases in carbon tetrachloride concentrations at locations MW02, PMP3, and PMP8 in the injection field were the most dramatic (Figure 4.10), at approximately 1-2 orders of magnitude. The decreases in carbon tetrachloride observed at PMP3 and PMP8 were accompanied by significant increases in the concentrations of associated chloroform at these locations. Methylene chloride became detectable at MW02 and PMP3 and was present at enhanced levels at PMP8. These observations together provide evidence for the probable occurrence of reductive dechlorination of carbon tetrachloride at these locations.

Decreased levels of carbon tetrachloride were also identified at monitoring wells PMP1, PMP5-PMP7, SB04, SB07R, SB08, and MW03 during the initial post-injection (January 8-10, 2008) and/or second post-injection (January 24, 2008) sampling event (Figures 4.9 and 4.10). Increases in associated concentrations of both chloroform and methylene chloride were detected at monitoring wells PMP1, PMP5, and PMP6; increased chloroform (only) was identified at SB04; and increased methylene chloride (only) was observed at PMP7, in conjunction with a decreased chloroform concentration. These observations also suggest the possible effects of reductive dechlorination; however, as noted in Section 4.2.2, the results of $\mathrm{Br}^{-}$tracer analyses do not support the direct presence of injected ISCR fluids at these locations. The results of the VOCs analyses at monitoring wells MW03, SB07R, and SB08 provide no evidence for reductive dechlorination as a possible mechanism for the initial (January 8-10, 2008) post-injection decreases in carbon tetrachloride observed at these locations.

Concentrations of carbon tetrachloride greater than the pre-injection baseline values were observed during the initial post-injection sampling event (January 8-10, 2008) at PMP9 and PMP2 (Figures 4.9 and 4.10). The absolute increase identified at PMP9 was small (results of "not detected" [ND] and $1.4 \mu \mathrm{g} / \mathrm{L}$ pre-injection, versus $1.9 \mu \mathrm{g} / \mathrm{L}$ post-injection). Levels of carbon tetrachloride (980 $\mu \mathrm{g} / \mathrm{L})$, chloroform $(951 \mu \mathrm{g} / \mathrm{L})$, and methylene chloride $(4.2 \mu \mathrm{g} / \mathrm{L})$ detected at PMP2, however, significantly exceed the average baseline levels of these contaminants at this location (estimated from results for nearby sampling points PSB1 and PSB2 at $378 \mu \mathrm{g} / \mathrm{L}$, $10.9 \mu \mathrm{g} / \mathrm{L}$, and ND, respectively, for carbon tetrachloride, chloroform, and methylene chloride). The analytical results for monitoring well PMP2 are discussed in greater detail below.

The results of extended monitoring of VOCs levels in the pilot test area are illustrated as time series data plots for the individual monitoring wells in Appendix D. Figures 4.11 and 4.12 present the concentrations of carbon tetrachloride and chloroform, respectively, identified in groundwater for the September 2008 sampling event. 
The time series diagrams in Appendix D show that the detailed trends in VOCs concentrations during the period of record differ significantly among the individual monitoring points, with relatively few consistent patterns. Review of the complete data set, however, suggests the following preliminary observations concerning the progress of the pilot groundwater restoration effort to date, in the context of the $\mathrm{Br}^{-}$tracer and inorganic geochemical observations presented in Sections 4.2.2-4.2.4.

- The carbon tetrachloride concentrations at all monitoring points have shown little or no further net decrease since the initial (January 8-10, 2008) postinjection sampling event.

- The dramatically lower carbon tetrachloride concentrations observed in the injection field at MW02, PMP3, and PMP8 increased somewhat during the monitoring period but remained near their initial post-injection levels, reflecting a persistent decrease of 96-99\% relative to the baseline concentrations at these points.

- As of the September 2008 sampling event, the concentrations of carbon tetrachloride (18-1,854 $\mu \mathrm{g} / \mathrm{L})$ at all monitoring locations in the injection field (PMP1, PMP2, PMP3, PMP8, MW02) exceeded the KDHE Tier 2 RBSL and the EPA maximum contaminant level of $5.0 \mu \mathrm{g} / \mathrm{L}$ for this contaminant in groundwater.

- Increasing levels of chloroform and methylene chloride have been observed at well MW02, while chloroform levels have decreased slowly in the injection field at PMP2, PMP3 and PMP8. The chloroform and methylene chloride concentrations at MW02, PMP2, PMP3, and PMP8 remained above the respective baseline levels as of the September 2008 sampling event. The concentrations of chloroform identified at wells PMP2 and PMP8 (318 $\mu \mathrm{g} / \mathrm{L}$ and $125 \mu \mathrm{g} / \mathrm{L}$, respectively) in September 2008 exceeded the KDHE Tier 2 RBSL of $80 \mu \mathrm{g} / \mathrm{L}$ for this contaminant in groundwater.

- Measured carbon tetrachloride concentrations at monitoring wells PMP1 (at the northern margin of the injection field) and PMP4-PMP7 (to the northeast, south, and southwest of the injection field) reached a transient minimum in the 
monitoring events from January 24, 2008, to March 2008. The carbon tetrachloride concentrations at these locations have since returned to levels observed during the baseline or initial (January 8-10, 2008) post-injection sampling events.

- Chloroform concentrations at PMP1 and PMP4-PMP7 have decreased slightly relative to more elevated values identified in the initial (January 8-10, 2008) post-injection sampling, but they remain comparable to or slightly higher than the corresponding baseline concentrations at these locations.

- The carbon tetrachloride concentrations observed at monitoring wells SB04, SB07R, SB08, and MW03 have shown little increase or decrease from their initial (January 8-10, 2008) post-injection levels (see Appendix D, Figures D.12-D.14 and D.2). Only trace concentrations $(<2 \mu \mathrm{g} / \mathrm{L})$ of chloroform or methylene chloride, comparable to baseline levels, have been detected at these locations.

Carbon tetrachloride and chloroform concentrations of $980 \mu \mathrm{g} / \mathrm{L}$ and $951 \mu \mathrm{g} / \mathrm{L}$, respectively, were detected at monitoring point PMP2 during the initial (January 8-10, 2008) post-injection sampling event. As noted above, chloroform levels at PMP2 have decreased slowly with time; however, the observed concentrations of carbon tetrachloride have generally increased since the initial post-injection sampling event, reaching a transient maximum of 2,873 $\mu \mathrm{g} / \mathrm{L}$ in June 2008 before decreasing to $1,315 \mu \mathrm{g} / \mathrm{L}$ in August, then rising to 1,854 $\mu \mathrm{g} / \mathrm{L}$ in September 2008. With two apparent exceptions (for the January 24 and February 2008 monitoring events), carbon tetrachloride concentrations at well PMP1 have also consistently been $>100 \mu \mathrm{g} / \mathrm{L}$ and have shown no decreasing trend. The carbon tetrachloride concentrations at these two locations represent the highest values consistently observed in the injection field; they contrast markedly with the reduced concentrations that have been documented at nearby monitoring points MW02, PMP3, and PMP8.

Possible cause(s) of the persistent high contaminant levels at PMP1 and PMP2 have not been conclusively identified; however, postulated factors that might influence the effectiveness of the ISCR treatment process at these points include the following: 
- Wells PMP1 and PMP2 are offset from the nearest pre-injection baseline sampling points (PSB1 and PSB2; Figure 4.1) by $\geq 7.5 \mathrm{ft}$. The observed carbon tetrachloride levels at PMP1 and PMP2 may therefore in part reflect highly localized, pre-existing heterogeneity in the original groundwater plume.

- With the KDHE's approval, three injection points (PT1, PT6, PT11) originally planned near PMP1 and PMP2 (Figure 2.2) were relocated (Figure 3.1), placing PMP1 and PMP2 along the northern margin of the injection field. In addition, a fourth injection point (PT8; Figure 2.2) originally planned near PMP1 and PMP2 was omitted to reduce the potential for daylighting at monitoring well MW02. Finally, significant daylighting of the ISCR fluids injected at point PT7 was observed toward the southeast and away from PMP1 and PMP2 (Section 3.2.1; Figure 3.2). These factors together might have limited the distribution of the ISCR amendments in the vicinity of PMP1 and PMP2, in comparison to the more central portions of the injection field.

- Injection of the ISCR amendments was performed in both vadose zone soils and the saturated zone at the injection points (PT2, PT7, PT12; Figure 3.1) nearest locations PMP1 and PMP2. Relatively low pre-injection levels of carbon tetrachloride were identified in the vadose zone soils near PMP1 and PMP2 (at PSB1 and PSB2; Section 2.2.2, Figure 2.8). The possibility that carbon tetrachloride was mobilized from these soils into the underlying groundwater as a result of the injection process cannot be ruled out, however, on the basis of presently available data. (See Section 4.3.)

\subsection{Post-Injection Data for VOCs in Vadose Zone Soil}

Vertical-profile soil coring and sampling for VOCs analyses were performed in conjunction with the August 2008 monitoring event, at a 3-ft offset (PSB13; Figure 3.4) from previously sampled locations PSB1 and SB12, to investigate the effects of the ISCR treatment in the vadose zone soils at this location. The soils were collected at 2-ft intervals, from $2 \mathrm{ft}$ to $40 \mathrm{ft}$ BGL. The results of these analyses (Table 4.4) are compared to the baseline analysis results for sampling point PSB1 in Figure 4.13. The carbon tetrachloride concentrations in the treated soils at PSB13 ranged from ND to $61 \mu \mathrm{g} / \mathrm{kg}$, all below the KDHE Tier 2 RBSL of $200 \mu \mathrm{g} / \mathrm{kg}$ for this 
contaminant in soils. The highest levels of carbon tetrachloride detected (10-61 $\mu \mathrm{g} / \mathrm{kg})$ were observed in the samples collected at the greatest depth (34-40 ft BGL). Quantifiable levels of chloroform (13-17 $\mu \mathrm{g} / \mathrm{kg}$ ) were identified at multiple depths from $14 \mathrm{ft}$ to $32 \mathrm{ft}$ BGL, and methylene chloride was detected $(11 \mu \mathrm{g} / \mathrm{kg})$ in one soil sample collected at $18 \mathrm{ft}$ BGL. The observed carbon tetrachloride levels in the treated soils at PSB13 showed an apparent decrease in concentration, at all depths, relative to the corresponding values identified in pre-treatment boring PSB1 (and earlier Argonne boring SB12; Figures 4.13 and 2.10).

As noted in Section 4.2.5, physical-chemical mobilization of contamination from the vadose zone soils (potentially into the underlying saturated zone soils and groundwater) in response to the injection of the ISCR fluids near borings PSB1, PSB12, and PSB13 cannot be ruled out as a possible mechanism affecting the carbon tetrachloride concentrations identified in the soils at PSB13. The increased (relative to baseline) levels of chloroform and the detection of methylene chloride at PSB13 suggest, however, that degradation of the carbon tetrachloride (by reductive dechlorination) in response to the ISCR amendments has also contributed to the reductions in carbon tetrachloride concentrations observed in the treated vadose zone soils.

\subsection{Estimated Costs of the Pilot ISCR Investigation}

The estimated costs for the pilot ISCR treatment study at Centralia, including materials, field operations, and required technical oversight, are as follows:

- $\quad$ Pre-injection baseline study

- Injection program

- Post-injection monitoring
$\$ 57,214$

$\$ 372,764$

$\$ 216,550$

The total cost for the pilot ISCR investigation, as of the end of August 2008, is $\$ 646,528$.

The pre-injection baseline study costs include charges for field sampling activities and laboratory analyses related to the pilot investigation in September-November 2007. The estimated costs of the injection program include remedial implementation charges for materials and services provided by Adventus and Redox, as well as additional required support staff, 
administration and oversight of the field operations by Argonne personnel, outside laboratory analyses, and other field work costs. Post-injection monitoring costs include charges for field sampling activities and laboratory analyses of soil and groundwater samples beginning in December 2007 and continuing through August 2008. Reporting costs are not included in the breakdown provided. 
TABLE 4.1 Results of bromide analyses for groundwater samples collected during the ISCR pilot study post-injection sampling events.

\begin{tabular}{|c|c|c|c|c|c|}
\hline Location & Sample & $\begin{array}{c}\text { Sample } \\
\text { Date }\end{array}$ & $\begin{array}{c}\text { Depth } \\
\text { (ft BGL) }\end{array}$ & $\begin{array}{c}\text { Bromide } \\
\text { (mg/L) }\end{array}$ & $\begin{array}{c}\text { Estimated Fraction }{ }^{\mathrm{a}} \\
\text { of Injected Fluid in } \\
\text { Sample } \\
(\%)\end{array}$ \\
\hline \multirow[t]{6}{*}{ MW02 } & CNMW02-W-26074 & $1 / 9 / 08$ & $49.5-59.5$ & 4.9 & 3.2 \\
\hline & CNMW02-W-26099 & 2/23/08 & & $5 U^{b}$ & $\mathrm{NC}^{\mathrm{C}}$ \\
\hline & CNMW02-W-26045 & $4 / 24 / 08$ & & $350^{d}$ & NC \\
\hline & CNMW02-W-26620 & 6/4/08 & & $490^{d}$ & NC \\
\hline & CNMW02-W-26639 & $7 / 8 / 08$ & & $5 U$ & $\mathrm{NC}$ \\
\hline & CNMW02-W-26658 & $8 / 6 / 08$ & & $250^{d}$ & NC \\
\hline \multirow[t]{4}{*}{ MW03 } & CNMW03-W-26078 & 1/10/08 & $50.5-60.5$ & 0.27 & 0.1 \\
\hline & CNMW03-W-26115 & $2 / 23 / 08$ & & 0.11 & $\mathrm{NC}$ \\
\hline & CNMW03-W-26046 & $4 / 23 / 08$ & & 0.27 & 0.1 \\
\hline & CNMW03-W-26659 & 8/6/08 & & 0.27 & 0.1 \\
\hline \multirow[t]{4}{*}{ PMP1 } & CNPMP1-W-26079 & 1/10/08 & $50-60$ & 0.43 & 0.2 \\
\hline & CNPMP1-W-25985 & $2 / 24 / 08$ & & 0.32 & 0.1 \\
\hline & CNPMP1-W-26050 & $4 / 24 / 08$ & & 0.36 & 0.1 \\
\hline & CNPMP1-W-26663 & 8/6/08 & & 0.52 & 0.3 \\
\hline \multirow[t]{4}{*}{ PMP2 } & CNPMP2-W-26081 & $1 / 10 / 08$ & $50-60$ & 1.4 & 0.8 \\
\hline & CNPMP2-W-25986 & $2 / 24 / 08$ & & 1.1 & 0.6 \\
\hline & CNPMP2-W-26051 & $4 / 24 / 08$ & & 1.2 & 0.7 \\
\hline & CNPMP2-W-26664 & 8/6/08 & & 0.84 & 0.5 \\
\hline \multirow[t]{4}{*}{ PMP3 } & CNPMP3-W-26071 & $1 / 9 / 08$ & $50-60$ & 32 & 21.3 \\
\hline & CNPMP3-W-25990 & $2 / 25 / 08$ & & 27 & 17.9 \\
\hline & CNPMP3-W-26052 & $4 / 24 / 08$ & & 2.1 & 1.3 \\
\hline & CNPMP3-W-26665 & 8/6/08 & & 1.1 & 0.6 \\
\hline \multirow[t]{4}{*}{ PMP4 } & CNPMP4-W-26068 & $1 / 9 / 08$ & $48.75-58.75$ & 0.27 & 0.1 \\
\hline & CNPMP4-W-25992 & $2 / 25 / 08$ & & 0.30 & 0.1 \\
\hline & CNPMP4-W-26053 & $4 / 24 / 08$ & & 0.37 & 0.2 \\
\hline & CNPMP4-W-26666 & 8/6/08 & & 0.36 & 0.1 \\
\hline \multirow[t]{4}{*}{ PMP5 } & CNPMP5-W-26069 & $1 / 9 / 08$ & $50-60$ & 0.30 & 0.1 \\
\hline & CNPMP5-W-25997 & $2 / 25 / 08$ & & 0.40 & 0.2 \\
\hline & CNPMP5-W-26054 & 4/24/08 & & 0.54 & 0.3 \\
\hline & CNPMP5-W-26667 & $8 / 6 / 08$ & & 0.61 & 0.3 \\
\hline \multirow[t]{4}{*}{ PMP6 } & CNPMP6-W-26065 & $1 / 8 / 08$ & $50-60$ & 0.28 & 0.1 \\
\hline & CNPMP6-W-25993 & $2 / 25 / 08$ & & 0.17 & 0.0 \\
\hline & CNPMP6-W-26055 & $4 / 24 / 08$ & & 0.32 & 0.1 \\
\hline & CNPMP6-W-26668 & 8/6/08 & & 0.28 & 0.1 \\
\hline \multirow[t]{4}{*}{ PMP7 } & CNPMP7-W-26066 & $1 / 8 / 08$ & $50-60$ & 0.59 & 0.3 \\
\hline & CNPMP7-W-25996 & $2 / 25 / 08$ & & 0.49 & 0.2 \\
\hline & CNPMP7-W-26056 & $4 / 24 / 08$ & & 0.52 & 0.3 \\
\hline & CNPMP7-W-26669 & $8 / 6 / 08$ & & 0.55 & 0.3 \\
\hline \multirow[t]{4}{*}{ PMP8 } & CNPMP8-W-26075 & $1 / 9 / 08$ & $50-60$ & 4.7 & 3.0 \\
\hline & CNPMP8-W-25994 & $2 / 25 / 08$ & & 1.3 & 0.8 \\
\hline & CNPMP8-W-26057 & 4/24/08 & & 11 & 7.2 \\
\hline & CNPMP8-W-26670 & 8/6/08 & & 9.7 & 6.4 \\
\hline
\end{tabular}


TABLE 4.1 (Cont.)

\begin{tabular}{|c|c|c|c|c|c|}
\hline Location & Sample & $\begin{array}{l}\text { Sample } \\
\text { Date }\end{array}$ & $\begin{array}{l}\text { Depth } \\
\text { (ft BGL) }\end{array}$ & $\begin{array}{c}\text { Bromide } \\
(\mathrm{mg} / \mathrm{L})\end{array}$ & $\begin{array}{l}\text { Estimated Fraction }{ }^{a} \\
\text { of Injected Fluid in } \\
\text { Sample } \\
(\%)\end{array}$ \\
\hline \multirow[t]{4}{*}{ PMP9 } & CNPMP9-W-26077 & 1/10/08 & \multirow[t]{4}{*}{$50-60$} & 0.14 & 0.0 \\
\hline & CNPMP9-W-25989 & $2 / 24 / 08$ & & $0.1 \cup$ & NC \\
\hline & CNPMP9-W-26058 & $4 / 24 / 08$ & & $0.1 \cup$ & NC \\
\hline & CNPMP9-W-26671 & 8/6/08 & & 0.13 & 0.0 \\
\hline \multirow[t]{4}{*}{ SB04 } & CNSB04-W-26076 & 1/10/08 & \multirow[t]{4}{*}{$51-61$} & 0.26 & 0.1 \\
\hline & CNSB04-W-26117 & 2/23/08 & & $0.1 \mathrm{U}$ & $\mathrm{NC}$ \\
\hline & CNSB04-W-26047 & $4 / 24 / 08$ & & $0.1 \mathrm{U}$ & NC \\
\hline & CNSB04-W-26660 & 8/6/08 & & $0.2 \mathrm{U}$ & $\mathrm{NC}$ \\
\hline \multirow[t]{4}{*}{ SB07R } & CNSB07R-W-26073 & $1 / 9 / 08$ & \multirow[t]{4}{*}{$45-60$} & $0.1 \mathrm{U}$ & $\mathrm{NC}$ \\
\hline & CNSB07R-W-25984 & $2 / 24 / 08$ & & $0.1 \mathrm{U}$ & NC \\
\hline & CNSB07R-W-26048 & $4 / 24 / 08$ & & $0.1 \mathrm{U}$ & $\mathrm{NC}$ \\
\hline & CNSB07R-W-26661 & 8/6/08 & & $0.2 \cup$ & NC \\
\hline \multirow[t]{4}{*}{ SB08 } & CNSB08-W-26070 & $1 / 9 / 08$ & \multirow[t]{4}{*}{$52-62$} & $0.1 \mathrm{U}$ & NC \\
\hline & CNSB08-W-25978 & 2/24/08 & & $0.1 \mathrm{U}$ & $\mathrm{NC}$ \\
\hline & CNSB08-W-26049 & $4 / 23 / 08$ & & $0.1 \mathrm{U}$ & NC \\
\hline & CNSB08-W-26662 & 8/6/08 & & $0.2 \cup$ & NC \\
\hline
\end{tabular}

a Mass balance calculation assumed a bromide concentration of $0.14 \mathrm{mg} / \mathrm{L}$ in the natural formation water.

b $U$, not detected at indicated reporting limit.

c NC, no value calculated.

d Analytical interference was reported in determination of values by TestAmerica. 
TABLE 4.2 Results of dissolved oxygen and oxidation-reduction potential analyses for groundwater samples collected during the ISCR pilot study postinjection sampling events.

\begin{tabular}{|c|c|c|c|c|c|}
\hline Location & Sample & $\begin{array}{l}\text { Sample } \\
\text { Date }\end{array}$ & $\begin{array}{l}\text { Depth } \\
\text { (ft BGL) }\end{array}$ & $\begin{array}{l}\text { Dissolved } \\
\text { Oxygen } \\
\text { (mg/L) }\end{array}$ & $\begin{array}{l}\text { ORP } \\
(\mathrm{mV})\end{array}$ \\
\hline \multirow[t]{9}{*}{ MW02 } & CNMW02-W-26074 & $1 / 9 / 08$ & $49.5-59.5$ & 0.17 & -218 \\
\hline & CNMW02-W-26097 & $1 / 24 / 08$ & & 0.96 & 89.0 \\
\hline & CNMW02-W-26099 & $2 / 23 / 08$ & & 2.40 & 44.7 \\
\hline & CNMW02-W-26000 & 3/12/08 & & 0.28 & -41.5 \\
\hline & CNMW02-W-26045 & $4 / 24 / 08$ & & 0.11 & -53.9 \\
\hline & CNMW02-W-26620 & $6 / 4 / 08$ & & 0.09 & -118 \\
\hline & CNMW02-W-26639 & $7 / 8 / 08$ & & 1.63 & -49.3 \\
\hline & CNMW02-W-26658 & $8 / 6 / 08$ & & 0.33 & -74.7 \\
\hline & CNMW02-W-26674 & 9/8/08 & & 0.40 & -73.5 \\
\hline \multirow[t]{8}{*}{ MW03 } & CNMW03-W-26078 & $1 / 10 / 08$ & $50.5-60.5$ & 10.31 & 113 \\
\hline & CNMW03-W-26115 & $2 / 23 / 08$ & & 7.19 & 246 \\
\hline & CNMW03-W-26001 & $3 / 12 / 08$ & & 7.90 & 88.5 \\
\hline & CNMW03-W-26046 & $4 / 23 / 08$ & & 5.46 & 177 \\
\hline & CNMW03-W-26621 & $6 / 4 / 08$ & & 5.61 & 123 \\
\hline & CNMW03-W-26640 & 7/7/08 & & 6.23 & 148 \\
\hline & CNMW03-W-26659 & 8/6/08 & & 1.98 & 208 \\
\hline & CNMW03-W-26675 & $9 / 9 / 08$ & & 9.60 & 66.0 \\
\hline \multirow{9}{*}{ PMP1 } & CNPMP1-W-26079 & $1 / 10 / 08$ & $50-60$ & 3.11 & -222 \\
\hline & CNPMP1-W-26083 & $1 / 24 / 08$ & & 1.01 & -357 \\
\hline & CNPMP1-W-25985 & $2 / 24 / 08$ & & 3.24 & 25.8 \\
\hline & CNPMP1-W-26005 & 3/13/08 & & - & - \\
\hline & CNPMP1-W-26050 & $4 / 24 / 08$ & & 0.21 & -95.1 \\
\hline & CNPMP1-W-26625 & $6 / 5 / 08$ & & 0.46 & -112 \\
\hline & CNPMP1-W-26644 & $7 / 8 / 08$ & & 0.47 & -53.1 \\
\hline & CNPMP1-W-26663 & 8/6/08 & & 3.52 & -48.2 \\
\hline & CNPMP1-W-26689 & 9/9/08 & & 1.37 & 39.9 \\
\hline \multirow[t]{9}{*}{ PMP2 } & CNPMP2-W-26081 & $1 / 10 / 08$ & $50-60$ & 0.18 & -556 \\
\hline & CNPMP2-W-26084 & $1 / 24 / 08$ & & 1.40 & -266 \\
\hline & CNPMP2-W-25986 & $2 / 24 / 08$ & & 2.05 & -86.9 \\
\hline & CNPMP2-W-26006 & 3/13/08 & & - & - \\
\hline & CNPMP2-W-26051 & $4 / 24 / 08$ & & 0.28 & -97.9 \\
\hline & CNPMP2-W-26626 & $6 / 5 / 08$ & & 0.34 & -101 \\
\hline & CNPMP2-W-26645 & $7 / 8 / 08$ & & 0.16 & -65.7 \\
\hline & CNPMP2-W-26664 & $8 / 6 / 08$ & & 2.87 & -66.2 \\
\hline & CNPMP2-W-26690 & 9/9/08 & & 0.05 & -41.1 \\
\hline \multirow[t]{9}{*}{ PMP3 } & CNPMP3-W-26071 & $1 / 9 / 08$ & $50-60$ & 0.15 & -550 \\
\hline & CNPMP3-W-26086 & $1 / 24 / 08$ & & 0.26 & -344 \\
\hline & CNPMP3-W-25990 & $2 / 25 / 08$ & & 0.62 & -17.4 \\
\hline & CNPMP3-W-26007 & 3/13/08 & & 0.33 & -88.3 \\
\hline & CNPMP3-W-26052 & $4 / 24 / 08$ & & 0.18 & -176 \\
\hline & CNPMP3-W-26627 & $6 / 5 / 08$ & & 0.03 & -193 \\
\hline & CNPMP3-W-26646 & $7 / 8 / 08$ & & 0.21 & -119 \\
\hline & CNPMP3-W-26665 & 8/6/08 & & 1.43 & -138 \\
\hline & CNPMP3-W-26691 & 9/9/08 & & 0.03 & -150 \\
\hline
\end{tabular}


TABLE 4.2 (Cont.)

\begin{tabular}{|c|c|c|c|c|c|}
\hline Location & Sample & $\begin{array}{c}\text { Sample } \\
\text { Date }\end{array}$ & $\begin{array}{c}\text { Depth } \\
\text { (ft BGL) }\end{array}$ & $\begin{array}{c}\text { Dissolved } \\
\text { Oxygen } \\
(\mathrm{mg} / \mathrm{L})\end{array}$ & $\begin{array}{l}\text { ORP } \\
(\mathrm{mV})\end{array}$ \\
\hline \multirow[t]{9}{*}{ PMP4 } & CNPMP4-W-26068 & $1 / 9 / 08$ & $48.75-58.75$ & 2.01 & -213 \\
\hline & CNPMP4-W-26087 & $1 / 24 / 08$ & & 2.35 & -306 \\
\hline & CNPMP4-W-25992 & $2 / 25 / 08$ & & 2.36 & -209 \\
\hline & CNPMP4-W-26008 & $3 / 13 / 08$ & & - & - \\
\hline & CNPMP4-W-26053 & $4 / 24 / 08$ & & 2.91 & 66.0 \\
\hline & CNPMP4-W-26628 & 6/5/08 & & 3.82 & 12.0 \\
\hline & CNPMP4-W-26647 & $7 / 7 / 08$ & & 12.13 & 97.4 \\
\hline & CNPMP4-W-26666 & $8 / 6 / 08$ & & 11.61 & 65.9 \\
\hline & CNPMP4-W-26692 & 9/9/08 & & 4.87 & 134 \\
\hline \multirow[t]{9}{*}{ PMP5 } & CNPMP5-W-26069 & $1 / 9 / 08$ & $50-60$ & 2.45 & -189 \\
\hline & CNPMP5-W-26088 & $1 / 24 / 08$ & & 2.23 & 33.0 \\
\hline & CNPMP5-W-25997 & $2 / 25 / 08$ & & 2.02 & 150 \\
\hline & CNPMP5-W-26009 & $3 / 13 / 08$ & & 1.83 & 221 \\
\hline & CNPMP5-W-26054 & $4 / 24 / 08$ & & 1.04 & 154 \\
\hline & CNPMP5-W-26629 & 6/4/08 & & 1.25 & -107 \\
\hline & CNPMP5-W-26648 & $7 / 8 / 08$ & & 3.93 & 111 \\
\hline & CNPMP5-W-26667 & $8 / 6 / 08$ & & 0.43 & 163 \\
\hline & CNPMP5-W-26693 & 9/10/08 & & 2.51 & 117 \\
\hline \multirow[t]{9}{*}{ PMP6 } & CNPMP6-W-26065 & $1 / 8 / 08$ & $50-60$ & 0.96 & -276 \\
\hline & CNPMP6-W-26090 & $1 / 24 / 08$ & & 3.36 & -155 \\
\hline & CNPMP6-W-25993 & $2 / 25 / 08$ & & 3.18 & 133 \\
\hline & CNPMP6-W-26010 & $3 / 13 / 08$ & & 3.31 & 231 \\
\hline & CNPMP6-W-26055 & $4 / 24 / 08$ & & 2.67 & 197 \\
\hline & CNPMP6-W-26630 & $6 / 5 / 08$ & & 2.94 & 99.9 \\
\hline & CNPMP6-W-26649 & $7 / 7 / 08$ & & 9.96 & 108 \\
\hline & CNPMP6-W-26668 & $8 / 6 / 08$ & & 9.13 & 88.6 \\
\hline & CNPMP6-W-26694 & $9 / 8 / 08$ & & 3.32 & 173 \\
\hline \multirow[t]{9}{*}{ PMP7 } & CNPMP7-W-26066 & $1 / 8 / 08$ & $50-60$ & 1.01 & -381 \\
\hline & CNPMP7-W-26091 & $1 / 24 / 08$ & & 2.9 & -201 \\
\hline & CNPMP7-W-25996 & $2 / 25 / 08$ & & 2.73 & 129 \\
\hline & CNPMP7-W-26011 & $3 / 13 / 08$ & & 3.20 & 218 \\
\hline & CNPMP7-W-26056 & $4 / 24 / 08$ & & 3.32 & 198 \\
\hline & CNPMP7-W-26631 & $6 / 5 / 08$ & & 3.77 & 94.6 \\
\hline & CNPMP7-W-26650 & $7 / 7 / 08$ & & 12.48 & 106 \\
\hline & CNPMP7-W-26669 & $8 / 6 / 08$ & & 9.67 & 85.0 \\
\hline & CNPMP7-W-26695 & 9/9/08 & & 2.18 & 14.7 \\
\hline \multirow[t]{9}{*}{ PMP8 } & CNPMP8-W-26075 & $1 / 9 / 08$ & $50-60$ & 0.08 & -548 \\
\hline & CNPMP8-W-26092 & $1 / 24 / 08$ & & 1.01 & -201 \\
\hline & CNPMP8-W-25994 & $2 / 25 / 08$ & & 0.31 & -121.8 \\
\hline & CNPMP8-W-26012 & $3 / 13 / 08$ & & - & - \\
\hline & CNPMP8-W-26057 & $4 / 24 / 08$ & & 0.18 & -186 \\
\hline & CNPMP8-W-26632 & $6 / 5 / 08$ & & 0.06 & -190 \\
\hline & CNPMP8-W-26651 & $7 / 8 / 08$ & & 0.08 & -135 \\
\hline & CNPMP8-W-26670 & $8 / 6 / 08$ & & 1.30 & -158 \\
\hline & CNPMP8-W-26696 & 9/9/08 & & 0.03 & -129 \\
\hline \multirow[t]{4}{*}{ PMP9 } & CNPMP9-W-26077 & $1 / 10 / 08$ & $50-60$ & 0.59 & -323 \\
\hline & CNPMP9-W-26109 & $1 / 24 / 08$ & & 2.64 & -244 \\
\hline & CNPMP9-W-25989 & $2 / 24 / 08$ & & 3.53 & -22.8 \\
\hline & CNPMP9-W-26013 & $3 / 13 / 08$ & & 4.18 & 75.2 \\
\hline
\end{tabular}


TABLE 4.2 (Cont.)

\begin{tabular}{|c|c|c|c|c|c|}
\hline Location & Sample & $\begin{array}{c}\text { Sample } \\
\text { Date }\end{array}$ & $\begin{array}{c}\text { Depth } \\
\text { (ft BGL) }\end{array}$ & $\begin{array}{c}\text { Dissolved } \\
\text { Oxygen } \\
(\mathrm{mg} / \mathrm{L})\end{array}$ & $\begin{array}{l}\text { ORP } \\
(\mathrm{mV})\end{array}$ \\
\hline & CNPMP9-W-26058 & $4 / 24 / 08$ & & 5.32 & 41.0 \\
\hline & CNPMP9-W-26633 & 6/5/08 & & 5.60 & 0.8 \\
\hline & CNPMP9-W-26652 & $7 / 8 / 08$ & & 5.67 & 34.4 \\
\hline & CNPMP9-W-26671 & 8/6/08 & & 18.65 & -10.0 \\
\hline & CNPMP9-W-26697 & $9 / 9 / 08$ & & 7.78 & 44.9 \\
\hline \multirow[t]{9}{*}{ SB04 } & CNSB04-W-26076 & 1/10/08 & $51-61$ & 8.73 & 89.0 \\
\hline & CNSB04-W-26094 & $1 / 24 / 08$ & & 6.32 & 184 \\
\hline & CNSB04-W-26117 & 2/23/08 & & 5.53 & 266 \\
\hline & CNSB04-W-26002 & $3 / 12 / 08$ & & 6.16 & 154 \\
\hline & CNSB04-W-26047 & $4 / 24 / 08$ & & 3.72 & 147 \\
\hline & CNSB04-W-26622 & 6/4/08 & & 4.73 & 128 \\
\hline & CNSB04-W-26641 & $7 / 7 / 08$ & & 3.88 & 164 \\
\hline & CNSB04-W-26660 & 8/6/08 & & 0.90 & 127 \\
\hline & CNSB04-W-26684 & 9/9/08 & & 6.48 & 69.9 \\
\hline \multirow[t]{9}{*}{ SB07R } & CNSB07R-W-26073 & 1/9/08 & $45-60$ & 6.64 & 130 \\
\hline & CNSB07R-W-26111 & $1 / 24 / 08$ & & 5.97 & 167 \\
\hline & CNSB07R-W-25984 & $2 / 24 / 08$ & & 4.64 & 276 \\
\hline & CNSB07R-W-26003 & $3 / 12 / 08$ & & 5.33 & 108 \\
\hline & CNSB07R-W-26048 & $4 / 24 / 08$ & & 1.93 & 158 \\
\hline & CNSB07R-W-26623 & 6/4/08 & & 4.06 & 111 \\
\hline & CNSB07R-W-26642 & $7 / 7 / 08$ & & 4.03 & 128 \\
\hline & CNSB07R-W-26661 & 8/6/08 & & 0.78 & 154 \\
\hline & CNSB07R-W-26686 & 9/9/08 & & 5.08 & 55.0 \\
\hline \multirow[t]{9}{*}{ SB08 } & CNSB08-W-26070 & $1 / 9 / 08$ & $52-62$ & 4.58 & 118 \\
\hline & CNSB08-W-26112 & $1 / 24 / 08$ & & 2.84 & 175 \\
\hline & CNSB08-W-25978 & $2 / 24 / 08$ & & 3.30 & 254 \\
\hline & CNSB08-W-26004 & $3 / 12 / 08$ & & 3.63 & 102 \\
\hline & CNSB08-W-26049 & 4/23/08 & & 2.55 & 168 \\
\hline & CNSB08-W-26624 & 6/4/08 & & 2.57 & 131 \\
\hline & CNSB08-W-26643 & $7 / 7 / 08$ & & 2.04 & 176 \\
\hline & CNSB08-W-26662 & 8/6/08 & & 0.64 & 102 \\
\hline & CNSB08-W-26687 & 9/8/08 & & 2.70 & 230 \\
\hline
\end{tabular}


TABLE 4.3 Results of organic analyses at the AGEM Laboratory for groundwater samples collected during the ISCR pilot study post-injection sampling events.

\begin{tabular}{|c|c|c|c|c|c|c|}
\hline \multirow[b]{2}{*}{ Location } & \multirow[b]{2}{*}{ Sample } & \multirow[b]{2}{*}{$\begin{array}{l}\text { Sample } \\
\text { Date }\end{array}$} & \multirow[b]{2}{*}{$\begin{array}{c}\text { Depth } \\
\text { (ft BGL) }\end{array}$} & \multicolumn{3}{|c|}{ Concentration $(\mu \mathrm{g} / \mathrm{L})$} \\
\hline & & & & $\begin{array}{c}\text { Carbon } \\
\text { Tetrachloride }\end{array}$ & Chloroform & $\begin{array}{l}\text { Methylene } \\
\text { Chloride }\end{array}$ \\
\hline \multirow[t]{9}{*}{ MW02 } & CNMW02-W-26074 & $1 / 9 / 08$ & $49.5-59.5$ & $0.3 \mathrm{Ja}$ & 23 & $N D^{b}$ \\
\hline & CNMW02-W-26097 & $1 / 24 / 08$ & & ND & 11 & 1.8 \\
\hline & CNMW02-W-26099 & 2/23/08 & & ND & 1.6 & 1.3 \\
\hline & CNMW02-W-26000 & 3/12/08 & & ND & 1.2 & 1.9 \\
\hline & CNMW02-W-26045 & 4/24/08 & & ND & 6.6 & 2.4 \\
\hline & CNMW02-W-26620 & $6 / 4 / 08$ & & 9.8 & 22 & 5.6 \\
\hline & CNMW02-W-26639 & $7 / 8 / 08$ & & 6.8 & 69 & 13 \\
\hline & CNMW02-W-26658 & 8/6/08 & & 21 & 72 & 12 \\
\hline & CNMW02-W-26674 & 9/8/08 & & 18 & 57 & 11 \\
\hline \multirow[t]{8}{*}{ MW03 } & CNMW03-W-26078 & 1/10/08 & $50.5-60.5$ & 2.9 & ND & ND \\
\hline & CNMW03-W-26115 & $2 / 23 / 08$ & & 2.1 & ND & ND \\
\hline & CNMW03-W-26001 & 3/12/08 & & 2.3 & ND & ND \\
\hline & CNMW03-W-26046 & $4 / 23 / 08$ & & 2.4 & ND & ND \\
\hline & CNMW03-W-26621 & 6/4/08 & & 2.7 & $0.2 \mathrm{~J}$ & ND \\
\hline & CNMW03-W-26640 & $7 / 7 / 08$ & & 3.3 & ND & ND \\
\hline & CNMW03-W-26659 & 8/6/08 & & 3.2 & $0.5 \mathrm{~J}$ & ND \\
\hline & CNMW03-W-26675 & 9/9/08 & & 3.2 & $0.3 \mathrm{~J}$ & ND \\
\hline \multirow[t]{9}{*}{ PMP1 } & CNPMP1-W-26079 & 1/10/08 & $50-60$ & 158 & 22 & ND \\
\hline & CNPMP1-W-26083 & $1 / 24 / 08$ & & $0.5 \mathrm{~J}$ & 92 & 6.8 \\
\hline & CNPMP1-W-25985 & 2/24/08 & & 71 & 87 & 6.0 \\
\hline & CNPMP1-W-26005 & 3/13/08 & & 131 & 49 & ND \\
\hline & CNPMP1-W-26050 & $4 / 24 / 08$ & & 113 & 41 & 1.2 \\
\hline & CNPMP1-W-26625 & 6/5/08 & & 150 & 39 & $0.7 \mathrm{~J}$ \\
\hline & CNPMP1-W-26644 & $7 / 8 / 08$ & & 145 & 33 & ND \\
\hline & CNPMP1-W-26663 & 8/6/08 & & 142 & 29 & ND \\
\hline & CNPMP1-W-26689 & 9/9/08 & & 136 & 30 & ND \\
\hline \multirow[t]{9}{*}{ PMP2 } & CNPMP2-W-26081 & 1/10/08 & $50-60$ & 980 & 951 & 4.2 \\
\hline & CNPMP2-W-26084 & $1 / 24 / 08$ & & 265 & 875 & 17 \\
\hline & CNPMP2-W-25986 & 2/24/08 & & 1249 & 715 & 24 \\
\hline & CNPMP2-W-26006 & 3/13/08 & & 1550 & 456 & Diluted \\
\hline & CNPMP2-W-26051 & $4 / 24 / 08$ & & 2254 & 476 & 8.7 \\
\hline & CNPMP2-W-26626 & 6/5/08 & & 2873 & 340 & 6.6 \\
\hline & CNPMP2-W-26645 & $7 / 8 / 08$ & & 1831 & 282 & 6.1 \\
\hline & CNPMP2-W-26664 & 8/6/08 & & 1315 & 246 & 5.2 \\
\hline & CNPMP2-W-26690 & 9/9/08 & & 1854 & 318 & 5.6 \\
\hline \multirow[t]{9}{*}{ PMP3 } & CNPMP3-W-26071 & $1 / 9 / 08$ & $50-60$ & 112 & 116 & 1.0 \\
\hline & CNPMP3-W-26086 & $1 / 24 / 08$ & & 4.2 & 79 & 3.4 \\
\hline & CNPMP3-W-25990 & 2/25/08 & & 3.1 & 147 & 6.5 \\
\hline & CNPMP3-W-26007 & 3/13/08 & & 3.9 & 110 & 10 \\
\hline & CNPMP3-W-26052 & 4/24/08 & & 16 & 89 & 28 \\
\hline & CNPMP3-W-26627 & 6/5/08 & & 46 & 129 & 32 \\
\hline & CNPMP3-W-26646 & $7 / 8 / 08$ & & 42 & 90 & 20 \\
\hline & CNPMP3-W-26665 & 8/6/08 & & 40 & 67 & 8.9 \\
\hline & CNPMP3-W-26691 & 9/9/08 & & 21 & 57 & 6.2 \\
\hline
\end{tabular}


TABLE 4.3 (Cont.)

\begin{tabular}{|c|c|c|c|c|c|c|}
\hline \multirow[b]{2}{*}{ Location } & \multirow[b]{2}{*}{ Sample } & \multirow[b]{2}{*}{$\begin{array}{l}\text { Sample } \\
\text { Date }\end{array}$} & \multirow[b]{2}{*}{$\begin{array}{c}\text { Depth } \\
\text { (ft BGL) }\end{array}$} & \multicolumn{3}{|c|}{ Concentration $(\mu \mathrm{g} / \mathrm{L})$} \\
\hline & & & & $\begin{array}{c}\text { Carbon } \\
\text { Tetrachloride }\end{array}$ & Chloroform & $\begin{array}{c}\text { Methylene } \\
\text { Chloride }\end{array}$ \\
\hline \multirow[t]{9}{*}{ PMP4 } & CNPMP4-W-26068 & $1 / 9 / 08$ & $48.75-58.75$ & 36 & 12 & ND \\
\hline & CNPMP4-W-26087 & $1 / 24 / 08$ & & 10 & 6.9 & 1.1 \\
\hline & CNPMP4-W-25992 & $2 / 25 / 08$ & & 42 & 5.8 & $0.6 \mathrm{~J}$ \\
\hline & CNPMP4-W-26008 & $3 / 13 / 08$ & & 31 & 4.4 & ND \\
\hline & CNPMP4-W-26053 & $4 / 24 / 08$ & & 33 & 6.8 & $0.8 \mathrm{~J}$ \\
\hline & CNPMP4-W-26628 & 6/5/08 & & 60 & 6.3 & ND \\
\hline & CNPMP4-W-26647 & $7 / 7 / 08$ & & 52 & 4.9 & ND \\
\hline & CNPMP4-W-26666 & $8 / 6 / 08$ & & 50 & 4.8 & ND \\
\hline & CNPMP4-W-26692 & $9 / 9 / 08$ & & 49 & 4.2 & ND \\
\hline \multirow[t]{9}{*}{ PMP5 } & CNPMP5-W-26069 & $1 / 9 / 08$ & $50-60$ & 321 & 97 & 2.6 \\
\hline & CNPMP5-W-26088 & $1 / 24 / 08$ & & 265 & 121 & 4.6 \\
\hline & CNPMP5-W-25997 & $2 / 25 / 08$ & & 421 & 62 & $0.7 \mathrm{~J}$ \\
\hline & CNPMP5-W-26009 & $3 / 13 / 08$ & & 70 & 13 & $0.7 \mathrm{~J}$ \\
\hline & CNPMP5-W-26054 & $4 / 24 / 08$ & & 357 & 101 & 2.9 \\
\hline & CNPMP5-W-26629 & 6/4/08 & & 365 & 91 & 2.7 \\
\hline & CNPMP5-W-26648 & $7 / 8 / 08$ & & 397 & 71 & 2.5 \\
\hline & CNPMP5-W-26667 & $8 / 6 / 08$ & & 476 & 55 & 1.7 \\
\hline & CNPMP5-W-26693 & 9/10/08 & & 418 & 46 & $1.6 \mathrm{~J}$ \\
\hline \multirow[t]{9}{*}{ PMP6 } & CNPMP6-W-26065 & $1 / 8 / 08$ & $50-60$ & 37 & 18 & ND \\
\hline & CNPMP6-W-26090 & $1 / 24 / 08$ & & 26 & 31 & 2.9 \\
\hline & CNPMP6-W-25993 & $2 / 25 / 08$ & & 111 & 8.8 & ND \\
\hline & CNPMP6-W-26010 & $3 / 13 / 08$ & & 94 & 10 & ND \\
\hline & CNPMP6-W-26055 & $4 / 24 / 08$ & & 103 & 13 & ND \\
\hline & CNPMP6-W-26630 & 6/5/08 & & 101 & 9.8 & ND \\
\hline & CNPMP6-W-26649 & $7 / 7 / 08$ & & 96 & 8.5 & ND \\
\hline & CNPMP6-W-26668 & $8 / 6 / 08$ & & 108 & 8.2 & ND \\
\hline & CNPMP6-W-26694 & $9 / 8 / 08$ & & 110 & 7.8 & ND \\
\hline \multirow[t]{9}{*}{ PMP7 } & CNPMP7-W-26066 & $1 / 8 / 08$ & $50-60$ & 119 & 26 & ND \\
\hline & CNPMP7-W-26091 & $1 / 24 / 08$ & & 62 & 40 & 1.6 \\
\hline & CNPMP7-W-25996 & $2 / 25 / 08$ & & 123 & 17 & $0.7 \mathrm{~J}$ \\
\hline & CNPMP7-W-26011 & $3 / 13 / 08$ & & 121 & 14 & $0.6 \mathrm{~J}$ \\
\hline & CNPMP7-W-26056 & $4 / 24 / 08$ & & 134 & 13 & ND \\
\hline & CNPMP7-W-26631 & $6 / 5 / 08$ & & 120 & 8.8 & ND \\
\hline & CNPMP7-W-26650 & $7 / 7 / 08$ & & 120 & 7.6 & ND \\
\hline & CNPMP7-W-26669 & $8 / 6 / 08$ & & 154 & 9.2 & ND \\
\hline & CNPMP7-W-26695 & 9/9/08 & & 119 & 13 & ND \\
\hline \multirow[t]{9}{*}{ PMP8 } & CNPMP8-W-26075 & $1 / 9 / 08$ & $50-60$ & 30 & 606 & 3.4 \\
\hline & CNPMP8-W-26092 & $1 / 24 / 08$ & & 31 & 430 & 28 \\
\hline & CNPMP8-W-25994 & $2 / 25 / 08$ & & 287 & 374 & 25 \\
\hline & CNPMP8-W-26012 & $3 / 13 / 08$ & & 122 & 292 & 20 \\
\hline & CNPMP8-W-26057 & $4 / 24 / 08$ & & 72 & 553 & 41 \\
\hline & CNPMP8-W-26632 & $6 / 5 / 08$ & & 73 & 364 & 24 \\
\hline & CNPMP8-W-26651 & $7 / 8 / 08$ & & 67 & 339 & 23 \\
\hline & CNPMP8-W-26670 & $8 / 6 / 08$ & & 105 & 317 & 18 \\
\hline & CNPMP8-W-26696 & 9/9/08 & & 72 & 125 & 3.4 \\
\hline
\end{tabular}


TABLE 4.3 (Cont.)

\begin{tabular}{|c|c|c|c|c|c|c|}
\hline \multirow[b]{2}{*}{ Location } & \multirow[b]{2}{*}{ Sample } & \multirow[b]{2}{*}{$\begin{array}{l}\text { Sample } \\
\text { Date }\end{array}$} & \multirow[b]{2}{*}{$\begin{array}{l}\text { Depth } \\
\text { (ft BGL) }\end{array}$} & \multicolumn{3}{|c|}{ Concentration $(\mu \mathrm{g} / \mathrm{L})$} \\
\hline & & & & $\begin{array}{c}\text { Carbon } \\
\text { Tetrachloride }\end{array}$ & Chloroform & $\begin{array}{l}\text { Methylene } \\
\text { Chloride }\end{array}$ \\
\hline \multirow[t]{9}{*}{ PMP9 } & CNPMP9-W-26077 & 1/10/08 & $50-60$ & 1.9 & $0.9 \mathrm{~J}$ & ND \\
\hline & CNPMP9-W-26109 & $1 / 24 / 08$ & & 3.1 & 1.1 & ND \\
\hline & CNPMP9-W-25989 & 2/24/08 & & 4.7 & $0.8 \mathrm{~J}$ & ND \\
\hline & CNPMP9-W-26013 & 3/13/08 & & 4.0 & $0.5 \mathrm{~J}$ & ND \\
\hline & CNPMP9-W-26058 & $4 / 24 / 08$ & & 5.7 & 1.8 & ND \\
\hline & CNPMP9-W-26633 & $6 / 5 / 08$ & & 6.3 & $0.6 \mathrm{~J}$ & ND \\
\hline & CNPMP9-W-26652 & $7 / 8 / 08$ & & 7.2 & 1.4 & ND \\
\hline & CNPMP9-W-26671 & $8 / 6 / 08$ & & 12 & 1.1 & ND \\
\hline & CNPMP9-W-26697 & 9/9/08 & & 7.6 & $0.4 \mathrm{~J}$ & ND \\
\hline \multirow[t]{9}{*}{ SB04 } & CNSB04-W-26076 & 1/10/08 & $51-61$ & 10 & 2.1 & ND \\
\hline & CNSB04-W-26094 & $1 / 24 / 08$ & & 15 & $0.2 \mathrm{~J}$ & ND \\
\hline & CNSB04-W-26117 & $2 / 23 / 08$ & & 42 & $0.4 \mathrm{~J}$ & ND \\
\hline & CNSB04-W-26002 & 3/12/08 & & 30 & $0.3 \mathrm{~J}$ & ND \\
\hline & CNSB04-W-26047 & $4 / 24 / 08$ & & 16 & $0.3 \mathrm{~J}$ & ND \\
\hline & CNSB04-W-26622 & $6 / 4 / 08$ & & 25 & $0.4 \mathrm{~J}$ & ND \\
\hline & CNSB04-W-26641 & 7/7/08 & & 14 & $0.2 \mathrm{~J}$ & ND \\
\hline & CNSB04-W-26660 & $8 / 6 / 08$ & & 11 & $0.2 \mathrm{~J}$ & ND \\
\hline & CNSB04-W-26684 & 9/9/08 & & 15 & $0.3 \mathrm{~J}$ & ND \\
\hline \multirow[t]{9}{*}{ SB07R } & CNSB07R-W-26073 & $1 / 9 / 08$ & $45-60$ & 30 & 1.8 & ND \\
\hline & CNSB07R-W-26111 & $1 / 24 / 08$ & & 19 & $0.9 \mathrm{~J}$ & ND \\
\hline & CNSB07R-W-25984 & $2 / 24 / 08$ & & 24 & 1.4 & ND \\
\hline & CNSB07R-W-26003 & 3/12/08 & & 13 & $0.9 \mathrm{~J}$ & ND \\
\hline & CNSB07R-W-26048 & $4 / 24 / 08$ & & 10 & $0.8 \mathrm{~J}$ & ND \\
\hline & CNSB07R-W-26623 & $6 / 4 / 08$ & & 17 & 1.0 & ND \\
\hline & CNSB07R-W-26642 & 7/7/08 & & 15 & $0.9 \mathrm{~J}$ & ND \\
\hline & CNSB07R-W-26661 & $8 / 6 / 08$ & & 15 & $0.9 \mathrm{~J}$ & ND \\
\hline & CNSB07R-W-26686 & 9/9/08 & & 21 & $1.4 \mathrm{~J}$ & ND \\
\hline \multirow[t]{9}{*}{ SB08 } & CNSB08-W-26070 & $1 / 9 / 08$ & $52-62$ & 32 & 1.5 & ND \\
\hline & CNSB08-W-26112 & $1 / 24 / 08$ & & 36 & 1.5 & ND \\
\hline & CNSB08-W-25978 & $2 / 24 / 08$ & & 44 & 1.5 & ND \\
\hline & CNSB08-W-26004 & 3/12/08 & & 28 & 1.1 & ND \\
\hline & CNSB08-W-26049 & $4 / 23 / 08$ & & 23 & 1.2 & ND \\
\hline & CNSB08-W-26624 & $6 / 4 / 08$ & & 24 & 1.4 & ND \\
\hline & CNSB08-W-26643 & 7/7/08 & & 17 & 1.0 & ND \\
\hline & CNSB08-W-26662 & $8 / 6 / 08$ & & 20 & 1.1 & ND \\
\hline & CNSB08-W-26687 & 9/8/08 & & 22 & $1.2 \mathrm{~J}$ & ND \\
\hline
\end{tabular}

a Qualifier $\mathrm{J}$ indicates as estimated concentration below the purge-and-trap method quantitation limit of $1.0 \mu \mathrm{g} / \mathrm{L}$.

b ND, not detected at an instrument detection limit of $0.1 \mu \mathrm{g} / \mathrm{L}$. 
TABLE 4.4 Results of purge-and-trap organic analyses at the AGEM Laboratory for soil samples collected at PSB13 during the August 2008 post-injection sampling event.

\begin{tabular}{|c|c|c|c|c|c|c|}
\hline \multirow[b]{2}{*}{ Location } & \multirow[b]{2}{*}{ Sample } & \multirow[b]{2}{*}{$\begin{array}{l}\text { Sample } \\
\text { Date }\end{array}$} & \multirow[b]{2}{*}{$\begin{array}{l}\text { Depth } \\
\text { (ft BGL) }\end{array}$} & \multicolumn{3}{|c|}{ Concentration $(\mu \mathrm{g} / \mathrm{kg})$} \\
\hline & & & & $\begin{array}{c}\text { Carbon } \\
\text { Tetrachloride }\end{array}$ & Chloroform & $\begin{array}{l}\text { Methylene } \\
\text { Chloride }\end{array}$ \\
\hline \multirow{20}{*}{ PSB13 } & CNPSB13-S-27120 & 8/21/08 & 2 & $N D^{a}$ & ND & ND \\
\hline & CNPSB13-S-27121 & $8 / 21 / 08$ & 4 & ND & ND & ND \\
\hline & CNPSB13-S-27122 & $8 / 21 / 08$ & 6 & ND & ND & ND \\
\hline & CNPSB13-S-27123 & $8 / 21 / 08$ & 8 & ND & ND & ND \\
\hline & CNPSB13-S-27124 & $8 / 21 / 08$ & 10 & ND & $5.1 \mathrm{~J}^{\mathrm{b}}$ & ND \\
\hline & CNPSB13-S-27125 & $8 / 21 / 08$ & 12 & ND & $3 \mathrm{~J}$ & ND \\
\hline & CNPSB13-S-27126 & $8 / 21 / 08$ & 14 & ND & 13 & ND \\
\hline & CNPSB13-S-27127 & $8 / 21 / 08$ & 16 & $6.5 \mathrm{~J}$ & $8.3 \mathrm{~J}$ & ND \\
\hline & CNPSB13-S-27128 & $8 / 21 / 08$ & 18 & ND & $3.4 \mathrm{~J}$ & 11 \\
\hline & CNPSB13-S-27129 & $8 / 21 / 08$ & 20 & ND & 13 & ND \\
\hline & CNPSB13-S-27130 & $8 / 21 / 08$ & 22 & $6.2 \mathrm{~J}$ & $6.9 \mathrm{~J}$ & ND \\
\hline & CNPSB13-S-27131 & $8 / 21 / 08$ & 24 & ND & $6.7 \mathrm{~J}$ & ND \\
\hline & CNPSB13-S-27132 & $8 / 21 / 08$ & 26 & $3 \mathrm{~J}$ & 16 & ND \\
\hline & CNPSB13-S-27133 & 8/21/08 & 28 & $4 \mathrm{~J}$ & 15 & ND \\
\hline & CNPSB13-S-27134 & $8 / 21 / 08$ & 30 & ND & 13 & ND \\
\hline & CNPSB13-S-27135 & $8 / 21 / 08$ & 32 & ND & 17 & ND \\
\hline & CNPSB13-S-27136 & $8 / 21 / 08$ & 34 & 46 & $4.8 \mathrm{~J}$ & ND \\
\hline & CNPSB13-S-27137 & $8 / 21 / 08$ & 36 & 61 & $9.6 \mathrm{~J}$ & ND \\
\hline & CNPSB13-S-27138 & $8 / 21 / 08$ & 38 & 32 & $7 \mathrm{~J}$ & ND \\
\hline & CNPSB13-S-27139 & $8 / 21 / 08$ & 40 & 10 & $3.6 \mathrm{~J}$ & ND \\
\hline
\end{tabular}

a ND, not detected at an instrument detection limit of $1 \mu \mathrm{g} / \mathrm{kg}$.

b Qualifier J indicates an estimated concentration below the purge-and-trap method quantitation limit of $10 \mu \mathrm{g} / \mathrm{kg}$. 


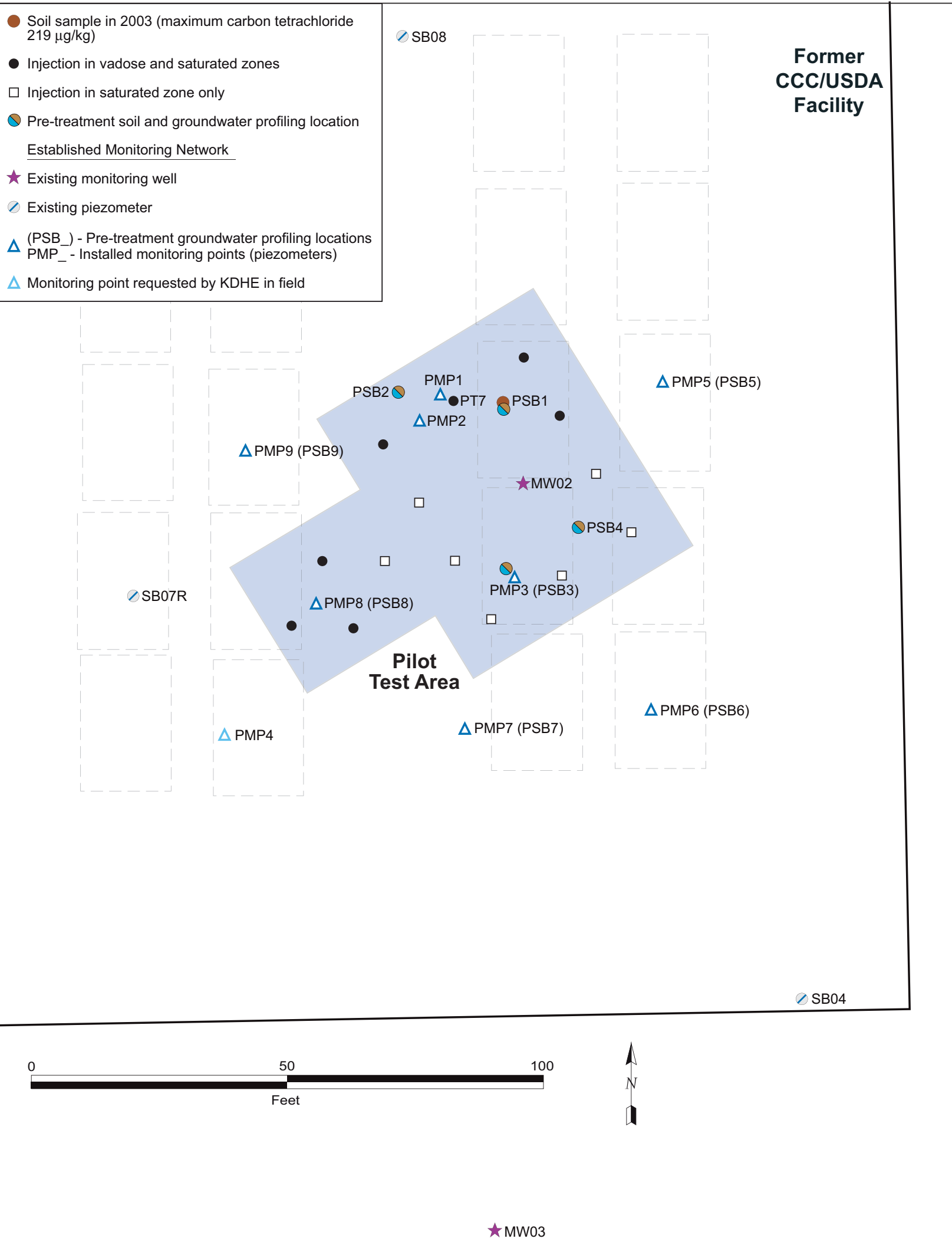

FIGURE 4.1 Locations of injection points and post-injection groundwater monitoring points. 


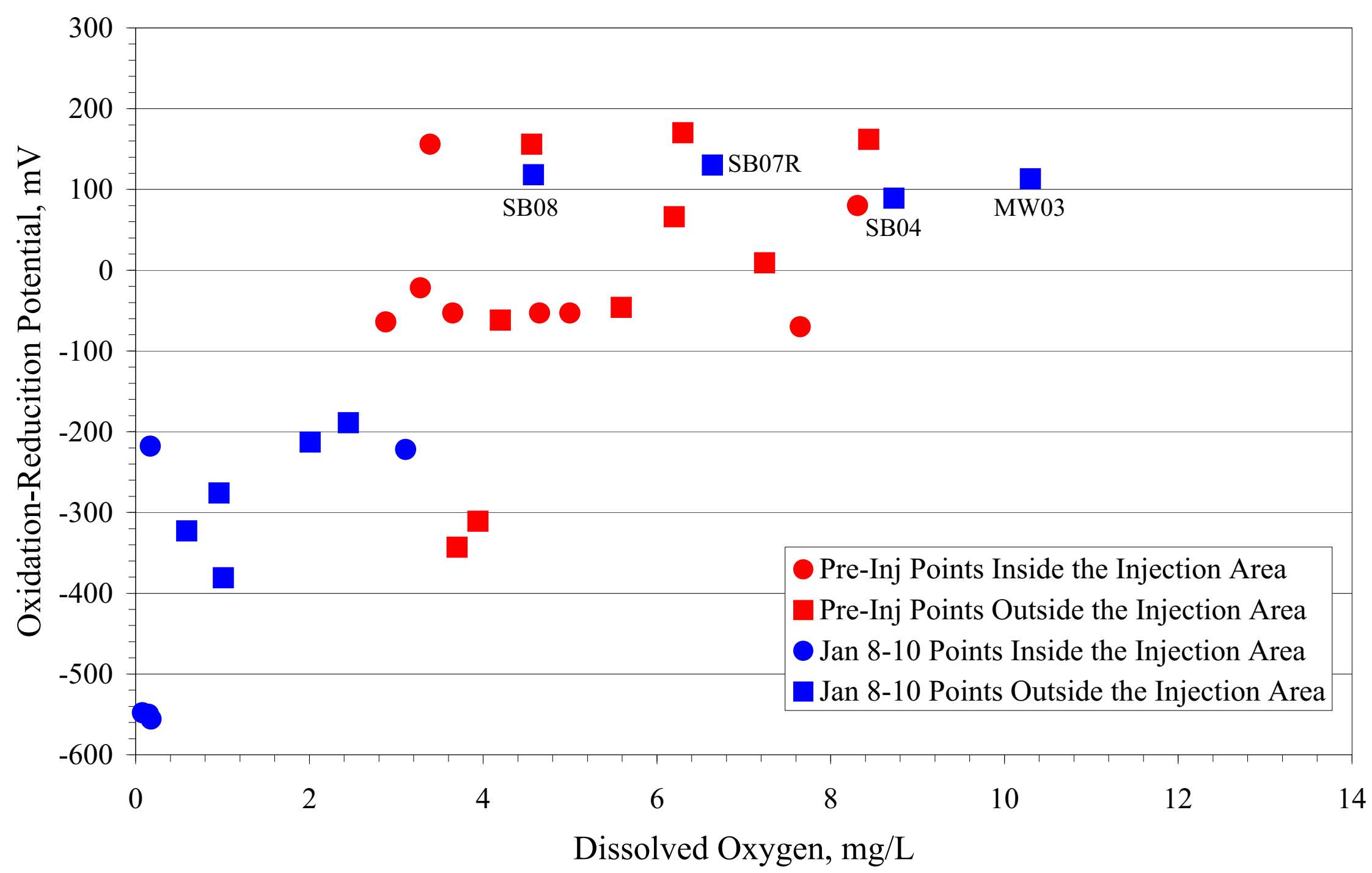

FIGURE 4.2 Dissolved oxygen concentrations and oxidation-reduction potential levels in groundwater samples collected at permanent monitoring points during the initial (January 8-10, 2008) post-injection sampling event. 


\section{Post-Injection Sampling Results \\ January 8-10, 2008}

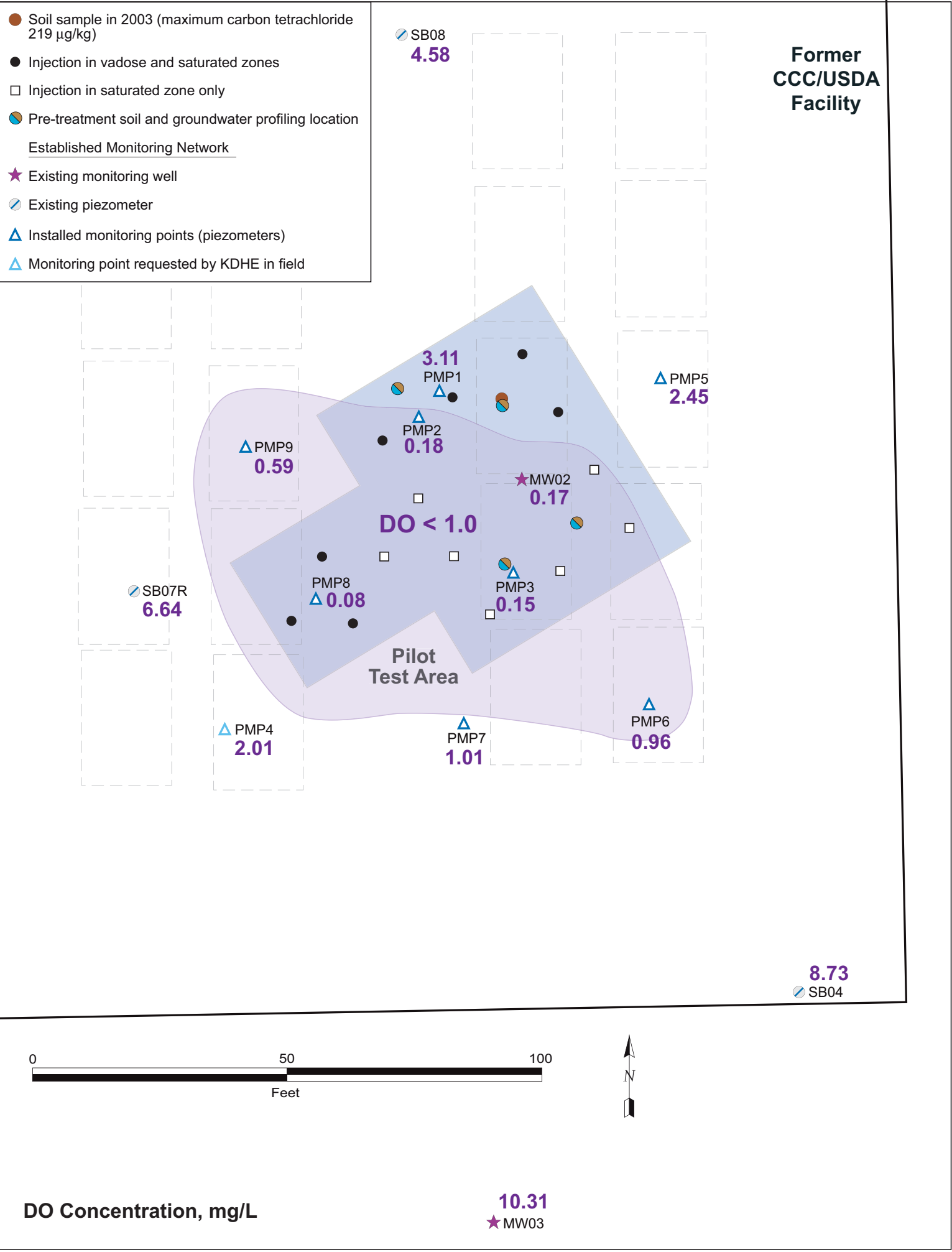

FIGURE 4.3 Dissolved oxygen concentrations in groundwater samples collected at permanent monitoring points during the initial (January 8-10, 2008) post-injection sampling event. 


\section{Post-Injection Sampling Results \\ January 8-10, 2008}

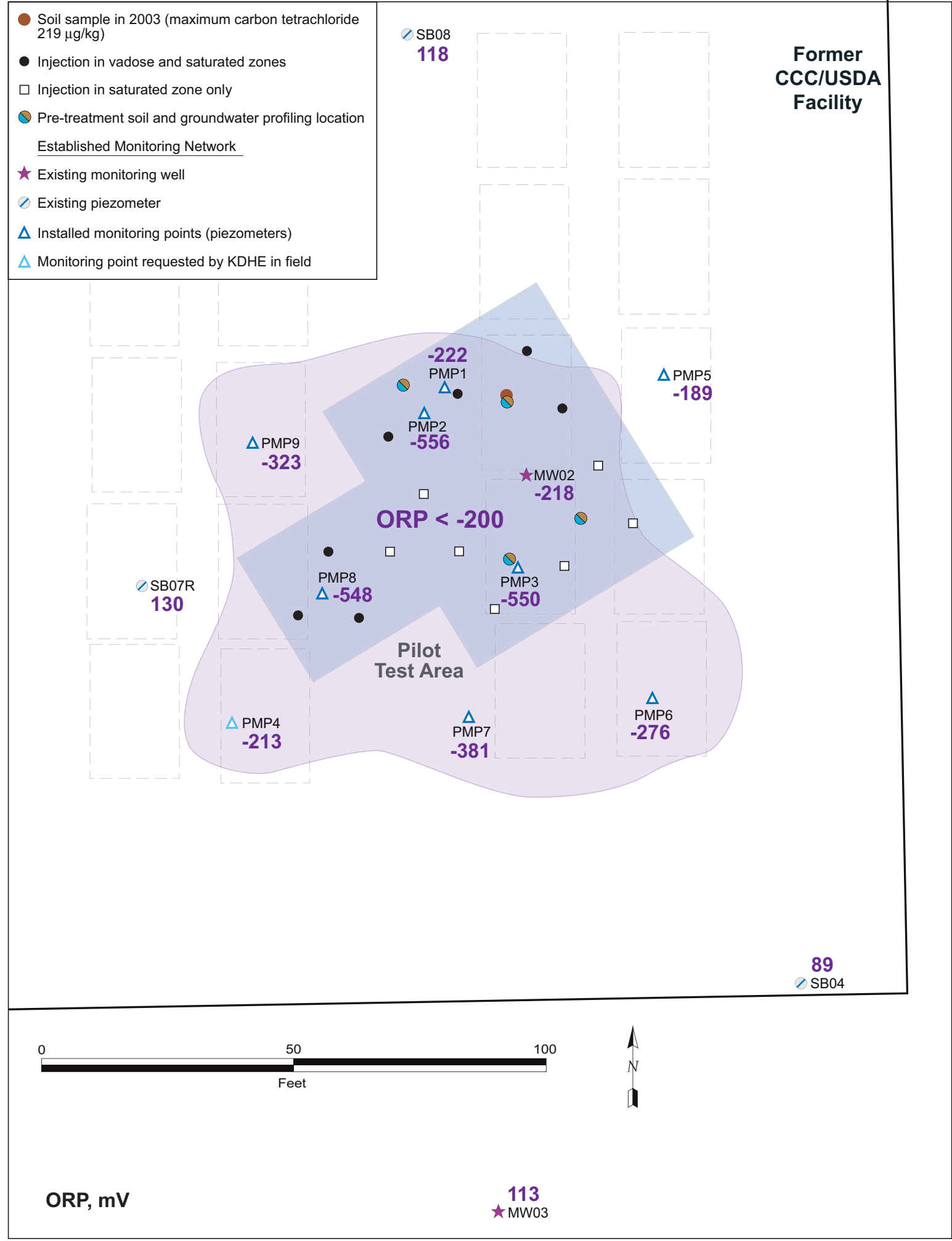

FIGURE 4.4 Oxidation-reduction potential levels in groundwater samples collected at permanent monitoring points during the initial (January 8-10, 2008) post-injection sampling event. 


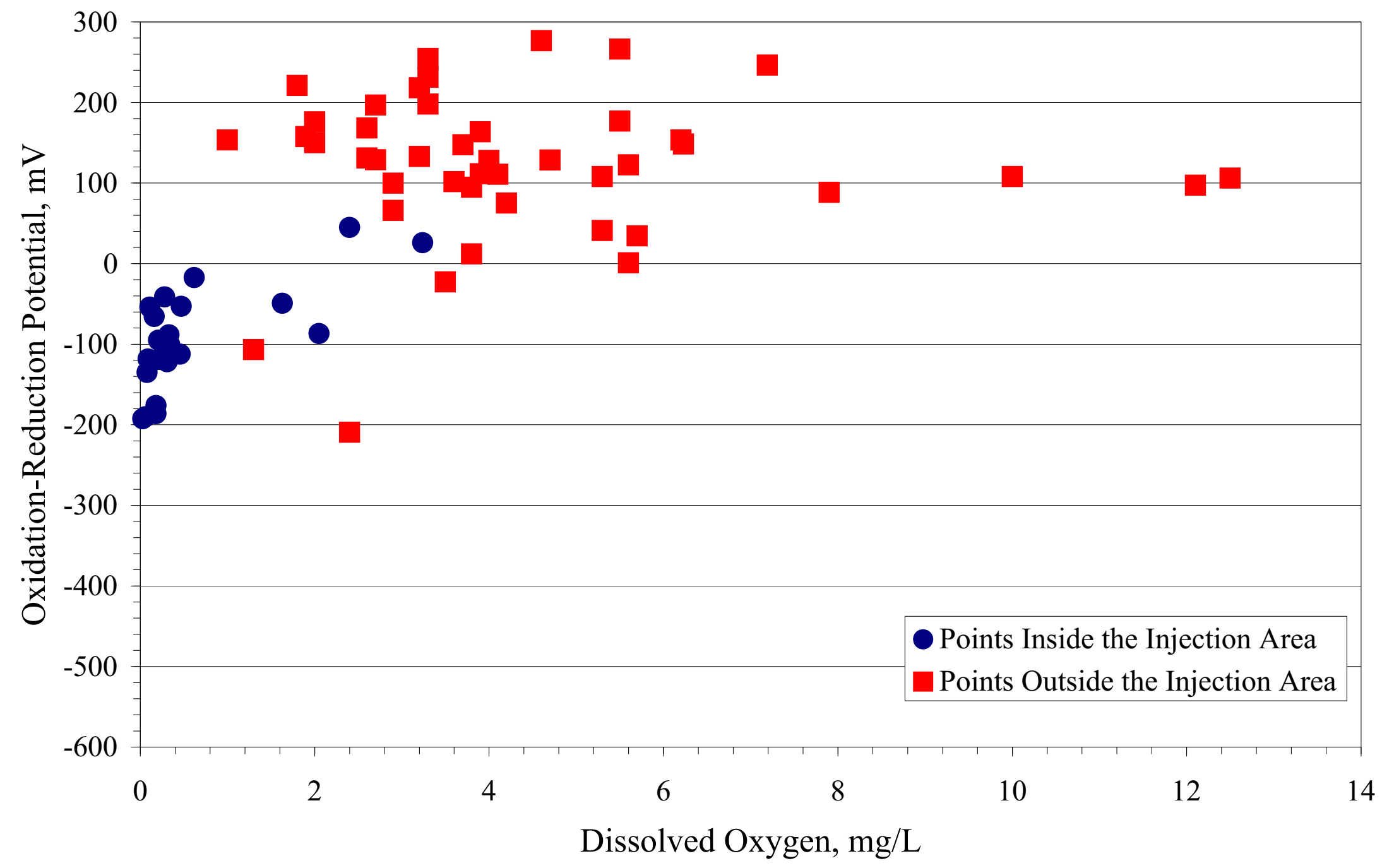

FIGURE 4.5 Dissolved oxygen concentrations and oxidation-reduction potential levels in groundwater samples collected at permanent monitoring points during post-injection sampling in February-September 2008. 


\section{Post-Injection Sampling Results \\ September 2008}

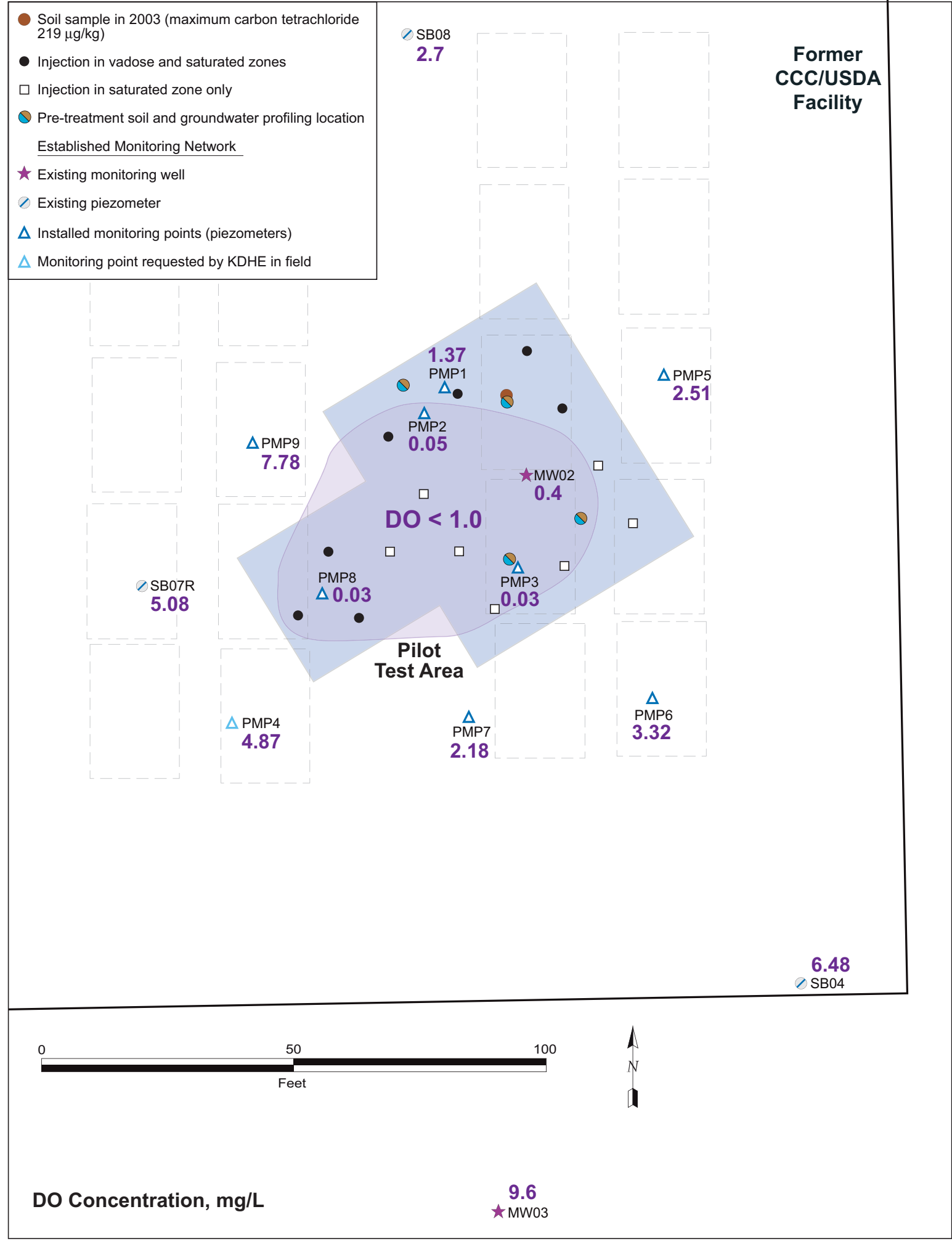

FIGURE 4.6 Dissolved oxygen concentrations in groundwater samples collected at permanent monitoring points during the September 2008 post-injection sampling event. 


\section{Post-Injection Sampling Results \\ September 2008}

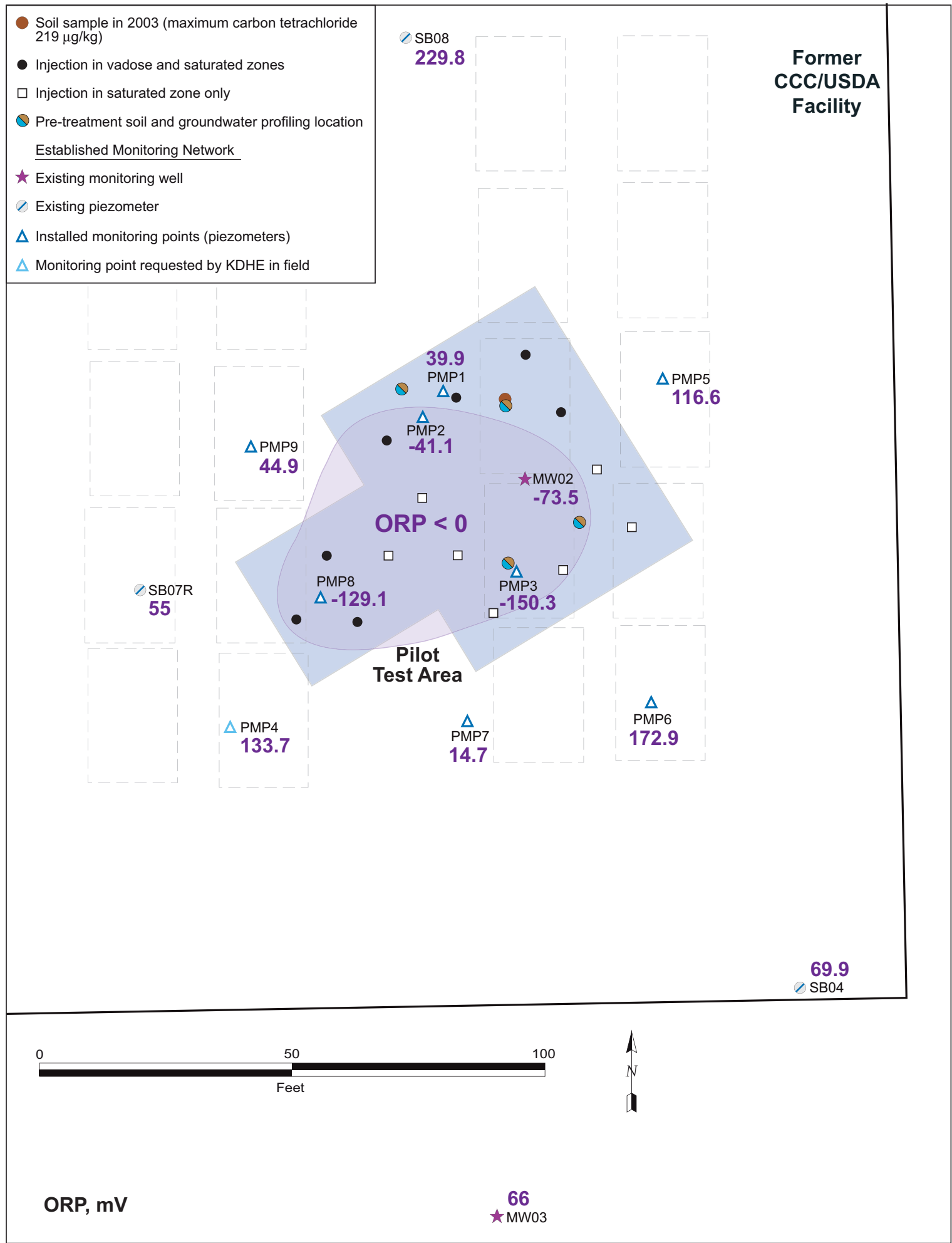

FIGURE 4.7 Oxidation-reduction potential levels in groundwater samples collected at permanent monitoring points during the September 2008 post-injection sampling event. 


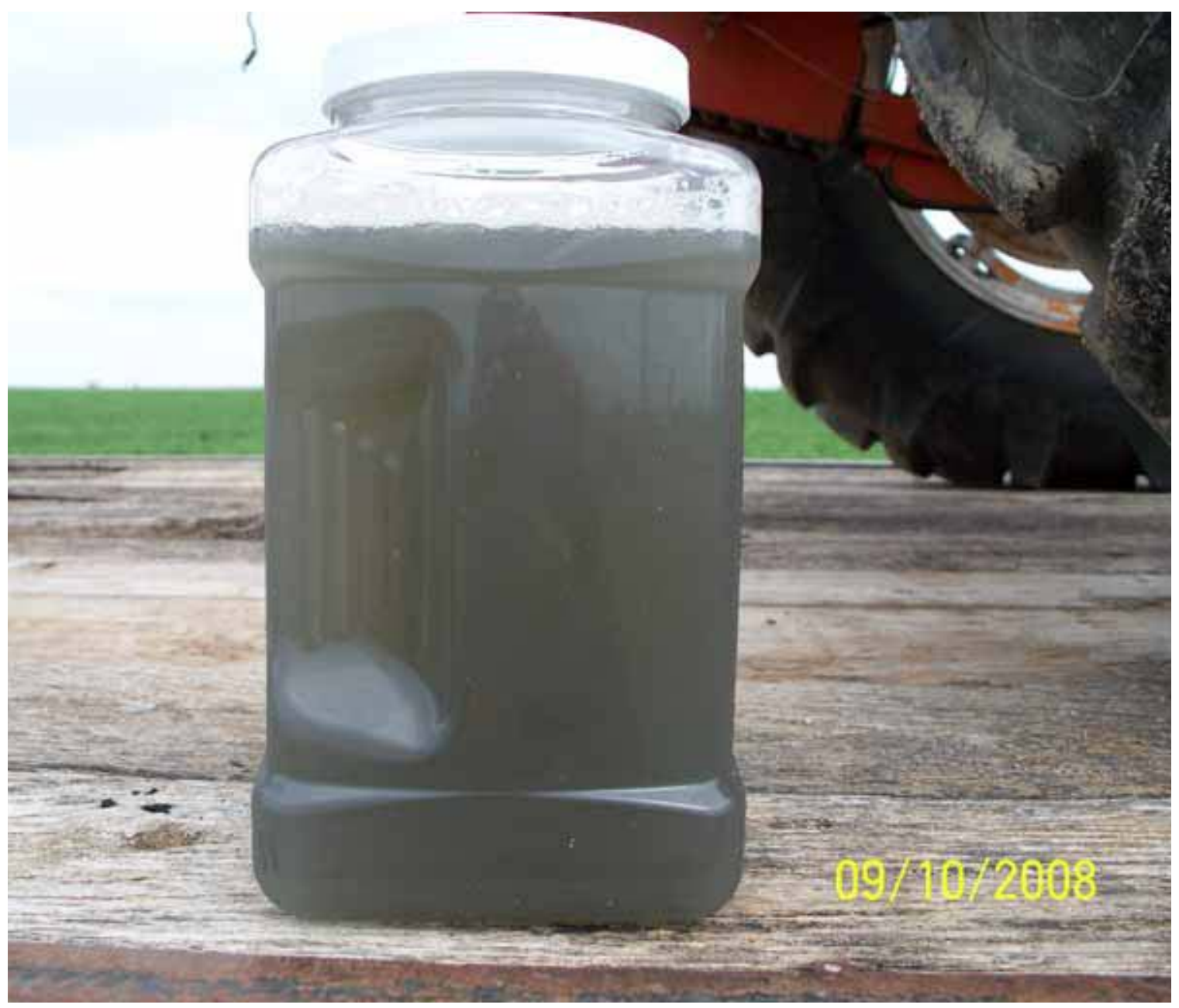

FIGURE 4.8 Appearance of groundwater collected from monitoring well MW02 during the September 2008 post-injection sampling event. 


\section{Post-Injection Sampling Results \\ January 8-10, 2008}

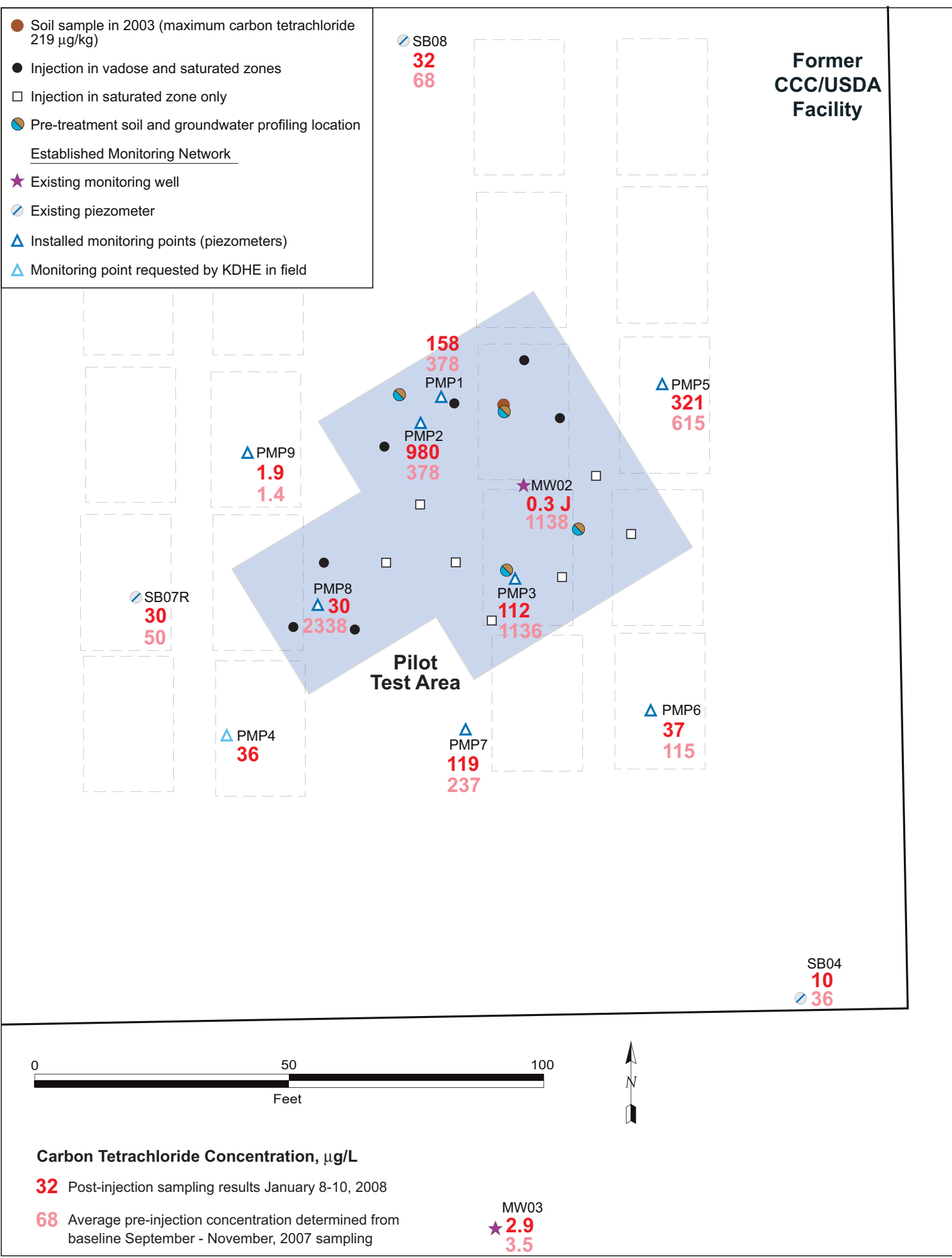

FIGURE 4.9 Carbon tetrachloride concentrations in groundwater samples collected at permanent monitoring points during the initial (January 8-10, 2008) post-injection sampling event, with preinjection values for comparison. 


\section{Post-Injection Sampling Results \\ January 8-10, 2008}

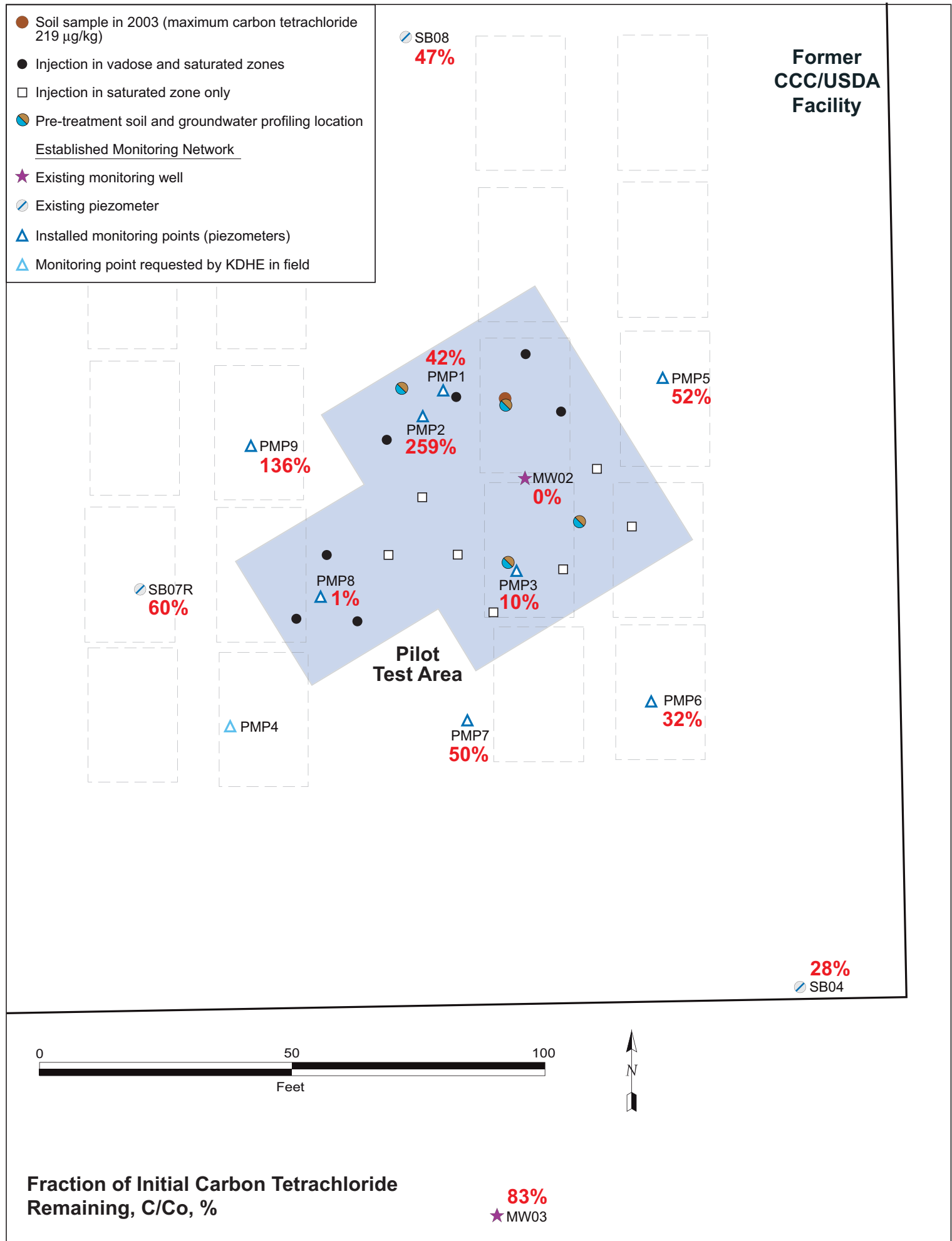

FIGURE 4.10 Calculated fraction of carbon tetrachloride remaining, in comparison to pre-injection concentrations, in groundwater samples collected at permanent monitoring points during the initial (January 8-10, 2008) post-injection sampling event. 


\section{Post-Injection Sampling Results September 2008}

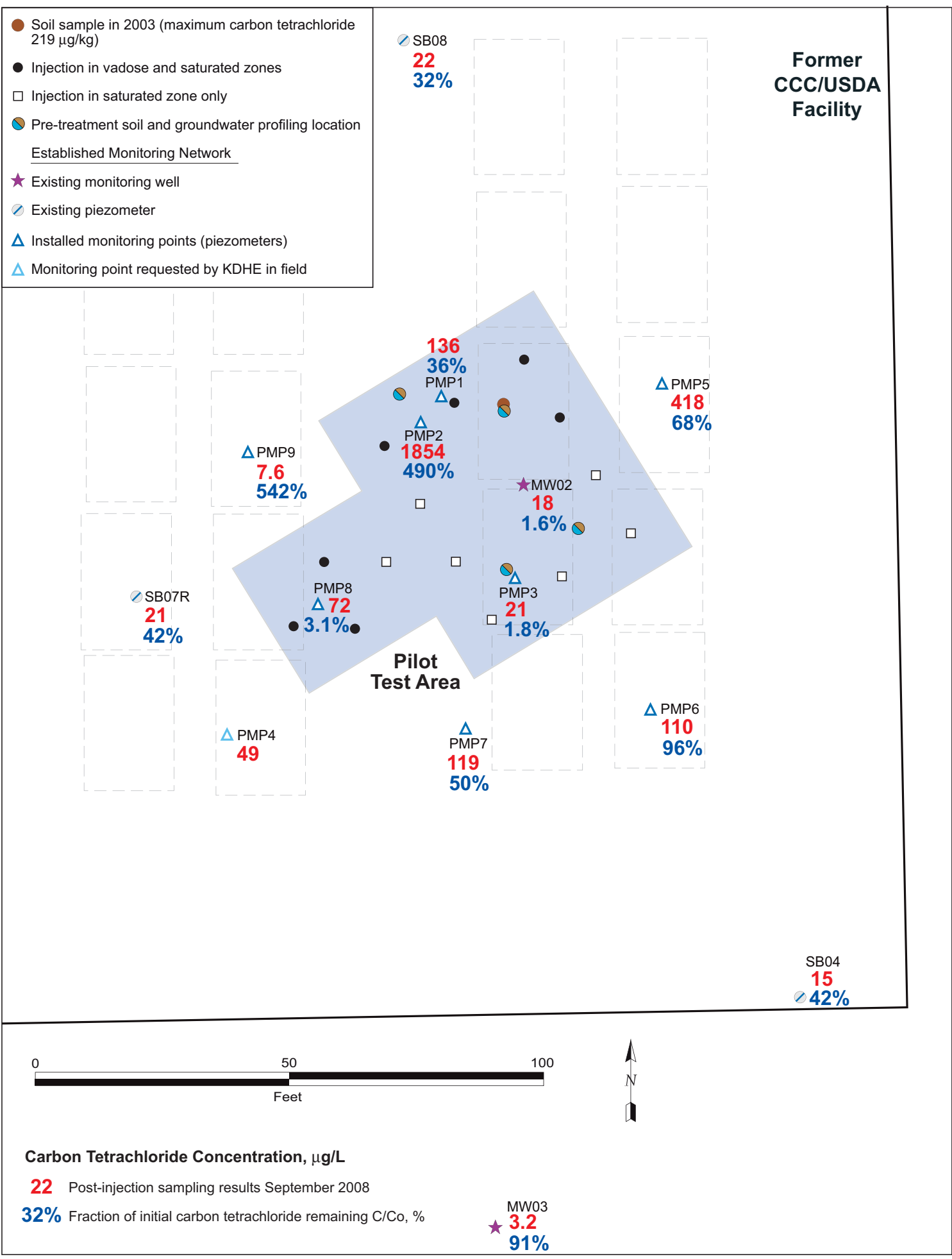

FIGURE 4.11 Carbon tetrachloride concentrations in groundwater samples collected at permanent monitoring points during the September 2008 post-injection sampling event, with calculated fraction of carbon tetrachloride remaining, in comparison to pre-injection concentrations. 


\section{Post-Injection Sampling Results \\ September 2008}

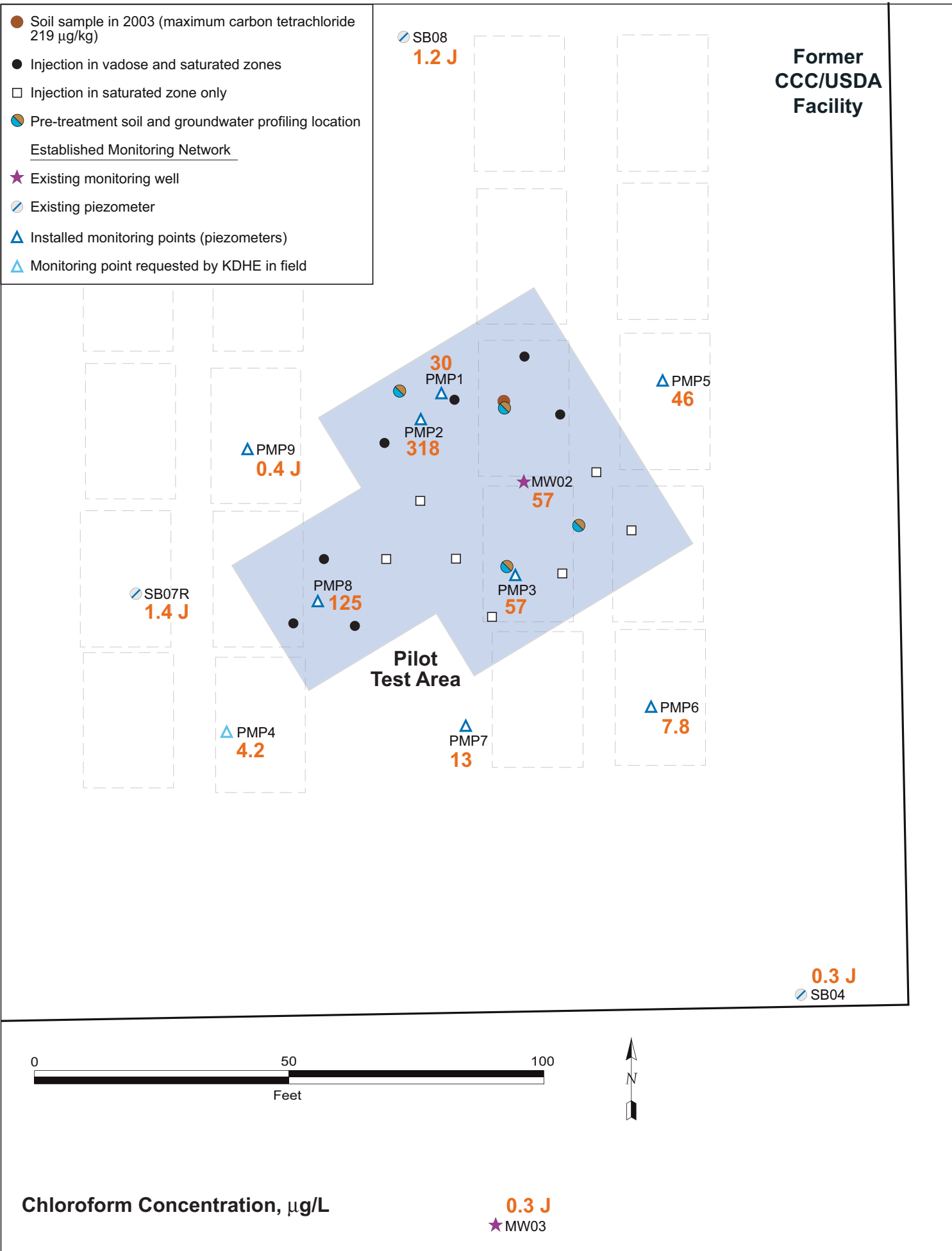

FIGURE 4.12 Chloroform concentrations in groundwater samples collected at permanent monitoring points during the September 2008 post-injection sampling event. 
PSB13
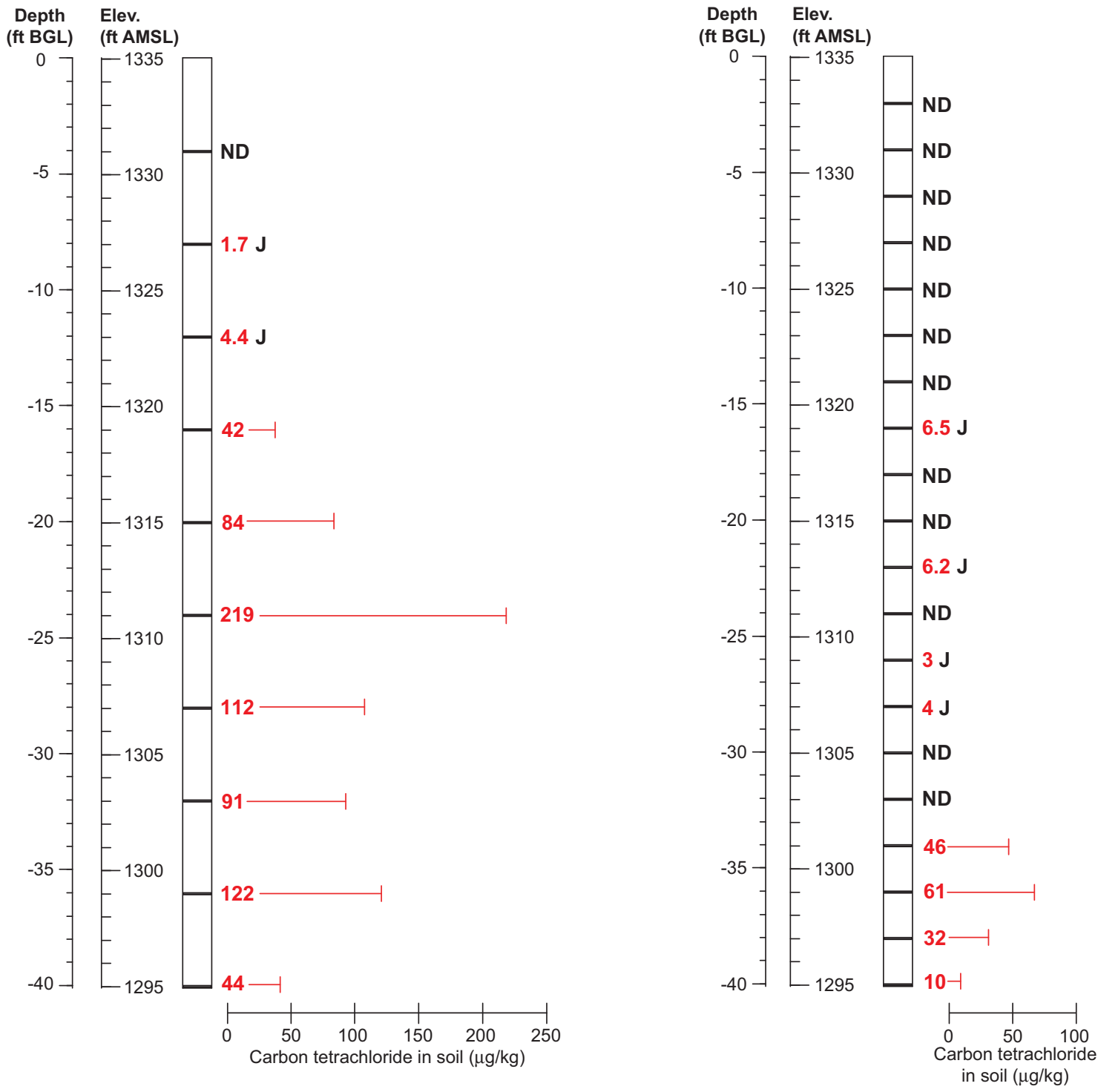

ND Not detected $(<10 \mu \mathrm{g} / \mathrm{kg})$

FIGURE 4.13 Comparison of carbon tetrachloride concentrations in the vadose zone soils at pre-injection sampling point PSB1 (November 2007) and corresponding post-injection sampling point PSB13 (August 2008). 


\section{Conclusions and Recommendations}

\subsection{Conclusions}

This report documents and provides a technical evaluation of the results of the Centralia ISCR pilot test as of September 2008. The primary observations and interpretations developed from these data are as follows:

- The ISCR technology was successfully implemented at the SB12-MW02 pilot test site; however, several technical and logistic concerns were identified during the injection program:

- Injection pressures exceeding 300 psi were required to achieve placement of the slurry.

- Frequent daylighting of the injected fluids to the surface occurred.

- Pre-existing borings in and near the injection area provided conduits for preferential daylighting of the injected fluids.

- The estimation of an effective injection radius of influence is problematic for the subsurface conditions at Centralia.

- The ISCR materials initially generated extremely reducing, oxygen-depleted groundwater conditions (with ORP values of $-200 \mathrm{mV}$ to $-550 \mathrm{mV}$ and $\mathrm{DO}$ $<1 \mathrm{mg} / \mathrm{L}$ ) within the injection field. Initial, less dramatic reductions in DO and ORP were also observed at monitoring points outside the treatment area.

- The DO and ORP values within the injection field have remained consistently lower than those at the monitoring points outside the injection area; however, the extremely low ORP levels observed initially were maintained only for approximately 5-7 weeks after injection. The continuing DO and ORP levels in the injection field should be conducive to enhanced biodegradation of 
carbon tetrachloride, but they are not sufficient to promote thermodynamic instability or the abiotic breakdown of carbon tetrachloride.

- A reduction of $96-99 \%$ in the levels of carbon tetrachloride in groundwater in the injection area was observed within the first 5-7 weeks after injection. Decreases in carbon tetrachloride concentrations of approximately 20-70\% were also observed at most monitoring points near the injection area in this time frame.

- Extended monitoring has shown that the dramatically lower carbon tetrachloride concentrations observed in the injection area have increased slightly but remain at or near the initial post-injection levels.

- Carbon tetrachloride, chloroform, and methylene chloride concentrations at all monitoring points have shown little or no clear trending indicating further contaminant decrease since the initial (January 8-10, 2008) post-injection sampling event.

- Persistent high carbon tetrachloride concentrations have been observed at monitoring points PMP1 and PMP2, at the northern margin of the injection field. The observed contaminant levels suggest that multiple possible factors, including lithologic heterogeneity, heterogeneity in the pre-injection groundwater carbon tetrachloride distribution, and unpredictable subsurface distribution of the injected fluids, might have influenced the potential effectiveness of the ISCR treatment approach at Centralia on a localized scale.

- Carbon tetrachloride concentrations in the vadose zone soils at pre-injection sampling point PSB1 (former sampling location SB12) showed an apparent decrease, at all sampled depths, in response to ISCR treatment of the vadose zone soils in the area. The carbon tetrachloride concentrations in the treated soils at this location decreased (from a maximum pre-injection concentration of $219 \mu \mathrm{g} / \mathrm{kg}$ ) to levels below the KDHE Tier 2 RBSL of $200 \mu \mathrm{g} / \mathrm{kg}$ for this contaminant in soils (maximum of $61 \mu \mathrm{g} / \mathrm{kg}$ at post-injection sampling point PSB13). 


\subsection{Recommendations}

The following recommendations, based on the technical findings summarized in this report, are presented for consideration with regard to the Centralia site:

- Adventus has estimated that the EHC materials might have an effect on the subsurface geochemical environment in the pilot test area for 1-5 years (Argonne 2007a). Monitoring of the ISCR pilot test area should continue, in order to assess the long-term impacts of the ISCR treatment on the groundwater at Centralia. A monitoring plan will be submitted to the KDHE for review. With the KDHE's approval, this plan will be implemented while a Corrective Action Study for the Centralia site is being prepared.

- Further implementation of the ISCR approach at Centralia in the context of an Interim Measure (Argonne 2007a) should not be pursued at this time. The factors influencing this determination include the following:

- The groundwater that has been treated at Centralia does not meet KDHE regulatory requirements for the contaminants (carbon tetrachloride and chloroform) targeted for remediation through use of ISCR technology. There is presently no indication that the concentrations of these contaminants will reach acceptable regulatory levels in the treated area in the future.

- Existing hydrogeologic data for the remainder of the Centralia site indicate that the subsurface conditions beneath much of this area are less favorable for the use of injection technologies than those encountered near SB12MW02.

- The expected areal influence of additional ISCR injections is limited. Very detailed internal characterization of the groundwater plume would therefore be required to support potential further injection efforts. A very closely spaced injection grid would also be required to address the identified lateral and vertical heterogeneity of the carbon tetrachloride distribution in the Centralia aquifer. 
- The future potential for beneficial use of groundwater at Centralia treated with the ISCR approach is questionable. The time frame required for return of the treated groundwater to acceptable general quality levels after ISCR treatment cannot presently be estimated.

- Further identification and critical evaluation of potential remedial alternatives for Centralia should be conducted as part of a Corrective Action Study for this site. 


\section{References}

Adventus, 2006, News Release: Adventus Outlines In Situ Chemical Reduction (ISCR) Advantages, Adventus Americas, Inc., Freeport, Illinois, http://www.adventusgroup.com/pdfs/ Release-ISCR\%20Oils.pdf, May 23, accessed October 28, 2008.

Adventus, 2008a, Remediation at the Former CCC/USDA Grain Storage Facility at Centralia, Kansas - Field Pilot Study Report: Treatment of Carbon Tetrachloride in Soil and Groundwater Using EHC and EHC-A ${ }^{T M}$ ISCR Technology, Adventus Project No. AA16-083b, prepared for the Environmental Science Division, Argonne National Laboratory, Argonne, Illinois, by Adventus Americas, Inc., Union, New Jersey, March.

Adventus, 2008b, EHC ${ }^{\circledR}$ Technology Overview — Field Applications, Adventus Americas, Inc., Freeport, Illinois, http://www.adventusgroup.com/pdfs/EHC_applications.pdf, accessed October 28, 2008.

Adventus, 2008c, EHC ${ }^{\circledR}$ for Carbon Tetrachloride, Adventus Americas, Inc., Freeport, Illinois, http://www.adventusgroup.com/pdfs/EHC_CT.pdf, accessed October 28, 2008.

Adventus, 2008d, Field Profile: EHC ${ }^{\circledR}$ Treatment of Groundwater Plume Containing Chlorinated Solvents, Adventus Americas, Inc., Freeport, Illinois, http://www.adventusgroup. com/pdfs/EHC/Chlorinated\%20Solvents.pdf, accessed October 28, 2008.

Adventus, 2008e, "Field Profile: Grain Silo Facility, Kansas," pp. 31-34 in Mueller, J., R. Srirangam, A. Seech, J. Vogan, A. Przepiora, and M. Martinson, Hydrogen Equivalents: Comparison of Simple Electron Donors to EHC ${ }^{T M}$ In Situ Chemical Reduction (ISCR) Reagent, Adventus Americas, Inc., Freeport, Illinois, http://www.adventusgroup.com/search/index. shtml?cx=010782173747260684302\%3Aiuzhufpl_cg\&cof=FORID\%3A11\&q=field+profile+ grain+silo\#181\f, accessed October 28, 2008.

Adventus, 2008f, EHC ${ }^{\circledR}$ Environmental Remediation Products, Adventus Americas, Inc., Freeport, Illinois, http://www.adventusgroup.com/products/ehc.shtml, accessed October 28, 2008. 
Argonne, 2002a, Final Work Plan: Phase I QuickSite Investigation, Centralia, Kansas, ANL/ER/TR-02/001, prepared for the Commodity Credit Corporation, U.S. Department of Agriculture, Washington, D.C., by Argonne National Laboratory, Argonne, Illinois, June.

Argonne, 2002b, Final Master Work Plan: Environmental Investigations at Former CCC/USDA Facilities in Kansas, 2002 Revision, ANL/ER/TR-02/004, prepared for the Commodity Credit Corporation, U.S. Department of Agriculture, Washington, D.C., by Argonne National Laboratory, Argonne, Illinois, December.

Argonne, 2003, Final Phase I Report and Phase II Work Plan: QuickSite Investigation, Centralia, Kansas, ANL/ER/TR-02/009, prepared for the Commodity Credit Corporation, U.S. Department of Agriculture, Washington, D.C., by Argonne National Laboratory, Argonne, Illinois, March.

Argonne, 2004, Final Phase II Report: QuickSite Investigation, Centralia, Kansas, ANL/ER/TR-03/006, prepared for the Commodity Credit Corporation, U.S. Department of Agriculture, Washington, D.C., by Argonne National Laboratory, Argonne, Illinois, March.

Argonne, 2005a, Final Work Plan: Groundwater Monitoring at Centralia, Kansas, ANL/ER/TR-05/004, prepared for the Commodity Credit Corporation, U.S. Department of Agriculture, Washington, D.C., by Argonne National Laboratory, Argonne, Illinois, August.

Argonne, 2005b, Final Report: 2004 Monitoring Well Installation and Sampling at Centralia, Kansas, ANL/ER/TR-04/011, prepared for the Commodity Credit Corporation, U.S. Department of Agriculture, Washington, D.C., by Argonne National Laboratory, Argonne, Illinois, October.

Argonne, 2005c, Results of Fall 2005 Sampling at Centralia, Kansas, and Recommendations for Expansion of the Monitoring Network, ANL/EVS/AGEM/CHRON-906, prepared for the Commodity Credit Corporation, U.S. Department of Agriculture, Washington, D.C., by Argonne National Laboratory, Argonne, Illinois, November 18.

Argonne, 2006a, Final Report: Groundwater Monitoring at Centralia, Kansas, in SeptemberOctober 2005 and March 2006, with Expansion of the Monitoring Network in January 2006, ANL/EVS/AGEM/TR-06-06, prepared for the Commodity Credit Corporation, U.S. Department of Agriculture, Washington, D.C., by Argonne National Laboratory, Argonne, Illinois, October. 
Argonne, 2006b, September Monitoring Results for Centralia, Kansas, ANL/EVS/AGEM/CHRON-1003, prepared for the Commodity Credit Corporation, U.S. Department of Agriculture, Washington, D.C., by Argonne National Laboratory, Argonne, Illinois, November 10.

Argonne, 2007a, Interim Measure Conceptual Design for Remediation at the Former CCC/USDA Grain Storage Facility at Centralia, Kansas: Pilot Test and Remedy Implementation, ANL/EVS/AGEM/TR-07-11, prepared for the Commodity Credit Corporation, U.S. Department of Agriculture, Washington, D.C., by Argonne National Laboratory, Argonne, Illinois, October.

Argonne, 2007b, March 2007 Monitoring Results for Centralia, Kansas, ANL/EVS/AGEM/TR/07-08, prepared for the Commodity Credit Corporation, U.S. Department of Agriculture, Washington, D.C., by Argonne National Laboratory, Argonne, Illinois, June.

Argonne, 2008a, September 2007 Monitoring Results for Centralia, Kansas, ANL/EVS/AGEM/TR/08-01, prepared for the Commodity Credit Corporation, U.S. Department of Agriculture, Washington, D.C., by Argonne National Laboratory, Argonne, Illinois, January.

Argonne, 2008b, letter from L. LaFreniere (Environmental Science Division, Argonne National Laboratory, Argonne, Illinois) to K. Hoeffner (Bureau of Water, Kansas Department of Health and Environment, Topeka, Kansas), ANL/EVS/AGEM/CHRON-1135, regarding transmittal of the Adventus document Remediation at the Former CCC/USDA Grain Storage Facility at Centralia, Kansas - Field Pilot Study Report: Treatment of Carbon Tetrachloride in Soil and Groundwater Using EHC and EHC-A ${ }^{T M}$ ISCR Technology, April 23.

EPA, 1995, Method 524.2: Measurement of Purgeable Organic Compounds in Water by Capillary Column Gas Chromatography/Mass Spectrometry, Revision 4.1, edited by J.W. Munch, National Exposure Research Laboratory, Office of Research and Development, U.S. Environmental Protection Agency, Cincinnati, Ohio.

EPA, 1998, Test Methods for Evaluation Solid Waste: Physical/Chemical Methods, EPA SW846, 3rd edition, Draft Update IVA, U.S. Environmental Protection Agency, January (available from National Technical Information Service, 5285 Port Royal Road, Springfield, VA, 22161). 
EPA, 2008, Drinking Water Contaminants, updated June 5, 2008, U.S. Environmental Protection Agency, Washington, D.C, http://www.epa.gov/safewater/contaminants/index.html, accessed October 28, 2008.

Kampbell, D.H., and S.A. Vandegrift, 1998, “Analysis of Dissolved Methane, Ethane, and Ethylene in Ground Water by a Standard Gas Chromatographic Technique,” Journal of Chromatographic Science 36:253-256.

KDHE, 2007a, letter from C. Carey (Bureau of Environmental Remediation, Kansas Department of Health and Environment, Topeka, Kansas) to C. Roe (Commodity Credit Corporation, U.S. Department of Agriculture, Washington, D.C.) regarding Interim Measure Conceptual Design for Remediation at the Former CCC/USDA Grain Storage Facility at Centralia, Kansas: Pilot Test and Remedy Implementation, November 9.

KDHE, 2007b, letter from K. Hoeffner (Bureau of Water, Kansas Department of Health and Environment, Topeka, Kansas) to L. LaFreniere (Environmental Science Division, Argonne National Laboratory, Argonne, Illinois) regarding the November 2, 2007, request by Argonne for UIC program authorization of 15 Class V injection points, November 20.

KDHE, 2008a, letter from D. Taylor (Bureau of Water, Kansas Department of Health and Environment, Topeka, Kansas) to D. Surgnier (Delta Environmental) regarding waiver request for flush mount monitoring wells at Centralia, February 19.

KDHE, 2008b, electronic mail message from E. McWilliams (Bureau of Environmental Remediation, Kansas Department of Health and Environment, Topeka, Kansas), to L. Larsen (Larsen and Associates, Lawrence, Kansas), regarding wastewater disposal, April 15.

KDHE, 2008c, electronic mail message from E. McWilliams (Bureau of Environmental Remediation, Kansas Department of Health and Environment, Topeka, Kansas), to L. Larsen (Larsen and Associates, Lawrence, Kansas), regarding wastewater disposal, July 31.

Puls, R.W., and Barcelona, M.J., 1996, "Low-Flow (Minimal Drawdown) Ground-Water Sampling Procedures,” EPA/540/S-95/504, in Ground Water Issue, Superfund Technology Support Center for Ground Water, National Risk Management Research Laboratory, Ada, Oklahoma, April (www.epa.gov/tio/tsp/download/lwflw2a.pdf). 


\section{Appendix A:}

Sequence of Pre-Injection Baseline Sampling Activities and Post-Injection Monitoring Activities 
TABLE A.1 Sequence of sampling activities during the Centralia pilot study, November 2007-September 2008.

\begin{tabular}{|c|c|c|c|c|c|c|c|c|c|}
\hline $\begin{array}{c}\text { Sample } \\
\text { Date }\end{array}$ & Time & Sample & $\begin{array}{l}\text { Sample } \\
\text { Medium }\end{array}$ & Type $^{a}$ & Location & $\begin{array}{c}\text { Depth } \\
\text { (ft BGL) }\end{array}$ & $\begin{array}{l}\text { Chain of } \\
\text { Custody }\end{array}$ & $\begin{array}{l}\text { Shipping } \\
\text { Date }\end{array}$ & Sample Description \\
\hline
\end{tabular}

Pre-injection baseline sampling, November 2007

11/12/07 13:59 CNPSB1-S-16338

11/12/07 14:17 CNPSB1-S-16339

11/12/07 14:24 CNPSB1-S-16340

11/12/07 14:53 CNPSB1-S-16341

11/12/07 15:11 CNPSB1-S-16342

11/12/07 15:27 CNPSB1-S-16343

11/12/07 15:46 CNPSB1-S-16344

11/12/07 16:08 CNPSB1-S-16345

11/12/07 16:21 CNQCTB-S-16347

11/12/07 16:28 CNPSB1-S-16346

11/12/07 16:49 CNPSB1-S-19946

11/12/07 18:30 CNPSB1-W-16245

$\begin{array}{ccccc}\text { Soil } & \text { CPT } & \text { PSB1 } & 4 & 6333 \\ \text { Soil } & \text { CPT } & \text { PSB1 } & 8 & 6333 \\ \text { Soil } & \text { CPT } & \text { PSB1 } & 12 & 6333 \\ \text { Soil } & \text { CPT } & \text { PSB1 } & 16 & 6333 \\ \text { Soil } & \text { CPT } & \text { PSB1 } & 20 & 6333 \\ \text { Soil } & \text { CPT } & \text { PSB1 } & 24 & 6333 \\ \text { Soil } & \text { CPT } & \text { PSB1 } & 28 & 6333 \\ \text { Soil } & \text { CPT } & \text { PSB1 } & 32 & 6333 \\ \text { Soil } & \text { TB } & \text { QC } & - & 6333 \\ & & & & \\ \text { Soil } & \text { CPT } & \text { PSB1 } & 36 & 6333 \\ \text { Soil } & \text { CPT } & \text { PSB1 } & 40 & 6333 \\ \text { Water } & \text { CPT } & \text { PSB1 } & 50-55 & 6348 \\ & & & & \end{array}$

$\begin{array}{rrl}11 / 13 / 07 & 7: 10 & \text { CNQCTB-W-19947b } \\ & & \\ 11 / 13 / 07 & 7: 13 & \text { CNQCTB-W-19948 } \\ & & \\ 11 / 13 / 07 & 8: 16 & \text { CNPSB1-W-16246 } \\ & & \\ 11 / 13 / 07 & 8: 20 & \text { CNPSB1-W-16246REP } \\ 11 / 13 / 07 & 10: 47 & \text { CNPSB2-S-19949 } \\ 11 / 13 / 07 & 10: 55 & \text { CNPSB2-S-19950 } \\ 11 / 13 / 07 & 11: 06 & \text { CNPSB2-S-19951 } \\ 11 / 13 / 07 & 11: 23 & \text { CNPSB2-S-19952 } \\ 11 / 13 / 07 & 11: 35 & \text { CNPSB2-S-19953 } \\ 11 / 13 / 07 & 11: 54 & \text { CNPSB2-S-19954 } \\ 11 / 13 / 07 & 11: 55 & \text { CNPSB2-S-19954REP b } \\ 11 / 13 / 07 & 12: 12 & \text { CNPSB2-S-19955 } \\ 11 / 13 / 07 & 13: 29 & \text { CNPSB2-S-19956 } \\ 11 / 13 / 07 & 13: 50 & \text { CNPSB2-S-19957 } \\ 11 / 13 / 07 & 14: 16 & \text { CNPSB2-S-19958 } \\ 11 / 13 / 07 & 16: 01 & \text { CNPSB2-W-16247 } \\ 11 / 13 / 07 & 17: 22 & \text { CNPSB2-W-16248 }\end{array}$

11/13/07 17:22 CNPSB2-W-16248

$\begin{array}{ccccc}\text { Water } & \text { TB } & \text { QC } & - & 6348 \\ & & & & \\ \text { Water } & \text { TB } & \text { QC } & - & 6346 \\ \text { Water } & \text { CPT } & \text { PSB1 } & 55-60 & 6348 \\ & & & & \\ \text { Water } & \text { CPT } & \text { PSB1 } & 55-60 & 6348 \\ \text { Soil } & \text { CPT } & \text { PSB2 } & 3.8 & 6349 \\ \text { Soil } & \text { CPT } & \text { PSB2 } & 8 & 6349 \\ \text { Soil } & \text { CPT } & \text { PSB2 } & 12 & 6349 \\ \text { Soil } & \text { CPT } & \text { PSB2 } & 16 & 6349 \\ \text { Soil } & \text { CPT } & \text { PSB2 } & 20 & 6349 \\ \text { Soil } & \text { CPT } & \text { PSB2 } & 24 & 6349 \\ \text { Soil } & \text { CPT } & \text { PSB2 } & 24 & 6349 \\ \text { Soil } & \text { CPT } & \text { PSB2 } & 28 & 6349 \\ \text { Soil } & \text { CPT } & \text { PSB2 } & 32 & 6349 \\ \text { Soil } & \text { CPT } & \text { PSB2 } & 36 & 6349 \\ \text { Soil } & \text { CPT } & \text { PSB2 } & 40 & 6349 \\ \text { Water } & \text { CPT } & \text { PSB2 } & 50-55 & 6348 \\ & & & & \\ \text { Water } & \text { CPT } & \text { PSB2 } & 55-60 & 6348\end{array}$

$1 / 13 / 07$ Vertical-profile soil sampling at location of former SB12

$11 / 13 / 07$ Vertical-profile soil sampling at location of former SB12.

$11 / 13 / 07$ Vertical-profile soil sampling at location of former SB12.

11/13/07 Vertical-profile soil sampling at location of former SB12.

11/13/07 Vertical-profile soil sampling at location of former SB12.

11/13/07 Vertical-profile soil sampling at location of former SB12.

11/13/07 Vertical-profile soil sampling at location of former SB12.

11/13/07 Vertical-profile soil sampling at location of former SB12.

$11 / 13 / 07$ Trip blank sent to the AGEM Laboratory for organic analysis with soil samples listed on COCs 6333 and 6349 .

$11 / 13 / 07$ Vertical-profile soil sampling at location of former SB12.

11/13/07 Vertical-profile soil sampling at location of former SB12.

$11 / 13 / 07$ Depth to water $=31 \mathrm{ft}$ from top of riser. Water level rising Depth of hole $=59.3 \mathrm{ft}$. Purged with Waterra pump. Sampled with bailer. Water very muddy. Difficult to preserve to $\mathrm{pH}<2$ for inorganic analysis because of high sediment content.

11/13/07 Trip blank sent to the AGEM Laboratory for organic analysis with water samples listed on COC 6348. Commercial distilled water.

11/13/07 Trip blank sent to Severn-Trent for methane analysis with water samples listed on COC 6349.

11/13/07 Sample collected by using bailer after purging with Waterra pump. Water very muddy.

11/13/07 Replicate of sample CNPSB1-W-16246.

11/13/07 Vertical-profile soil sampling.

11/13/07 Vertical-profile soil sampling.

11/13/07 Vertical-profile soil sampling.

11/13/07 Vertical-profile soil sampling.

11/13/07 Vertical-profile soil sampling.

11/13/07 Vertical-profile soil sampling.

11/13/07 Replicate of sample CNPSB2-S-19954.

11/13/07 Vertical-profile soil sampling.

11/13/07 Vertical-profile soil sampling.

11/13/07 Vertical-profile soil sampling.

11/13/07 Vertical-profile soil sampling.

$11 / 13 / 07$ Depth to water $=29.2 \mathrm{ft}$ from top of riser. Depth of hole $=$ $61.38 \mathrm{ft}$. Immediate water, muddy.

11/13/07 Depth to water $=29.2 \mathrm{ft}$ from top of riser. Depth of hole $=$ $66.1 \mathrm{ft}$. Immediate water, muddy. 
TABLE A.1 (Cont.)

\begin{tabular}{|c|c|c|c|c|c|c|c|c|c|}
\hline $\begin{array}{l}\text { Sample } \\
\text { Date }\end{array}$ & Time & Sample & $\begin{array}{l}\text { Sample } \\
\text { Medium }\end{array}$ & Type $^{a}$ & Location & $\begin{array}{l}\text { Depth } \\
\text { (ft BGL) }\end{array}$ & $\begin{array}{l}\text { Chain of } \\
\text { Custody }\end{array}$ & $\begin{array}{l}\text { Shipping } \\
\text { Date }\end{array}$ & Sample Description \\
\hline \multicolumn{10}{|c|}{ Pre-injection baseline sampling, November 2007 (cont.) } \\
\hline $11 / 13 / 07$ & 19:00 & CNPSB3-W-16249 & Water & СРТ & PSB3 & $50-55$ & 6352 & $11 / 14 / 07$ & $\begin{array}{l}\text { Depth to water }=30.1 \mathrm{ft} \text { from top of riser. Depth of hole }= \\
61.5 \mathrm{ft} . \text { Water is muddy. }\end{array}$ \\
\hline $11 / 14 / 07$ & 8:04 & CNQCTB-W-19959b & Water & TB & QC & - & 6352 & $11 / 14 / 07$ & $\begin{array}{l}\text { Trip blank sent to the AGEM Laboratory for organic analysis } \\
\text { with water samples listed on COC } 6352 \text {. }\end{array}$ \\
\hline $11 / 14 / 07$ & 8:05 & CNQCTB-W-19960 & Water & TB & QC & - & 6355 & $11 / 14 / 07$ & $\begin{array}{l}\text { Trip blank sent to Severn-Trent for methane analysis with } \\
\text { water samples listed on COC } 6355 .\end{array}$ \\
\hline $11 / 14 / 07$ & $8: 24$ & CNQCTB-W-19961 b & Water & TB & QC & - & 6347 & $11 / 14 / 07$ & $\begin{array}{l}\text { Trip blank sent to Envirosystems, Inc., for verification organic } \\
\text { analysis with water samples listed on COC } 6347 \text {. }\end{array}$ \\
\hline $11 / 14 / 07$ & $8: 35$ & CNPSB3-W-16250 & Water & СРТ & PSB3 & $55-60$ & 6352 & $11 / 14 / 07$ & $\begin{array}{l}\text { Depth to water }=\text { approx } 32 \mathrm{ft} \text { from top of riser. Water level } \\
\text { rising. Depth of hole }=66.3 \mathrm{ft} . \text { Immediate water, muddy. }\end{array}$ \\
\hline $11 / 14 / 07$ & $8: 40$ & CNPSB3-W-16250REPb & Water & СРT & PSB3 & $55-60$ & 6352 & $11 / 14 / 07$ & Replicate of sample CNPSB3-W- 16250. \\
\hline $11 / 14 / 07$ & $9: 24$ & CNQCBR-W-19962 ${ }^{b}$ & Water & $\mathrm{RI}$ & QC & - & 6352 & $11 / 14 / 07$ & $\begin{array}{l}\text { Rinsate of decontaminated sampling bailer after collection of } \\
\text { sample CNPSB3-W-16250 and replicate CNPSB3-W- } \\
\text { 16250DUP. }\end{array}$ \\
\hline $11 / 14 / 07$ & $10: 44$ & CNPSB4-W-16251 & Water & СРТ & PSB4 & $50-55$ & 6352 & $11 / 14 / 07$ & $\begin{array}{l}\text { Depth to water }=29.9 \mathrm{ft} \text { from top of riser. Depth of hole }= \\
60.9 \mathrm{ft} \text {. Immediate water, muddy. }\end{array}$ \\
\hline $11 / 14 / 07$ & $11: 44$ & CNPSB4-W-16252 & Water & СРТ & PSB4 & $55-60$ & 6352 & $11 / 14 / 07$ & $\begin{array}{l}\text { Depth to water }=30.9 \mathrm{ft} \text { from top of riser. Water level rising. } \\
\text { Depth of hole }=65.68 \mathrm{ft} \text {. Immediate water, very muddy. }\end{array}$ \\
\hline $11 / 14 / 07$ & $14: 26$ & CNPSB6-W-16253 & Water & СРТ & PSB6 & $50-55$ & 6352 & $11 / 14 / 07$ & $\begin{array}{l}\text { Depth to water }=30.3 \mathrm{ft} \text { from top of riser. Depth of hole }= \\
61.04 \mathrm{ft} . \text { Immediate water, muddy. }\end{array}$ \\
\hline $11 / 14 / 07$ & $15: 33$ & CNPSB6-W-16254 & Water & СРТ & PSB6 & $55-60$ & 6352 & $11 / 14 / 07$ & $\begin{array}{l}\text { Depth to water }=30.44 \mathrm{ft} \text { from top of riser. Depth of hole }= \\
66.0 \mathrm{ft} \text {. Immediate water, muddy. }\end{array}$ \\
\hline $11 / 14 / 07$ & $16: 42$ & CNPSB5-W-16255 & Water & СРТ & PSB5 & $50-55$ & 6352 & $11 / 14 / 07$ & $\begin{array}{l}\text { Depth to water }=30.6 \mathrm{ft} \text { from top of riser. Water level rising. } \\
\text { Depth of hole }=61.1 \mathrm{ft} \text {. }\end{array}$ \\
\hline $11 / 14 / 07$ & $18: 00$ & CNPSB5-W-16256 & Water & СРТ & PSB5 & $55-60$ & 6364 & $11 / 15 / 07$ & $\begin{array}{l}\text { Depth to water }=34.4 \mathrm{ft} \text { from top of riser. Water level rising. } \\
\text { Depth of hole }=65.9 \mathrm{ft} \text {. }\end{array}$ \\
\hline $11 / 15 / 07$ & $8: 14$ & CNQCTB-W-19963 ${ }^{b}$ & Water & TB & QC & - & 6364 & $11 / 15 / 07$ & $\begin{array}{l}\text { Trip blank sent to the AGEM Laboratory for organic analysis } \\
\text { with water samples listed on COC } 6364 \text {. }\end{array}$ \\
\hline $11 / 15 / 07$ & $8: 15$ & CNQCTB-W-19964 & Water & TB & QC & - & 6363 & $11 / 15 / 07$ & $\begin{array}{l}\text { Trip blank sent to Severn-Trent for methane analysis with } \\
\text { water samples listed on COC } 6363 \text {. }\end{array}$ \\
\hline $11 / 15 / 07$ & $9: 36$ & CNPSB7-W-16257 & Water & СРТ & PSB7 & $55-60$ & 6364 & $11 / 15 / 07$ & $\begin{array}{l}\text { Depth to water }=29.7 \mathrm{ft} \text { from top of riser. Water level rising. } \\
\text { Depth of hole }=65.85 \mathrm{ft} . \text { Immediate water, muddy. }\end{array}$ \\
\hline $11 / 15 / 07$ & $10: 58$ & CNPSB7-W-16258 & Water & СРТ & PSB7 & $50-55$ & 6364 & $11 / 15 / 07$ & $\begin{array}{l}\text { Set as temporary riser with sand and Benseal in the morning. } \\
\text { ORP reading erratic, from }-211 \text { to }-611 \mathrm{mV} \text {. }\end{array}$ \\
\hline $11 / 15 / 07$ & $12: 51$ & CNPSB8-W-26060 & Water & CPT & PSB8 & $50-55$ & 6364 & $11 / 15 / 07$ & Sampled via riser. \\
\hline $11 / 15 / 07$ & $13: 27$ & CNQCFB-W-19965 & Water & FB & QC & - & 6364 & $11 / 15 / 07$ & Blank of water used during field event. \\
\hline $11 / 15 / 07$ & $13: 54$ & CNPSB8-W-26061 & Water & СРТ & PSB8 & $55-60$ & 6364 & $11 / 15 / 07$ & $\begin{array}{l}\text { Depth to water }=28.55 \mathrm{ft} \text { from top of riser. Depth of hole }= \\
65.6 \mathrm{ft} \text {. Actual interval is } 54.63 \text { to } 59.63 \mathrm{ft} \text { BGL. Could not } \\
\text { push to } 60 \mathrm{ft} \text {. }\end{array}$ \\
\hline $11 / 15 / 07$ & $13: 55$ & CNPSB8-W-26061REPb & Water & СРТ & PSB8 & $55-60$ & 6364 & $11 / 15 / 07$ & Replicate of sample CNPSB8-W-26061. \\
\hline
\end{tabular}


TABLE A.1 (Cont.)

\begin{tabular}{|c|c|c|c|c|c|c|c|c|c|}
\hline $\begin{array}{c}\text { Sample } \\
\text { Date }\end{array}$ & Time & Sample & $\begin{array}{l}\text { Sample } \\
\text { Medium }\end{array}$ & Type ${ }^{a}$ & Location & $\begin{array}{c}\text { Depth } \\
\text { (ft BGL) }\end{array}$ & $\begin{array}{l}\text { Chain of } \\
\text { Custody }\end{array}$ & $\begin{array}{l}\text { Shipping } \\
\text { Date }\end{array}$ & Sample Description \\
\hline
\end{tabular}

Pre-injection baseline sampling, November 2007 (cont.)

\begin{tabular}{|c|c|c|c|c|c|c|c|}
\hline $11 / 15 / 07$ & $14: 01$ & CNPUBL-W-26062 ${ }^{b}$ & Water & FB & QC & - & $\begin{array}{l}6366 \\
6362\end{array}$ \\
\hline $11 / 15 / 07$ & $15: 15$ & CNPSB9-W-26063 & Water & CPT & PSB9 & $50-55$ & 6364 \\
\hline $11 / 15 / 07$ & $16: 08$ & CNPSB9-W-26064 & Water & CPT & PSB9 & $55-60$ & 6364 \\
\hline $11 / 16 / 07$ & $7: 48$ & CNPSB4-S-19966 & Soil & CPT & PSB4 & 4 & 6368 \\
\hline $11 / 16 / 07$ & $8: 04$ & CNPSB4-S-19967 & Soil & CPT & PSB4 & 8 & 6368 \\
\hline $11 / 16 / 07$ & 8:09 & CNQCTB-S-19969b & Soil & TB & QC & - & 6368 \\
\hline $11 / 16 / 07$ & $8: 19$ & CNPSB4-S-19968 & Soil & CPT & PSB4 & 12 & 6368 \\
\hline $11 / 16 / 07$ & $8: 36$ & CNPSB4-S-19970 & Soil & CPT & PSB4 & 16 & 6368 \\
\hline $11 / 16 / 07$ & $8: 50$ & CNPSB4-S-19971 & Soil & CPT & PSB4 & 20 & 6368 \\
\hline $11 / 16 / 07$ & $9: 19$ & CNPSB4-S-19972 & Soil & CPT & PSB4 & 24 & 6368 \\
\hline $11 / 16 / 07$ & $9: 20$ & CNPSB4-S-19972REPb & Soil & CPT & PSB4 & 24 & 6368 \\
\hline $11 / 16 / 07$ & $9: 38$ & CNPSB4-S-19973 & Soil & CPT & PSB4 & 28 & 6368 \\
\hline $11 / 16 / 07$ & $9: 57$ & CNPSB4-S-19974 & Soil & CPT & PSB4 & 32 & 6368 \\
\hline $11 / 16 / 07$ & $10: 19$ & CNPSB4-S-19975 & Soil & CPT & PSB4 & 36 & 6368 \\
\hline $11 / 16 / 07$ & $10: 51$ & CNPSB4-S-25960 & Soil & CPT & PSB4 & 40 & 6368 \\
\hline $11 / 16 / 07$ & $11: 45$ & CNPSB3-S-25961 & Soil & CPT & PSB3 & 8 & 6369 \\
\hline $11 / 16 / 07$ & $11: 58$ & CNPSB3-S-25962 & Soil & CPT & PSB3 & 12 & 6369 \\
\hline $11 / 16 / 07$ & $13: 24$ & CNPSB3-S-25963 & Soil & CPT & PSB3 & 16 & 6369 \\
\hline $11 / 16 / 07$ & $13: 34$ & CNPSB3-S-25964 & Soil & CPT & PSB3 & 20 & 6369 \\
\hline $11 / 16 / 07$ & $14: 05$ & CNPSB3-S-25965 & Soil & CPT & PSB3 & 24 & 6369 \\
\hline $11 / 16 / 07$ & $14: 23$ & CNPSB3-S-25966 & Soil & CPT & PSB3 & 28 & 6369 \\
\hline $11 / 16 / 07$ & $14: 24$ & CNPSB3-S-25966REPb & Soil & CPT & PSB3 & 28 & 6369 \\
\hline $11 / 16 / 07$ & $14: 42$ & CNPSB3-S-25967 & Soil & CPT & PSB3 & 32 & 6369 \\
\hline $11 / 16 / 07$ & $14: 55$ & CNPSB3-S-25968 & Soil & CPT & PSB3 & 36 & 6369 \\
\hline $11 / 16 / 07$ & $15: 15$ & CNPSB3-S-25969 & Soil & CPT & PSB3 & 40 & 6369 \\
\hline $11 / 16 / 07$ & $15: 35$ & CNPSB3-S-25970 & Soil & CPT & PSB3 & 4 & 6369 \\
\hline $11 / 27 / 07$ & $9: 40$ & CN-S-MeohBlank-27Nov07 & Soil & TB & QC & - & 3441 \\
\hline
\end{tabular}

11/15/07 Blank of water used during field event, sent to Severn-Trent for attenuation parameter analysis and to Envirosystems for VOCs analyses including carbon disulfide.

11/15/07 Depth to water = approx $42 \mathrm{ft}$ from top of riser. Water level rising. Depth of hole $=61.2 \mathrm{ft}$. Water entered rods more slowly than at all previous locations. Muddy.

$11 / 15 / 07$ Depth to water $=$ approx $38 \mathrm{ft}$ from top of riser. Water level rising. Depth of hole $=66.34 \mathrm{ft}$.

$11 / 16 / 07$ Vertical profile subsurface soil sampling.

11/16/07 Vertical profile subsurface soil sampling.

11/16/07 Trip blank sent to the AGEM Laboratory for organic analysis with soil samples listed on COCs 6368 and 6369.

$11 / 16 / 07$ Vertical profile subsurface soil sampling.

11/16/07 Vertical profile subsurface soil sampling.

11/16/07 Vertical profile subsurface soil sampling.

$11 / 16 / 07$ Vertical profile subsurface soil sampling.

11/16/07 Replicate of sample CNPSB4-S-19972.

11/16/07 Vertical profile subsurface soil sampling.

11/16/07 Vertical profile subsurface soil sampling.

$11 / 16 / 07$ Vertical profile subsurface soil sampling.

$11 / 16 / 07$ Vertical profile subsurface soil sampling.

$11 / 16 / 07$ Vertical profile subsurface soil sampling.

$11 / 16 / 07$ Vertical profile subsurface soil sampling.

$11 / 16 / 07$ Vertical profile subsurface soil sampling.

11/16/07 Vertical profile subsurface soil sampling.

11/16/07 Vertical profile subsurface soil sampling.

11/16/07 Vertical profile subsurface soil sampling.

11/16/07 Replicate of sample CNPSB3-S-25966.

$11 / 16 / 07$ Vertical profile subsurface soil sampling.

11/16/07 Vertical profile subsurface soil sampling.

$11 / 16 / 07$ Vertical profile subsurface soil sampling.

11/16/07 Vertical profile subsurface soil sampling.

11/27/07 Trip blank sent to TestAmerica for verification organic analysis with soil samples listed on COC 3441.

Post-injection sampling, December 2007

12/6/07 13:05 CNPSB11-W-25971

12/7/07 11:46 CNPSB11-W-25972
Water CPT PSB11 26-30

Water CPT PSB11 54-58
6023

6023 
TABLE A.1 (Cont.)

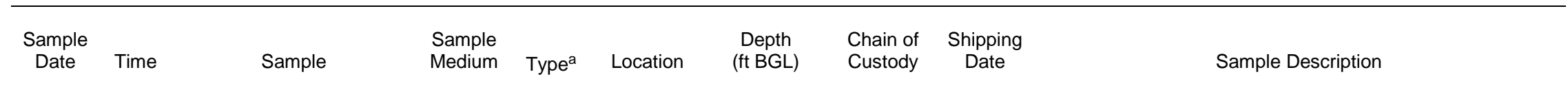

Post-injection sampling, December 2007 (cont.)

12/17/07 15:15 CNPSB12-W-25973

Post-injection sampling, January 8-10, 2008

11:00 CNPMP6-W-26065

Water

CPT/P

$1 / 8 / 08$

16:56 CNPMP7-W-26066

$1 / 8 / 08$

$1 / 8 / 08$

17:03 CNPMP7-W-26067

17:12 CNQCBR-W-25974 ${ }^{\mathrm{b}}$

$1 / 9 / 08$

8:18 CNQCTB-W-25975

$1 / 9 / 08$

8:21 CNQCTB-W-25976

$1 / 9 / 08$

9:31 CNPMP5-W-26069

$1 / 9 / 08$

9:41 CNPMP4-W-26068

Water

CPT/P

PMP4

$48.75-58.75$

$1 / 9 / 08$

11:09 CNSB08-W-26070
Water $\quad \mathrm{CPT} / \mathrm{P} \quad \mathrm{SB} 08$
$54-58$

6023
6379

$50-60$

$50-60$

6379

6379

6379

6379

6380

6379

$2-62$

12/18/07 Post-injection. Water recovered from core barrel to analyze for bromide tracer. Free water $(500 \mathrm{~mL})$ with small amount of mud.

1/10/08 New 0.5-in. piezometer at pre-injection location PSB6.

Measured inside crawler: water level $=30.7 \mathrm{ft}$, total depth $=$ $66.05 \mathrm{ft}$. Silt in screen. Immediate water, muddy, brown with sediment. Collected without purging by using bailer for VOCs and Waterra pump for bromide.

1/10/08 New 0.5-in. piezometer at pre-injection location PSB7.

Measured inside crawler: water level $=28.9 \mathrm{ft}$ and rising, total depth $=65.6 \mathrm{ft}$. Immediate water. Very muddy, tan/brown, with sediment. Collected without purging by using bailer for VOCs and Waterra pump for bromide.

1/10/08 Replicate of sample CNPMP7-W-26066.

1/10/08 Rinsate of decontaminated sampling bailer after collection of sample CNPMP7-W-26066 and replicate CNPMP7-W26067.

1/10/08 Trip blank sent to the AGEM Laboratory for organic analysis with water samples listed on COCs 6379 and 6381.

1/10/08 Trip blank sent to Envirosystems for verification organic analysis with water samples listed on COC 6380.

1/10/08 New 1-in. piezometer at pre-injection location PSB5.

Measured from stickup ( $0.7 \mathrm{ft}$ above grade level) water level $=23.59 \mathrm{ft}$. Water muddy, brown, with sediment. Collected without purging by using bailer for VOCs and Waterra pump for bromide.

1/10/08 New 0.5-in. piezometer southeast of SB07R as requested by $\mathrm{KDHE}$. Measured inside cone penetrometer: water level = $27.35 \mathrm{ft}$ and rising, total depth $=63.83 \mathrm{ft}$. Immediate water, muddy, brown sediment. Collected without purging by using bailer for VOCs and Waterra pump for bromide.

$1 / 10 / 08$ Depth to water from TOC $=19.57 \mathrm{ft}$. Depth of 1 -in. well $=$ $59.81 \mathrm{ft}$. Water slightly cloudy/tan. Collected without purging by using bailer for VOCs and Waterra pump for bromide. 
TABLE A.1 (Cont.)

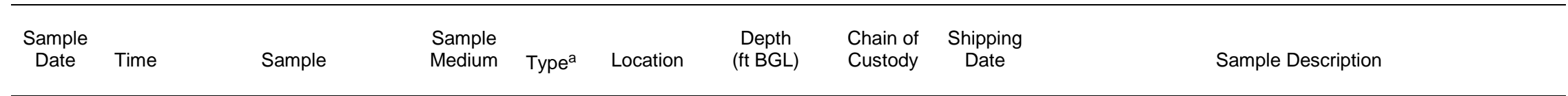

Post-injection sampling, January 8-10, 2008 (cont.)

\begin{tabular}{|c|c|c|c|c|c|c|c|}
\hline $1 / 9 / 08$ & $12: 44$ & CNPMP3-W-26071 & Water & $\mathrm{CPT} / \mathrm{P}$ & PMP3 & $50-60$ & 6379 \\
\hline $1 / 9 / 08$ & $12: 52$ & CNPMP3-W-26072b & Water & $\mathrm{CPT} / \mathrm{P}$ & PMP3 & $50-60$ & 6379 \\
\hline $1 / 9 / 08$ & $13: 52$ & CNSB07R-W-26073 & Water & $\mathrm{CPT} / \mathrm{P}$ & SB07R & $45-60$ & 6379 \\
\hline $1 / 9 / 08$ & $15: 21$ & CNMW02-W-26074 & Water & MW & MW02 & $49.5-59.5$ & 6379 \\
\hline $1 / 9 / 08$ & $16: 04$ & CNQCBR-W-25977 & Water & $\mathrm{RI}$ & QC & - & 6379 \\
\hline $1 / 9 / 08$ & $16: 19$ & CNPMP8-W-26075 & Water & $\mathrm{CPT} / \mathrm{P}$ & PMP8 & $50-60$ & 6379 \\
\hline $1 / 10 / 08$ & $8: 26$ & CNSB04-W-26076 & Water & CPT/P & SB04 & $51-61$ & 6381 \\
\hline $1 / 10 / 08$ & $9: 31$ & CNPMP9-W-26077 & Water & CPT/P & PMP9 & $50-60$ & 6381 \\
\hline $1 / 10 / 08$ & $10: 44$ & CNMW03-W-26078 & Water & MW & MW03 & $50.5-60.5$ & 6381 \\
\hline $1 / 10 / 08$ & $13: 46$ & CNPMP1-W-26079 & Water & CPT/P & PMP1 & $50-60$ & 6381 \\
\hline $1 / 10 / 08$ & $13: 50$ & CNPMP1-W-26080 & Water & $\mathrm{CPT} / \mathrm{P}$ & PMP1 & $50-60$ & 6381 \\
\hline
\end{tabular}

1/10/08 New 0.5-in. piezometer at pre-injection location PSB3. Measured inside crawler: water level $=28.60 \mathrm{ft}$, total depth $=65.50 \mathrm{ft}$. Immediate water, muddy, brown. Unpleasant odor. Magnetic black fine sediment in water. Iron is average of 0.48 and $0.77 \mathrm{mg} / \mathrm{L}$.

1/10/08 Replicate of sample CNPMP3-W-26071.

$1 / 10 / 08$ Depth to water from TOC $=18.60 \mathrm{ft}$. Water almost clear, faintly cloudy. Collected without purging by using bailer for VOCs and Waterra pump for bromide.

1/10/08 Depth to water from TOC $=20.95 \mathrm{ft}$. Depth of well $=61.28 \mathrm{ft}$. Water cloudy gray. Smells bad, like sewage. Magnetic fine black sediment. Collected without purging by using bailer for VOCs and Waterra pump for bromide.

1/10/08 Rinsate of decontaminated sampling bailer after collection of sample CNPMP3-W-26071 and replicate CNPMP3-W26072 and prior to collection of sample CNPMP8-W26075.

1/10/08 New piezometer at pre-injection location PSB8. Measured inside crawler: water level $=35.3 \mathrm{ft}$ and rising, total depth $=$ $65.5 \mathrm{ft}$. Water, muddy, brown. Faint smell. Magnetic fine black sediment. Collected without purging by using bailer for VOCs and Waterra pump for bromide.

$1 / 10 / 08$ Depth to water from TOC $=22.58 \mathrm{ft}$. Depth of well $=59.37 \mathrm{ft}$. Water pale muddy brown. Collected without purging by using bailer for VOCs and Waterra pump for bromide.

1/10/08 New 0.5-in. piezometer at pre-injection location PSB9. Measured inside crawler: water level $=22.6 \mathrm{ft}$, total depth $=$ $65.9 \mathrm{ft}$. Immediate water, muddy, brown. Magnetic fine black sediment. Collected without purging by using bailer for VOCs and Waterra pump for bromide.

1/10/08 Depth to water from TOC $=21.46 \mathrm{ft}$. Depth of well $=62.45 \mathrm{ft}$. Water slightly cloudy tan. Collected without purging by using bailer for VOCs and Waterra pump for bromide.

1/10/08 New piezometer $3 \mathrm{ft}$ offset from PT7. Measured inside crawler: water level $=32.6 \mathrm{ft}$ and rising, total depth $=$ $65.78 \mathrm{ft}$. Immediate water, muddy, brown. Magnetic fine black sediment. Collected without purging by using bailer for VOCs and Waterra pump for bromide. Replicate of sample CNPMP1-W-26079. 
TABLE A.1 (Cont.)

\begin{tabular}{|c|c|c|c|c|c|c|c|c|c|}
\hline $\begin{array}{l}\text { Sample } \\
\text { Date }\end{array}$ & Time & Sample & $\begin{array}{l}\text { Sample } \\
\text { Medium }\end{array}$ & Type $^{\mathrm{a}}$ & Location & $\begin{array}{l}\text { Depth } \\
\text { (ft BGL) }\end{array}$ & $\begin{array}{l}\text { Chain of } \\
\text { Custody }\end{array}$ & $\begin{array}{l}\text { Shipping } \\
\text { Date }\end{array}$ & Sample Description \\
\hline
\end{tabular}

Post-injection sampling, January 8-10, 2008 (cont.)

1/10/08 16:52 CNPMP2-W-26081

CPT/P PMP2 50-60

6381

1/10/08 New piezometer midway between PT2 and PT7. Measured inside crawler: water level $=38.0 \mathrm{ft}$ and rising, total depth $=$ $66.00 \mathrm{ft}$. Water muddy, brown. Magnetic sediment. Collected without purging by using bailer for VOCs and Waterra pump for bromide.

Post-injection sampling, January 24, 2008

\begin{tabular}{|c|c|c|c|c|c|c|c|}
\hline $1 / 24 / 08$ & $8: 28$ & CNPMP1-W-26083 & Water & $\mathrm{CPT} / \mathrm{P}$ & PMP1 & $50-60$ & 6030 \\
\hline $1 / 24 / 08$ & $9: 41$ & CNPMP2-W-26084 & Water & $\mathrm{CPT} / \mathrm{P}$ & PMP2 & $50-60$ & 6030 \\
\hline $1 / 24 / 08$ & $9: 42$ & CNPMP2-W-26085 & Water & $\mathrm{CPT} / \mathrm{P}$ & PMP2 & $50-60$ & 6030 \\
\hline $1 / 24 / 08$ & $10: 48$ & CNPMP3-W-26086 & Water & $\mathrm{CPT} / \mathrm{P}$ & PMP3 & $50-60$ & 6030 \\
\hline $1 / 24 / 08$ & $11: 27$ & CNPMP4-W-26087 & Water & $\mathrm{CPT} / \mathrm{P}$ & PMP4 & $48.75-58.75$ & 6030 \\
\hline $1 / 24 / 08$ & $11: 56$ & CNPMP5-W-26088 & Water & $\mathrm{CPT} / \mathrm{P}$ & PMP5 & $50-60$ & 6030 \\
\hline $1 / 24 / 08$ & $11: 57$ & CNPMP5-W-26089b & Water & $\mathrm{CPT} / \mathrm{P}$ & PMP5 & $50-60$ & 6030 \\
\hline $1 / 24 / 08$ & $12: 45$ & CNPMP6-W-26090 & Water & $\mathrm{CPT} / \mathrm{P}$ & PMP6 & $50-60$ & 6030 \\
\hline $1 / 24 / 08$ & $13: 10$ & CNPMP7-W-26091 & Water & $\mathrm{CPT} / \mathrm{P}$ & PMP7 & $50-60$ & 6030 \\
\hline $1 / 24 / 08$ & $13: 45$ & CNPMP8-W-26092 & Water & $\mathrm{CPT} / \mathrm{P}$ & PMP8 & $50-60$ & 6030 \\
\hline $1 / 24 / 08$ & $14: 25$ & CNPMP9-W-26109 & Water & $\mathrm{CPT} / \mathrm{P}$ & PMP9 & $50-60$ & 6031 \\
\hline $1 / 24 / 08$ & $14: 49$ & CNSB07R-W-26111 & Water & $\mathrm{CPT} / \mathrm{P}$ & SB07R & $45-60$ & 6031 \\
\hline $1 / 24 / 08$ & $15: 19$ & CNSB04-W-26094 & Water & $\mathrm{CPT} / \mathrm{P}$ & SB04 & $51-61$ & 6030 \\
\hline $1 / 24 / 08$ & $15: 40$ & CNSB08-W-26112 & Water & $\mathrm{CPT} / \mathrm{P}$ & SB08 & $52-62$ & 6031 \\
\hline $1 / 24 / 08$ & $15: 45$ & CNQCTB-W-25980 & Water & TB & QC & - & 6030 \\
\hline $1 / 24 / 08$ & $15: 46$ & CNQCTB-W-25981 & Water & TB & QC & - & 6028 \\
\hline $1 / 24 / 08$ & $15: 50$ & CNQCRI-W-25982b & Water & $\mathrm{RI}$ & QC & - & 6032 \\
\hline $1 / 24 / 08$ & $16: 30$ & CNMW02-W-26097 & Water & MW & MW02 & $49.5-59.5$ & 6031 \\
\hline
\end{tabular}

1/24/08 Depth to water from ground $=21.90 \mathrm{ft}$. Depth to bottom $=$ $57.72 \mathrm{ft}$. Sample collected by using Waterra tube.

1/24/08 Depth to water from ground $=21.90 \mathrm{ft}$. Depth to bottom $=$ $58.40 \mathrm{ft}$. Sample collected by using Waterra tube.

1/24/08 Replicate of sample CNPMP2-W-26084.

$1 / 24 / 08$ Depth to water from ground $=22.96 \mathrm{ft}$. Depth to bottom $=$ $58.10 \mathrm{ft}$. Sample collected by using Waterra tube.

$1 / 24 / 08$ Depth to water from ground $=19.95 \mathrm{ft}$. Depth to bottom $=$ $56.80 \mathrm{ft}$. Sample collected by using Waterra tube.

1/24/08 Depth to water from ground $=23.18 \mathrm{ft}$. Depth to bottom $=$ $59.30 \mathrm{ft}$. Sample collected by using Waterra tube.

1/24/08 Replicate of sample CNPMP5-W-26088.

$1 / 24 / 08$ Depth to water from ground $=23.20 \mathrm{ft}$. Depth to bottom $=$ $59.18 \mathrm{ft}$. Sample collected by using Waterra tube.

1/24/08 Depth to water from ground $=21.11 \mathrm{ft}$. Depth to bottom $=$ $58.23 \mathrm{ft}$. Sample collected by using Waterra tube.

1/24/08 Depth to water from ground $=21.00 \mathrm{ft}$. Depth to bottom $=$ $56.10 \mathrm{ft}$. Sample collected by using Waterra tube.

$1 / 24 / 08$ Depth to water from ground $=16.46 \mathrm{ft}$. Depth to bottom $=$ $58.65 \mathrm{ft}$. Sample collected by using Waterra tube.

$1 / 24 / 08$ Depth to water from ground $=18.75 \mathrm{ft}$. Depth to bottom $=$ $58.48 \mathrm{ft}$. Sample collected by using Waterra tube.

1/24/08 Depth to water from ground $=23.03 \mathrm{ft}$. Depth to bottom $=$ $59.36 \mathrm{ft}$. Sample collected by using Waterra tube.

$1 / 24 / 08$ Sample collected by using Waterra tube.

$1 / 24 / 08$ Trip blank sent to the AGEM Laboratory for organic analysis with water samples listed on COCs 6030, 6031, and 6032

1/24/08 Trip blank sent to Envirosystems for verification organic analysis with water samples listed on COC 6028.

1/24/08 Rinsate of decontaminated sampling tube after collection of sample CNSB08-W-26112.

$1 / 24 / 08$ Depth to water from ground $=22.07 \mathrm{ft}$. Depth to bottom $=$ $61.24 \mathrm{ft}$. Sample collected by using Waterra tube. 
TABLE A.1 (Cont.)

\begin{tabular}{|c|c|c|c|c|c|c|c|c|c|}
\hline $\begin{array}{l}\text { Sample } \\
\text { Date }\end{array}$ & Time & Sample & $\begin{array}{l}\text { Sample } \\
\text { Medium }\end{array}$ & Type $^{a}$ & Location & $\begin{array}{l}\text { Depth } \\
\text { (ft BGL) }\end{array}$ & $\begin{array}{l}\text { Chain of } \\
\text { Custody }\end{array}$ & $\begin{array}{l}\text { Shipping } \\
\text { Date }\end{array}$ & Sample Description \\
\hline \multicolumn{10}{|c|}{ Post-injection sampling, January 24, 2008 (cont.) } \\
\hline $1 / 24 / 08$ & $16: 31$ & CNMW02-W-26098 & Water & MW & MW02 & $49.5-59.5$ & 6031 & $1 / 24 / 08$ & Replicate of sample CNMW02-W-26097. \\
\hline \multicolumn{10}{|c|}{ Post-injection sampling, February 2008} \\
\hline 2/23/08 & $10: 51$ & CNMW02-W-26099 & Water & MW & MW02 & $49.5-59.5$ & 6212 & $2 / 25 / 08$ & $\begin{array}{l}\text { Depth to water }=22.37 \mathrm{ft} \text {. Depth of } 4 \text {-in. well }=61.2 \mathrm{ft} \text {. } \\
\text { Sample collected by using low-flow bladder pump after } \\
\text { purging of } 3.9 \mathrm{~L} \text {. }\end{array}$ \\
\hline 2/23/08 & $14: 38$ & CNMW03-W-26115 & Water & MW & MW03 & $50.5-60.5$ & 6212 & $2 / 25 / 08$ & $\begin{array}{l}\text { Depth to water }=21.81 \mathrm{ft} \text {. Depth of } 4 \text {-in. well }=62.4 \mathrm{ft} \text {. } \\
\text { Sample collected by using low-flow bladder pump after } \\
\text { purging of } 5 \mathrm{~L} \text {. }\end{array}$ \\
\hline 2/23/08 & $16: 38$ & CNSB04-W-26117 & Water & $\mathrm{CPT} / \mathrm{P}$ & SB04 & $51-61$ & 6212 & $2 / 25 / 08$ & $\begin{array}{l}\text { Depth to water }=22.97 \mathrm{ft} \text {. Depth of } 1 \text {-in. well }=59.35 \mathrm{ft} \text {. } \\
\text { Sample collected by using low-flow bladder pump after } \\
\text { purging of } 5.6 \mathrm{~L} \text {. }\end{array}$ \\
\hline $2 / 24 / 08$ & $8: 30$ & CNQCTB-W-26118 ${ }^{\mathrm{b}}$ & Water & TB & QC & - & 6212 & $2 / 25 / 08$ & $\begin{array}{l}\text { Trip blank sent to the AGEM Laboratory for organic analysis } \\
\text { with water samples listed on COCs } 6212 \text { and } 6215 \text {. }\end{array}$ \\
\hline $2 / 24 / 08$ & $8: 33$ & CNQCTB-W-25983 ${ }^{b}$ & Water & TB & QC & - & 6213 & $2 / 25 / 08$ & $\begin{array}{l}\text { Trip blank sent to Envirosystems for verification organic } \\
\text { analysis with water samples listed on COC } 6213 \text {. }\end{array}$ \\
\hline $2 / 24 / 08$ & $8: 43$ & CNQCPR-W-26119 b & Water & $\mathrm{RI}$ & QC & - & 6212 & $2 / 25 / 08$ & $\begin{array}{l}\text { Rinsate of decontaminated low-flow bladder pump after } \\
\text { sampling at SB04. }\end{array}$ \\
\hline 2/24/08 & $9: 38$ & CNSB08-W-25978 & Water & $\mathrm{CPT} / \mathrm{P}$ & SB08 & $52-62$ & 6212 & $2 / 25 / 08$ & $\begin{array}{l}\text { Depth to water }=19.78 \mathrm{ft} \text {. Depth of } 1 \text {-in. well }=59.76 \mathrm{ft} \text {. } \\
\text { Sample collected by using low-flow bladder pump after } \\
\text { purging of } 3.5 \mathrm{~L} \text {. }\end{array}$ \\
\hline 2/24/08 & $9: 42$ & CNSB08-W-25979b & Water & $\mathrm{CPT} / \mathrm{P}$ & SB08 & $52-62$ & 6212 & $2 / 25 / 08$ & Replicate of sample CNSB08-W-25978. \\
\hline $2 / 24 / 08$ & $12: 22$ & CNSB07R-W-25984 & Water & $\mathrm{CPT} / \mathrm{P}$ & SB07R & $45-60$ & 6212 & $2 / 25 / 08$ & $\begin{array}{l}\text { Depth to water }=18.89 \mathrm{ft} \text {. Depth of } 2 \text {-in. well }=58.53 \mathrm{ft} \text {. } \\
\text { Sample collected by using low-flow bladder pump after } \\
\text { purging of } 4 \mathrm{~L} \text {. }\end{array}$ \\
\hline $2 / 24 / 08$ & $15: 19$ & CNPMP1-W-25985 & Water & MW & PMP1 & $50-60$ & 6212 & $2 / 25 / 08$ & $\begin{array}{l}\text { Depth to water }=22.09 \mathrm{ft} \text {. Depth of } 0.5 \text {-in. well }=60 \mathrm{ft} \text {. } \\
\text { Sample collected by using Waterra pump tubing as bailer } \\
\text { after purging of } 1.2 \mathrm{~L} \text {. }\end{array}$ \\
\hline $2 / 24 / 08$ & 16:00 & CNPMP2-W-25986 & Water & MW & PMP2 & $50-60$ & 6212 & $2 / 25 / 08$ & $\begin{array}{l}\text { Depth to water }=22.50 \mathrm{ft} \text {. Depth of } 0.5 \text {-in. well }=60 \mathrm{ft} \text {. } \\
\text { Sample collected by using Waterra pump tubing as bailer } \\
\text { after purging of } 410 \mathrm{~mL} \text {. }\end{array}$ \\
\hline 2/24/08 & $16: 04$ & CNPMP2-W-25988 ${ }^{\mathrm{b}}$ & Water & MW & PMP2 & $50-60$ & 6212 & $2 / 25 / 08$ & Replicate of sample CNPMP2-W-25986. \\
\hline $2 / 24 / 08$ & 18:04 & CNPMP9-W-25989 & Water & MW & PMP9 & $50-60$ & 6212 & $2 / 25 / 08$ & $\begin{array}{l}\text { Depth to water }=17.12 \mathrm{ft} \text {. Depth of } 0.5 \text {-in. well }=60 \mathrm{ft} \text {. } \\
\text { Sample collected by using Waterra pump tubing as bailer } \\
\text { after purging of } 7 \mathrm{~L} \text {. }\end{array}$ \\
\hline $2 / 25 / 08$ & $9: 44$ & CNPMP3-W-25990 & Water & MW & PMP3 & $50-60$ & 6215 & $2 / 25 / 08$ & $\begin{array}{l}\text { Depth to water }=22.84 \mathrm{ft} \text {. Depth of } 0.5 \text {-in. well }=60 \mathrm{ft} \text {. } \\
\text { Sample collected by using Waterra pump tubing as bailer } \\
\text { after purging of } 3 \mathrm{~L} \text {. Water pale cloudy brown. }\end{array}$ \\
\hline
\end{tabular}


TABLE A.1 (Cont.)

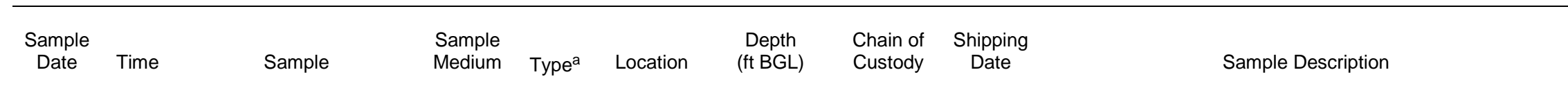

Post-injection sampling, February 2008 (cont.)

\begin{tabular}{|c|c|c|c|c|c|c|c|}
\hline $2 / 25 / 08$ & $11: 49$ & CNPMP4-W-25992 & Water & MW & PMP4 & $48.75-58.75$ & 6215 \\
\hline $2 / 25 / 08$ & $13: 19$ & CNPMP6-W-25993 & Water & MW & PMP6 & $50-60$ & 6215 \\
\hline $2 / 25 / 08$ & $14: 50$ & CNPMP8-W-25994 & Water & MW & PMP8 & $50-60$ & 6215 \\
\hline $2 / 25 / 08$ & $16: 11$ & CNPMP7-W-25996 & Water & MW & PMP7 & $50-60$ & 6215 \\
\hline $2 / 25 / 08$ & $17: 28$ & CNPMP5-W-25997 & Water & MW & PMP5 & $50-60$ & 6215 \\
\hline 2/25/08 & $18: 13$ & CNWAS-W-25998b & Water & BT & QC & - & 6215 \\
\hline \multicolumn{8}{|c|}{ Post-injection sampling, March 2008} \\
\hline 3/12/08 & $10: 07$ & CNMW02-W-26000 & Water & MW & MW02 & $49.5-59.5$ & 6078 \\
\hline 3/12/08 & $12: 06$ & CNMW03-W-26001 & Water & MW & MW03 & $50.5-60.5$ & 6078 \\
\hline 3/12/08 & $12: 07$ & CNMW03DUP-W-26021b & Water & MW & MW03 & $50.5-60.5$ & 6078 \\
\hline 3/12/08 & $14: 09$ & CNSB07R-W-26003 & Water & $\mathrm{CPT} / \mathrm{P}$ & SB07R & $45-60$ & 6078 \\
\hline 3/12/08 & $14: 32$ & CNQCIR-W-26017 & Water & $\mathrm{RI}$ & QC & - & 6078 \\
\hline 3/12/08 & $15: 51$ & CNSB08-W-26004 & Water & $\mathrm{CPT} / \mathrm{P}$ & SB08 & $52-62$ & 6078 \\
\hline 3/12/08 & $17: 14$ & CNSB04-W-26002 & Water & $\mathrm{CPT} / \mathrm{P}$ & SB04 & $51-61$ & 6078 \\
\hline
\end{tabular}

2/25/08 Depth to water $=20.36 \mathrm{ft}$. Depth of 0.5 -in. well $=58.75 \mathrm{ft}$. Sample collected by using Waterra pump as bailer after purging of $5 \mathrm{~L}$. Water muddy brown.

2/25/08 Depth to water $=23.68 \mathrm{ft}$. Depth of 0.5 -in. well $=60 \mathrm{ft}$. Sample collected by using Waterra pump as bailer after purging of $4.5 \mathrm{~L}$. Water muddy brown.

$2 / 25 / 08$ Depth to water $=21.49 \mathrm{ft}$. Depth of 0.5 -in. well $=60 \mathrm{ft}$. Sample collected by using Waterra pump as bailer after purging of $3 \mathrm{~L}$.

2/25/08 Depth to water $=22.47 \mathrm{ft}$. Depth of 0.5 -in. well $=60 \mathrm{ft}$. Sample collected by using Waterra pump as bailer after purging of $2.5 \mathrm{~L}$.

2/25/08 Depth to water $=23.72 \mathrm{ft}$. Depth of 1 -in. well $=60 \mathrm{ft}$. Sample collected by using low-flow bladder pump after purging of $3 \mathrm{~L}$.

2/25/08 Sample from drum containing waste purge water collected during low-flow sampling of wells.

3/13/08 Depth to water $=21.85 \mathrm{ft}$. Depth of 4 -in. well $=59.50 \mathrm{ft}$. Sample collected by using low-flow bladder pump after purging of $3.05 \mathrm{~L}$. Purge water had greenish color and had an odor.

3/13/08 Depth to water $=21.22 \mathrm{ft}$. Depth of 4 -in. well $=60.50 \mathrm{ft}$. Sample collected by using low-flow bladder pump after purging of $3.51 \mathrm{~L}$. Purge water was clear.

3/13/08 Replicate of sample CNMW03-W-26001.

$3 / 13 / 08$ Depth to water $=18.23 \mathrm{ft}$. Depth of 2-in. well $=60 \mathrm{ft}$. Sample collected by using low-flow bladder pump after purging of $2.26 \mathrm{~L}$. Purge water was clear.

3/13/08 Rinsate of decontaminated bladder pump after collection of sample CNSB07R-W-26003.

3/13/08 Depth to water $=19.24 \mathrm{ft}$. Depth of 1 -in. well $=59.86 \mathrm{ft}$ Sample collected by using low-flow bladder pump after purging of $1.92 \mathrm{~L}$. Purge water was clear.

3/13/08 Depth to water $=22.34 \mathrm{ft}$. Depth of 1 -in. well $=59.41 \mathrm{ft}$. Sample collected by using low-flow bladder pump after purging of 3.21 L. Purge water was clear. 
TABLE A.1 (Cont.)

\begin{tabular}{|c|c|c|c|c|c|c|c|c|c|}
\hline $\begin{array}{l}\text { Sample } \\
\text { Date }\end{array}$ & Time & Sample & $\begin{array}{l}\text { Sample } \\
\text { Medium }\end{array}$ & Type ${ }^{a}$ & Location & $\begin{array}{c}\text { Depth } \\
\text { (ft BGL) }\end{array}$ & $\begin{array}{l}\text { Chain of } \\
\text { Custody }\end{array}$ & $\begin{array}{l}\text { Shipping } \\
\text { Date }\end{array}$ & Sample Description \\
\hline \multicolumn{10}{|c|}{ Post-injection sampling, March 2008 (cont.) } \\
\hline $3 / 13 / 08$ & $8: 34$ & CNPMP5-W-26009 & Water & MW & PMP5 & $50-60$ & 6080 & $3 / 13 / 08$ & $\begin{array}{l}\text { Depth to water }=23.20 \mathrm{ft} \text {. Depth of } 1 \text {-in. well }=59.96 \mathrm{ft} \text {. } \\
\text { Sample collected by using low-flow bladder pump after } \\
\text { purging of } 1.24 \mathrm{~L} \text {. Purge water was clear. }\end{array}$ \\
\hline $3 / 13 / 08$ & $9: 05$ & CNQCIR-W-26018 ${ }^{\mathrm{b}}$ & Water & $\mathrm{RI}$ & QC & - & 6080 & $3 / 13 / 08$ & $\begin{array}{l}\text { Rinsate of decontaminated bladder pump after collection of } \\
\text { sample CNPMP5-W-26009. }\end{array}$ \\
\hline $3 / 13 / 08$ & $10: 15$ & CNPMP6-W-26010 & Water & MW & PMP6 & $50-60$ & 6080 & $3 / 13 / 08$ & $\begin{array}{l}\text { Depth to water }=23.20 \mathrm{ft} \text {. Depth of } 0.5 \text {-in. well }=58.45 \mathrm{ft} \text {. } \\
\text { Sample collected by using Waterra pump tubing as bailer } \\
\text { after purging of } 4.5 \mathrm{~L} \text {. Purge water was muddy brown. }\end{array}$ \\
\hline $3 / 13 / 08$ & $11: 22$ & CNPMP7-W-26011 & Water & MW & PMP7 & $50-60$ & 6080 & $3 / 13 / 08$ & $\begin{array}{l}\text { Depth to water }=21.09 \mathrm{ft} \text {. Depth of } 0.5 \text {-in. well }=58.65 \mathrm{ft} \text {. } \\
\text { Sample collected by using Waterra pump tubing as bailer } \\
\text { after purging of } 4.45 \mathrm{~L} \text {. Purge water was cloudy tan. }\end{array}$ \\
\hline $3 / 13 / 08$ & $12: 58$ & CNPMP3-W-26007 & Water & MW & PMP3 & $50-60$ & 6080 & $3 / 13 / 08$ & $\begin{array}{l}\text { Depth to water }=21.83 \mathrm{ft} \text {. Depth of } 0.5 \text {-in. well }=56.70 \mathrm{ft} \text {. } \\
\text { Sample collected by using Waterra pump tubing as bailer } \\
\text { after purging of } 3.04 \mathrm{~L} \text {. Purge water was mousy gray }\end{array}$ \\
\hline 3/13/08 & $12: 59$ & CNPMP3DUP-W-26022b & Water & MW & PMP3 & $50-60$ & 6080 & $3 / 13 / 08$ & Replicate of sample CNPMP3-W-26007. \\
\hline $3 / 13 / 08$ & $14: 20$ & CNPMP4-W-26008 & Water & MW & PMP4 & $48.75-58.75$ & 6080 & $3 / 13 / 08$ & $\begin{array}{l}\text { Depth to water }=19.90 \mathrm{ft} \text {. Well silted in. Used Waterra pump } \\
\text { to remove sandy material and purge } 3 \text { well volumes, then } \\
\text { sampled. Purge water was dark tan with no odor. No field } \\
\text { measurements. }\end{array}$ \\
\hline $3 / 13 / 08$ & $14: 30$ & CNPMP9-W-26013 & Water & MW & PMP9 & $50-60$ & 6080 & $3 / 13 / 08$ & $\begin{array}{l}\text { Depth to water }=16.10 \mathrm{ft} \text {. Depth of } 0.5 \text {-in. well }=58.0 \mathrm{ft} \text {. } \\
\text { Sample collected by using Waterra pump tubing as bailer } \\
\text { after purging of } 5.3 \mathrm{~L} \text {. Purge water was tan; no odor. }\end{array}$ \\
\hline $3 / 13 / 08$ & $14: 45$ & CNQCTB-W-26014 & Water & TB & QC & - & 6080 & $3 / 13 / 08$ & $\begin{array}{l}\text { Trip blank sent to the AGEM Laboratory for organic analysis } \\
\text { with water samples listed on COCs } 6078 \text { and } 6080 \text {. }\end{array}$ \\
\hline $3 / 13 / 08$ & $14: 46$ & CNQCTB-W-26014ab & Water & TB & QC & - & 6081 & $3 / 13 / 08$ & $\begin{array}{l}\text { Trip blank sent to Envirosystems for verification organic } \\
\text { analysis with water samples listed on COC } 6081 \text {. }\end{array}$ \\
\hline $3 / 13 / 08$ & 16:06 & CNPMP8-W-26012 & Water & MW & PMP8 & $50-60$ & 6082 & $3 / 13 / 08$ & $\begin{array}{l}\text { Depth to water }=20.95 \mathrm{ft} \text {. Well silted in. Used Waterra pump } \\
\text { to remove sand and gray-colored material (with odor) and } \\
\text { purge } 3 \text { well volumes, then sampled. Only iron measured } \\
\text { in field. }\end{array}$ \\
\hline $3 / 13 / 08$ & $16: 39$ & CNPMP2-W-26006 & Water & MW & PMP2 & $50-60$ & 6082 & $3 / 13 / 08$ & $\begin{array}{l}\text { Depth to water }=21.80 \mathrm{ft} \text {. Well silted in. Used Waterra pump } \\
\text { to remove sand and brown material (with odor) and purge } \\
3 \text { well volumes, then sampled. Only iron measured in field. }\end{array}$ \\
\hline $3 / 13 / 08$ & $17: 15$ & CNPMP1-W-26005 & Water & MW & PMP1 & $50-60$ & 6082 & $3 / 13 / 08$ & $\begin{array}{l}\text { Depth to water }=21.45 \mathrm{ft} \text {. Well silted in. Used Waterra pump } \\
\text { to remove sand and brown material (with odor) and purge } \\
3 \text { well volumes, then sampled. Only iron measured in field. }\end{array}$ \\
\hline $3 / 13 / 08$ & $17: 30$ & CNQCTB-W-26016 & Water & TB & QC & - & 6082 & $3 / 13 / 08$ & $\begin{array}{l}\text { Trip blank sent to the AGEM Laboratory for organic analysis } \\
\text { with water samples listed on COC } 6082 \text {. }\end{array}$ \\
\hline $3 / 19 / 08$ & $9: 13$ & CNMW01-W-26023 & Water & MW & MW01 & $54.5-64.5$ & 6083 & $3 / 19 / 08$ & $\begin{array}{l}\text { Depth to water }=11.89 \mathrm{ft} \text {. Depth of } 4 \text {-in. well }=69.55 \mathrm{ft} \text {. } \\
\text { Sample collected by using low-flow bladder pump after } \\
\text { purging of } 4.31 \mathrm{~L} \text {. Purge water clear, no odor. }\end{array}$ \\
\hline
\end{tabular}


TABLE A.1 (Cont.)

\begin{tabular}{|c|c|c|c|c|c|c|c|c|c|}
\hline $\begin{array}{l}\text { Sample } \\
\text { Date }\end{array}$ & Time & Sample & $\begin{array}{l}\text { Sample } \\
\text { Medium }\end{array}$ & Type $^{\mathrm{a}}$ & Location & $\begin{array}{l}\text { Depth } \\
\text { (ft BGL) }\end{array}$ & $\begin{array}{l}\text { Chain of } \\
\text { Custody }\end{array}$ & $\begin{array}{l}\text { Shipping } \\
\text { Date }\end{array}$ & Sample Description \\
\hline \multicolumn{10}{|c|}{ Post-injection sampling, March 2008 (cont.) } \\
\hline $3 / 19 / 08$ & $9: 47$ & CNQCIR-W-26034 & Water & $\mathrm{RI}$ & QC & - & 6083 & $3 / 19 / 08$ & $\begin{array}{l}\text { Rinsate of decontaminated bladder pump after collection of } \\
\text { sample CNMW01-W-26023. }\end{array}$ \\
\hline $3 / 19 / 08$ & $11: 46$ & CNMW04-W-26024 & Water & MW & MW04 & $37.5-47.5$ & 6083 & $3 / 19 / 08$ & $\begin{array}{l}\text { Depth to water }=24.23 \mathrm{ft} \text {. Depth of } 4 \text {-in. well }=49.25 \mathrm{ft} \text {. } \\
\text { Sample collected by using low-flow bladder pump after } \\
\text { purging of } 3.72 \mathrm{~L} \text {. }\end{array}$ \\
\hline $3 / 19 / 08$ & $11: 47$ & CNMW04DUP-W-26036 & Water & MW & MW04 & $37.5-47.5$ & 6083 & $3 / 19 / 08$ & Replicate of sample CNMW04-W-26024. \\
\hline $3 / 19 / 08$ & 13:56 & CNMW05-W-26025 & Water & MW & MW05 & $34.5-44.5$ & 6083 & $3 / 19 / 08$ & $\begin{array}{l}\text { Depth to water }=7.32 \mathrm{ft} \text {. Depth of } 4 \text {-in. well }=47.58 \mathrm{ft} \text {. } \\
\text { Sample collected by using low-flow bladder pump after } \\
\text { purging of } 4.25 \mathrm{~L} \text {. }\end{array}$ \\
\hline $3 / 19 / 08$ & 15:30 & CNMW06-W-26026 & Water & MW & MW06 & $46.5-56.5$ & 6083 & $3 / 19 / 08$ & $\begin{array}{l}\text { Depth to water }=34.70 \mathrm{ft} \text {. Depth of } 4 \text {-in. well }=60.03 \mathrm{ft} \text {. } \\
\text { Sample collected by using low-flow bladder pump after } \\
\text { purging of } 2.12 \mathrm{~L} \text {. Purge water was tan. }\end{array}$ \\
\hline 3/19/08 & $15: 31$ & CNMW06DUP-W-26037 b & Water & MW & MW06 & $46.5-56.5$ & 6083 & $3 / 19 / 08$ & Replicate of sample CNMW06-W-26026. \\
\hline $3 / 19 / 08$ & $16: 57$ & CNQCTB-W-26035 & Water & TB & QC & - & 6083 & $3 / 19 / 08$ & $\begin{array}{l}\text { Trip blank sent to the AGEM Laboratory for organic analysis } \\
\text { with water samples listed on COC } 6083 \text {. }\end{array}$ \\
\hline 3/19/08 & $16: 58$ & CNQCTB-W-26035a ${ }^{b}$ & Water & TB & QC & - & 6081 & $3 / 19 / 08$ & $\begin{array}{l}\text { Trip blank sent to Envirosystems for verification organic } \\
\text { analysis with water samples listed on COCs } 6079 \text { and } \\
6081 .\end{array}$ \\
\hline $3 / 19 / 08$ & $18: 22$ & CNMW07-W-26027 & Water & MW & MW07 & $45-55$ & 6085 & $3 / 20 / 08$ & $\begin{array}{l}\text { Depth to water }=28.32 \mathrm{ft} \text {. Depth of } 2 \text {-in. well }=58.50 \mathrm{ft} \text {. } \\
\text { Sample collected by using low-flow bladder pump after } \\
\text { purging of } 2.5 \mathrm{~L} \text {. Purge water was cloudy. }\end{array}$ \\
\hline $3 / 19 / 08$ & $14: 00$ & Centralia-WW ${ }^{b}$ & Water & BT & QC & - & - & $3 / 20 / 08$ & $\begin{array}{l}\text { Composite sample of accumulated waste purge water for } \\
\text { analysis at Pace Analytical Services, Lenexa, Kansas. }\end{array}$ \\
\hline 3/20/08 & $8: 46$ & CNMW10-W-26030 & Water & MW & MW10 & $30-45$ & 6085 & $3 / 20 / 08$ & $\begin{array}{l}\text { Depth to water }=20.90 \mathrm{ft} \text {. Depth of } 2 \text {-in. well }=47.73 \mathrm{ft} \text {. } \\
\text { Sample collected by using low-flow bladder pump after } \\
\text { purging of } 4.0 \mathrm{~L} \text {. }\end{array}$ \\
\hline $3 / 20 / 08$ & $9: 44$ & CNSB09-W-26033 & Water & $\mathrm{CPT} / \mathrm{P}$ & SB09 & $32-42$ & 6085 & $3 / 20 / 08$ & $\begin{array}{l}\text { Depth to water }=2.90 \mathrm{ft} \text {. Depth of } 1 \text {-in. well }=35.50 \mathrm{ft} \text {. } \\
\text { Sample collected by using low-flow bladder pump after } \\
\text { purging of } 2.36 \mathrm{~L} \text {. }\end{array}$ \\
\hline $3 / 20 / 08$ & $10: 12$ & CNMW08-W-26028 & Water & MW & MW08 & $38-53$ & 6085 & $3 / 20 / 08$ & $\begin{array}{l}\text { Depth to water }=18.85 \mathrm{ft} \text {. Depth of } 2 \text {-in. well }=57.15 \mathrm{ft} \text {. } \\
\text { Sample collected by using low-flow bladder pump after } \\
\text { purging of } 4.1 \mathrm{~L} \text {. }\end{array}$ \\
\hline $3 / 20 / 08$ & $11: 40$ & CNMW09-W-26029 & Water & MW & MW09 & $25-35$ & 6085 & $3 / 20 / 08$ & $\begin{array}{l}\text { Depth to water }=0 \mathrm{ft} \text {. Depth of } 2 \text {-in. well }=39.15 \mathrm{ft} \text {. Sample } \\
\text { collected by using low-flow bladder pump after purging of } \\
9 \mathrm{~L} \text {. Water flowed out of casing when the plug was } \\
\text { removed. }\end{array}$ \\
\hline $3 / 20 / 08$ & $11: 45$ & CNQCIR-W-26039 & Water & $\mathrm{RI}$ & QC & - & 6085 & $3 / 20 / 08$ & $\begin{array}{l}\text { Rinsate of decontaminated bladder pump after collection of } \\
\text { sample CNMW09-W-26029. }\end{array}$ \\
\hline $3 / 20 / 08$ & $13: 23$ & CNSB01-W-26031 & Water & $\mathrm{CPT} / \mathrm{P}$ & SB01 & $40-50$ & 6085 & $3 / 20 / 08$ & $\begin{array}{l}\text { Depth to water }=15.87 \mathrm{ft} \text {. Depth of } 1 \text {-in. well }=48.90 \mathrm{ft} \text {. } \\
\text { Sample collected by using low-flow bladder pump after } \\
\text { purging of } 5.4 \mathrm{~L} \text {. }\end{array}$ \\
\hline
\end{tabular}


TABLE A.1 (Cont.)

\begin{tabular}{|c|c|c|c|c|c|c|c|c|c|}
\hline $\begin{array}{c}\text { Sample } \\
\text { Date }\end{array}$ & Time & Sample & $\begin{array}{l}\text { Sample } \\
\text { Medium }\end{array}$ & Type ${ }^{a}$ & Location & $\begin{array}{l}\text { Depth } \\
\text { (ft BGL) }\end{array}$ & $\begin{array}{l}\text { Chain of } \\
\text { Custody }\end{array}$ & $\begin{array}{c}\text { Shipping } \\
\text { Date }\end{array}$ & Sample Description \\
\hline
\end{tabular}

Post-injection sampling, March 2008 (cont.)

\begin{tabular}{|c|c|c|c|c|c|c|c|}
\hline $3 / 20 / 08$ & $14: 50$ & CNSB05-W-26032 & Water & $\mathrm{CPT} / \mathrm{P}$ & SB05 & $32-42$ & 6085 \\
\hline $3 / 20 / 08$ & $14: 55$ & CNQCTB-W-26038 & Water & TB & QC & - & 6085 \\
\hline $3 / 20 / 08$ & $14: 55$ & CNQCTB-W-26038ab & Water & TB & QC & - & 6130 \\
\hline \multicolumn{8}{|c|}{ Post-injection sampling, April 2008} \\
\hline $4 / 23 / 08$ & $16: 51$ & CNSB08-W-26049 & Water & $\mathrm{CPT} / \mathrm{P}$ & SB08 & $52-62$ & 4793 \\
\hline $4 / 23 / 08$ & $18: 18$ & CNMW03-W-26046 & Water & MW & MW03 & $50.5-60.5$ & 4793 \\
\hline $4 / 24 / 08$ & 9:03 & CNSB04-W-26047 & Water & $\mathrm{CPT} / \mathrm{P}$ & SB04 & $51-61$ & 4794 \\
\hline $4 / 24 / 08$ & 9:04 & CNSB04DUP-W-26062 & Water & $\mathrm{CPT} / \mathrm{P}$ & SB04 & $51-61$ & 4793 \\
\hline $4 / 24 / 08$ & $9: 18$ & CNQCIR-W-26060 & Water & $\mathrm{RI}$ & QC & - & 4793 \\
\hline $4 / 24 / 08$ & $10: 26$ & CNPMP6-W-26055 & Water & MW & PMP6 & $50-60$ & 4794 \\
\hline $4 / 24 / 08$ & $10: 37$ & CNPMP5-W-26054 & Water & MW & PMP5 & $50-60$ & 4794 \\
\hline $4 / 24 / 08$ & $10: 38$ & CNPMP5DUP-W-26063 & Water & MW & PMP5 & $50-60$ & 4793 \\
\hline $4 / 24 / 08$ & $10: 52$ & CNQCIR-W-26061 ${ }^{\mathrm{b}}$ & Water & $\mathrm{RI}$ & QC & - & 4793 \\
\hline $4 / 24 / 08$ & $11: 48$ & CNPMP7-W-26056 & Water & MW & PMP7 & $50-60$ & 4794 \\
\hline
\end{tabular}

3/20/08 Depth to water $=7.43 \mathrm{ft}$. Depth of 1 -in. well $=41.02 \mathrm{ft}$. Sample collected by using Waterra pump tubing as bailer after purging of $10 \mathrm{~L}$. Observation: the well casing seemed to be broken at $2 \mathrm{ft} 3 \mathrm{in}$. below grade.

3/20/08 Trip blank sent to the AGEM Laboratory for organic analysis with water samples listed on COC 6085.

3/20/08 Trip blank sent to Envirosystems for verification organic analysis with water samples listed on COC 6130.

4/24/08 Depth to water $=18.10 \mathrm{ft}$. Depth of 1 -in. well $=59.80 \mathrm{ft}$. Sample collected by using low-flow bladder pump after purging of $1.77 \mathrm{~L}$. Purge water slightly cloudy.

$4 / 24 / 08$ Depth to water $=20.15 \mathrm{ft}$. Depth of 4 -in. well $=62.20 \mathrm{ft}$. Sample collected by using low-flow bladder pump after purging of $3.365 \mathrm{~L}$. Purge water clear.

4/24/08 Depth to water $=21.30 \mathrm{ft}$. Depth of 1 -in. well $=59.40 \mathrm{ft}$. Sample collected by using low-flow bladder pump after purging of $1.64 \mathrm{~L}$. Purge water clear.

4/24/08 Replicate of sample CNSB04-W-26047.

$4 / 24 / 08$ Rinsate of decontaminated bladder pump after collection of sample CNSB04-W-26047 and replicate CNSB04DUP-W 26062.

4/24/08 Depth to water $=20.80 \mathrm{ft}$. Depth of 0.5 -in. well $=57.10 \mathrm{ft}$. Sample collected by using Waterra pump tubing as bailer after purging of $5.5 \mathrm{~L}$ (3 well volumes). Purge water light brown.

4/24/08 Depth to water $=20.79 \mathrm{ft}$. Depth of 1 -in. well $=58.90 \mathrm{ft}$. Sample collected by using Waterra pump tubing as bailer after purging of $1.5 \mathrm{~L}$. Purge water gray looking.

4/24/08 Replicate of sample CNPMP5-W-26054.

4/24/08 Rinsate of decontaminated bladder pump after collection of sample CNPMP5-W-26054 and replicate CNPMP5DUPW-26063.

4/24/08 Depth to water $=19.70 \mathrm{ft}$. Depth of 0.5 -in. well $=56.85 \mathrm{ft}$. Sample collected by using Waterra pump tubing as bailer after purging of $5.1 \mathrm{~L}$ (3 well volumes). Purge water cloudy tan. 
TABLE A.1 (Cont.)

\begin{tabular}{|c|c|c|c|c|c|c|c|c|c|}
\hline $\begin{array}{l}\text { Sample } \\
\text { Date }\end{array}$ & Time & Sample & $\begin{array}{l}\text { Sample } \\
\text { Medium }\end{array}$ & Type $^{a}$ & Location & $\begin{array}{l}\text { Depth } \\
\text { (ft BGL) }\end{array}$ & $\begin{array}{l}\text { Chain of } \\
\text { Custody }\end{array}$ & $\begin{array}{l}\text { Shipping } \\
\text { Date }\end{array}$ & Sample Description \\
\hline \multicolumn{10}{|c|}{ Post-injection sampling, April 2008 (cont.) } \\
\hline $4 / 24 / 08$ & $12: 06$ & CNSB07R-W-26048 & Water & $\mathrm{CPT} / \mathrm{P}$ & SB07R & $45-60$ & 4794 & $4 / 24 / 08$ & $\begin{array}{l}\text { Depth to water }=17.87 \mathrm{ft} \text {. Depth of } 2 \text {-in. well }=58.50 \mathrm{ft} \text {. } \\
\text { Sample collected by using low-flow bladder pump after } \\
\text { purging of } 2.82 \mathrm{~L} \text {. }\end{array}$ \\
\hline $4 / 24 / 08$ & $12: 40$ & CNPMP4-W-26053 & Water & MW & PMP4 & $48.75-58.75$ & 4794 & $4 / 24 / 08$ & $\begin{array}{l}\text { Depth to water }=17.70 \mathrm{ft} \text {. Depth of } 0.5 \text {-in. well }=57.40 \mathrm{ft} \text {. } \\
\text { Sample collected by using Waterra pump as bailer after } \\
\text { purging of } 5.5 \mathrm{~L} \text { ( } 3 \text { well volumes). Water brown and sandy. }\end{array}$ \\
\hline $4 / 24 / 08$ & $13: 41$ & CNPMP8-W-26057 & Water & MW & PMP8 & $50-60$ & 4794 & $4 / 24 / 08$ & $\begin{array}{l}\text { Depth to water }=18.60 \mathrm{ft} \text {. Depth of } 0.5 \text {-in. well }=58.8 \mathrm{ft} \text {. } \\
\text { Sample collected by using Waterra pump as bailer after } \\
\text { purging of } 5 \mathrm{~L} \text { ( } 3 \text { well volumes). Purge water brown. }\end{array}$ \\
\hline $4 / 24 / 08$ & $13: 46$ & CNMW02-W-26045 & Water & MW & MW02 & $49.5-59.5$ & 4794 & $4 / 24 / 08$ & $\begin{array}{l}\text { Depth to water }=20.72 \mathrm{ft} \text {. Depth of } 4 \text {-in. well }=61.40 \mathrm{ft} \text {. } \\
\text { Sample collected by using low-flow bladder pump after } \\
\text { purging of } 2.5 \mathrm{~L} \text {. Purge water black; tubing and probes } \\
\text { covered with slimy substance with strong odor. Iron sample } \\
\text { bronze color. }\end{array}$ \\
\hline $4 / 24 / 08$ & $14: 40$ & CNPMP3-W-26052 & Water & MW & PMP3 & $50-60$ & 4794 & $4 / 24 / 08$ & $\begin{array}{l}\text { Depth to water }=20.30 \mathrm{ft} \text {. Depth of } 0.5 \text {-in. well }=56.10 \mathrm{ft} \text {. } \\
\text { Sample collected by using Waterra pump as bailer after } \\
\text { purging of } 5.5 \mathrm{~L} \text { ( } 3 \text { well volumes). Purge water light brown } \\
\text { to greenish color, with odor. Iron sample turned red } \\
\text { orange. }\end{array}$ \\
\hline $4 / 24 / 08$ & $15: 25$ & CNPMP9-W-26058 & Water & MW & PMP9 & $50-60$ & 4794 & $4 / 24 / 08$ & $\begin{array}{l}\text { Depth to water }=13.68 \mathrm{ft} \text {. Depth of } 0.5 \text {-in. well }=56.50 \mathrm{ft} \text {. } \\
\text { Sample collected by using Waterra pump as bailer after } \\
\text { purging of } 5.5 \mathrm{~L} \text { ( } 3 \text { well volumes). Purge water tan. }\end{array}$ \\
\hline $4 / 24 / 08$ & $15: 49$ & CNQCTB-W-26059 & Water & TB & QC & - & 4793 & $4 / 24 / 08$ & $\begin{array}{l}\text { Trip blank sent to the AGEM Laboratory for organic analysis } \\
\text { with water samples listed on COCs } 4793 \text { and } 4794 \text {. }\end{array}$ \\
\hline $4 / 24 / 08$ & $15: 50$ & CNQCTB-W-26059a ${ }^{b}$ & Water & TB & QC & - & 6084 & $4 / 24 / 08$ & $\begin{array}{l}\text { Trip blank sent to Envirosystems for verification organic } \\
\text { analysis with water samples listed on COC } 6084 \text {. }\end{array}$ \\
\hline $4 / 24 / 08$ & 16:06 & CNPMP2-W-26051 & Water & MW & PMP2 & $50-60$ & 4794 & $4 / 24 / 08$ & $\begin{array}{l}\text { Depth to water }=19.61 \mathrm{ft} \text {. Depth of } 0.5 \text {-in. well }=59.05 \mathrm{ft} \text {. } \\
\text { Sample collected by using Waterra pump as bailer after } \\
\text { purging of } 5.4 \mathrm{~L} \text { ( } 3 \text { well volumes). Purge water dark tan. }\end{array}$ \\
\hline $4 / 24 / 08$ & $16: 39$ & CNPMP1-W-26050 & Water & MW & PMP1 & $50-60$ & 4794 & 4/24/08 & $\begin{array}{l}\text { Depth to water }=19.46 \mathrm{ft} \text {. Depth of } 0.5 \text {-in. well }=58.68 \mathrm{ft} \text {. } \\
\text { Sample collected by using Waterra pump as bailer after } \\
\text { purging of } 5 \mathrm{~L} \text { ( } 3 \text { well volumes). Purge water dark tan. }\end{array}$ \\
\hline \multicolumn{10}{|c|}{ Post-injection sampling, June 2008} \\
\hline $6 / 4 / 08$ & $11: 44$ & CNSB04-W-26622 & Water & $\mathrm{CPT} / \mathrm{P}$ & SB04 & $51-61$ & 6128 & $6 / 5 / 08$ & $\begin{array}{l}\text { Depth to water }=20.8 \mathrm{ft} \text {. Depth of } 1 \text {-in. well }=59.35 \mathrm{ft} \text {. } \\
\text { Sample collected by using low-flow bladder pump after } \\
\text { purging of } 2.5 \mathrm{~L} \text {. Purge water clear. }\end{array}$ \\
\hline $6 / 4 / 08$ & $12: 58$ & CNMW03-W-26621 & Water & MW & MW03 & $50.5-60.5$ & 6128 & $6 / 5 / 08$ & $\begin{array}{l}\text { Depth to water }=19.7 \mathrm{ft} \text {. Depth of } 4 \text {-in. well }=62.41 \mathrm{ft} \text {. } \\
\text { Sample collected by using low-flow bladder pump after } \\
\text { purging of } 6 \mathrm{~L} \text {. Purge water clear. }\end{array}$ \\
\hline
\end{tabular}


TABLE A.1 (Cont.)

\begin{tabular}{|c|c|c|c|c|c|c|c|c|c|}
\hline $\begin{array}{l}\text { Sample } \\
\text { Date }\end{array}$ & Time & Sample & $\begin{array}{l}\text { Sample } \\
\text { Medium }\end{array}$ & Type $^{a}$ & Location & $\begin{array}{l}\text { Depth } \\
\text { (ft BGL) }\end{array}$ & $\begin{array}{l}\text { Chain of } \\
\text { Custody }\end{array}$ & $\begin{array}{l}\text { Shipping } \\
\text { Date }\end{array}$ & Sample Description \\
\hline \multicolumn{10}{|c|}{ Post-injection sampling, June 2008 (cont.) } \\
\hline $6 / 4 / 08$ & $14: 06$ & CNSB07R-W-26623 & Water & $\mathrm{CPT} / \mathrm{P}$ & SB07R & $45-60$ & 6128 & $6 / 5 / 08$ & $\begin{array}{l}\text { Depth to water }=17.9 \mathrm{ft} \text {. Depth of } 2 \text {-in. well }=58.53 \mathrm{ft} . \\
\text { Sample collected by using low-flow bladder pump after } \\
\text { purging of } 6.2 \text { L. Purge water clear. }\end{array}$ \\
\hline $6 / 4 / 08$ & 14:08 & CNSB07RDUP-W-26636 & Water & $\mathrm{CPT} / \mathrm{P}$ & SB07R & $45-60$ & 6128 & $6 / 5 / 08$ & Replicate of sample CNSB07R-W-26623. \\
\hline $6 / 4 / 08$ & $14: 40$ & CNQCIR-W-26634 & Water & $\mathrm{RI}$ & QC & - & 6128 & $6 / 5 / 08$ & $\begin{array}{l}\text { Rinsate of decontaminated bladder pump after collection of } \\
\text { sample CNSB07R-W-26623 and replicate CNSB07RDUP- } \\
\text { W-26636. }\end{array}$ \\
\hline $6 / 4 / 08$ & 17:32 & CNSB08-W-26624 & Water & $\mathrm{CPT} / \mathrm{P}$ & SB08 & $52-62$ & 6128 & $6 / 5 / 08$ & $\begin{array}{l}\text { Depth to water }=19 \mathrm{ft} \text {. Depth of } 1 \text {-in. well }=59.76 \mathrm{ft} \text {. Sample } \\
\text { collected by using low-flow bladder pump after purging of } \\
2.5 \text { L. Purge water clear. }\end{array}$ \\
\hline $6 / 4 / 08$ & 18:36 & CNMW02-W-26620 & Water & MW & MW02 & $49.5-59.5$ & 6128 & $6 / 5 / 08$ & $\begin{array}{l}\text { Depth to water }=20.4 \mathrm{ft} \text {. Depth of } 4 \text {-in. well }=61.2 \mathrm{ft} \text {. Sample } \\
\text { collected by using low-flow bladder pump after purging of } \\
6.1 \mathrm{~L} \text {. Purge water grayish tint with manure-like odor. }\end{array}$ \\
\hline $6 / 4 / 08$ & $20: 18$ & CNPMP5-W-26629 & Water & MW & PMP5 & $50-60$ & 6128 & $6 / 5 / 08$ & $\begin{array}{l}\text { Depth to water }=20.5 \mathrm{ft} \text {. Depth of } 1 \text {-in. well }=60 \mathrm{ft} \text {. Sample } \\
\text { collected by using low-flow bladder pump after purging of } \\
3 \mathrm{~L} \text {. Purge water cloudy to greenish tint with odor. }\end{array}$ \\
\hline $6 / 4 / 08$ & $20: 40$ & CNQCIR-W-26635 & Water & $\mathrm{RI}$ & QC & - & 6128 & $6 / 5 / 08$ & $\begin{array}{l}\text { Rinsate of decontaminated bladder pump after collection of } \\
\text { sample CNPMP5-W-26629. }\end{array}$ \\
\hline $6 / 5 / 08$ & $9: 52$ & CNPMP6-W-26630 & Water & MW & PMP6 & $50-60$ & 6129 & $6 / 5 / 08$ & $\begin{array}{l}\text { Depth to water }=20.2 \mathrm{ft} \text {. Depth of } 0.5 \text {-in. well }=60 \mathrm{ft} \text {. Sample } \\
\text { collected by using Waterra pump tubing as bailer after } \\
\text { purging of } 6 \mathrm{~L} \text { ( } 3 \text { well volumes). Purge water brown. }\end{array}$ \\
\hline $6 / 5 / 08$ & $10: 28$ & CNPMP7-W-26631 & Water & MW & PMP7 & $50-60$ & 6129 & $6 / 5 / 08$ & $\begin{array}{l}\text { Depth to water }=19 \mathrm{ft} \text {. Depth of } 0.5 \text {-in. well }=60 \mathrm{ft} \text {. Sample } \\
\text { collected by using Waterra pump tubing as bailer after } \\
\text { purging of } 6.5 \mathrm{~L} \text { ( } 3 \text { well volumes). Purge water brown. }\end{array}$ \\
\hline $6 / 5 / 08$ & $11: 27$ & CNPMP4-W-26628 & Water & MW & PMP4 & $48.75-58.75$ & 6129 & $6 / 5 / 08$ & $\begin{array}{l}\text { Depth to water }=17 \mathrm{ft} \text {. Depth of } 0.5 \text {-in. well }=58.75 \mathrm{ft} \text {. } \\
\text { Sample collected by using Waterra pump tubing as bailer } \\
\text { after purging of } 6.5 \mathrm{~L} \text { ( } 3 \text { well volumes). Purge water brown. }\end{array}$ \\
\hline $6 / 5 / 08$ & $12: 18$ & CNPMP8-W-26632 & Water & MW & PMP8 & $50-60$ & 6129 & $6 / 5 / 08$ & $\begin{array}{l}\text { Depth to water }=17.7 \mathrm{ft} \text {. Depth of } 0.5 \text {-in. well }=60 \mathrm{ft} \text {. Sample } \\
\text { collected by using Waterra pump tubing as bailer after } \\
\text { purging of } 6.5 \mathrm{~L} \text {. Purge water brownish gray with odor. }\end{array}$ \\
\hline $6 / 5 / 08$ & $13: 18$ & CNPMP3-W-26627 & Water & MW & PMP3 & $50-60$ & 6129 & $6 / 5 / 08$ & $\begin{array}{l}\text { Depth to water }=19.8 \mathrm{ft} \text {. Depth of } 0.5 \text {-in. well }=60 \mathrm{ft} \text {. Sample } \\
\text { collected by using Waterra pump tubing as bailer after } \\
\text { purging of } 6 \mathrm{~L} \text {. Purge water brownish gray with odor. }\end{array}$ \\
\hline $6 / 5 / 08$ & $13: 50$ & CNQCTB-W-26638 & Water & TB & QC & - & 6129 & $6 / 5 / 08$ & $\begin{array}{l}\text { Trip blank sent to the AGEM Laboratory for organic analysis } \\
\text { with water samples listed on COCs } 6128 \text { and } 6129 \text {. }\end{array}$ \\
\hline $6 / 5 / 08$ & $13: 51$ & CNQCTB-W-26638ab & Water & TB & QC & - & 6130 & $6 / 5 / 08$ & $\begin{array}{l}\text { Trip blank sent to Envirosystems for verification organic } \\
\text { analysis with water samples listed on COC } 6130 \text {. }\end{array}$ \\
\hline $6 / 5 / 08$ & $13: 58$ & CNPMP9-W-26633 & Water & MW & PMP9 & $50-60$ & 6129 & $6 / 5 / 08$ & $\begin{array}{l}\text { Depth to water }=13.2 \mathrm{ft} \text {. Depth of } 0.5 \text {-in. well }=60 \mathrm{ft} \text {. Sample } \\
\text { collected by using Waterra pump tubing as bailer after } \\
\text { purging of } 7 \mathrm{~L} \text {. Purge water cloudy to brownish. }\end{array}$ \\
\hline
\end{tabular}


TABLE A.1 (Cont.)

\begin{tabular}{|c|c|c|c|c|c|c|c|c|c|}
\hline $\begin{array}{l}\text { Sample } \\
\text { Date }\end{array}$ & Time & Sample & $\begin{array}{l}\text { Sample } \\
\text { Medium }\end{array}$ & Type $^{a}$ & Location & $\begin{array}{l}\text { Depth } \\
\text { (ft BGL) }\end{array}$ & $\begin{array}{l}\text { Chain of } \\
\text { Custody }\end{array}$ & $\begin{array}{c}\text { Shipping } \\
\text { Date }\end{array}$ & Sample Description \\
\hline
\end{tabular}

Post-injection sampling, June 2008 (cont.)

$6 / 5 / 08$

$6 / 5 / 08$

14:36 CNPMP2DUP-W-26637

15:17 CNPMP1-W-26625

Water$$
50-60
$$

$50-60$

6129

6129

Post-injection sampling, July 2008

\begin{tabular}{|c|c|c|c|c|c|c|c|}
\hline $7 / 7 / 08$ & $14: 26$ & CNSB04-W-26641 & Water & $\mathrm{CPT} / \mathrm{P}$ & SB04 & $51-61$ & 6131 \\
\hline 7/7/08 & $14: 28$ & CNSB04DUP-W-26655 & Water & $\mathrm{CPT} / \mathrm{P}$ & SB04 & $51-61$ & 6131 \\
\hline $7 / 7 / 08$ & $15: 40$ & CNMW03-W-26640 & Water & MW & MW03 & $50.5-60.5$ & 6131 \\
\hline $7 / 7 / 08$ & $16: 52$ & CNSB07R-W-26642 & Water & $\mathrm{CPT} / \mathrm{P}$ & SB07R & $45-60$ & 6131 \\
\hline $7 / 7 / 08$ & $17: 22$ & CNPMP4-W-26647 & Water & MW & PMP4 & $48.75-58.75$ & 6131 \\
\hline $7 / 7 / 08$ & $18: 16$ & CNPMP7-W-26650 & Water & MW & PMP7 & $50-60$ & 6131 \\
\hline $7 / 7 / 08$ & $18: 26$ & CNSB08-W-26643 & Water & $\mathrm{CPT} / \mathrm{P}$ & SB08 & $52-62$ & 6131 \\
\hline 7/7/08 & 19:01 & CNPMP6-W-26649 & Water & MW & PMP6 & $50-60$ & 6131 \\
\hline $7 / 8 / 08$ & $12: 02$ & CNPMP5-W-26648 & Water & MW & PMP5 & $50-60$ & 6125 \\
\hline 7/8/08 & $12: 30$ & CNPMP3-W-26646 & Water & MW & PMP3 & $50-60$ & 6125 \\
\hline $7 / 8 / 08$ & $13: 09$ & CNPMP8-W-26651 & Water & MW & PMP8 & $50-60$ & 6125 \\
\hline
\end{tabular}

6/5/08 Depth to water $=19 \mathrm{ft}$. Depth of 0.5 -in. well $=60 \mathrm{ft}$. Sample collected by using Waterra pump tubing as bailer after purging of $9 \mathrm{~L}$. Purge water brownish with odor.

6/5/08 Replicate of sample CNPMP2-W-26626.

$6 / 5 / 08$ Depth to water $=19.2 \mathrm{ft}$. Depth of 0.5 -in. well $=60 \mathrm{ft}$. Sample collected by using Waterra pump tubing as bailer after purging of $7 \mathrm{~L}$. Purge water brown with an odor.

7/8/08 Depth to water $=20.75 \mathrm{ft}$. Depth of 1 -in. well $=59.35 \mathrm{ft}$. Sample collected by using low-flow bladder pump after purging of $2.5 \mathrm{~L}$. Water clear.

7/8/08 Replicate of sample CNSB04-W-26641.

$7 / 8 / 08$ Depth to water $=20.1 \mathrm{ft}$. Depth of 4 -in. well $=62.41 \mathrm{ft}$ Sample collected by using low-flow bladder pump after purging of $6.9 \mathrm{~L}$.

$7 / 8 / 08$ Depth to water $=17.3 \mathrm{ft}$. Depth of 2 -in well $=58.53 \mathrm{ft}$ Sample collected by using low-flow bladder pump after purging of $6 \mathrm{~L}$. Water clear.

$7 / 8 / 08$ Depth to water $=17.4 \mathrm{ft}$. Depth of 0.5 -in. well $=58.75 \mathrm{ft}$ Sample collected by using Waterra pump tubing as bailer after purging of $5.9 \mathrm{~L}$. Water brown.

$7 / 8 / 08$ Depth to water $=19.3 \mathrm{ft}$. Depth of 0.5 -in. well $=60 \mathrm{ft}$. Sample collected by using Waterra pump tubing as bailer after purging of $6.2 \mathrm{~L}$. Water cloudy.

$7 / 8 / 08$ Depth to water $=17.7 \mathrm{ft}$. Depth of 1 -in. well $=59.76 \mathrm{ft}$. Sample collected by using low-flow bladder pump after purging of $3.5 \mathrm{~L}$. Water clear.

7/8/08 Depth to water $=20.4 \mathrm{ft}$. Depth of 0.5 -in. well $=60 \mathrm{ft}$. Sample collected by using Waterra pump tubing as bailer after purging of $6.5 \mathrm{~L}$. Water tan.

$7 / 8 / 08$ Depth to water $=20.4 \mathrm{ft}$. Depth of 1 -in. well $=60 \mathrm{ft}$. Sample collected by using low-flow bladder pump after purging of 3.7 L. Water muddy.

$7 / 8 / 08$ Depth to water $=19.8 \mathrm{ft}$. Depth of 0.5 -in. well $=60 \mathrm{ft}$. Sample collected by using Waterra pump tubing as bailer after purging of $7.5 \mathrm{~L}$.

7/8/08 Depth to water $=18.2 \mathrm{ft}$. Depth of 0.5 -in. well $=60 \mathrm{ft}$. Sample collected by using Waterra pump tubing as bailer after purging of $8 \mathrm{~L}$. 
TABLE A.1 (Cont.)

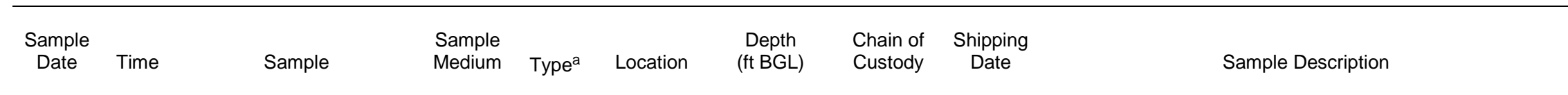

Post-injection sampling, July 2008 (cont.)

\begin{tabular}{|c|c|c|c|c|c|c|c|}
\hline $7 / 8 / 08$ & $13: 16$ & CNMW02-W-26639 & Water & MW & MW02 & $49.5-59.5$ & 6125 \\
\hline $7 / 8 / 08$ & 13:54 & CNPMP9-W-26652 & Water & MW & PMP9 & $50-60$ & 6125 \\
\hline $7 / 8 / 08$ & $13: 55$ & CNPMP9DUP-W-26656 b & Water & MW & PMP9 & $50-60$ & 6125 \\
\hline $7 / 8 / 08$ & $14: 00$ & CNQCIR-W-26653 & Water & $\mathrm{RI}$ & QC & - & 6125 \\
\hline $7 / 8 / 08$ & $15: 15$ & CNPMP2-W-26645 & Water & MW & PMP2 & $50-60$ & 6125 \\
\hline $7 / 8 / 08$ & $15: 55$ & CNPMP1-W-26644 & Water & MW & PMP1 & $50-60$ & 6125 \\
\hline $7 / 8 / 08$ & $16: 05$ & CNQCIR-W-26654 ${ }^{\mathrm{b}}$ & Water & $\mathrm{RI}$ & QC & - & 6125 \\
\hline $7 / 8 / 08$ & $16: 30$ & CNQCTB-W-26657b & Water & TB & QC & - & 6125 \\
\hline $7 / 8 / 08$ & $16: 31$ & CNQCTB-W-26657ab & Water & TB & QC & - & 6132 \\
\hline $7 / 16 / 08$ & $13: 30$ & $C N-W W^{b}$ & Water & BT & QC & - & - \\
\hline \multicolumn{8}{|c|}{ Post-injection sampling, August 2008} \\
\hline $8 / 6 / 08$ & $12: 34$ & CNPMP6-W-26668 & Water & MW & PMP6 & $50-60$ & 6115 \\
\hline $8 / 6 / 08$ & $12: 34$ & CNPMP9-W-26671 & Water & MW & PMP9 & $50-60$ & 6115 \\
\hline $8 / 6 / 08$ & $12: 50$ & CNSB08-W-26662 & Water & $\mathrm{CPT} / \mathrm{P}$ & SB08 & $52-62$ & 6115 \\
\hline $8 / 6 / 08$ & $13: 10$ & CNPMP7-W-26669 & Water & MW & PMP7 & $50-60$ & 6115 \\
\hline
\end{tabular}

7/8/08 Depth to water $=19.7 \mathrm{ft}$. Depth of 4 -in. well $=61.2 \mathrm{ft}$. Sample collected by using low-flow bladder pump after purging of $6 \mathrm{~L}$.

$7 / 8 / 08$ Depth to water $=13.2 \mathrm{ft}$. Depth of 0.5 -in. well $=60 \mathrm{ft}$. Sample collected by using Waterra pump tubing as bailer after purging of $8 \mathrm{~L}$.

7/8/08 Replicate of sample CNPMP9-W-26652.

$7 / 8 / 08$ Rinsate of decontaminated sampling tube after collection of sample CNPMP9-W-26652 and replicate CNPMP9DUPW-26656.

$7 / 8 / 08$ Depth to water $=19.2 \mathrm{ft}$. Depth of 0.5 -in. well $=60 \mathrm{ft}$. Sample collected by using Waterra pump tubing as bailer after purging of $7 \mathrm{~L}$. Water tannish color.

$7 / 8 / 08$ Depth to water $=19.3 \mathrm{ft}$. Depth of 0.5 -in. well $=60 \mathrm{ft}$. Sample collected by using Waterra pump tubing as bailer after purging of $7 \mathrm{~L}$. Water milky brown.

7/8/08 Rinsate of decontaminated sampling tube after collection of sample CNPMP1-W-26644.

7/8/08 Trip blank with water samples listed on COCs 6125 and 6131 to the AGEM Laboratory for organic analysis.

7/8/08 Trip blank sent to TestAmerica for verification organic analysis with water samples listed on COC 6132.

7/17/08 Composite sample of accumulated waste purge water for analysis at Pace Analytical Services, Lenexa, Kansas.

8/6/08 Depth to water $=21.5 \mathrm{ft}$. Depth of 0.5 -in. well $=60 \mathrm{ft}$. Sample collected by using Waterra pump tubing as bailer after purging of $6 \mathrm{~L}$. Water tan in color.

$8 / 6 / 08$ Depth to water $=15.1 \mathrm{ft}$. Depth of 0.5 -in. well $=60 \mathrm{ft}$. Sample collected by using Waterra pump tubing as bailer after purging of $7 \mathrm{~L}$.

8/6/08 Depth to water $=18.2 \mathrm{ft}$. Depth of 1 -in. well $=59.76 \mathrm{ft}$ Sample collected by using low-flow bladder pump after

purging of $3 \mathrm{~L}$.
$8 / 6 / 08$ Depth to water $=19.9 \mathrm{ft}$. Depth of 0.5 -in. well $=60 \mathrm{ft}$. Sample collected by using Waterra pump tubing as bailer after purging of $6 \mathrm{~L}$. Water clear to cloudy. 
TABLE A.1 (Cont.)

\begin{tabular}{|c|c|c|c|c|c|c|c|}
\hline $\begin{array}{c}\text { Sample } \\
\text { Date }\end{array}$ & Time & Sample & $\begin{array}{l}\text { Sample } \\
\text { Medium }\end{array}$ & Type ${ }^{a}$ & Location & $\begin{array}{c}\text { Depth } \\
\text { (ft BGL) }\end{array}$ & $\begin{array}{l}\text { Chain of } \\
\text { Custody }\end{array}$ \\
\hline \multicolumn{8}{|c|}{ Post-injection sampling, August 2008 (cont.) } \\
\hline $8 / 6 / 08$ & $13: 56$ & CNSB07R-W-26661 & Water & $\mathrm{CPT} / \mathrm{P}$ & SB07R & $45-60$ & 6115 \\
\hline $8 / 6 / 08$ & $14: 10$ & CNPMP4-W-26666 & Water & MW & PMP4 & $48.75-58.75$ & 6115 \\
\hline 8/6/08 & $15: 46$ & CNPMP8-W-26670 & Water & MW & PMP8 & $50-60$ & 6115 \\
\hline $8 / 6 / 08$ & $16: 00$ & CNMW03-W-26659 & Water & MW & MW03 & $50.5-60.5$ & 6115 \\
\hline $8 / 6 / 08$ & $16: 21$ & CNPMP3-W-26665 & Water & MW & PMP3 & $50-60$ & 6115 \\
\hline $8 / 6 / 08$ & $17: 08$ & CNSB04-W-26660 & Water & $\mathrm{CPT} / \mathrm{P}$ & SB04 & $51-61$ & 6115 \\
\hline $8 / 6 / 08$ & $18: 06$ & CNPMP2-W-26664 & Water & MW & PMP2 & $50-60$ & 6115 \\
\hline 8/6/08 & $18: 24$ & CNPMP5-W-26667 & Water & MW & PMP5 & $50-60$ & 6115 \\
\hline $8 / 6 / 08$ & $18: 44$ & CNPMP1-W-26663 & Water & MW & PMP1 & $50-60$ & 6115 \\
\hline $8 / 6 / 08$ & $19: 10$ & CNMW02-W-26658 & Water & MW & MW02 & $49.5-59.5$ & 6115 \\
\hline $8 / 6 / 08$ & $19: 20$ & CNQCTB-W-26672 & Water & TB & QC & - & 6115 \\
\hline $8 / 6 / 08$ & $19: 21$ & CNQCTB-W-26672ab & Water & TB & QC & - & 6116 \\
\hline $8 / 21 / 08$ & $10: 55$ & CNPSB13-S-27120 & Soil & СРT & PSB13 & 2 & 4309 \\
\hline $8 / 21 / 08$ & $10: 56$ & CNPSB13-S-27121 & Soil & СРT & PSB13 & 4 & 4309 \\
\hline $8 / 21 / 08$ & $11: 17$ & CNPSB13-S-27122 & Soil & СPT & PSB13 & 6 & 4309 \\
\hline $8 / 21 / 08$ & $11: 18$ & CNPSB13-S-27123 & Soil & СРТ & PSB13 & 8 & 4309 \\
\hline $8 / 21 / 08$ & $11: 23$ & CNPSB13-S-27124 & Soil & СPT & PSB13 & 10 & 4309 \\
\hline $8 / 21 / 08$ & $11: 24$ & CNPSB13-S-27125 & Soil & СРT & PSB13 & 12 & 4309 \\
\hline $8 / 21 / 08$ & $11: 29$ & CNPSB13-S-27126 & Soil & СРT & PSB13 & 14 & 4309 \\
\hline
\end{tabular}
collected by using low-flow bladder pump after purging of $5.3 \mathrm{~L}$.

8/6/08 Depth to water $=19.1 \mathrm{ft}$. Depth of 0.5 -in. well $=58.75 \mathrm{ft}$. Sample collected by using Waterra pump tubing as bailer after purging of $6.2 \mathrm{~L}$. Water tannish in color.

8/6/08 Depth to water $=19.7 \mathrm{ft}$. Depth of 0.5 -in. well $=60 \mathrm{ft}$. Sample collected by using Waterra pump tubing as bailer after purging of $6.5 \mathrm{~L}$. Water greenish brown in color with odor

8/6/08 Depth to water $=20.2 \mathrm{ft}$. Depth of $4-\mathrm{in}$. well $=62.41 \mathrm{ft}$. Sample collected by using low-flow bladder pump after purging of $8 \mathrm{~L}$. Water muggy.

8/6/08 Depth to water $=21.1 \mathrm{ft}$. Depth of 0.5 -in. well $=60 \mathrm{ft}$. Sample collected by using Waterra pump tubing as bailer after purging of $6.2 \mathrm{~L}$.

8/6/08 Depth to water $=21.5 \mathrm{ft}$. Depth of 1 -in. well $=59.35 \mathrm{ft}$. Sample collected by using low-flow bladder pump after purging of $3.2 \mathrm{~L}$.

8/6/08 Depth to water $=20.5 \mathrm{ft}$. Depth of 0.5 -in. well $=60 \mathrm{ft}$. Sample collected by using Waterra pump tubing as bailer after purging of $6 \mathrm{~L}$. Water tannish brown in color with odor.

8/6/08 Depth to water $=21 \mathrm{ft}$. Depth of 1 -in. well $=60 \mathrm{ft}$. Sample collected by using low-flow bladder pump after purging of $3.5 \mathrm{~L}$.

8/6/08 Depth to water $=20.1 \mathrm{ft}$. Depth of 0.5 -in. well $=60 \mathrm{ft}$. Sample collected by using Waterra pump tubing as bailer after purging of $6 \mathrm{~L}$. Water brownish in color with odor.

8/6/08 Depth to water $=20.4 \mathrm{ft}$. Depth of 4 -in. well $=61.2 \mathrm{ft}$. Sample collected by using low-flow bladder pump after purging of $5.3 \mathrm{~L}$

8/6/08 Trip blank sent to the AGEM Laboratory for organic analysis with water samples listed on COC 6115.

8/6/08 Trip blank sent to TestAmerica for verification organic analysis with water samples listed on COC 6116 .

$8 / 21 / 08$ Vertical profile soil sampling at location near PSB1.

$8 / 21 / 08$ Vertical profile soil sampling at location near PSB1.

$8 / 21 / 08$ Vertical profile soil sampling at location near PSB1.

8/21/08 Vertical profile soil sampling at location near PSB1.

$8 / 21 / 08$ Vertical profile soil sampling at location near PSB1.

8/21/08 Vertical profile soil sampling at location near PSB1.

8/21/08 Vertical profile soil sampling at location near PSB1. 
TABLE A.1 (Cont.)

\begin{tabular}{|c|c|c|c|c|c|c|c|c|c|}
\hline $\begin{array}{l}\text { Sample } \\
\text { Date }\end{array}$ & Time & Sample & $\begin{array}{l}\text { Sample } \\
\text { Medium }\end{array}$ & Type ${ }^{a}$ & Location & $\begin{array}{l}\text { Depth } \\
\text { (ft BGL) }\end{array}$ & $\begin{array}{l}\text { Chain of } \\
\text { Custody }\end{array}$ & $\begin{array}{c}\text { Shipping } \\
\text { Date }\end{array}$ & Sample Description \\
\hline
\end{tabular}

Post-injection sampling, August 2008 (cont.)

$\begin{array}{llll}\text { 8/21/08 } & 11: 30 & \text { CNPSB13-S-27127 } & \text { S } \\ \text { 8/21/08 } & 11: 35 & \text { CNPSB13-S-27128 } & \text { S } \\ \text { 8/21/08 } & 11: 36 & \text { CNPSB13-S-27129 } & \text { S } \\ \text { 8/21/08 } & 12: 01 & \text { CNPSB13-S-27130 } & \text { Soll } \\ \text { 8/21/08 } & 12: 02 & \text { CNPSB13-S-27131 } & \text { S } \\ \text { 8/21/08 } & 14: 29 & \text { CNPSB13-S-27132 } & \text { S } \\ \text { 8/21/08 } & 14: 30 & \text { CNPSB13-S-27133 } & \text { S } \\ \text { 8/21/08 } & 15: 25 & \text { CNPSB13-S-27134 } & \text { S } \\ \text { 8/21/08 } & 16: 10 & \text { CNPSB13-S-27135 } & \text { S } \\ \text { 8/21/08 } & 17: 48 & \text { CNPSB13-S-27136 } & \text { S } \\ \text { 8/21/08 } & 18: 45 & \text { CNPSB13-S-27137 } & \text { S } \\ \text { 8/21/08 } & 20: 02 & \text { CNPSB13-S-27138 } & \text { S } \\ \text { 8/21/08 } & 21: 20 & \text { CNPSB13-S-27139 } & \text { S }\end{array}$

$\begin{array}{lll}\text { Soil } & \text { CPT } & \text { PSB13 } \\ \text { Soil } & \text { CPT } & \text { PSB13 } \\ \text { Soil } & \text { CPT } & \text { PSB13 } \\ \text { Soil } & \text { CPT } & \text { PSB13 } \\ \text { Soil } & \text { CPT } & \text { PSB13 } \\ \text { Soil } & \text { CPT } & \text { PSB13 } \\ \text { Soil } & \text { CPT } & \text { PSB13 } \\ \text { Soil } & \text { CPT } & \text { PSB13 } \\ \text { Soil } & \text { CPT } & \text { PSB13 } \\ \text { Soil } & \text { CPT } & \text { PSB13 } \\ \text { Soil } & \text { CPT } & \text { PSB13 } \\ \text { Soil } & \text { CPT } & \text { PSB13 } \\ \text { Soil } & \text { CPT } & \text { PSB13 }\end{array}$

Post-injection sampling, September 2008

\begin{tabular}{|c|c|c|c|c|c|c|c|}
\hline $9 / 8 / 08$ & $12: 04$ & CNSB08-W-26687 & Water & $\mathrm{CPT} / \mathrm{P}$ & SB08 & $52-62$ & 6122 \\
\hline 9/8/08 & $13: 16$ & CNMW02-W-26674 & Water & MW & MW02 & $49.5-59.5$ & 6122 \\
\hline 9/8/08 & $13: 19$ & CNPMP6-W-26694 & Water & MW & PMP6 & $50-60$ & 6124 \\
\hline 9/9/08 & $9: 30$ & CNMW01-W-26673 & Water & MW & MW01 & $54.5-64.5$ & 6123 \\
\hline 9/9/08 & $10: 52$ & CNSB07R-W-26686 & Water & $\mathrm{CPT} / \mathrm{P}$ & SB07R & $45-60$ & 6123 \\
\hline 9/9/08 & $11: 27$ & CNPMP7-W-26695 & Water & MW & PMP7 & $50-60$ & 6123 \\
\hline 9/9/08 & $12: 04$ & CNSB04-W-26684 & Water & $\mathrm{CPT} / \mathrm{P}$ & SB04 & $51-61$ & 6123 \\
\hline 9/9/08 & $12: 33$ & CNPMP4-W-26692 & Water & MW & PMP4 & $48.75-58.75$ & 6123 \\
\hline
\end{tabular}

4309

4309

4309

4309

4309

4309

4309

4309
4310

4310

4310

4311

4311

6122

9/10/08 Depth to water $=18.6 \mathrm{ft}$. Depth of 1 -in. well $=62 \mathrm{ft}$. Sample collected by using low-flow bladder pump after purging of $3 \mathrm{~L}$. Water clear.

9/10/08 Depth to water $=21.9 \mathrm{ft}$. Depth of 4 -in. well $=59.5 \mathrm{ft}$. Sample collected by using low-flow bladder pump after purging of $6.7 \mathrm{~L}$. Water tannish in color with strong manure-like odor. Carbon dioxide difficult to read.

9/10/08 Depth to water $=20.9 \mathrm{ft}$. Depth of 0.5 -in. well $=60 \mathrm{ft}$. Sample collected by using Waterra pump after purging of $5.2 \mathrm{~L}$. Water brown to tannish in color.

9/10/08 Depth to water $=13.6 \mathrm{ft}$. Depth of 4 -in. well $=64.5 \mathrm{ft}$. Sample collected by using low-flow bladder pump after purging of 10 L. Water clear.

9/10/08 Depth to water $=18.4 \mathrm{ft}$. Depth of 2 -in. well $=60 \mathrm{ft}$. Sample collected by using low-flow bladder pump after purging of $6 \mathrm{~L}$. Water clear.

9/10/08 Depth to water $=20 \mathrm{ft}$. Depth of 0.5 -in. well $=60 \mathrm{ft}$. Sample collected by using Waterra pump. Water has light brown tint.

9/10/08 Depth to water $=21.9 \mathrm{ft}$. Depth of 1 -in. well $=61 \mathrm{ft}$. Sample collected by using low-flow bladder pump after purging of 3.2 L. Water clear.

9/10/08 Depth to water $=19.15 \mathrm{ft}$. Depth of 0.5 -in. well $=58.75 \mathrm{ft}$. Sample collected by using Waterra pump after purging of

$5.5 \mathrm{~L}$. Water tannish-brown in color.

$8 / 21 / 08$ Vertical profile soil sampling at location near PSB1.

8/21/08 Vetical profile soil sampling at location near PSB1.

Vertical profile soil sampling at location near PSB1.

Vertical profile soil sampling at location near PSB1. 
TABLE A.1 (Cont.)

\begin{tabular}{|c|c|c|c|c|c|c|c|c|c|}
\hline $\begin{array}{l}\text { Sample } \\
\text { Date }\end{array}$ & Time & Sample & $\begin{array}{l}\text { Sample } \\
\text { Medium }\end{array}$ & Type $^{a}$ & Location & $\begin{array}{l}\text { Depth } \\
\text { (ft BGL) }\end{array}$ & $\begin{array}{l}\text { Chain of } \\
\text { Custody }\end{array}$ & $\begin{array}{l}\text { Shipping } \\
\text { Date }\end{array}$ & Sample Description \\
\hline \multicolumn{10}{|c|}{ Post-injection sampling, September 2008 (cont.) } \\
\hline 9/9/08 & $12: 54$ & CNMW03-W-26675 & Water & MW & MW03 & $50.5-60.5$ & 6123 & $9 / 10 / 08$ & $\begin{array}{l}\text { Depth to water }=20.8 \mathrm{ft} \text {. Depth of } 4 \text {-in. well }=60.5 \mathrm{ft} \text {. Sample } \\
\text { collected by using low-flow bladder pump after purging of } \\
6.3 \mathrm{~L} \text {. Water clear. }\end{array}$ \\
\hline $9 / 9 / 08$ & $13: 10$ & CNPMP8-W-26696 & Water & MW & PMP8 & $50-60$ & 6123 & $9 / 10 / 08$ & $\begin{array}{l}\text { Depth to water }=20.3 \mathrm{ft} \text {. Depth of } 0.5 \text {-in. well }=60 \mathrm{ft} \text {. Sample } \\
\text { collected by using Waterra pump after purging of } 5.5 \mathrm{~L} \text {. } \\
\text { Water tannish in color with odor. }\end{array}$ \\
\hline $9 / 9 / 08$ & $13: 40$ & CNPMP3-W-26691 & Water & MW & PMP3 & $50-60$ & 6123 & $9 / 10 / 08$ & $\begin{array}{l}\text { Depth to water }=21.6 \mathrm{ft} \text {. Depth of } 0.5 \text {-in. well }=60 \mathrm{ft} \text {. Sample } \\
\text { collected by using Waterra pump after purging of } 5.2 \mathrm{~L} \text {. } \\
\text { Water cloudy to grayish tint in color with odor. }\end{array}$ \\
\hline $9 / 9 / 08$ & $13: 48$ & CNMW10-W-26682 & Water & MW & MW10 & $30-45$ & 6123 & 9/10/08 & $\begin{array}{l}\text { Depth to water }=21.1 \mathrm{ft} \text {. Depth of } 2 \text {-in. well }=45 \mathrm{ft} \text {. Sample } \\
\text { collected by using low-flow bladder pump after purging of } \\
7 \mathrm{~L} \text {. Water clear. }\end{array}$ \\
\hline $9 / 9 / 08$ & $14: 12$ & CNPMP9-W-26697 & Water & MW & PMP9 & $50-60$ & 6122 & $9 / 10 / 08$ & $\begin{array}{l}\text { Depth to water }=16.2 \mathrm{ft} \text {. Depth of } 0.5 \text {-in. well }=60 \mathrm{ft} \text {. Sample } \\
\text { collected by using Waterra pump after purging of } 5.4 \mathrm{~L} \text {. } \\
\text { Water clear to cloudy. }\end{array}$ \\
\hline $9 / 9 / 08$ & $14: 42$ & CNPMP2-W-26690 & Water & MW & PMP2 & $50-60$ & 6123 & $9 / 10 / 08$ & $\begin{array}{l}\text { Depth to water }=20.9 \mathrm{ft} \text {. Depth of } 0.5 \text {-in. well }=60 \mathrm{ft} \text {. Sample } \\
\text { collected by using Waterra pump after purging of } 5.1 \mathrm{~L} \text {. } \\
\text { Water clear to cloudy with strong odor. }\end{array}$ \\
\hline $9 / 9 / 08$ & $15: 10$ & CNMW06-W-26678 & Water & MW & MW06 & $46.5-56.5$ & 6123 & 9/10/08 & $\begin{array}{l}\text { Depth to water }=36.6 \mathrm{ft} \text {. Depth of } 4 \text {-in. well }=56.5 \mathrm{ft} \text {. Sample } \\
\text { collected by using low-flow bladder pump after purging of } \\
6.4 \mathrm{~L} \text {. Water tannish in color. }\end{array}$ \\
\hline $9 / 9 / 08$ & $15: 12$ & CNPMP1-W-26689 & Water & MW & PMP1 & $50-60$ & 6123 & $9 / 10 / 08$ & $\begin{array}{l}\text { Depth to water }=20.9 \mathrm{ft} \text {. Depth of } 0.5 \text {-in. well }=60 \mathrm{ft} \text {. Sample } \\
\text { collected by using Waterra pump after purging of } 5 \mathrm{~L} \text {. } \\
\text { Water brown-tan in color with odor. }\end{array}$ \\
\hline $9 / 9 / 08$ & $16: 18$ & CNMW07-W-26679 & Water & MW & MW07 & $45-55$ & 6123 & $9 / 10 / 08$ & $\begin{array}{l}\text { Depth to water }=28.2 \mathrm{ft} \text {. Depth of } 2 \text {-in. well }=55 \mathrm{ft} \text {. Sample } \\
\text { collected by using low-flow bladder pump after purging of } \\
7 \mathrm{~L} \text {. Water clear. }\end{array}$ \\
\hline $9 / 9 / 08$ & $16: 59$ & CNSB05-W-26685 & Water & $\mathrm{CPT} / \mathrm{P}$ & SB05 & $32-42$ & 6123 & $9 / 10 / 08$ & $\begin{array}{l}\text { Depth to water }=10.6 \mathrm{ft} \text {. Depth of } 1 \text {-in. well }=42 \mathrm{ft} \text {. Sample } \\
\text { collected by using Waterra pump after purging of } 15 \mathrm{~L} \text {. } \\
\text { Water clear to cloudy. }\end{array}$ \\
\hline $9 / 9 / 08$ & $17: 10$ & CNMW04-W-26676 & Water & MW & MW04 & $37.5-47.5$ & 6123 & $9 / 10 / 08$ & $\begin{array}{l}\text { Depth to water }=24 \mathrm{ft} \text {. Depth of } 4 \text {-in. well }=47.5 \mathrm{ft} \text {. Sample } \\
\text { collected by using low-flow bladder pump after purging of } \\
6.2 \mathrm{~L} \text {. Water clear. }\end{array}$ \\
\hline $9 / 10 / 08$ & $9: 22$ & CNMW05-W-26677 & Water & MW & MW05 & $34.5-44.5$ & 6124 & $9 / 10 / 08$ & $\begin{array}{l}\text { Depth to water }=10 \mathrm{ft} \text {. Depth of } 4 \text {-in. well }=44.5 \mathrm{ft} \text {. Sample } \\
\text { collected by using low-flow bladder pump after purging of } \\
5 \mathrm{~L} \text {. Water clear. }\end{array}$ \\
\hline $9 / 10 / 08$ & 9:23 & CNMW05DUP-W-26698 ${ }^{\mathrm{b}}$ & Water & MW & MW05 & $34.5-44.5$ & 6124 & $9 / 10 / 08$ & Replicate of sample CNMW05-W-26677. \\
\hline $9 / 10 / 08$ & $10: 22$ & CNPMP5-W-26693 & Water & MW & PMP5 & $50-60$ & 6124 & $9 / 10 / 08$ & $\begin{array}{l}\text { Depth to water }=21.4 \mathrm{ft} \text {. Depth of } 1 \text {-in. well }=60 \mathrm{ft} \text {. Sample } \\
\text { collected by using low-flow bladder pump after purging of } \\
3 \mathrm{~L} \text {. Water brownish color. }\end{array}$ \\
\hline
\end{tabular}


TABLE A.1 (Cont.)

\begin{tabular}{|c|c|c|c|c|c|c|c|c|c|}
\hline $\begin{array}{l}\text { Sample } \\
\text { Date }\end{array}$ & Time & Sample & $\begin{array}{l}\text { Sample } \\
\text { Medium }\end{array}$ & Type ${ }^{a}$ & Location & $\begin{array}{l}\text { Depth } \\
\text { (ft BGL) }\end{array}$ & $\begin{array}{l}\text { Chain of } \\
\text { Custody }\end{array}$ & $\begin{array}{l}\text { Shipping } \\
\text { Date }\end{array}$ & Sample Description \\
\hline \multicolumn{10}{|c|}{ Post-injection sampling, September 2008 (cont.) } \\
\hline 9/10/08 & $11: 38$ & CNSB01-W-26683 & Water & $\mathrm{CPT} / \mathrm{P}$ & SB01 & $40-50$ & 6124 & $9 / 10 / 08$ & $\begin{array}{l}\text { Depth to water }=20.1 \mathrm{ft} \text {. Depth of } 1 \text {-in. well }=50 \mathrm{ft} \text {. Sample } \\
\text { collected by using low-flow bladder pump after purging of } \\
3 \mathrm{~L} \text {. Water clear. }\end{array}$ \\
\hline $9 / 10 / 08$ & $11: 45$ & CNQCIR-W-26700 b & Water & RI & QC & - & 6124 & $9 / 10 / 08$ & $\begin{array}{l}\text { Rinsate of decontaminated pump purge line after collection of } \\
\text { sample CNSB01-W-26683. }\end{array}$ \\
\hline $9 / 10 / 08$ & $12: 22$ & CNMW09-W-26681 & Water & MW & MW09 & $25-35$ & 6124 & $9 / 10 / 08$ & $\begin{array}{l}\text { Depth to water }=2.5 \mathrm{ft} \text {. Depth of } 2 \text {-in. well }=35 \mathrm{ft} \text {. Sample } \\
\text { collected by using low-flow bladder pump after purging of } \\
6 \mathrm{~L} \text {. }\end{array}$ \\
\hline $9 / 10 / 08$ & $13: 34$ & CNSB09-W-26688 & Water & $\mathrm{CPT} / \mathrm{P}$ & SB09 & $32-42$ & 6124 & 9/10/08 & $\begin{array}{l}\text { Depth to water }=6.6 \mathrm{ft} \text {. Depth of } 1 \text {-in. well }=42 \mathrm{ft} \text {. Sample } \\
\text { collected by using low-flow bladder pump after purging of } \\
3 \mathrm{~L} \text {. }\end{array}$ \\
\hline $9 / 10 / 08$ & $13: 45$ & CNQCIR-W-26701 & Water & RI & QC & - & 6124 & 9/10/08 & $\begin{array}{l}\text { Rinsate of decontaminated pump purge line after collection of } \\
\text { sample CNMW09-W-26681. }\end{array}$ \\
\hline $9 / 10 / 08$ & $14: 28$ & CNMW08-W-26680 & Water & MW & MW08 & $38-53$ & 6124 & $9 / 10 / 08$ & $\begin{array}{l}\text { Depth to water }=18.6 \mathrm{ft} \text {. Depth of } 2 \text {-in. well }=53 \mathrm{ft} \text {. Sample } \\
\text { collected by using low-flow bladder pump after purging of } \\
6.5 \mathrm{~L} \text {. Water clear. }\end{array}$ \\
\hline 9/10/08 & $14: 29$ & CNMW08DUP-W-26699b & Water & MW & MW08 & $38-53$ & 6124 & $9 / 10 / 08$ & Replicate of sample CNMW08-W-26680. \\
\hline $9 / 10 / 08$ & $15: 00$ & CNQCTB-W-26702 & Water & TB & QC & - & 6124 & $9 / 10 / 08$ & $\begin{array}{l}\text { Trip blank sent to the AGEM Laboratory for organic analysis } \\
\text { with water samples listed on COCs } 6122,6123 \text {, and } 6124 \text {. }\end{array}$ \\
\hline 9/10/08 & 15:01 & CNQCTB-W-26702a & Water & TB & QC & - & 6134 & 9/10/08 & $\begin{array}{l}\text { Trip blank sent to TestAmerica for verification organic } \\
\text { analysis with water samples listed on COC } 6134 \text {. }\end{array}$ \\
\hline
\end{tabular}

a Sample types: BT, wastewater composite; CPT, cone penetrometer; CPT/P, piezometer; FB, field blank; MW, monitoring well; RI, rinsate; TB, trip blank.

b Quality control sample collected to monitor sample collection and handling activities. 


\section{Appendix B:}

Photographs of Sediment Cores Collected in December 2007 at Post-Injection Investigation Points PSB10, PSB11, and PSB12 


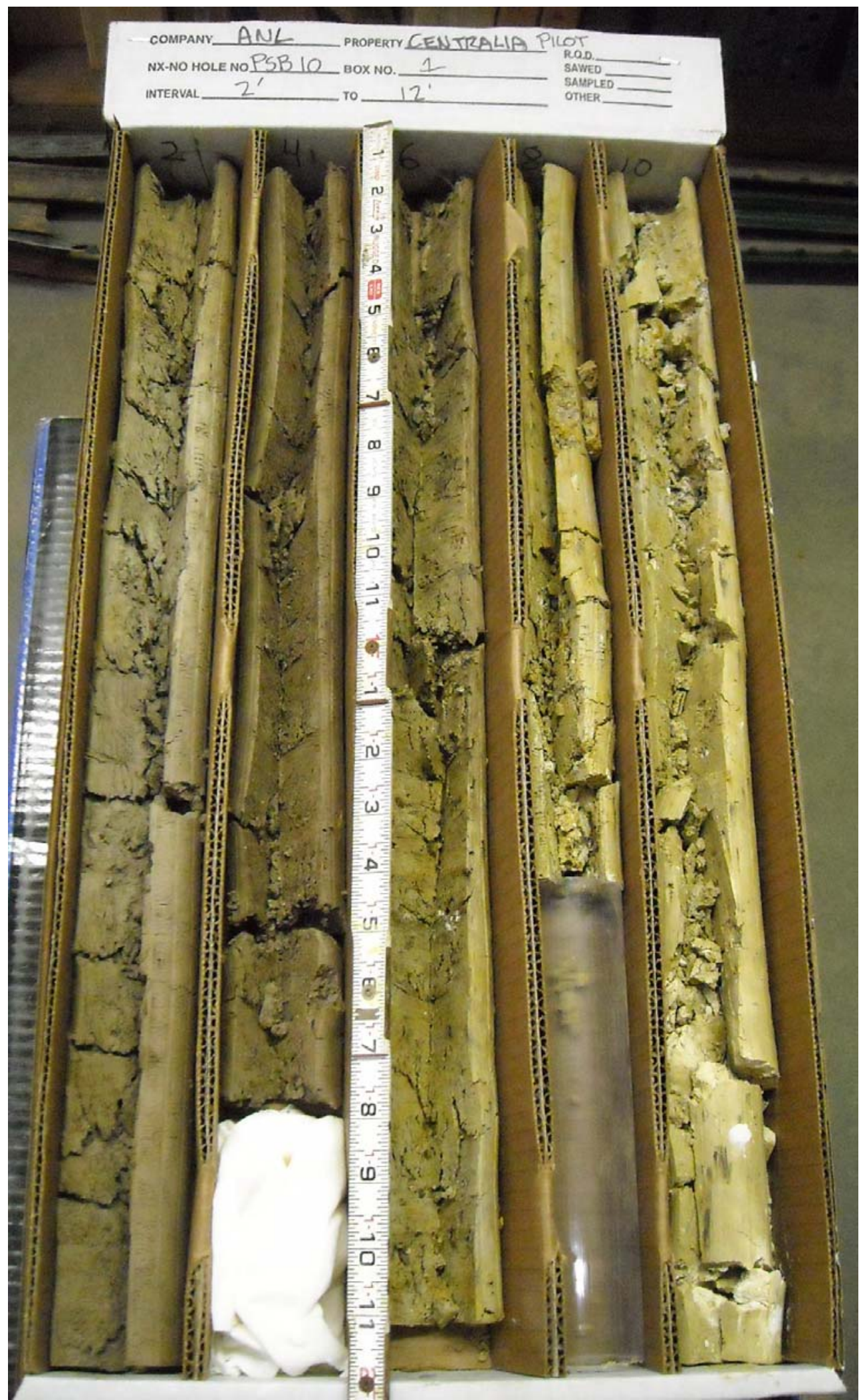

FIGURE B.1 Photographs of the soil core collected from boring PSB10 approximately two weeks after injection, in December 2007. 


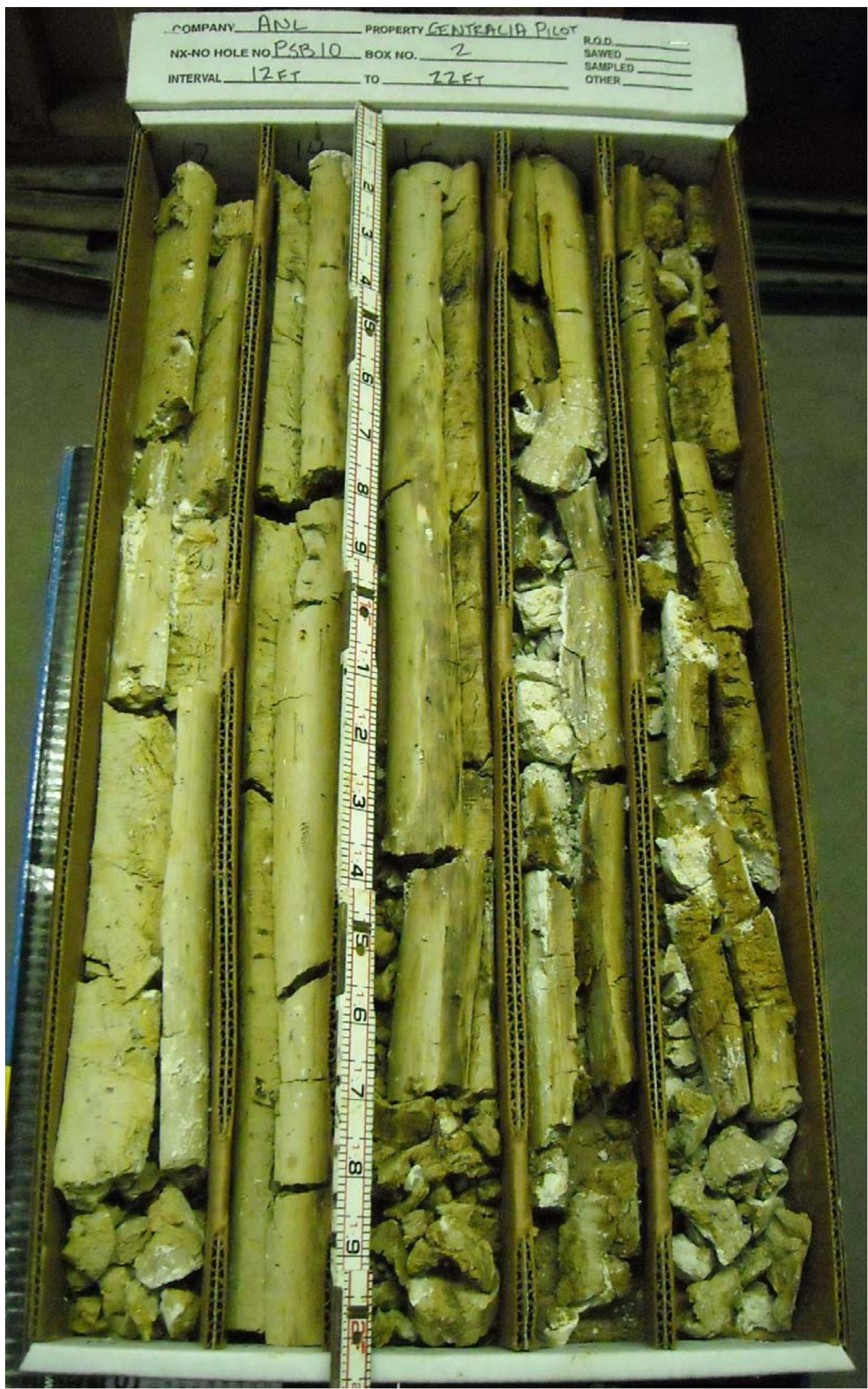

FIGURE B.1 (Cont.) 


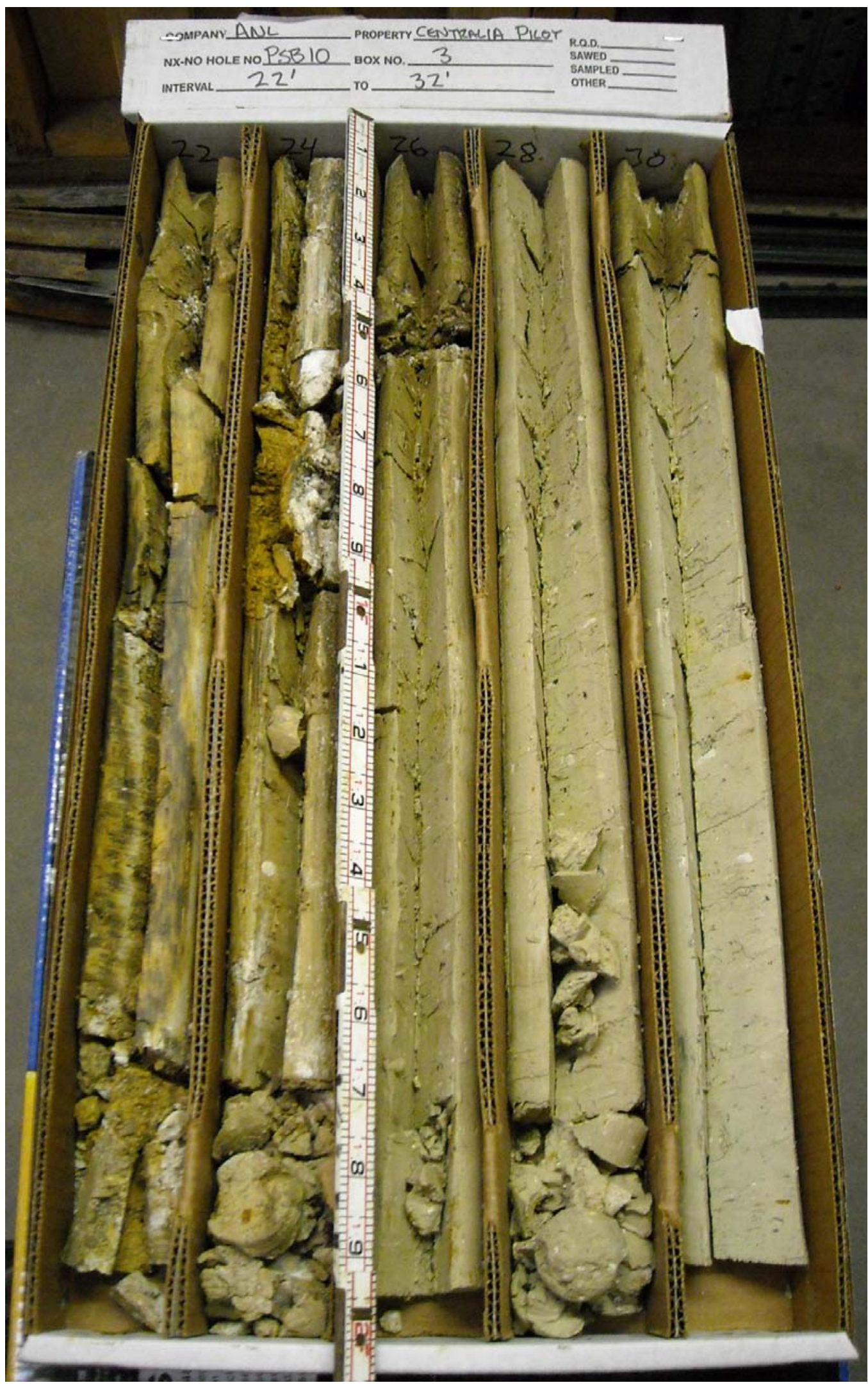

FIGURE B.1 (Cont.) 


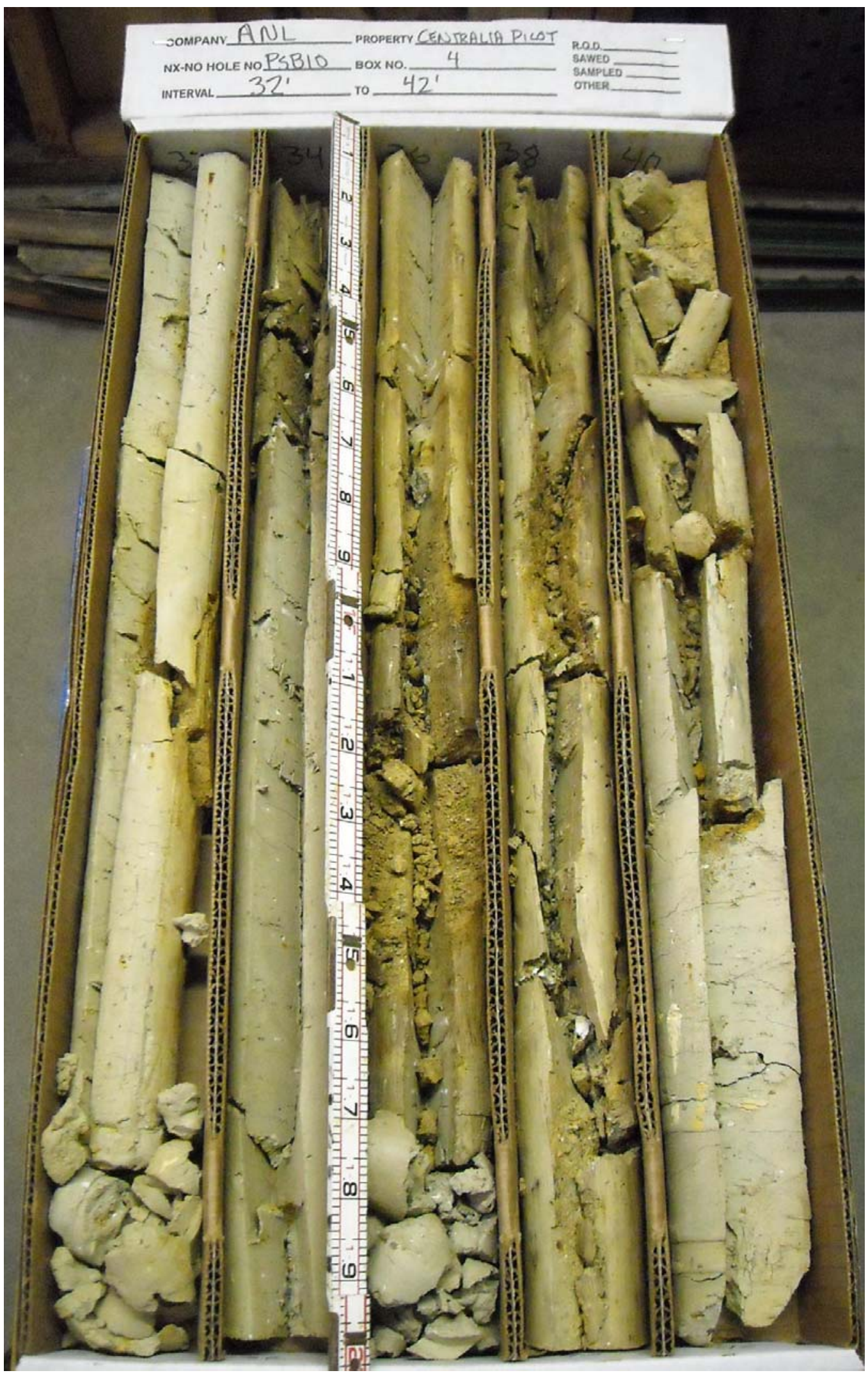

FIGURE B.1 (Cont.) 


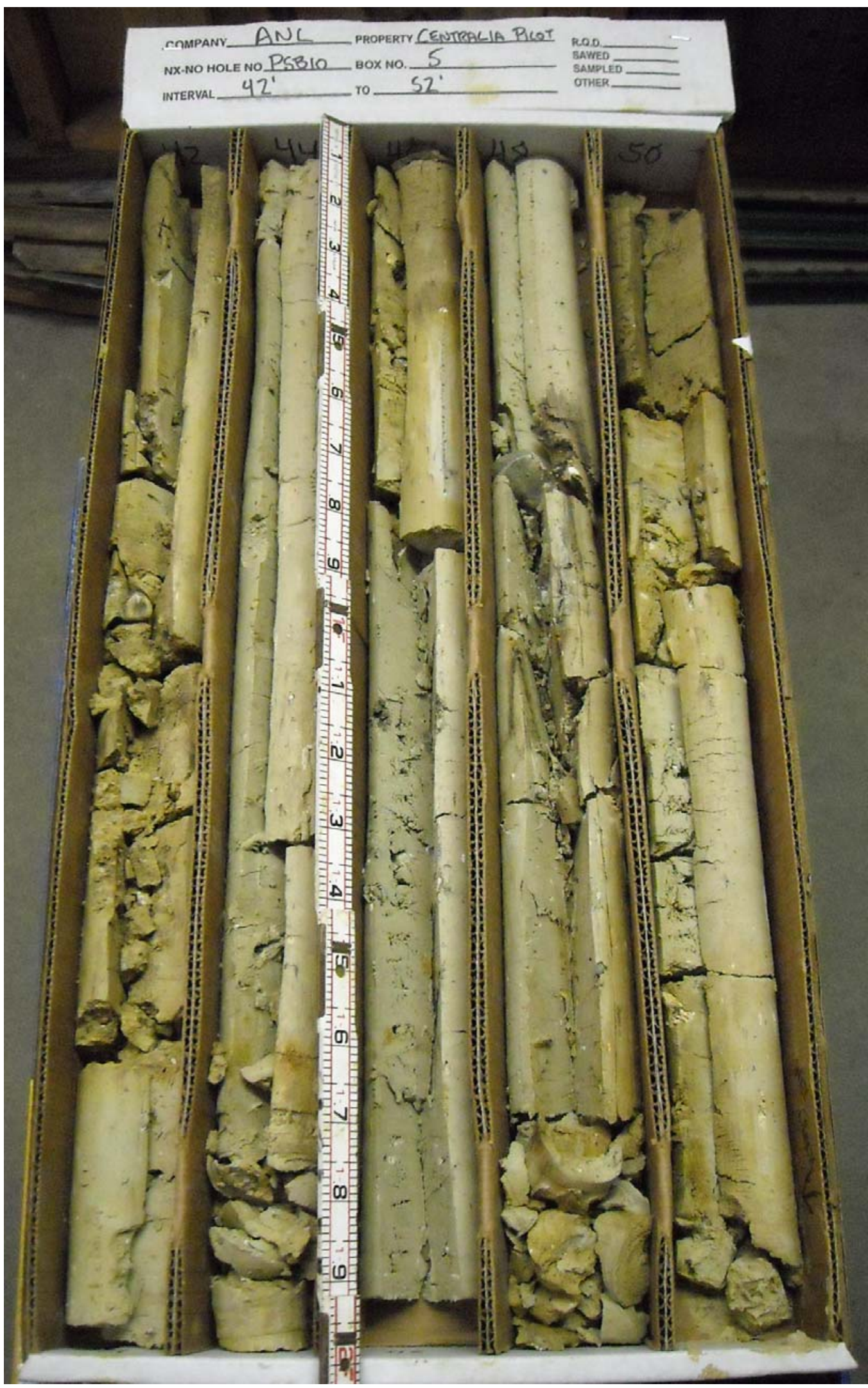

FIGURE B.1 (Cont.) 


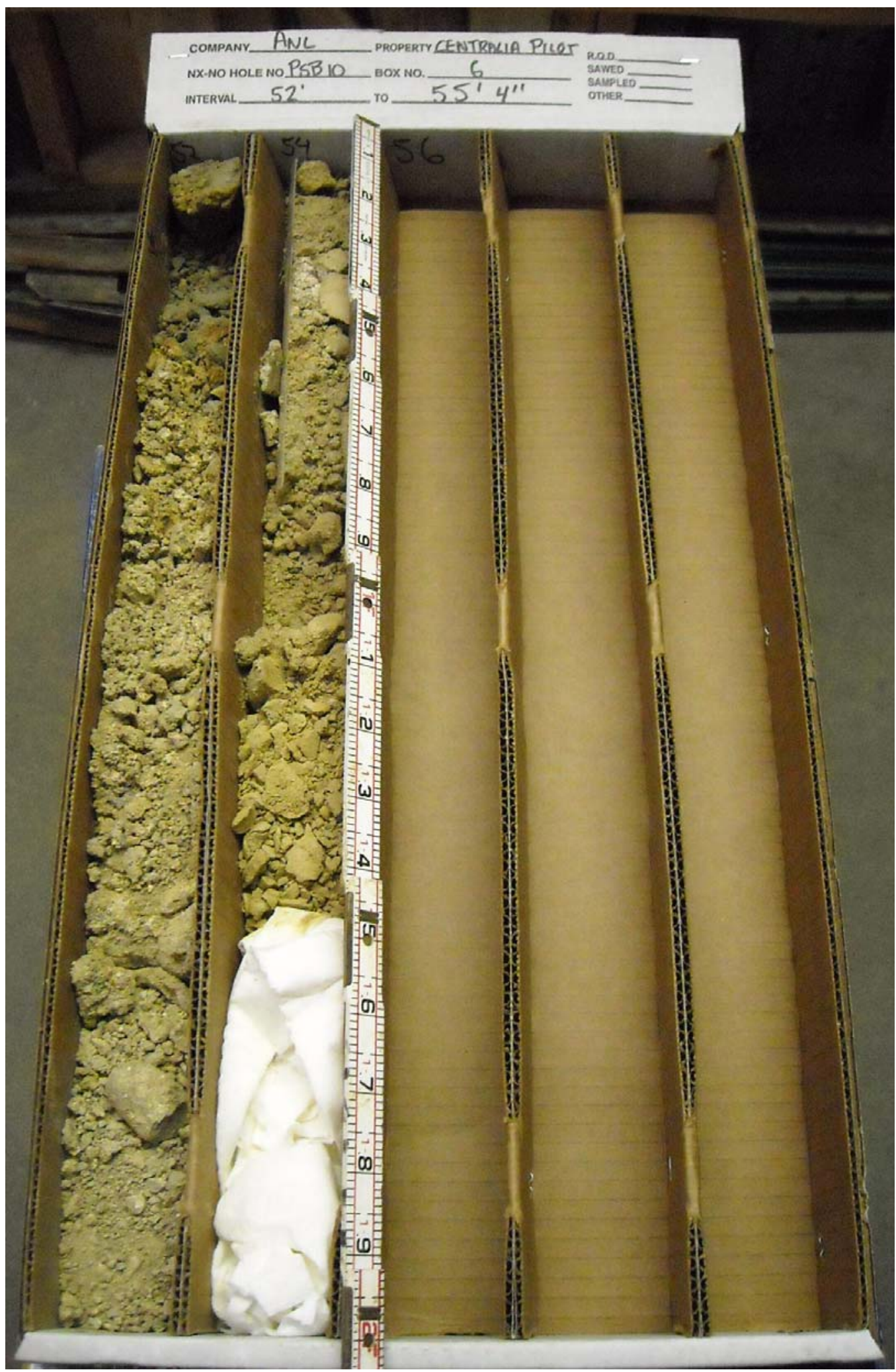

FIGURE B.1 (Cont.) 


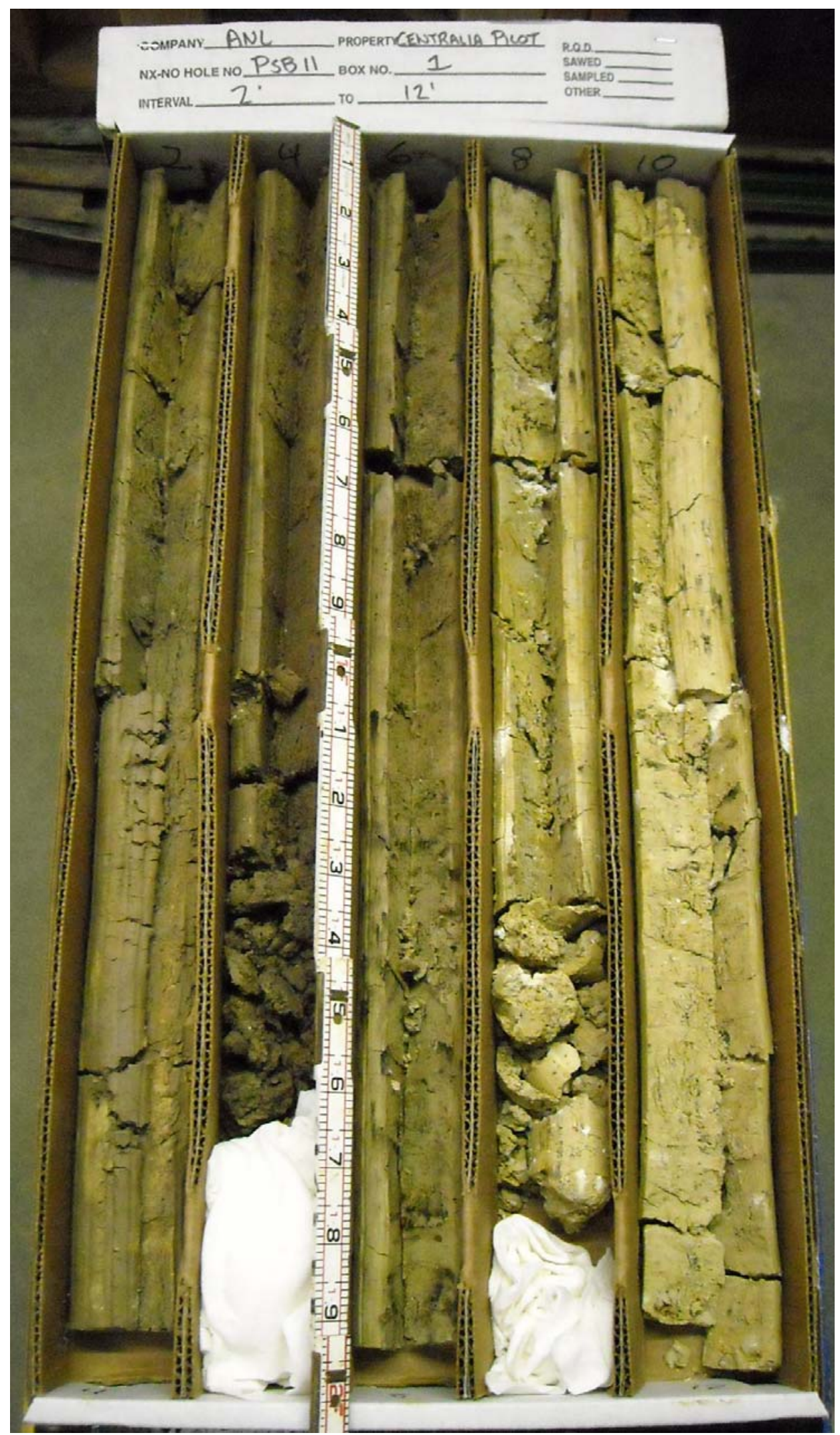

FIGURE B.2 Photographs of the soil core collected from boring PSB11 approximately two weeks after injection, in December 2007. 


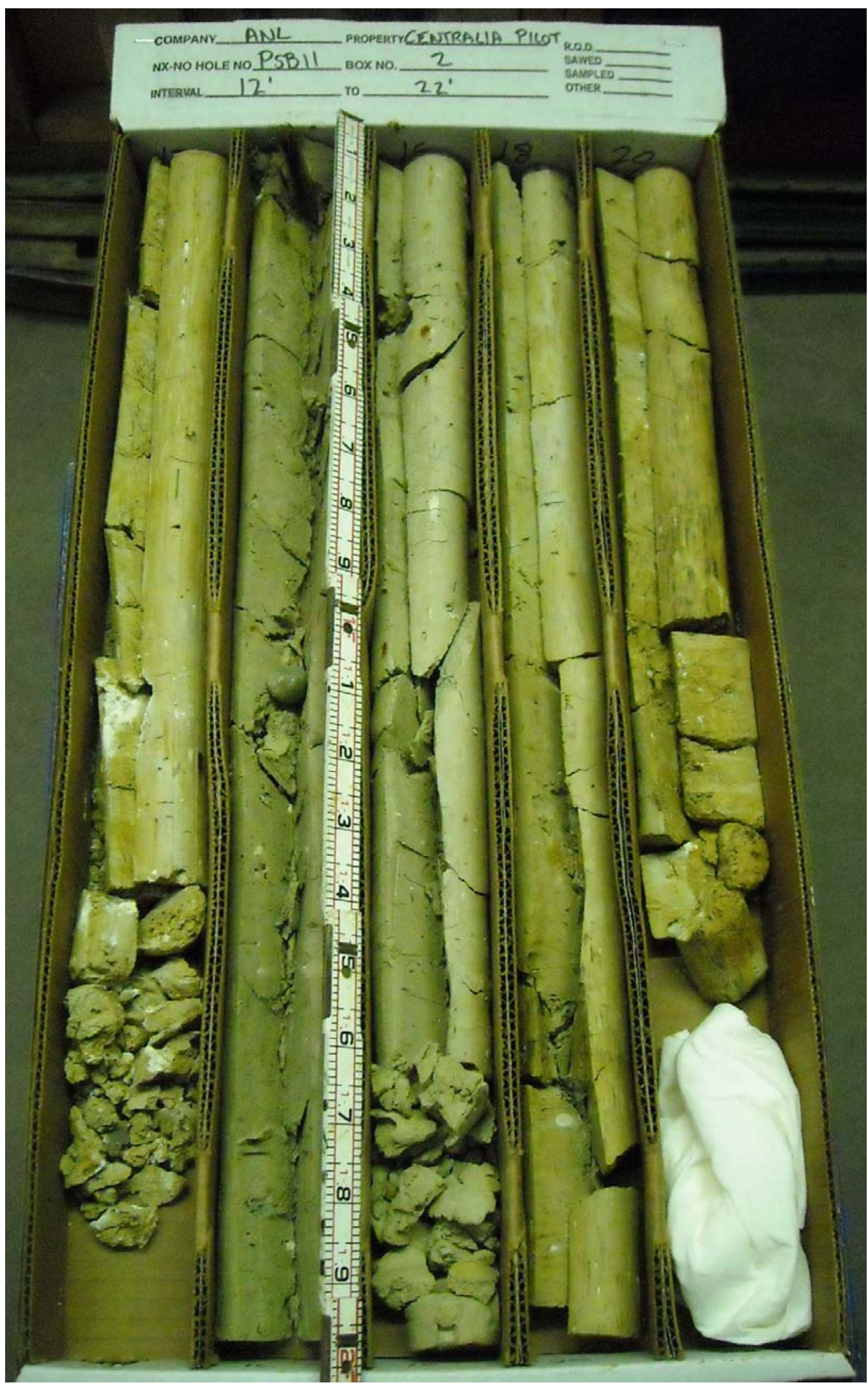

FIGURE B.2 (Cont.) 


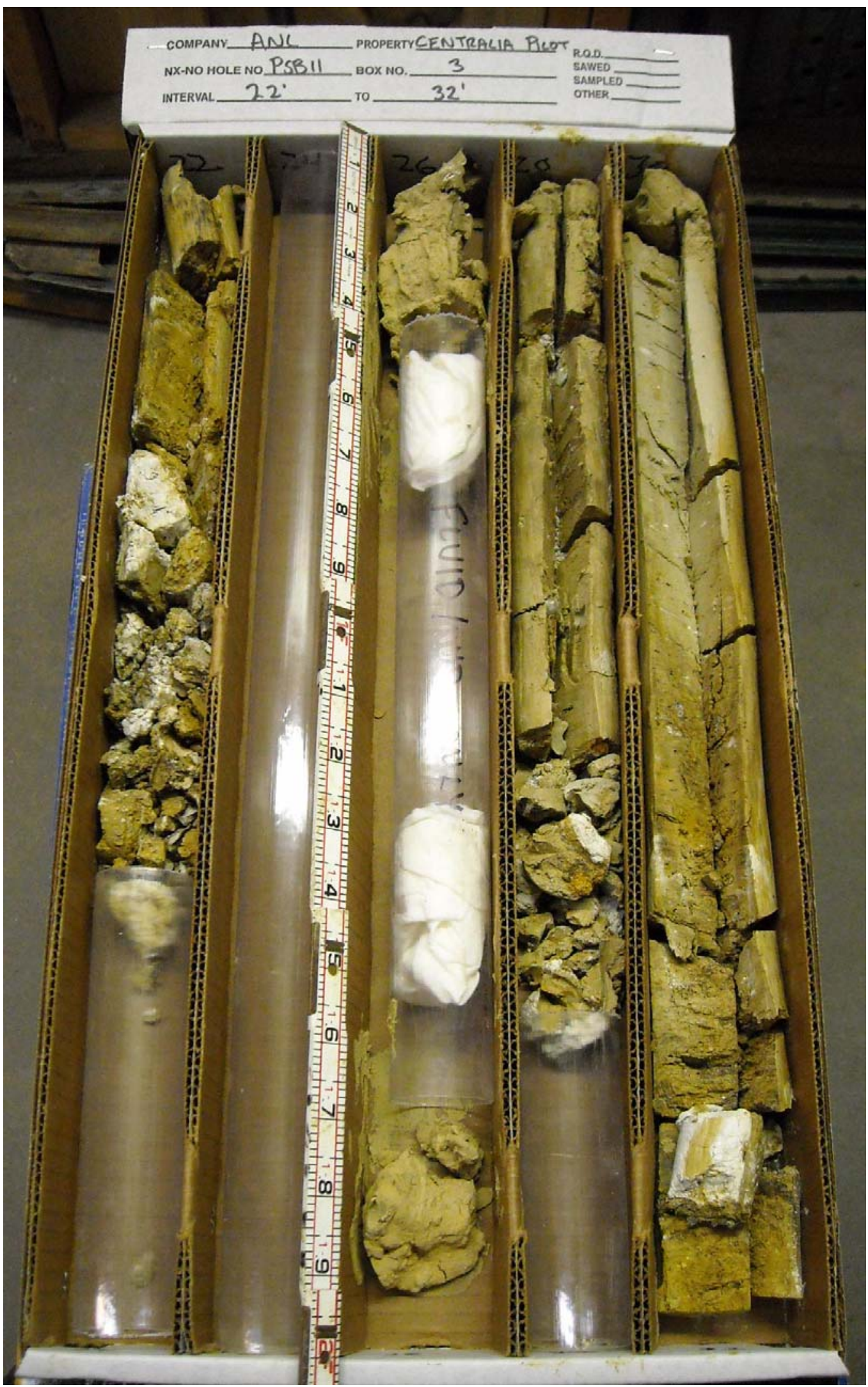

FIGURE B.2 (Cont.) 


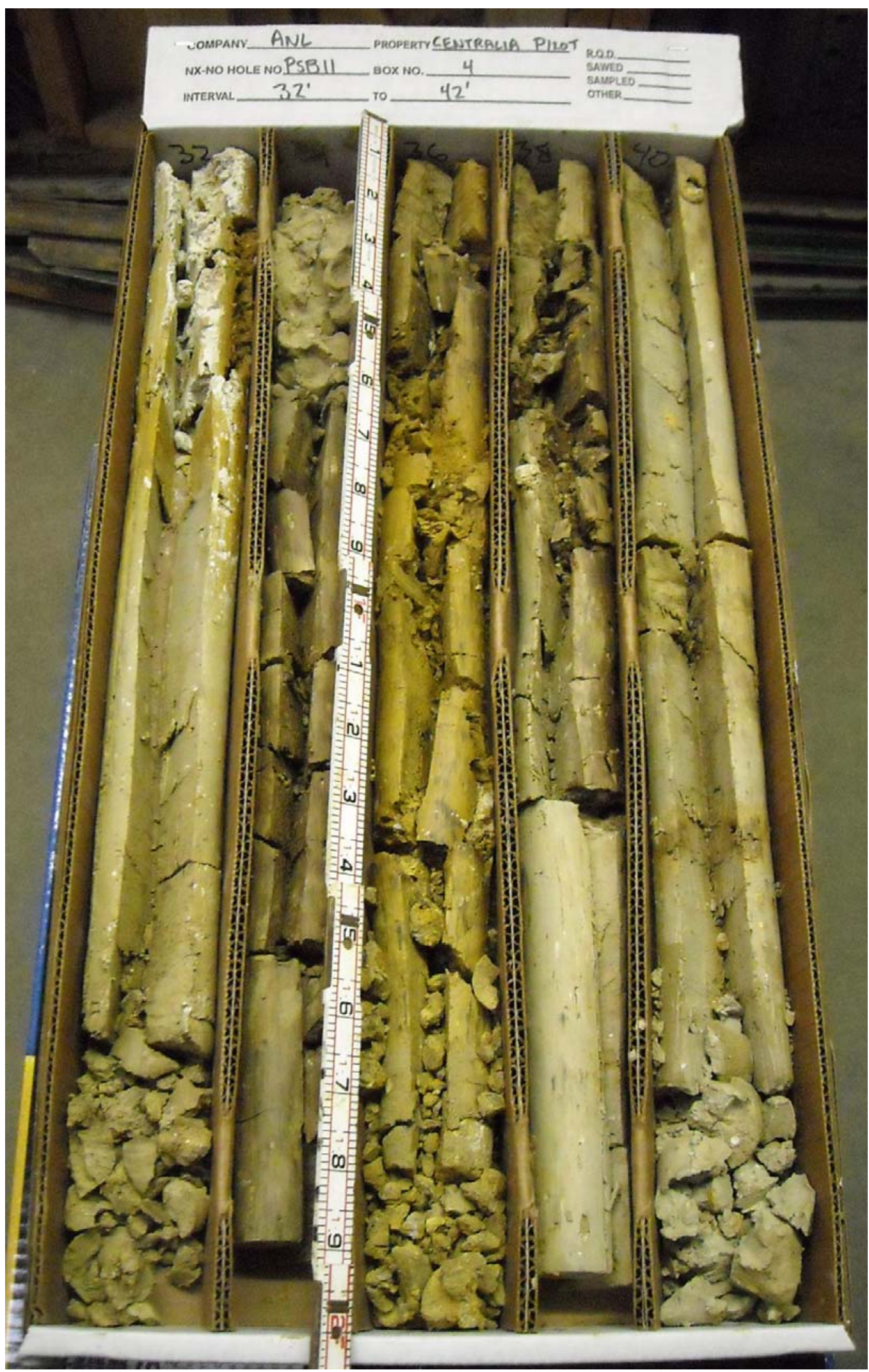

FIGURE B.2 (Cont.) 


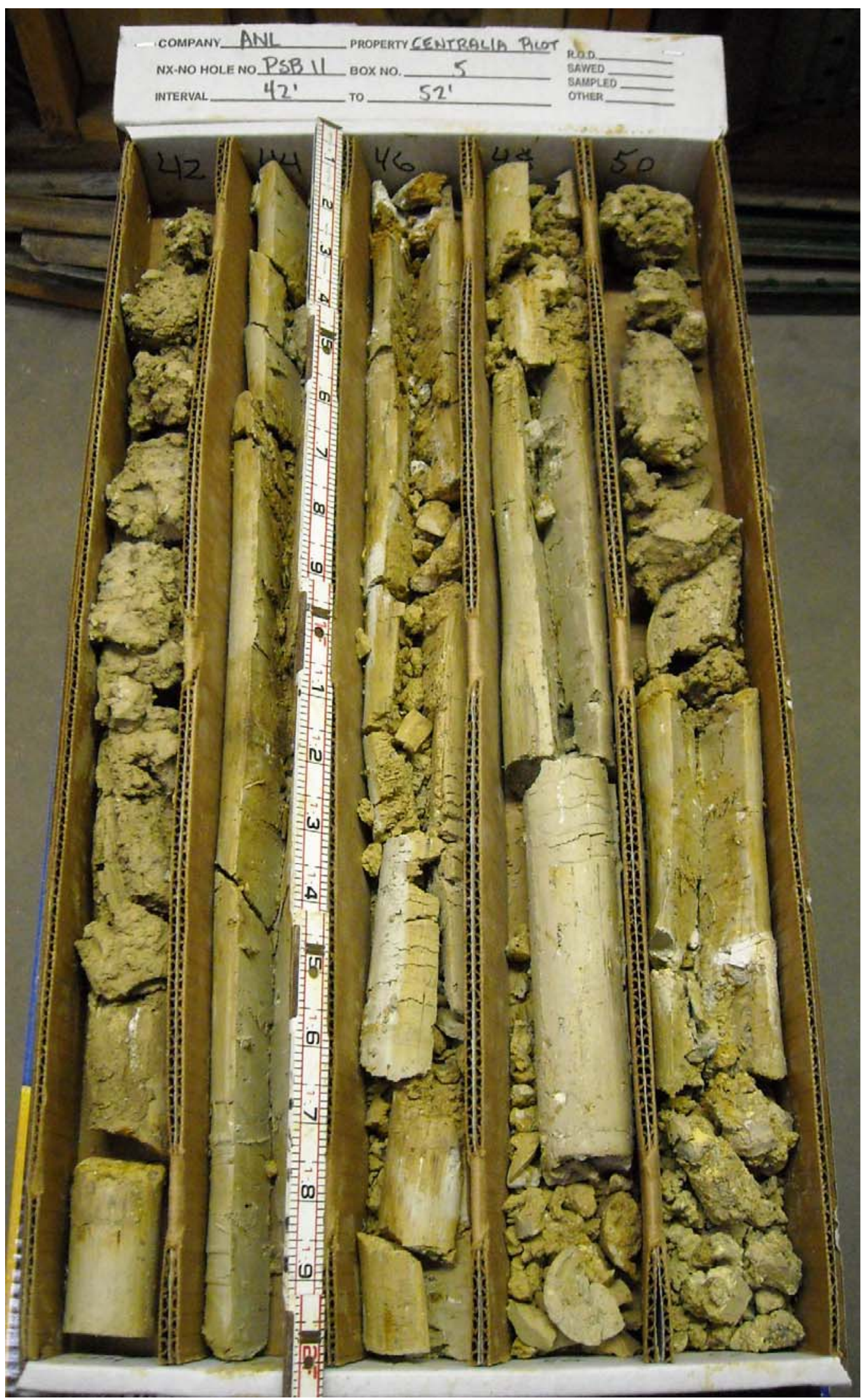

FIGURE B.2 (Cont.) 


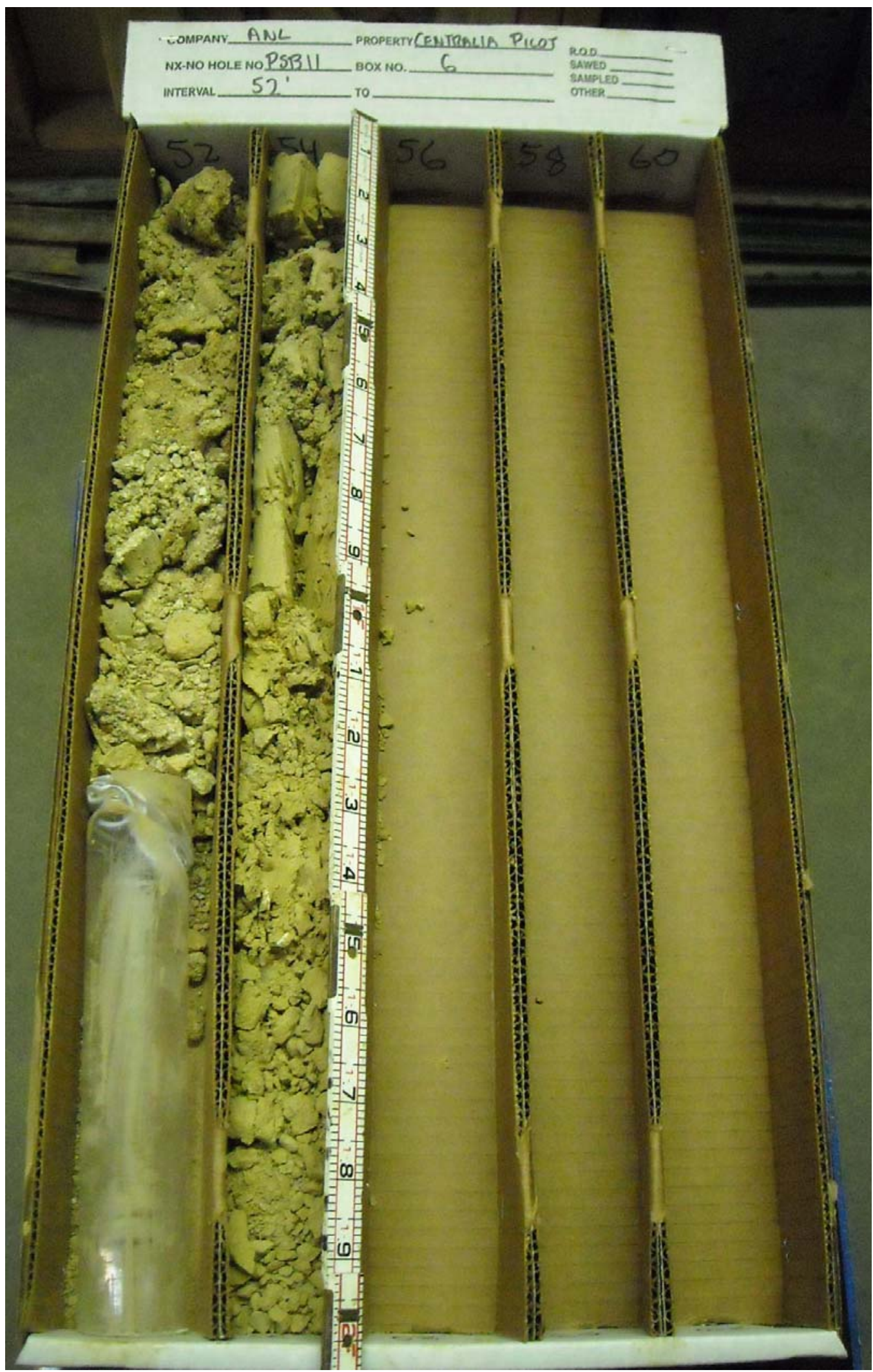

FIGURE B.2 (Cont.) 


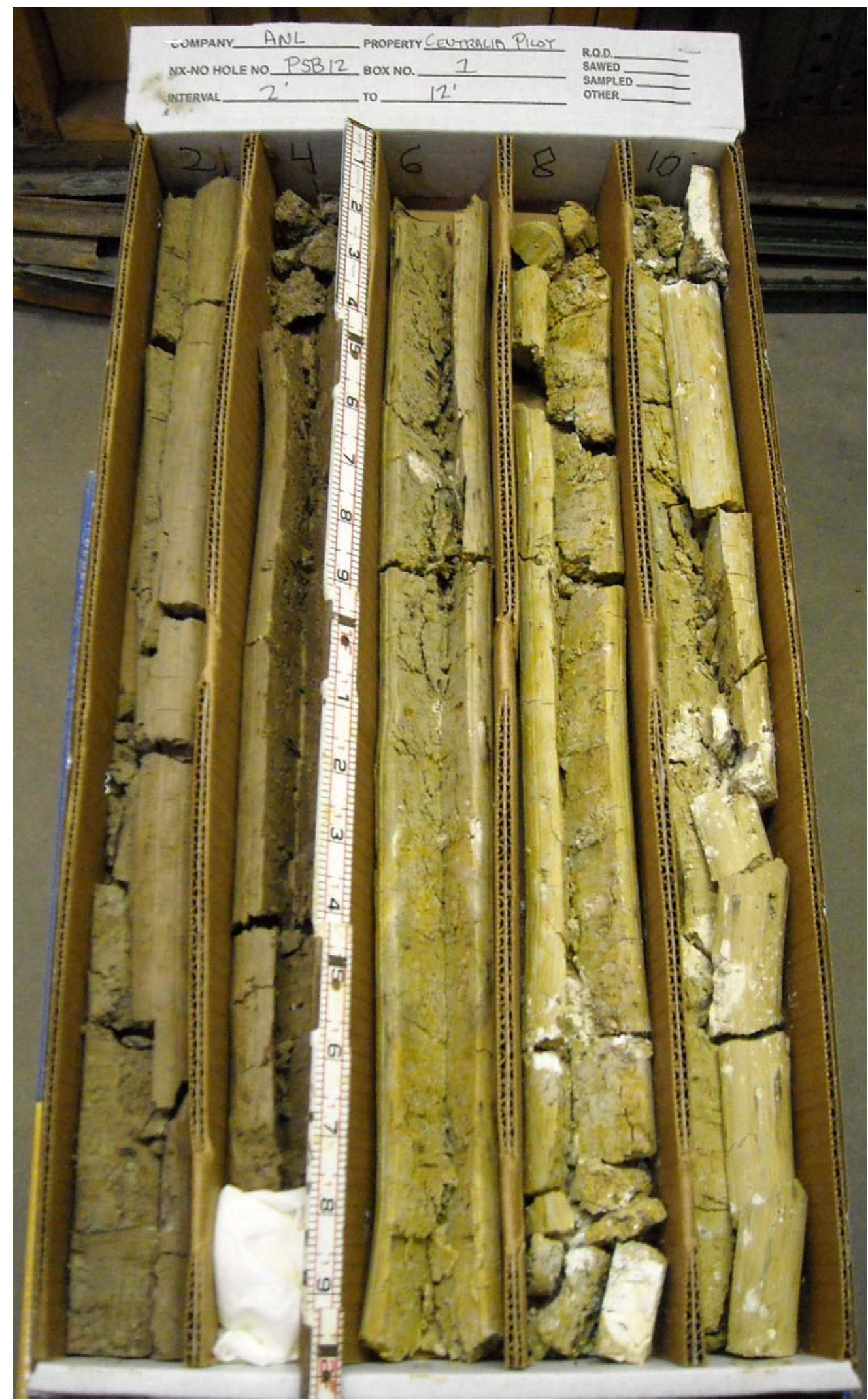

FIGURE B.3 Photographs of the soil core collected from boring PSB12 approximately two weeks after injection, in December 2007. 


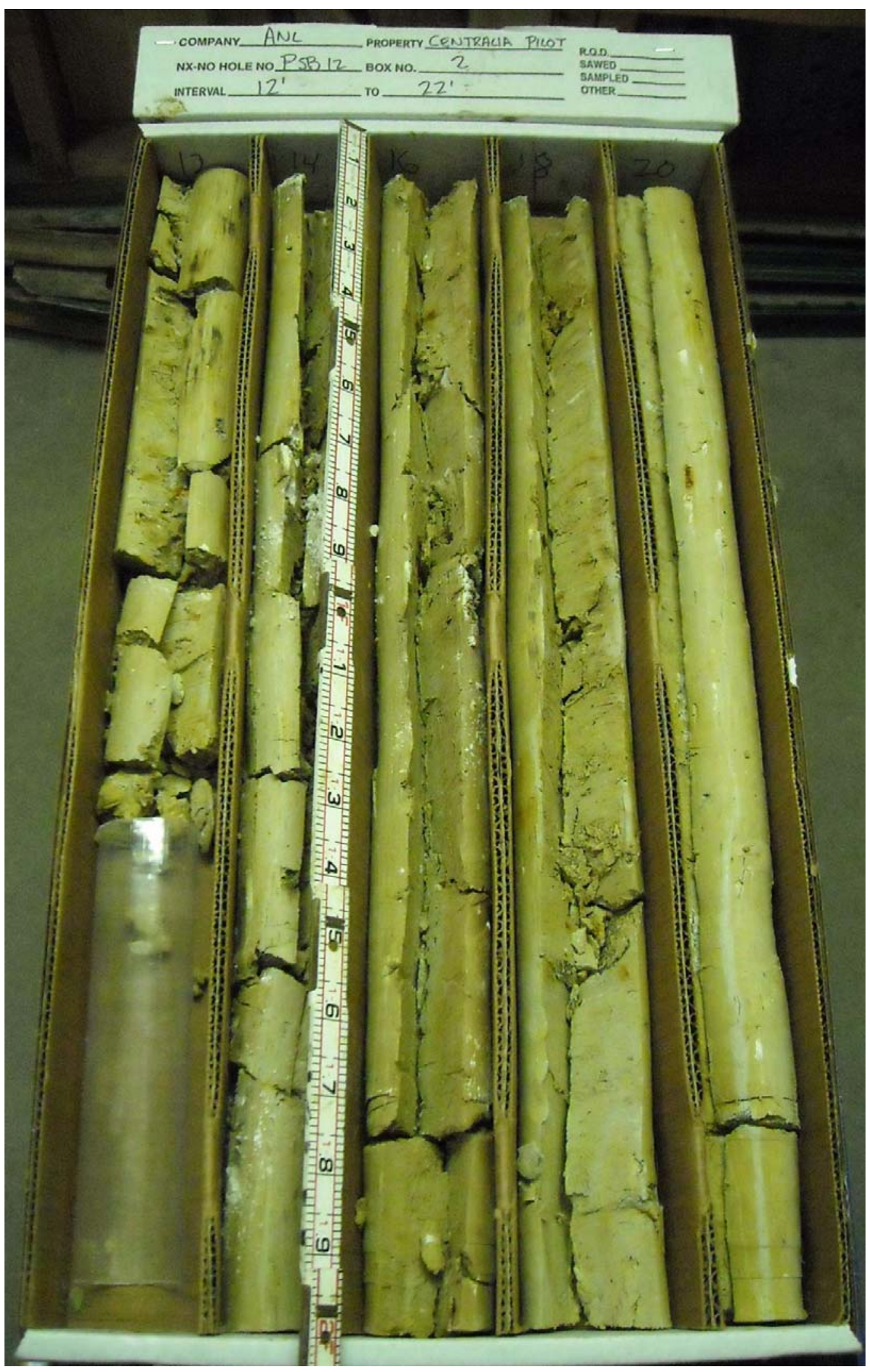

FIGURE B.2 (Cont.) 


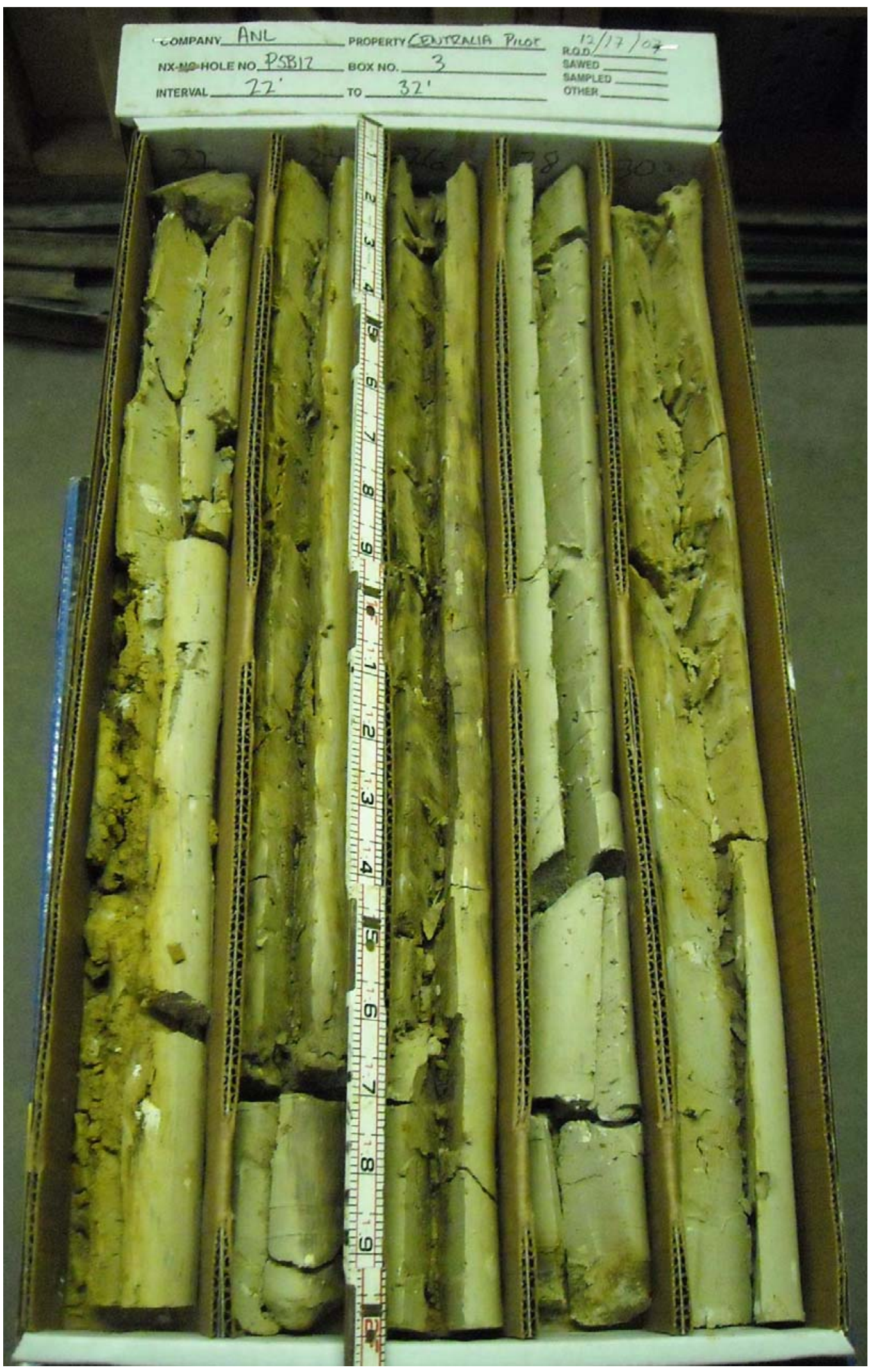

FIGURE B.2 (Cont.) 


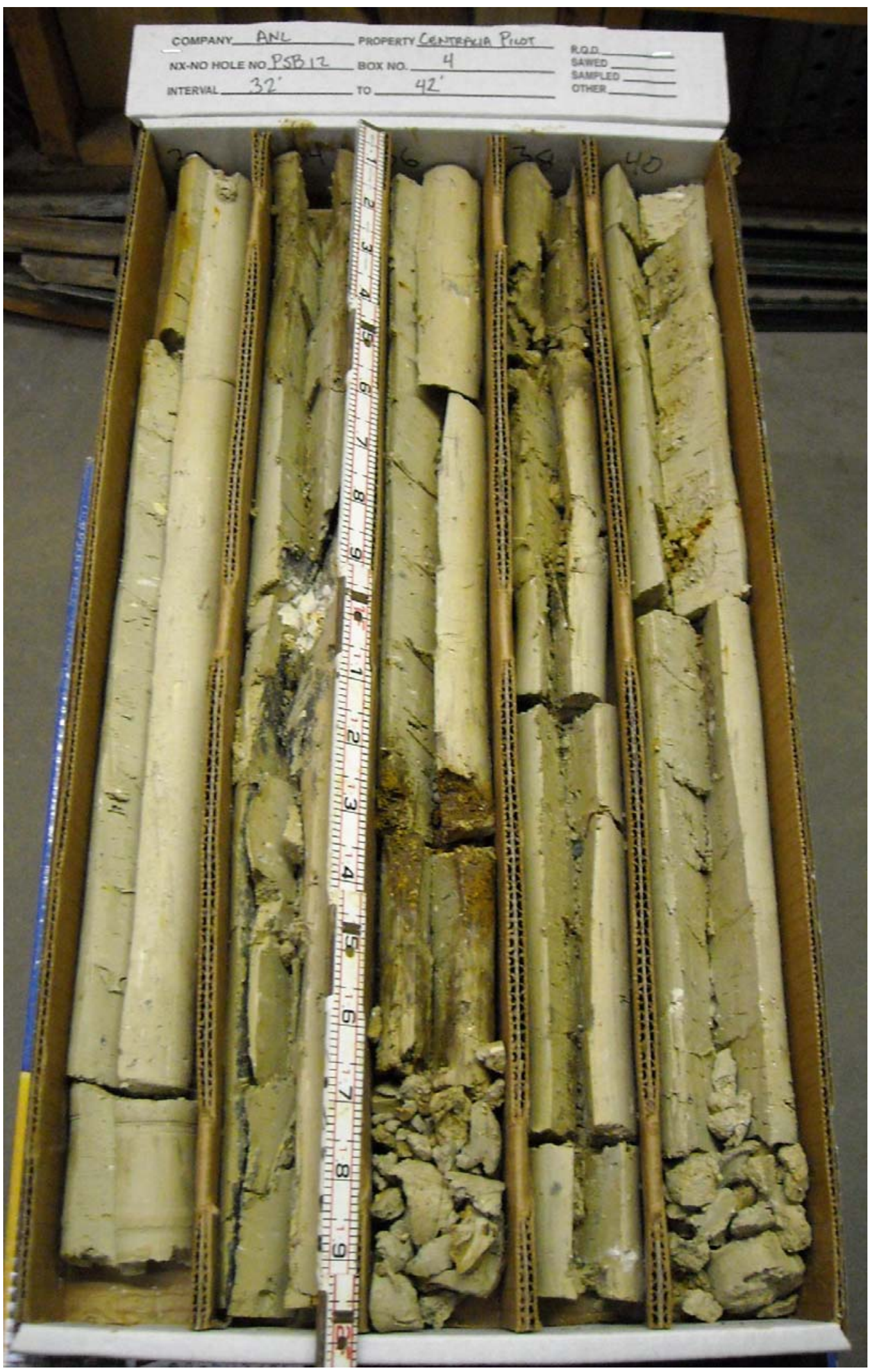

FIGURE B.2 (Cont.) 


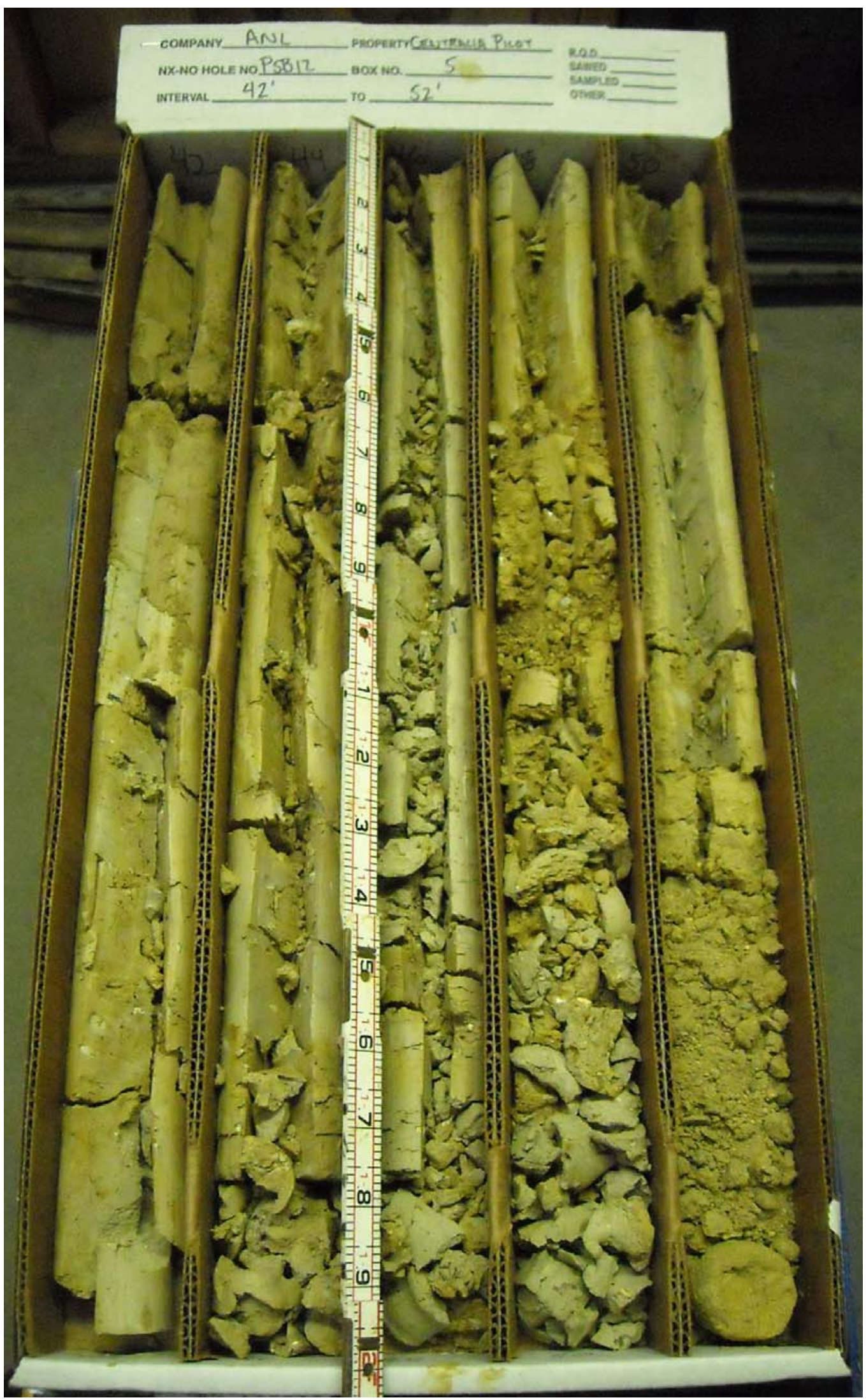

FIGURE B.2 (Cont.) 


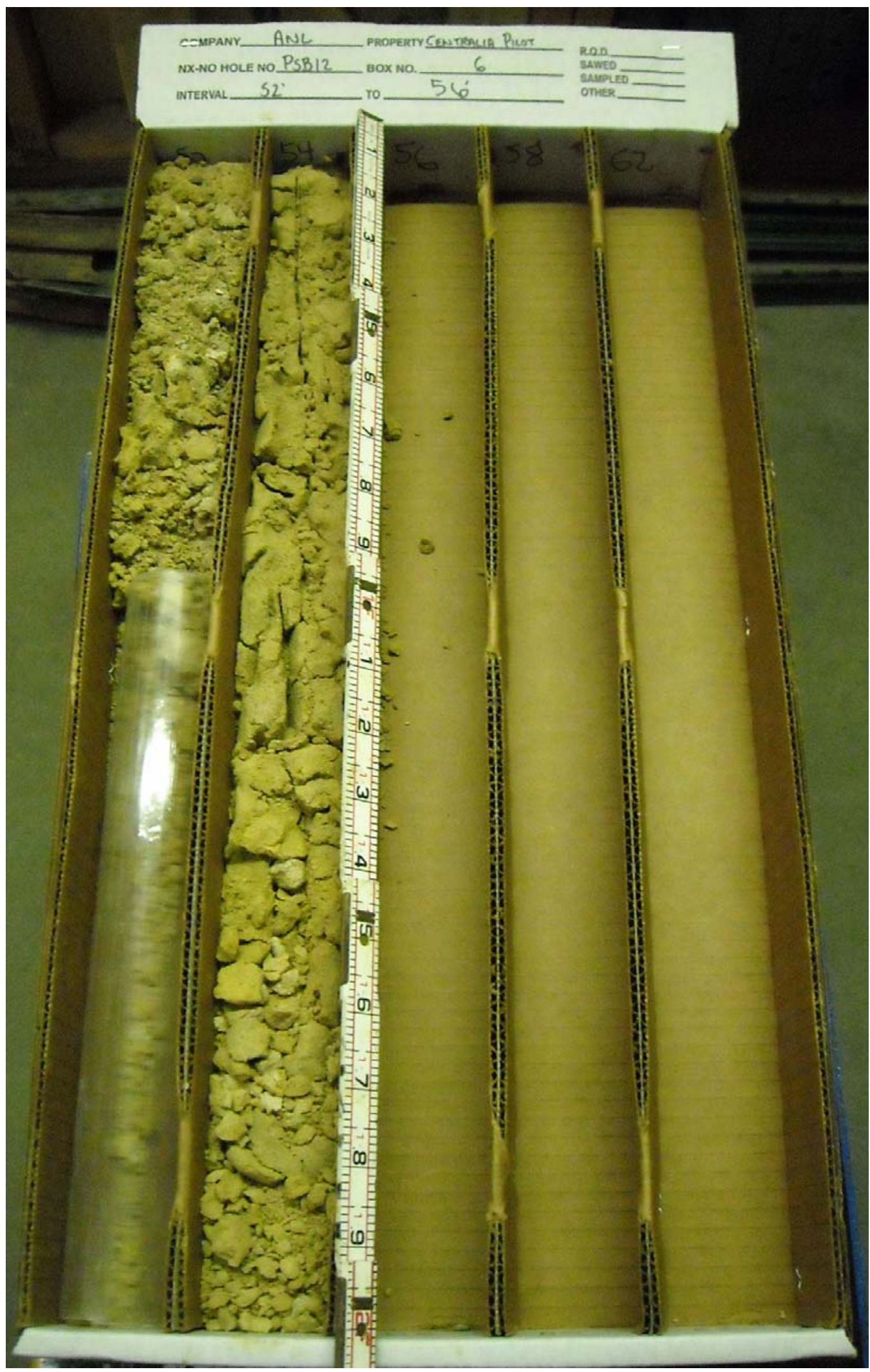

FIGURE B.2 (Cont.) 


\section{Appendix C:}

KDHE Completion Variance and Well Registration Forms (WWC-5) 


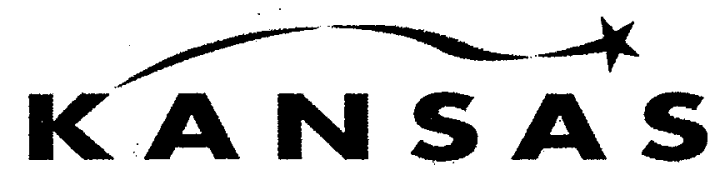

Kathleen Sebelius, Governor

Roderick L. Bremby, Secretary

DEPARTMENT OF HEALTH

AND ENVIRONMENT

www.kdheks.gov

Division of Environment

February 19, 2008

Delta Environmental

David Surgnier

\% Argonne National Laboratory

EVS \# 203, B-133

9700 South Cass, Ave.

Lemont, IL 60439

Re: Waiver request for flush mount monitoring wells for USDA/CCC; Centralia, Kansas. Located in the NE 1/4 of the NE 1/4 of the SE 1/4 of Section 1, Township 4 South, Range 11 East, Nemaha County, Kansas.

Dear Mr. Surgnier:

In accordance with Kansas Administrative Regulations (K.A.R.) 28-30-9, appealing regulations as stated in Article 30, your request for an exception to K.A.R. 2830-6(b)(1),(c) and (e) for authorization of 20 geotechnical wells to be constructed less than 12 inches above the finished ground level and less than 20 feet of grout if ground water encountered less than $\mathbf{2 0}^{\prime}$ below the surface, at the above-mentioned site is hereby granted subject to the following stipulations:

1. Monitoring wells included in this request will be grouted from a maximum of two feet below a ground surface to within one foot above the screened section.

2. The wellhead will be encased in an approved water resistant/proof manhole. The manhole will be encased in cement, which is to be domed or sloped to allow drainage away from the manhole, (refer to the attached diagram page 2).

BUREAU OF WATER - GEOLOGY SECTION

CURTIS STATE OFFICE BUILDING, 1000 SW JACKSON ST., STE. 420, TOPEKA, KS 66612-1367

Voice 785-296-5524 Fax 785-296-5509 Web http://kdheks/gov/geo 
February 19, 2008

Page two

3. The casing of the monitoring well will be sealed with an approved watertight lock able monitoring well caps (refer to the attached diagram).

4. A copy of this KDHE letter, approving your request for waiver of K.A.R. 28-30-6 (b)(1),(c) and (e), will be sent to KDHE attached to the water well record (WWC5 Form) of the first well drilled under the granted waiver.

5. Upon completion of the project the wells covered by the waiver shall be plugged in accordance with K.A.R. 28-30-7 (d).

The decision to grant this waiver is based almost entirely on the data provided in your request. This waiver is to cover only the $\mathbf{2 0}$ wells mentioned and reportedly will be drilled by Delta Environmental. Kansas Water Well Contractor License Number $\mathbf{6 8 0}$. This waiver will become null and void if any of the information submitted in the request is found to be false or if the wells are not constructed in strict conformity to Kansas rules and regulations and the above mentioned, stipulations. Please contact Don Taylor, (785) 296-5522, if you have any questions or concerns pertaining to this matter.

Sincerely,

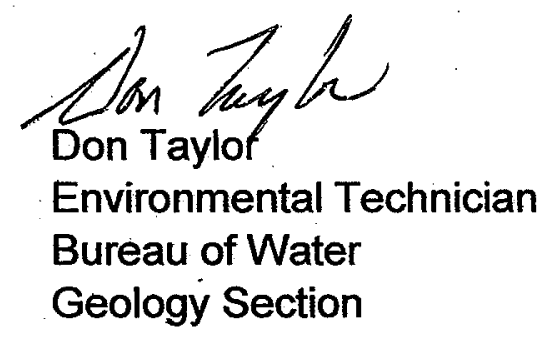

DT:jf

cc: Harper

North East District Office 


\section{Flush-Mount Well Head Completion:}

K.A.R 28-30-6(e) does not allow well casing to be terminated less than one foot above finished ground surface. Because state trust fund site investigations are often conducted in areas where completing monitoring well heads above grade is not practical, consideration must be given to completing flush-mount monitoring well heads.

If monitoring wells must be completed with a flush-mount well head design, a waiver of K.A.R. 28-30-6(e) must be requested in writing. The procedures for requesting a waiver of this regulation are described as follows:

1. Prior to the monitoring well installation, the written request must be submitted to Mr. Don Taylor at the address indicated below.

2. The request must contain the following information:

a. Facility name and street address;

b. Legal description of the property where the wells are proposed to be located; $1 / 4 \quad 1 / 4 \quad 1 / 4$ Sec. Town. Range

c. Number of wells to be installed with flush-mount well heads;

d. Reason(s) why the regulation should be waived;

e. Approximate depth to groundwater in the local area;

f. The general geology or lithologies expected to be encountered in drilling; and

g. Specifications and/or diagrams of the vault proposed to be installed including the manufacturer's name and any other descriptive information such as a manufacturer's trade sheet.

3. Wait for approval of the waiver request before completing monitoring wells.

4. When waivers are approved and monitoring wells are installed with a flush-mount well head design, the well head completion must be indicated accordingly in the lithologic section of the WWC-5 water well record form. The name of the KDHE contact person that approved the waiver must also be provided in the lithologic section of the WWC-5 form.

5. Kansas licensed water well contractor and number.

Any waiver of regulations applies only to the wells and information indicated in the written request. A verbal request for waiver of regulations may be approved on any additional wells needed for the same area or site. The verbal request must be directed to Mr. Don Taylor.

Monitoring Well Grouting Requirements:

K.A.R. 28-30-6, part (b) requires that constructed or reconstructed wells be sealed by grouting the annular space between the casing and the well bore from ground level to a minimum of 20 feet or to a minimum of five feet into the first clay layer, whichever is greater. Part (c) of the same regulation specifies if groundwater is encountered at a depth less than the minimum grouting requirements, the grouting requirement may be modified to meet local conditions if approved by the department.

If modifications to the grouting requirements are necessary solely because of shallow groundwater, a waiver of the regulations is not needed; however, the reason for modifying the grouting requirements must be indicated accordingly on the WWC-5 water well record form. In situations where grouting modifications are required for reasons other than shallow groundwater, a waive of K.A.R. 28-30-6(b) must be obtained following the same procedures as described for flush-mount well heads above.

Submit requests for waivers and direct any questions on well design regulations to:

Mr. Don Taylor

Kansas Department of Health \& Environment

Bureau of Water - Geology Section

1000 S.W. Jackson Street, Suite \#420

Topeka, Kansas 66612-1367

Phone: (785) 296-5522 


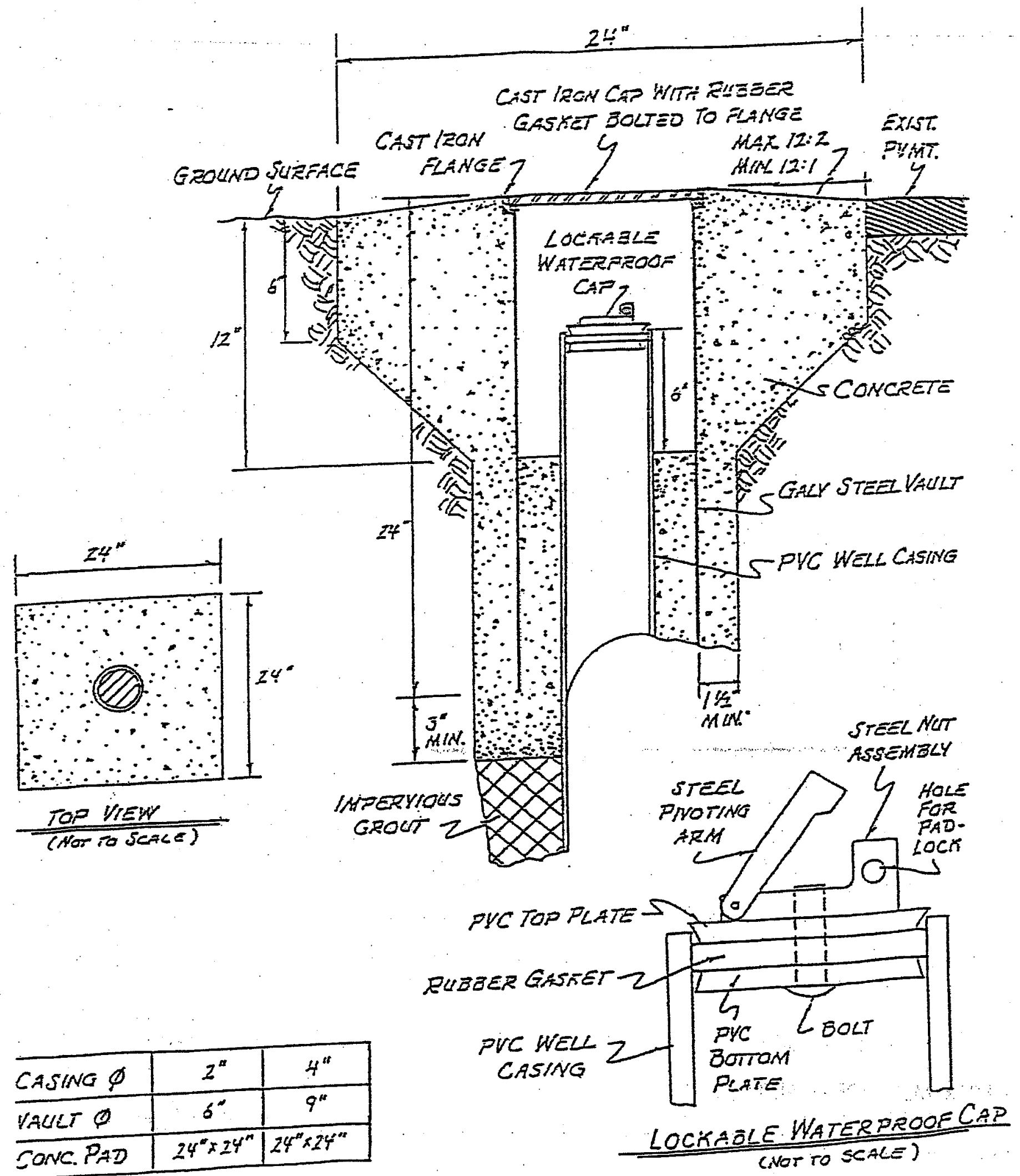

$10, r \div 3$ 
WATER WELL RECORD Form WWC-5 KSA 82a-1212 ID No. PMP-1

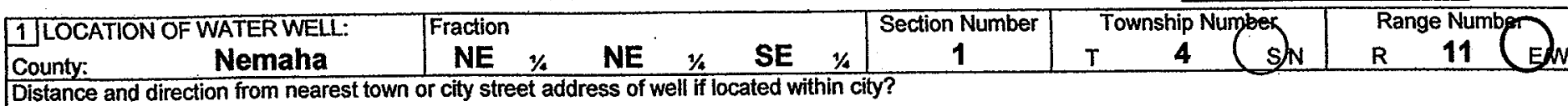

\section{WATER WELL OWNER: USDA/CCC}

RR\#, St. Address, Box\# : Stop 0513, Room 4717-S/ 1400 Independence Ave SW Board of Agriculture, Division of Water Resources City, State, ZIP Code : Washington, DC 20250-0513

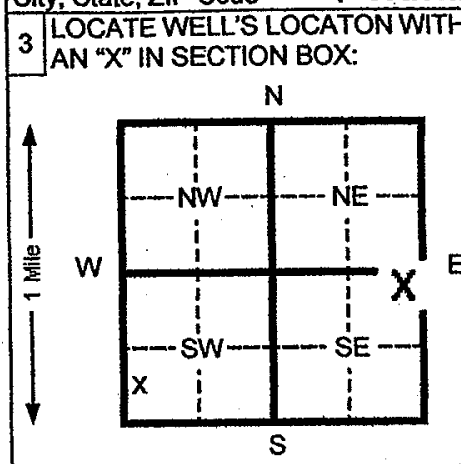

5 TYPE OF BLANK CASING USED:
1 Steel
3 RMP (SR)
(2) PVC
4 ABS

Blank casing diameter $1 / 2 "$ in. to 60 ........ Dia .... N/A

4 DEPTH OF COMPLETED WELL

Depth(s) Groundwater Encountered

WELL'S STATIC WATER LEVEL

Pump test data: Well water was

Est. Yield N.N/A gpm: Well water was

E Bore Hole Diameter 4.25 in. to

WELL WATER TO BE ÜSÉĒ ĀS: 5 Püblic water supply

1 Domestic 3 Feed lot 6 Oil field water supply

2 Irrigation 4 Industrial

submitted N/A

Application Number.

Blank casing diameter ...1/2" in. to 60 ft., Dia ... N/A

in. to 7 Fiberglass

60 ft. Elevation:

ft. 2

Nì̈

$1,334.40$

ft. $3 \ldots$ N/A ft. N/A ft. after N/A hours pumping N/A gpm
N/A las NiA ft. after N N $/ \bar{A}$ hours pumping N/A gpm N/A _... ft. after N/A hours pumping $-60 \mathrm{gpm}$ 8 Air conditioning

in Injection weil

9 Dewatering

(1) Monitoring well

2) Other (Specify below)

Sand Point MW

Casing height above land surface Flush Mount in., weight

TYPE OF SCREEN OR PERFORATION MATERIAL:
1 Steel
3 Stainless steel
5 Fiberglass
6 Concrete tile

\section{Brass}
4 Galvanized steel

Sche No $X$ If

If yes, mo/day/yr sample was Water Well Disinfected? Yes No $X$

SCREEN OR PERFORATION OPRTINGS ARE:

1 Continuous slot

2 Louvered shutter

3. Mill slot

4 Key punched
5 Gauzed wrapped

6 Wire wrapped

7 Torch cut
8 Concrete tile

9 Other (specify below)

A
CASING JOINTS: Glued

Clamped

Welded

Threaded

NIA ded
$\overline{\mathrm{X}}$

SCREEN-PERFORATED INTERVALS: From 50 ft: to 60

GRAVEL PACK INTERVALS: From .......48 ft. to

ft. to 60

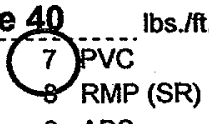

(7) 7 RMP (SR) ft., Dia

\section{Asbestos-cement \\ 11 Other (specify)}

12 None used (open hole)

8 Saw cut
9 Drilled holes
10 Other (specify)

11 None (open hole)

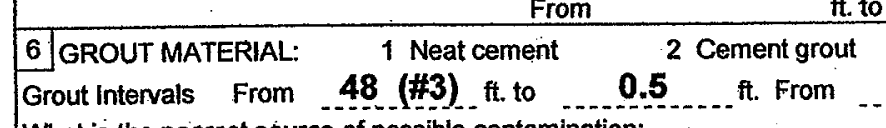

What is the nearest source of possible contamination:
4 Septictank
2 Sewer lines
3. Watertight sewer lines
$\begin{array}{ll}4 & \text { Lateral lines } \\ 5 & \text { Cess pool } \\ 6 & \text { Seepage pit }\end{array}$

ft. From

ft. From

ft. to

ft. to

ft. to

ft. From
$109^{3}$ ft. 


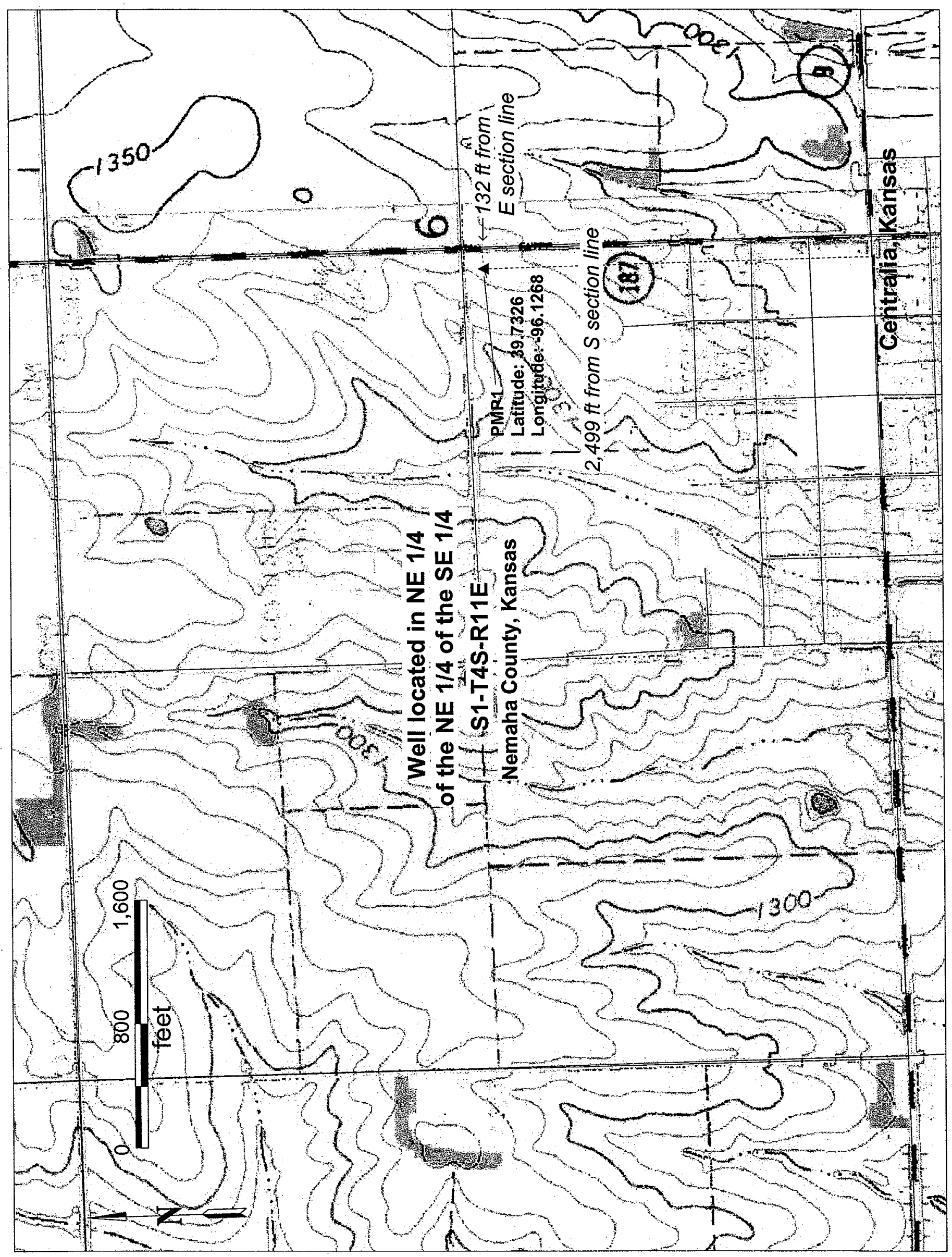


WATER WELL RECORD Form WWC-5 KSA 82a-1212 ID No. PMP-2

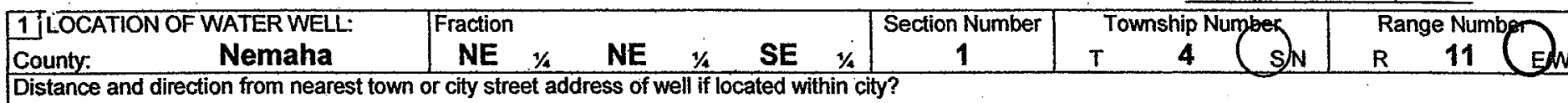

\section{WATER WELL OWNER: USDAICCC}

RR\#, St. Address, Box\# : Stop 0513, Room 4717-S/ 1400 Independence Ave SW

City, State, ZIP Code : Washington, DC 20250-0513

3 LOCATE WELL'S LOCATON WITH 4

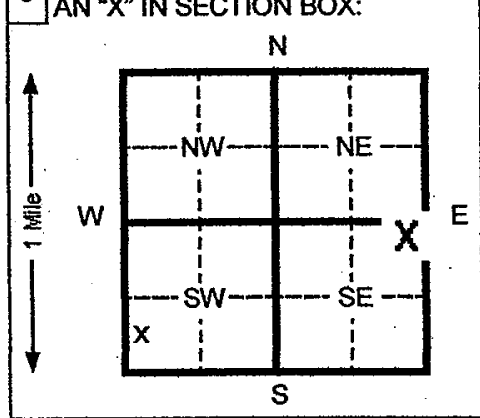

5 TYPE OF BLANK CASING USED:
1 Steel
3 RMP (SR)
(2) PVC
4 ABS

Blank casing diameter $\ldots 1 / 2 "$ in, to 60 ft. Dia

4 DEPTH OF COMPLETED WELL.

Depth(s) Groundwater Encountered

WELL'S STATIC WATER LEVEL

Pump test data: Well water was

Est. Yield N/A gpm: Well water was

Bore Hole Diameter 4.25 in to
WELL WATER TO BE USEDAS: 5 Pülic water supply

1 Domestic 3 Feed lot 6 Oil field water supply

2 Irrigation 4 Industrial

7 Lawn and garden (domestic)

Was a chemicalbacteriological sample submitted to Department? Yes submitted N/A
Water Well Disinfected? Yes

Board of Agriculture,
Application Number:

Applicatit

\section{$1,334.33$}
5 Wrought iron
8 Concrete tile
9 Other (specify below)
6 Asbestos-Cement

A. in. to N/A ft. Dia

CASING JOINTS: Glued

Welded.

Threaded

N/A
Casing height above land surface Flush Mount
TYPE OF SCREEN OR PERFORATION MATERIAL:
1 . Steel
3 Stainless steel
4 Galvanized steel

2 Brass

SCREEN OR PERFORATION OPZIVINGS ARE:
1 Continuous slot
2 Louvered shutter
3 Mill slot
4 Key punched

5 Fiberglass

6 Concrete tile

5 Gauzed wrapped

6 Wire wrapped

7 Torch cut

SCREEN-PERFORATED INTERVALS: From

50 ft. to

From

GRAVEL PACK INTERVALS:

From $48^{-1}$ ft. to

1 Neat cement

2 Cement grout

6 GROUT MATERIAL:

From

Grout Intervals From 48 (\#3) ft. to

What is the nearest source of possible contamination:
4 Septic tank
2 Sewer lines
3 Watertight sewer lines
4 Lateral lines
5 Cess pool
6 Seepage pit

ft. From

to 60

$40.1 \mathrm{~d}$.Ift.
7 RVC
RMP (SR)

9 ABS all thickness or gauge No.

10 Asbestos-cement

11 Other (specify)

12 None used (open hole)

8 Saw cut

9 Drilled holes

10 Other (specify)
11 None (open hole)

ft. From

ft. From

ft. From

ft. From
......... (n)

2 Other (Specify below)

Sand Point MW

$09 / 23108$ 3
0

Direction from well?

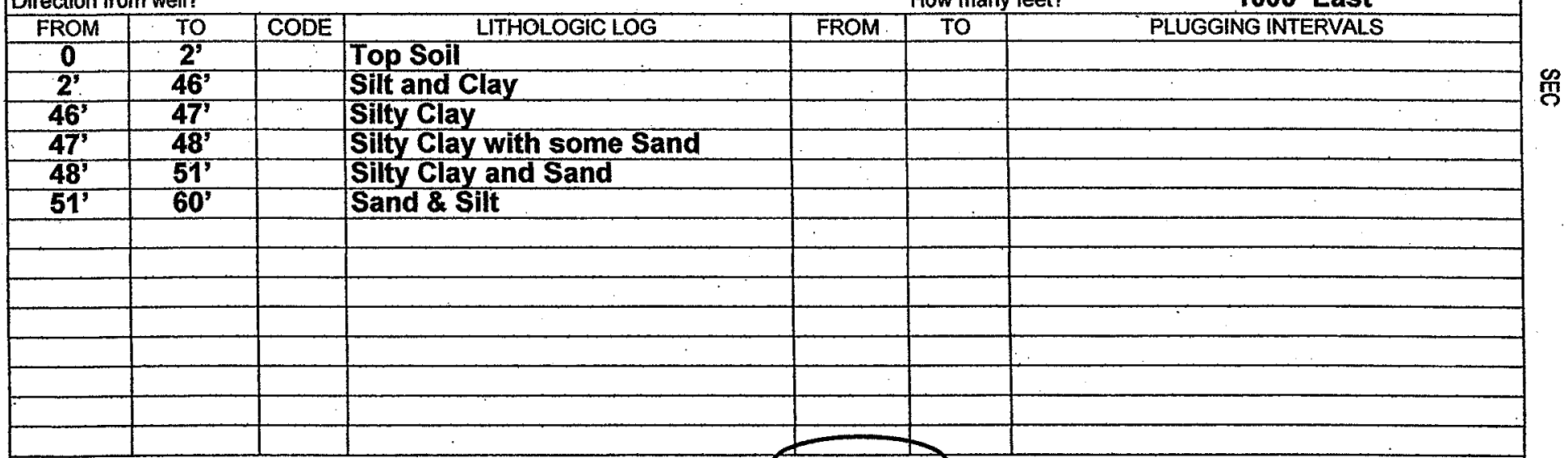

7 CONTRACTOR'S OR LANDOWNER'S CERTIFICATION: This water well wa (1) constructed (2) reconstructed, or (3) plugged under my jurisdiction and was completed on (mo/day/yr) $\quad 09 / 23 / 08$

Water Well Contractor's License No.

under the business name of
7 Pit privy
8 Sewage lagoon
8 Feedyard

3 Bentonite 4

Other BenSeal Chips No $X$

Clamped 


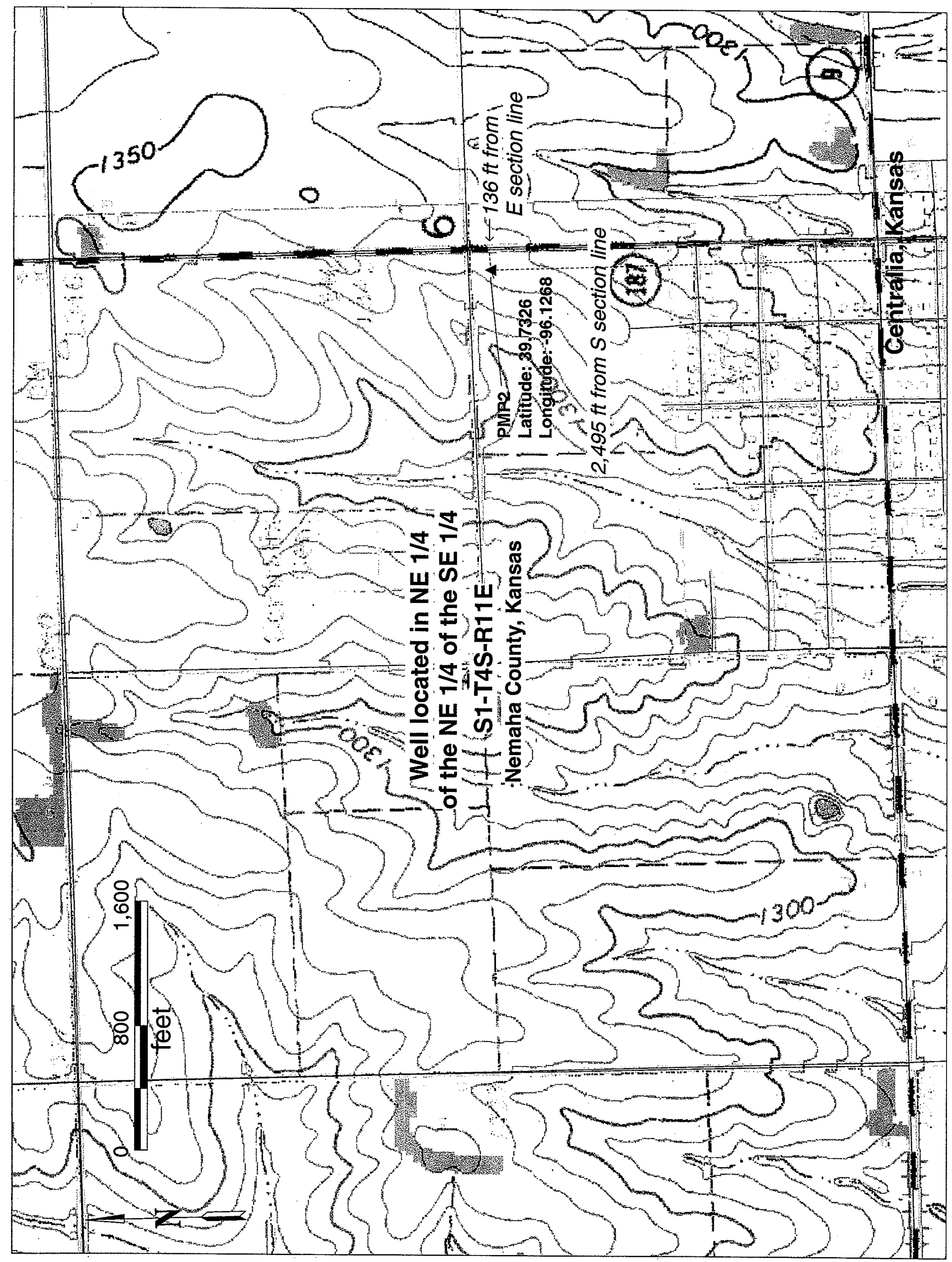


WATER WELL RECORD Form WWC-5 KSA 82a-1212 ID No. PMP-3

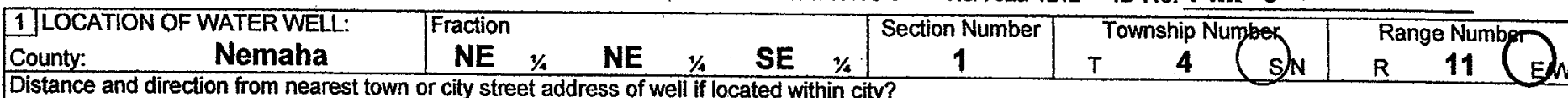

2 WATER WELL OWNER: USDAVCC

RR\#, St. Address, Box \# : Stop 0513, Room 4717-S/ 1400 Independence Ave SW City, State, ZIP Code : Washington, DC 20250-0513

\begin{tabular}{l|l|}
\hline 3 & LOCATE WELL'S LOCATON WITH \\
AN “ $X^{n}$ IN SECTION BOX:
\end{tabular}

Board of Agriculture, Division of Water Resources Application Number:

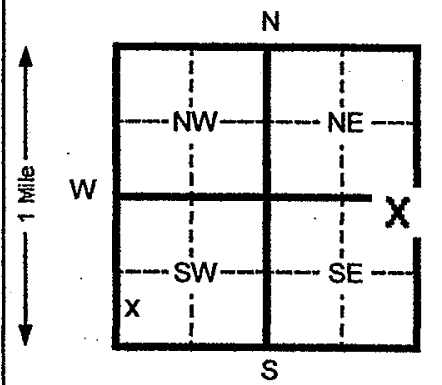

5 TYPE OF BLANK CASING USED:
(2) PvC
3 RMP (SR)
4 ABS

Blank casing diameter $\ldots .1 / 23$ in. to

TYPE OF SCREEN OR PERFORATION MATERIAL:
1 Steel
2 Brass
3 Stainless steel
4 Galvanized steel

1 Continuous slot

2 Louvered shutter

(3) Mill slot

4 Key punched
SCREEN OR PERFORATION OPETIIYGS ARE:
DEPTH OF COMPLETED WELL $\quad \mathbf{6 0}$. ft. ELEVATION: 51 f. 2
$1,335.26$ NIA 21.1 t. below land surface measured on mo/day/yr $09 / 23 / 08$

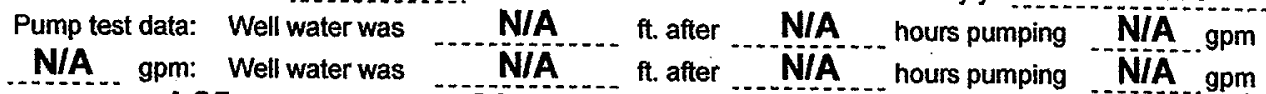
21 . t. after $1 .-25$ Pilot in to Bore Hole Diameter 4.25 in. to
WELL WATER TO BE USEED ÁS: 5 Püblic water supply 1 Domestic 3 Feed lot 6 Oil field water supply 9 Dewatering

2 Irrigation 4 industrial 7 Lawn and garden (domestic) (1) Monitoring well

2) Other (Specify below) Sand Point MW No $X$ If yes, mo/day/yr sample was Water Well Disinfected? Yes No $X$
5 Wrought Iron
8 Concrete tile
6 Asbestos-Cement 9 Other (specify below)
7 Fiberglass

60 fi., Dia

$\mathbf{N} / \mathbf{A}_{\text {in, to }} \mathbf{N} / \mathbf{A}$ ft., Dia

CASING JOINTS: Glued ....... Clamped

SCREEN-PERFORATED INTERVALS:

GRAVEL PACK INTERVALS:

From
From
From
From

5 Fiberglass

6 Concrete tile

5 Gauzed wrapped

6 Wire wrapped

7 Torch cut

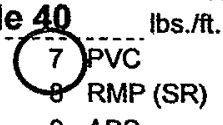

7 PVC
RMP (SR)

9 ABS
N/A$$
\text { Welded }
$$

Threaded
10 Asbestos-cement

12 None used (open hole)

8 Saw cut

9 Drilled holes

10 Other (specify)
11 None (open hole)

60 ft. From

ft. From

ft. From

ft. From ft. to to
ft. to
ft. to

\section{GROUT MATERIAL: \\ 1 Neat cement} 2 Cement grout
$0.5 \mathrm{ft}$. From 0.5 ft. From 60 to : 48 (\#4) 3 Bentonite 45 Other Ben What is the nearest source of possible contamination:
4 Septic tank $>$
2 Sewer lines
3 Watertight sewer lines

\begin{abstract}
4 Lateral lines
5 . Cess pool

6 Seepage pit
\end{abstract}

7 Pit privy

8 Sewage lagoon

9 Feedyard
10 Livestock pens

11 Fuel storage

12 Fertilizer storage

13 Insecticide storage

How many feet?

Direction from well?

\begin{tabular}{|c|c|c|c|c|c|c|}
\hline \multicolumn{5}{|c|}{ Direction from well? } & \multirow{2}{*}{\multicolumn{2}{|c|}{$\begin{array}{l}\text { How many feet? } \\
\text { TO }\end{array}$}} \\
\hline FROM & TO & CODE & LITHOLOGIC LOG & FROM & & \\
\hline 0 & $2^{\prime}$ & & Top Soll & & & \\
\hline $2^{5}$ & $46^{\prime}$ & & Silt and Clay & & & \\
\hline $46^{3}$ & $47^{\prime}$ & & Silty Clay & & & \\
\hline $47^{\prime}$ & $48^{3}$ & & Silty Clay with some Sand & & & \\
\hline $48^{5}$ & 51 & & Silty Clay and Sand & & & \\
\hline $51^{3}$ & $60^{\prime}$ & & Sand \& Silt & & & \\
\hline & & & & & & \\
\hline & & & & & & \\
\hline & & & . & & & \\
\hline & & & & & & \\
\hline & & & & & & \\
\hline & & & & & & \\
\hline & & & & & & \\
\hline & & & & & & \\
\hline
\end{tabular}

7 CONTRACTOR'S OR LANDOWNER'S CERTIFICATION: This water well wa (1) constructed 2 (2) reconstructed, or (3) plugged under my jurisdiction and was completed on (mo/day/yr)

Water Well Contractor's License No. 680

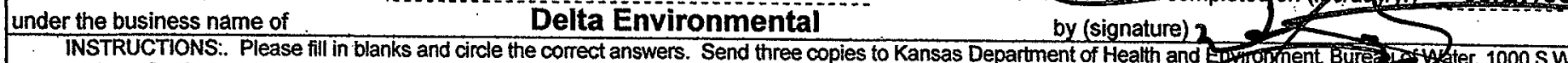
INSTRUCTIONS: Please fill in blanks and circle the correct Enswers. Send three copies to Kansas Department of Heatth and to bela (signature) and this record is true to the bestormy knowledgeg filef. Kansas Jackson St., Ste. 420, Topeka, Kansas 66612-1367. Telephone: 913-296-5545. Send one to WATER WELL OWNER and re eain one for your recof rds. 


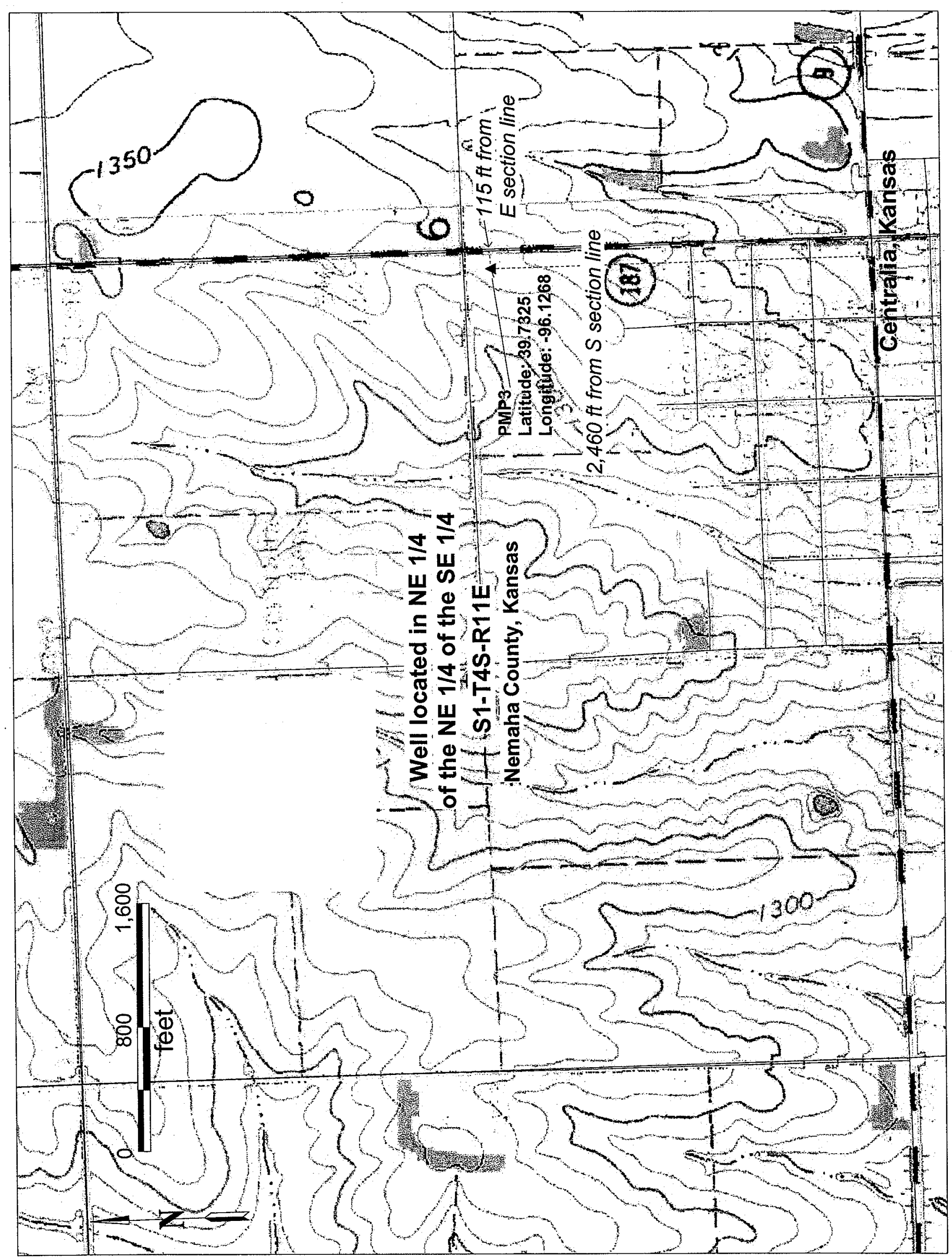


WATER WELL RECORD Form WWG-5 KSA 82a-1212 ID No. PMP-4

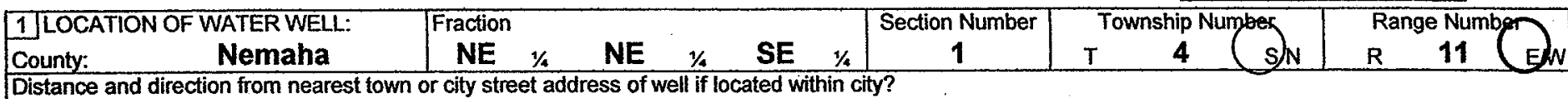

\section{WATER WELL OWNER: USDA/CCC}

RR\#, St. Address, Box\# . : Stop 0513, Room 4717-S/ 1400 Independence Ave SW Board of Agriculture, Division of Water Resources City, State, ZIP Code : Washington, DC 20250-0513

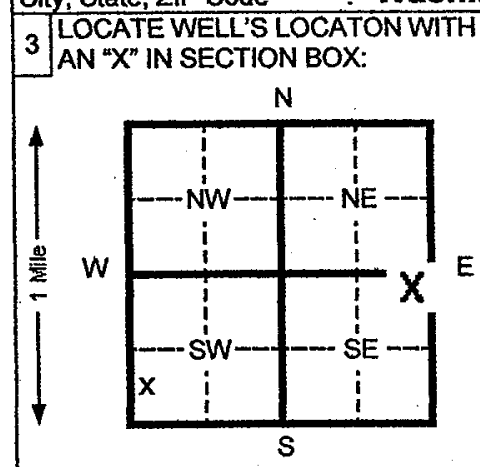

5 TYPE OF BLANK CASING USED:

$$
\begin{array}{ll}
1 \text { Steel } & 3 \mathrm{RMP} \text { (SR) } \\
\text { (2) PVC } & 4 \mathrm{ABS}
\end{array}
$$

Blank casing diameter

in. to

4 DEPTH OF COMPLETED WELL

Depth(s) Groundwater Encountered 1

WELL'S STATIC WATER LEVEL

Pump test data: Well water was

Est. Yield ..N/A gpm: Well water was

E Bore Hole Diameter 4.25 in. to WELL WATER TO BE USEAD ÄS.:
1 Domestic 3 Feed lot

2 Irrigation 4 Industrial submitted N/A
58.75 ft. ELEVATION: 51

ft. 2
Application Number:

$1,332.78$

NiA $\mathrm{At.} 3 \mathrm{~N} / \mathrm{A}$

ft. below land surface measured on mo/day/yr

$09 / 23 / 08$

$\mathbf{N} / \mathbf{A}$. ft. after ... N/A hours pumping . N/A. $\mathrm{gpm}$ NiA ft. after NiA hours pumping N/A gpm 21

ft. and 3.25 Pilot in. to

$60^{f t}$

8 Air conditioning

9 Dewatering

it Injection weil

(2) Other (Specify below)

6 Oil field water supply

(1) Monitoring well

Sand Point MW

Casing height above land surface Flush Mount in., weight Schedule 40

TYPE OF SCREEN OR PERFORATION MATERIAL:
1 Steel
3 Stainless steel
2. Brass
4 Galvanized steel

5 Fiberglass

6 Concrete tile

5 Wrought Iron 8 Concrete tile

Water Well Disinfected? Yes

No $X$

6 Asbestos-Cement 9 Other (specify below)

7 Fiberglass

CASING JOINTS: Glued

Clamped

SCREEN OR PERFORATION OPRTIYGS ARE:

1 Continuous slot

2 Louvered shutter

(3) Mill slot

4 Key punched
5 Gauzed wrapped

6 Wire wrapped

7 Torch cut

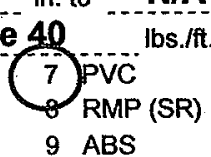

7 PVC
9 RMP (SR)
9 ABS

SCREEN-PERFORATED INTERVALS: From

GRAVEL PACK INTERVALS: From .......47.... ft. to

58.75

8 Saw cut

9 Drilled holes

10 Other (specify)

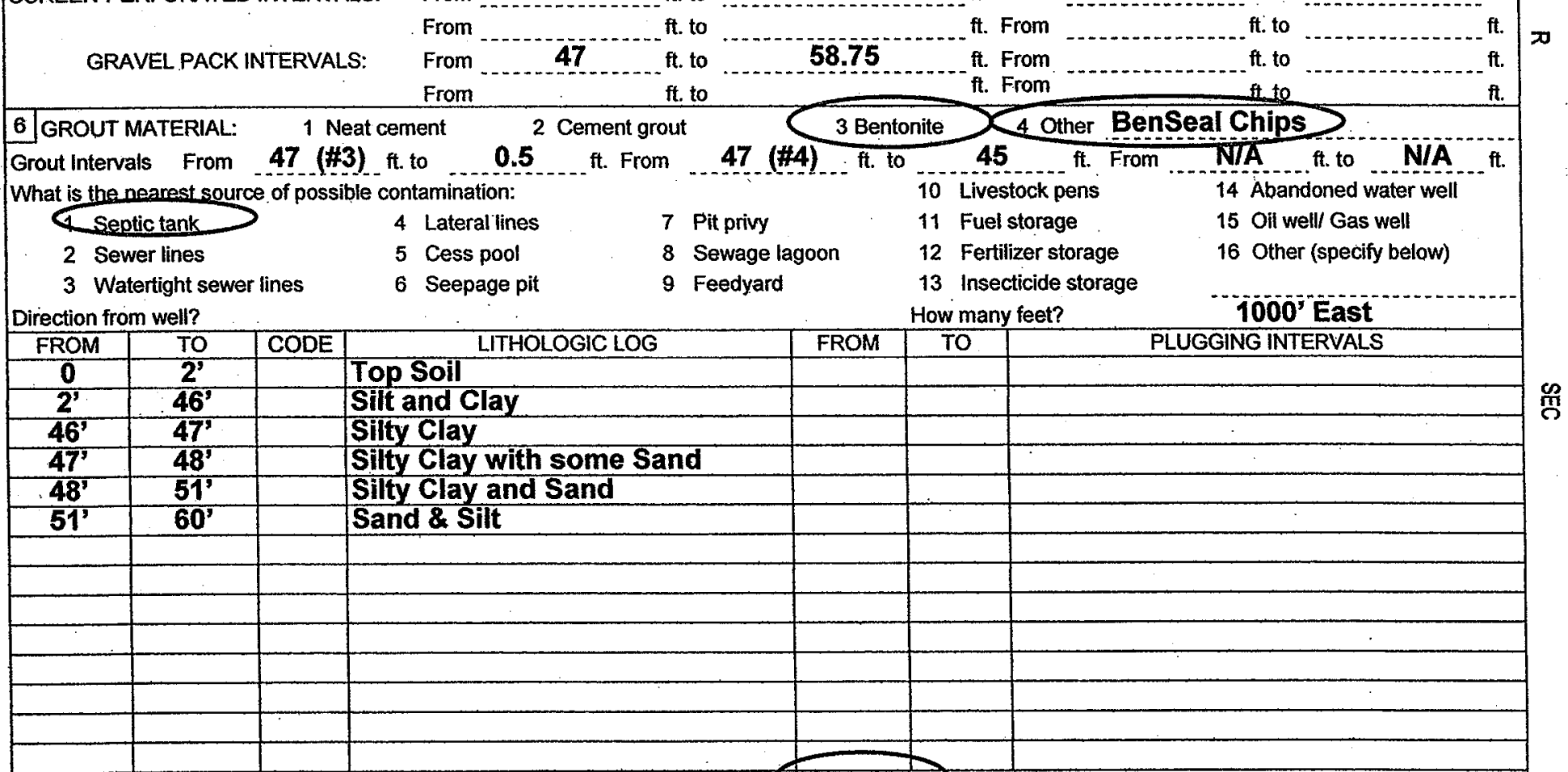

7 CONTRACTOR'S OR LANDOWNER'S CERTIFICATION: This water well wa (1) constructed, (2) reconstructed, or (3) plugged under my jurisdiction and was completed on (mo/day/yr) ...... 09/23/08 Water Well Contractor's License No. and this record is true to the best of my knowledgen pelief. Kansas under the business name of 


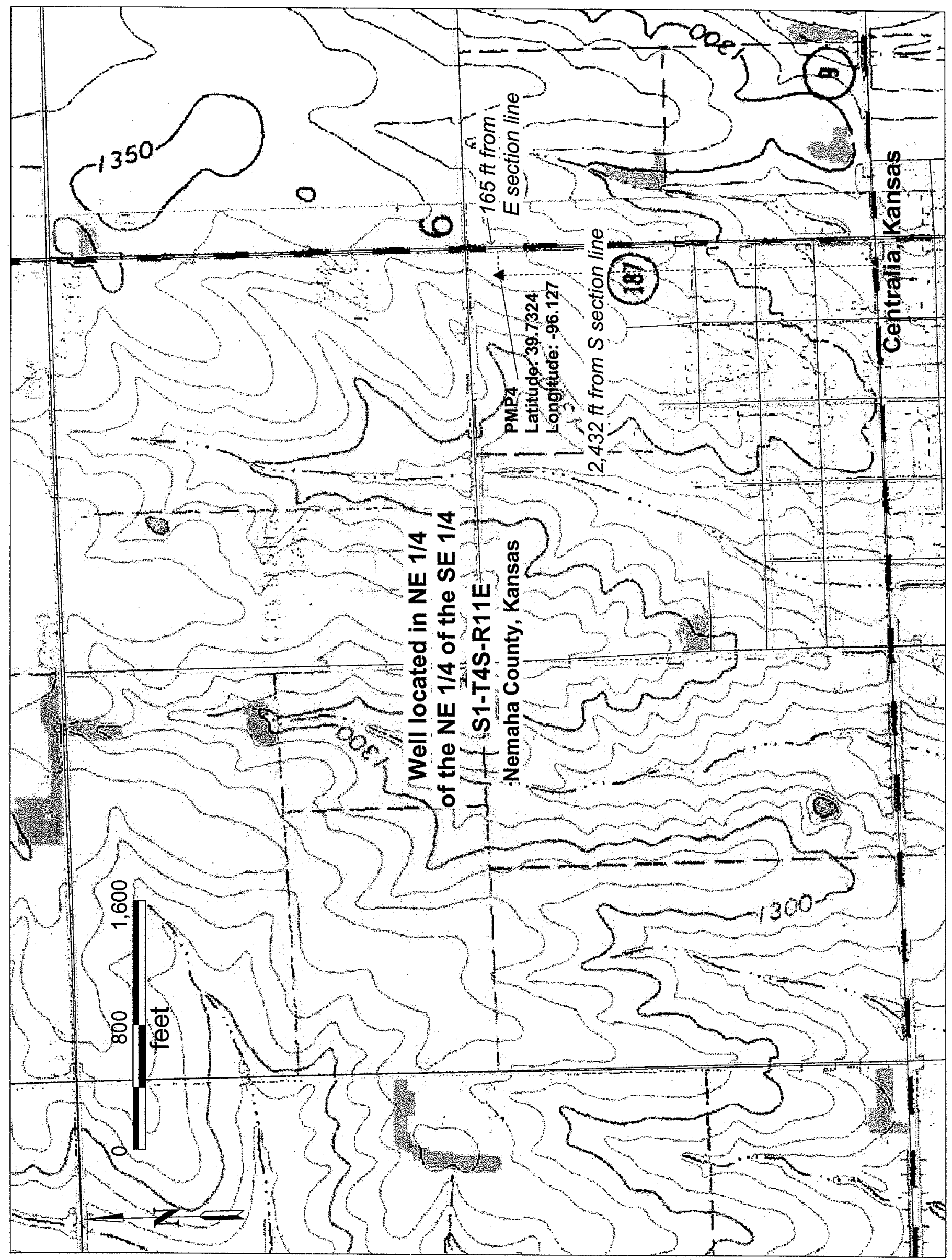


WATER WELl RECORD Form WWC-5 KSA 82a-1212 ID No. PMP-5

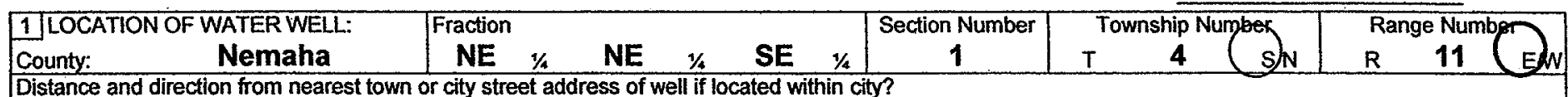

2 WATER WELL OWNER: USDAJCCC

RR\#, St. Address, Box\# : Stop 0513, Room 4717-S/ 1400 Independence Ave SW Board of Agriculture, Division of Water Resources City, State, ZIP Code : Washington, DC 20250-0513

3 LOCATE WELL'S LOCATON WITH

3 AN " $X$ " IN SECTION BOX:

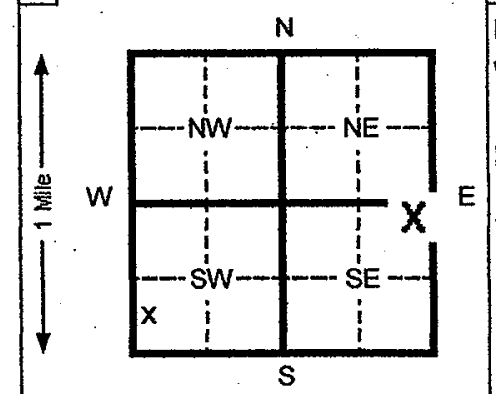

4 DEPTH OF COMPLETED WELL

Depth(s) Groundwater Encountered 1

60 ft. ELEVATION:

Application Number:

WELL'S STATIC WATER LEVEL

1.51

51 ft. 2. Est. Yield ..N/A gpm: Well water was ... N/A ... ft. after ... N/A hours pumping . N/A gpm Bore Hole Diameter 4.25 in. to 21 21 ft. and 3.25 Pilot in to

60

injection weil

8 Air conditionin

9 Dewatering

2. Other (Specify below)

1 Domestic 3 Feed lot 6 Oil field water supply

2 Irrigation 4 Industrial 7 Lawn and garden (domestic) 11 Monitoring well Sand Point MW Was a chemical/bacteriological sample submitted to Department? Yes ....... No . $X$... If yes, mo/day/yr sample was

5 TYPE OF BLANK CASING USED: submitted N/A

Water Well Disinfected? Yes

No $X$
1 Steel
3 RMP (SR)
5 Wrought Iron
8 Concrete tile
CASING JOINTS: Glued
Clamped ......
4 ABS
6. Asbestos-Cement 9 Other (specify below)
7 Fiberglass
Blank casing diameter 1 "'
in. to
60
ft. Dia
N/A in to N/A ft., Dia N/A
Welded
Threaded
NiA TYPE OF SCREEN OR PERFORATION MATERIAL:

1 Steel

2 Brass

3 Stainless steel

4 Galvanized steel

SCREEN OR PERFORATION OPZNINGS ARE:

1 Continuous slot

2 Louvered shutter

(3) Mill slot

4 Key punched

SCREEN-PERFORATED INTERVALS: From $\quad \mathbf{5 0} \quad \mathrm{ft}$ to 60 ft. From

GRAVEL PACK INTERVALS. From .......... $\mathrm{ft}$ to

5 Fiberglass

6 Concrete tile

5 Gauzed wrapped
6 Wire wrapped

7 Torch cut

Schedule 40 los./f. Wall thickness or gauge No.

11 Other (specify)

12 None used (open hole)

$.113^{\prime \prime}$

8 Saw cut

11 None (open hole)

9 Drilled holes

10 Other (specify)

ft. to

GRAVEL PACK INTERVALS: $\quad$ From .......48

From

6 GROUT MATERIAL:

1 Neat cement

2 Cement grout

Grout intervals From $48(\# 3)$ ft. to 0.5 ft: From

What is the nearest source of possible contamination:

$\begin{array}{ll}4 \text { Septic tank } & 4 \text { Lateral lines } \\ 2 \text { Sewer lines } & 5 \text { Cess pool } \\ 3 \text { Watertight sewer lines } & 6 \text { Seepage pit }\end{array}$

3 Watertight sewer lines

6 Seepage pit

to

60

ft. From

ft. From

ft. From

ft. From

3 Bentonite 4 Other BenSeal Chips

fit. to

ft. to

Direction from well?

\begin{tabular}{|c|c|c|l|l|l|}
\hline FROM & TO & CODE & \multicolumn{1}{|c|}{ LITHOLOGIC LOG } & FROM & \\
\hline 0 & $2^{\prime}$ & & & \\
\hline $2^{\prime}$ & $46^{\prime}$ & Top Soll & & \\
\hline $46^{\prime}$ & $47^{\prime}$ & Silt and Clay & & \\
\hline $47^{\prime}$ & $48^{\prime}$ & Silty Clay & & \\
\hline $48^{\prime}$ & $5^{\prime}$ & Silty Clay with some Sand & & \\
\hline $51^{\prime}$ & $60^{\prime}$ & Silty Clay and Sand & & \\
\hline & & Sand \& Silt & & \\
\hline & & & & & \\
\hline & & & & & \\
\hline & & & & & \\
\hline & & & & & \\
\hline
\end{tabular}

$\begin{array}{llll}7 & \text { Pit privy } & 11 & \text { Fuel storage } \\ 8 & \text { Sewage lagoon } & 12 & \text { Fertilizer storage } \\ 9 & \text { Feedyard } & 13 \text { Insecticide storage }\end{array}$

How many feet?

\begin{abstract}
45 ft. From .... N/A ft to N/A
15 Oil well/ Gas well

16 Other (specify below)
\end{abstract}

1000 'East

7 CONTRACTOR'S OR LANDOWNER'S CERTIFICATION: This water well wa (1) constructed, (2) reconstructed, or (3) plugged under my jurisdiction and was completed on (mo/day/yr)

Water Well Contractor's License No. 680 under the business name of

Delta Environmental and this record is the best of my knowle ons INSTRUCTIONS: Please fill in blanks and circle the correct answers. Send three copies to Kansas Department of Health artentirctiment, Bure of of Water, Joco SW Jackson St. Ste. 420, Topeka, Kansas 66612-1367. Telephone: 913-296-5545. Send one to WATER WELL OWNER and setaintynetoryenty 


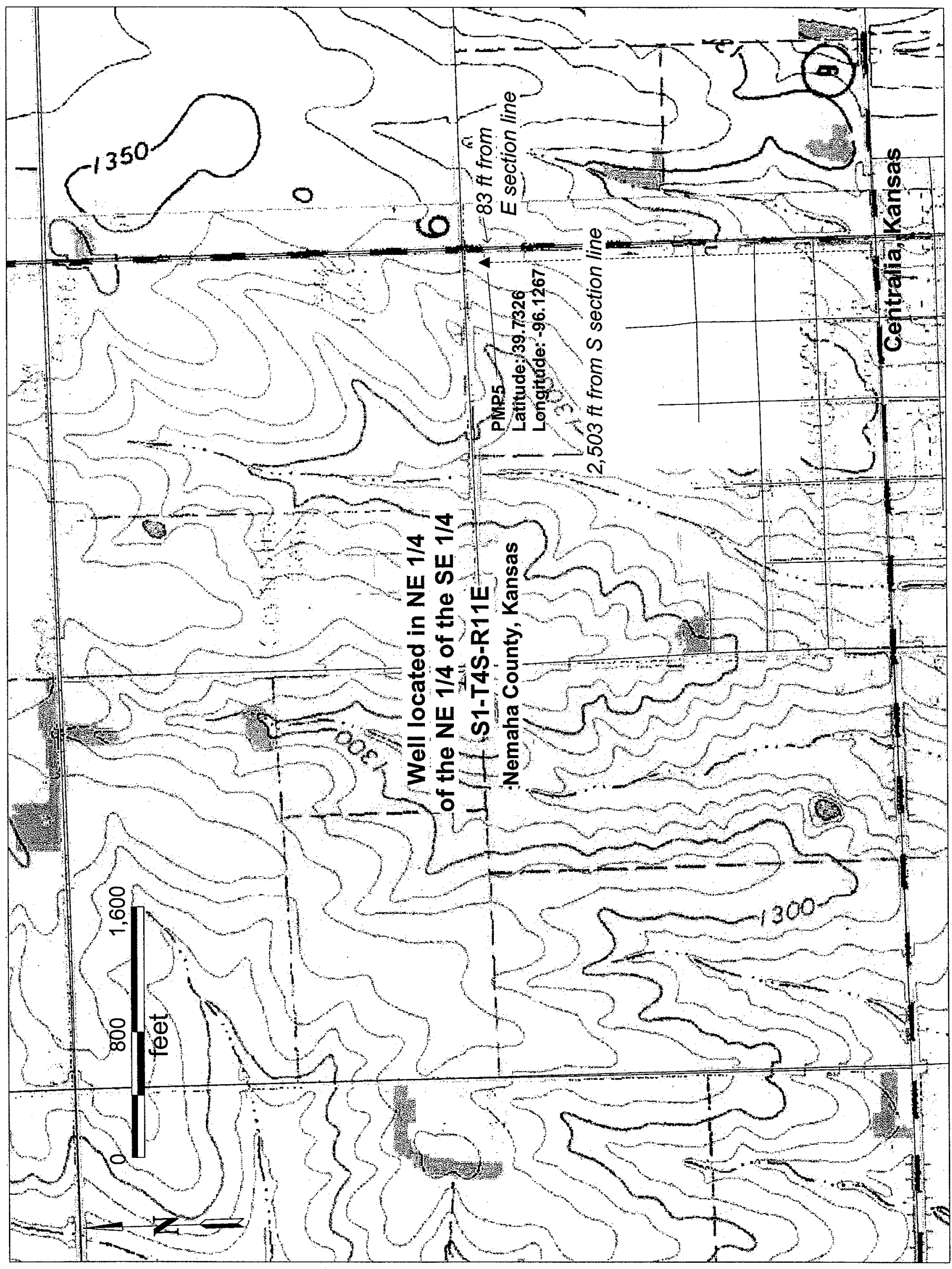


WATER WELL RECORD Form WWC-5 KSA 82a-1212 ID No. PMP-6

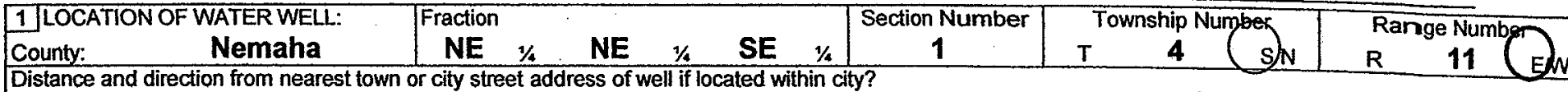

\section{WATER WELL OWNER: USDA/CCC}

RR\#, St. Address, Box\# : Stop 0513, Room 4717-S/ 1400 Independence Ave SW City, State, ZIP Code : Washington, DC 20250-0513

3 LOCATE WELL'S LOCATON WITH 4

3 AN " $X^{n}$ IN SECTION BOX:

$\mathbf{N}$

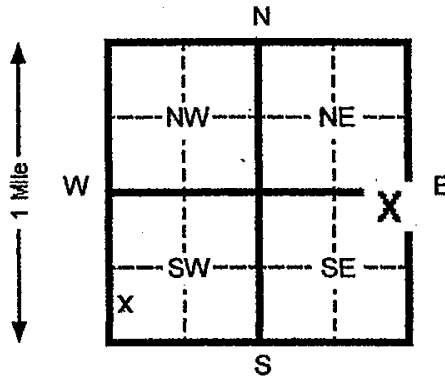

4 DEPTH OF COMPLETED WELL

60

ft. ELEVATION:

Application Number:

Depth(s) Groundwater Encountered $1 \ldots \ldots .51$ ft. 2

NiA $1,335.19$

Est. Yield ...N/A .. gpm: Well water was .... N/A ... ft. after .... N/A hours pumping E Bore Hole Diameter. 4.25 in to 21 ft. and 3.25 Pilot

WELL WATER TO BE ÜSËDĀS: 5 Public water supply $\quad 8$ Air conditioning

1 Domestic 3 Feed lot 6 Oil field water supply

9 Aewatering

2 Irrigation 4 Industrial 7 Lawn and garden (domestic) (1) Monitoring well

injection weil

$60^{-10.98 m}$

2) Other (Specify below)

Sand Point MW a chemical/bacteriological sample submitted to Department? Yes ...... No . X If yes, mo/day/yr sample was submitted $\mathbf{N} / \mathbf{A}$

5 TYPE OF BLANK CASING USED:
1 Steel
3 RMP (SR)
(2) PVC
4 ABS

Blank casing diameter 1/2" in.
5 Wrought Iron
7 Fiberglass

8 Concrete tile

Water

6 Asbestos-Cement 9 Other (specify below)

\section{0}

ft., Dia N/A

N/A in to N/A ft. Dia

CASING JOINTS: Glued

No $X$ TYPE OF SCREEN OR PERFORATION MATERIAL:
1 Steel
2 Brass
3 Stainless steel
4 Galvanized steel

SCREEN OR PERFORATION OPRIIYGS ARE:

1 Continuous slot

2 Louvered shutter

3 Mill slot
4 Key punched

\section{Fiberglass}

6 Concrete tile

5 Gauzed wrapped

6 Wire wrapped

7 Torch cut

SCREEN-PERFORATED INTERVALS: From ........50 ...... ft. to

GRAVEL PACK INTERVALS:

From

From

60

60 ft. From

8 Saw cut

9 Drilled holes

10 Other (specify)

Clamped

Welded

Threaded $\overline{\mathbf{X}}$

ft. $109^{\prime \prime}$

\section{GROUT MATERIAL: \\ 1 Neat cement \\ 2 Cement grout}

From

60

ft. From

ft. From

ft. From

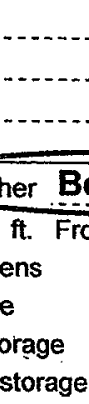

ft. From 45

10 Livestock pens

11 Fuel storage

12 Fertilizer storage

13 Insecticide storage How many feet?
11 None (open hole) ..
What is the nearestsource of possible contamination:

2 Sewer lines

3 Watertight sewer lines

Direction from well?

\author{
5 Cess pool
}

6 Seepage pit
7 Pit privy

8 Sewage lagoon

9 Feedyard

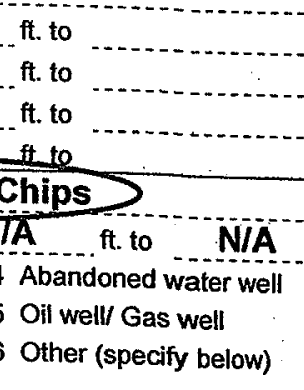

16 Other (specify below)

\begin{tabular}{|c|c|c|l|c|c|c|}
\hline FROM & TO & CODE & \multicolumn{1}{|c|}{ LITHOLOGIC LOG } & FROM & TO & PLUGGING INTERVALS \\
\hline 0 & $2^{\prime}$ & Top Soll & & & \\
\hline $2^{\prime}$ & $46^{\prime}$ & Silt and Clay & & & \\
\hline $46^{\prime}$ & $47^{\prime}$ & Silty Clay & & \\
\hline $47^{\prime}$ & $48^{\prime}$ & Silty Clay with some Sand & & & \\
\hline $48^{\prime}$ & $51^{\prime}$ & Silty Clay and Sand & & & \\
\hline $51^{\prime}$ & $60^{\prime}$ & Sand \& Silt & & & \\
\hline
\end{tabular}

7 CONTRACTOR'S OR LANDOWWER'S CERTIFICATION: This water well wa (1) constructed, 2 ) reconstructed, or (3) plugged under my jurisdiction and was completed on (mo/day/yr)

Water Well Contractor's License No.

under the business name of Jackson St, Ste. 420. Topeka, Kansas 66612-1367. Telephone: 913-296-5545. Send one to WATER WELL OWNER and retain one for you ecords.

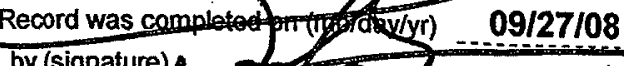
t. 


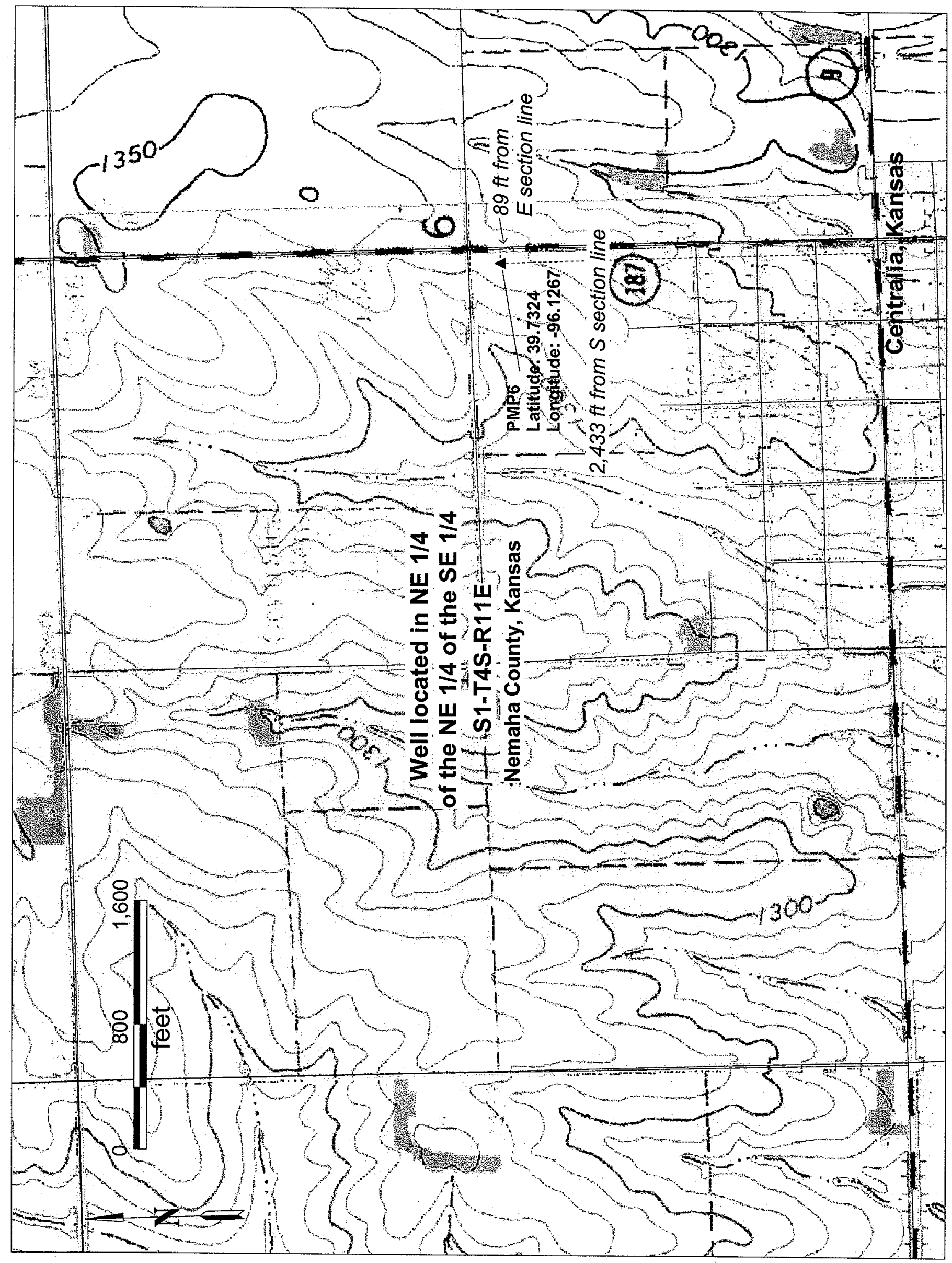


WATER WELL RECORD Form WWC-5 KSA 82a-1212 ID No. PMP-7

\begin{tabular}{|c|c|c|c|c|c|c|c|c|c|c|}
\hline $1 / \mathrm{LOCA}$ & WATER WELL: & Fraction & & & & & & Section Number & Township Nun & Range Nun \\
\hline County: & Nemaha & NE & $1 / 4$ & NE & $1 / 4$ & SE & $1 / 4$ & 1 & $T$ & \\
\hline
\end{tabular}

Distance and direction from nearest town or city street address of well if located within city?

2 WATER WELL OWNER: USDA/CCC

RR\#, St. Address, Box\# : Stop 0513, Room 4717-S/ 1400 Independence Ave SW Board of Agriculture, Division of Water Resources City, State, ZIP Code : Washington, DC 20250-0513

3 LOCATE WELL'S LOCATON WITH

3 AN " $X$ " IN SECTION BOX:

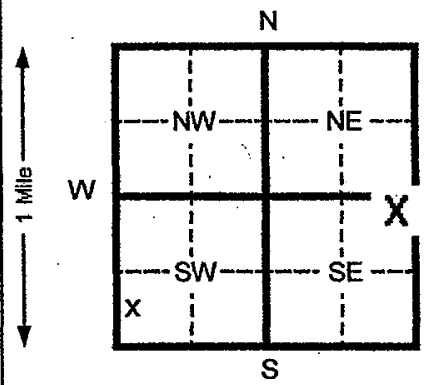
1 Steel
3 RMP (SR)
(2) PVC
4 ABS

Blank casing diameter 1/2"
4 DEPTH OF COMPLETED WELL

Depth(s) Groundwater Encountered

WELL'S STATIC WATER LEVEL

Pump test data: Well water was

Est. Yield N/A _. gpm: Well water was

E Bore Hole Diameter 4.25 in. to

WELL WATER TO BE ÜS̈̈Ē ĀS:

1 Domestic 3 Feed lot

2 Irrigation 4 industrial

6 Public water supply

7 Lawn and garden (dome

60

51

ft. ELEVATION:

ft. 2

NiA $\mathrm{ft.} 3$ N/A

N/A

Was a chemicai/bacteriological sample submitted to Department? Yes ....... No . X ... If yes, mo/day/yr sample was submitted N/A

Water Well Disinfected? Yes

No $X$

5 TYPE OF BLANK CASING USED:

Casing height above land surface Flush Mount in., weight

TYPE OF SCREEN OR PERFORATION MATERIAL:

1 Steel

2 Brass

3 Stainless steel

4 Galvanized steel

SCREEN OR PERFORATION OPRTINGS ARE:

1 Continuous slot

2 Louvered shutter

(3) Mill slot

4 Key punched

SCREEN-PERFORATED INTERVALS: From

GRAVEL PACK INTERVALS:

From

From

From

1. Neat cement

6 GROUT MATERIAL:

Grout Intervals From 48 (\#3) ft. to ... 0.5

What is the nearest source of possible contamination:

$\begin{array}{ll}4 \text { Septictank } & 4 \text { Lateral lines } \\ 2 \text { Sewer lines } & 5 \text { Cess pool } \\ 3 \text { Watertight sewer lines } & 6 \text { Seepage pit }\end{array}$

\section{Fiberglass}

6 Concrete tile

5 Gauzed wrapped

6 Wire wrapped

7 Torch cut
8 Concrete tile

9 Other (specify below)

/A
CASING JOINTS: Glued

Welded

Threaded
Clamped

Schedule 40

N/A ft., Dia N/A

d

3 Watertight sewer lines

6 Seepage pit

50 ft. to

60 ft. From

Ibs.ft. Wall thickness or gauge No.

10 Asbestos-cement

11 Other (specify)

12 None used (open hole)

N/A ft. 109

.109

8 Saw cut

11 None (open hole)

9 Drilled holes

10 Other (specify)

ft. to ft.

ft. to

ft. From

48 ft. to

60

ft. From

ft. to

2 Cement grout 3 Bentonite ft. From

ft. to

fi: to

fito

4 Other BenSeal Chips
7 Pit privy

8 Sewage lagoon

9 Feedyard 45

10 Livestock pens

11 Fuel storage

12 Fertilizer storage

13 Insecticide storage How many feet?
NIA ft. to $\mathbf{N} / \mathbf{A}$ ft.

14 Abandoned water well

15 Oil well/ Gas well

16 Other (specify below)

$1000^{3}$ East

Direction from well?

\begin{tabular}{|c|c|c|}
\hline FROM & TO & CODE \\
\hline 0 & 2 & \\
\hline $2^{3}$ & $46^{\prime}$ & \\
\hline $46^{5}$ & $47^{\prime}$ & \\
\hline $47^{5}$ & $48^{\prime}$ & \\
\hline $48^{3}$ & $51^{\prime}$ & \\
\hline $51^{\prime}$ & $60^{\prime}$ & \\
\hline
\end{tabular}

LITHOLOGIC LOG

Top Soil

Silt and Clay

Silty Clay

Silty Clay with some Sand

Silty Clay and Sand

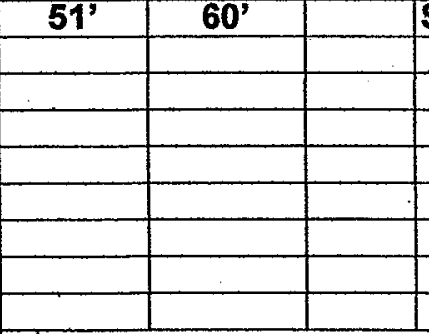
Sand \& Silt

7 CONTRACTOR'S OR LANDOWNER'S CERTIFICATION: This water well wa (1)

completed on (mo/day/yr)

Water Well Contractor's License No.

680

under the business name of

Delta Environmental and this record is true to the best of my knowly $1 \mathrm{~d}$ belief. Kansas INSTRUCTIONS: Please fill in blanks and circle the correct answers. Send three copies to Kansas Department of Health and) Jackson St., Ste. 420. Topeka, Kansas 66612-1367. Telephone: 913-296-5545. Send one to WATER WELL OWNER and re ain on for your reco.s. 


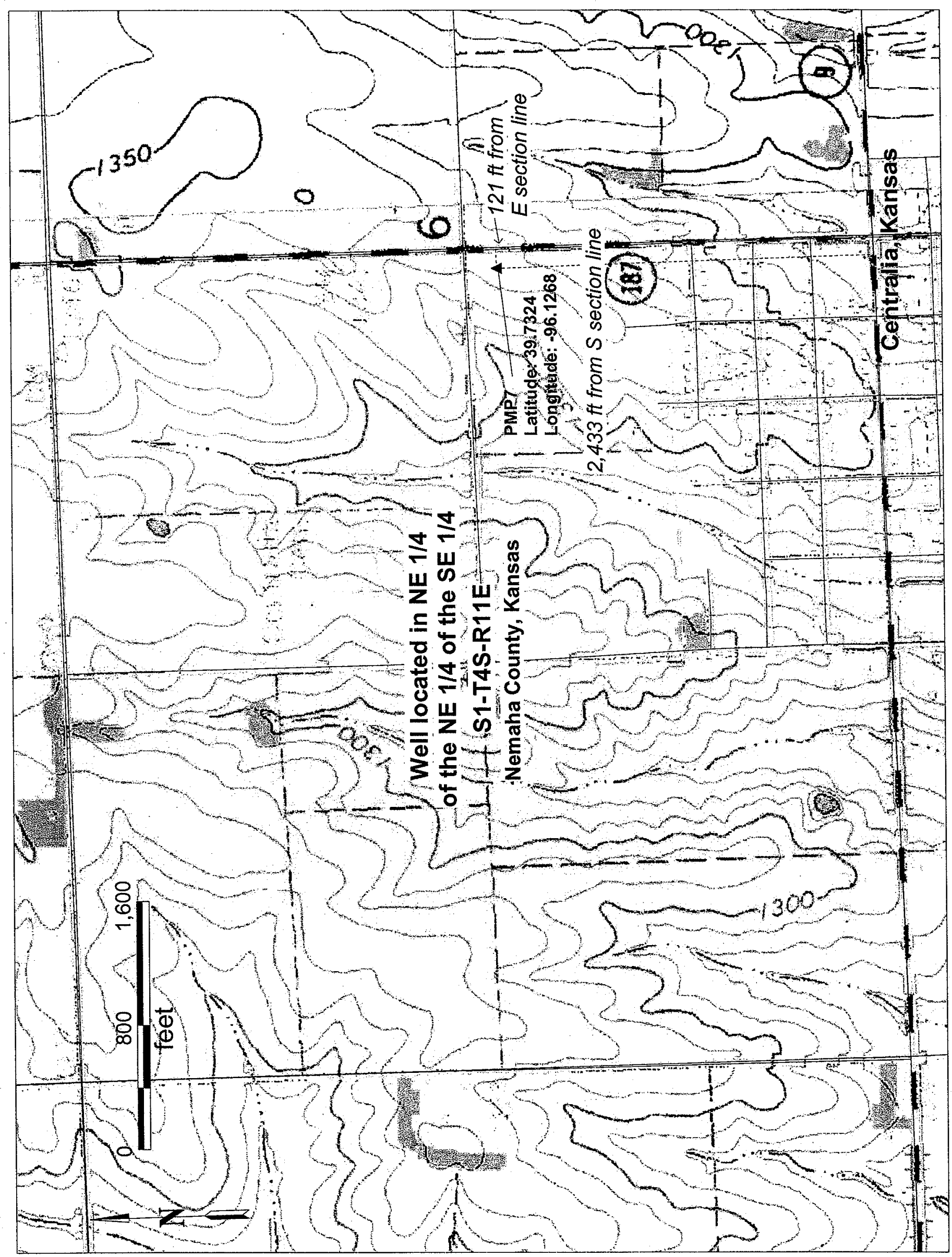


WATER WELL RECORD Form WWC-5 KSA 82a-1212 ID No. PMP-8

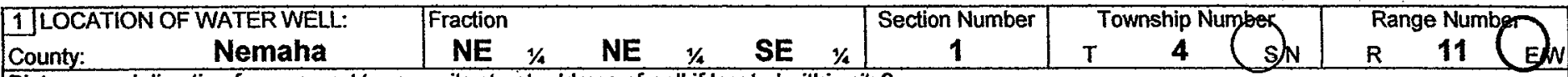

Distance and direction from nearest town or city street address of well if located within city?

\section{WATER WELL OWNER: USDA/CCC}

RR\#, St. Address, Box\# : Stop 0513, Room 4717-S/ 1400 Independence Ave SW City, State, ZIP Code : Washington, DC 20250-0513

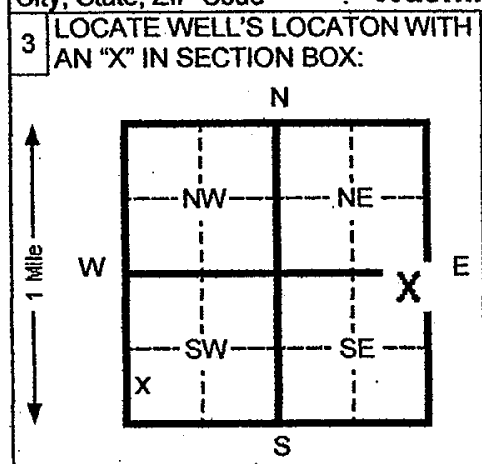

4 DEPTH OF COMPLETED WELL

Depth(s) Groundwater Encountered

60 ft. ELEVATION:

Board of Agriculture, Division of Water Resources Application Number:

5 TYPE OF BLANK CASING USED:
1 Steel
3 RMP (SR)
(2) PVC
4 ABS

WELL'S STATIC WATER LEVEL

20.1 ft below land surface measured on mo/day/yr $099 / 23 / 08$

$1,333.62$

Pump test data: Well water was ..... N/A ... ft. after ....N/A hours pumping ... N/A ...gpm

Est. Yield N/A gpm: Well water was ............ ft. after N/A hours pumping N/A Bore Hole Diameter 4.25 in. to 21

WELL WATER TO BE ÜSÉ ÁS̃: 5 Püblic water supplỳ

1 Domestic 3 Feed lot 6 Oil field water supply

2 Irrigation 4 Industrial 7 Lawn and garden (domestic)

8 Air conditioning

Manitoring well Sand Point MW

Was a chemical/bacteriological sample submitted to Deparment? Yes ...... No . $X$... If yes, mo/day/yr sample was submitted N/A

Water Well Disinfected? Yes

No $X$

5 Wrought Iron 8 Concrete tile $\quad$ CASING JOINTS: Glued .......... Clamped

$\begin{array}{llll}5 & \text { Wrought Iron } & 8 & \text { Concrete tile } \\ 6 & \text { Asbestos-Cement } & 9 & \text { Other (specify below) }\end{array}$

7 Fiberglass

CASING JOINTS: Glued

Clamped

Blank casing diameter $1 / 2$ in to
Casing height above land surface Flush Mount

TYPE OF SCREEN OR PERFORATION MATERIAL:

60 ft. Dia

N/A in. to N/A fi., Dia

N/A Threaded in to ................ Schedule 49 bs./ft. Wall thickness or gauge $\mathrm{No}$ $109^{35}$
1 Steel
3 Stainless steel
2 Brass
4 Galvanized steel \\ SCREEN OR PERFORATION OPRTINGS ARE: \\ 1 Continuous slot \\ 2 Louvered shutter

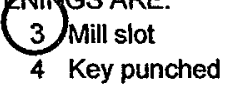

5 Fiberglass

6 Concrete tile

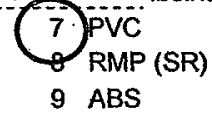

10 Asbestos-cernent
11 Other (specify)

12 None used (open hole)

8 Saw.cut

9 Drilled holes

10 Other (specify)

SCREEN-PERFORATED INTERVALS: From 50

GRAVEL PACK INTERVALS: From .............. th to

ft. From

ft. From

ft. to
ft. to

From $\quad \mathrm{ft}$ to

6 GROUT MATERIAL: 1 Neat cement 2 Cement grout

Grout Intervals From 48 (\#3) ft. to 0.5 ft. From

What is the nearest source of possible contamination:
4 Septictank
4 Lateral lines
5 Cess pool
2 Sewer lines
3 Watertight sewer lines
6 Seepage pit

ft. to 60 ft. From

me fto to

11 None (open hole)

Direction from well?

\begin{tabular}{|c|c|c|l|c|c|c|}
\hline FROM & TO & CODE & \multicolumn{1}{|c|}{ LITHOLOGICLOG } & FROM & TO & PLUGGING INTERVALS \\
\hline 0 & $2^{\prime}$ & & TOP Soll & & & \\
\hline $2^{\prime}$ & $46^{\prime}$ & Silt and Clay & & & \\
\hline $46^{\prime}$ & $47^{\prime}$ & Silty Clay & & & \\
\hline $47^{\prime}$ & $48^{\prime}$ & Silty Clay with some Sand & & & \\
\hline $48^{\prime}$ & $51^{\prime}$ & Silty Clay and Sand & & & & \\
\hline $51^{\prime}$ & $60^{\prime}$ & Sand \& Silt & & & \\
\hline
\end{tabular}

48 (\#4) fientonite

4 Other BenSeal Chips

7 CONTRACTOR'S OR LANDOWNER'S CERTIFICATION: This water well wa (1) constructed, 2 ) reconstructed, or (3) plugged under myjurisdiction and was

completed on (mo/day/yr) 09/23/08

Water Well Contractor's License No. 


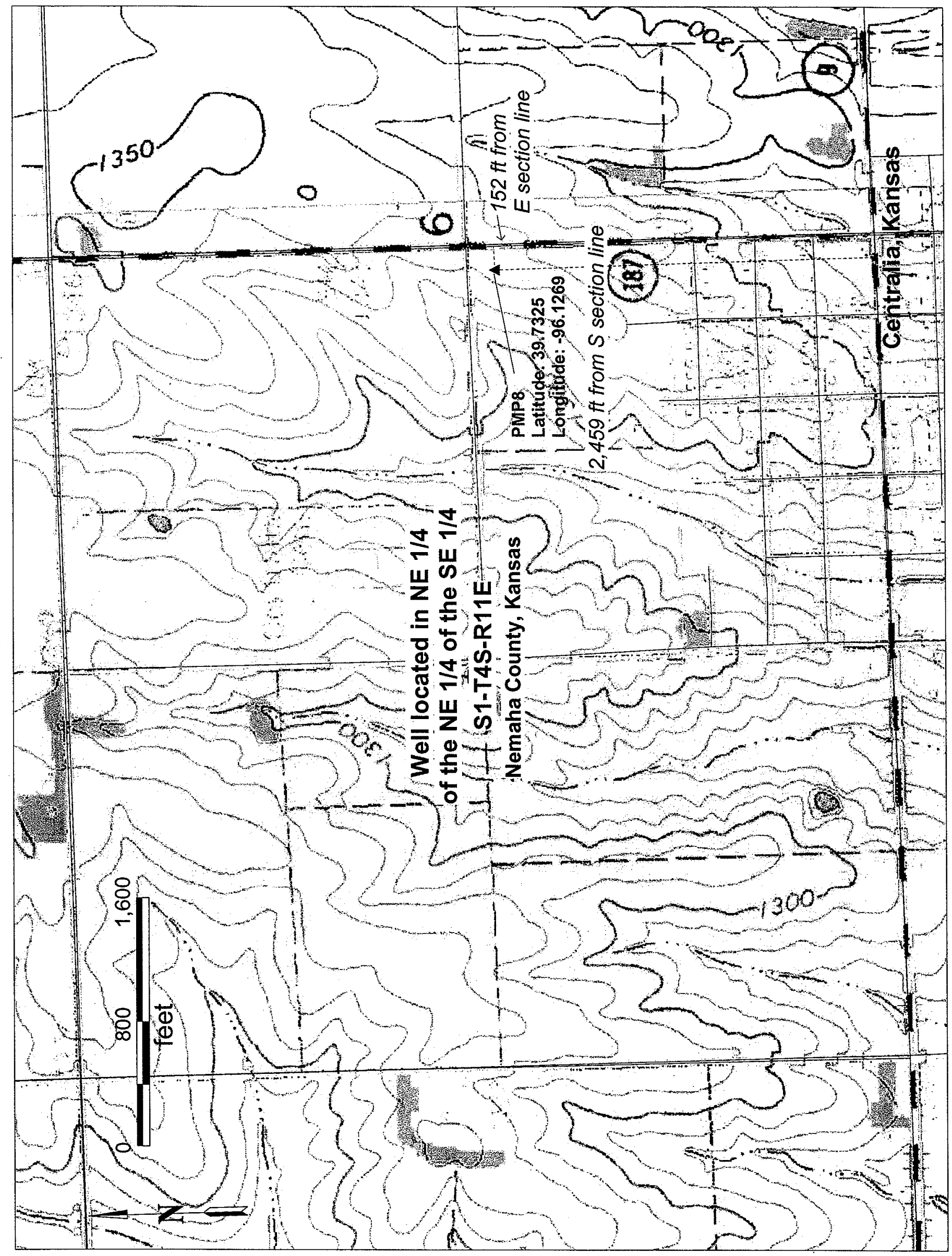




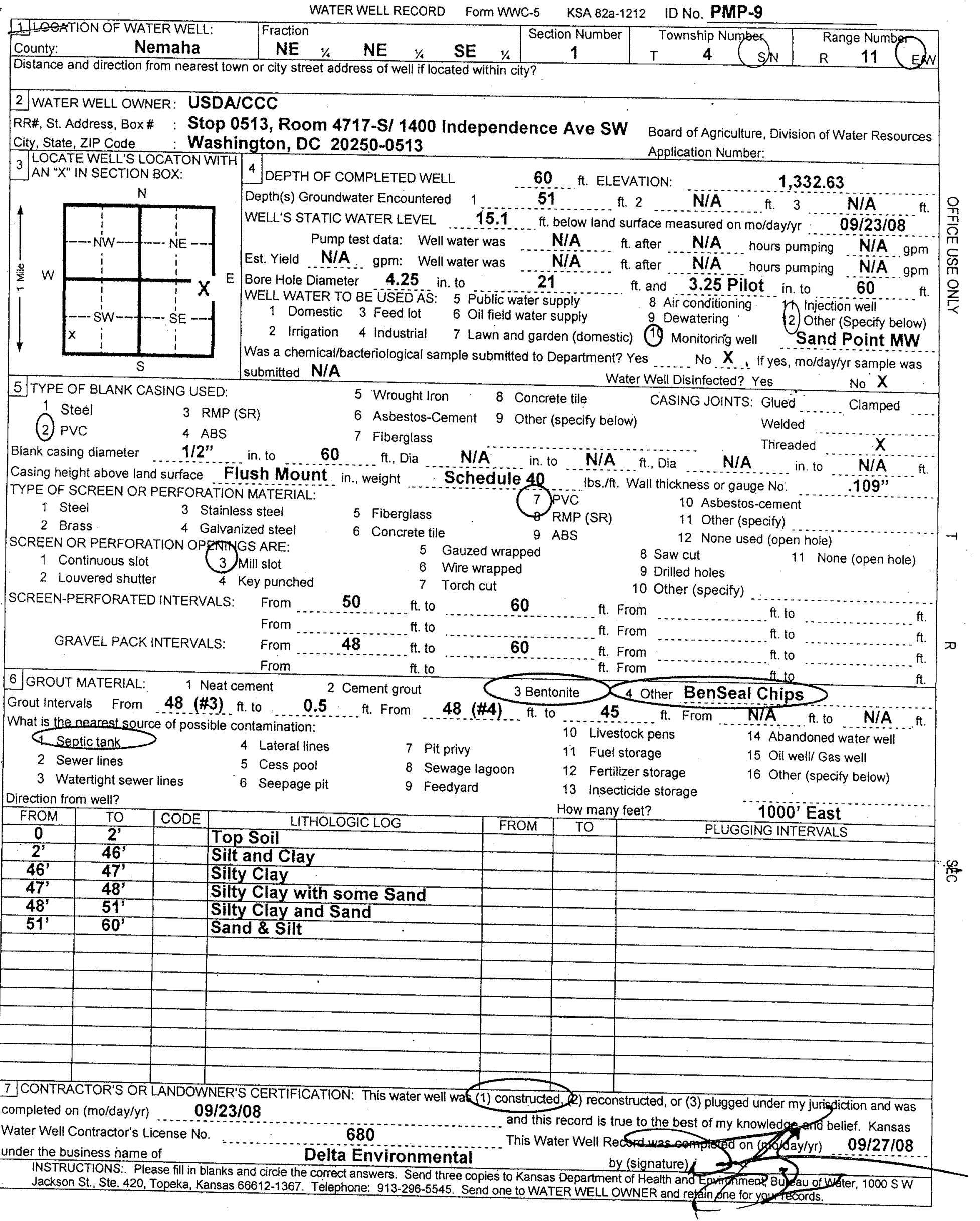




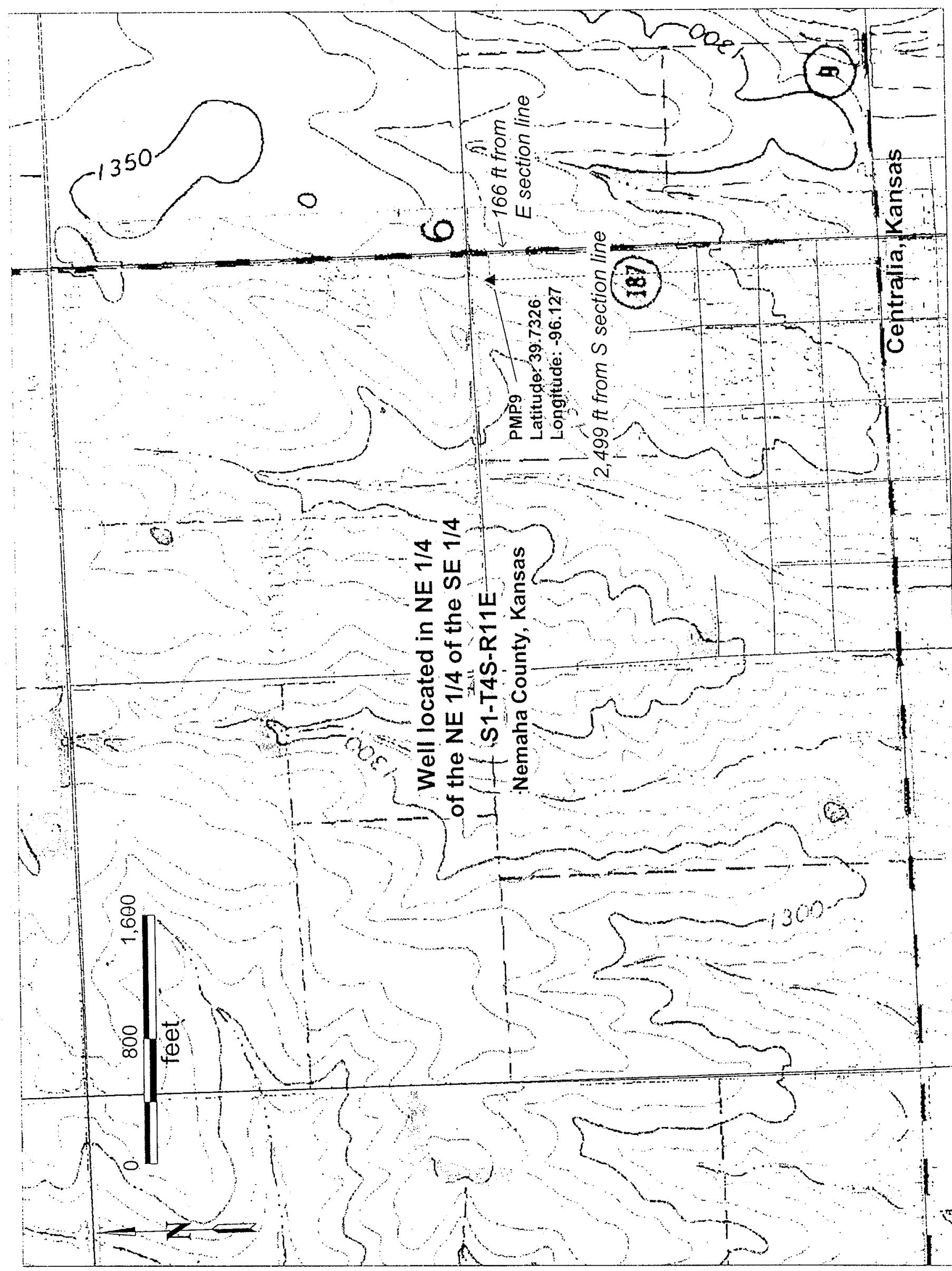




\section{Appendix D:}

Time Series Diagrams Presenting the Results of VOCs, DO, and ORP Analyses for Groundwater Monitoring Samples Collected during the ISCR Pilot Test 

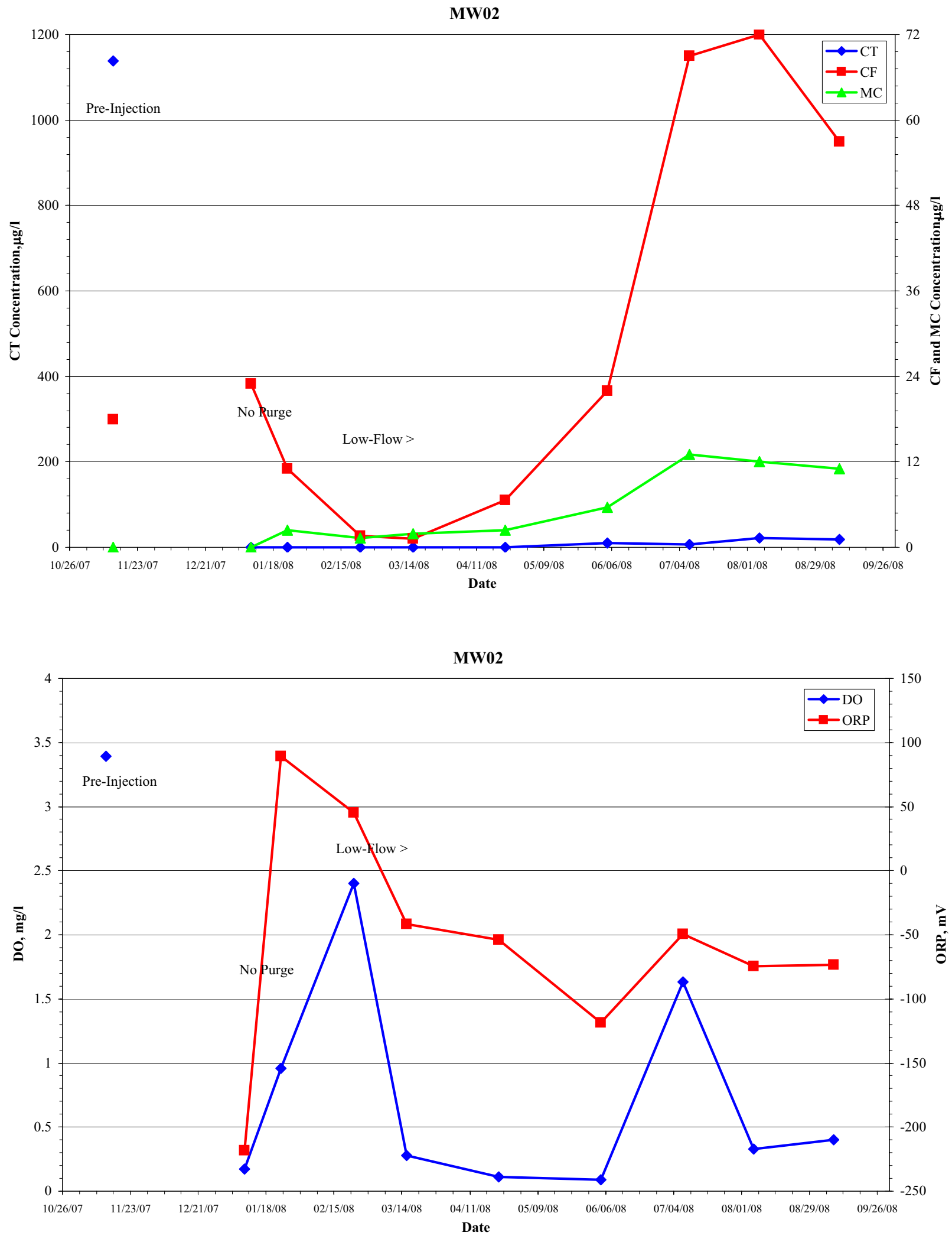

FIGURE D.1 Analytical results for volatile organic compounds, dissolved oxygen, and oxidation-reduction potential in groundwater samples collected at location MW02, November 2007 to September 2008. 

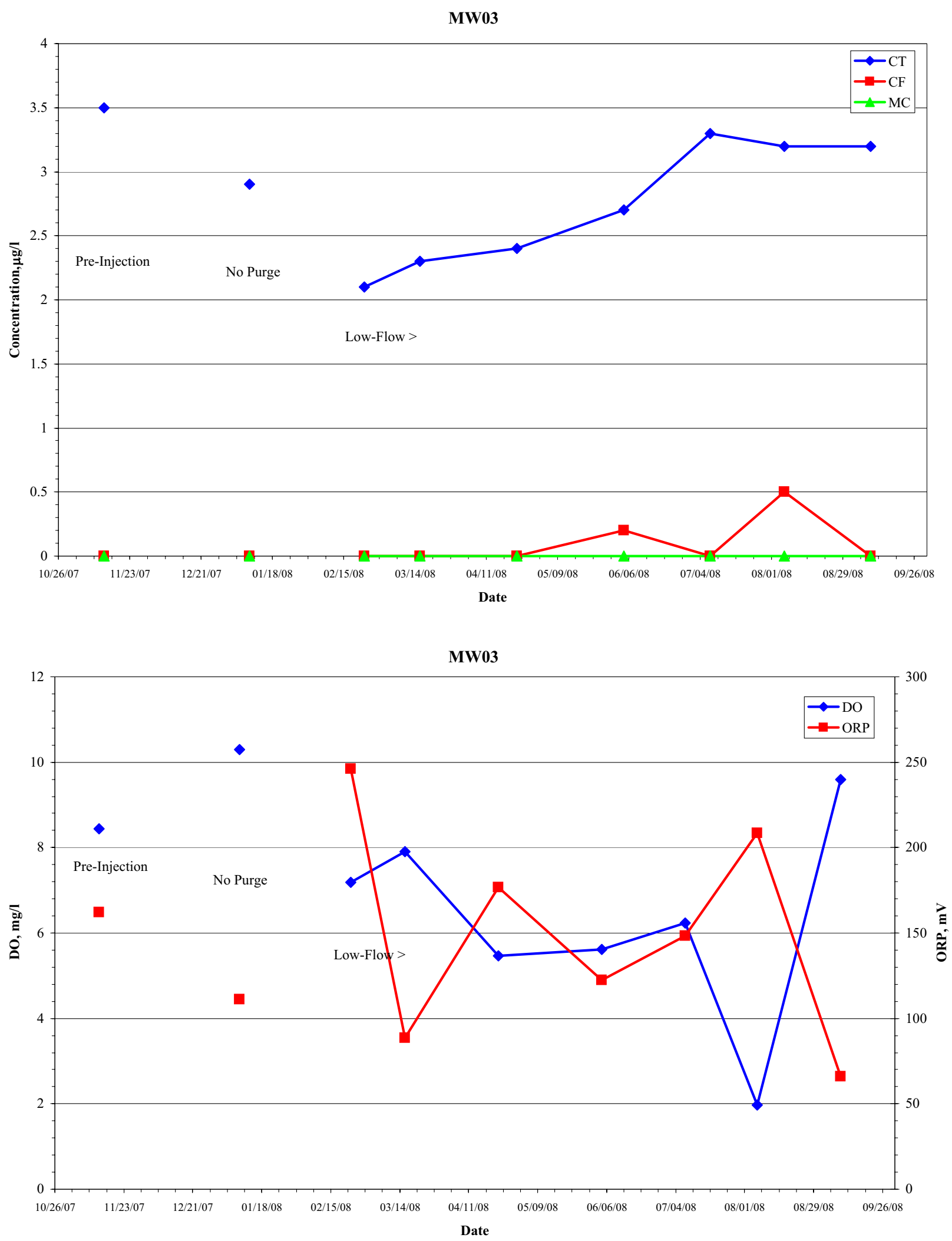

FIGURE D.2 Analytical results for volatile organic compounds, dissolved oxygen, and oxidation-reduction potential in groundwater samples collected at location MW03, November 2007 to September 2008. 
PMP1
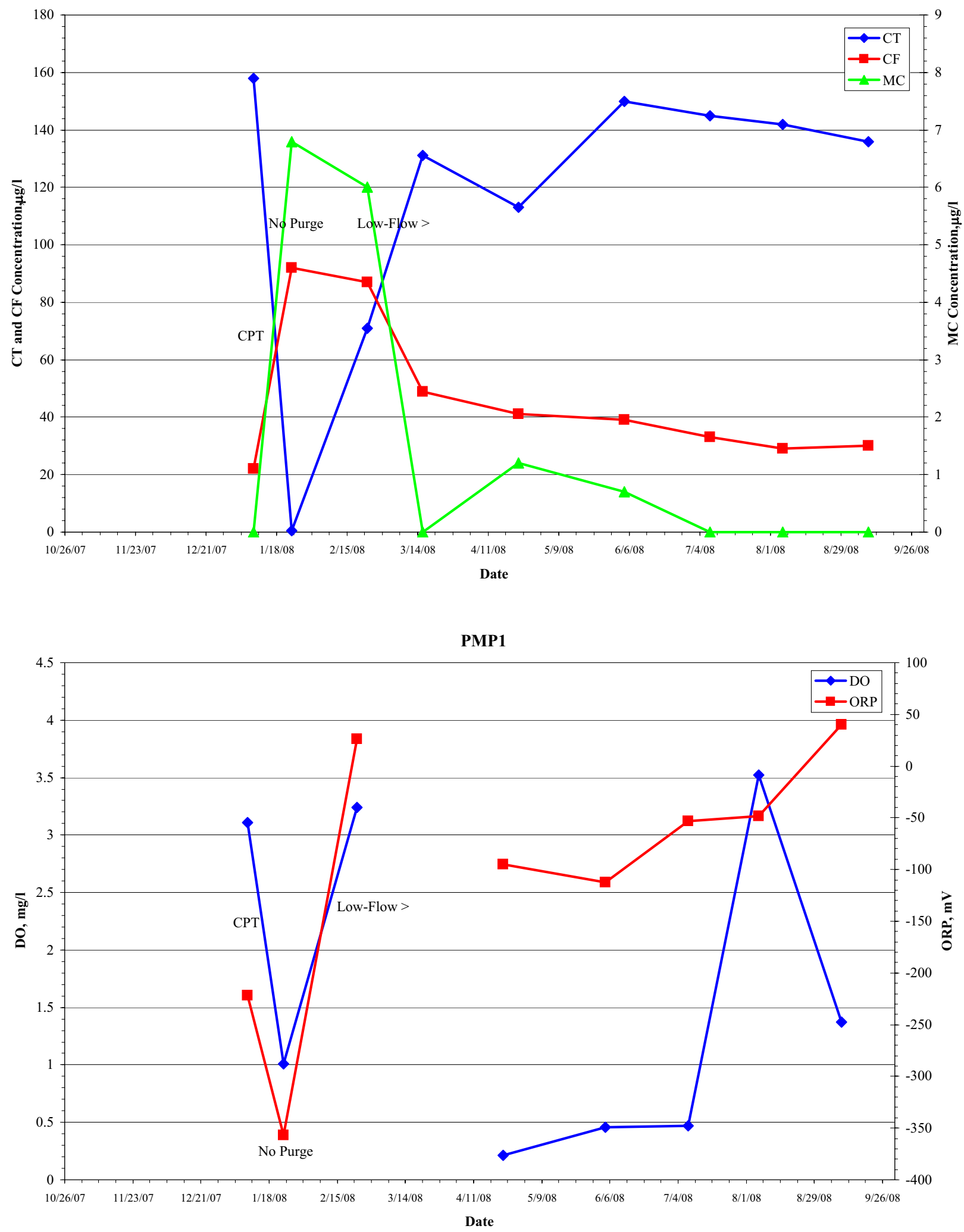

FIGURE D.3 Analytical results for volatile organic compounds, dissolved oxygen, and oxidation-reduction potential in groundwater samples collected at location PMP1, January 2008 to September 2008. 
PSB2-PMP2
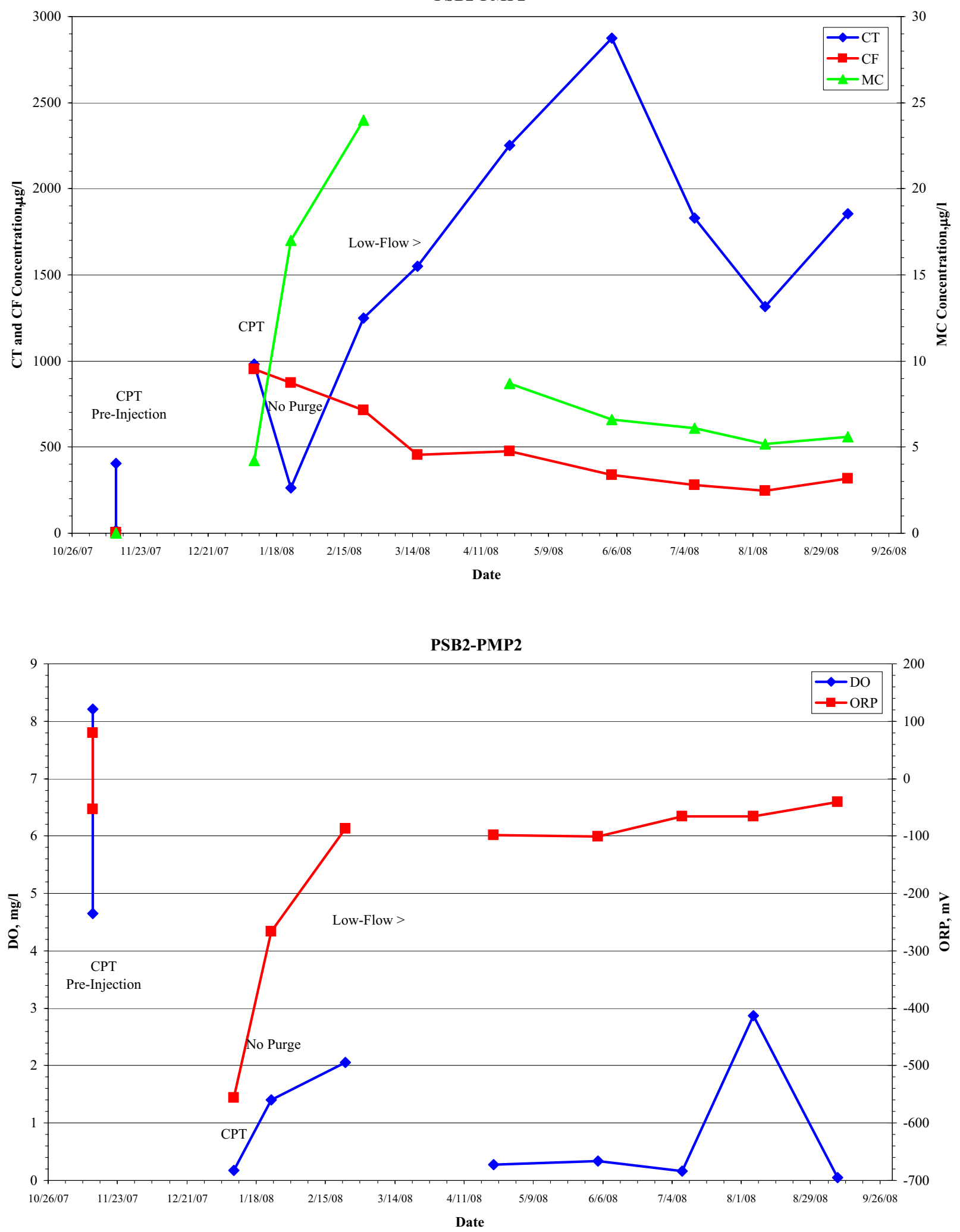

FIGURE D.4 Analytical results for volatile organic compounds, dissolved oxygen, and oxidation-reduction potential in groundwater samples collected at locations PSB2 and PMP2, November 2007 to September 2008. 

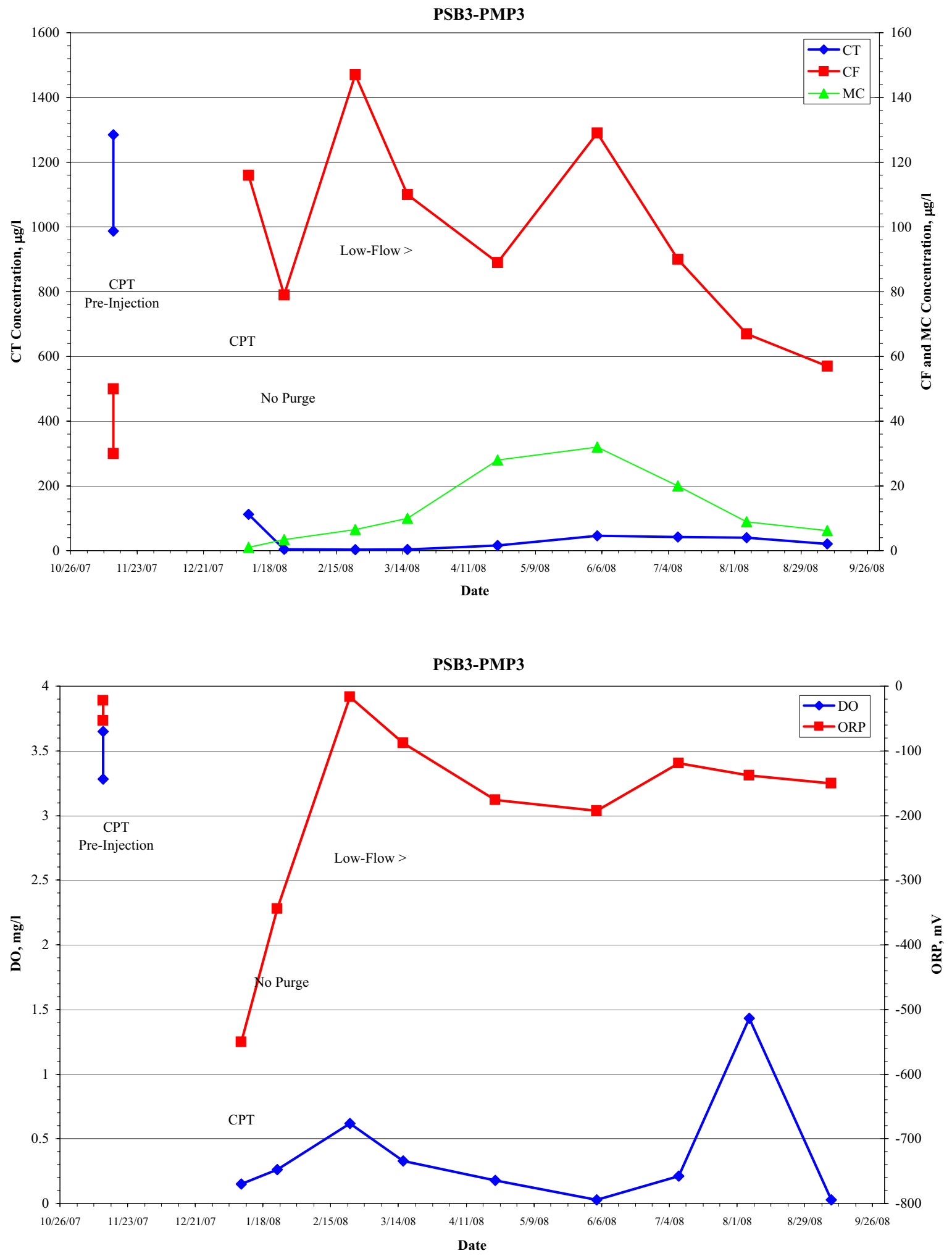

FIGURE D.5 Analytical results for volatile organic compounds, dissolved oxygen, and oxidation-reduction potential in groundwater samples collected at locations PSB3 and PMP3, November 2007 to September 2008. 
PMP4
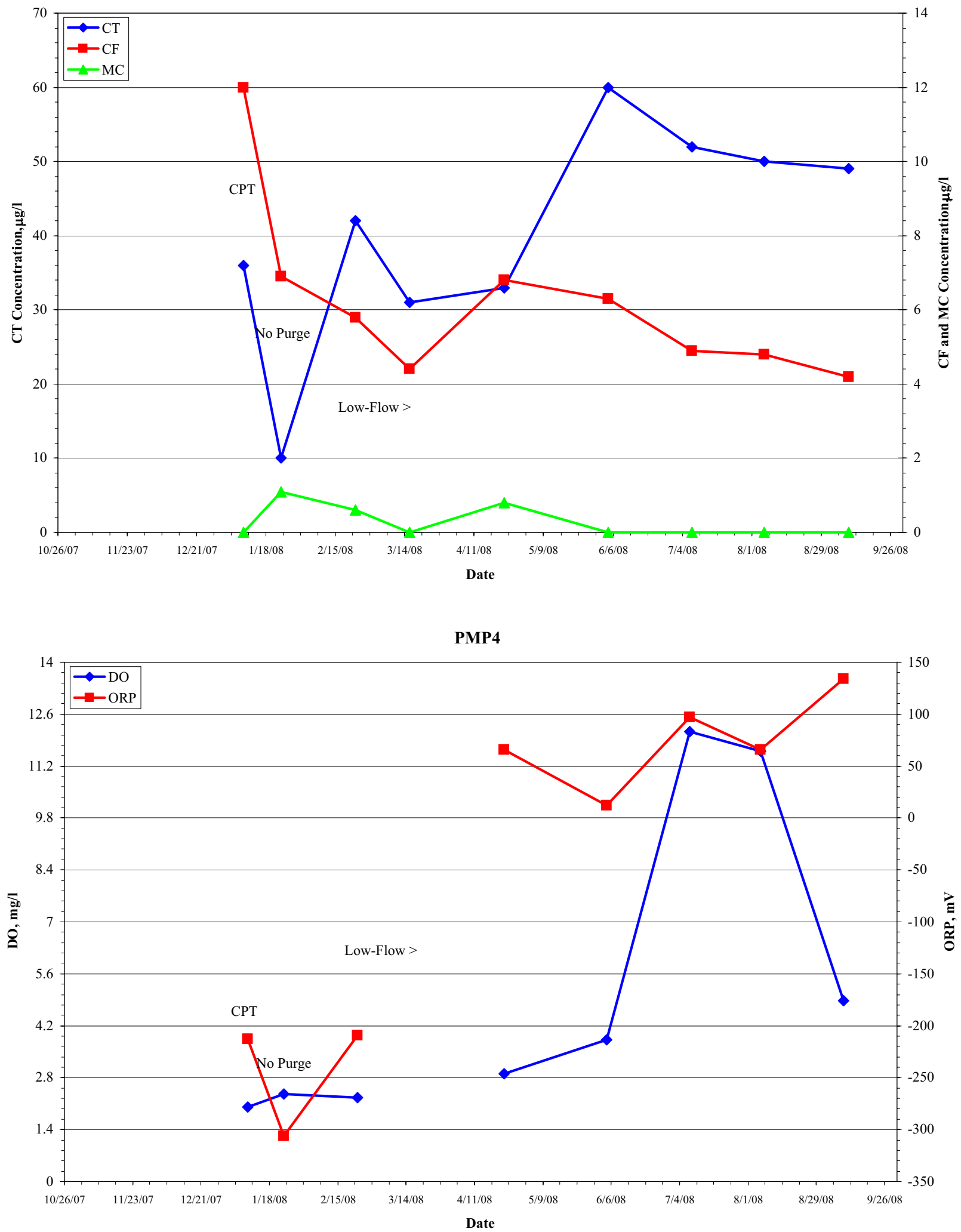

FIGURE D.6 Analytical results for volatile organic compounds, dissolved oxygen, and oxidation-reduction potential in groundwater samples collected at location PMP4, January 2008 to September 2008. 

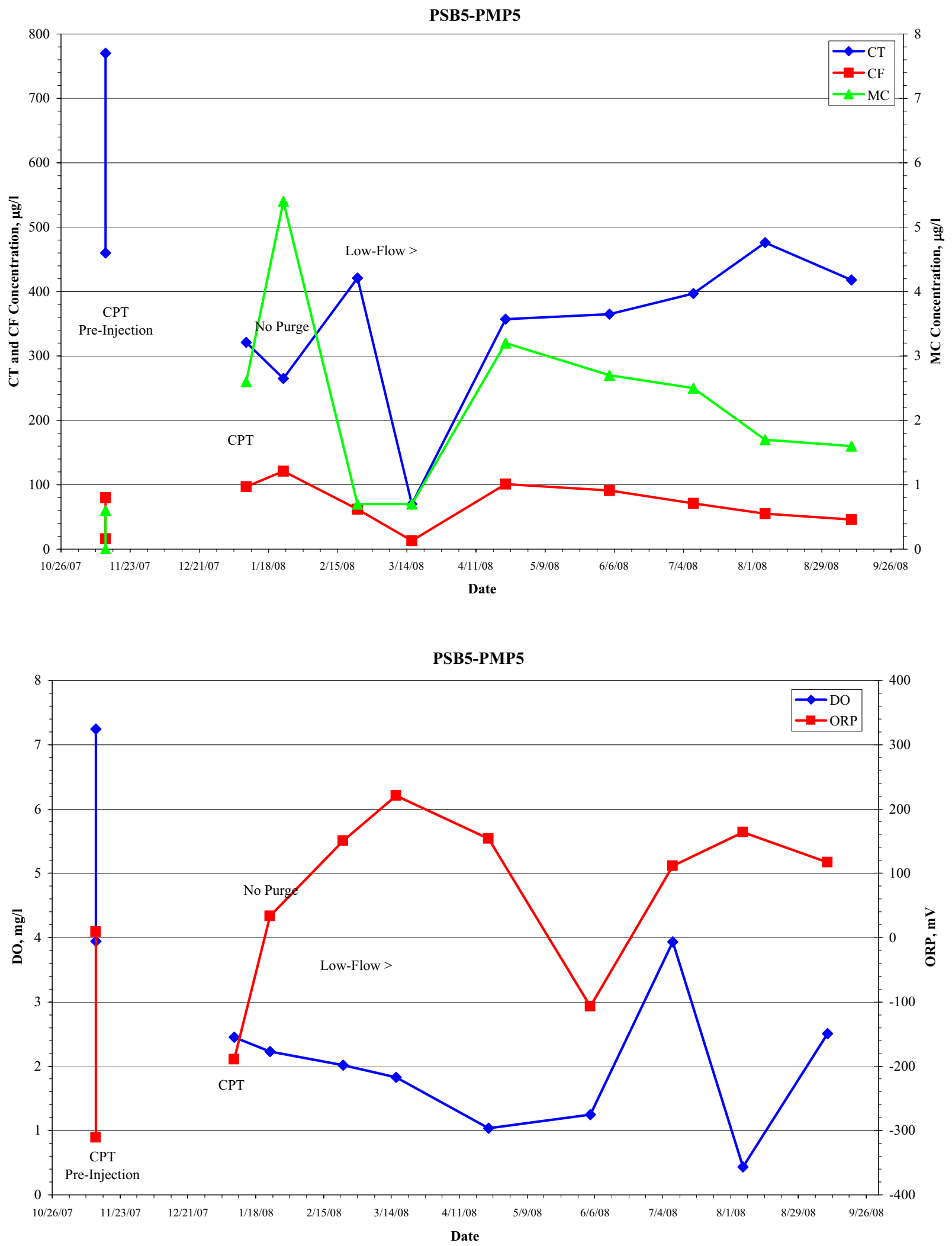

FIGURE D.7 Analytical results for volatile organic compounds, dissolved oxygen, and oxidation-reduction potential in groundwater samples collected at locations PSB5 and PMP5, November 2007 to September 2008. 
PSB6-PMP6
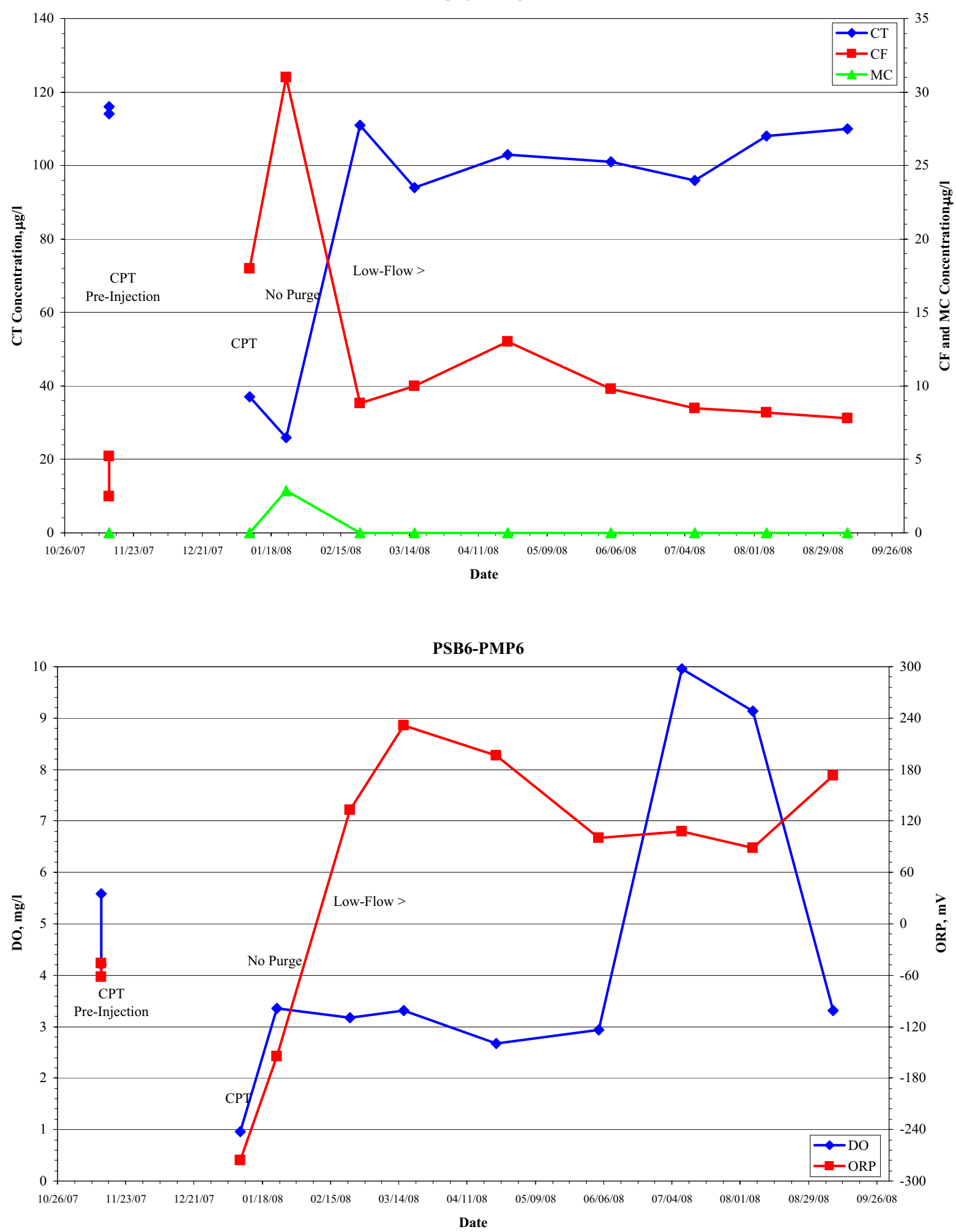

FIGURE D.8 Analytical results for volatile organic compounds, dissolved oxygen, and oxidation-reduction potential in groundwater samples collected at locations PSB6 and PMP6, November 2007 to September 2008. 
PSB7-PMP7

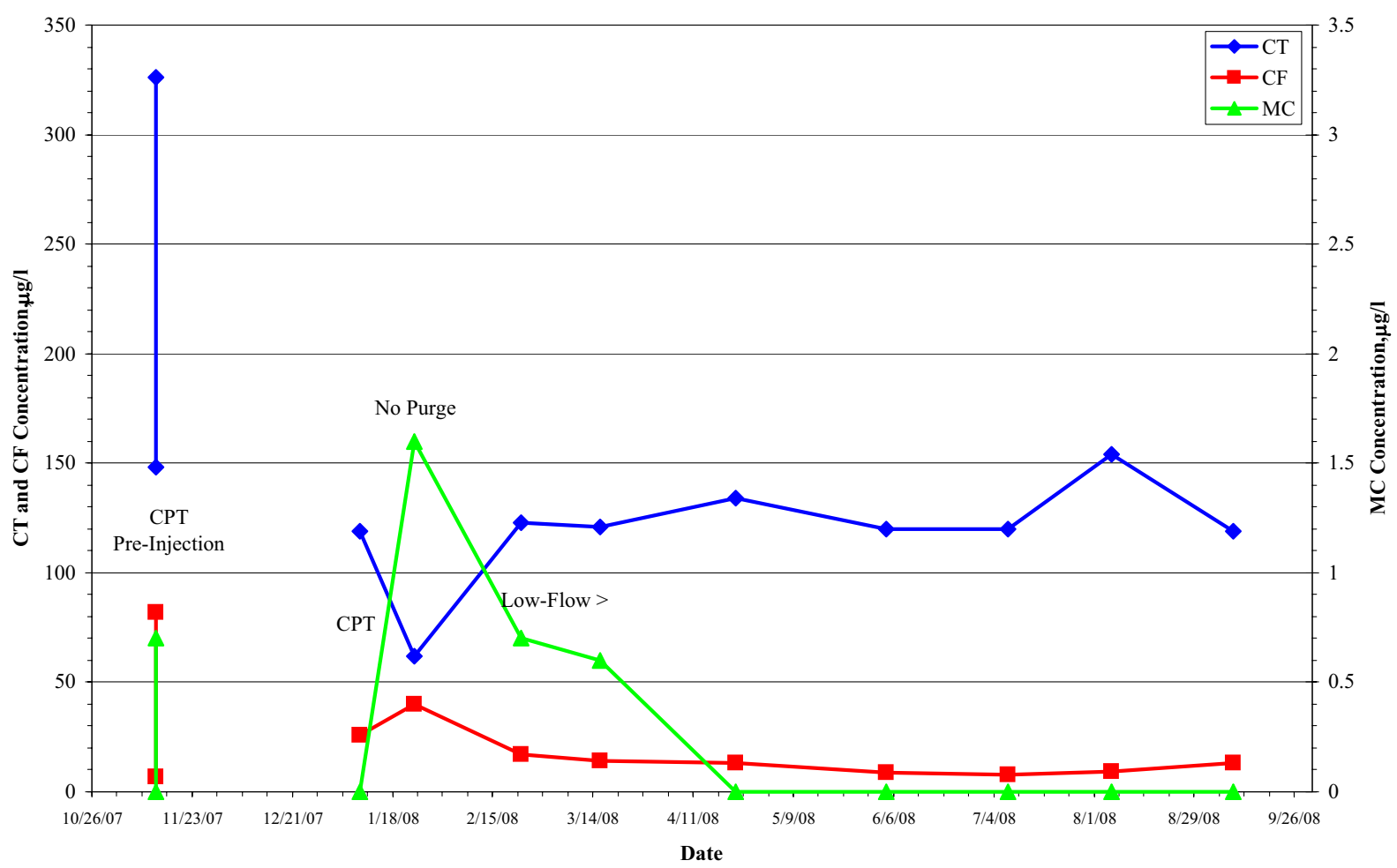

PSB7-PMP7

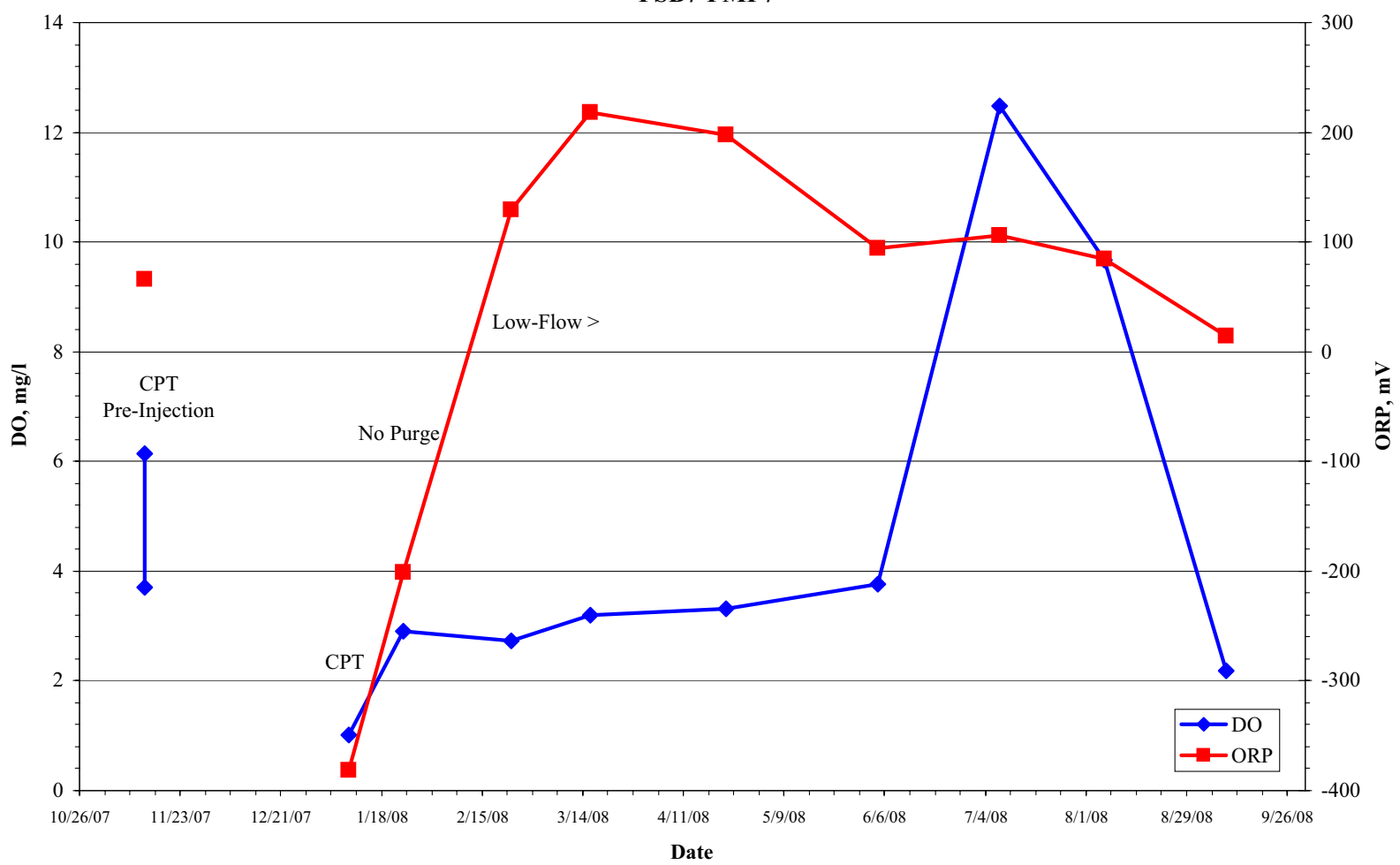

FIGURE D.9 Analytical results for volatile organic compounds, dissolved oxygen, and oxidation-reduction potential in groundwater samples collected at locations PSB7 and PMP7, November 2007 to September 2008. 
PSB8-PMP8
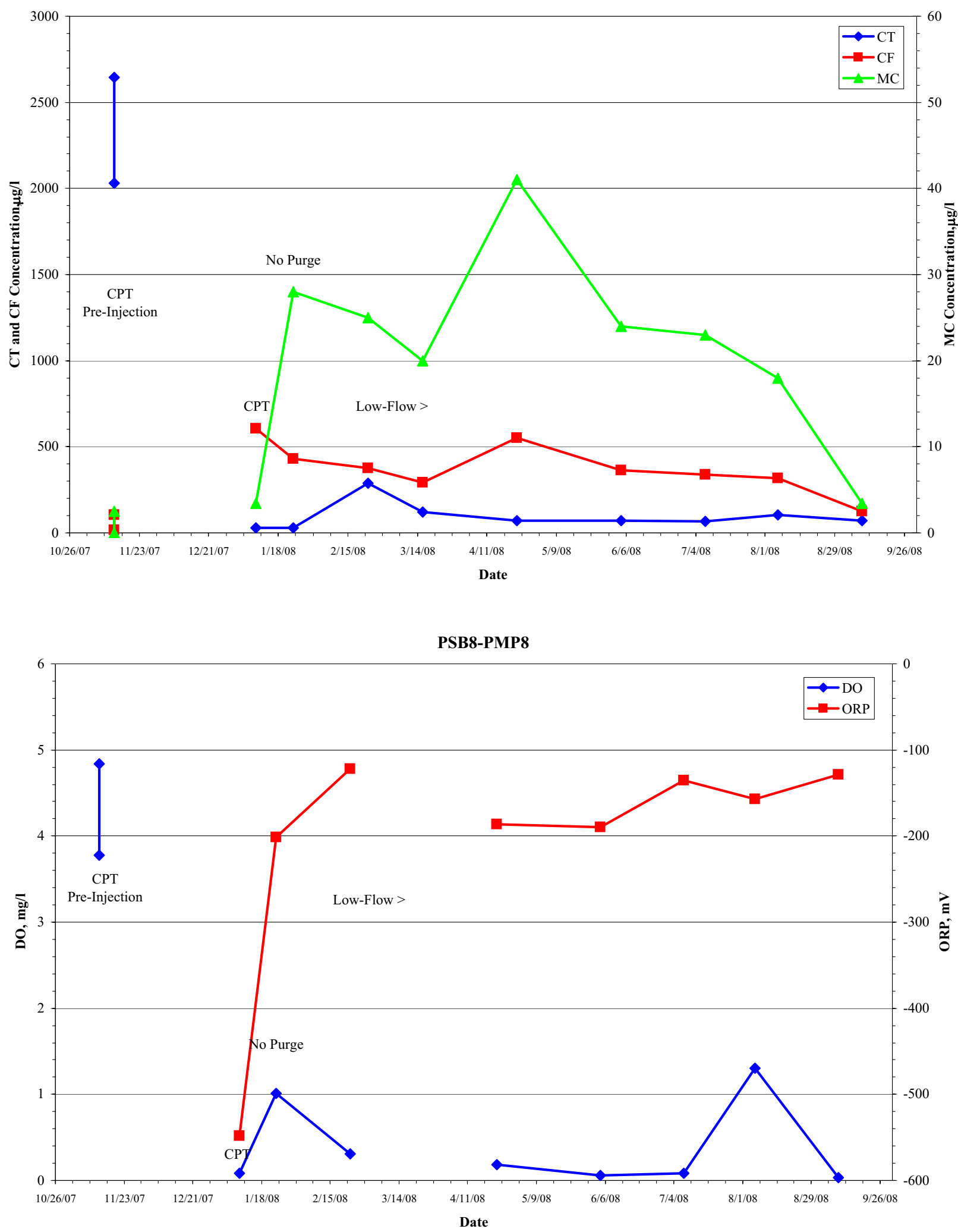

FIGURE D.10 Analytical results for volatile organic compounds, dissolved oxygen, and oxidationreduction potential in groundwater samples collected at locations PSB8 and PMP8, November 2007 to September 2008. 
PSB9-PMP9
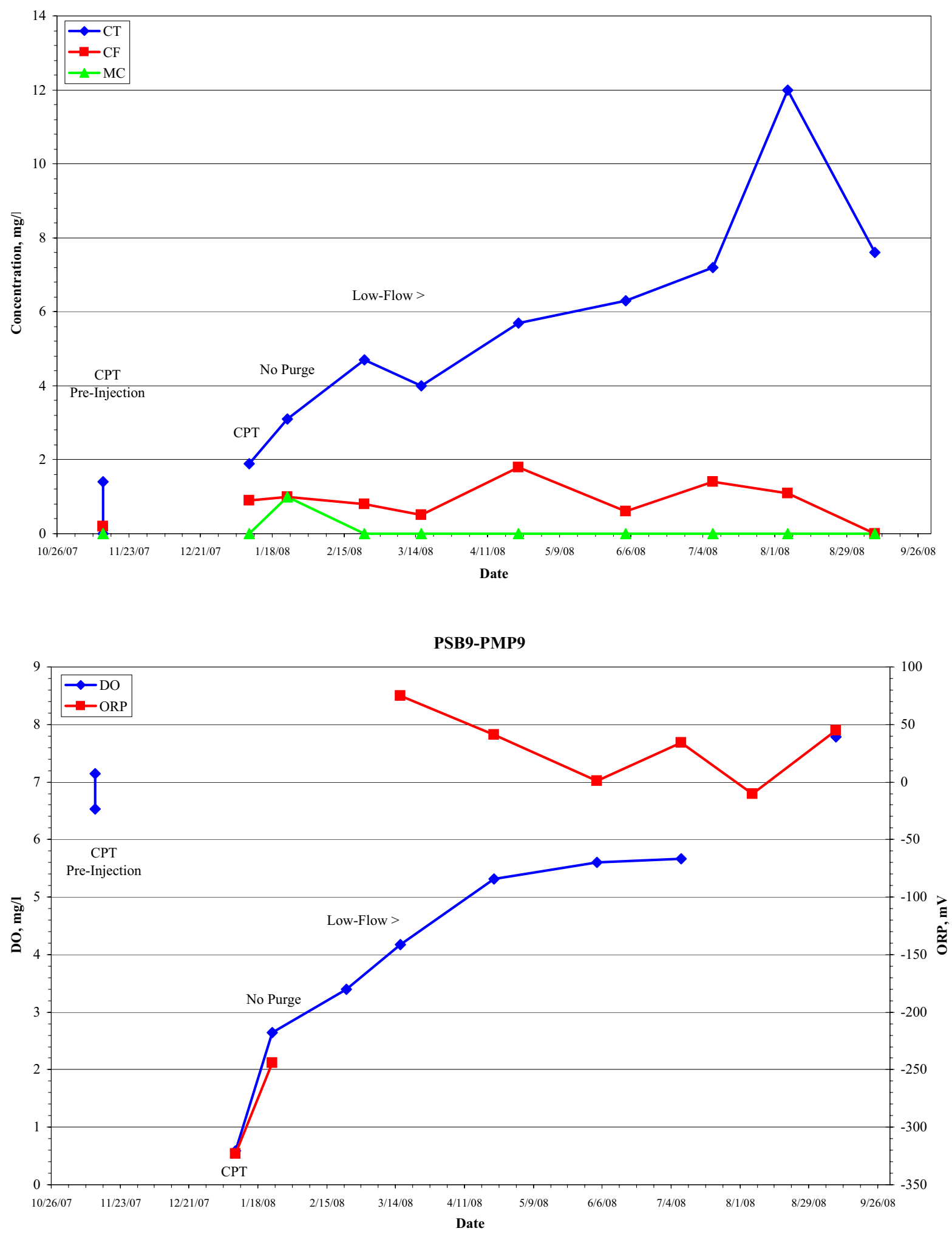

FIGURE D.11 Analytical results for volatile organic compounds, dissolved oxygen, and oxidationreduction potential in groundwater samples collected at locations PSB9 and PMP9, November 2007 to September 2008. 
SB04
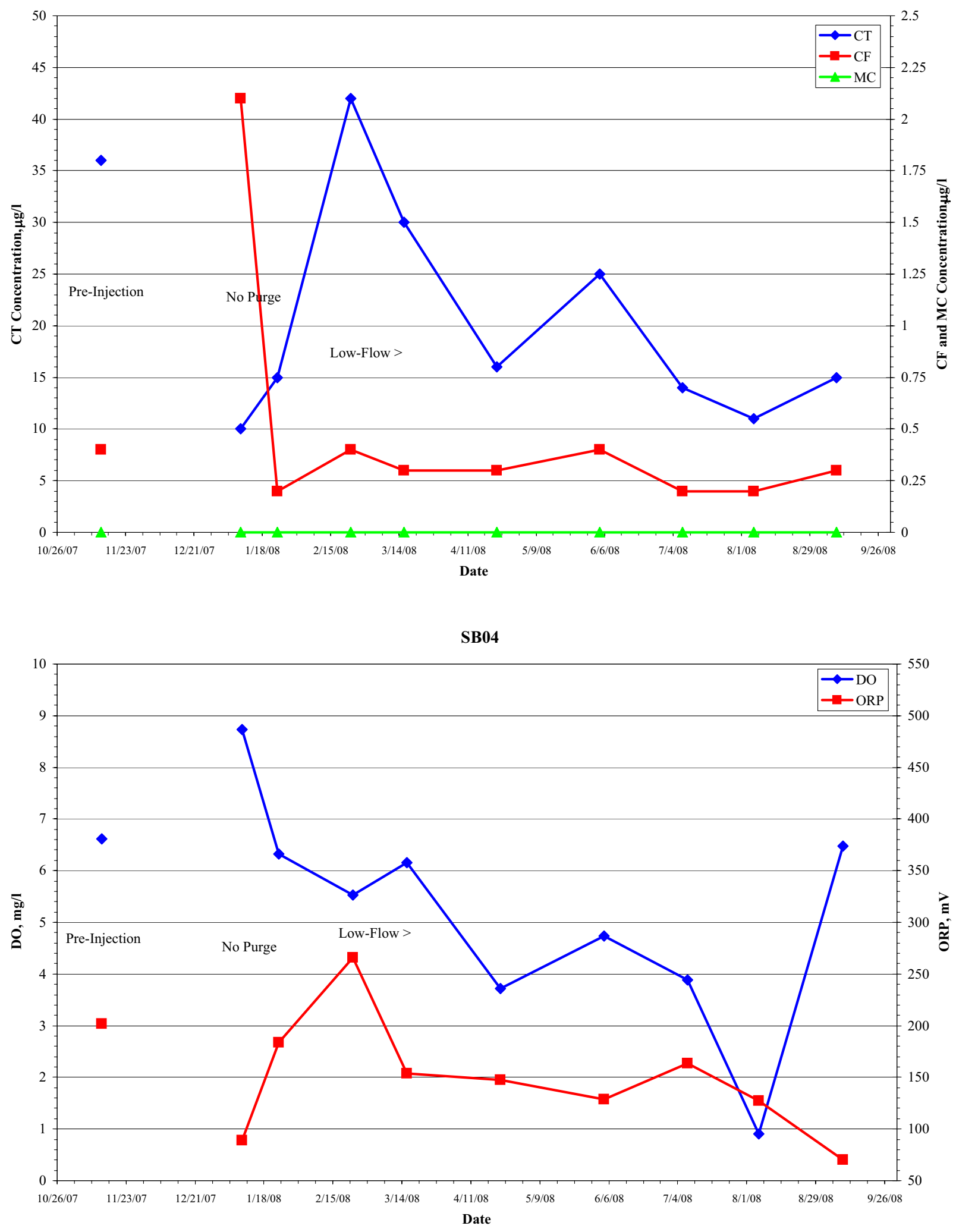

FIGURE D.12 Analytical results for volatile organic compounds, dissolved oxygen, and oxidationreduction potential in groundwater samples collected at location SB04, November 2007 to September 2008. 

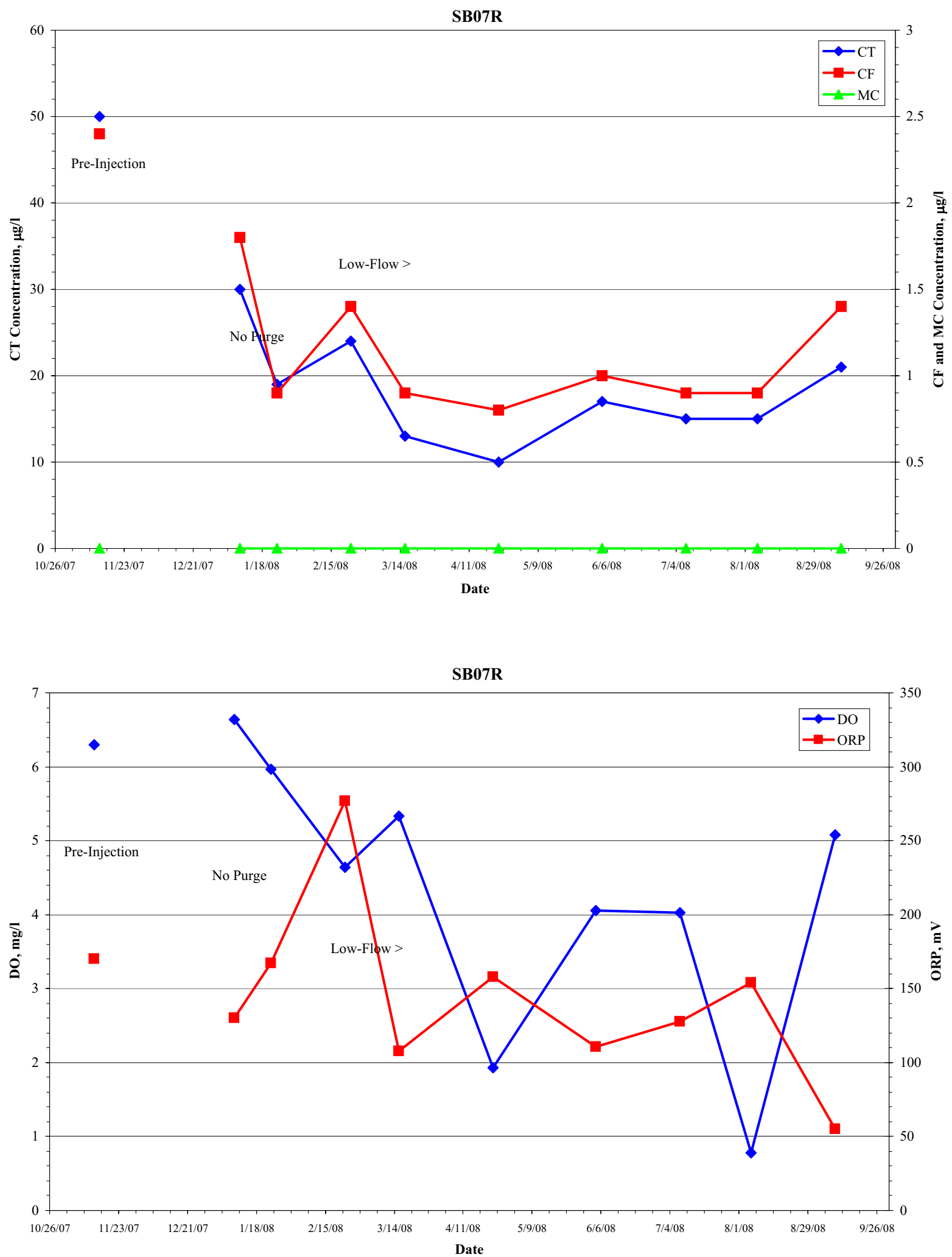

FIGURE D.13 Analytical results for volatile organic compounds, dissolved oxygen, and oxidationreduction potential in groundwater samples collected at location SB07R, November 2007 to September 2008. 

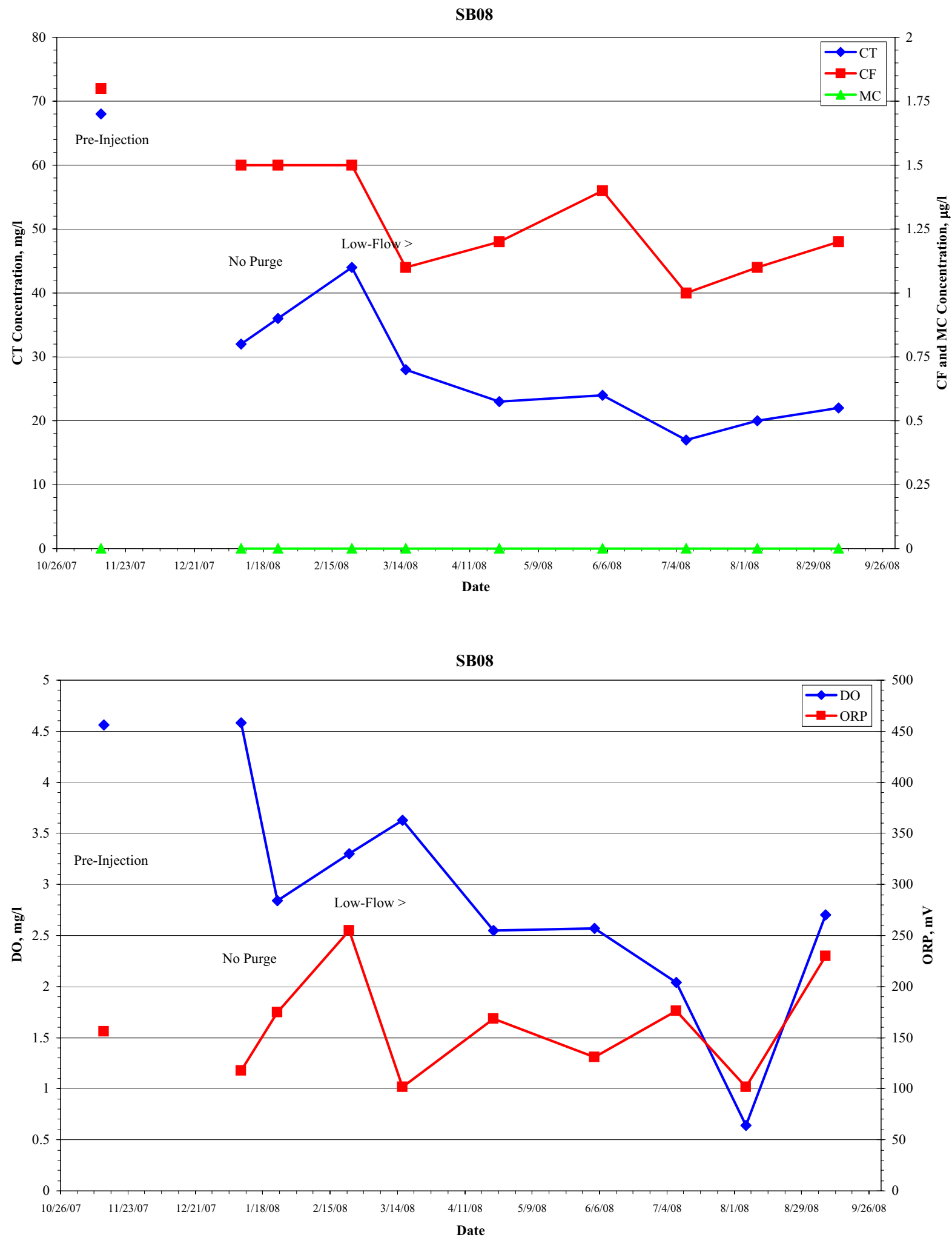

FIGURE D.14 Analytical results for volatile organic compounds, dissolved oxygen, and oxidationreduction potential in groundwater samples collected at location SB08, November 2007 to September 2008. 


\section{Progress Report and Technical Evaluation of the ISCR Pilot Test Conducted at the Former CCC/USDA Grain Storage Facility in Centralia, Kansas}

Applied Geosciences and Environmental Management Section, Environmental Science Division,

Argonne National Laboratory, 9700 South Cass Avenue, Argonne, Illinois 60439

\section{Contents:}

S1_wastehandling.pdf Supplement 1:

Waste Handling, Characterization, and Disposal and Quality Control for Sample Collection, Handling, and Analysis

S2_analyses-pre.pdf Supplement 2:

Analytical Results for Groundwater and Soil Samples Collected during the Pre-Injection Baseline Sampling Activities

S3_injection-rept.pdf Supplement 3:

Injection Program Summary Report for the Centralia ISCR Pilot Test, Prepared by Adventus Americas, Inc., March 4, 2008

S4_analyses-post.pdf Supplement 4:

Analytical Results for Groundwater and Soil Samples Collected during the Extended Post-Injection Monitoring Program

S5_outsideanal.pdf Supplement 5:

Outside Laboratory Data for Verification Organic Analyses

January 2009

Work sponsored by Commodity Credit Corporation, United States Department of Agriculture 


\section{Supplement 1:}

Waste Handling, Characterization, and Disposal and Quality Control for Sample Collection, Handling, and Analysis 


\section{Supplement 1:}

\section{Waste Handling, Characterization, and Disposal and Quality Control for Sample Collection, Handling, and Analysis}

\section{S1.1 Handling, Characterization, and Disposal of Investigation-Derived Waste}

With the approval of the KDHE/BER, sampling during the two monitoring events in January 2008 was performed without purging to preclude possible remobilization of the injected ISCR fluids near pumping monitoring wells. Subsequent monitoring events were performed by using a low-flow purging and sampling technique. Purge water generated as potentially contaminated investigation-derived waste during the periodic monitoring events since January 2008 has been containerized on-site for subsequent sampling. The accumulated purge water was sampled on two occasions and analyzed by Pace Analytical Services, Inc., Lenexa, Kansas, with EPA Method 5030/8260 for VOCs, EPA Method 504.1 for ethylene dibromide (EDB), and EPA Method 300 for nitrate as nitrogen. Results were as follows:

- In sampling of accumulated wastewater on March 19, 2008, after the February and March 2008 monitoring events, carbon tetrachloride was detected at $6.6 \mu \mathrm{g} / \mathrm{L}$ and chloroform at $20 \mu \mathrm{g} / \mathrm{L}$. No EDB was detected. Nitrate was present at $1.2 \mathrm{mg} / \mathrm{L}$. With the approval of the KDHE (2008b), the wastewater was taken to the Sabetha publicly owned treatment works on May 21, 2008, for disposal.

- In sampling of accumulated wastewater on July 16, 2008, following the April, June, and July 2008 monitoring events, carbon tetrachloride was detected at $12.6 \mu \mathrm{g} / \mathrm{L}$ and chloroform at $22.2 \mu \mathrm{g} / \mathrm{L}$. Nitrate and EDB were not detected. With the approval of the KDHE (2008c), the wastewater was taken to the Sabetha publicly owned treatment works on August 1, 2008, for disposal.

Wastewater generated during the August and September monitoring events has been containerized and remains on-site. This wastewater will be sampled for analysis, and the results will be transmitted to KDHE after the next monitoring event. Subsequent disposal of the wastewater will be subject to KDHE authorization. 


\section{S1.2 Quality Control for Sample Collection, Handling, and Analysis}

Quality control/quality assurance (QA/QC) procedures followed during the Centralia pilot study were described in detail in the Master Work Plan (Argonne 2002b). The results of the QA/AC activities are summarized as follows:

- Sample collection and handling activities were monitored by the documentation of samples as they were collected and the use of chain-ofcustody (COC) forms and custody seals to ensure sample integrity during the handling and shipment of samples for analysis.

- Samples collected for VOCs analyses were received at the laboratory with custody seals intact and at the appropriate preservation temperature. All samples were analyzed within required holding times.

- The QC samples collected to monitor sample collection and handling activities included field blanks, equipment rinsates, and trip blanks. Method blanks were analyzed with the samples to monitor analytical methodologies. Chloroform was present at low concentrations $(<2.6 \mu \mathrm{g} / \mathrm{L})$ in waters used during the pilot study. Carbon tetrachloride was present at low concentration $(<2 \mu \mathrm{g} / \mathrm{L})$ in 2 of 17 equipment rinsates collected. Qualification of associated samples was not warranted. Trip blanks were free of carbon tetrachloride contamination. Methylene chloride present in some method blanks associated with verification organic analyses by Envirosystems, Inc., resulted in qualification of the associated data. Analytical results for the samples collected to monitor sample collection and handling activities are in Table S1.1. These samples are described in Appendix A, Table A.1.

- Groundwater and soil samples were analyzed for VOCs at the AGEM Laboratory by the purge-and-trap method on a gas chromatograph-mass spectrometer system. Calibration checks with each sample delivery group were required to be within $\pm 20 \%$ of the standard. Surrogate standard determinations performed on samples and blanks were within the specified range of $80-120 \%$ for all samples in either the initial analysis or a successful reanalysis. 
- To monitor sample collection and analytical methodologies, replicate samples were collected, and samples were selected by the AGEM Laboratory for duplicate analyses. The analytical results for these secondary QA/QC samples (Table S1.2) compare well overall, with an average relative percent difference values between the primary and secondary QC analyses of $14.5 \%$ for carbon tetrachloride, $22.6 \%$ for chloroform, and $2.5 \%$ for methylene chloride. Some heterogeneity is evident in discrete pre-injection groundwater samples collected within the contaminant zone and in the initial samples collected from the monitoring wells without purging.

- In accordance with the QA/QC procedures defined in the Master Work Plan (Argonne 2002b), the analyses of samples at the AGEM Laboratory were verified at a second laboratory. The results for these verification organic analyses (Table S1.3) support the results from the AGEM Laboratory, with average relative percent difference values of $39.4 \%$ for carbon tetrachloride, $18.3 \%$ for chloroform, and $10.1 \%$ for methylene chloride. Summary pages for the verification organic analyses are in Supplement 5 (on CD).

- Samples shipped to TestAmerica for geochemical and attenuation parameter analyses were received with custody seals intact and at the appropriate preservation conditions. Results for laboratory QC samples prepared and analyzed with the samples to evaluate accuracy and precision were within acceptable limits. In the bromide analysis of two samples from monitoring well MW02 (CNMW02-W-26099 collected on 2/23/08 and CNMW02-W26639 collected on 7/8/08), interfering peaks eluting where bromide would normally elute prevented detection of bromide. Matrix spike/matrix spike duplicate recoveries did not meet QC criteria. Reported bromide concentrations for samples collected in April, June, and August exceeded the maximum initial bromide concentration $(150 \mathrm{mg} / \mathrm{L})$ in the injected ISCR fluids, and hence they are also questionable. 
TABLE S1.1 Results for quality control samples analyzed to monitor sample collection and handling activities during the pilot test at Centralia.

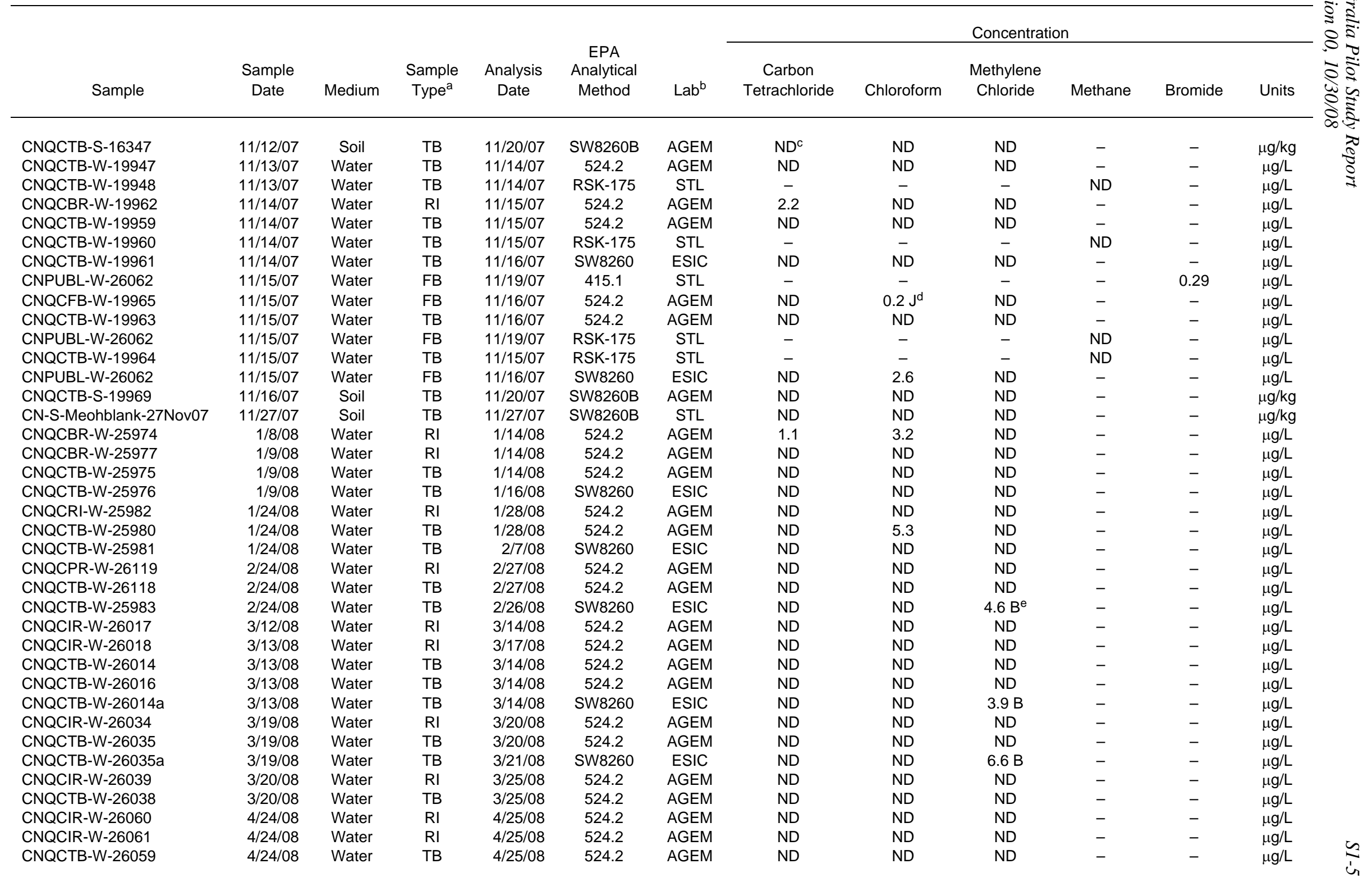




\begin{tabular}{|c|c|c|c|c|c|c|c|c|c|c|c|c|}
\hline CNQCTB-W-26059a & $4 / 24 / 08$ & Water & TB & $5 / 5 / 08$ & SW8260 & ESIC & ND & ND & $7.7 \mathrm{~B}$ & - & - & $\mu \mathrm{g} / \mathrm{L}$ \\
\hline CNQCIR-W-26634 & $6 / 4 / 08$ & Water & RI & 6/6/08 & 524.2 & AGEM & ND & ND & ND & - & - & $\mu \mathrm{g} / \mathrm{L}$ \\
\hline CNQCIR-W-26635 & $6 / 4 / 08$ & Water & $\mathrm{RI}$ & $6 / 6 / 08$ & 524.2 & AGEM & ND & ND & ND & - & - & $\mu \mathrm{g} / \mathrm{L}$ \\
\hline CNQCTB-W-26638 & $6 / 5 / 08$ & Water & TB & 6/9/08 & 524.2 & AGEM & ND & ND & ND & - & - & $\mu \mathrm{g} / \mathrm{L}$ \\
\hline CNQCTB-W-26638a & $6 / 5 / 08$ & Water & TB & $7 / 11 / 08$ & SW8260 & ESIC & ND & ND & ND & - & - & $\mu \mathrm{g} / \mathrm{L}$ \\
\hline CNQCIR-W-26653 & $7 / 8 / 08$ & Water & $\mathrm{RI}$ & 7/10/08 & 524.2 & AGEM & ND & ND & ND & - & - & $\mu \mathrm{g} / \mathrm{L}$ \\
\hline CNQ̈CIR-W-26654 & $7 / 8 / 08$ & Water & $\mathrm{RI}$ & $7 / 10 / 08$ & 524.2 & AGEM & ND & ND & ND & - & - & $\mu \mathrm{g} / \mathrm{L}$ \\
\hline CNQCTB-W-26657 & $7 / 8 / 08$ & Water & TB & $7 / 10 / 08$ & 524.2 & AGEM & ND & $0.5 \mathrm{~J}$ & ND & - & - & $\mu \mathrm{g} / \mathrm{L}$ \\
\hline CNQCTB-W-26657a & $7 / 8 / 08$ & Water & TB & $7 / 14 / 08$ & SW8260 & STL & ND & ND & ND & - & - & $\mu \mathrm{g} / \mathrm{L}$ \\
\hline CNQCTB-W-26672 & 8/6/08 & Water & TB & 8/11/08 & 524.2 & AGEM & ND & ND & ND & - & - & $\mu \mathrm{g} / \mathrm{L}$ \\
\hline CNQCTB-W-26672a & $8 / 6 / 08$ & Water & TB & 8/11/08 & SW8260 & STL & ND & ND & ND & - & - & $\mu \mathrm{g} / \mathrm{L}$ \\
\hline CNQCIR-W-26700 & 9/10/08 & Water & $\mathrm{RI}$ & 9/17/08 & 524.2 & AGEM & ND & ND & ND & - & - & $\mu \mathrm{g} / \mathrm{L}$ \\
\hline CNQCIR-W-26701 & 9/10/08 & Water & $\mathrm{RI}$ & 9/18/08 & 524.2 & AGEM & ND & ND & ND & - & - & $\mu \mathrm{g} / \mathrm{L}$ \\
\hline CNQCTB-W-26702 & 9/10/08 & Water & TB & 9/18/08 & 524.2 & AGEM & ND & ND & ND & - & - & $\mu \mathrm{g} / \mathrm{L}$ \\
\hline CNQCTB-W-26702a & 9/10/08 & Water & TB & $9 / 12 / 08$ & SW8260 & STL & ND & ND & ND & - & - & $\mu \mathrm{g} / \mathrm{L}$ \\
\hline
\end{tabular}

a Sample types: FB, field blank; RI, rinsate; TB, trip blank.

b Laboratories: AGEM, Applied Geosciences and Environmental Management; ESIC, Envirosystems; STL, Severn-Trent/TestAmerica.

c ND, not detected.

d Qualifier J indicates an estimated concentration below the method quantitation limit.

e Qualifier B indicates that methylene chloride was present in the associated method blank. 
TABLE S1.2 Results from the AGEM Laboratory for primary soil and groundwater samples and associated replicate samples and duplicate analyses.

\begin{tabular}{|c|c|c|c|c|c|c|c|c|c|c|c|}
\hline \multirow[b]{2}{*}{$\begin{array}{l}\text { Sample } \\
\text { Date }\end{array}$} & \multirow[b]{2}{*}{ Location } & \multirow[b]{2}{*}{$\begin{array}{l}\text { Sample } \\
\text { Depth } \\
\text { (ft BGL) }\end{array}$} & \multirow[b]{2}{*}{$\begin{array}{l}\text { Sample } \\
\text { Medium }\end{array}$} & \multirow[b]{2}{*}{$\begin{array}{l}\text { Sample } \\
\text { Type }\end{array}$} & \multirow[b]{2}{*}{$\begin{array}{c}\text { EPA } \\
\text { Analytical } \\
\text { Method }\end{array}$} & \multirow[b]{2}{*}{ Sample } & \multirow[b]{2}{*}{$\begin{array}{l}\text { Analysis } \\
\text { Type }\end{array}$} & \multicolumn{4}{|c|}{ Concentration } \\
\hline & & & & & & & & $\begin{array}{c}\text { Carbon } \\
\text { Tetrachloride }\end{array}$ & Chloroform & $\begin{array}{l}\text { Methylene } \\
\text { Chloride }\end{array}$ & Units \\
\hline \multirow[t]{2}{*}{$11 / 12 / 07$} & PSB1 & 40 & Soil & СРТ & SW8260B & CNPSB1-S-19946 & Primary & 44 & $4.6 \mathrm{~J}^{\mathrm{a}}$ & $N D^{b}$ & $\mu \mathrm{g} / \mathrm{kg}$ \\
\hline & & & & & & CNPSB1-S-19946DUP & Duplicate & 53 & $5.3 \mathrm{~J}$ & ND & $\mu \mathrm{g} / \mathrm{kg}$ \\
\hline \multirow[t]{2}{*}{$11 / 13 / 07$} & PSB2 & 24 & Soil & CPT & SW8260B & CNPSB2-S-19954 & Primary & 103 & $6.1 \mathrm{~J}$ & ND & $\mu \mathrm{g} / \mathrm{kg}$ \\
\hline & & & & & & CNPSB2-S-19954REP & Replicate & 76 & 12 & ND & $\mu \mathrm{g} / \mathrm{kg}$ \\
\hline \multirow[t]{2}{*}{$11 / 13 / 07$} & PSB1 & $55-60$ & Water & CPT & 524.2 & CNPSB1-W-16246 & Primary & 309 & 11 & ND & $\mu g / L$ \\
\hline & & & & & & CNPSB1-W-16246REP & Replicate & 627 & 25 & ND & $\mu \mathrm{g} / \mathrm{L}$ \\
\hline \multirow[t]{2}{*}{$11 / 14 / 07$} & PSB3 & $55-60$ & Water & CPT & 524.2 & CNPSB3-W-16250 & Primary & 1285 & 30 & ND & $\mu g / L$ \\
\hline & & & & & & CNPSB3-W-16250REP & Replicate & 1397 & 26 & ND & $\mu g / L$ \\
\hline \multirow[t]{2}{*}{$11 / 15 / 07$} & PSB8 & $55-60$ & Water & CPT & 524.2 & CNPSB8-W-26061 & Primary & 2029 & 18 & ND & $\mu \mathrm{g} / \mathrm{L}$ \\
\hline & & & & & & CNPSB8-W-26061REP & Replicate & 1835 & 41 & ND & $\mu \mathrm{g} / \mathrm{L}$ \\
\hline \multirow[t]{2}{*}{$11 / 16 / 07$} & PSB3 & 8 & Soil & СРТ & SW8260B & CNPSB3-S-25961 & Primary & ND & ND & ND & $\mu \mathrm{g} / \mathrm{kg}$ \\
\hline & & & & & & CNPSB3-S-25961DUP & Duplicate & ND & ND & ND & $\mu \mathrm{g} / \mathrm{kg}$ \\
\hline \multirow[t]{2}{*}{$11 / 16 / 07$} & PSB3 & 28 & Soil & CPT & SW8260B & CNPSB3-S-25966 & Primary & $2.7 \mathrm{~J}$ & $1.2 \mathrm{~J}$ & ND & $\mu \mathrm{g} / \mathrm{kg}$ \\
\hline & & & & & & CNPSB3-S-25966REP & Replicate & $5.4 \mathrm{~J}$ & $1.1 \mathrm{~J}$ & ND & $\mu \mathrm{g} / \mathrm{kg}$ \\
\hline \multirow{2}{*}{$11 / 16 / 07$} & PSB3 & 32 & Soil & CPT & SW8260B & CNPSB3-S-25967 & Primary & 13 & $1.7 \mathrm{~J}$ & ND & $\mu \mathrm{g} / \mathrm{kg}$ \\
\hline & & & & & & CNPSB3-S-25967DUP & Duplicate & 12 & $1.2 \mathrm{~J}$ & ND & $\mu \mathrm{g} / \mathrm{kg}$ \\
\hline \multirow[t]{2}{*}{$11 / 16 / 07$} & PSB4 & 24 & Soil & CPT & SW8260B & CNPSB4-S-19972 & Primary & $1.4 \mathrm{~J}$ & $1.4 \mathrm{~J}$ & ND & $\mu \mathrm{g} / \mathrm{kg}$ \\
\hline & & & & & & CNPSB4-S-19972REP & Replicate & $2.7 \mathrm{~J}$ & $1.1 \mathrm{~J}$ & ND & $\mu \mathrm{g} / \mathrm{kg}$ \\
\hline \multirow[t]{2}{*}{$1 / 8 / 08$} & PMP7 & $50-60$ & Water & MW & 524.2 & CNPMP7-W-26066 & Primary & 119 & 26 & ND & $\mu \mathrm{g} / \mathrm{L}$ \\
\hline & & & & & & CNPMP7-W-26067 & Replicate & 121 & 17 & ND & $\mu \mathrm{g} / \mathrm{L}$ \\
\hline \multirow[t]{2}{*}{$1 / 9 / 08$} & PMP3 & $50-60$ & Water & MW & 524.2 & CNPMP3-W-26071 & Primary & 112 & 116 & 1.0 & $\mu g / L$ \\
\hline & & & & & & CNPMP3-W-26072 & Replicate & 52 & 115 & 1.0 & $\mu g / L$ \\
\hline \multirow[t]{2}{*}{$1 / 9 / 08$} & SB08 & $52-62$ & Water & MW & 524.2 & CNSB08-W-26070 & Primary & 32 & 1.5 & ND & $\mu \mathrm{g} / \mathrm{L}$ \\
\hline & & & & & & CNSB08-W-26070DUP & Duplicate & 33 & 1.2 & ND & $\mu g / L$ \\
\hline \multirow[t]{2}{*}{$1 / 10 / 08$} & PMP1 & $50-60$ & Water & MW & 524.2 & CNPMP1-W-26079 & Primary & 158 & 22 & ND & $\mu g / L$ \\
\hline & & & & & & CNPMP1-W-26080 & Replicate & 171 & 26 & ND & $\mu g / L$ \\
\hline \multirow[t]{2}{*}{ 1/10/08 } & PMP2 & $50-60$ & Water & MW & 524.2 & CNPMP2-W-26081 & Primary & 980 & 951 & 4.2 & $\mu g / L$ \\
\hline & & & & & & CNPMP2-W-26081DUP & Duplicate & 860 & 816 & 3.9 & $\mu \mathrm{g} / \mathrm{L}$ \\
\hline \multirow[t]{2}{*}{$1 / 24 / 08$} & MW02 & $49.5-59.5$ & Water & MW & 524.2 & CNMW02-W-26097 & Primary & ND & 11 & 1.8 & $\mu g / L$ \\
\hline & & & & & & CNMW02-W-26098 & Replicate & ND & 11 & 2.4 & $\mu g / L$ \\
\hline \multirow[t]{2}{*}{$1 / 24 / 08$} & PMP2 & $50-60$ & Water & MW & 524.2 & CNPMP2-W-26084 & Primary & 265 & 875 & 17 & $\mu \mathrm{g} / \mathrm{L}$ \\
\hline & & & & & & CNPMP2-W-26085 & Replicate & 256 & 816 & 17 & $\mu \mathrm{g} / \mathrm{L}$ \\
\hline $1 / 24 / 08$ & PMP5 & $50-60$ & Water & MW & 524.2 & CNPMP5-W-26088 & Primary & 265 & 121 & 4.6 & $\mu \mathrm{g} / \mathrm{L}$ \\
\hline & & & & & & CNPMP5-W-26089 & Replicate & 229 & 140 & 5.4 & $\mu g / L$ \\
\hline
\end{tabular}


TABLE S1.2 (Cont.)

\begin{tabular}{|c|c|c|c|c|c|c|c|c|c|c|c|}
\hline \multirow[b]{2}{*}{$\begin{array}{l}\text { Sample } \\
\text { Date }\end{array}$} & \multirow[b]{2}{*}{ Location } & \multirow{2}{*}{$\begin{array}{l}\text { Sample } \\
\text { Depth } \\
\text { (ft BGL) }\end{array}$} & \multirow[b]{2}{*}{$\begin{array}{l}\text { Sample } \\
\text { Medium }\end{array}$} & \multirow[b]{2}{*}{$\begin{array}{l}\text { Sample } \\
\text { Type }\end{array}$} & \multirow{2}{*}{$\begin{array}{c}\text { EPA } \\
\text { Analytical } \\
\text { Method }\end{array}$} & \multirow[b]{2}{*}{ Sample } & \multirow[b]{2}{*}{$\begin{array}{l}\text { Analysis } \\
\text { Type }\end{array}$} & \multicolumn{4}{|c|}{ Concentration } \\
\hline & & & & & & & & $\begin{array}{c}\text { Carbon } \\
\text { Tetrachloride }\end{array}$ & Chloroform & $\begin{array}{l}\text { Methylene } \\
\text { Chloride }\end{array}$ & Units \\
\hline $1 / 24 / 08$ & PMP6 & $50-60$ & Water & MW & 524.2 & $\begin{array}{l}\text { CNPMP6-W-26090 } \\
\text { CNPMP6-W-26090DUP }\end{array}$ & $\begin{array}{l}\text { Primary } \\
\text { Duplicate }\end{array}$ & $\begin{array}{l}26 \\
24\end{array}$ & $\begin{array}{l}31 \\
25\end{array}$ & $\begin{array}{l}2.9 \\
2.4\end{array}$ & $\begin{array}{l}\mu \mathrm{g} / \mathrm{L} \\
\mu \mathrm{g} / \mathrm{L}\end{array}$ \\
\hline $2 / 24 / 08$ & PMP2 & $50-60$ & Water & MW & 524.2 & $\begin{array}{l}\text { CNPMP2-W-25986 } \\
\text { CNPMP2-W-25988 }\end{array}$ & $\begin{array}{l}\text { Primary } \\
\text { Replicate }\end{array}$ & $\begin{array}{c}1249 \\
934\end{array}$ & $\begin{array}{l}715 \\
662\end{array}$ & $\begin{array}{l}24 \\
25\end{array}$ & $\begin{array}{l}\mu \mathrm{g} / \mathrm{L} \\
\mu \mathrm{g} / \mathrm{L}\end{array}$ \\
\hline $2 / 24 / 08$ & PMP9 & $50-60$ & Water & MW & 524.2 & $\begin{array}{l}\text { CNPMP9-W-25989 } \\
\text { CNPMP9-W-25989DUP }\end{array}$ & $\begin{array}{l}\text { Primary } \\
\text { Duplicate }\end{array}$ & $\begin{array}{l}4.7 \\
4.6\end{array}$ & $\begin{array}{l}0.8 \mathrm{~J} \\
0.8 \mathrm{~J}\end{array}$ & $\begin{array}{l}\text { ND } \\
\text { ND }\end{array}$ & $\begin{array}{l}\mu \mathrm{g} / \mathrm{L} \\
\mu \mathrm{g} / \mathrm{L}\end{array}$ \\
\hline $2 / 24 / 08$ & SB08 & $52-62$ & Water & MW & 524.2 & $\begin{array}{l}\text { CNSB08-W-25978 } \\
\text { CNSB08-W-25979 }\end{array}$ & $\begin{array}{l}\text { Primary } \\
\text { Replicate }\end{array}$ & $\begin{array}{l}44 \\
43\end{array}$ & $\begin{array}{l}1.5 \\
1.4\end{array}$ & $\begin{array}{l}\text { ND } \\
\text { ND }\end{array}$ & $\begin{array}{l}\mu \mathrm{g} / \mathrm{L} \\
\mu \mathrm{g} / \mathrm{L}\end{array}$ \\
\hline $3 / 12 / 08$ & MW03 & $50.5-60.5$ & Water & MW & 524.2 & $\begin{array}{l}\text { CNMW03-W-26001 } \\
\text { CNMW03DUP-W-26021 }\end{array}$ & $\begin{array}{l}\text { Primary } \\
\text { Replicate }\end{array}$ & $\begin{array}{l}2.3 \\
2.1\end{array}$ & $\begin{array}{l}\text { ND } \\
\text { ND }\end{array}$ & $\begin{array}{l}\text { ND } \\
\text { ND }\end{array}$ & $\begin{array}{l}\mu \mathrm{g} / \mathrm{L} \\
\mu \mathrm{g} / \mathrm{L}\end{array}$ \\
\hline 3/13/08 & PMP3 & $50-60$ & Water & MW & 524.2 & $\begin{array}{l}\text { CNPMP3-W-26007 } \\
\text { CNPMP3-W-26007DUP } \\
\text { CNPMP3DUP-W-26022 }\end{array}$ & $\begin{array}{l}\text { Primary } \\
\text { Duplicate } \\
\text { Replicate }\end{array}$ & $\begin{array}{l}3.9 \\
3.9 \\
4.2\end{array}$ & $\begin{array}{l}110 \\
114 \\
109\end{array}$ & $\begin{array}{l}10 \\
11 \\
9.2\end{array}$ & $\begin{array}{l}\mu \mathrm{g} / \mathrm{L} \\
\mu \mathrm{g} / \mathrm{L} \\
\mu \mathrm{g} / \mathrm{L}\end{array}$ \\
\hline $3 / 13 / 08$ & PMP4 & $48.75-58.75$ & Water & MW & 524.2 & $\begin{array}{l}\text { CNPMP4-W-26008 } \\
\text { CNPMP4-W-26008DUP }\end{array}$ & $\begin{array}{l}\text { Primary } \\
\text { Duplicate }\end{array}$ & $\begin{array}{l}31 \\
28\end{array}$ & $\begin{array}{l}4.4 \\
4.0\end{array}$ & $\begin{array}{l}\text { ND } \\
\text { ND }\end{array}$ & $\begin{array}{l}\mu \mathrm{g} / \mathrm{L} \\
\mu \mathrm{g} / \mathrm{L}\end{array}$ \\
\hline 3/19/08 & MW04 & $37.5-47.5$ & Water & MW & 524.2 & $\begin{array}{l}\text { CNMW04-W-26024 } \\
\text { CNMW04DUP-W-26036 }\end{array}$ & $\begin{array}{l}\text { Primary } \\
\text { Replicate }\end{array}$ & $\begin{array}{l}1.3 \\
1.5\end{array}$ & $\begin{array}{l}\text { ND } \\
\text { ND }\end{array}$ & $\begin{array}{l}\text { ND } \\
\text { ND }\end{array}$ & $\begin{array}{l}\mu \mathrm{g} / \mathrm{L} \\
\mu \mathrm{g} / \mathrm{L}\end{array}$ \\
\hline 3/19/08 & MW06 & $46.5-56.5$ & Water & MW & 524.2 & $\begin{array}{l}\text { CNMW06-W-26026 } \\
\text { CNMW06DUP-W-26037 }\end{array}$ & $\begin{array}{l}\text { Primary } \\
\text { Replicate }\end{array}$ & $\begin{array}{l}\text { ND } \\
\text { ND }\end{array}$ & $\begin{array}{l}\text { ND } \\
\text { ND }\end{array}$ & $\begin{array}{l}\text { ND } \\
\text { ND }\end{array}$ & $\begin{array}{l}\mu \mathrm{g} / \mathrm{L} \\
\mu \mathrm{g} / \mathrm{L}\end{array}$ \\
\hline $4 / 23 / 08$ & SB08 & $52-62$ & Water & MW & 524.2 & $\begin{array}{l}\text { CNSB08-W-26049 } \\
\text { CNSB08-W-26049DUP }\end{array}$ & $\begin{array}{l}\text { Primary } \\
\text { Duplicate }\end{array}$ & $\begin{array}{l}23 \\
24\end{array}$ & $\begin{array}{l}1.2 \\
1.4\end{array}$ & $\begin{array}{l}\text { ND } \\
\text { ND }\end{array}$ & $\begin{array}{l}\mu \mathrm{g} / \mathrm{L} \\
\mu \mathrm{g} / \mathrm{L}\end{array}$ \\
\hline $4 / 24 / 08$ & PMP2 & $50-60$ & Water & MW & 524.2 & $\begin{array}{l}\text { CNPMP2-W-26051 } \\
\text { CNPMP2-W-26051DUP }\end{array}$ & $\begin{array}{l}\text { Primary } \\
\text { Duplicate }\end{array}$ & $\begin{array}{l}2254 \\
2739\end{array}$ & $\begin{array}{l}476 \\
515\end{array}$ & $\begin{array}{l}8.7 \\
8.4\end{array}$ & $\begin{array}{l}\mu \mathrm{g} / \mathrm{L} \\
\mu \mathrm{g} / \mathrm{L}\end{array}$ \\
\hline $4 / 24 / 08$ & PMP5 & $50-60$ & Water & MW & 524.2 & $\begin{array}{l}\text { CNPMP5-W-26054 } \\
\text { CNPMP5DUP-W-26063 }\end{array}$ & $\begin{array}{l}\text { Primary } \\
\text { Replicate }\end{array}$ & $\begin{array}{l}357 \\
391\end{array}$ & $\begin{array}{l}101 \\
105\end{array}$ & $\begin{array}{l}2.9 \\
3.2\end{array}$ & $\begin{array}{l}\mu \mathrm{g} / \mathrm{L} \\
\mu \mathrm{g} / \mathrm{L}\end{array}$ \\
\hline $4 / 24 / 08$ & PMP9 & $50-60$ & Water & MW & 524.2 & $\begin{array}{l}\text { CNPMP9-W-26058 } \\
\text { CNPMP9-W-26058DUP }\end{array}$ & $\begin{array}{l}\text { Primary } \\
\text { Duplicate }\end{array}$ & $\begin{array}{l}5.7 \\
5.7\end{array}$ & $\begin{array}{l}1.8 \\
0.5 \mathrm{~J}\end{array}$ & $\begin{array}{l}\text { ND } \\
\text { ND }\end{array}$ & $\begin{array}{l}\mu \mathrm{g} / \mathrm{L} \\
\mu \mathrm{g} / \mathrm{L}\end{array}$ \\
\hline $4 / 24 / 08$ & SB04 & $51-61$ & Water & MW & 524.2 & $\begin{array}{l}\text { CNSB04-W-26047 } \\
\text { CNSB04DUP-W-26062 }\end{array}$ & $\begin{array}{l}\text { Primary } \\
\text { Replicate }\end{array}$ & $\begin{array}{l}16 \\
22\end{array}$ & $\begin{array}{l}0.3 \mathrm{~J} \\
0.3 \mathrm{~J}\end{array}$ & $\begin{array}{l}\text { ND } \\
\text { ND }\end{array}$ & $\begin{array}{l}\mu \mathrm{g} / \mathrm{L} \\
\mu \mathrm{g} / \mathrm{L}\end{array}$ \\
\hline $6 / 4 / 08$ & SB07R & $45-60$ & Water & MW & 524.2 & $\begin{array}{l}\text { CNSB07R-W-26623 } \\
\text { CNSB07RDUP-W-26636 }\end{array}$ & $\begin{array}{l}\text { Primary } \\
\text { Replicate }\end{array}$ & $\begin{array}{l}17 \\
13\end{array}$ & $\begin{array}{l}1.0 \\
0.8 \mathrm{~J}\end{array}$ & $\begin{array}{l}\text { ND } \\
\text { ND }\end{array}$ & $\begin{array}{l}\mu \mathrm{g} / \mathrm{L} \\
\mu \mathrm{g} / \mathrm{L}\end{array}$ \\
\hline 6/5/08 & PMP2 & $50-60$ & Water & MW & 524.2 & $\begin{array}{l}\text { CNPMP2-W-26626 } \\
\text { CNPMP2DUP-W-26637 }\end{array}$ & $\begin{array}{l}\text { Primary } \\
\text { Replicate }\end{array}$ & $\begin{array}{l}2873 \\
2155\end{array}$ & $\begin{array}{l}340 \\
272\end{array}$ & $\begin{array}{l}6.6 \\
6.1\end{array}$ & $\begin{array}{l}\mu \mathrm{g} / \mathrm{L} \\
\mu \mathrm{g} / \mathrm{L}\end{array}$ \\
\hline 6/5/08 & PMP3 & $50-60$ & Water & MW & 524.2 & $\begin{array}{l}\text { CNPMP3-W-26627 } \\
\text { CNPMP3-W-26627DUP }\end{array}$ & $\begin{array}{l}\text { Primary } \\
\text { Duplicate }\end{array}$ & $\begin{array}{l}46 \\
45\end{array}$ & $\begin{array}{l}129 \\
127\end{array}$ & $\begin{array}{l}32 \\
34\end{array}$ & $\begin{array}{l}\mu \mathrm{g} / \mathrm{L} \\
\mu \mathrm{g} / \mathrm{L}\end{array}$ \\
\hline
\end{tabular}


TABLE S1.2 (Cont.)

\begin{tabular}{|c|c|c|c|c|c|c|c|c|c|c|c|}
\hline \multirow[b]{2}{*}{$\begin{array}{l}\text { Sample } \\
\text { Date }\end{array}$} & \multirow[b]{2}{*}{ Location } & \multirow[b]{2}{*}{$\begin{array}{l}\text { Sample } \\
\text { Depth } \\
\text { (ft BGL) }\end{array}$} & \multirow[b]{2}{*}{$\begin{array}{l}\text { Sample } \\
\text { Medium }\end{array}$} & \multirow[b]{2}{*}{$\begin{array}{l}\text { Sample } \\
\text { Type }\end{array}$} & \multirow[b]{2}{*}{$\begin{array}{c}\text { EPA } \\
\text { Analytical } \\
\text { Method }\end{array}$} & \multirow[b]{2}{*}{ Sample } & \multirow[b]{2}{*}{$\begin{array}{l}\text { Analysis } \\
\text { Type }\end{array}$} & \multicolumn{4}{|c|}{ Concentration } \\
\hline & & & & & & & & $\begin{array}{c}\text { Carbon } \\
\text { Tetrachloride }\end{array}$ & Chloroform & $\begin{array}{l}\text { Methylene } \\
\text { Chloride }\end{array}$ & Units \\
\hline \multirow[t]{2}{*}{$6 / 5 / 08$} & PMP7 & $50-60$ & Water & MW & 524.2 & CNPMP7-W-26631 & Primary & 120 & 8.8 & ND & $\mu \mathrm{g} / \mathrm{L}$ \\
\hline & & & & & & CNPMP7-W-26631DUP & Duplicate & 129 & 9.7 & ND & $\mu \mathrm{g} / \mathrm{L}$ \\
\hline \multirow[t]{2}{*}{$7 / 7 / 08$} & SB04 & $51-61$ & Water & MW & 524.2 & CNSB04-W-26641 & Primary & 14 & $0.2 \mathrm{~J}$ & ND & $\mu \mathrm{g} / \mathrm{L}$ \\
\hline & & & & & & CNSB04DUP-W-26655 & Replicate & 13 & $0.2 \mathrm{~J}$ & ND & $\mu \mathrm{g} / \mathrm{L}$ \\
\hline \multirow[t]{2}{*}{$7 / 7 / 08$} & SB08 & $52-62$ & Water & MW & 524.2 & CNSB08-W-26643 & Primary & 17 & 1 & ND & $\mu \mathrm{g} / \mathrm{L}$ \\
\hline & & & & & & CNSB08-W-26643DUP & Duplicate & 15 & 1 & ND & $\mu \mathrm{g} / \mathrm{L}$ \\
\hline \multirow[t]{2}{*}{$7 / 8 / 08$} & PMP9 & $50-60$ & Water & MW & 524.2 & CNPMP9-W-26652 & Primary & 7.2 & 1.4 & ND & $\mu \mathrm{g} / \mathrm{L}$ \\
\hline & & & & & & CNPMP9DUP-W-26656 & Replicate & 8.7 & $0.6 \mathrm{~J}$ & ND & $\mu \mathrm{g} / \mathrm{L}$ \\
\hline \multirow[t]{2}{*}{$8 / 6 / 08$} & PMP6 & $50-60$ & Water & MW & 524.2 & CNPMP6-W-26668 & Primary & 108 & 8.2 & ND & $\mu \mathrm{g} / \mathrm{L}$ \\
\hline & & & & & & CNPMP6-W-26668DUP & Duplicate & 107 & 8.9 & ND & $\mu \mathrm{g} / \mathrm{L}$ \\
\hline \multirow[t]{2}{*}{$8 / 21 / 08$} & PSB13 & 32 & Soil & CPT & SW8260B & CNPSB13-S-27135 & Primary & ND & 17 & ND & $\mu \mathrm{g} / \mathrm{kg}$ \\
\hline & & & & & & CNPSB13-S-27135DUP & Duplicate & ND & 15 & ND & $\mu \mathrm{g} / \mathrm{kg}$ \\
\hline \multirow[t]{2}{*}{$8 / 21 / 08$} & PSB13 & 36 & Soil & СРT & SW8260B & CNPSB13-S-27137 & Primary & 61 & $9.6 \mathrm{~J}$ & ND & $\mu \mathrm{g} / \mathrm{kg}$ \\
\hline & & & & & & CNPSB13-S-27137DUP & Duplicate & 62 & 10 & ND & $\mu \mathrm{g} / \mathrm{kg}$ \\
\hline \multirow[t]{2}{*}{$9 / 9 / 08$} & PMP9 & $50-60$ & Water & MW & 524.2 & CNPMP9-W-26697 & Primary & 7.6 & $0.4 \mathrm{~J}$ & ND & $\mu \mathrm{g} / \mathrm{L}$ \\
\hline & & & & & & CNPMP9-W-26697DUP & Duplicate & 7.8 & $0.4 \mathrm{~J}$ & ND & $\mu \mathrm{g} / \mathrm{L}$ \\
\hline \multirow[t]{2}{*}{ 9/9/08 } & SB04 & $51-61$ & Water & MW & 524.2 & CNSB04-W-26684 & Primary & 15 & $0.3 \mathrm{~J}$ & ND & $\mu \mathrm{g} / \mathrm{L}$ \\
\hline & & & & & & CNSB04-W-26684DUP & Duplicate & 14 & $0.2 \mathrm{~J}$ & ND & $\mu \mathrm{g} / \mathrm{L}$ \\
\hline \multirow{2}{*}{ 9/10/08 } & MW05 & $34.5-44.5$ & Water & MW & 524.2 & CNMW05-W-26677 & Primary & 13 & $0.7 \mathrm{~J}$ & ND & $\mu \mathrm{g} / \mathrm{L}$ \\
\hline & & & & & & CNMW05DUP-W-26698 & Replicate & 12 & $0.6 \mathrm{~J}$ & ND & $\mu \mathrm{g} / \mathrm{L}$ \\
\hline \multirow[t]{2}{*}{ 9/10/08 } & MW08 & $38-53$ & Water & MW & 524.2 & CNMW08-W-26680 & Primary & ND & ND & ND & $\mu \mathrm{g} / \mathrm{L}$ \\
\hline & & & & & & CNMW08DUP-W-26699 & Replicate & ND & ND & ND & $\mu \mathrm{g} / \mathrm{L}$ \\
\hline
\end{tabular}

a Qualifier J indicates an estimated concentration below the method quantitation limit of $10 \mu \mathrm{g} / \mathrm{kg}$ for soil samples or $1.0 \mu \mathrm{g} / \mathrm{L}$ for water samples.

b ND, not detected at an instrument detection limit of $1.0 \mu \mathrm{g} / \mathrm{kg}$ for soil samples or $0.1 \mu \mathrm{g} / \mathrm{L}$ for water samples. 
TABLE S1.3 Results for verification organic analyses of soil and groundwater samples collected during the pilot test at Centralia.

\begin{tabular}{|c|c|c|c|c|c|c|c|c|c|c|c|c|c|}
\hline \multirow[b]{2}{*}{$\begin{array}{l}\text { Sample } \\
\text { Date }\end{array}$} & \multirow[b]{2}{*}{ Location } & \multirow[b]{2}{*}{$\begin{array}{l}\text { Sample } \\
\text { Depth } \\
\text { (ft Bgl) }\end{array}$} & \multirow[b]{2}{*}{$\begin{array}{l}\text { Sample } \\
\text { Medium }\end{array}$} & \multirow[b]{2}{*}{ Sample } & \multicolumn{4}{|c|}{ AGEM Laboratory } & \multicolumn{4}{|c|}{ Secondary Laboratory } & \multirow[b]{2}{*}{ Units } \\
\hline & & & & & $\begin{array}{l}\text { Carbon } \\
\text { Tetrachloride }\end{array}$ & Chloroform & $\begin{array}{l}\text { Methylene } \\
\text { Chloride }\end{array}$ & $\begin{array}{l}\text { Quantification } \\
\text { Limit }\end{array}$ & $\begin{array}{l}\text { Carbon } \\
\text { Tetrachloride }\end{array}$ & Chloroform & $\begin{array}{l}\text { Methylene } \\
\text { Chloride }\end{array}$ & $\begin{array}{l}\text { Quantification } \\
\text { Limit }\end{array}$ & \\
\hline $11 / 12 / 07$ & PSB1 & 20 & Soil & CNPSB1-S-16342 & 84 & 14 & $N D^{a}$ & 10 & 180 & $7 \mathrm{~J}^{\mathrm{b}}$ & ND & 8.8 & $\mu \mathrm{g} / \mathrm{kg}$ \\
\hline $11 / 13 / 07$ & PSB2 & 8 & Soil & CNPSB2-S-19950 & ND & ND & ND & 10 & $2.2 \mathrm{~J}$ & $2.8 \mathrm{~J}$ & 23 & 7.8 & $\mu \mathrm{g} / \mathrm{kg}$ \\
\hline $11 / 13 / 07$ & PSB1 & $55-60$ & Water & CNPSB1-W-16246 & 309 & 11 & ND & 1.0 & 570 & 28 & ND & 5 & $\mu \mathrm{g} / \mathrm{L}$ \\
\hline $11 / 14 / 07$ & PSB3 & $55-60$ & Water & CNPSB3-W-16250 & 1285 & 30 & ND & 1.0 & 1200 & 25 & ND & 5 & $\mu \mathrm{g} / \mathrm{L}$ \\
\hline $11 / 14 / 07$ & PSB6 & $55-60$ & Water & CNPSB6-W-16254 & 116 & 5.2 & ND & 1.0 & 57 & 5.6 & ND & 5 & $\mu \mathrm{g} / \mathrm{L}$ \\
\hline $11 / 16 / 07$ & PSB3 & 28 & Soil & CNPSB3-S-25966REP & $5.4 \mathrm{~J}$ & $1.1 \mathrm{~J}$ & ND & 10 & $8.2 \mathrm{~J}$ & ND & ND & 8.4 & $\mu \mathrm{g} / \mathrm{kg}$ \\
\hline $11 / 16 / 07$ & PSB4 & 24 & Soil & CNPSB4-S-19972 & $1.4 \mathrm{~J}$ & $1.4 \mathrm{~J}$ & ND & 10 & $4.2 \mathrm{~J}$ & ND & ND & 8.3 & $\mu \mathrm{g} / \mathrm{kg}$ \\
\hline $1 / 9 / 08$ & PMP3 & $55-60$ & Water & CNPMP3-W-26071 & 112 & 116 & 1 & 1.0 & ND & 150 & ND & 5 & $\mu \mathrm{g} / \mathrm{L}$ \\
\hline $1 / 9 / 08$ & PMP4 & $48.75-58.75$ & Water & CNPMP4-W-26068 & 36 & 12 & ND & 1.0 & 7.9 & 22 & ND & 5 & $\mu \mathrm{g} / \mathrm{L}$ \\
\hline $1 / 9 / 08$ & PMP8 & $55-60$ & Water & CNPMP8-W-26075 & 30 & 606 & 3.4 & 1.0 & 5.1 & 720 & $4.1 \mathrm{~J}$ & 5 & $\mu \mathrm{g} / \mathrm{L}$ \\
\hline $1 / 24 / 08$ & PMP2 & $50-60$ & Water & CNPMP2-W-26084 & 265 & 875 & 17 & 1.0 & 140 & $930 \mathrm{E}^{\mathrm{c}}$ & 11 & 5 & $\mu \mathrm{g} / \mathrm{L}$ \\
\hline $1 / 24 / 08$ & PMP5 & $50-60$ & Water & CNPMP5-W-26088 & 265 & 121 & 4.6 & 1.0 & ND & 100 & $3.2 \mathrm{~J}$ & 5 & $\mu \mathrm{g} / \mathrm{L}$ \\
\hline $2 / 24 / 08$ & SB08 & $52-62$ & Water & CNSB08-W-25978 & 44 & 1.5 & ND & 1.0 & 43 & ND & $3 \mathrm{~J} \mathrm{~B}^{\mathrm{d}}$ & 5 & $\mu \mathrm{g} / \mathrm{L}$ \\
\hline 2/24/08 & PMP2 & $50-60$ & Water & CNPMP2-W-25986 & 1249 & 715 & 24 & 1.0 & $720 \mathrm{E}$ & $570 \mathrm{E}$ & 13 & 5 & $\mu \mathrm{g} / \mathrm{L}$ \\
\hline $3 / 12 / 08$ & MW03 & $50.5-60.5$ & Water & CNMW03-W-26001 & 2.3 & ND & ND & 1.0 & ND & ND & $3.5 \mathrm{JB}$ & 5 & $\mu \mathrm{g} / \mathrm{L}$ \\
\hline 3/13/08 & PMP3 & $50-60$ & Water & CNPMP3-W-26007 & 3.9 & 110 & 10 & 1.0 & ND & 140 & 8.9 & 5 & $\mu \mathrm{g} / \mathrm{L}$ \\
\hline 3/13/08 & PMP7 & $50-60$ & Water & CNPMP7-W-26011 & 121 & 14 & $0.6 \mathrm{~J}$ & 1.0 & 67 & 16 & $4.4 \mathrm{~J} \mathrm{~B}$ & 5 & $\mu \mathrm{g} / \mathrm{L}$ \\
\hline 3/19/08 & MW04 & $37.5-47.4$ & Water & CNMW04-W-26024 & 1.3 & ND & ND & 1.0 & ND & ND & $6.8 \mathrm{~B}$ & 5 & $\mu \mathrm{g} / \mathrm{L}$ \\
\hline 3/19/08 & MW06 & $46.5-56.5$ & Water & CNMW06-W-26026 & ND & ND & ND & 1.0 & ND & ND & $7 \mathrm{~B}$ & 5 & $\mu \mathrm{g} / \mathrm{L}$ \\
\hline $4 / 24 / 08$ & PMP5 & $50-60$ & Water & CNPMP5-W-26054 & 357 & 101 & 2.9 & 1.0 & 280 & 81 & $20 \mathrm{~B}$ & 5 & $\mu \mathrm{g} / \mathrm{L}$ \\
\hline $4 / 24 / 08$ & SB04 & $51-61$ & Water & CNSB04-W-26047 & 16 & $0.3 \mathrm{~J}$ & ND & 1.0 & ND & ND & $9 \mathrm{JB}$ & 5 & $\mu \mathrm{g} / \mathrm{L}$ \\
\hline $6 / 4 / 08$ & SB04 & $51-61$ & Water & CNSB04-W-26622 & 25 & $0.4 \mathrm{~J}$ & ND & 1.0 & 16 & ND & ND & 5 & $\mu \mathrm{g} / \mathrm{L}$ \\
\hline $6 / 5 / 08$ & PMP7 & $50-60$ & Water & CNPMP7-W-26631 & 120 & 8.8 & ND & 1.0 & 130 & 9.1 & ND & 5 & $\mu \mathrm{g} / \mathrm{L}$ \\
\hline 7/7/08 & MW03 & $50.5-60.5$ & Water & CNMW03-W-26640 & 3.3 & ND & ND & 1.0 & 2.5 & ND & ND & 0.5 & $\mu \mathrm{g} / \mathrm{L}$ \\
\hline $7 / 8 / 08$ & PMP3 & $50-60$ & Water & CNPMP3-W-26646 & 42 & 90 & 20 & 1.0 & 33 & 92 & 17 & 0.5 & $\mu \mathrm{g} / \mathrm{L}$ \\
\hline $8 / 6 / 08$ & PMP6 & $50-60$ & Water & CNPMP6-W-26668 & 108 & 8.2 & ND & 1.0 & 110 & 8.5 & $0.29 \mathrm{~J}$ & 0.5 & $\mu \mathrm{g} / \mathrm{L}$ \\
\hline $8 / 6 / 08$ & SB07R & $45-60$ & Water & CNSB07R-W-26661 & 15 & $0.9 \mathrm{~J}$ & ND & 1.0 & 15 & 1.1 & ND & 0.5 & $\mu \mathrm{g} / \mathrm{L}$ \\
\hline 9/9/08 & MW01 & $54.5-64.5$ & Water & CNMW01-W-26673 & ND & ND & ND & 1.0 & ND & ND & ND & 0.5 & $\mu \mathrm{g} / \mathrm{L}$ \\
\hline 9/9/08 & MW04 & $37.5-47.4$ & Water & CNMW04-W-26676 & 2 & ND & ND & 1.0 & 1.8 & ND & ND & 0.5 & $\mu \mathrm{g} / \mathrm{L}$ \\
\hline 9/9/08 & PMP1 & $50-60$ & Water & CNPMP1-W-26689 & 136 & 30 & ND & 1.0 & 120 & 24 & $0.3 \mathrm{~J}$ & 0.5 & $\mu \mathrm{g} / \mathrm{L}$ \\
\hline
\end{tabular}

a ND, contaminant not detected.

b J, estimated concentraion below indicated quantification limit.

c $E$, concentration exceeded calibration range for analysis.

d B, methylene chloride was present in the associated laboratory blank. 
Supplement 2:

Analytical Results for Groundwater and Soil Samples Collected during the Pre-Injection

Baseline Sampling Activities 
TABLE S2.1 Baseline characterization: Field measurements made prior to collection of groundwater samples within and adjacent to the pilot test area.

\begin{tabular}{|c|c|c|c|c|c|c|c|c|c|c|}
\hline \multirow[b]{2}{*}{ Location } & \multirow[b]{2}{*}{ Sample } & \multirow[b]{2}{*}{$\begin{array}{l}\text { Sample } \\
\text { Date }\end{array}$} & \multirow[b]{2}{*}{$\begin{array}{l}\text { Depth } \\
\text { (ft BGL) }\end{array}$} & \multirow[b]{2}{*}{$\begin{array}{c}\text { Temperature } \\
\left({ }^{\circ} \mathrm{C}\right)\end{array}$} & \multirow[b]{2}{*}{$\mathrm{pH}$} & \multirow[b]{2}{*}{$\begin{array}{l}\text { Conductivity } \\
(\mu \mathrm{S} / \mathrm{cm})\end{array}$} & \multicolumn{3}{|c|}{ Concentration (mg/L) } & \multirow[b]{2}{*}{$\begin{array}{l}\text { ORP } \\
(\mathrm{mV})\end{array}$} \\
\hline & & & & & & & $\begin{array}{l}\text { Dissolved } \\
\text { Oxygen }\end{array}$ & $\begin{array}{l}\text { Carbon } \\
\text { Dioxide }\end{array}$ & Iron(II) & \\
\hline \multicolumn{11}{|c|}{ Samples from existing monitoring wells within and adjacent to the treatment zone } \\
\hline MW02 & CNMW02-W-16227 & $9 / 26 / 07$ & $49.5-59.5$ & 15.4 & 7.04 & 763 & 3.39 & 25 & 0 & 156 \\
\hline MW03 & CNMW03-W-16223 & $9 / 25 / 07$ & $50.5-60.5$ & 14.3 & 6.97 & 738 & 8.44 & 30 & 0 & 162 \\
\hline SB04 & CNSB04-W-16230 & $9 / 26 / 07$ & 51-61 & 19.8 & 7.03 & 760 & 6.61 & 30 & 0 & 202 \\
\hline SB07R & CNSB07R-W-16225 & $9 / 25 / 07$ & $45-60$ & 17.4 & 7.06 & 642 & 6.30 & 35 & 0.11 & 170 \\
\hline SB08 & CNSB08-W-16229 & $9 / 26 / 07$ & $52-62$ & 17.4 & 7.11 & 617 & 4.56 & 40 & 0.77 & 156 \\
\hline \multicolumn{11}{|c|}{ Direct-push samples collected within and adjacent to the treatment zone } \\
\hline PSB1 & CNPSB1-W-16245 & $11 / 12 / 07$ & $50-55$ & 18.0 & 6.89 & 630 & - & 65 & 0 & 34 \\
\hline PSB1 & CNPSB1-W-16246 & $11 / 13 / 07$ & $55-60$ & 13.4 & 6.93 & 703 & 7.65 & 55 & 0.25 & -70 \\
\hline PSB2 & CNPSB2-W-16247 & $11 / 13 / 07$ & $50-55$ & 18.3 & 6.89 & 611 & 8.31 & 50 & 0 & 80 \\
\hline PSB2 & CNPSB2-W-16248 & $11 / 13 / 07$ & $55-60$ & 17.1 & 6.97 & 625 & 4.65 & 35 & 0 & -53 \\
\hline PSB3 & CNPSB3-W-16249 & $11 / 13 / 07$ & $50-55$ & 17.2 & 6.88 & 708 & 3.65 & - & 0 & -53 \\
\hline PSB3 & CNPSB3-W-16250 & $11 / 14 / 07$ & $55-60$ & 16.7 & 6.87 & 699 & 3.28 & 65 & 0.50 & -22 \\
\hline PSB4 & CNPSB4-W-16251 & $11 / 14 / 07$ & $50-55$ & 16.2 & 6.88 & 702 & 5.00 & 50 & 0.26 & -53 \\
\hline PSB4 & CNPSB4-W-16252 & $11 / 14 / 07$ & $55-60$ & 16.8 & 6.90 & 712 & 2.88 & 50 & 0.20 & -64 \\
\hline PSB5 & CNPSB5-W-16255 & $11 / 14 / 07$ & $50-55$ & 16.9 & 6.85 & 729 & 7.24 & 50 & 0 & 9 \\
\hline PSB5 & CNPSB5-W-16256 & $11 / 14 / 07$ & $55-60$ & 17.3 & 6.94 & 811 & 3.94 & 45 & 0.98 & -311 \\
\hline PSB6 & CNPSB6-W-16253 & $11 / 14 / 07$ & $50-55$ & 17.3 & 6.86 & 705 & 5.59 & 60 & 0.73 & -46 \\
\hline PSB6 & CNPSB6-W-16254 & $11 / 14 / 07$ & $55-60$ & 17.0 & 6.92 & 717 & 4.20 & 50 & 0 & -62 \\
\hline PSB7 & CNPSB7-W-16258 & $11 / 15 / 07$ & $50-55$ & 15.3 & 6.88 & 685 & 3.70 & 45 & 1.31 & -343 \\
\hline PSB7 & CNPSB7-W-16257 & $11 / 15 / 07$ & $55-60$ & 16.9 & 6.85 & 706 & - & 55 & 2.69 & 66 \\
\hline PSB8 & CNPSB8-W-26060 & $11 / 15 / 07$ & $50-55$ & 15.9 & 6.95 & 678 & 3.78 & 30 & 0.54 & - \\
\hline PSB8 & CNPSB8-W-26061 & $11 / 15 / 07$ & $55-60$ & 16.7 & 6.87 & 649 & 4.84 & 50 & 0.77 & - \\
\hline PSB9 & CNPSB9-W-26063 & $11 / 15 / 07$ & $50-55$ & 16.9 & 6.94 & 534 & 6.53 & 55 & 0 & - \\
\hline PSB9 & CNPSB9-W-26064 & $11 / 15 / 07$ & $55-60$ & 17.7 & 6.92 & 531 & 7.14 & 35 & 0.25 & - \\
\hline
\end{tabular}


TABLE S2.2 Baseline characterization: Results of organic analyses of groundwater and soil samples collected within and adjacent to the pilot test area.

\begin{tabular}{|c|c|c|c|c|c|c|c|c|}
\hline \multirow[b]{2}{*}{ Location } & \multirow[b]{2}{*}{ Sample } & \multirow[b]{2}{*}{$\begin{array}{l}\text { Sample } \\
\text { Date }\end{array}$} & \multirow[b]{2}{*}{$\begin{array}{l}\text { Depth } \\
\text { (ft BGL) }\end{array}$} & \multirow[b]{2}{*}{ Medium } & \multicolumn{4}{|c|}{ Concentration } \\
\hline & & & & & $\begin{array}{c}\text { Carbon } \\
\text { Tetrachloride }\end{array}$ & Chloroform & $\begin{array}{c}\text { Methylene } \\
\text { Chloride }\end{array}$ & Units \\
\hline
\end{tabular}

Samples from existing monitoring wells within and adjacent to the treatment zone

$\begin{array}{lllllcccc}\text { MW02 } & \text { CNMW02-W-16227 } & 9 / 26 / 07 & 49.5-59.5 & \text { Water } & 1138 & 18 & \text { ND } & \mu \mathrm{g} / \mathrm{L} \\ \text { MW03 } & \text { CNMW03-W-16223 } & 9 / 25 / 07 & 50.5-60.5 & \text { Water } & 3.5 & \text { ND } & \text { ND } & \mu \mathrm{g} / \mathrm{L} \\ \text { SB04 } & \text { CNSB04-W-16230 } & 9 / 26 / 07 & 51-61 & \text { Water } & 36 & 0.4 \mathrm{~J} & \text { ND } & \mu \mathrm{g} / \mathrm{L} \\ \text { SB07R } & \text { CNSB07R-W-16225 } & 9 / 25 / 07 & 45-60 & \text { Water } & 50 & 2.4 & \text { ND } & \mu \mathrm{g} / \mathrm{L} \\ \text { SB08 } & \text { CNSB08-W-16229 } & 9 / 26 / 07 & 52-62 & \text { Water } & 68 & 1.8 & \text { ND } & \mu \mathrm{g} / \mathrm{L}\end{array}$

Direct-push samples collected within and adjacent to the treatment zone

\begin{tabular}{|c|c|c|c|c|c|c|c|c|}
\hline PSB1 & CNPSB1-S-16338 & $11 / 12 / 07$ & 4 & Soil & ND & ND & ND & $\mu \mathrm{g} / \mathrm{kg}$ \\
\hline PSB1 & CNPSB1-S-16339 & $11 / 12 / 07$ & 8 & Soil & $1.7 \mathrm{~J}$ & $1.2 \mathrm{~J}$ & ND & $\mu \mathrm{g} / \mathrm{kg}$ \\
\hline PSB1 & CNPSB1-S-16340 & $11 / 12 / 07$ & 12 & Soil & $4.4 \mathrm{~J}$ & $3 \mathrm{~J}$ & ND & $\mu \mathrm{g} / \mathrm{kg}$ \\
\hline PSB1 & CNPSB1-S-16341 & $11 / 12 / 07$ & 16 & Soil & 42 & $6.4 \mathrm{~J}$ & ND & $\mu \mathrm{g} / \mathrm{kg}$ \\
\hline PSB1 & CNPSB1-S-16342 & $11 / 12 / 07$ & 20 & Soil & 84 & 14 & ND & $\mu \mathrm{g} / \mathrm{kg}$ \\
\hline PSB1 & CNPSB1-S-16343 & $11 / 12 / 07$ & 24 & Soil & 219 & $3.7 \mathrm{~J}$ & ND & $\mu \mathrm{g} / \mathrm{kg}$ \\
\hline PSB1 & CNPSB1-S-16344 & $11 / 12 / 07$ & 28 & Soil & 112 & 11 & ND & $\mu \mathrm{g} / \mathrm{kg}$ \\
\hline PSB1 & CNPSB1-S-16345 & $11 / 12 / 07$ & 32 & Soil & 91 & $8.3 \mathrm{~J}$ & ND & $\mu \mathrm{g} / \mathrm{kg}$ \\
\hline PSB1 & CNPSB1-S-16346 & $11 / 12 / 07$ & 36 & Soil & 122 & $3 \mathrm{~J}$ & ND & $\mu \mathrm{g} / \mathrm{kg}$ \\
\hline PSB1 & CNPSB1-S-19946 & $11 / 12 / 07$ & 40 & Soil & 44 & $4.6 \mathrm{~J}$ & ND & $\mu \mathrm{g} / \mathrm{kg}$ \\
\hline PSB1 & CNPSB1-W-16245 & $11 / 12 / 07$ & $50-55$ & Water & 782 & 27 & ND & $\mu \mathrm{g} / \mathrm{L}$ \\
\hline PSB1 & CNPSB1-W-16246 & $11 / 13 / 07$ & $55-60$ & Water & 309 & 11 & ND & $\mu \mathrm{g} / \mathrm{L}$ \\
\hline PSB2 & CNPSB2-S-19949 & $11 / 13 / 07$ & 3.8 & Soil & ND & ND & ND & $\mu \mathrm{g} / \mathrm{kg}$ \\
\hline PSB2 & CNPSB2-S-19950 & $11 / 13 / 07$ & 8 & Soil & ND & ND & ND & $\mu \mathrm{g} / \mathrm{kg}$ \\
\hline PSB2 & CNPSB2-S-19951 & $11 / 13 / 07$ & 12 & Soil & ND & ND & ND & $\mu \mathrm{g} / \mathrm{kg}$ \\
\hline PSB2 & CNPSB2-S-19952 & $11 / 13 / 07$ & 16 & Soil & ND & ND & ND & $\mu \mathrm{g} / \mathrm{kg}$ \\
\hline PSB2 & CNPSB2-S-19953 & $11 / 13 / 07$ & 20 & Soil & $9 \mathrm{~J}$ & $3.5 \mathrm{~J}$ & ND & $\mu \mathrm{g} / \mathrm{kg}$ \\
\hline PSB2 & CNPSB2-S-19954 & $11 / 13 / 07$ & 24 & Soil & 103 & $6.1 \mathrm{~J}$ & ND & $\mu \mathrm{g} / \mathrm{kg}$ \\
\hline PSB2 & CNPSB2-S-19955 & $11 / 13 / 07$ & 28 & Soil & 66 & $8.8 \mathrm{~J}$ & ND & $\mu \mathrm{g} / \mathrm{kg}$ \\
\hline PSB2 & CNPSB2-S-19956 & $11 / 13 / 07$ & 32 & Soil & 152 & $5.3 \mathrm{~J}$ & ND & $\mu \mathrm{g} / \mathrm{kg}$ \\
\hline PSB2 & CNPSB2-S-19957 & $11 / 13 / 07$ & 36 & Soil & 173 & $3.5 \mathrm{~J}$ & ND & $\mu \mathrm{g} / \mathrm{kg}$ \\
\hline PSB2 & CNPSB2-S-19958 & $11 / 13 / 07$ & 40 & Soil & 62 & $2.9 \mathrm{~J}$ & ND & $\mu \mathrm{g} / \mathrm{kg}$ \\
\hline PSB2 & CNPSB2-W-16247 & $11 / 13 / 07$ & $50-55$ & Water & 405 & 3.4 & ND & $\mu \mathrm{g} / \mathrm{L}$ \\
\hline PSB2 & CNPSB2-W-16248 & $11 / 13 / 07$ & $55-60$ & Water & 16 & 2.2 & ND & $\mu \mathrm{g} / \mathrm{L}$ \\
\hline PSB3 & CNPSB3-S-25970 & $11 / 16 / 07$ & 4 & Soil & ND & ND & ND & $\mu \mathrm{g} / \mathrm{kg}$ \\
\hline PSB3 & CNPSB3-S-25961 & $11 / 16 / 07$ & 8 & Soil & ND & ND & ND & $\mu \mathrm{g} / \mathrm{kg}$ \\
\hline PSB3 & CNPSB3-S-25962 & $11 / 16 / 07$ & 12 & Soil & ND & ND & ND & $\mu \mathrm{g} / \mathrm{kg}$ \\
\hline PSB3 & CNPSB3-S-25963 & $11 / 16 / 07$ & 16 & Soil & ND & ND & ND & $\mu \mathrm{g} / \mathrm{kg}$ \\
\hline PSB3 & CNPSB3-S-25964 & $11 / 16 / 07$ & 20 & Soil & ND & ND & ND & $\mu \mathrm{g} / \mathrm{kg}$ \\
\hline PSB3 & CNPSB3-S-25965 & $11 / 16 / 07$ & 24 & Soil & $1.2 \mathrm{~J}$ & ND & ND & $\mu \mathrm{g} / \mathrm{kg}$ \\
\hline PSB3 & CNPSB3-S-25966 & $11 / 16 / 07$ & 28 & Soil & $2.7 \mathrm{~J}$ & $1.2 \mathrm{~J}$ & ND & $\mu \mathrm{g} / \mathrm{kg}$ \\
\hline PSB3 & CNPSB3-S-25967 & $11 / 16 / 07$ & 32 & Soil & 13 & $1.7 \mathrm{~J}$ & ND & $\mu \mathrm{g} / \mathrm{kg}$ \\
\hline PSB3 & CNPSB3-S-25968 & $11 / 16 / 07$ & 36 & Soil & 20 & $2.4 \mathrm{~J}$ & ND & $\mu \mathrm{g} / \mathrm{kg}$ \\
\hline PSB3 & CNPSB3-S-25969 & $11 / 16 / 07$ & 40 & Soil & 15 & $4.3 \mathrm{~J}$ & ND & $\mu \mathrm{g} / \mathrm{kg}$ \\
\hline PSB3 & CNPSB3-W-16249 & $11 / 13 / 07$ & $50-55$ & Water & 987 & 50 & ND & $\mu \mathrm{g} / \mathrm{L}$ \\
\hline PSB3 & CNPSB3-W-16250 & $11 / 14 / 07$ & $55-60$ & Water & 1285 & 30 & ND & $\mu \mathrm{g} / \mathrm{L}$ \\
\hline PSB4 & CNPSB4-S-19966 & $11 / 16 / 07$ & 4 & Soil & ND & ND & ND & $\mu \mathrm{g} / \mathrm{kg}$ \\
\hline PSB4 & CNPSB4-S-19967 & $11 / 16 / 07$ & 8 & Soil & ND & ND & ND & $\mu \mathrm{g} / \mathrm{kg}$ \\
\hline PSB4 & CNPSB4-S-19968 & $11 / 16 / 07$ & 12 & Soil & ND & ND & ND & $\mu \mathrm{g} / \mathrm{kg}$ \\
\hline PSB4 & CNPSB4-S-19970 & $11 / 16 / 07$ & 16 & Soil & ND & ND & ND & $\mu \mathrm{g} / \mathrm{kg}$ \\
\hline PSB4 & CNPSB4-S-19971 & $11 / 16 / 07$ & 20 & Soil & ND & ND & ND & $\mu \mathrm{g} / \mathrm{kg}$ \\
\hline PSB4 & CNPSB4-S-19972 & $11 / 16 / 07$ & 24 & Soil & $1.4 \mathrm{~J}$ & $1.4 \mathrm{~J}$ & ND & $\mu \mathrm{g} / \mathrm{kg}$ \\
\hline PSB4 & CNPSB4-S-19973 & $11 / 16 / 07$ & 28 & Soil & 24 & $4.7 \mathrm{~J}$ & ND & $\mu \mathrm{g} / \mathrm{kg}$ \\
\hline
\end{tabular}


TABLE S2.2 (Cont.).

\begin{tabular}{|c|c|c|c|c|c|c|c|c|}
\hline \multirow[b]{2}{*}{ Location } & \multirow[b]{2}{*}{ Sample } & \multirow[b]{2}{*}{$\begin{array}{l}\text { Sample } \\
\text { Date }\end{array}$} & \multirow[b]{2}{*}{$\begin{array}{l}\text { Depth } \\
\text { (ft BGL) }\end{array}$} & \multirow[b]{2}{*}{ Medium } & \multicolumn{4}{|c|}{ Concentration } \\
\hline & & & & & $\begin{array}{c}\text { Carbon } \\
\text { Tetrachloride }\end{array}$ & Chloroform & $\begin{array}{l}\text { Methylene } \\
\text { Chloride }\end{array}$ & Units \\
\hline \multicolumn{9}{|c|}{ Direct-push samples collected within and adjacent to the treatment zone (cont.) } \\
\hline PSB4 & CNPSB4-S-19974 & $11 / 16 / 07$ & 32 & Soil & 89 & $7.8 \mathrm{~J}$ & ND & $\mu \mathrm{g} / \mathrm{kg}$ \\
\hline PSB4 & CNPSB4-S-19975 & $11 / 16 / 07$ & 36 & Soil & 58 & $6 \mathrm{~J}$ & ND & $\mu \mathrm{g} / \mathrm{kg}$ \\
\hline PSB4 & CNPSB4-S-25960 & $11 / 16 / 07$ & 40 & Soil & 57 & $9.7 \mathrm{~J}$ & ND & $\mu \mathrm{g} / \mathrm{kg}$ \\
\hline PSB4 & CNPSB4-W-16251 & $11 / 14 / 07$ & $50-55$ & Water & 908 & 17 & ND & $\mu \mathrm{g} / \mathrm{L}$ \\
\hline PSB4 & CNPSB4-W-16252 & $11 / 14 / 07$ & $55-60$ & Water & 830 & 25 & ND & $\mu \mathrm{g} / \mathrm{L}$ \\
\hline PSB5 & CNPSB5-W-16255 & $11 / 14 / 07$ & $50-55$ & Water & 770 & 16 & ND & $\mu \mathrm{g} / \mathrm{L}$ \\
\hline PSB5 & CNPSB5-W-16256 & $11 / 14 / 07$ & $55-60$ & Water & 460 & 80 & $0.6 \mathrm{~J}$ & $\mu \mathrm{g} / \mathrm{L}$ \\
\hline PSB6 & CNPSB6-W-16253 & $11 / 14 / 07$ & $50-55$ & Water & 114 & 2.5 & ND & $\mu \mathrm{g} / \mathrm{L}$ \\
\hline PSB6 & CNPSB6-W-16254 & $11 / 14 / 07$ & $55-60$ & Water & 116 & 5.2 & ND & $\mu \mathrm{g} / \mathrm{L}$ \\
\hline PSB7 & CNPSB7-W-16258 & $11 / 15 / 07$ & $50-55$ & Water & 326 & 82 & $0.7 \mathrm{~J}$ & $\mu \mathrm{g} / \mathrm{L}$ \\
\hline PSB7 & CNPSB7-W-16257 & $11 / 15 / 07$ & $55-60$ & Water & 148 & 6.6 & ND & $\mu \mathrm{g} / \mathrm{L}$ \\
\hline PSB8 & CNPSB8-W-26060 & $11 / 15 / 07$ & $50-55$ & Water & 2646 & 103 & 2.5 & $\mu \mathrm{g} / \mathrm{L}$ \\
\hline PSB8 & CNPSB8-W-26061 & $11 / 15 / 07$ & $55-60$ & Water & 2029 & 18 & ND & $\mu \mathrm{g} / \mathrm{L}$ \\
\hline PSB9 & CNPSB9-W-26063 & $11 / 15 / 07$ & $50-55$ & Water & ND & $0.2 \mathrm{~J}$ & ND & $\mu \mathrm{g} / \mathrm{L}$ \\
\hline PSB9 & CNPSB9-W-26064 & $11 / 15 / 07$ & $55-60$ & Water & 1.4 & $0.2 \mathrm{~J}$ & ND & $\mu \mathrm{g} / \mathrm{L}$ \\
\hline
\end{tabular}

a ND, not detected at instrument detection limits of $0.1 \mu \mathrm{g} / \mathrm{L}$ for water samples and $1.0 \mu \mathrm{g} / \mathrm{kg}$ for soil samples.

b Qualifier J indicates an estimated concentration below the purge-and-trap method quantitation limit of $1.0 \mu \mathrm{g} / \mathrm{L}$ for water samples or $10 \mu \mathrm{g} / \mathrm{kg}$ for soil samples. 
TABLE S2.3 Baseline characterization: Results for geochemical and attenuation parameter analyses of groundwater samples collected within and adjacent to the treatment zone.

\begin{tabular}{|c|c|c|c|c|c|c|c|c|c|c|c|c|c|}
\hline \multirow[b]{2}{*}{ Location } & \multirow[b]{2}{*}{ Sample } & \multirow[b]{2}{*}{$\begin{array}{l}\text { Sample } \\
\text { Date }\end{array}$} & \multirow[b]{2}{*}{$\begin{array}{l}\text { Depth } \\
\text { (ft BGL) }\end{array}$} & \multicolumn{10}{|c|}{ Concentration ${ }^{\mathrm{a}}(\mathrm{mg} / \mathrm{L})$} \\
\hline & & & & Alkalinity & Aluminum & Bromide & Calcium & Chloride & Iron & Magnesium & Manganese & $\begin{array}{l}\text { Nitrate } \\
\text { as N }\end{array}$ & $\begin{array}{l}\text { Nitrate-Nitrite } \\
\text { Nitrogen }\end{array}$ \\
\hline \multicolumn{14}{|c|}{ Samples from existing monitoring wells within and adjacent to the treatment zone } \\
\hline MW02 & CNMW02-W-16227 & 9/26/07 & $49.5-59.5$ & 340 & $0.2 \mathrm{U}$ & - & 69.4 & 7.5 & $0.1 \mathrm{U}$ & 26.6 & $0.015 \mathrm{U}$ & 8.0 & 9.1 \\
\hline MW03 & CNMW03-W-16223 & $9 / 25 / 07$ & $50.5-60.5$ & 330 & $0.2 \mathrm{U}$ & - & 77.9 & 25 & $0.1 \mathrm{U}$ & 30.0 & $0.015 \mathrm{U}$ & 7.9 & - \\
\hline SB04 & CNSB04-W-16230 & $9 / 26 / 07$ & $51-61$ & 370 & $0.2 \mathrm{U}$ & - & 77.4 & 42 & $0.1 \mathrm{U}$ & 29.5 & $0.015 \mathrm{U}$ & 1.3 & 2.0 \\
\hline SB08 & CNSB08-W-16229 & $9 / 26 / 07$ & $52-62$ & 300 & $0.2 \mathrm{U}$ & - & 71.7 & 14 & $0.1 \mathrm{U}$ & 30.1 & $0.015 \mathrm{U}$ & 0.94 & 1.1 \\
\hline \multicolumn{14}{|c|}{ Direct-push samples collected within and adjacent to the treatment zone } \\
\hline PSB1 & CNPSB1-W-16245 & $11 / 12 / 07$ & $50-55$ & 530 & $0.2 \mathrm{U}$ & 0.24 & 73 & 13 & $0.1 \mathrm{U}$ & 26.5 & 0.147 & 7.6 & 7.8 \\
\hline PSB1 & CNPSB1-W-16246 & $11 / 13 / 07$ & $55-60$ & 340 & $0.2 \mathrm{U}$ & 0.14 & 72.5 & 12 & $0.1 \mathrm{U}$ & 27.9 & 0.0816 & 8.4 & 8.3 \\
\hline PSB2 & CNPSB2-W-16247 & $11 / 13 / 07$ & $50-55$ & 330 & $0.2 \mathrm{U}$ & 0.19 & 77.8 & 15 & $0.1 \mathrm{U}$ & 27.7 & 0.0523 & 6.7 & 5.8 \\
\hline PSB2 & CNPSB2-W-16248 & $11 / 13 / 07$ & $55-60$ & 340 & $0.2 \mathrm{U}$ & 0.40 & 72.3 & 21 & $0.1 \mathrm{U}$ & 28.4 & 0.595 & 3.3 & 2.8 \\
\hline PSB3 & CNPSB3-W-16249 & $11 / 13 / 07$ & $50-55$ & - & $0.2 \mathrm{U}$ & 0.49 & 84.5 & 25 & $0.2 \mathrm{U}$ & 30.0 & 0.19 & 8.9 & 9.1 \\
\hline PSB3 & CNPSB3-W-16250 & $11 / 14 / 07$ & $55-60$ & 360 & $0.2 \mathrm{U}$ & 0.49 & 79.7 & 17 & $0.2 \mathrm{U}$ & 29.7 & 0.299 & 11 & 10 \\
\hline PSB4 & CNPSB4-W-16252 & $11 / 14 / 07$ & $55-60$ & 350 & $0.2 \mathrm{U}$ & 0.31 & 80.8 & 14 & $0.2 \mathrm{U}$ & 30.3 & 0.69 & 10 & 11 \\
\hline PSB5 & CNPSB5-W-16255 & $11 / 14 / 07$ & $50-55$ & 350 & $0.2 \mathrm{U}$ & 0.95 & 78.6 & 51 & $0.2 \mathrm{U}$ & 28.5 & 0.0763 & 3.4 & 3.1 \\
\hline PSB5 & CNPSB5-W-16256 & $11 / 14 / 07$ & $55-60$ & 340 & $0.2 \mathrm{U}$ & 2.0 & 80.9 & 96 & $0.2 \mathrm{U}$ & 30.9 & 1.29 & 2.4 & 2.5 \\
\hline PSB6 & CNPSB6-W-16253 & $11 / 14 / 07$ & $50-55$ & 330 & $0.2 \mathrm{U}$ & 0.72 & 92.4 & 41 & $0.2 \mathrm{U}$ & 33.6 & 0.35 & 8.1 & 8.6 \\
\hline PSB6 & CNPSB6-W-16254 & $11 / 14 / 07$ & $55-60$ & 350 & $0.2 \mathrm{U}$ & 0.26 & 88.3 & 19 & $0.2 \mathrm{U}$ & 33.6 & 0.506 & 11 & 12 \\
\hline PSB7 & CNPSB7-W-16258 & $11 / 15 / 07$ & $50-55$ & 340 & $0.2 \mathrm{U}$ & 0.49 & 77.1 & 24 & $0.2 \mathrm{U}$ & 28.2 & 1.05 & 8.7 & 8.5 \\
\hline PSB7 & CNPSB7-W-16257 & $11 / 15 / 07$ & $55-60$ & 400 & $0.2 \mathrm{U}$ & 0.43 & 85.1 & 19 & $0.2 \mathrm{U}$ & 30.8 & $0.015 \mathrm{U}$ & 11 & 10 \\
\hline PSB8 & CNPSB8-W-26060 & $11 / 15 / 07$ & $50-55$ & 360 & $0.2 \mathrm{U}$ & 1.0 & 81.8 & 36 & $0.2 \mathrm{U}$ & 29.5 & 0.219 & 5.0 & 4.4 \\
\hline PSB8 & CNPSB8-W-26061 & $11 / 15 / 07$ & $55-60$ & 350 & $0.2 \mathrm{U}$ & 0.78 & 78.4 & 37 & $0.2 \mathrm{U}$ & 30.6 & 0.704 & 4.1 & 3.4 \\
\hline PSB9 & CNPSB9-W-26063 & $11 / 15 / 07$ & $50-55$ & 330 & $0.2 \mathrm{U}$ & 0.15 & 71.4 & 9.2 & $0.2 \mathrm{U}$ & 27.6 & 0.0795 & 0.27 & 0.15 \\
\hline PSB9 & CNPSB9-W-26064 & $11 / 15 / 07$ & $55-60$ & 330 & $0.2 \mathrm{U}$ & 0.15 & 70.4 & 8.7 & $0.2 \mathrm{U}$ & 28.1 & $0.015 \mathrm{U}$ & 0.21 & 0.12 \\
\hline \multicolumn{14}{|c|}{ Water used for preparation of EHC injection mixture } \\
\hline $\mathrm{QC}$ & CNPUBL-W-26062 & $11 / 15 / 07$ & - & 350 & $0.2 \mathrm{U}$ & 0.29 & 74.4 & 12 & $0.14 \mathrm{~B}$ & 26.7 & 0.0981 & 0.15 & - \\
\hline
\end{tabular}


TABLE S2.3 (Cont.)

\begin{tabular}{|c|c|c|c|c|c|c|c|c|c|c|c|c|c|}
\hline \multirow[b]{2}{*}{ Location } & \multirow[b]{2}{*}{ Sample } & \multirow{2}{*}{$\begin{array}{l}\text { Sample } \\
\text { Date }\end{array}$} & \multirow{2}{*}{$\begin{array}{l}\text { Depth } \\
\text { (ft BGL) }\end{array}$} & \multicolumn{9}{|c|}{ Concentration $^{\mathrm{a}}(\mathrm{mg} / \mathrm{L})$} & \multirow{2}{*}{$\begin{array}{c}\text { Methane } \\
(\mu \mathrm{g} / \mathrm{L})\end{array}$} \\
\hline & & & & Phosphate & Phosphorus & Potassium & Silicon & Sodium & Sulfate & Sulfide & Zinc & TOC $^{b}$ & \\
\hline \multicolumn{14}{|c|}{ Sample from existing monitoring wells within and adjacent to the treatment zone } \\
\hline MW02 & CNMW02-W-16227 & 9/26/07 & $49.5-59.5$ & $0.2 \mathrm{U}$ & $0.25 \mathrm{U}$ & $5 \mathrm{U}$ & 14.4 & 57.0 & 17 & $0.02 \mathrm{U}$ & $0.02 \mathrm{U}$ & 1.3 & 13 \\
\hline MW03 & CNMW03-W-16223 & $9 / 25 / 07$ & $50.5-60.5$ & $0.2 \mathrm{U}$ & $0.25 \mathrm{U}$ & $5 \mathrm{U}$ & 15.6 & 44.2 & 7.6 & $0.02 \mathrm{U}$ & $0.02 \mathrm{U}$ & 1.5 & $<2^{\mathrm{C}}$ \\
\hline SB04 & CNSB04-W-16230 & $9 / 26 / 07$ & $51-61$ & $0.2 \mathrm{U}$ & $0.25 \mathrm{U}$ & $5 \mathrm{U}$ & 16.4 & 58.0 & 8.5 & $0.02 \mathrm{U}$ & $0.02 \mathrm{U}$ & 1.2 & $<2$ \\
\hline SB07R & CNSB07R-W-16225 & $9 / 25 / 07$ & $45-60$ & $0.2 \mathrm{U}$ & $0.25 \mathrm{U}$ & $5 \mathrm{U}$ & 14.9 & 33.7 & 14 & $0.02 \mathrm{U}$ & 0.0546 & 1.0 & $<2$ \\
\hline SB08 & CNSB08-W-16229 & $9 / 26 / 07$ & $52-62$ & $0.2 \mathrm{U}$ & $0.25 U$ & $5 \mathrm{U}$ & 16.7 & 26.8 & 6.9 & $0.02 \mathrm{U}$ & $0.02 \mathrm{U}$ & 1.3 & $<2$ \\
\hline \multicolumn{14}{|c|}{ Direct-push samples collected within and adjacent to the treatment zone } \\
\hline PSB1 & CNPSB1-W-16245 & $11 / 12 / 07$ & $50-55$ & $0.2 \mathrm{U}$ & $0.25 \mathrm{U}$ & $5 \mathrm{U}$ & 13.8 & 59.6 & 8.3 & 0.082 & $0.02 \mathrm{U}$ & 10.4 & $<2$ \\
\hline PSB1 & CNPSB1-W-16246 & $11 / 13 / 07$ & $55-60$ & $0.2 \mathrm{U}$ & $0.25 \mathrm{U}$ & $5 U$ & 14.5 & 57.6 & 6.6 & $0.02 \mathrm{U}$ & $0.02 \mathrm{U}$ & 11 & 2 \\
\hline PSB2 & CNPSB2-W-16247 & $11 / 13 / 07$ & $50-55$ & $0.2 \mathrm{U}$ & $0.25 \mathrm{U}$ & $5 \mathrm{U}$ & 15.3 & 46.2 & 3.8 & $0.02 \mathrm{U}$ & $0.02 \mathrm{U}$ & 5.2 & $<2$ \\
\hline PSB2 & CNPSB2-W-16248 & $11 / 13 / 07$ & $55-60$ & $0.2 \mathrm{U}$ & $0.25 \mathrm{U}$ & $5 \mathrm{U}$ & 13.8 & 40.9 & 4.7 & 0.19 & $0.02 \mathrm{U}$ & 2.4 & 2.7 \\
\hline PSB3 & CNPSB3-W-16249 & $11 / 13 / 07$ & $50-55$ & $0.2 \mathrm{U}$ & $0.25 \mathrm{U}$ & $5 \mathrm{U}$ & 12.0 & 53.7 & 21 & $0.02 \mathrm{U}$ & $0.02 \mathrm{U}$ & 2.8 & $<2$ \\
\hline PSB3 & CNPSB3-W-16250 & $11 / 14 / 07$ & $55-60$ & $0.2 \mathrm{U}$ & $0.25 \mathrm{U}$ & $5 \mathrm{U}$ & 14.7 & 65.8 & 20 & $0.02 \mathrm{U}$ & $0.02 \mathrm{U}$ & 4.1 & $<2$ \\
\hline PSB4 & CNPSB4-W-16251 & $11 / 14 / 07$ & $50-55$ & $0.2 \mathrm{U}$ & $0.25 \mathrm{U}$ & $5 \mathrm{U}$ & 15.8 & 56.9 & 22 & 0.024 & $0.02 \mathrm{U}$ & 1.7 & $<2$ \\
\hline PSB4 & CNPSB4-W-16252 & $11 / 14 / 07$ & $55-60$ & $0.2 \mathrm{U}$ & $0.25 \mathrm{U}$ & $5 \mathrm{U}$ & 12.6 & 66.4 & 23 & 0.026 & $0.02 \mathrm{U}$ & 2.2 & 3.8 \\
\hline PSB5 & CNPSB5-W-16255 & $11 / 14 / 07$ & $50-55$ & $0.2 \mathrm{U}$ & $0.25 \mathrm{U}$ & $5 U$ & 16.1 & 72.9 & 6.1 & $0.02 \mathrm{U}$ & $0.02 \mathrm{U}$ & 1.8 & $<2$ \\
\hline PSB5 & CNPSB5-W-16256 & $11 / 14 / 07$ & $55-60$ & $0.2 \mathrm{U}$ & $0.25 \mathrm{U}$ & $5 U$ & 11.7 & 74.6 & 10 & $0.02 \mathrm{U}$ & $0.02 \mathrm{U}$ & 2.2 & $<2$ \\
\hline PSB6 & CNPSB6-W-16253 & $11 / 14 / 07$ & $50-55$ & $0.2 \mathrm{U}$ & $0.25 \mathrm{U}$ & $5 U$ & 13.4 & 40.1 & 9.3 & $0.02 \mathrm{U}$ & $0.02 \mathrm{U}$ & 3.6 & $<2$ \\
\hline PSB6 & CNPSB6-W-16254 & $11 / 14 / 07$ & $55-60$ & $0.2 \mathrm{U}$ & $0.25 \mathrm{U}$ & $5 \mathrm{U}$ & 15.6 & 52.1 & 16 & $0.02 \mathrm{U}$ & $0.02 \mathrm{U}$ & 5.1 & 4.1 \\
\hline PSB7 & CNPSB7-W-16258 & 11/15/07 & $50-55$ & $0.2 \mathrm{U}$ & $0.25 U$ & 5.98 & 13.2 & 54.5 & 13 & $0.02 \mathrm{U}$ & $0.02 \mathrm{U}$ & 3.6 & 3.4 \\
\hline PSB7 & CNPSB7-W-16257 & 11/15/07 & $55-60$ & $0.2 \mathrm{U}$ & $0.25 \mathrm{U}$ & $5 \mathrm{U}$ & 14.7 & 58.6 & 16 & $0.02 \mathrm{U}$ & $0.02 \mathrm{U}$ & 4.8 & $<2$ \\
\hline PSB8 & CNPSB8-W-26060 & $11 / 15 / 07$ & $50-55$ & $0.2 \mathrm{U}$ & $0.25 \mathrm{U}$ & $5 \mathrm{U}$ & 14.0 & 56.5 & 18 & $0.02 \mathrm{U}$ & $0.02 \mathrm{U}$ & 2.3 & 2.5 \\
\hline PSB8 & CNPSB8-W-26061 & $11 / 15 / 07$ & $55-60$ & $0.2 \mathrm{U}$ & $0.25 \mathrm{U}$ & $5 U$ & 12.6 & 45.0 & 5.1 & $0.02 \mathrm{U}$ & $0.02 \mathrm{U}$ & 2.6 & $<2$ \\
\hline PSB9 & CNPSB9-W-26063 & $11 / 15 / 07$ & $50-55$ & $0.2 \mathrm{U}$ & $0.25 \mathrm{U}$ & $5 \mathrm{U}$ & 14.5 & 32.4 & 1.8 & $0.02 \mathrm{U}$ & $0.02 \mathrm{U}$ & 5.7 & $<2$ \\
\hline PSB9 & CNPSB9-W-26064 & $11 / 15 / 07$ & $55-60$ & $0.2 \mathrm{U}$ & $0.25 \mathrm{U}$ & $5 \mathrm{U}$ & 14.6 & 31.8 & 1.7 & $0.02 \mathrm{U}$ & $0.02 \mathrm{U}$ & 1.0 & $<2$ \\
\hline \multicolumn{14}{|c|}{ Water used for preparation of EHC injection mixture } \\
\hline QC & CNPUBL-W-26062 & $11 / 15 / 07$ & - & $0.2 \mathrm{U}$ & 0.377 & 11.7 & 12.7 & 45.6 & 40 & $0.02 \mathrm{U}$ & $0.02 \mathrm{U}$ & 2.0 & $<2$ \\
\hline
\end{tabular}

a Qualifiers: B, constituent detected in associated blank; $U$, constituent not detected at the indicated reporting limit.

b TOC, total organic carbon.

c Not detected at the reporting limit of $2.0 \mu \mathrm{g} / \mathrm{L}$. 


\section{Supplement 3:}

Injection Program Summary Report for the Centralia ISCR Pilot Test, Prepared by Adventus Americas, Inc., March 4, 2008 


\section{Argonne}

April 23, 2008
Lorraine M. LaFreniere, Ph.D.

Manager, Applied Geosciences and Environmental Management Section

Environmental Science Division Argonne National Laboratory 9700 South Cass Avenue, BIdg. 203 Argonne, IL 60439-4843

1-630-252-7969 phone 1-630-252-5747 fax lafreniere@anl.gov

Kirk Hoeffner, Professional Geologist

Underground Injection Control Program

Geology Section, Bureau of Water

Kansas Department of Health and Environment

1000 SW Jackson

Topeka, KS 66612-1367

Subject: Field Pilot Study Report for Remediation at the Former CCC/USDA Grain Storage Facility at Centralia, Kansas, ANL/EVS/AGEM/CHRON-1135

Dear Mr. Hoeffner:

Attached, at the request of Caroline Roe of the Commodity Credit Corporation, U.S. Department of Agriculture (CCC/USDA), is the document Remediation at the Former CCC/USDA Grain Storage Facility at Centralia, Kansas - Field Pilot Study Report: Treatment of Carbon Tetrachloride in Soil and Groundwater Using EHC and EHC-A ${ }^{T M}$ ISCR Technology. This report was prepared by the Adventus Group for Argonne National Laboratory, which provides technical support for the CCC/USDA's remediation project at Centralia. The work is reported as Adventus Project No. AA16-083b.

The attached report is part of the permitting process related to the remediation project at Centralia. Please direct questions and comments to me. Let me know if we can do anything to facilitate your review.

Sincerely,

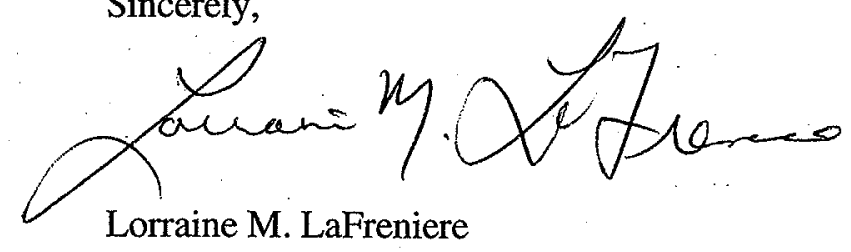

LML:rs

Attachment: Adventus Field Pilot Study Report and Appendices

cc (with attachment):

C. Roe (CCC/USDA)

G. Fremerman (CCC/USDA)

S. Gilmore (CCC/USDA)

D. Steck (CCC/USDA)

Chron 1135 


\section{(1) ADVENTUS}

Proven Soil, Sediment, and Groundwater Remediation Technologies

VIA E-MAIL: lafreniere@anl.gov

March 4, 2008

Lorraine M. LaFreniere

Argonne National Laboratory

9700 South Cass Avenue

Argonne, IL 60439

\section{Subject: $\quad$ Remediation at the Former CCC/USDA Grain Storage Facility at Centralia, Kansas - Field Pilot Study Report \\ Treatment of Carbon Tetrachloride in Soil and Groundwater using EHC and EHC-A ${ }^{\mathrm{TM}}$ ISCR Technology Adventus Project No. AAl6-083b}

Dear Ms. LaFreniere:

This report summarizes pilot-scale field injection activities conducted from November 27 to December 5, 2007. Activities were generally conducted in accordance with the injection permit application, amendment, and KDHE approval letter (Appendix A). However, as is generally the case with remedial injection projects, some adjustments to plans are made in the field. Accordingly, this report is intended to provide sufficient information for your files to be complete with regard to the actual activities conducted. As you are aware, we had some email correspondence during the course of the injection work to keep KDHE apprised of observed injection pressures as well as the occurrence of daylighting during the course of the work. In order to make this report complete, this email correspondence is included as Appendix B,

\section{SITE BACKGROUND}

Investigations conducted on behalf of the CCC/USDA by Argonne National Laboratory (ANL) have demonstrated that groundwater at the Centralia site is contaminated with carbon tetrachloride at levels that exceed the Kansas Tier 2 Risk-Based Screening Level (RBSL) and the U.S. Environmental Protection Agency's maximum contaminant level of $5.0 \mu \mathrm{g} / \mathrm{L}$ for this compound. Groundwater sampling and analyses conducted by Argonne under a monitoring program approved by the Kansas Department of Health and Environment (KDHE) indicated that the carbon tetrachloride levels at several locations in the groundwater plume have increased since twice yearly monitoring of the site began in September 2005. The identified groundwater contamination currently poses no unacceptable health risks, in view of the absence of potential human receptors in the vicinity of the former CCC/USDA facility. 
Carbon tetrachloride contamination has also been identified at Centralia in subsurface soils at concentrations on the order of the Kansas Tier 2 RBSL of $200 \mu \mathrm{g} / \mathrm{kg}$ in soil for the soil togroundwater protection pathway.

\section{PILOT SCALE DESIGN}

This section describes the Adventus Group design for the treatment of both soils and groundwater at the site, as planned prior to injection activities being conducted.

The area selected for the short-term field-scale pilot test is approximately centered on the locations of existing monitoring well MW02 and former investigative boring SB12 (Figure 1). These two borings penetrated the highest concentrations of carbon tetrachloride identified in both vadose zone soils and groundwater at the Centralia investigation site. The two borings define a hot-spot area that has demonstrated sustained high carbon tetrachloride levels. The pilot test activities were to be confined to a rectangular area around MW02 and SB12. The surface of the rectangular area is approximately $45 \mathrm{ft}$ wide by $75 \mathrm{ft}$ long, oriented approximately orthogonally to the apparent direction of groundwater flow in this portion of the site.

Figure 1. Original Field Injection Layout

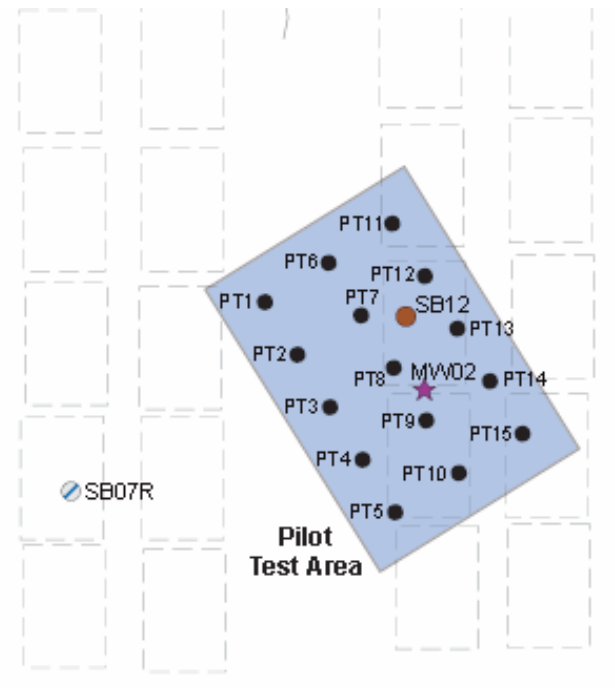

\section{Treatment of Groundwater in Saturated Zone}

EHC was to be injected in the fifteen borings locations shown in Figure 1 over the depth interval of 40 to 60 feet bgs, with allowances provided for the grid spacing being adjusted in the field a bit near MW02. EHC was to be applied at an application rate of approximately 
$0.1 \%$ to soil mass. Table 1 gives the amended injection plan for treatment of contaminants in the saturated zone.

Table 1: EHC Mass Requirements and Injection Details - Pilot Study

\begin{tabular}{|c|c|c|}
\hline & Value & Unit \\
\hline \multicolumn{3}{|l|}{ Treatment Area Dimensions: } \\
\hline Length of Treatment Area & 75 & $\mathrm{ft}$ \\
\hline Width of Treatment Area & 45 & $\mathrm{ft}$ \\
\hline Depth to Top of Treatment Area & 50 & $\mathrm{ft}$ \\
\hline Depth to Bottom of Treatment Area & 60 & $\mathrm{ft}$ \\
\hline Treatment Area Thickness & 10 & $\mathrm{ft}$ \\
\hline Treatment Area Volume & 33,750 & $\mathrm{ft} 3$ \\
\hline Mass of Soil in Treatment Area & 1,856 & U.S. tons \\
\hline Volume pore space & 10,125 & $\mathrm{ft} 3$ \\
\hline \multicolumn{3}{|l|}{ EHC mass calculations: } \\
\hline Percentage EHC by soil mass & $0.20 \%$ & \\
\hline Mass of EHC Required & 7,500 & Ibs \\
\hline \multicolumn{3}{|l|}{ Preparation of EHC Slurry: } \\
\hline Percent solids in slurry (can be altered) & $23.1 \%$ & \\
\hline Volume Water Required & 3,000 & U.S. gallons \\
\hline Slurry Volume to Inject & 3,386 & U.S. gallons \\
\hline \multicolumn{3}{|l|}{ Injection details: } \\
\hline Injection spacing & 15 & $\mathrm{ft}$ \\
\hline Number of injection points & 15 & points \\
\hline Mass EHC per point & 500 & lbs \\
\hline Slurry volume per point & 226 & U.S. gallons \\
\hline \multicolumn{3}{|l|}{ Application rates for reference: } \\
\hline Slurry volume to pore space volume & $4.5 \%$ & \\
\hline EHC concentration in groundwater & 0.7 & $\mathrm{Ibs} / \mathrm{ft} 3$ \\
\hline
\end{tabular}

\section{Treatment of Soil in Vadose Zone}

The goal was to reduce the COI mass in the soil to below the treatment standard and reduce leaching to groundwater. This was to be achieved via in-situ reductive dechlorination. For this application an aqueous formulation of EHC, EHC-A, was used. The aqueous formulation was utilized to increase potential contact between the CT-impacted soil and the injected EHC product. to allow for movement in the subsurface. EHC-A is composed of a water soluble organic amendment and soluble iron $(\mathrm{Fe}(\mathrm{II}))$. Two design alternatives were provided to ANL with regard to the treatment of site vadose soils (from 20 to 40 feet bgs). The first of these plans was provided in an April 5, 2007 proposal, as reflected in Table 2. The targeted area was four borings within a targeted area of approximately $30 \mathrm{ft}$ long $\times 30 \mathrm{ft}$ wide $\times 20 \mathrm{ft}$ deep (from 20 to $40 \mathrm{ft}$ bgs). EHC-A was to be applied at an application rate of approximately $0.1 \%$ to soil mass. 
Table 2. EHC-A mass requirements and injection volumes.

\begin{tabular}{|c|c|c|}
\hline & Value & Unit \\
\hline \multicolumn{3}{|l|}{ Treatment Area Dimensions: } \\
\hline Length of Treatment Area & 30 & $\mathrm{ft}$ \\
\hline Width of Treatment Area & 30 & $\mathrm{ft}$ \\
\hline Depth to Top of Treatment Area & 20 & $\mathrm{ft}$ \\
\hline Depth to Bottom of Treatment Area & 40 & $\mathrm{ft}$ \\
\hline Treatment Area Thickness & 20 & $\mathrm{ft}$ \\
\hline Treatment Area Volume & 18,000 & $\mathrm{ft} 3$ \\
\hline Mass of Soil in Treatment Area & 990 & U.S. tons \\
\hline Volume pore space & 5,400 & $\mathrm{ft3}$ \\
\hline \multicolumn{3}{|l|}{ EHC mass calculations: } \\
\hline Percentage EHC-A by soil mass & $0.10 \%$ & \\
\hline Mass of EHC-A Required & 2,000 & Ibs \\
\hline \multicolumn{3}{|l|}{ Preparation of EHC Slurry: } \\
\hline Percent solids in slurry (can be altered) & $10 \%$ & \\
\hline Volume Water Required & 2,160 & U.S. gallons \\
\hline Slurry Volume to Inject & 2,222 & U.S. gallons \\
\hline \multicolumn{3}{|l|}{ Injection details: } \\
\hline Injection spacing & 15 & $\mathrm{ft}$ \\
\hline Number of injection points & 4 & points \\
\hline Mass EHC per point & 500 & Ibs \\
\hline Slurry volume per point & 556 & U.S. gallons \\
\hline \multicolumn{3}{|l|}{ Application rates for reference: } \\
\hline Slurry volume to pore space volume & $5.5 \%$ & \\
\hline
\end{tabular}

The second of these was provided in an April 6, 2007 proposal, is reflected in the injection permit application, and presented as Table 3.

Table 3. EHC-A mass requirements and injection volumes.

\begin{tabular}{|l|c|c|}
\hline & Value & Unit \\
\hline Treatment Area Dimensions: & & \\
\hline Length of Treatment Area & 50 & $\mathrm{ft}$ \\
\hline Width of Treatment Area & 45 & $\mathrm{ft}$ \\
\hline Depth to Top of Treatment Area & 20 & $\mathrm{ft}$ \\
\hline Depth to Bottom of Treatment Area & 40 & $\mathrm{ft}$ \\
\hline Treatment Area Thickness & 20 & $\mathrm{ft}$ \\
\hline Treatment Area Volume & 45,000 & $\mathrm{ft} 3$ \\
\hline Mass of Soil in Treatment Area & 2,475 & $\mathrm{U.S.} \mathrm{tons}$ \\
\hline Volume pore space & 13,500 & $\mathrm{ft} 3$ \\
\hline EHC mass calculations: & & \\
\hline Percentage EHC by soil mass & $0.10 \%$ & \\
\hline Mass of EHC Required & 5,000 & $\mathrm{lbs}$ \\
\hline Preparation of EHC Slurry: & & \\
\hline Percent solids in slurry (can be altered) & $10 \%$ & \\
\hline
\end{tabular}




\begin{tabular}{|l|c|c|}
\hline Volume Water Required & 5,399 & U.S. gallons \\
\hline Slurry Volume to Inject & 5,555 & U.S. gallons \\
\hline Injection details: & & \\
\hline Injection spacing & 15 & $\mathrm{ft}$ \\
\hline Number of injection points & 9 & points \\
\hline Mass EHC per point & 556 & Ibs \\
\hline Slurry volume per point & 617 & U.S. gallons \\
\hline Application rates for reference: & & \\
\hline Slurry volume to pore space volume & $5.5 \%$ & \\
\hline EHC concentration in groundwater & 0.4 & lbs/ft3 \\
\hline
\end{tabular}

\section{Field Design Modifications}

Prior to the start of pilot injection work, ANL conducted some pre-injection boring activities to develop current site data. These activities will be reported elsewhere. However, as a consequence of these data collection activities, the following general observations were made:

- Although the water table elevation is much shallower, the target saturated zone is confined and permeable from 50 to 60 feet. (The initial design basis was to inject within a saturated zone from 40 to 60 feet.)

- The extent of carbon tetrachloride in groundwater is greater in concentration and extent than previously observed.

- The extent of carbon tetrachloride in vadose soils is essentially the same as previously observed.

Based on these observations, the injection approach and mass requirements were modified in the field in order to fulfill the requirements of the KDHE Bureau of Environmental Remediation (BER). The modified injection layout is shown in Figure 2 where the injection points from the original plan have been modified to account for hot spots in both vadose and saturated zones. According the modified area of injections, PT 1, 6, and 11 shown on Figure 1 were moved around pre-injection boring PSB8 due to the higher concentrations of carbon tetrachloride detected there. The final injection design plans were divided into borings where only saturated zone (i.e., groundwater) injections were to be performed and borings where both vadose (i.e., soil) and groundwater injections were to be performed. The divisions are as listed below:

Soil and Groundwater borings (Vadose (20-50 ft) and saturated zone (50-60 ft)

\section{PT 1, 2, 6, 7, 8, 11, 12, and 13}

$>$ EHC-A was to be applied at an application rate of $0.1 \%$ to soil mass in the vadose zone (20-40 ft) (Table 4).

$>$ EHC was to be applied at an application rate of $0.06 \%$ to soil mass in the lower vadose zone between 40 and 50 feet, using a thin slurry to enhance distribution efficiency (Table 5). 
> EHC was to be applied at an application rate of $0.14 \%$ to soil mass in the more permeable saturated zone (50 -60 ft) (Table 6).

Figure 2. Field-Modified Injection Layout

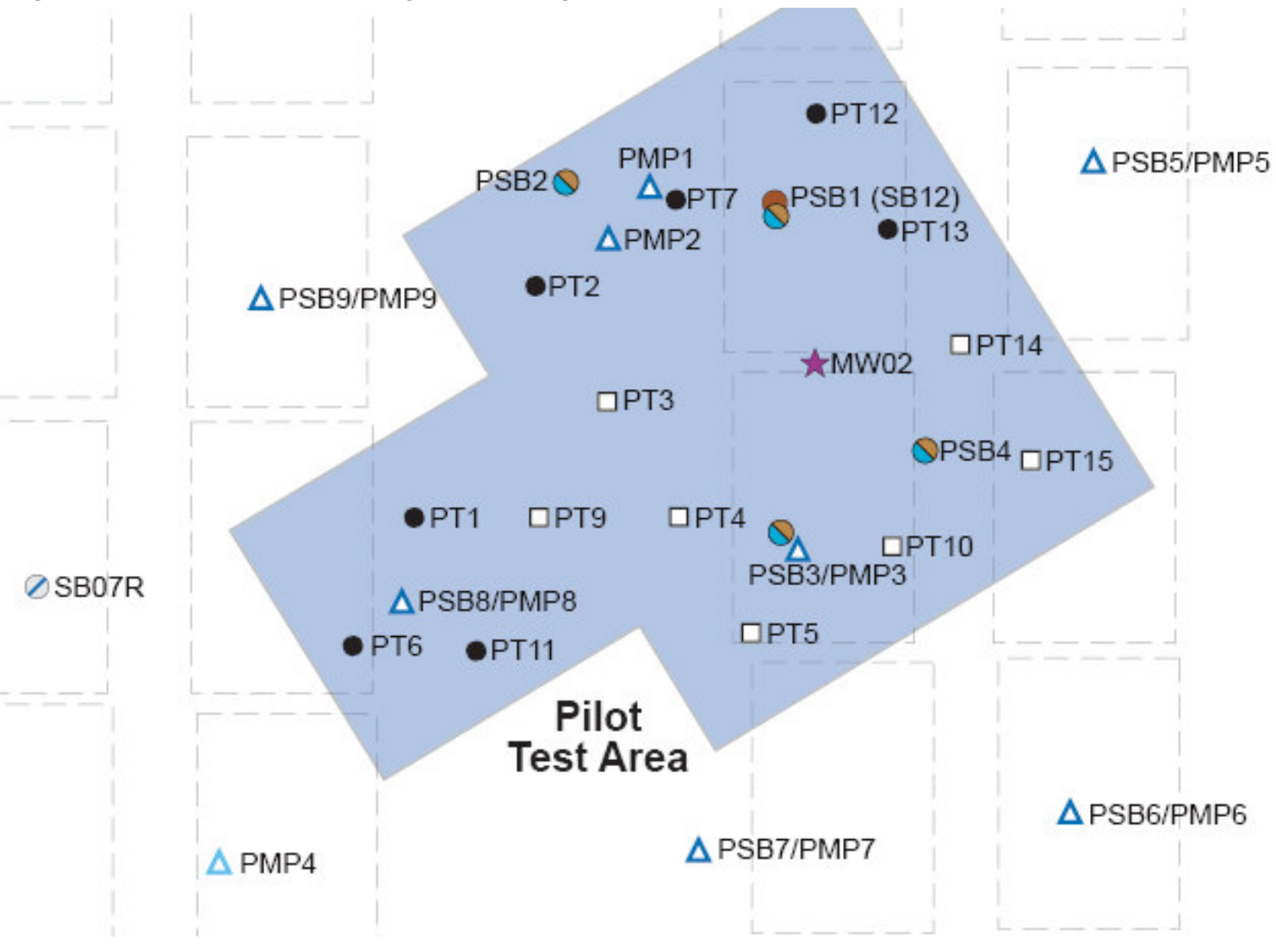

Table 4: EHC-A - 8 borings from 20 to 40 feet

\begin{tabular}{|c|c|}
\hline Injected depth (feet) & $\begin{array}{c}\text { EHC-A Mass/Interval } \\
\text { (Ibs) }\end{array}$ \\
\hline 21 & 50 \\
\hline 23 & 50 \\
\hline 25 & 50 \\
\hline 27 & 50 \\
\hline 29 & 50 \\
\hline 31 & 50 \\
\hline 33 & 50 \\
\hline 35 & 50 \\
\hline 37 & 50 \\
\hline 39 & 50 \\
Total & $\mathbf{5 0 0}$ \\
\hline
\end{tabular}

* Target water to material ratio is 54 Gallons / 50 lbs which is approximately 10 wt\% 
Table 5: EHC -8 borings from 40 to 50 feet

\begin{tabular}{|c|c|}
\hline Injected depth (feet) & EHC Mass/Interval (Ibs) \\
\hline 42 & 50 \\
\hline 45 & 50 \\
\hline 48 & 50 \\
\hline Total & $\mathbf{1 5 0}$ \\
\hline
\end{tabular}

* Target water to material ratio is 54 Gallons / 50 lbs which is approximately $10 \mathrm{wt} \%$

Table 6: EHC - 8 borings from 50 to 60 feet

\begin{tabular}{|c|c|}
\hline Injected depth (feet) & EHC Mass/Interval (lbs) \\
\hline 51 & 50 \\
\hline 53 & 100 \\
\hline 55 & 50 \\
\hline 57 & $\mathbf{1 0 0}$ \\
\hline 59 & $\mathbf{5 0}$ \\
\hline Total & $\mathbf{3 5 0}$ \\
\hline
\end{tabular}

*Target water to material ratio is 20 Gallons / 50 lbs which is approximately 23 wt\%

Groundwater borings (Saturated zone 50-60 ft)

PT 3, 4, 5, 9, 10, 14, and 15

$>$ EHC was to be applied at an application rate of $0.2 \%$ to soil mass to treat the saturated zone (50 -60 ft) (Table 7).

Table 7: EHC - 7 borings from 50 to 60 feet

\begin{tabular}{|c|c|}
\hline Injected depth (feet) & EHC Mass/Interval (Ibs) \\
\hline 51 & 100 \\
\hline 53 & 100 \\
\hline 55 & 100 \\
\hline 57 & $\mathbf{1 0 0}$ \\
\hline 59 & $\mathbf{1 0 0}$ \\
\hline Total & $\mathbf{5 0 0}$ \\
\hline
\end{tabular}

*Target water to material ratio is 20 Gallons / 50 lbs which is approximately 23 wt\%

\section{Field Observations}

The EHC and EHC-A were delivered as a dry powder in 50-lb bags (Figure 3), and mixed with water on site into a slurry (Figure 4), to be injected via direct push technology (Figure 5) by Redox Tech, LLC using a Chem-Grout pump (Figure 6). Field observations are summarized by way of both daily and general observations (below), as well as by detailed injection interval information (Appendix C). 


\section{(6) ADVENTUS}

Figure 3. EHC Pallets on Site

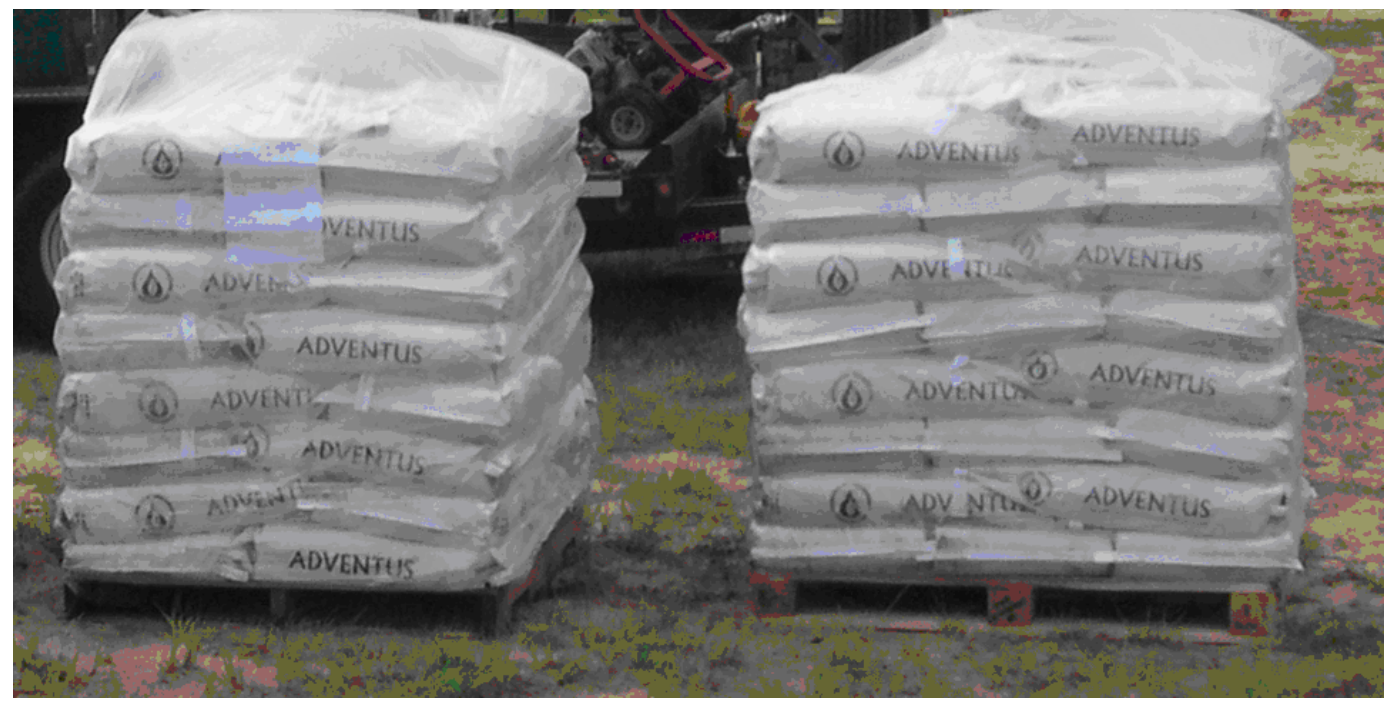

Figure 4. Mixing of EHC and EHC-A on Site
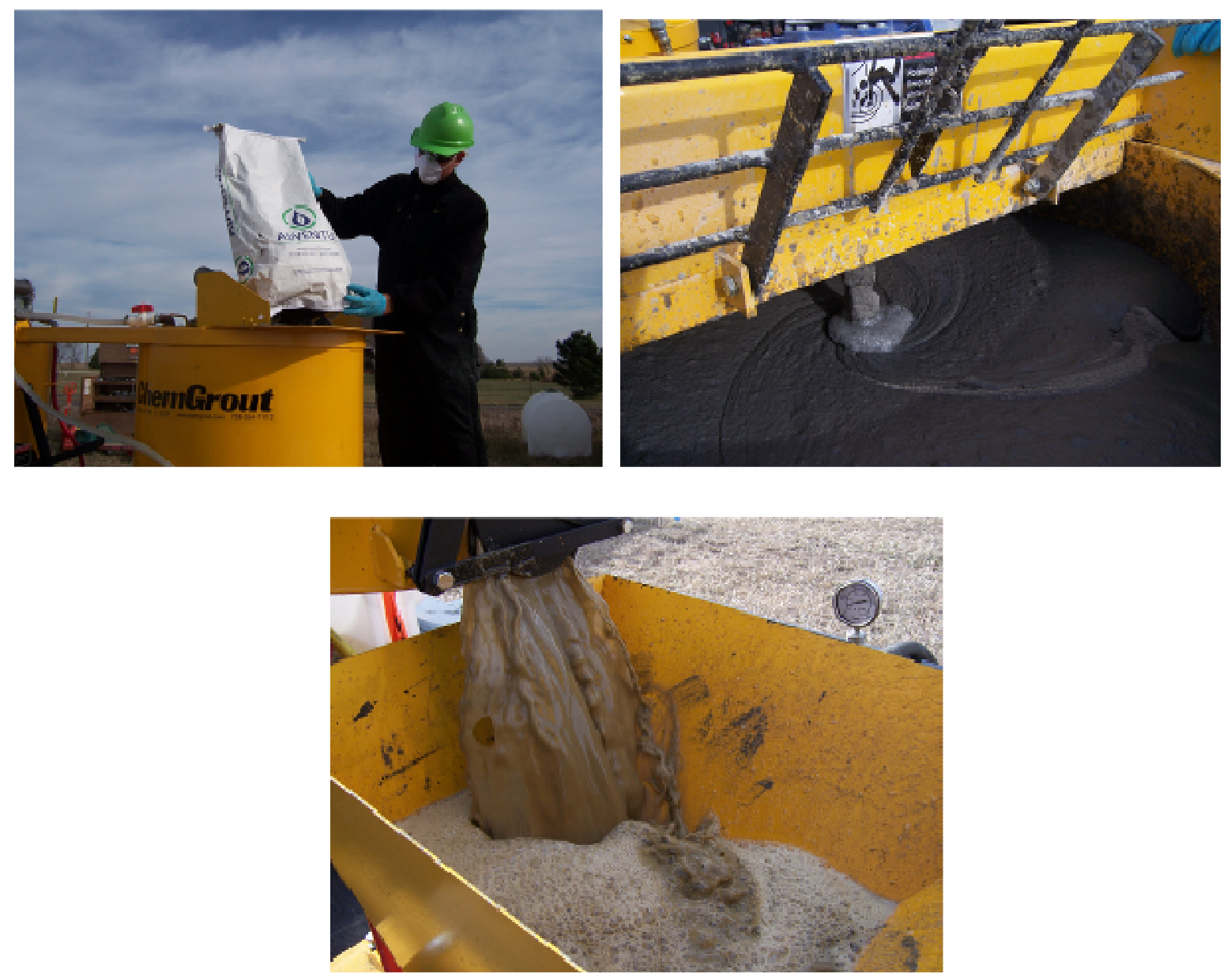


\section{(b) ADVENTUS}

\section{Figure 5. Direct Push Rig Used for EHC Injections}
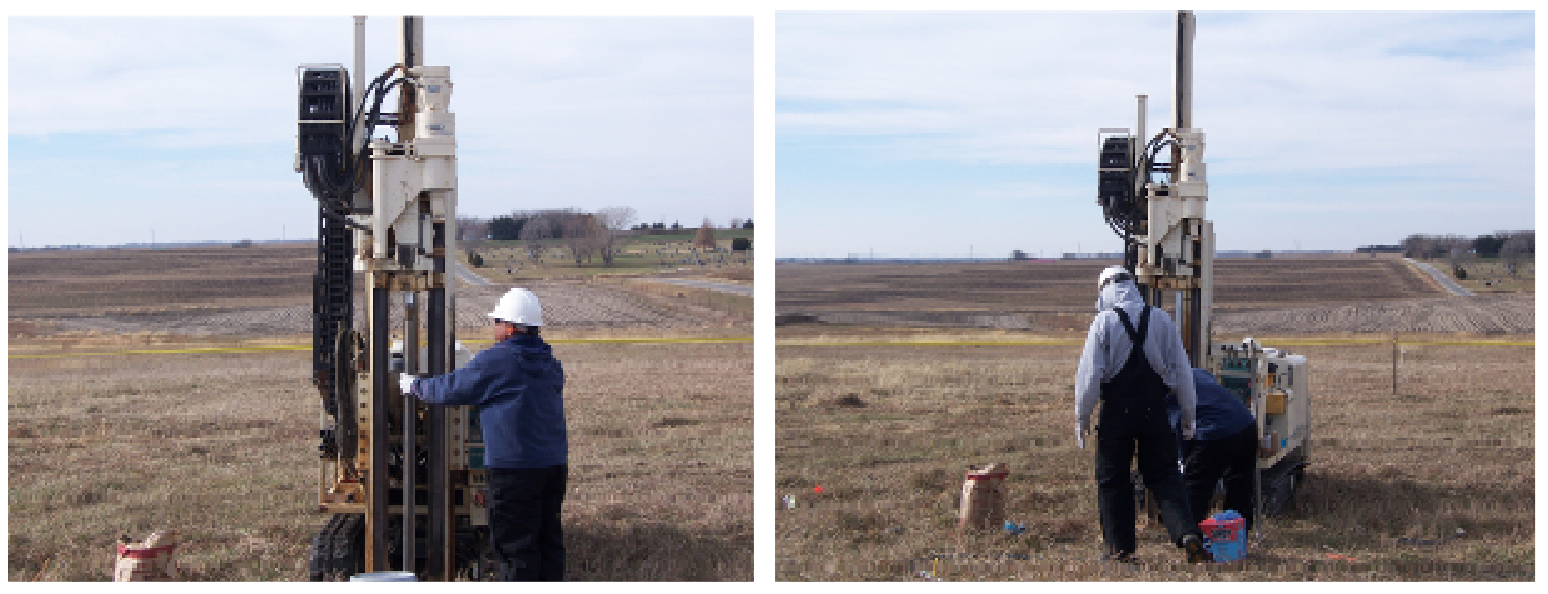

Figure 6. Chem Grout Pump

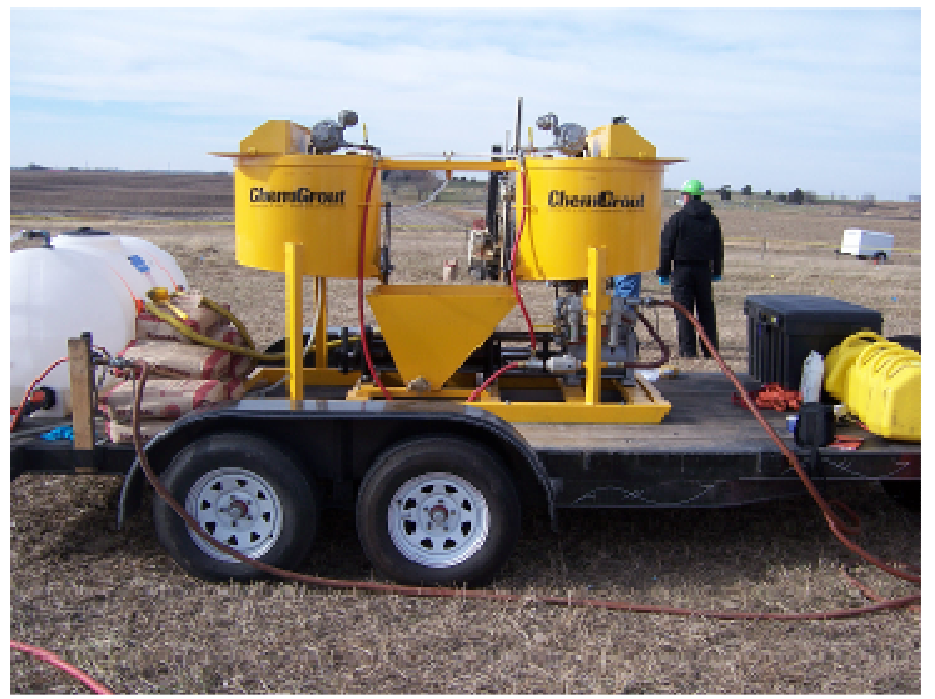

November 27, 2007

Initially, Redox Tech had planned to use an expendable injection tip to perform bottom-up injections, as reflected in the injection permit (see bottom-out injection diagram, page 4 of the October 30, 2007 permit application in Appendix A). However, KDHE BER requested that injections be performed from the top-down. Accordingly, Redox Tech utilized the expendable tip in top-down fashion while a Geoprobe ${ }^{\mathrm{TM}}$ tool designed for top-down injections was obtained. Information on the Geoprobe ${ }^{\mathrm{TM}}$ pressure-activated tip is available on their website: http://www.geoprobe.com/products/geoprobe accessories/injprobedesc.htm. Use of the expendable tip necessarily required 'tripping' in and out of the borehole to replace the tip for each successive injection interval. 
Initially, the EHC slurry ( 40 gallons water to 100\# EHC) was injected at PT-5 for treating the saturated zone. With increasing depth in injections, there was significant back pressure in the injections rods which could be attributed to the tight formation at the site and/or the thickness of the EHC mix. From this point forward, the Redox Tech injection operators elected to use slightly more than the design water volume of 20 gallons of water to each 50 pound bag of $\mathrm{EHC}$, based on the perception that the mixing equipment may not have adequately broken up all clumps of EHC powder. In addition, Redox Tech routinely flushed water through the injection lines after each injection interval. Because the injection tooling was in the ground when this flushing action was conducted, this additional water volume was injected in to the ground. Accordingly, the water volumes presented in Appendix C for each interval are approximations. As a means of avoiding potential EHC powder clump issues, Redox Tech eventually installed a grate in the Chem Grout pump hopper, in order to avoid potential clogging issues (Figure 7)

Figure 7. Grate Installed in ChemGrout Pump Hopper

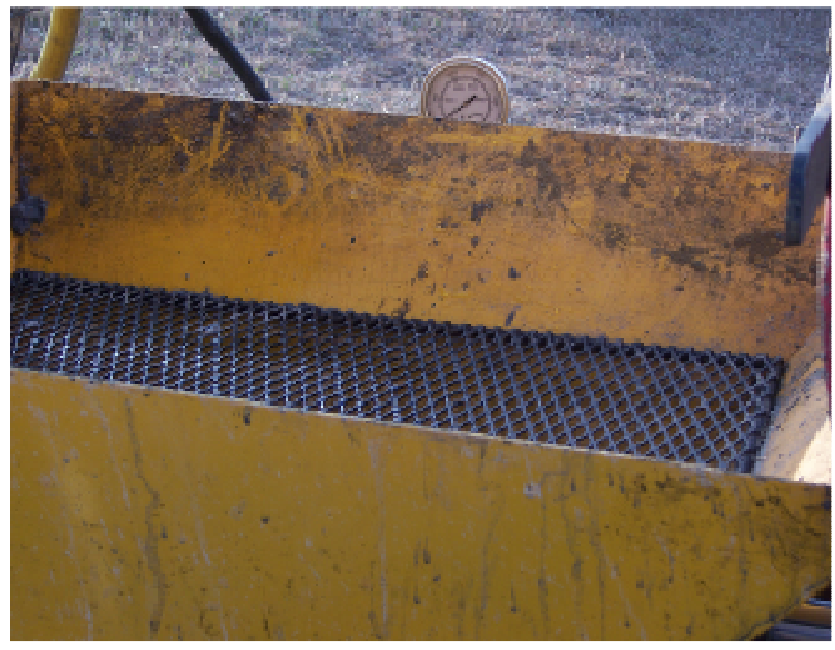

\section{November 28, 2007}

The injection intervals remaining at boring PT-5 were completed successfully using the GeoProbe tool. Borings PT-3 (groundwater) and PT-13 (both soil and groundwater treatment) were also conducted on this day. Where additional boreholes were utilized to accomplish this (as occurred at PT-5), Appendix C indicates this through addition of a letter after the boring ID. Such additional boreholes were used whenever needed to enable injection at the target zones. Using PT-5 as an example, this occurred when high injection back pressures were observed. Because of challenging drilling conditions for the Redox Tech direct push rig, ANL staff utilized the ANL cone penetrometer (CPT) rig (Figure 8) to advance a borehole to 50 feet bgs for some of the groundwater only borings (such as PT-3). 


\section{(b) ADVENTUS}

Given the proximity of PT-13 to MW-02, the well was protected through the use of a packer assembly to decrease the potential for entry of injected material into the well screen (Figure 9). The injections at PT-13 were successfully completed through a depth of 42 feet with injection pressures less than 250 psi. When a thin EHC slurry was injected at $45 \mathrm{ft}$ (vadose zone), EHC material started coming around the annulus of MW-02 (Figure 10). Use of a thicker slurry was attempted at 48 and 51 feet, and additional daylighting occurred, which led to skipping the remaining intervals temporarily at this particular boring. As a corrective measure, the well seal was later repaired (Figure 11). No evidence of plugging of the well screen by EHC material was observed in MW-02 when checked by ANL staff.

Figure 8. ANL CPT Rig

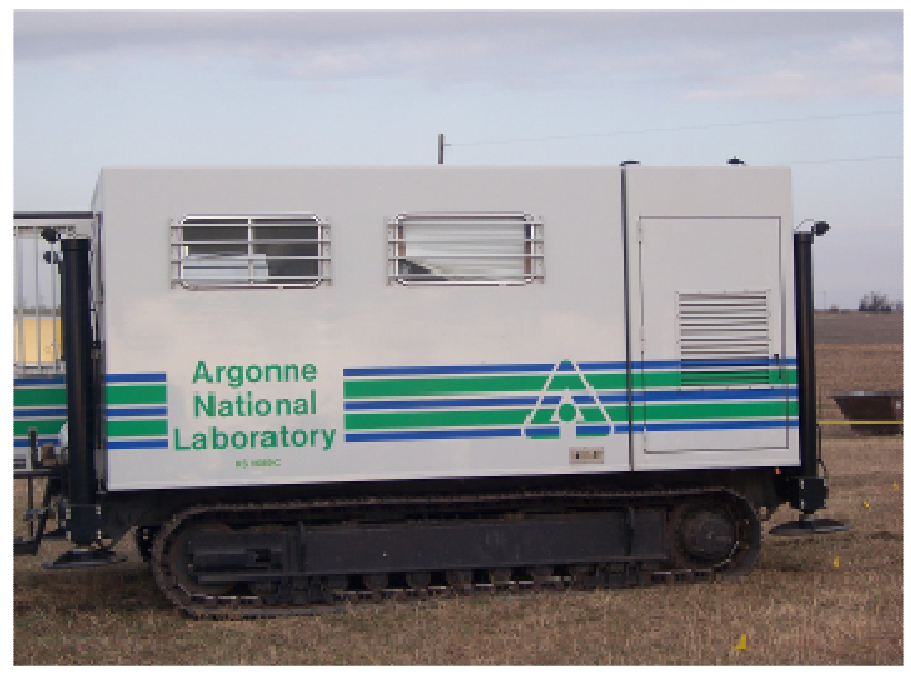

Figure 9. Packer Assembly at MW-02

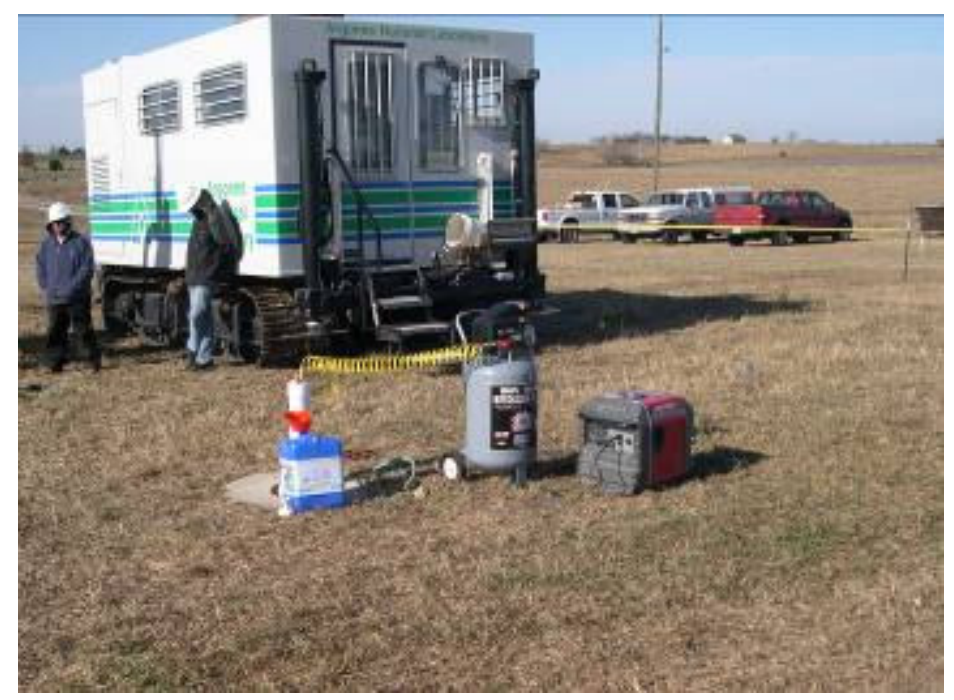




\section{Figure 10. Annular Leakage of Thin EHC Slurry}

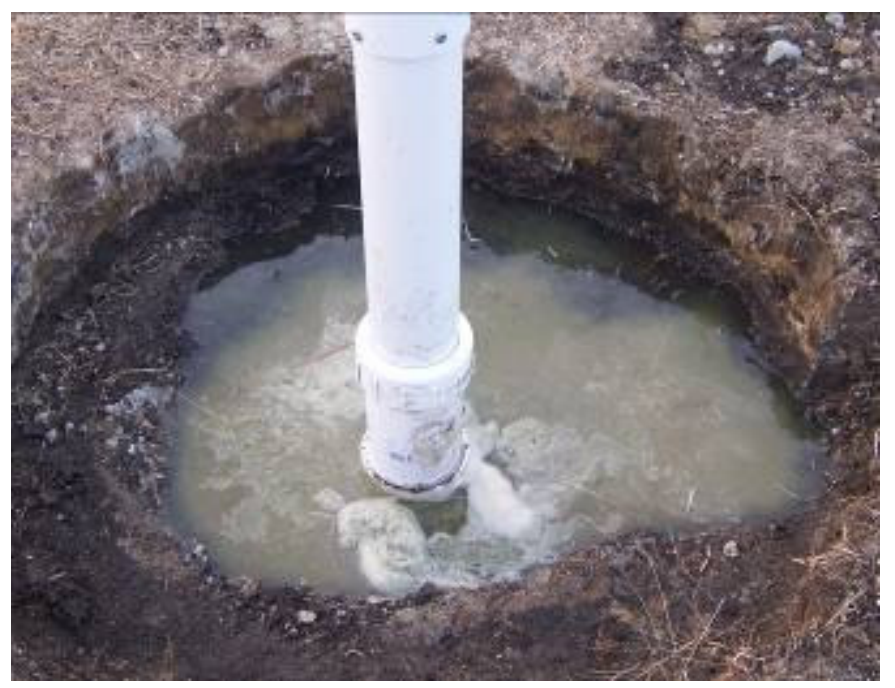

Figure 11. Well Seal Repair at MW-02

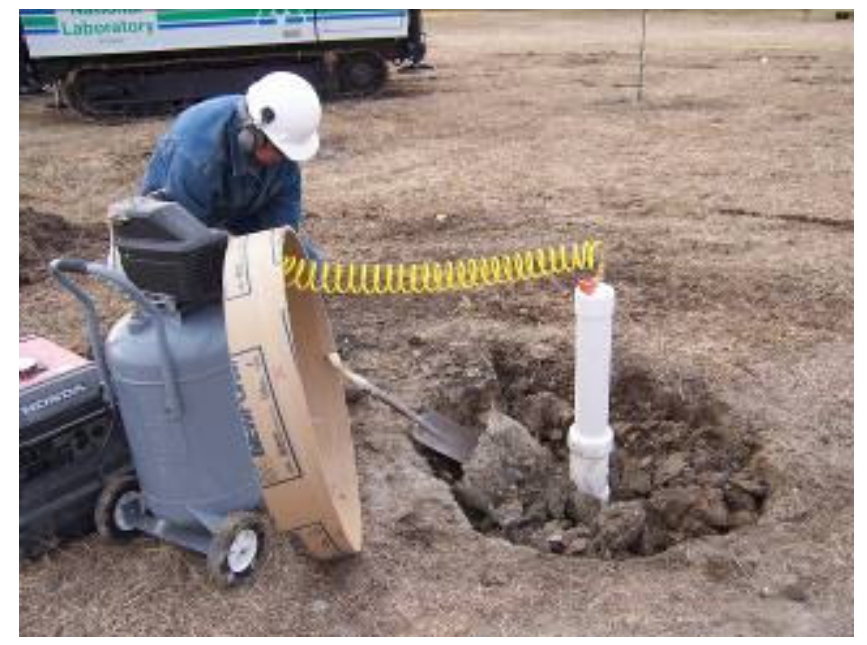

\section{November 29, 2007}

Injections were conducted at PT-15 (groundwater) and PT-1 (soil and groundwater). While Injecting at PT-15, there was a need to slightly vary the depths of EHC injections to enable injections into and around tight zones of clay and high back pressures encountered at particular depths. The back pressures may have resulted directly from the formation or from plugging of the Geoprobe tip. In addition, some daylighting of EHC product occurred to the surface while injecting at PT-15-57' and 59'. This was effectively addressed by slowing the rate of injection and by applying pressure to observed daylighting points at the surface. A variety of means were used to apply pressure at the surface, including use of the CPT rig 
stabilizer pads and using a 'spud'. Occurrence of surficial daylighting at shallow depths also occurred and was similarly addressed during injections at PT-1 (Figure 12).

When injections were done at a depth of $42 \mathrm{ft}$ at PT-1, there was a rise in pressure apparently due to tip clogging in the tight formation. There was also a need to start a hole adjacent to PT-1 once injections at the saturated zone commenced due to a rise in back pressures. This allowed some time for pressures to dissipate at the one location while injection commenced at the adjacent location. High pressures were also addressed by putting off the deeper intervals to a later date to allow pressure in and around PT-1 to dissipate.

Figure 12. Daylighting of Thin EHC-A Slurry at PT-1

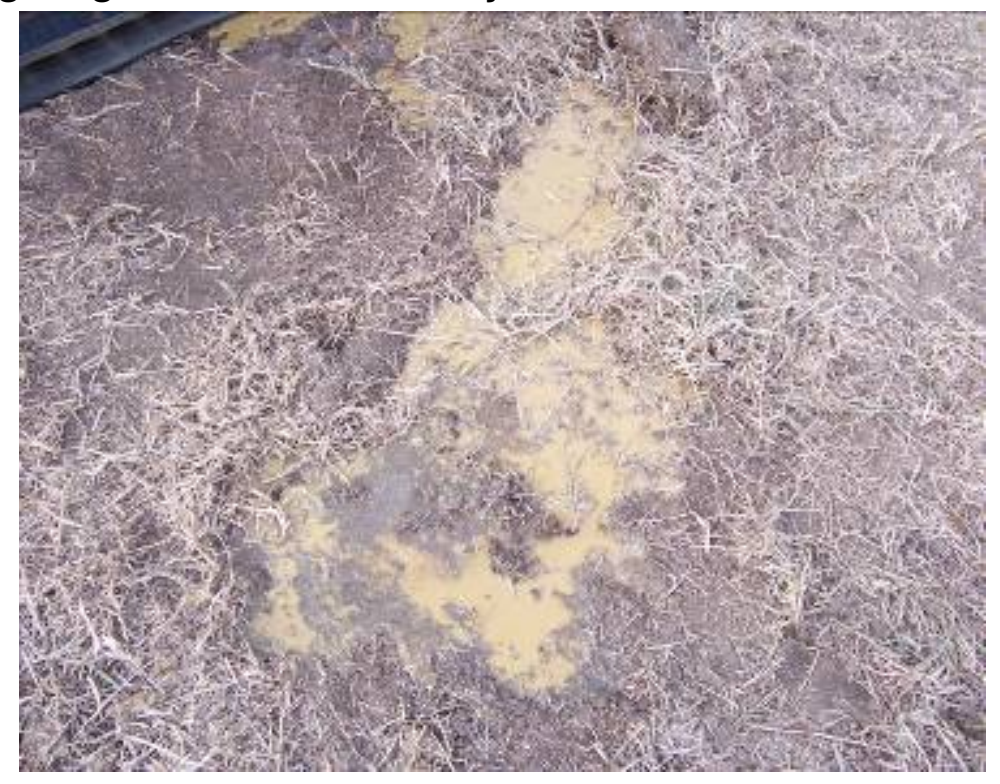

\section{November 30, 2007}

Injections were conducted at PT-12, PT-2, and PT-6, and were restricted to the vadose zone (20-50 feet) in all the three borings. Injections at PT-12 were completed to 35 feet due to high back pressures at greater depths. Daylighting of EHC-A occurred near SB07R when injecting at PT-6. This was observed at multiple surficial points beginning at a depth of 25 feet, and fading away with increasing depth. EHC material started coming out of the annulus between the plastic well casing and the flush mount housing when injections were carried out at a depth of 48 feet at PT-6. This could be attributed to a preferential pathway of EHC material in a tight clay formation at the site (Figure 13). Daylighting was addressed by discontinuing injection, applying pressure at the surface, and/or slowing the injection flow rate. 
Figure 13: Daylighting at SB07R during injection at PT-6

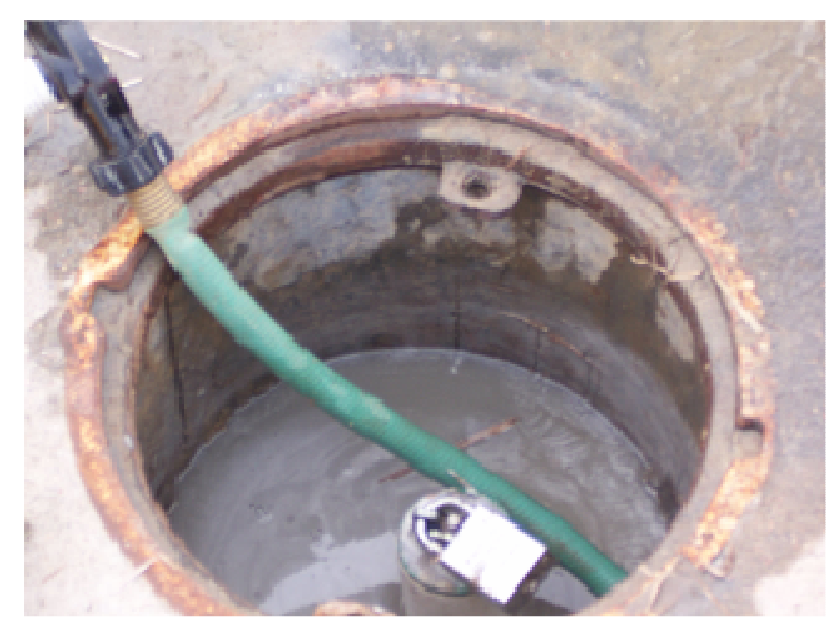

December 1, 2007

No site work was conducted this day due to very unfavorable weather conditions (ice storms).

\section{December 2, 2007}

Injections were conducted at PT-7 and PT-10, in both the vadose and saturated zones. Some daylighting occurred during the injections. Daylighting was observed at the surface near MW-02. (Figure 14) Again, daylighting was addressed by discontinuing injection, applying pressure at the surface, and/or slowing the injection flow rate. In addition, a slightly thicker EHC slurry was used to minimize injected volume in an effort to minimize daylighting.

Figure 14: Surficial Daylighting near PT-7 and PT-10
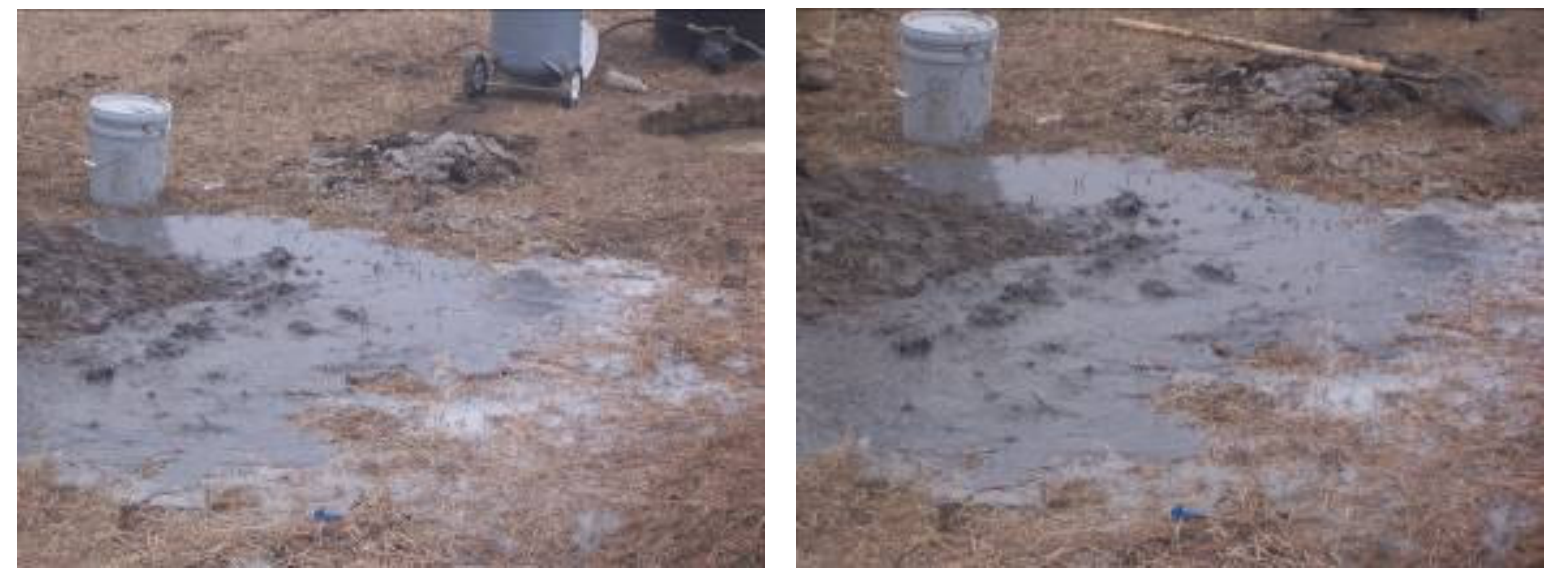


\section{December 3, 2007}

Injections were conducted at PT-4 and PT-14 (groundwater borings), and PT-2 (completion of prior skipped soil intervals and completion of groundwater intervals). The injection tip was initially advanced without the check valve spring in place at PT-14. This resulted in a clog which was resolved by pulling the drill string out of the ground and correcting the problem. Intervals in the saturated zone between 50-60 feet at PT-2 were altered owing to EHC daylighting. (Figure 15). Vertical injection spacing in the saturated zone between 50 and 60 feet was increased as needed as daylighting was observed at the surface.

Figure 15: Daylighting at multiple points around PT-14 and PT-2
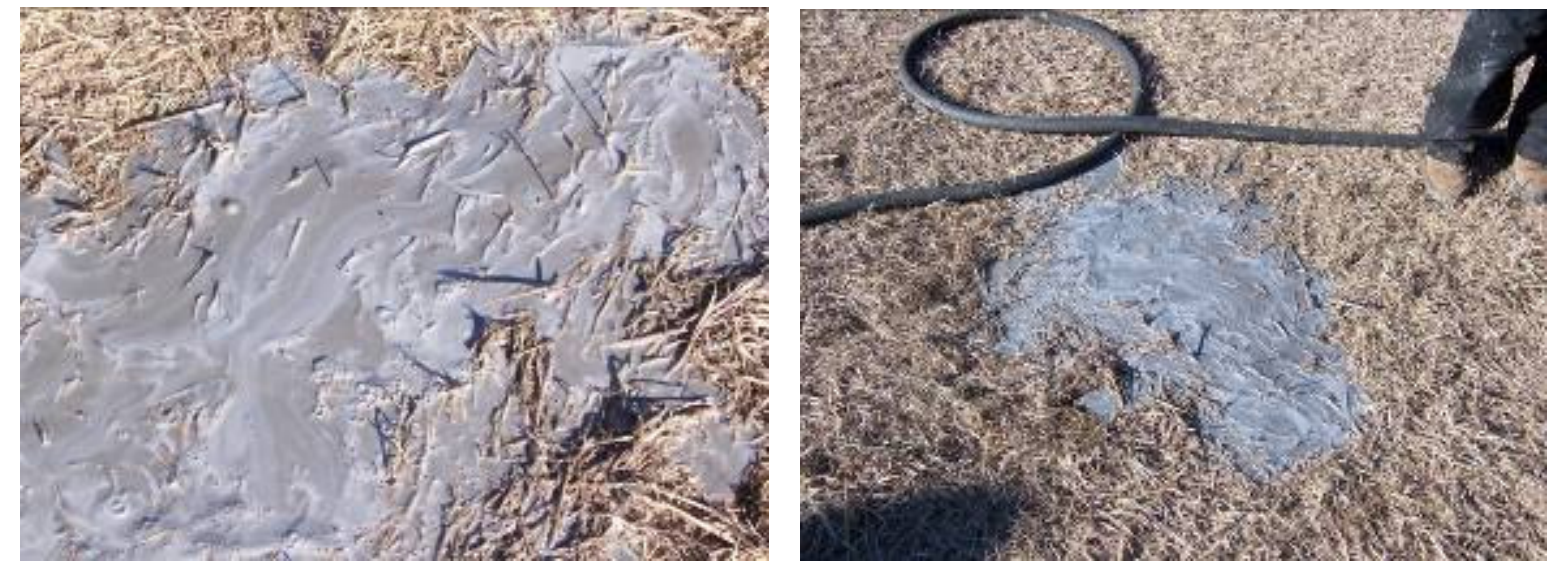

\section{December 4, 2007}

Injections were conducted at PT-9 and PT-11 (groundwater), at PT-1 (remaining groundwater intervals), and at PT-12 (remaining soil and groundwater intervals). PT-9 was moved from the original location to a point southwest of PT-3 and PT-4 due to its proximity to MW-02. No daylighting was observed, but some evidence of tight formation (high pressures) was seen. Accordingly, vertical injection spacing and injected volumes in the saturated zone between 50 and 60 feet were altered as needed resulting in more material being emplaced at greater depths.

\section{December 5, 2007}

Injections were conducted at PT-11 (remaining soil intervals) and at PT-6 (groundwater). Some minimal surficial daylighting was witnessed at shallow depths when injecting at PT-11. This was addressed by adjusting the volumes at each depth to minimize daylighting. 


\section{Observations Summary - General Field Observation}

- EHC-A and EHC injections were not performed at PT-8 as it was very close to MW-02. ANL consulted with KDHE to determine that sufficient injections had been done and enough material distributed for treatment of carbon tetrachloride in the areas of interest to make for a credible pilot test.

- Upon completion of injection activities and removal of the Geoprobe injection rods, boreholes were abandoned with Holeblok+ (Figure 16). HoleBlok+ was poured slowly ensure good filling of boreholes and to avoid bridging. In addition, a small size (\#9) HoleBlok + was used and the HoleBlok+ was compacted with a 'spud' to ensure good compaction. Around $500 \mathrm{lbs}$ of Holeblok+ was used for abandonment of boreholes. Additional information on HoleBlok and HoleBlok+ is included as test reports in Appendix D.

Figure 16: Hole Blok+ usage for bore-hole abandonment
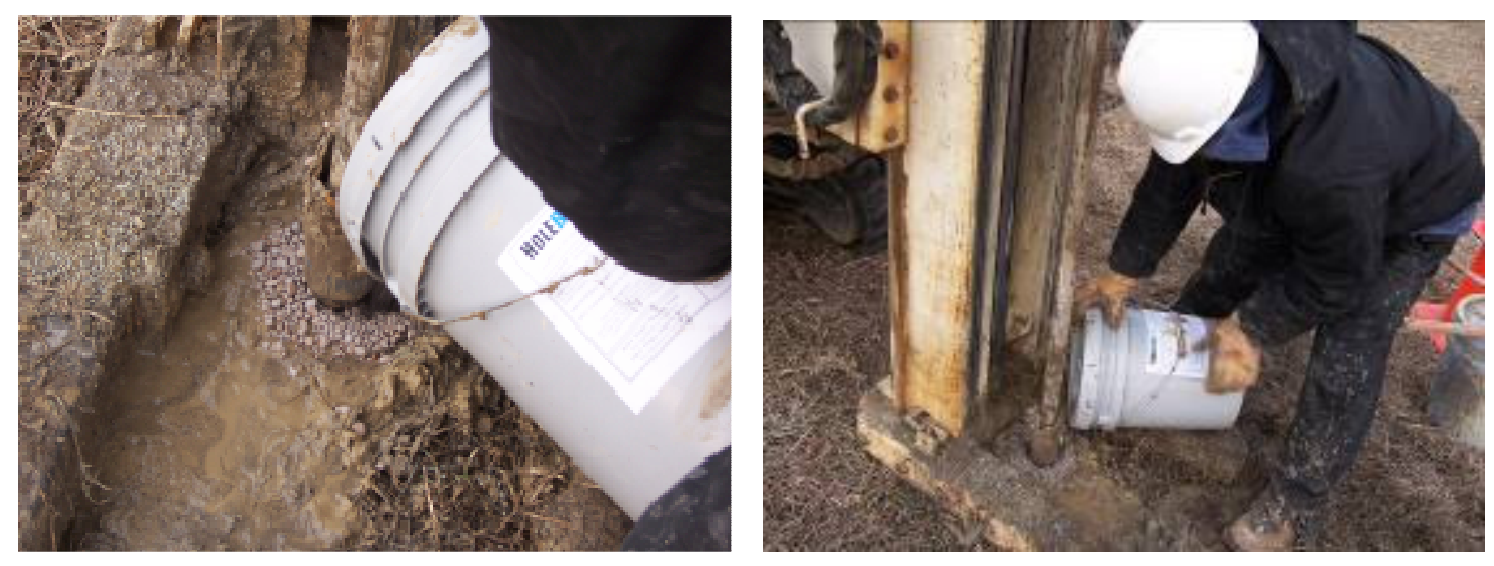

- During the entire duration of injection activities, the injection equipment (ChemGrout pump) had to be heated everyday for sometime before commencement due to cold conditions encountered at the site. In addition, ANL staff arranged for off-site storage of the equipment to limit winter weather exposure. Cold weather definitely slowed work progress overall due to freezing conditions, as well as a lost day due to an ice storm.

- The amount of water used for injections was higher in comparison with actual design at every injection point. This resulted in part from the use of additional water to flush out any material present in the line and rods as well as to clear material that might clog the injection tip at the next interval. In addition, the water used in the 50 to 60 foot interval was at higher than the initial design ratio. The design called for a 23.1 wt\% slurry, or 20 gallons per 50 pound bag of EHC. As discussed above, more water was generally used in most EHC batches from 50 to 60 feet, in order to avoid EHC 
powder clumps from causing injection problems.. Also, water quantities in Appendix C are approximations for each interval, although the total volume is also provided.

- Bromide tracer usage was tied to the volume of water injected with EHC. The target bromide concentration of $150 \mathrm{mg} / \mathrm{L}(223 \mathrm{mg} / \mathrm{L} \mathrm{KBr}$ ) was achieved by adding $84.3 \mathrm{~g}$ $\mathrm{KBr} / 100$ gallons of water used. Given a total water usage of 8650 gallons, this amounts to approximately $7.3 \mathrm{~kg}$ of $\mathrm{KBr}$ added to the water as a tracer.

- High pressures observed during injection are necessarily not indicative of injection pressures within the formation, especially as observed at the injection tip. Resistances within the injection equipment, pressures required to overcome the check valve, etc, play a role in this, as well as the measurement point for the pressure having been at the ChemGrout pump. Pressure resulting from resistance in the hoses or clogging of injection tip may have played a role in some of the transient high pressures observed at the site.

- EHC and EHC-A were found to daylight out of the annulus of monitoring wells MW-02 and SB07R. There was no observation or evidence of EHC material coming from within the wells.

- Challenges in the form of daylighting during EHC injections were avoided by either skipping an interval, immediately plugging points where surfacing occurred by applying pressure at the surface, or injecting a thicker EHC slurry when possible.

- Redox Tech used expendable injection tips whenever necessary. Typically, this was done when the GeoProbe tip may have been clogged or when the formation was very tight, and high pressures were observed. Regardless, top-down injection were performed throughout as per KDHE BER request.

- Exposure monitoring was assessed through the use of a Photo-ionization Detector, or PID. Periodic checks were made daily in the breathing zone of on-site personnel to check for potential exposure to carbon tetrachloride. Potential exposure to these constituents may arise through contact with the Geoprobe rods that are in contact with the groundwater, or if there is any mixing between groundwater and injection slurry that surfaces at the ground surface. PID measurements corrected for background were always $0 \mathrm{ppm}$ indicating that no carbon tetrachloride was detected in the air around the working zone throughout the injection period.

- Prepunching of selected boring location using the Argonne cone penetrometer (CPT) rig was done at borings (PT 3, 4, 5, 9, 10, 14 and 15) where treatment was required in the saturated zone only in order to hasten the injection process.

- The application rate of EHC in the saturated zones of borings where both soil and groundwater treatment was required alternates every interval between $0.06 \%$ and $0.14 \%$. This is done to treat the unsaturated zone between $40-50$ feet using EHC as per KDHE requirements.

EHC-A and EHC dosages were actually offset by slight variances during field implementation. This occurred in due to a combination of resistance due to tight formations, back pressures in injection tips and daylighting at multiple points. The approximate amount of 
material injected at various borings is tabulated in Table 8, below. Again, more detailed information relating to the injection event is provided in Appendix $\mathbf{C}$.

Table 8: Actual Material Injected at Borings

\begin{tabular}{|c|c|c|c|}
\hline $\begin{array}{c}\text { Location } \\
\text { (borings }\end{array}$ & $\begin{array}{c}\text { Vadose (20 to } \\
\text { 40' bgs) zone } \\
\text { EHC-A (lbs) }\end{array}$ & $\begin{array}{c}\text { Vadose (40 to } \\
\text { 50' bgs) zone } \\
\text { EHC (lbs) }\end{array}$ & $\begin{array}{c}\text { Saturated (50 to 60' } \\
\text { bgs) zone } \\
\text { EHC (lbs) }\end{array}$ \\
\hline PT-1 & 500 & 150 & 350 \\
\hline PT-2 & 500 & 150 & 250 \\
\hline PT-3 & & & 500 \\
\hline PT-4 & 500 & 150 & 500 \\
\hline PT-5 & 500 & 150 & 300 \\
\hline PT-6 & & & 350 \\
\hline PT-7 & & & 500 \\
\hline PT-8 & & 300 & 300 \\
\hline PT-9 & 650 & 150 & 350 \\
\hline PT-10 & 500 & 150 & 350 \\
\hline PT-11 & 500 & 100 & 50 \\
\hline PT-12 & & & 500 \\
\hline PT-13 & & & 500 \\
\hline PT-14 & & & \\
\hline PT-15 & & & \\
\hline
\end{tabular}

Based on the injected masses identified in Table 8 (above) and the injected water volumes by interval (Appendix C), an estimate of the EHC dosages applied and total pore volumes injected by interval was developed and is presented in Tables 9 and 10. The designed area was developed based on the planned boring spacing of 15 borings over a $45 \times 75$ foot area. Although boring locations were moved about, the assumed area of influence of each boring as part of the design (i.e., one-fifteenth of 45 feet $\times 75$ feet, or 225 square feet) was used to provide a consistent basis for comparing actual versus intended injection mass ratios.

In Table 9, the water volumes used for the pore volume calculation do not include the total volume of water used, which is on the order of 1500 gallons more than shown in the table. The reason for this is that the precise volumes and intervals of additional water usage are not definitively known. Thus, the Table 9 values should be considered as a minimum in evaluating \% pore volume versus design. As previously discussed, this additional water was used to flush injection tips between intervals. 
Table 9. Estimated EHC Dosage and Injected Pore Volumes

\begin{tabular}{|c|c|c|c|}
\hline Area & $\begin{array}{l}\text { Upper Vadose } \\
\left(20 \text { to } 40^{\prime}\right)\end{array}$ & $\begin{array}{l}\text { Lower Vadose } \\
\quad\left(40 \text { to } 50^{\prime}\right)\end{array}$ & $\begin{array}{c}\text { Saturated } \\
\text { Zone (50 to } \\
\left.60^{\prime}\right)\end{array}$ \\
\hline \# Borings & 7 & 7 & 14 \\
\hline Mass Injected (\#) & 3650 & 1300 & 5300 \\
\hline Presumed Area (ft^2) & 1575 & 1575 & 3150 \\
\hline $\begin{array}{l}\text { Presumed Volume } \\
\left(\mathrm{ft}^{\wedge} 3\right)\end{array}$ & 31500 & 15750 & 31500 \\
\hline Soil Porosity & $30 \%$ & $30 \%$ & $30 \%$ \\
\hline $\begin{array}{l}\text { Pore Space Volume } \\
\left(\mathrm{ft}^{\wedge} 3\right)\end{array}$ & 9450 & 4725 & 9450 \\
\hline $\begin{array}{l}\text { Inferred Soil Mass } \\
\text { (Tons) }\end{array}$ & 1732.5 & 866.25 & 1732.5 \\
\hline $\begin{array}{l}\text { Inferred EHC Dosage } \\
\text { (wt \%) }\end{array}$ & $0.11 \%$ & $0.08 \%$ & $0.15 \%$ \\
\hline $\begin{array}{l}\text { Estimated Water } \\
\text { Dosage* (USG) }^{\star}\end{array}$ & 3626 & 942 & 2555 \\
\hline $\begin{array}{l}\text { Total Mass Injected (\#, } \\
\text { as EHC + water) }\end{array}$ & 33891 & 9156 & 26609 \\
\hline $\begin{array}{l}\text { Slurry concentration } \\
\text { (\% wt/wt) }\end{array}$ & $11 \%$ & $14 \%$ & $20 \%$ \\
\hline $\begin{array}{l}\text { Estimated Slurry } \\
\text { Density (\#/gal) }\end{array}$ & 8.8 & 9.2 & 9.5 \\
\hline $\begin{array}{l}\text { Estimated Volume } \\
\text { Injected (gallons) }\end{array}$ & 3843 & 998 & 2799 \\
\hline $\begin{array}{l}\text { Estimated Volume } \\
\text { Injected }\left(\mathrm{ft}^{\wedge} 3\right)\end{array}$ & 514 & 133 & 374 \\
\hline $\begin{array}{l}\text { Estimated \% Pore } \\
\text { Volume Occupied by } \\
\text { Injection }\end{array}$ & $5.4 \%$ & $2.8 \%$ & $4.0 \%$ \\
\hline
\end{tabular}

* - Excluding flush water

Alternately, Table 10 provides the estimated water volume usage assuming a proportional usage of the additional (estimated) injection water volume (1500 gallons) based on the total mass injected with in each categorized zone.

Table 10. Estimated EHC Dosage and Injected Pore Volumes

\begin{tabular}{|l|r|r|r|}
\hline Area & $\begin{array}{c}\text { Upper Vadose } \\
(\mathbf{2 0} \text { to } \mathbf{4 0})\end{array}$ & $\begin{array}{c}\text { Lower Vadose } \\
(\mathbf{4 0} \text { to } \mathbf{5 0})\end{array}$ & $\begin{array}{c}\text { Saturated } \\
\text { Zone (50 to } \\
\mathbf{6 0})\end{array}$ \\
\hline \# Borings & 7 & 7 & 14 \\
\hline Mass Injected (\#) & 3650 & 1300 & 5300 \\
\hline Presumed Area (ft^2) & 1575 & 1575 & 3150 \\
\hline $\begin{array}{l}\text { Presumed Volume } \\
\left(\mathbf{f t}^{\wedge} \mathbf{3}\right)\end{array}$ & 31500 & 15750 & 31500 \\
\hline Soil Porosity & $30 \%$ & $30 \%$ & $30 \%$ \\
\hline
\end{tabular}




\begin{tabular}{|l|r|r|r|}
\hline $\begin{array}{l}\text { Pore Space Volume } \\
\text { (ft^3) }\end{array}$ & 9450 & 4725 & 9450 \\
\hline $\begin{array}{l}\text { Inferred Soil Mass } \\
\text { (Tons) }\end{array}$ & 1732.5 & 866.25 & 1732.5 \\
\hline $\begin{array}{l}\text { Inferred EHC Dosage } \\
\text { (wt \%) }\end{array}$ & $0.11 \%$ & $0.08 \%$ & $0.15 \%$ \\
\hline $\begin{array}{l}\text { Estimated Water } \\
\text { Dosage (USG) }\end{array}$ & 3626 & 942 & 2555 \\
\hline $\begin{array}{l}\text { Estimated Flush Water } \\
\text { Dosage (USG) }\end{array}$ & 534 & 190 & 776 \\
\hline $\begin{array}{l}\text { Estimated Total Water } \\
\text { Dosage }\end{array}$ & 4160 & 1132 & 3331 \\
\hline $\begin{array}{l}\text { Total Mass Injected (\#, } \\
\text { as EHC + water) }\end{array}$ & 38346 & 10743 & 33077 \\
\hline $\begin{array}{l}\text { Slurry concentration } \\
\text { (\% wt/wt) }\end{array}$ & $10 \%$ & $12 \%$ & $16 \%$ \\
\hline $\begin{array}{l}\text { Estimated Slurry } \\
\text { Density (\#/gal) }\end{array}$ & 8.8 & 9.2 & 9.5 \\
\hline $\begin{array}{l}\text { Estimated Volume } \\
\text { Injected (gallons) }\end{array}$ & 4376 & 1165 & 3479 \\
\hline $\begin{array}{l}\text { Estimated Volume } \\
\text { Injected (ft^3) }\end{array}$ & 585 & 156 & 465 \\
\hline $\begin{array}{l}\text { Estimated \% Pore } \\
\text { Volume Occupied by } \\
\text { Injection }\end{array}$ & & & \\
\hline
\end{tabular}

On behalf of Adventus, I thank you for your interest in our products and technologies. Please contact us by email at Ravi.Srirangam@adventusgroup.com or John.Valkenburg@Adventusgroup.com if you have any questions regarding this report.

Yours truly,

Adventus Americas, Inc.

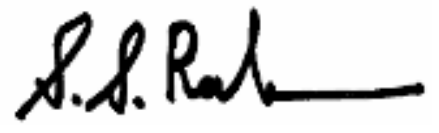

Ravikumar Srirangam

Environmental Engineer

cc: $\quad \operatorname{Jim}$ Mueller, Adventus

$\mathrm{EHC}^{\circledR}{ }^{\circledR}$ is a trademark of Adventus Intellectual Property Inc. 


\section{Appendix A:}

Proposal to Inject Remedial Compounds into a Class V Underground Injection Control Well (Injection Point): Former CCC/USDA Grain Storage Facility, Centralia, Kansas 


\section{Proposal to Inject Remedial Compounds into a Class V Underground Injection Control Well (Injection Point) \\ Former CCC/USDA Grain Storage Facility, Centralia, Kansas}

\section{Name of facility and facility owner.}

Facility Name: Centralia - USDA (KDHE project C406670940)

The facility was on property formerly leased by the Commodity Credit Corporation of the U.S. Department of Agriculture (CCC/USDA) for grain storage. No structures remain on the property. The proposed treatment area lies within the former CCC/USDA leasehold.

Mrs. Jean Burdett Lackey is the current owner of the contaminated part of the former grain storage facility, while Mr. Bob Cross owns the remainder of the former facility (surrounding and adjacent to the Burdett Lackey property). Injection is proposed on the portion owned by Mrs. Burdett Lackey. Monitoring is proposed on both the portion owned by Mrs. Burdett Lackey and the portion owned by Mr. Cross.

\section{Name, address and telephone number of facility owner.}

\section{CCC/USDA:}

Ms. Caroline Roe

Stop 0513 - Room 4715

1400 Independence Avenue, SW

Washington, DC 20250

202-720-9964

\section{Current Owners:}

Bob Cross

105 Main Street

Centralia, KS 66415

785-857-3511

- Permission for monitoring is required from Mr. Cross.

Jean Burdett Lackey

$7 \mathrm{E} \mathrm{Elk}$

Seneca, KS 66538

785-336-3698

- Permission for both injection and monitoring is required from Mrs. Burdett Lackey. 
3. Site legal description of the injection points, injection point identification numbers and a facility map with the location of the injection points depicted in relation to water supply wells and monitoring wells located at and near the facility.

The proposed 15 injection points, PT01-PT15, are located in the NE quarter of the NE quarter of the SE quarter of Section 1, Township 4 South, Range 11 East. One monitoring well, MW02, lies within the area targeted for injection. The four additional monitoring points nearest to the injection area are MW03, SB04, SB07R, and SB08. All of these locations are shown in Figure 1. Nemaha County Rural Water District 3 provides a public water supply to the city of Centralia and surrounding residences. The closest public water supply wells are approximately $6 \mathrm{mi}$ southwest of the former CCC/USDA facility location, as indicated in Figure 1.

Figure 1 Locations of proposed injection points, existing monitoring wells and piezometers, and the nearest PWS wells.

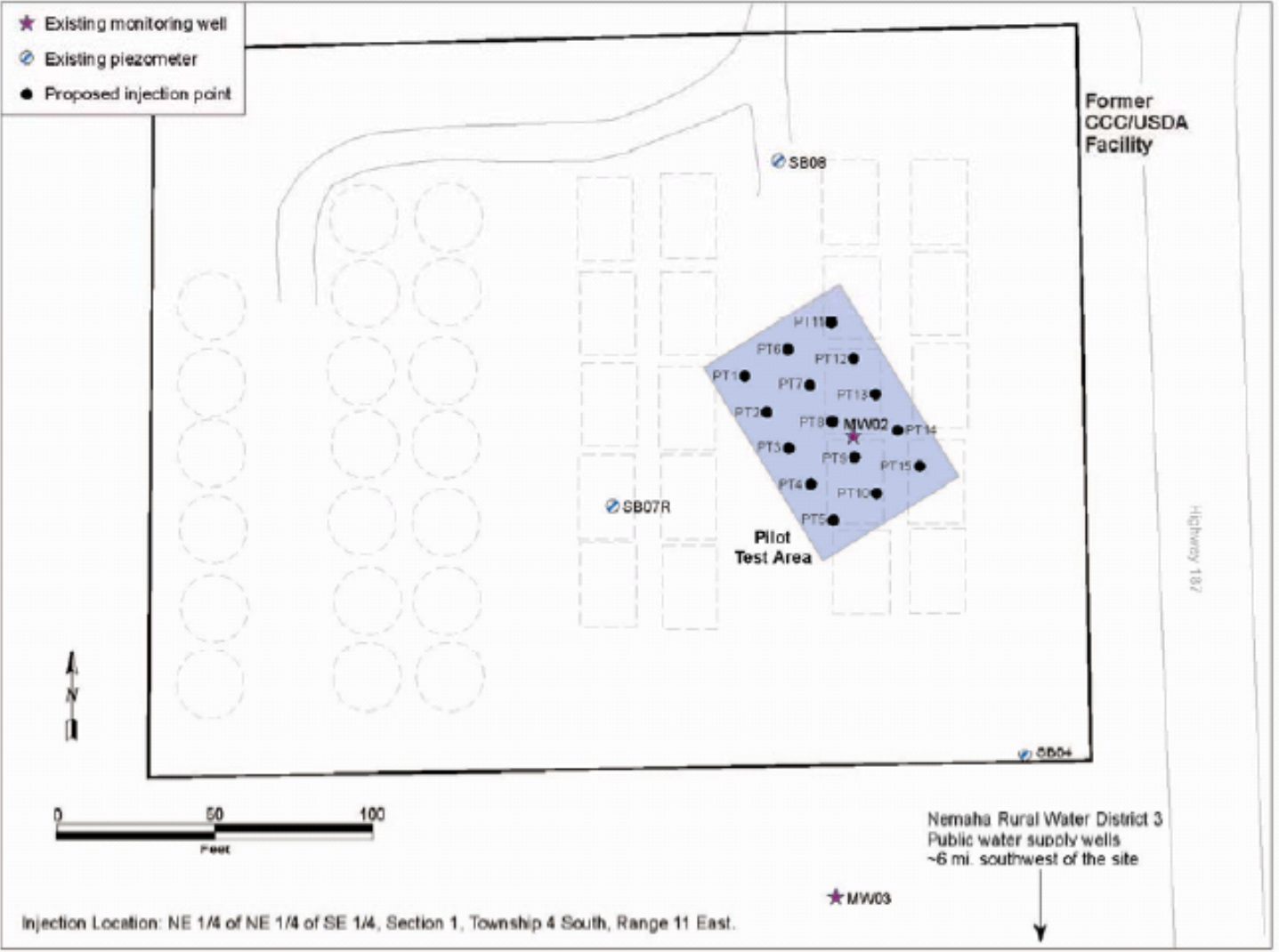

4. Documentation KDHE's Bureau of Environmental Remediation approves the injection of the remedial compounds for the remediation project.

The proposed remediation project, which involves injecting of remediation compounds, has been described in a document that is under review by the KDHE's Bureau of 
Environmental Remediation, on an expedited schedule. When approval is received from the $\mathrm{KDHE}$, the documentation will be sent separately to the permitting agency

\section{A description of the contamination and contamination source.}

The CCC/USDA operated a grain storage facility from 1949 until 1971. The Site is currently vacant (no structures remain on site) but two existing subsurface foundations are present that relate to subsequent concrete mixing operations. Two additional grain storage facilities are located within the vicinity of the former CCC/USDA facility: 1) The Nehama County Co-op located about 4,000 ft south (downgradient) of the Site, and 2) a private grain storage facility located about 3,500 north (upgradient) of the site.

Prior to 1986, CT was utilized as a grain fumigant at the Site. In 1998, CT was detected in groundwater from the Private Grain storage facility. Subsequent Site investigations identified CT impacts at the former CCC/USDA Site but these were limited to the subsurface soil and the upper shallow aquifer and were generally confined to the Site.

Investigations conducted on behalf of the Commodity Credit Corporation of the U.S. Department of Agriculture (CCC/USDA) by Argonne National Laboratory have demonstrated that groundwater at the Centralia site is contaminated with carbon tetrachloride at levels that exceed the Kansas Tier 2 Risk-Based Screening Level (RBSL) and the U.S. Environmental Protection Agency's maximum contaminant level of $5.0 \mathrm{~g} / \mathrm{L}$ for this compound. Groundwater sampling and analyses conducted by Argonne under a monitoring program approved by the Kansas Department of Health and Environment (KDHE) indicated that the carbon tetrachloride levels at several locations in the groundwater plume have increased since twice yearly monitoring of the site began in September 2005. The identified groundwater contamination currently poses no unacceptable health risks, in view of the absence of potential human receptors in the vicinity of the former CCC/USDA facility.

Carbon tetrachloride contamination has also been identified at Centralia in subsurface soils at concentrations on the order of the Kansas Tier 2 RBSL of $200 \mathrm{~g} / \mathrm{kg}$ in soil for the soil-to-groundwater protection pathway. 


\section{Schematic of a typical injection point design}

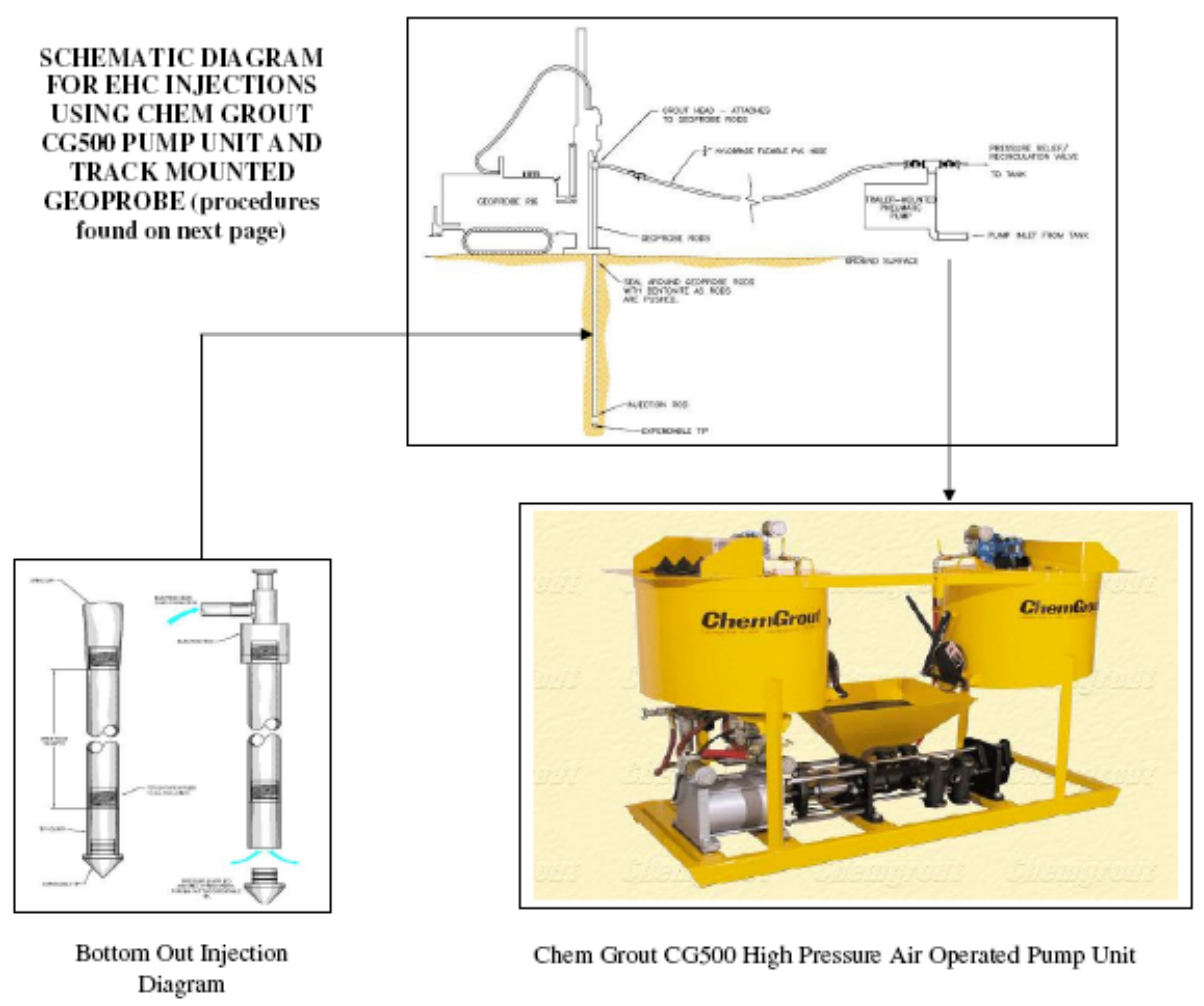

\section{Name and description of the geologic formation into which the remedial}

compound will be injected.

Groundwater CT impacts are generally confined to the area of the former CCC/USDA grain storage facility, and they are limited vertically to the shallow aquifer within the glacial outwash deposits of the Upper Pleistocene Independence Formation.

The aquifer is generally composed of glacial outwash consisting of coarser-grained sand deposits to finer-grained silt and clay. Groundwater is located at a depth of about 40 to $60 \mathrm{ft}$ bgs and flows to the south/southwest. The aquifer conductivity is very low, ranging from $10^{-3}$ to $10^{-9} \mathrm{~cm} / \mathrm{sec}$. As such, the groundwater CT impacts are stable (not moving horizontally or vertically), limited to the upper portion of the shallow aquifer, and they remain very localized on Site.

\section{Approximate depth below ground surface of injection interval.}

Two pilot study areas have been selected, as shown on Figure 2. 
Figure 2. Locations of proposed monitoring wells upgradient and downgradient of the treatment area. (Two monitoring wells inside of treatment area are not shown and will be placed at 3-4 $\mathrm{ft}$ from one injection point and midway between two injection points.)

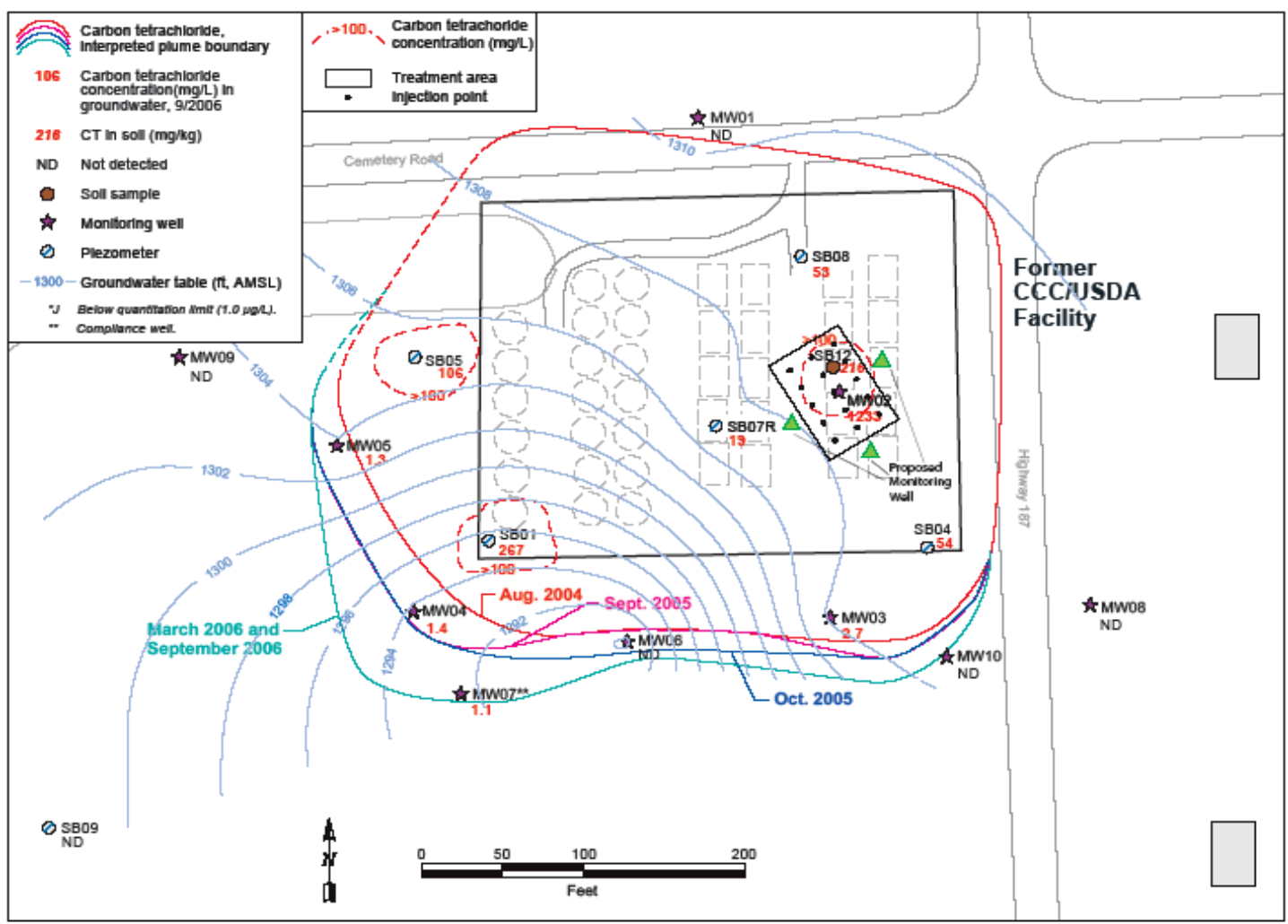

1. Treatment of Groundwater near SB-12 / MW-02

To perform the pilot study, EHC will be injected in a gridded area of $45 \times 75$ feet around SB-12 (designated as SB-12/Grid) over the depth interval of 40 to 60 feet bgs, with the grid spacing being adjusted in the field a bit near MW-02 and another monitoring well. In addition, three new monitoring wells will be installed. One of these new wells will be installed up- and two down- gradient of SB-12/Grid. Figure 1 depicts the approximate layout of SB-12/Grid and the new monitoring wells. Positive effects on the quality of groundwater will be observed in the areas generally downgradient of SB-12/Grid, and at the wells located within SB-12/Grid itself.

\section{Treatment of Soil in Vadose Zone}

This will be achieved via enhanced natural attenuation using an aqueous formulation of EHC, EHC-A, to allow for movement in the subsurface. EHC-A is composed of a water soluble organic amendment and soluble iron (Fe(II)). Soil concentrations above the treatment criteria of $200 \mathrm{mg} / \mathrm{kg}$ were only detected in one sample. However, EHC-A will 
be injected in ten of the fifteen geoprobe boring locations. The targeted area will hence cover approximately $2 / 3$ of SB-12/Grid, or 2,250 square feet over the depth range of 20 to 40 feet.

\section{Detailed description of the injection procedure, including proposed injection pressure.}

The injection procedure is described in detail below. The injection pressure may rise to 200 pounds per square inch or higher, as needed to deliver the injectate into the formation.

\section{EHC Injection Procedure}

1. Inspect all hoses, air and injection lines for any cracks or potential spots that could break and leak.

2. Read any MSDS sheets prior to chemical handling for any risks involved and advised PPE by chemical manufacturer.

3. Inspect tank for any residual chemicals. If needed flush out tank prior to injections to mitigate any adverse chemical reactions.

4. When attaching air hoses use whip-stops on both connections at the compressor and the pump.

5. While attaching injection lines check for locking cam locks. If the cam locks are not locking wrap connections in duct tape to keep attachments from coming loose.

6. Don level D PPE and a respirator prior to adding chemicals to the tank.

7. Add $\mathrm{EHC}$ to one of the two 50 -gal tanks.

8. Add water to the EHC in first tank. Wear all proper PPE and safety glasses during this process. Splashing will occur if mixing paddles are rotated too quickly. Keep hands away from the mixing paddles

9. Mix the chemicals using the mixing paddles adjusting the rotation with the control level. Once complete, reduce rotation revolutions.

10. Prepare for injection by closing off the re-circulating valve and opening up the valve to the injection line.

11. Open the valve at the injection head and charge the line at approx 20 to 60 psi. The pump will not stop when the line is charged; therefore, control the pressure by stokes of the piston pump (2-3 strokes). Once the line is charged lift the GeoProbe rods and continue injections. If tight conditions are encountered, fracture the soil. Increase the pressure with the pump control until injections 
12. In between injection intervals, shut off the pump using the control level to stop injections.

13. Relieve any backpressure by opening the injection valves and the re-circulation valves together to relieve any pressure back into the tank.

14. Once the backpressure has been relieved close the re-circulation lines and wait for the drillers signal to resume injections.

15. Continue mixing the $\mathrm{EHC}$ solution with the mixing paddles. Mix the $\mathrm{EHC}$ in the second tank while the injections are occurring in the first tank.

16. Upon completion of all injections of a single chemical, clean out the tank, lines, pump and plumbing before adding a new chemical to the tank. This is best done by rinsing the tank as the last injection is finishing, the water will clean the insides of the tank and will flush the lines. Once this is complete, drain the lines and the pumps of all residual liquids in an approved area.

17. When all injection activities are completed, completely decontaminate all equipment prior to leaving the site.

\section{Description of the contents and characteristics of the compounds to be injected.}

Two compounds are proposed to be injected at the site, Adventus EHC ${ }^{\mathrm{TM}}$ and EHC-A ${ }^{\mathrm{TM}}$. They are described in detail below, and Material Safety Data Sheets (MSDS) for the products are attached.

EHC technology describes a family of remediation products used for the in situ treatment of groundwater and saturated soil impacted by heavy metals and persistent organic compounds such as chlorinated solvents, pesticides and energetics. The technology is a modification of the Adventus DARAMEND $®$ technology which has been used since 1992 to treat over 2,500,000 tons of similarly effected soil and sediment. Both EHC and DARAMEND are the subjects of numerous patents owned by Adventus Intellectual Properties, Inc. (Adventus).

$\mathrm{EHC}$ is a controlled-release, integrated carbon and zero valent iron (ZVI) source that yields redox potential (Eh) in the -500 to $-650 \mathrm{mV}$ range. This Eh is significantly lower than that achieved when using either organic materials (lactate, molasses, and sugars) or reduced metal alone. Eh potentials in this range facilitate the timely and effective removal of normally recalcitrant chlorinated organics (e.g., CT, PCE) and other persistent compounds (e.g., perchlorate) without the formation of potentially problematic intermediates, such as DCE/VC from the anaerobic degradation of PCE/TCE or CF/DCM from the anaerobic degradation of CT (See below Figure 6). 
The organic component of EHC (fibrous organic material) is nutrient-rich, hydrophilic, and has high surface area; thus, it is an ideal support for growth of bacteria in the groundwater environment. As they grow on EHC particle surfaces, indigenous heterotrophic bacteria consume dissolved oxygen, thereby reducing the redox potential in groundwater. In addition, as the bacteria grow on the organic particles, they ferment carbon and release a variety of volatile fatty acids (VFAs, for example acetic, propionic, butyric), which diffuse from the site of fermentation into the groundwater plume and serve as electron donors for other bacteria, including dehalogenators and halorespiring species. Finally, the soluble ferrous sulfate particles provide substantial reactive surface area that stimulates direct chemical dechlorination and an additional drop in the redox potential of the groundwater via chemical oxygen scavenging. These physical, chemical, and biological processes combine to create an extremely reduced environment that stimulates chemical and microbiological dechlorination of otherwise persistent compounds.

EHC-A is a cold-water soluble formulation for application by injection or to existing wells or other networks. EHC-A is composed of a water soluble organic amendment and soluble iron $(\mathrm{Fe}(\mathrm{II}))$. Similarly to $\mathrm{EHC}$, physical, chemical, and biological processes combine to create an extremely reduced environment that stimulates dechlorination.

\section{The amount of remedial compound to be injected.}

Calculated application rates and total amounts of EHC and EHC-A to be injected are detailed in Tables 1 and 2, below.

Table 1. EHC Mass Requirements and Injection Details

\begin{tabular}{|l|c|c|}
\hline & Value & Unit \\
\hline Treatment Area Dimensions: & & $\mathrm{ft}$ \\
\hline Length of Treatment Area & 75 & $\mathrm{ft}$ \\
\hline Width of Treatment Area & 45 & $\mathrm{ft}$ \\
\hline Depth to Top of Treatment Area & 40 & $\mathrm{ft}$ \\
\hline Depth to Bottom of Treatment Area & 60 & $\mathrm{ft}$ \\
\hline Treatment Area Thickness & 20 & $\mathrm{ft} 3$ \\
\hline Treatment Area Volume & 67,500 & U.S. tons \\
\hline Mass of Soil in Treatment Area & 3,713 & $\mathrm{ft} 3$ \\
\hline Volume pore space & 20,250 & \\
\hline EHC mass calculations: & & Ibs \\
\hline Percentage EHC by soil mass & $0.10 \%$ & \\
\hline Mass of EHC Required & 7,500 & \\
\hline Preparation of EHC Slurry: & & $30 \%$ \\
\hline Percent solids in slurry (can be altered) & 2,100 & U.S. gallons \\
\hline Volume Water Required & 2,604 & U.S. gallons \\
\hline Slurry Volume to Inject & & \\
\hline Injection details: & & \\
\hline
\end{tabular}




\begin{tabular}{|l|c|c|}
\hline Injection spacing & 15 & $\mathrm{ft}$ \\
\hline Number of injection points & 15 & points \\
\hline Mass EHC per point & 500 & $\mathrm{lbs}$ \\
\hline Slurry volume per point & 174 & U.S. gallons \\
\hline Application rates for reference: & & \\
\hline Slurry volume to pore space volume & $1.7 \%$ & \\
\hline EHC concentration in groundwater & 0.4 & $\mathrm{lbs} / \mathrm{ft} 3$ \\
\hline
\end{tabular}

Table 2. EHC-A mass requirements and injection volumes.

\begin{tabular}{|l|c|c|}
\hline & Value & Unit \\
\hline Treatment Area Dimensions: & & $\mathrm{ft}$ \\
\hline Length of Treatment Area & 50 & $\mathrm{ft}$ \\
\hline Width of Treatment Area & 45 & $\mathrm{ft}$ \\
\hline Depth to Top of Treatment Area & 20 & $\mathrm{ft}$ \\
\hline Depth to Bottom of Treatment Area & 40 & $\mathrm{ft}$ \\
\hline Treatment Area Thickness & 20 & $\mathrm{ft} 3$ \\
\hline Treatment Area Volume & 45,000 & U.S. tons \\
\hline Mass of Soil in Treatment Area & 2,475 & $\mathrm{ft} 3$ \\
\hline Volume pore space & 13,500 & \\
\hline EHC mass calculations: & & $\mathrm{lbs}$ \\
\hline Percentage EHC by soil mass & $0.10 \%$ & \\
\hline Mass of EHC Required & 5,000 & $\mathrm{Ibs} / \mathrm{ft} 3$ \\
\hline Preparation of EHC Slurry: & & U.S. gallons \\
\hline Percent solids in slurry (can be altered) & $10 \%$ & U.S. gallons \\
\hline Volume Water Required & 5,399 & \\
\hline Slurry Volume to Inject & 5,555 & $\mathrm{ft}$ \\
\hline Injection details: & & points \\
\hline Injection spacing & 15 & $9.5 \%$ gallons \\
\hline Number of injection points & 556 & \\
\hline Mass EHC per point & 617 & \\
\hline Slurry volume per point & & \\
\hline Application rates for reference: & & \\
\hline Slurry volume to pore space volume & & \\
\hline EHC concentration in groundwater & & \\
\hline
\end{tabular}

\section{Frequency of Injection}

The intent of this pilot test is to inject these compounds only once. 
13. Plugging procedure for the injection point including a schematic of the injection point after plugging.

\section{Borehole Plugging Procedure}

1. Upon completion of injection activities and removal of the GeoProbe injection rods, fill the borehole with bentonite Hole Plug+ (Figure 3). Note: Bentonite Hole Plug does not hydrate very quickly and is dense enough to fall below the water table surface and will provide a good seal. Cement grout should be avoided since it is possible to displace the grout in subsequent injections nearby.

Figure 3. Schematic of borehole plugging procedure.

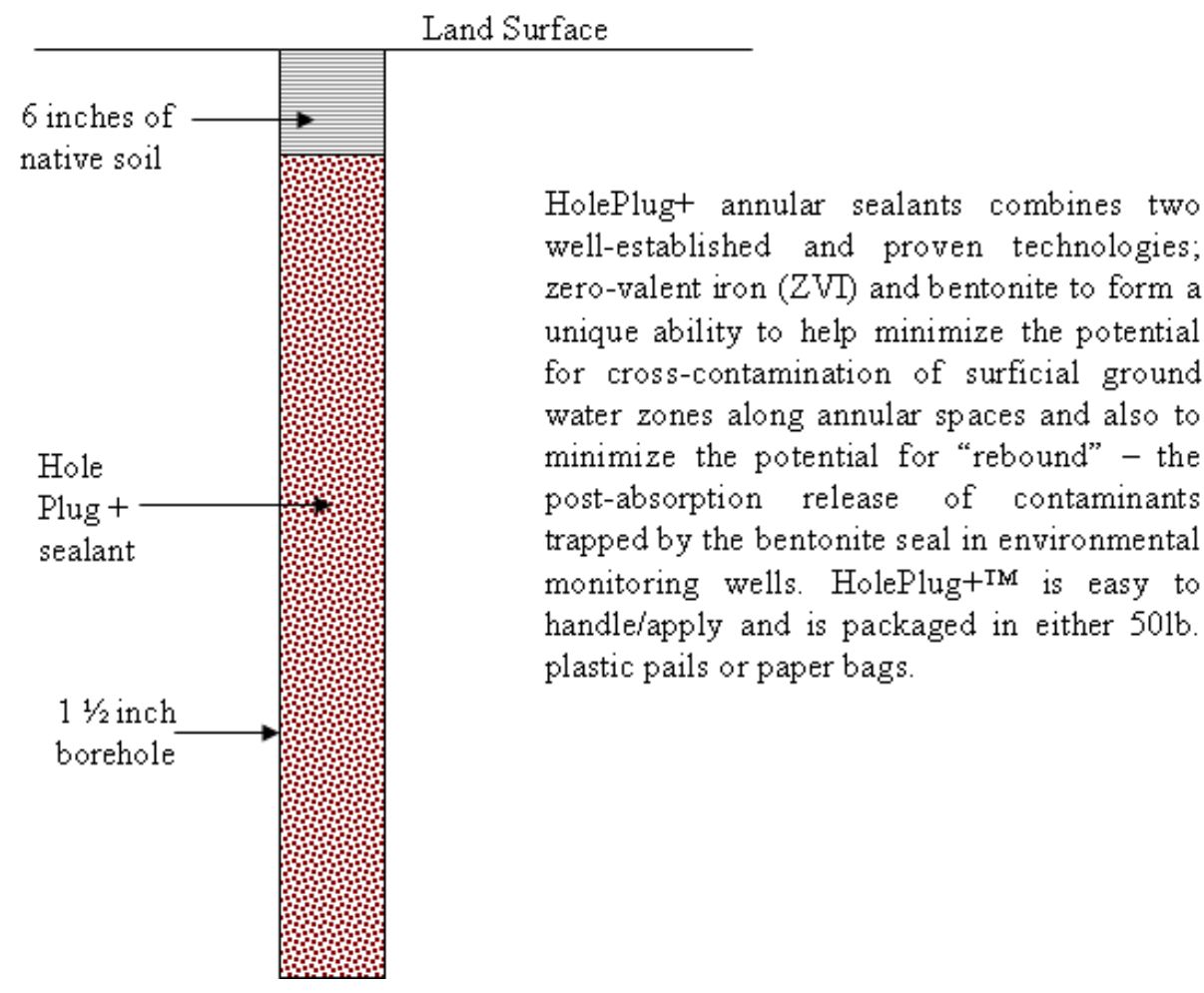

2. Bentonite granular material may be placed in the borehole above the water table surface and is preferred when a fast-hydration is needed. Do not use below the water table because bridging will occur.

3. Fill the last few inches of the borehole with native soils or asphalt patch or cement patch in paved areas. 


\section{Description of the basic chemistry of the remediation process, including products and byproducts.}

Following placement of EHC into the subsurface environment, a number of physical, chemical and microbiological processes combine to create very strong reducing conditions that stimulate rapid and complete dechlorination of organic solvents and other recalcitrant compounds. First, the organic component of EHC (fibrous organic material) is nutrient rich, hydrophilic and has high surface area; thus, it is an ideal support for growth of bacteria in the groundwater environment. As they grow on EHC particle surfaces, indigenous heterotrophic bacteria consume dissolved oxygen thereby reducing the redox potential in groundwater. In addition, as the bacteria grow on the organic particles, they ferment carbon and release a variety of volatile fatty acids (acetic, propionic, butyric) which diffuse from the site of fermentation into the groundwater plume and serve as electron donors for other bacteria, including dehalogenators and halorespiring species. Finally, the small ZVI particles ( $<5$ to $45 \mu \mathrm{m})$ provide substantial reactive surface area that stimulates direct chemical dechlorination and an additional drop in the redox potential of the groundwater via chemical oxygen scavenging.

These physical, chemical and biological processes combine to create an extremely reduced environment that stimulates chemical and microbiological dechlorination of otherwise persistent compounds. Redox potentials as low as $-550 \mathrm{mV}$ are commonly observed in groundwater after EHC application. At these Eh levels, many organic constituents of interest $(\mathrm{COI})$ are thermodynamically unstable and they will readily degrade via pathways more typical of physical destruction processes (minimum production and no accumulation of typically recognized biodegradation intermediates). Hence, the ISCR technology is microbiologically based in that we rely on indigenous microbes to biodegrade the EHC carbon (refined plant materials), but we do not require the presence or activity of special or otherwise unique bacteria for complete and effective remediation.

In either event, the fibrous organic carbon and ZVI or other reduced metal that comprises the slow release EHC will remain in the location where it is injected. It will not only treat COI that migrates into the treated area, but it will also have a 'halo' or 'zone of influence' of low redox conditions that will extend beyond its physical space, greatly increasing its effectiveness. Figure 4 provides an example of how a small fracture of EHC creates a wide zone of influence outside of its immediate location. The native soil color is the yellow visible on the right hand side of the core. The orange discoloration is due to the low redox conditions created by the $\mathrm{EHC}$, which became apparent after exposure to the air for 2 hours. 
Figure 4. Photograph of a soil core, from $30 \mathrm{ft}$ to $33 \mathrm{ft} \mathrm{bgs,} \mathrm{showing} \mathrm{a} \mathrm{1-inch}$ fracture

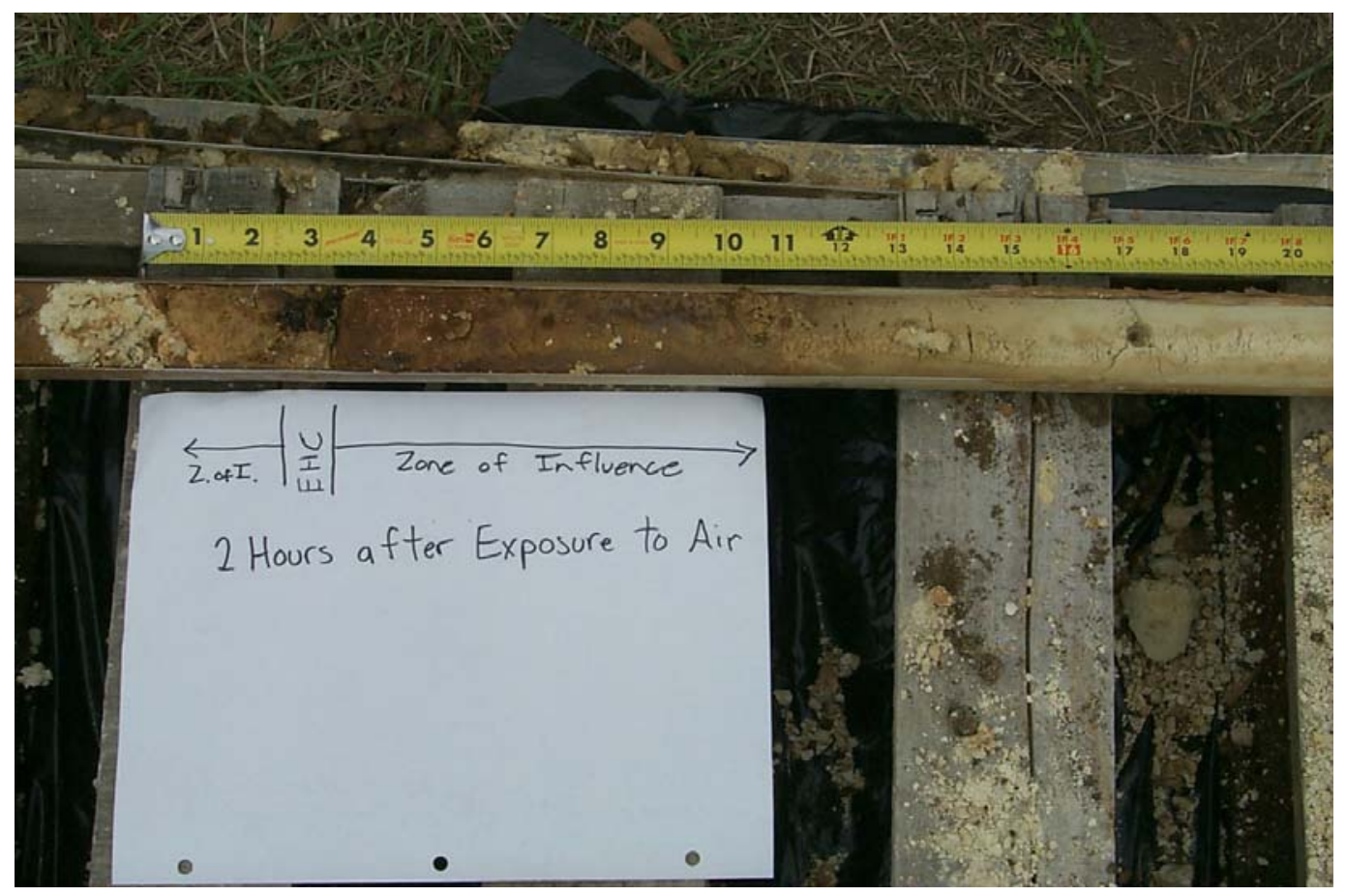

During aerobic respiration, dissolved oxygen concentrations decrease. After depletion of dissolved oxygen, anaerobic microorganisms utilize nitrate as an electron acceptor, followed by iron (III), sulfate, and finally carbon dioxide (methanogenesis). Each sequential reaction drives the oxidation reduction potential further down in to the range in which reductive dechlorination can occur. Reductive dechlorination is most effective in the ORP range corresponding to sulfate reduction and methanogenesis. When such conditions exist, the alternate pathway to carbon disulfide shown below can be realized. (Criddle, et. al., 1990; Freedman et. al., 1995; Devlin and Muller, 1999; Hashsham and Freedman, 1999).

It is critical to understand that the processes of COI destruction under ISCR conditions are different from the typical pathways. As an example, it is well documented for PCE and TCE that under ISCR conditions (Eh $<-550 \mathrm{mV}$ ), these pathways are avoided and terminal destruction / mineralization proceeds along the lines of the recognized betaelimination pathways (Figure 5). These differences have been described by various experts in the field of biotransformation processes (e.g., Dr. John Wilson, US EPA as reported in the AFCEE Technology Transfer Seminar, 2003; Dr. Mark Ferry, MPCAA; J, Szecsody and J. Fruchter et al., Battelle Pacific Northwest National Laboratory). For Carbon Tetrachloride and related compounds, the ISCR pathway primarily entails the direct mineralization to innocuous terminal end products formate and carbon monoxide (Figures 6a and 6b). 
Figure 5. PCE ITCE Degradation Schematic - Representative Reactions for Mineralization under ISCR Conditions

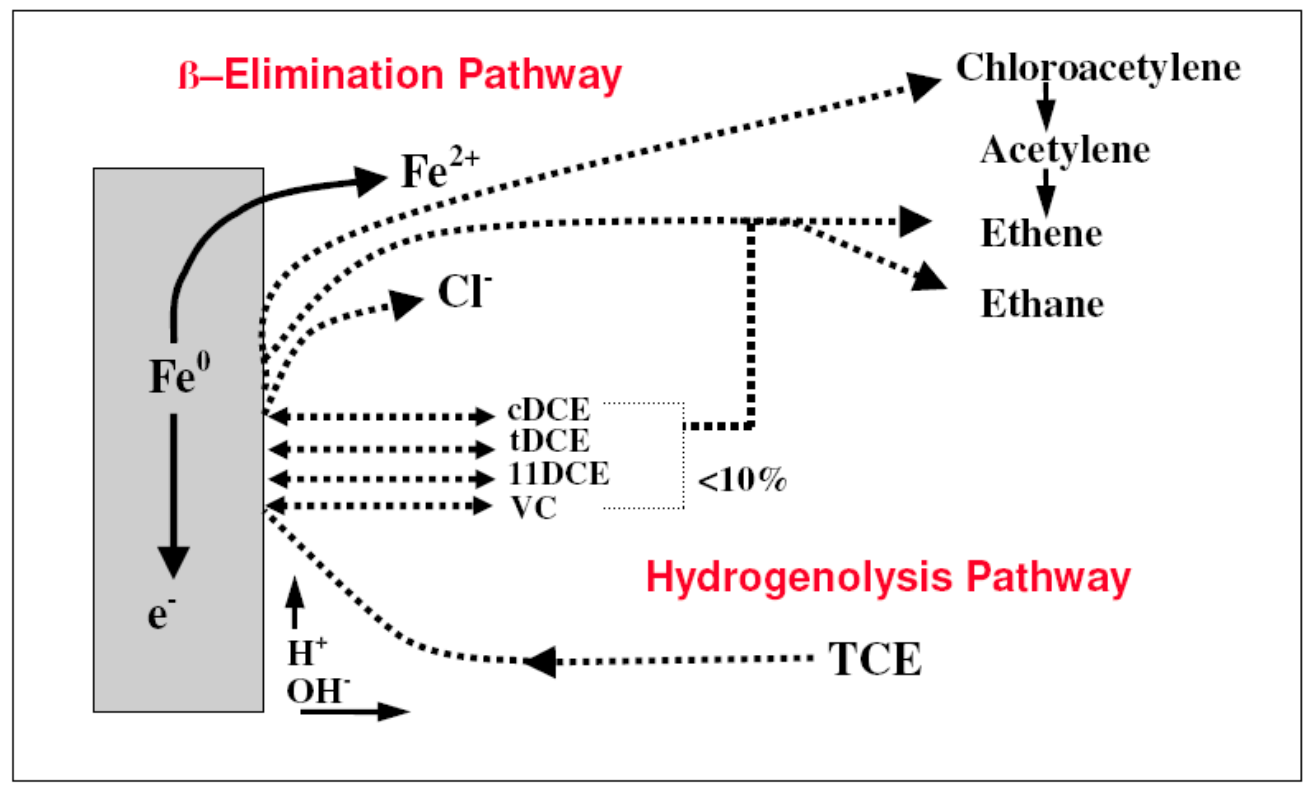

Figure 6a. CT Degradation Schematic - Sequential Reductive Dehalogenation and Sulfate Reduction Reactions under Typical Anaerobic Conditions

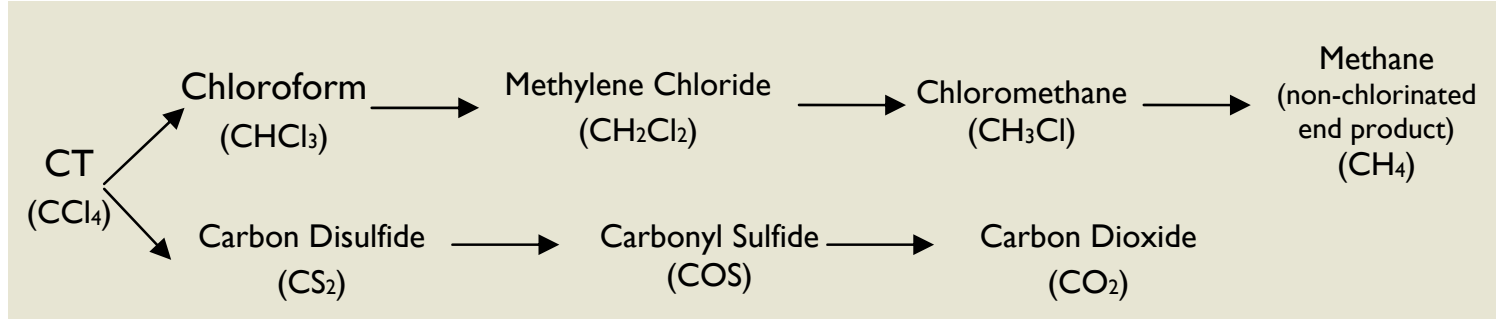

Note that many of these end products (methane, carbonyl sulfide and carbon dioxide) are gases under standard conditions. 
Figure 6b. CT Degradation Schematic Under ISCR Conditions (Middle Column) as compared to typical Sequential Reductive Dehalogenation (left column) and dichloroelimination reactions, which DO NOT occur under high iron, ISCR conditions (right column).

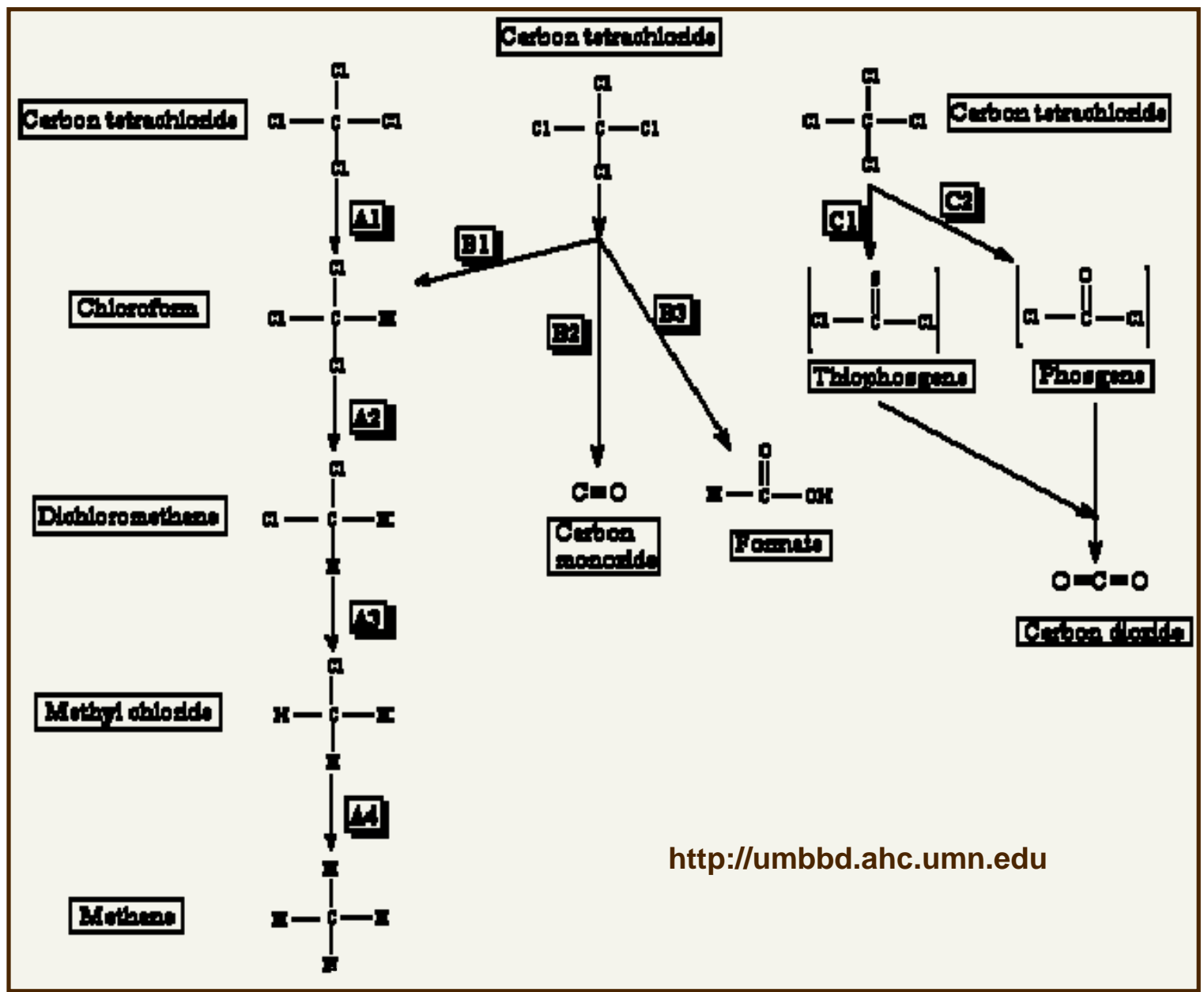

\section{References}

Biteman, S., J. Valkenburg, S. MacFabe, J. Mueller and J. Molin. 2007. Pilot-Scale Reductive Dechlorination of Carbon Tetrachloride in Groundwater. Battelle's Ninth International In Situ and On-Site Bioremediation Symposium, May 7-10, 2007 Baltimore, MD.

Criddle, C.S., DeWitt, J.T., and McCarty , P.L., Reductive Dehalogenation of Carbon Tetrachloride by Escherichia coli K-12, in Applied and Environmental Microbiology, November 1990, p. 3247-3254.

Devlin, J.F., and Muller, D., Field and Laboratory Studies of Carbon Tetrachloride Transformation in a Sandy Aquifer under Sulfate Reducing Conditions, in Environmental Science and Technology, 1999, v. 33, p. 1021-1027. 
Dolfing, J. M. van Eekert, A. Seech, J. Vogan and J. Mueller (2008).In Situ Chemical Reduction (ISCR) Technologies - Significance of Low Eh Reactions. International Journal of Soil \& Sediment Contamination (in press).

Freedman, D.L., Lasecki, M., Hashsham, S.A., and Scholze, R. In Bioremediation of Chlorinated Solvents; Hinchee, R. E. Leeson, A., Semprini, L., Eds., Battelle Press: Columbus, OH 1995, p. 123-137.

Hashsham, S.A., and Freedman, D.L., Enhanced Biotransformation of Carbon Tetrachloride by Acetobacterium wodii upon Addition of Hydroxocobalamin and Fructose, in Applied and Environmental Microbiology, October 1999, p. 4537-4542.

Mueller, J., J. Molin. A .Seech, K. Bolanos-Shaw and D. Hill and A. Seech. 2006. Effect of $\mathrm{EHC}^{\mathrm{TM}}$ In Situ Chemical Reduction (ISCR) on Chlorinated Compounds under Laboratory and Field Conditions. AEHS 16th Annual West Coast Conference on Soils, Sediments and Water March 13-16, 2006 San Diego, CA 


\section{Argonne}

November 12, 2007

Mr. Kirk Hoeffer

Kansas Departmetnt of Health and Environment

khoeffer@kdhe.ks.us

Subject: Amendment to Injection Permit-Centralia, $K S$
Jorge S. Alvarado, Ph.D.

Applied Geosciences and Environmental Management Section

Environmental Science Division Argonne National Laboratory 9700 South Cass Avenue, Bldg. 203 Argonne, IL 60439-4843

1-630-252-5267 phone

1-630-252-5747 fax

jsalvarado@anl.gov

\section{Dear Mr. Hoeffer:}

Following instructions from Mr. Christopher C. Carey of the Kansas Department of Health and Environment $(\mathrm{KDHE})$ for the implementation of the pilot plan in the remediation of the former CCC/USDA site at Centralia, KS, he requested the introduction of a new compound to be use as tracer during the injection of $\mathrm{EHC}^{\mathrm{TM}}$ product.

We have selected to introduce bromide $\left(\mathrm{Br}^{-}\right)$as tracer for this project as potassium bromide $(\mathrm{KBr})$ at a concentration of $\mathrm{Br}$ - not to exceed $200 \mathrm{mg} / \mathrm{L}$. Enclosed you will find the Material Safety Data Sheet of Potassium Bromide. The product is stable under normal temperature and pressures, solid, colorless to white, odorless, neutral in solution and not listed as carcinogenic. This product is not listed as Hazardous Substance under the Clean Water Act (CWA). None of the chemicals in this product are listed as Priority Pollutants or toxic pollutants under CWA. None of the chemicals in this product are considered highly hazardous by OSHA. This material not contains any hazardous air pollutants.

Due to the request of KDHE and the need to use a tracer in this project, I would like to request an amendment to the injection permit to include potassium bromide in the list of chemicals to be injected in the remediation project at Centralia, KS.

If you have any questions, please do not hesitate to call me at the number above, Sincerely,

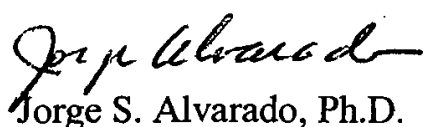

Environmental Science Division

JSA:rs 


\section{Amendment to Injection Permit}

Kirk:

This note has been prepared In accordance with Chris Carey's request that we add a tracer to the EHC slurry that we will be injecting at the CCC/ANL site in Centralia.

We are looking to inject the bromide along with a slightly more dilute mix of EHC to improve injectability and potentially decrease injection pressures. Accordingly, this table replaces the original Table 1 provided in the October 30, 2007 Proposal to Inject:

\begin{tabular}{|c|c|c|}
\hline & Value & Unit \\
\hline \multicolumn{3}{|l|}{ Treatment Area Dimensions: } \\
\hline Length of Treatment Area & 75 & $\mathrm{ft}$ \\
\hline Width of Treatment Area & 45 & $\mathrm{ft}$ \\
\hline Depth to Top of Treatment Area & 40 & $\mathrm{ft}$ \\
\hline Depth to Bottom of Treatment Area & 60 & $\mathrm{ft}$ \\
\hline Treatment Area Thickness & 20 & $\mathrm{ft}$ \\
\hline Treatment Area Volume & 67,500 & $\mathrm{ft}^{3}$ \\
\hline Mass of Soil in Treatment Area & 3,713 & U.S. tons \\
\hline Volume pore space & 20,250 & $\mathrm{ft}^{3}$ \\
\hline \multicolumn{3}{|l|}{ EHC mass calculations: } \\
\hline Percentage EHC by soil mass. & $0.10 \%$ & \\
\hline Mass of EHC Required & 7,500 & Ibs \\
\hline \multicolumn{3}{|l|}{ Preparation of EHC Slurry: } \\
\hline Percent solids in slurry (can be altered) & $23.1 \%$ & \\
\hline Volume Water Required & 3,000 & U.S. gallons \\
\hline Slurry Volume to Inject & 3,386 & U.S. gallons \\
\hline \multicolumn{3}{|l|}{ Injection details: } \\
\hline Injection spacing & 15 & $\mathrm{ft}$ \\
\hline Number of injection points & 15 & points \\
\hline Mass EHC per point & 500 & Ibs \\
\hline Slurry volume per point & 226 & U.S. gallons \\
\hline \multicolumn{3}{|l|}{ Application rates for reference: } \\
\hline Slurry volume to pore space volume & $2.2 \%$ & \\
\hline EHC concentration in groundwater & 0.4 & $\mathrm{Ibs} / \mathrm{ft}^{3}$ \\
\hline
\end{tabular}

Starting with this information, here are some of the relevant factors we have used in selecting a bromide tracer dosage:

1). Assume that we like to reach the surrounding 5 newly installed piezometers, which will cover an additional area $22.5 \mathrm{ft}$ from edge of the treatment area. So the maximum area to be covered is $90 \times 120 \mathrm{ft}^{2}$

2). Assume that the maximum dilution vertically will extend $5 \mathrm{ft}$ above and below. So thickness will be $30 \mathrm{ft}$.

3). The entire soil volume will be $324,000 \mathrm{ft}^{3}$ and pore volume will be $97,200 \mathrm{ft}^{3}$ based on a maximum effective porosity of 0.3 . The total groundwater will be about 800,000 gallon. 
4). The proposed total injection slurry ( $\sim 3400$ gallon) is made of 3000 -gallon water mixed with EHC.

5). The following estimation is based on the initial bromide concentration in -3000 gallon of water:

\begin{tabular}{|r|r|}
\hline $\begin{array}{l}\text { Initial } \\
\mathrm{Br}^{-1} \\
\text { conc } \\
\text { (mg/L) }\end{array}$ & $\begin{array}{l}\text { Diluted } \\
\mathrm{Br}^{-1} \\
(\mathrm{mg} / \mathrm{L})\end{array}$ \\
\hline 400 & 1.70 \\
\hline 200 & 0.85 \\
\hline 150 & 0.63 \\
\hline 100 & 0.42 \\
\hline
\end{tabular}

The analytical method has a detection limit ranging from 0.0025 to $0.014 \mathrm{mg} / \mathrm{L}$. Considering the highest detection limit and by trying to be at least one order of magnitude higher than the method detection limits, then we choose to use $150 \mathrm{mg} / \mathrm{L}$ concentration of $\mathrm{Br}^{-}(223 \mathrm{mg} / \mathrm{L}$ as KBr$)$. For 3000 gallons of water to be mixed with the $\mathrm{EHC}$, we would need $2530 \mathrm{~g}$ of $\mathrm{KBr}$ to make a 150 $\mathrm{mg} / \mathrm{L}$ concentration.

Bromide concentrations in drinking water are around $0.1 \mathrm{mg} / \mathrm{L}$. Because we are going to be above this level, regular drinking water will be fine for this application.

Thanks,

John Valkenburg, P.E. / Senior Engineer / Adventus Americas Inc. / 1493 West Pratt Road / DeWitt, Ml 48820

Phone: 5176695400 / Fax: 5176695455 


\title{
MATERIAL SAFETY DATA SHEET
}

\author{
POTASSIUM BROMIDE, STANDARD INFRARED GRADE, \\ MATERIAL SAFETY DATA SHEET \\ NSN: 681000 N037212 \\ Manufacturer's CAGE: 60928 \\ Part No. Indicator: A \\ Part Number/Trade Name: POTASSIUM BROMIDE, STANDARD INFRARED GRADE,

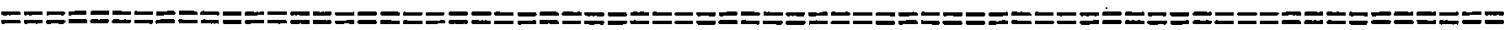 \\ Ingredients/ldentity Information

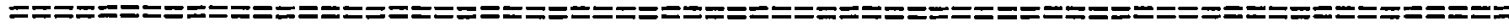 \\ Proprietary: NO \\ Ingredient: POTASSIUM BROMIDE; (POTASSIUM BROMIDE, STANDARD INFRARED \\ GRADE) \\ Ingredient Sequence Number: 01 \\ NIOSH (RTECS) Number: TS7650000 \\ CAS Number: 7758-02-3 \\ OSHA PEL: NOT APPI_ICABLE \\ ACGIH TLV: NOT APPLICABLE

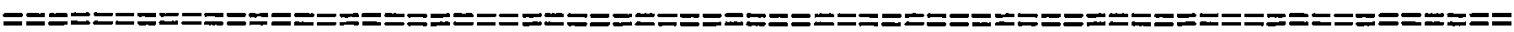

Physical/Chemical Characteristics

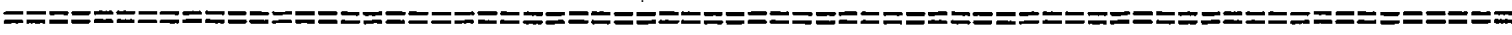

Appearance and Odor: NONE SPECIFIED BY MANUFACTURER.

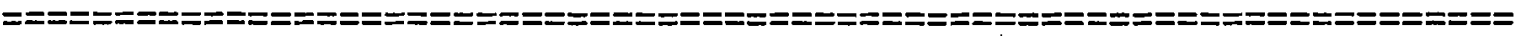

Fire and Explosion Hazard Data

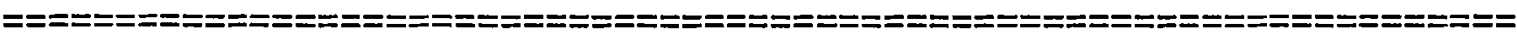

Extinguishing Media: CARBON DIOXIDE, DRY CHEMICAL POWDER, ALCOHOL OR

POLYMER FOAM.

Special Fire Fighting Proc: WEAR NIOSH/MSHA APPROVED SCBA AND FULL

PROTECTIVE EQUIPMENT (FP N). PREVENT CONTACT WITH SKIN AND EYES.

Unusual Fire And Expl Hazards: EMITS TOXIC FUMES UNDER FIRE CONDITIONS.

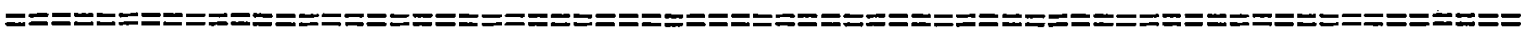

Reactivity Data

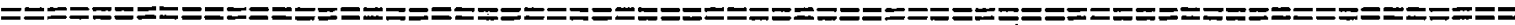

Stability: YES

Cond to Avoid (Stability): NONE SPECIFIED BY MANUFACTURER.

Materials to Avoid: STRONG OXIDIZING AGENTS, STRONG ACIDS, HEAVY METAL

SALTS.

Hazardous Decomp Products: HYDROGEN BROMIDE GAS.

Hazardous Poly Occur: NO

Conditions to Avoid (Poly): NOT RELEVANT

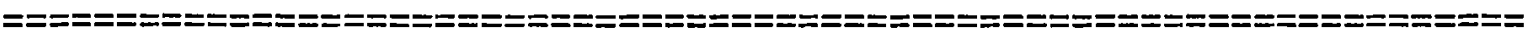


Health Hazard Data

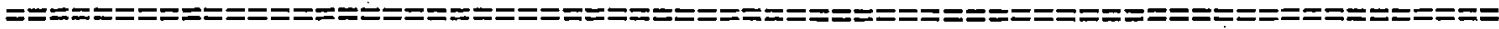

LD50-LC50 Mixture: NONE SPECIFIED BY MANUFACTURER.

Route of Entry - Inhalation: YES

Route of Entry - Skin: YES

Route of Entry - Ingestion: NO

Health Haz Acute And Chronic: ACUTE: HARMFUL IF SWALLOWED. MAY BE HARMFUL IF ABSORBED THRU SKIN. MAY BE HARMFUL IF INHALED. CAUSES EYE AND SKIN IRRITATION. MATERIAL IS IRRITATING TO MUCOUS MEMBRANES AND UPPER

RESPIRATORY TRACT. CAN CAUSE CNS DEPRESSION. POTASSIUM BROMIDE ON PROLONGED

CONTACT W/MOIST SKIN CAN PRODUCE SEVERE (EFTS OF OVEREXP)

Carcinogenicity - NTP: NO

Carcinogenicity - IARC: NO

Carcinogenicity - OSHA: NO

Explanation Carcinogenicity: NOT RELEVANT

Signs/Symptoms of Overexp: HLTH HAZ:IRRIT/BURNS. PRLNGD INHAL OF DUST CAN

PRDCE BRONCHITIS. INGEST OF LG QTY CAN CAUSE IRRITABILITY, CONFUSION, TREMORS, ACNE-LIKE ERUPTIOINS, MEMORY LOSS, HDCH, SLURRED SPEECH \& ANOREXIA.

TO THE BEST OF OUR KNOWLEDGE, THE CHEMICAL, PHYSICAL, \& TOXICOLOGICAL PROPERTIES HAVE NOT BEEN THOROUGHLY INVESTIGATED.

Med Cond Aggravated By Exp: NONE SPECIFIED BY MANUFACTURER.

Emergency/First Aid Proc: EYES: IMMED FLUSH WITH COPIOUS AMOUNTS OF WATER FOR AT LEAST 15 MINUTES. SKIN: IMMED WASH WITH SOAP AND COPIOUS AMOUNTS OF

WATER. WASH CONTAMINATED CLOTHING BEFORE REUSE. INHAL: REMOVE TO FRESH AIR.

IF NOT BREATHING GIVE ARTIFICIAL RESPIRATION. IF BREATHING IS DIFFICULT, GIVE OXYGEN. INGEST: WASH OUT MOUTH WITH WATER PROVIDED PERSON IS CONSCIOUS. CALL A PHYSICIAN.

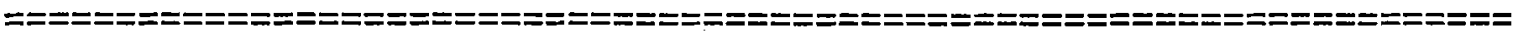

Precautions for Safe Handling and Use

Steps If Matl Released/Spill: EVACUATE AREA. WEAR NIOSH/MSHA APPROVED

RESPIRATOR, CHEMICAL SAFETY GOGGLES, RUBBER BOOTS \& HEAVY RUBBER GLOVES.

SWEEP UP, PLACE IN A BAG \& HOLD FOR WASTE DISPOSAL. AVOID RAISING DUST.

VENTILATE AREA AND WASH SPILL SITE AFTER MATL PICKUP IS COMPLETE.

Neutralizing Agent: NONE SPECIFIED BY MANUFACTURER.

Waste Disposal Method: FOR SMALL QTY:CAUTIOUSLY ADD TO A LARGE STIRRED

EXCESS OF WATER. ADJUST PH TO NEUTRAL, SEPARATE ANY INSOL SOLIDS/LIQ \& PKG

THEM FOR HAZ-WASTE DISP. FLUSH AQUEOUS SOLN DOWN DRAIN W/PLENTY OF WATER.

HYDROLYSIS \& NEUT RXN MAY GENERATE HEAT \& (SUPP DATA)

Precautions-Handling/Storing: DO NOT BREATHE DUST. AVOID CONT W/EYES/SKIN/

CLTHG. AVOID PRLNGD/RPTD EXPOSURE. IRRITANT. KEEP TIGHTTLY CLOSED.

HYGROSCOPIC. STORE IN A COOL DRY PLACE.

Other Precautions: NONE SPECIFIED BY MANUFACTURER. 
Control Measures

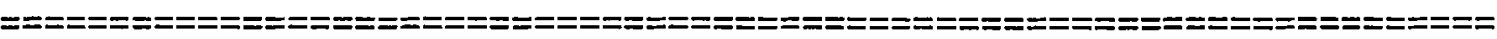

Respiratory Protection: WEAR APPROPRIATE NIOSH/MSHA APPROVED RESPIRATOR. Ventilation: MECHANICAL EXHAUST REQUIRED.

Protective Gloves: CHEMICAL-RESISTANT GLOVES.

Eye Protection: CHEMICAL WORKERS GOGGLES (FP N).

Other Protective Equipment: SAFETY SHOWER AND EYE BATH. RUBBER BOOTS. WEAR

OTHER PROTECTIVE CLOTHING.

Work Hygienic Practices: WASH THOROUGHLY AFTER HANDLING.

Suppl. Safety \& Health Data: WASTE DISP METH: FUMES WHICH CAN BE

CONTROLLED BY THE RATE OF ADDITION. OBSERVE ALL FEDERAL, STATE AND LOCAL

ENVIRONMENTAL REGULATIONS.

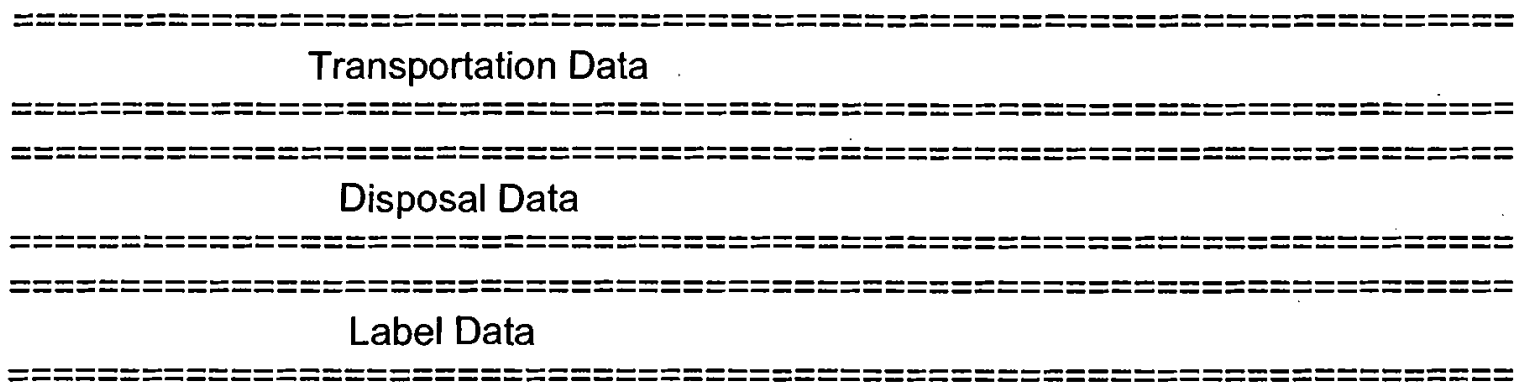

Label Required: YES

Technical Review Date: 02DEC92

Label Date: 02DEC92

Label Status: $\mathrm{G}$

Common Name: POTASSIUM BROMIDE, STANDARD INFRARED GRADE, 34650-0

Chronic Hazard: YES

Signal Word: WARNING!

Acute Health Hazard-Moderate: $X$

Contact Hazard-Slight: $X$

Fire Hazard-None: $X$

Reactivity Hazard-None: $X$

Special Hazard Precautions: ACUTE: INGESTION, SKIN ABSORPTION OR

INHALATION MAY BE HARMFUL. EYE AND SKIN CONTACT MAY CAUSE IRRITATION.

MATERIAL MAY BE IRRITATING TO MUCOUS MEMBRANES AND UPPER RESPIRATORY TRACT

\& MAY CAUSE CNS DEPRESSION. PROLONGED CONTACT W/MOIST SKIN MAY PRODUCE

SEVERE IRRITATION OR BURNS. INGESTION OF LARGE QUANTITIES MAY CAUSE

IRRITABILITY, CONFUSION, TREMORS, ACNE-LIKE SKIN ERUPTIONS, MEMORY LOSS,

HEADACHE, SLURRED SPEECH AND ANOREXIA. CHRONIC: PROLONGED INHALATION OF

DUST MAY PRODUCE BRONCHITIS.

Protect Eye: $Y$

Protect Skin: $Y$

Protect Respiratory: $Y$

Label Country: US

Label Emergency Number: 414-273-3850 


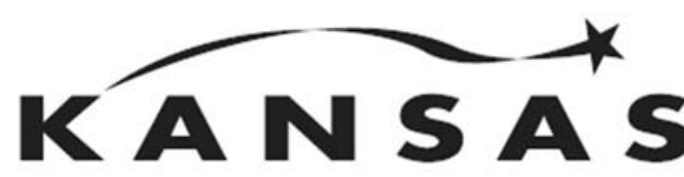

Kathleen Sebelius, Governor

Roderick L. Bremby, Secretary

DEPARTMENT OF HEALTH

AND ENVIRONMENT

www.kdheks.gov

Division of Environment

November 20, 2007

Mr. Lorraine M. LaFreniere, Ph.D.

Argonne National Laboratory

9700 South Cass Avenue, Bldg. 203

Argonne, IL 60439-4843

RE: CCC/USDA Site at Centralia

Class V UIC Authorization

15 Injection Points

Dear: Mr. LaFreniere:

The Kansas Department of Health \& Environment's Geology Section KDHE administers the Underground Injection Control (UIC) program. The UIC program has oversite of Class V injection points and has completed its review of this injection proposal submitted under your letter dated November 2, 2007, for compliance with the Underground Injection Control (UIC) Program Requirements. We have determined the proposal complies with the UIC Program requirements. This letter serves as the UIC Program authorization for the injection points.

This proposal was only reviewed for compliance with the UIC Program requirements. BER has oversight authority for this project. Your attached letter, from Chris Carey with BER, indicates approval to install and operate the injection points.

The following conditions required by the UIC Program apply:

The injection points shall not endanger public health or the environment.

This authorization is valid only for this proposal.

This authorization is only for the injection of EHC, EHC-A, and bromide tracer solution.

BUREAU OF WATER - GEOLOGY SECTION

CURTIS STATE OFFICE BUILDING, 1000 SW JACKSON ST., STE. 420, TOPEKA, KS 66612-1367

Voice 785-296-5524 Fax 785-296-5509 Web http://kdheks/gov/geo 
Mr. Lorraine LaFreniere, Ph.D.

November 2, 2007

Pg. 2

Injection pressure must be kept below 300 psi, unless prior approval is received from KDHE.

Proposed significant changes of the injection proposal must be submitted to KDHE in writing, with supportive information, and have the approval of both KDHE=s Bureau of Environmental Remediation (BER) and the UIC program prior to implementation.

If you have any questions, please call me at (785) 296-1843 or email at khoeffne@kdhe.state.ks.us.

Sincerely,

Kirk Hoeffner, L.G.

Unit Chief, Underground Injection Control Program

Geology Section

Bureau of Water

C: $\quad$ Julie Coleman - KDHE/NEDO

Mike Cochran $\rightarrow$ File: USDA Centralia, Nemaha Co. Class V - General 
Appendix B:

Correspondence 


\section{John Valkenburg}

From: John Valkenburg [john.valkenburg@adventusgroup.com]

Sent: Tuesday, November 27, 2007 8:07 PM

To: KHoeffner@kdhe.state.ks.us

Cc: $\quad$ 'LaFreniere, Lorraine M.'; 'jim mueller@adventusgroup.com'; 'Ravi'; 'John Valkenburg'; 'Greg Powers'; 'haselow@redox-tech.com'; 'John Valkenburg'

Subject: Centralia Injection Status Update (11/27/07)

Kirk -

As discussed by phone today, we have observed these average injection pressures at our first boring (PT-5) today, as follows:

- PT-5-51'-200 psi

- PT-5-53'-250 psi

- PT-5-55 - 400 psi, with a peak of 500 psi.

As discussed, given the depth of these injections, the lack of any evidence of product surfacing, or any harm resulting from the injections, this is not of particular concern at this site. Accordingly, we will advise you if we should significantly exceed 500 psi at subsequent locations.

Thanks,

John Valkenburg, P.E. / Senior Engineer / Adventus Americas Inc. / 1493 West Pratt Road / DeWitt, MI 48820 Phone: 5176695400 / Fax: 5176695455

www adventusgroup com / www aquablokinfo.com / www.adventus.us / www.eti.ca

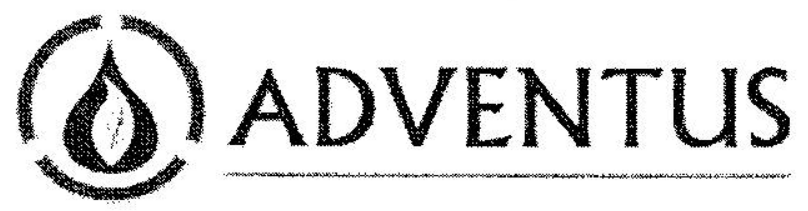

"North American Environmental Remediation Product Innovation of the Year" from Frost \& Sullivan, May 2007 "Innovation Award for Technology 2007" from ICU, May 2007 
From:

Sent:

To:

Cc:

Subject:

Attachments:
KHoeffner@kdhe.state.ks.us

Wednesday, November 28, 2007 9:10 AM

John Valkenburg

CKhan@kdhe.state.ks.us; MCochran@kdhe.state.ks.us

Re: Centralia injection Status Update (11/27/07)

1)

pic22929.gif (11

KB)

pic22929.gif

Thanks for the email documentation and for letting us know if there are additional pressure increases above 500 psi during the injection.

Kirk Hoeffner

Unit Chief, Underground Injection Control, Geology Section Kansas Department of Health \& Environment 1000 SW Jackson St., Suite 420 Topeka, KS 66612-1367

Telephone: (785) 296-1843

Fax: (785) 296-5509

www . kdheks. gov/geo

Website: www.kdheks.gov/geo

File: USDA Centralia, Nemaha Co. Class V - General

"John

Valkenburg"

$<j o h n$.valkenburg

dadventusgroup.c

om>

<KHoeffner@kdhe.state.ks.us>

To

$11 / 27 / 2007 \quad 07: 06$

$\mathrm{PM}$

"'LaEreniere, Lorraine M. "

$\mathrm{CC}$

<lafrenieredanl.gov>, "'jim

muellereadventusgroup. com" "

$<$ jim.muellereadventusgroup.com>,

"'Ravi" "

<Ravi.SrirangamaAdventusGroup. com>,

" John Valkenburg" "

<john. valkenburgeadventusgroup.com>,

"Greg Powers" "

$<$ powersaredox-tech. com>,

<haseloweredox-tech.com>, "'John

Valkenburg'"

<john. val kenburgeadventusgroup. com> Subject

Centralia Injection Status Update

(11/27/07) 
As discussed by phone today, we have observed these average injection pressures at our first boring (PT-5) today, as follows:

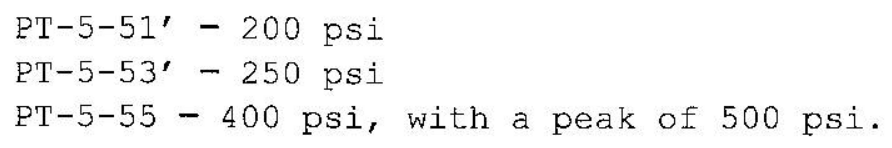

As discussed, given the depth of these injections, the lack of any evidence of product surfacing, or any harm resulting from the injections, this is not of particular concern at this site. Accordingly, we will advise you if we should significantly exceed 500 psi at subsequent locations.

Thanks,

John Valkenburg, P.E. / Senior Engineer / Adventus Americas Inc. / 1493 West Pratt Road / DeWitt, MI 48820

Phone: 5176695400 / Fax: 5176695455

www. adventusgroup.com / www.aquablokinfo.com / www.adventus.us / www.eti.ca

(Embedded image moved to file: pic22929.gif)

"North American Environmental Remediation Product Innovation of the Year" from Frost \& Sullivan, May 2007

"Innovation Award for Technology 2007" from ICU, May 2007 


\section{John Valkenburg}

From: John Valkenburg [john.valkenburg@adventusgroup.com]

Sent: Wednesday, November 28, 2007 11:58 AM

To: KHoeffner@kdhe.state.ks.us

Cc: $\quad$ Chris Carey; 'jim mueller@adventusgroup.com'; 'LaFreniere, Lorraine M.'; 'DAVID SURGNIER'; 'Ravi'; 'John Valkenburg'; Sedivy, Robert A.

Subject: Centralia Injection Pressure Update (11/28/07)

Kirk -

We observed slightly higher pressure at PT-3-55', with an average injection pressure of $450 \mathrm{psi}$, and a peak of 600 psi. We will continue to advise you if we see higher pressures than this. Again, we have seen no evidence of any problems arising from operating at these pressures.

Was unable to reach you by phone, as your number (785) 296-1673 seemingly currently rings at the number of one Carrie Bacon (sp?) of the Kansas Department of Disability Concerns. Please call my cell phone to discuss this matter as/if desired at (517) 927-3752.

Thanks,

John Valkenburg, P.E. / Senior Engineer / Adventus Americas Inc. / 1493 West Pratt Road / DeWitt, MI 48820 Phone: 5176695400 / Fax: 5176695455

www.adventusgroup.com / www.aquablokinfo.com / www.adventus.us / www.eti.ca

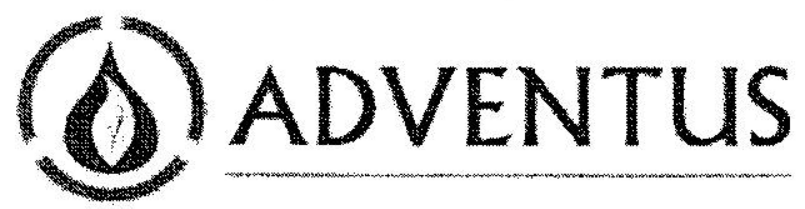

"North American Environmental Remediation Product Innovation of the Year" from Frost \& Sullivan, May 2007 "Innovation Award for Technology 2007" from ICU, May 2007 


\section{John Valkenburg}

From: John Valkenburg [john.valkenburg@adventusgroup.com]

Sent: Thursday, November 29, 2007 12:37 PM

To: KHoeffner@kdhe.state.ks.us

Cc: $\quad$ 'LaFreniere, Lorraine M.'; Sedivy, Robert A.; 'DAVID SURGNIER'; 'John Valkenburg'; 'Ravi'; 'jim mueller@adventusgroup.com'

Subject: Centralia Injection Pressure Update (11/29/07)

Kirk -

Just a note to advise you of some transient high pressures we have observed. At PT-15-53', we observed a pressure of 900 to $1000 \mathrm{psi}$. However, there was little or no injected material flow at this pressure. This suggests a plugged injection tip or tight formation. After some assessment of the condition in the field, we advanced the boring an additional 8" and had no problem injecting at normal pressures (in this case approximately 150 psi). We also observed a transient high pressure of 600 to $700 \mathrm{psi}$ at PT-15-55' (for one or two seconds). After this the pressure stabilized to $450 \mathrm{psi}$ and ultimately stabilized to 300 psi by the end of the injection.

Thanks,

John Valkenburg, P.E. / Senior Engineer / Adventus Americas Inc. / 1493 West Pratt Road / DeWitt, MI 48820 Phone: 5176695400 / Fax: 5176695455

www.adventusgroup.com / www.aquablokinfo.com / www.adventus. us / www.eti.ca

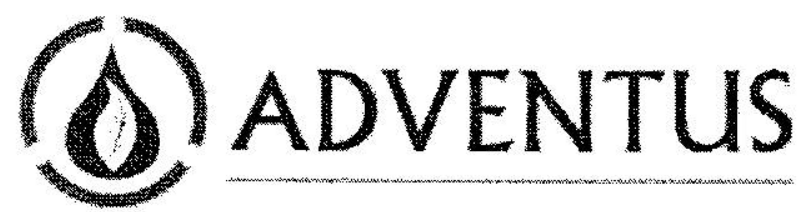

"North American Environmental Remediation Product Innovation of the Year" from Frost \& Sullivan, May 2007 "Innovation Award for Technology 2007" from ICU, May 2007 
From:

Sent:

To:

Cc:

Subject:

Attachments:
KHoeffner@kdhe.state.ks.us Thursday, November 29, 2007 5:14 PM John Valkenburg RHarper@kdhe.state.ks.us; DFTaylor@kdhe.state.ks.us; MCochran@kdhe.state.ks.us Re: Centralia Injection Pressure Update (11/29/07)

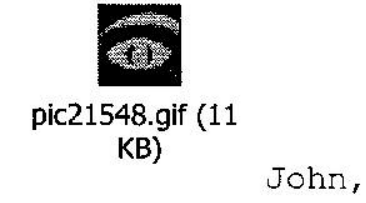

As per our discussions regarding the plugging of the injection probe boreholes, we do not require grouting with cement. The bentonite pellets you are currently using are an approved grout. As we also discussed, if you cannot use a pvc pipe to tremie the pellets down the well, then slowly pouring them down and then making sure their is no bridging with a probe rod will be sufficient. You indicated that all the boreholes so far have taken enough material to properly plug the interval above the water table without any indicates of bridging.

Kirk Hoeffner

Unit Chief, Underground Injection Control, Geology Section Kansas Department of Health \& Environment $1000 \mathrm{SW}$ Jackson St., Suite 420 Topeka, KS 66612-1367

Telephone: (785) 296-1843

Fax: (785) 296-5509

WWW. kdheks.gov/geo

Website: Ww. kdheks.gov/geo

Eile: USDA Centralia, Nemaha Co. Class V - General

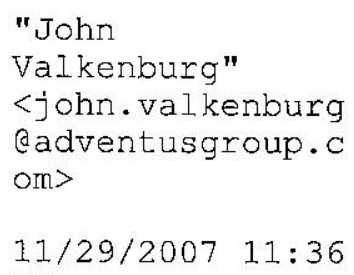

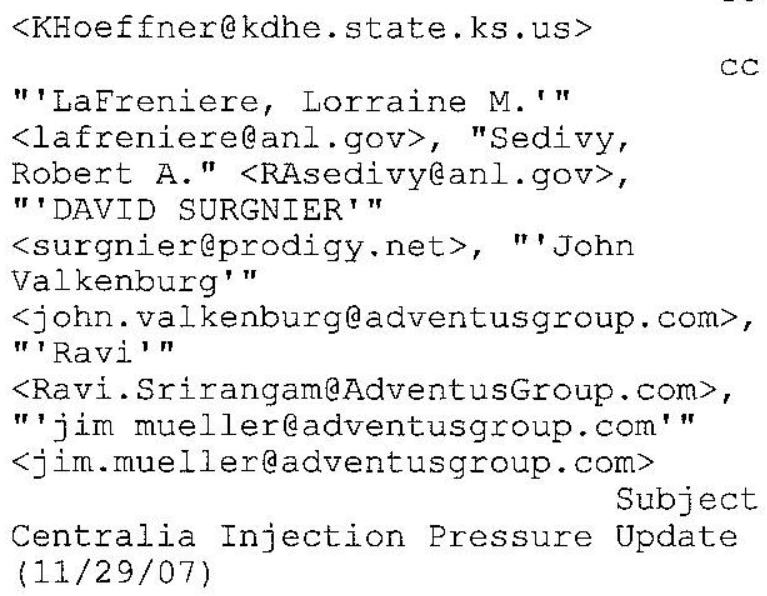


Kirk -

Just a note to advise you of some transient high pressures we have observed. At PT-15-53', we observed a pressure of 900 to 1000 psi. However, there was little or no injected material flow at this pressure. This suggests a plugged injection tip or tight formation. After some assessment of the condition in the field, we advanced the boring an additional $8^{\prime \prime}$ and had no problem injecting at normal pressures (in this case approximately 150 psi). We also observed a transient high pressure of 600 to 700 psi at PT-15-55' (for one or two seconds). After this the pressure stabilized to 450 psi and ultimately stabilized to 300 psi by the end of the injection.

Thanks,

John Valkenburg, P.E. / Senior Engineer / Adventus Americas Inc. / 1493 west Pratt Road / Dewitt, MI 48820

Phone: 5176695400 / Fax: 5176695455

ww. adventusgroup.com / www.aquablokinfo.com / www.adventus.us / www.eti.ca

(Embedded image moved to file: pic21548.gif)

"North American Environmental Remediation Product Innovation of the Year" from Frost \& Sullivan, May 2007

"Innovation Award for Technology 2007" from ICU, May 2007 


\section{John Valkenburg}

From: John Valkenburg [john.valkenburg@adventusgroup.com]

Sent: Tuesday, December 04, 2007 9:17 AM

To: KHoeffner@kdhe.state.ks.us

Cc: $\quad$ 'LaFreniere, Lorraine M.'; 'John Valkenburg'; Chris Carey; 'jim mueller@adventusgroup.com'; 'Ravi'

Subject: FW: Centralia, KS -12/4/07

Kirk -

Please see the note below with regard to some of our observations on injection pressures in the field. The saturated zone is quite tight in some locations.

Thanks,

John Valkenburg, P.E. / Senior Engineer / Adventus Americas Inc. / 1493 West Pratt Road / DeWitt, Ml 48820 Phone: 5176695400 / Fax: 5176695455

www. adventusgroup.com / www.aquablokinfo.com / www.adventus.us / www.eti.ca

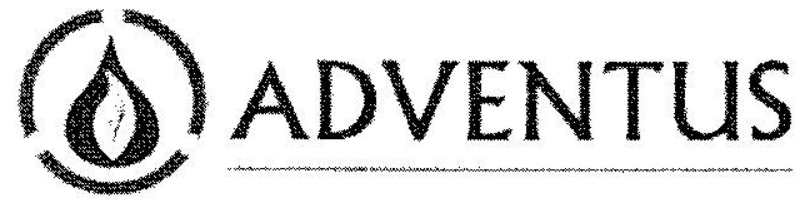

"North American Environmental Remediation Product Innovation of the Year" from Frost \& Sullivan, May 2007 "Innovation Award for Technology 2007" from ICU, May 2007

From: Ravi [mailto:Ravi.Srirangam@AdventusGroup.com]

Sent: Tuesday, December 04, 2007 9:04 AM

To: John Valkenburg

Cc: '"jim mueller@adventusgroup.com"'

Subject: Centralia, KS -12/4/07

John,

I think Lotrraine is quite sick and is not going to be on site. I hope she checks her email from her room.

I wanted to just mention that we went up to 900 psi a couple of times when we were injecting in the saturated zone

The average for the borings in the saturated zone could be between $450-550 \mathrm{psi}$

Again , I do not believe that high pressures are necessarily indicative of the pressure that we inject, It could be a combination of pressure building up in the hoses and clogging of the injection tip ( 1 just wanted to brief you so that you know and can inform Kirk).

The weather today is going to be the last of the good days. Wednesday and Thursday are going to be pretty cold and there is a good possibility for rain or snow.

Greg said he is going to leave tomorrow but Dave and Elias are going to take care of the operation after he leaves.

Thanks 


\section{Ravi}

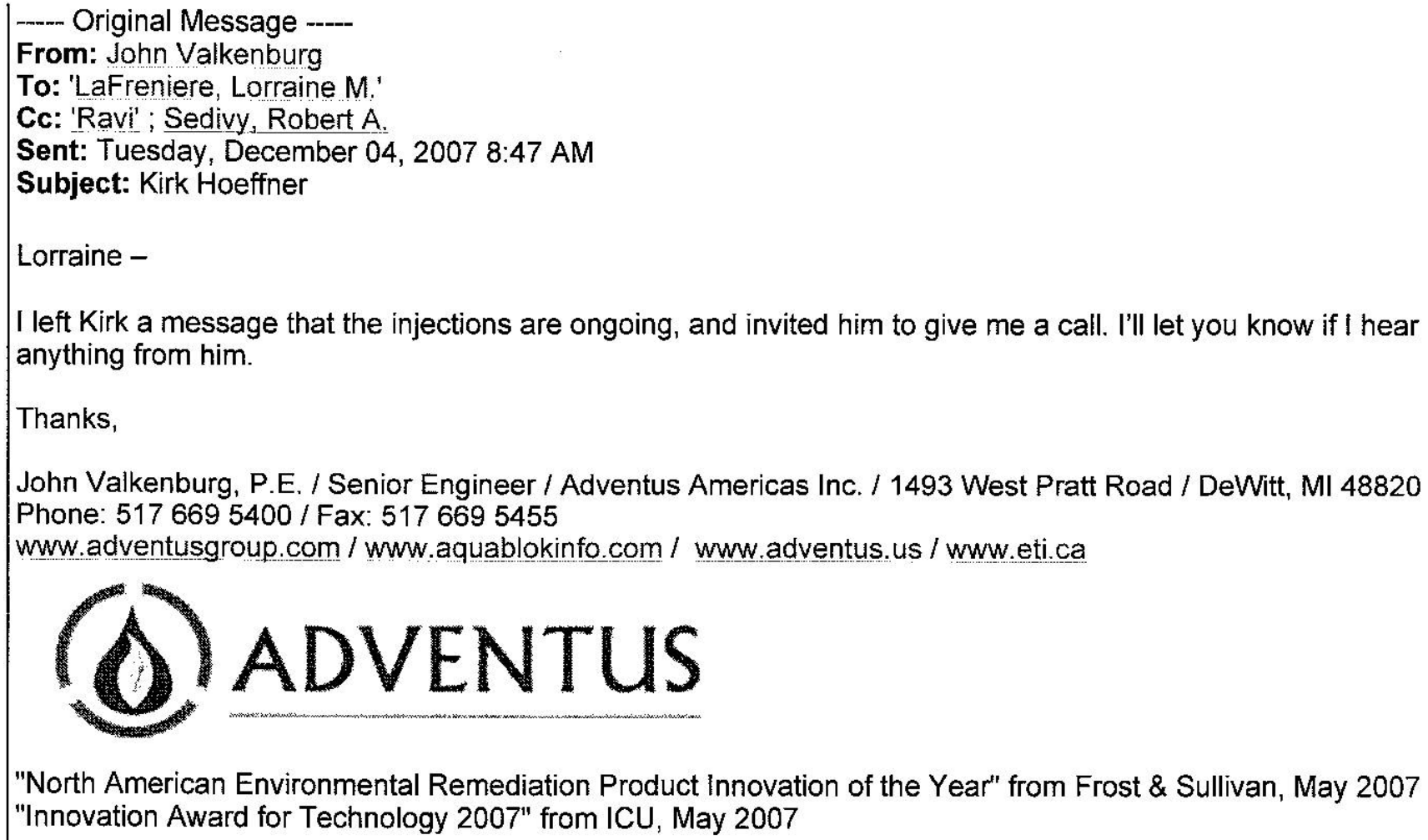

"North American Environmental Remediation Product Innovation of the Year" from Frost \& Sullivan, May 2007 "Innovation Award for Technology 2007" from ICU, May 2007 


\section{John Valkenburg}

From: John Valkenburg [john.valkenburg@adventusgroup.com]

Sent: Tuesday, December 04, 2007 11:29 AM

To: KHoeffner@kdhe.state.ks.us

Cc: $\quad$ 'LaFreniere, Lorraine M.'; 'Ravi'; Sedivy, Robert A.; 'John Valkenburg'; 'jim mueller@adventusgroup.com'; Chris Carey

Subject: Centralia Phone Conversation w/KDHE (12/04/07)

Kirk -

We welcome your possible trip out to the ANL site on Wednesday this week.

Also as discussed, we have observed some 'daylighting' or 'short-circuiting' of product to the surface at some locations. When we observe this, we have attempted to abate the problem (such as plugging the point from which surfacing is occurring) immediately. In cases where we are unable to easily abate the problem, we have stopped injecting at that interval and worked from another interval or location.

Please let us know if you have any questions or comments.

Thanks,

John Valkenburg, P.E. / Senior Engineer / Adventus Americas Inc. / 1493 West Pratt Road / DeWitt, MI 48820 Phone: 5176695400 / Fax: 5176695455

www.adventusgroup.com / www.aquablokinfo.com / www.adventus.us / www.eti.ca

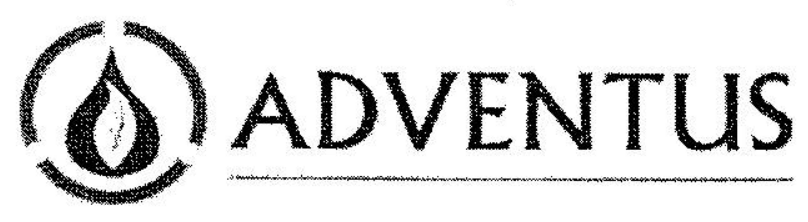

"North American Environmental Remediation Product Innovation of the Year" from Frost \& Sullivan, May 2007 "Innovation Award for Technology 2007" from ICU, May 2007 
From:

Sent:

To:

Subject:

Attachments:
KHoeffner@kdhe.state.ks.us

Tuesday, December 04, 2007 11:39 AM

John Valkenburg

Re: Centralia Phone Conversation w/KDHE (12/04/07)

pic07958.gif

pic07958.gif (11

$\mathrm{KB})$

John,

Thanks for the email with the information. I will plan to come out to the site tomorrow morning probably around 8:30 or 9:00 a.m.

Kirk Hoeffner

Unit Chief, Underground Injection Control, Geology Section Kansas Department of Health \& Environment $1000 \mathrm{SW}$ Jackson St., Suite 420 Topeka, KS 66612-1367

Telephone: (785) 296-1843

Fax: (785) 296-5509

www. kdheks.gov/geo

Website: www.kdheks.gov/geo

"John Valkenburg"
<john. valkenburge
adventusgroup.com
>

$12 / 04 / 2007 \quad 10: 29$ $\mathrm{AM}$
<KHoeffner@kdhe.state.ks.us>

To

$\mathrm{CC}$

"'LaFreniere, Lorraine M." "

<lafrenieredanl.gov>, "'Ravi""

<Ravi.SrirangameAdventusGroup.com>,

"Sedivy, Robert A."

<RAsedivy@anl.gov>, "'John

Valkenburg' "

<john. val kenburgeadventusgroup.com>

, "'jim mueller@adventusgroup.com" "

<jim.mueller@adventusgroup.com>,

"Chris Carey"

<ccareyakdhe.state.ks.us>

Subject

Centralia Phone Conversation w/KDHE (12/04/07)

Kirk - 
We welcome your possible trip out to the ANL site on wednesday this week.

Also as discussed, we have observed some 'daylighting' or 'short-circuiting' of product to the surface at some locations. When we observe this, we have attempted to abate the problem (such as plugging the point from which surfacing is occurring) immediately. In cases where we are unable to easily abate the problem, we have stopped injecting at that interval and worked from another interval or location.

Please let us know if you have any questions or comments.

Thanks,

John Valkenburg, P.E. / Senior Engineer / Adventus Americas Inc. / 1493 West Pratt Road / Dewitt, MI 48820

Phone: 5176695400 / Fax: 5176695455

www.adventusgroup.com / wWw.aquablokinfo.com / wWw.adventus.us / wWw.eti.ca

(Embedded image moved to file: pic07958.gif)

"North American Environmental Remediation Product Innovation of the Year" from Frost \& Sullivan, May 2007

"Innovation Award for Technology 2007" from ICU, May 2007 
Appendix C:

Field Injection Log 


\section{Appendix C. Field Injection Log \\ USDA/CCC Argonne Labs Site \\ Centralia, Kansas}

\begin{tabular}{|c|c|c|c|c|c|c|c|c|}
\hline $\begin{array}{c}\text { Boring } \\
\text { ID }\end{array}$ & $\begin{array}{l}\text { Injection } \\
\text { Depth (ft) }\end{array}$ & Date & $\begin{array}{l}\text { Time } \\
\text { (CST) }\end{array}$ & $\begin{array}{l}\text { EHC or } \\
\text { EHC-A }\end{array}$ & $\begin{array}{c}\text { Water } \\
\text { (gallons) }\end{array}$ & $\begin{array}{c}\text { EHC or } \\
\text { EHC-A } \\
\text { (lbs) }\end{array}$ & $\begin{array}{l}\text { Pressure } \\
\quad \text { (psi) }\end{array}$ & Comments \\
\hline PT-5 & 51 & $11 / 27 / 2007$ & $12: 20$ & EHC-A & 40 & 100 & $200-250$ & $\begin{array}{l}\text { EHC-A Injected } \\
\text { instead of EHC }\end{array}$ \\
\hline PT-5 & 53 & $11 / 27 / 2007$ & $12: 50$ & $\mathrm{EHC}$ & 40 & 100 & $250-300$ & $\begin{array}{c}\text { Pressure remained } \\
\text { on rods after } \\
\text { injection } \\
\text { completed }\end{array}$ \\
\hline PT-5A & 55 & $11 / 27 / 2007$ & $13: 15$ & $\mathrm{EHC}$ & 40 & 100 & $400-450$ & $\begin{array}{l}\text { High back } \\
\text { pressure due to } \\
\text { tight formation }\end{array}$ \\
\hline PT-5B & 57 & $11 / 28 / 2007$ & $8: 30$ & $\mathrm{EHC}$ & 40 & 100 & $300-350$ & $\begin{array}{c}\text { Shifted to new } \\
\text { hole }\end{array}$ \\
\hline PT-5B & 59 & $11 / 28 / 2007$ & $9: 10$ & $\mathrm{EHC}$ & 40 & 100 & $350-400$ & $\begin{array}{l}\text { Back Pressure in } \\
\text { the Injection rods }\end{array}$ \\
\hline PT-3 & 51 & $11 / 28 / 2007$ & $9: 40$ & $\mathrm{EHC}$ & 40 & 100 & $350-400$ & \\
\hline PT-3 & 53 & $11 / 28 / 2007$ & $10: 10$ & $\mathrm{EHC}$ & 40 & 100 & $450-500$ & $\begin{array}{c}\text { Maximum } \\
\text { pressure of } 600 \\
\text { psi for a short time }\end{array}$ \\
\hline PT-3 & 55 & $11 / 28 / 2007$ & $10: 40$ & $\mathrm{EHC}$ & 40 & 100 & $350-400$ & $\begin{array}{l}\text { Reduction of } \\
\text { pressure at greater } \\
\text { depths }\end{array}$ \\
\hline PT-3 & 57 & $11 / 28 / 2007$ & $11: 15$ & $\mathrm{EHC}$ & 40 & 100 & $300-350$ & \\
\hline PT-3 & 59 & $11 / 28 / 2007$ & $13: 15$ & $\mathrm{EHC}$ & 40 & 100 & $300-350$ & \\
\hline & & & & & & & & \\
\hline PT-13 & 21 & $11 / 28 / 2007$ & $14: 20$ & EHC-A & 54 & 50 & $50-75$ & \\
\hline PT-13 & 23 & $11 / 28 / 2007$ & $14: 30$ & EHC-A & 54 & 50 & $50-75$ & \\
\hline PT-13 & 25 & $11 / 28 / 2007$ & $14: 40$ & EHC-A & 54 & 50 & $50-75$ & \\
\hline PT-13 & 27 & $11 / 28 / 2007$ & $14: 50$ & EHC-A & 54 & 50 & $50-75$ & \\
\hline PT-13 & 29 & $11 / 28 / 2007$ & $14: 55$ & EHC-A & 54 & 50 & $50-75$ & \\
\hline PT-13 & 31 & $11 / 28 / 2007$ & $15: 02$ & EHC-A & 54 & 50 & $50-75$ & \\
\hline PT-13 & 33 & $11 / 28 / 2007$ & $15: 07$ & EHC-A & 54 & 50 & $100-150$ & \\
\hline PT-13 & 35 & $11 / 28 / 2007$ & $15: 15$ & EHC-A & 54 & 50 & $100-150$ & \\
\hline PT-13 & 37 & $11 / 28 / 2007$ & $15: 27$ & EHC-A & 54 & 50 & $100-150$ & \\
\hline PT-13 & 39 & $11 / 28 / 2007$ & $15: 40$ & EHC-A & 54 & 50 & $100-150$ & \\
\hline PT-13 & 42 & $11 / 28 / 2007$ & $16: 10$ & $\mathrm{EHC}$ & 54 & 50 & $150-200$ & \\
\hline PT-13 & 45 & $11 / 28 / 2007$ & $16: 20$ & $\mathrm{EHC}$ & 54 & 50 & $200-250$ & $\begin{array}{c}\text { Daylighting at MW- } \\
02\end{array}$ \\
\hline PT-13 & 48 & $11 / 28 / 2007$ & $16: 30$ & $\mathrm{EHC}$ & 54 & 50 & $400-450$ & $\begin{array}{c}\text { Daylighting at MW- } \\
02 \text { continues }\end{array}$ \\
\hline PT-13 & 51 & $11 / 28 / 2007$ & $16: 40$ & $\mathrm{EHC}$ & 54 & 50 & $400-450$ & $\begin{array}{l}\text { Further Daylighting } \\
\text { at MW-02 resulted } \\
\text { in skipping the } \\
\text { deeper saturated } \\
\text { zone }\end{array}$ \\
\hline & & & & & & & & \\
\hline
\end{tabular}




\section{Appendix C. Field Injection Log \\ USDA/CCC Argonne Labs Site \\ Centralia, Kansas}

\begin{tabular}{|c|c|c|c|c|c|c|c|c|}
\hline $\begin{array}{c}\text { Boring } \\
\text { ID }\end{array}$ & $\begin{array}{l}\text { Injection } \\
\text { Depth (ft) }\end{array}$ & Date & $\begin{array}{l}\text { Time } \\
\text { (CST) }\end{array}$ & $\begin{array}{l}\text { EHC or } \\
\text { EHC-A }\end{array}$ & $\begin{array}{c}\text { Water } \\
\text { (gallons) }\end{array}$ & $\begin{array}{c}\text { EHC or } \\
\text { EHC-A } \\
\text { (lbs) }\end{array}$ & $\begin{array}{l}\text { Pressure } \\
\quad(p s i)\end{array}$ & Comments \\
\hline PT-15 & 51.5 & $11 / 29 / 2007$ & 9:00 & $\mathrm{EHC}$ & 40 & 100 & $150-200$ & $\begin{array}{l}\text { Delay due to } \\
\text { clogging of tips } \\
\text { during injection }\end{array}$ \\
\hline PT-15 & 53.8 & $11 / 29 / 2007$ & $9: 45$ & $\mathrm{EHC}$ & 40 & 100 & $100-150$ & $\begin{array}{l}\text { Less injection } \\
\text { Pressure at } 8 \\
\text { inches down } \\
\text {-attributed to } \\
\text { formation at 53' }\end{array}$ \\
\hline PT-15 & 55 & $11 / 29 / 2007$ & $10: 10$ & $\mathrm{EHC}$ & 40 & 100 & $200-250$ & $\begin{array}{c}\text { Drilling tough due } \\
\text { to tight zone of } \\
\text { clay }\end{array}$ \\
\hline PT-15 & 57 & $11 / 29 / 2007$ & $10: 25$ & $\mathrm{EHC}$ & 40 & 100 & $300-400$ & $\begin{array}{l}600-700 \text { psi for } \\
\text { short period, slight } \\
\text { daylighting at } \\
\text { surface }\end{array}$ \\
\hline PT-15 & 59 & $11 / 29 / 2007$ & $10: 45$ & $\mathrm{EHC}$ & 40 & 100 & $200-250$ & $\begin{array}{c}\text { Minimal } \\
\text { Daylighting } \\
\text { recorded }\end{array}$ \\
\hline PT-1 & 21 & $11 / 29 / 2007$ & 12.45 & EHC-A & 54 & 5 & $75-100$ & \\
\hline PT-1 & 23 & $11 / 29 / 2007$ & $12: 55$ & EHC-A & 54 & 50 & $75-100$ & $\begin{array}{c}\text { Occurrence of } \\
\text { surficial daylighting }\end{array}$ \\
\hline PT-1 & 25 & $11 / 29 / 2007$ & $13: 00$ & EHC-A & 54 & 50 & $75-100$ & $\begin{array}{c}\text { Surficial } \\
\text { daylighting } \\
\text { continues }\end{array}$ \\
\hline PT-1 & 27 & $11 / 29 / 2007$ & $13: 20$ & EHC-A & 54 & 50 & $75-100$ & \\
\hline PT-1 & 29 & $11 / 29 / 2007$ & $13: 30$ & EHC-A & 54 & 50 & $75-100$ & \\
\hline PT-1 & 31 & $11 / 29 / 2007$ & $13: 40$ & EHC-A & 54 & 50 & $75-100$ & \\
\hline PT-1 & 33 & $11 / 29 / 2007$ & $13: 46$ & EHC-A & 54 & 50 & $75-100$ & \\
\hline PT-1 & 35 & $11 / 29 / 2007$ & $13: 53$ & EHC-A & 54 & 50 & $75-100$ & \\
\hline PT-1 & 37 & $11 / 29 / 2007$ & $14: 00$ & EHC-A & 54 & 50 & $100-200$ & \\
\hline PT-1 & 39 & $11 / 29 / 2007$ & $14: 08$ & EHC-A & 54 & 50 & $100-200$ & \\
\hline $\begin{array}{l}\text { PT-1 } \\
\end{array}$ & 42 & $11 / 29 / 2007$ & $14: 13$ & $\mathrm{EHC}$ & 54 & 50 & $450-600$ & $\begin{array}{l}\text { Pressure stabilized } \\
\text { after clogging } \\
\text { removal }\end{array}$ \\
\hline PT-1 & 44.3 & $11 / 29 / 2007$ & $14: 25$ & $\mathrm{EHC}$ & 54 & 50 & $400-600$ & $\begin{array}{c}\text { Back pressure } \\
\text { reduction by going } \\
\text { up } 0.7 \mathrm{ft}\end{array}$ \\
\hline PT-1 & 48.5 & $11 / 29 / 2007$ & & & & & & $\begin{array}{l}\text { Had flow around } \\
\text { rods (annulus), so } \\
\text { moved to new } \\
\text { location for 49' }\end{array}$ \\
\hline
\end{tabular}




\section{Appendix C. Field Injection Log \\ USDA/CCC Argonne Labs Site \\ Centralia, Kansas}

\begin{tabular}{|c|c|c|c|c|c|c|c|c|}
\hline $\begin{array}{c}\text { Boring } \\
\text { ID }\end{array}$ & $\begin{array}{l}\text { Injection } \\
\text { Depth (ft) }\end{array}$ & Date & $\begin{array}{l}\text { Time } \\
\text { (CST) }\end{array}$ & $\begin{array}{l}\text { EHC or } \\
\text { EHC-A }\end{array}$ & $\begin{array}{c}\text { Water } \\
\text { (gallons) }\end{array}$ & $\begin{array}{c}\text { EHC or } \\
\text { EHC-A } \\
\text { (lbs) }\end{array}$ & $\begin{array}{l}\text { Pressure } \\
\text { (psi) }\end{array}$ & Comments \\
\hline PT-1A & 49 & $11 / 29 / 2007$ & $14: 35$ & $\mathrm{EHC}$ & 54 & 50 & $150-250$ & $\begin{array}{l}\text { Back pressure } \\
\text { reduction by going } \\
\text { up } 1 \mathrm{ft} \text { - still } \\
\text { treated as a GW } \\
\text { zone injection }\end{array}$ \\
\hline PT-1A & 51 & $11 / 29 / 2007$ & $16: 40$ & $\mathrm{EHC}$ & 54 & 50 & $150-250$ & \\
\hline PT-12 & 21 & $11 / 30 / 2007$ & $8: 42$ & EHC-A & 54 & 50 & $50-100$ & $\begin{array}{c}\text { Preheating } \\
\text { required due to } \\
\text { weather condition }\end{array}$ \\
\hline PT-12 & 23 & $11 / 30 / 2007$ & $8: 49$ & EHC-A & 54 & 50 & 50 & \\
\hline PT-12 & 25 & $11 / 30 / 2007$ & $8: 55$ & EHC-A & 54 & 50 & $50-100$ & \\
\hline PT-12 & 27 & $11 / 30 / 2007$ & 9:02 & EHC-A & 54 & 50 & $50-100$ & \\
\hline PT-12 & 29 & $11 / 30 / 2007$ & $9: 10$ & EHC-A & 54 & 50 & $50-100$ & \\
\hline PT-12 & 31 & $11 / 30 / 2007$ & $9: 17$ & EHC-A & 54 & 50 & $250-300$ & \\
\hline PT-12 & 33 & $11 / 30 / 2007$ & $9: 24$ & EHC-A & 54 & 50 & $250-300$ & $\begin{array}{l}\text { Geoprobe tip } \\
\text { plugging/jams }\end{array}$ \\
\hline PT-12 & 35 & $11 / 30 / 2007$ & $9: 32$ & EHC-A & 54 & 50 & $\sim 450$ & $\begin{array}{l}\text { Backpressure } \\
\text { prevented } \\
\text { proceeding further }\end{array}$ \\
\hline & & & & & & & & \\
\hline PT-2 & 21 & $11 / 30 / 2007$ & $11: 00$ & EHC-A & 54 & 50 & 50 & \\
\hline PT-2 & 23 & $11 / 30 / 2007$ & 11:07 & EHC-A & 54 & 50 & $50-100$ & \\
\hline PT-2 & 25 & $11 / 30 / 2007$ & $11: 14$ & EHC-A & 54 & 50 & 50 & \\
\hline PT-2 & 27 & $11 / 30 / 2007$ & $11: 18$ & EHC-A & 54 & 50 & $50-100$ & \\
\hline PT-2 & 29 & $11 / 30 / 2007$ & $11: 23$ & EHC-A & 54 & 50 & $100-200$ & \\
\hline PT-2 & 31 & $11 / 30 / 2007$ & $11: 26$ & EHC-A & 54 & 50 & $50-100$ & \\
\hline PT-2 & 33 & $11 / 30 / 2007$ & $11: 30$ & EHC-A & 54 & 50 & $50-100$ & \\
\hline PT-2 & 35 & $11 / 30 / 2007$ & $11: 38$ & EHC-A & 54 & 50 & $100-200$ & \\
\hline PT-2 & 37 & $11 / 30 / 2007$ & $11: 47$ & EHC-A & 54 & 50 & $100-150$ & \\
\hline PT-2 & 39 & $11 / 30 / 2007$ & $13: 10$ & EHC-A & 54 & 50 & $50-100$ & \\
\hline PT-6 & 21 & $11 / 30 / 2007$ & $13: 26$ & EHC-A & 54 & 50 & 50 & Small daylights \\
\hline PT-6 & 23 & $11 / 30 / 2007$ & $13: 32$ & EHC-A & 54 & 50 & $100-300$ & \\
\hline PT-6 & 25 & $11 / 30 / 2007$ & $13: 40$ & EHC-A & 54 & 50 & $150-250$ & $\begin{array}{l}\text { Daylighting of EHC } \\
\text { A observed from } \\
\text { this depth to } 35 \\
\text { feet. }\end{array}$ \\
\hline PT-6 & 27 & $11 / 30 / 2007$ & $13: 46$ & EHC-A & 54 & 50 & $100-200$ & \\
\hline PT-6 & 29 & $11 / 30 / 2007$ & 13:52 & EHC-A & 54 & 50 & $100-200$ & \\
\hline PT-6 & 31 & $11 / 30 / 2007$ & 13:58 & EHC-A & 54 & 50 & $250-300$ & $\begin{array}{l}\text { Daylights at probe } \\
\text { annulus. }\end{array}$ \\
\hline PT-6 & 33 & $11 / 30 / 2007$ & $14: 07$ & EHC-A & 54 & 50 & $150-250$ & \\
\hline
\end{tabular}




\section{Appendix C. Field Injection Log \\ USDA/CCC Argonne Labs Site \\ Centralia, Kansas}

\begin{tabular}{|c|c|c|c|c|c|c|c|c|}
\hline $\begin{array}{l}\text { Boring } \\
\text { ID }\end{array}$ & $\begin{array}{l}\text { Injection } \\
\text { Depth (ft) }\end{array}$ & Date & $\begin{array}{l}\text { Time } \\
\text { (CST) }\end{array}$ & $\begin{array}{l}\text { EHC or } \\
\text { EHC-A }\end{array}$ & $\begin{array}{c}\text { Water } \\
\text { (gallons) }\end{array}$ & $\begin{array}{c}\text { EHC or } \\
\text { EHC-A } \\
\text { (lbs) }\end{array}$ & $\begin{array}{l}\text { Pressure } \\
\quad \text { (psi) }\end{array}$ & Comments \\
\hline PT-6 & 35 & $11 / 30 / 2007$ & $14: 13$ & EHC-A & 54 & 50 & $100-200$ & $\begin{array}{c}\text { Daylighting } \\
\text { beyond SB-07R }\end{array}$ \\
\hline $\begin{array}{l}\text { PT-6 } \\
\end{array}$ & 37 & $11 / 30 / 2007$ & $14: 19$ & EHC-A & 54 & 50 & $150-200$ & \\
\hline PT-6 & 39 & $11 / 30 / 2007$ & $14: 24$ & EHC-A & 54 & 50 & $150-250$ & \\
\hline PT-6 & 42 & $11 / 30 / 2007$ & $14: 30$ & $\mathrm{EHC}$ & 54 & 50 & $150-250$ & \\
\hline PT-6 & 45 & $11 / 30 / 2007$ & $14: 40$ & $\mathrm{EHC}$ & 54 & 50 & $150-250$ & \\
\hline PT-6 & 48 & $11 / 30 / 2007$ & $14: 50$ & $\mathrm{EHC}$ & 54 & 50 & $150-200$ & $\begin{array}{c}\text { EHC daylighting } \\
\text { from the annulus } \\
\text { of SB07R }\end{array}$ \\
\hline$\overline{\text { PT-7 }}$ & 21 & $12 / 2 / 2007$ & $8: 32$ & EHC-A & 54 & 50 & $150-200$ & \\
\hline PT-7 & 23 & $12 / 2 / 2007$ & $8: 40$ & EHC-A & 54 & 50 & $200-250$ & \\
\hline PT-7 & 25 & $12 / 2 / 2007$ & $8: 47$ & EHC-A & 54 & 50 & $200-300$ & \\
\hline $\begin{array}{l}\text { PT-7 } \\
\end{array}$ & 27 & $12 / 2 / 2007$ & $8: 55$ & EHC-A & 54 & 50 & $200-250$ & \\
\hline PT-7 & 29 & $12 / 2 / 2007$ & $9: 00$ & EHC-A & 54 & 50 & $200-250$ & $\begin{array}{l}\text { Daylighting of EHC } \\
\text { A at MW-02 }\end{array}$ \\
\hline PT-7 & 31 & $12 / 2 / 2007$ & $9: 20$ & EHC-A & 54 & 50 & $200-250$ & \\
\hline PT-7 & 33 & $12 / 2 / 2007$ & $9: 32$ & EHC-A & 54 & 50 & $200-250$ & \\
\hline PT-7 & 35 & $12 / 2 / 2007$ & $9: 43$ & EHC-A & 54 & 50 & $200-250$ & $\begin{array}{c}\text { Some Daylighting } \\
\text { at MW-02 }\end{array}$ \\
\hline PT-7 & 37 & $12 / 2 / 2007$ & $9: 55$ & EHC-A & 54 & 50 & $250-300$ & \\
\hline PT-7 & 39 & $12 / 2 / 2007$ & $10: 07$ & EHC-A & 54 & 50 & $250-300$ & \\
\hline PT-7 & 42 & $12 / 2 / 2007$ & $10: 45$ & $\mathrm{EHC}$ & 54 & 50 & $250-300$ & \\
\hline PT-7 & 45 & $12 / 2 / 2007$ & $11: 15$ & $\mathrm{EHC}$ & & & & $\begin{array}{l}\text { Some daylighting, } \\
\text { ceased injection }\end{array}$ \\
\hline PT-7 & 49 & $12 / 2 / 2007$ & $11: 45$ & $\mathrm{EHC}$ & 40 & 100 & $250-300$ & $\begin{array}{l}\text { Back pressure was } \\
\text { offset by going } 1 \\
\text { foot deeper }\end{array}$ \\
\hline PT-7 & 52 & $12 / 2 / 2007$ & $1: 15$ & $\mathrm{EHC}$ & 40 & 100 & $350-400$ & \\
\hline PT-7 & 55 & $12 / 2 / 2007$ & $1: 24$ & $\mathrm{EHC}$ & 60 & 150 & $350-400$ & $\begin{array}{l}\text { Skipped interval in } \\
\text { between due to } \\
\text { high pressure } \\
\text { (Dosage } \\
\text { increased) }\end{array}$ \\
\hline PT-7 & 58 & $12 / 2 / 2007$ & $1: 31$ & $\mathrm{EHC}$ & 40 & 100 & $300-350$ & $\begin{array}{l}\text { Injected at } 58 \text { to } \\
\text { account for tight } \\
\text { formation }\end{array}$ \\
\hline PT-10 & 52 & $12 / 2 / 2007$ & $13: 45$ & $\mathrm{EHC}$ & 40 & 100 & $250-300$ & $\begin{array}{l}\text { Elevated back } \\
\text { pressure at } 51 \text { ' } \\
\text { was offset by } \\
\text { going deeper }\end{array}$ \\
\hline
\end{tabular}




\section{Appendix C. Field Injection Log \\ USDA/CCC Argonne Labs Site \\ Centralia, Kansas}

\begin{tabular}{|c|c|c|c|c|c|c|c|c|}
\hline $\begin{array}{l}\text { Boring } \\
\text { ID }\end{array}$ & $\begin{array}{l}\text { Injection } \\
\text { Depth (ft) }\end{array}$ & Date & $\begin{array}{l}\text { Time } \\
\text { (CST) }\end{array}$ & $\begin{array}{l}\text { EHC or } \\
\text { EHC-A }\end{array}$ & $\begin{array}{c}\text { Water } \\
\text { (gallons) }\end{array}$ & $\begin{array}{c}\text { EHC or } \\
\text { EHC-A } \\
\text { (lbs) }\end{array}$ & $\begin{array}{l}\text { Pressure } \\
\text { (psi) }\end{array}$ & Comments \\
\hline PT-10 & 53 & $12 / 2 / 2007$ & $14: 10$ & $\mathrm{EHC}$ & 40 & 100 & $300-350$ & $\begin{array}{l}\text { Some daylighting, } \\
\text { ceased injection }\end{array}$ \\
\hline PT-10 & 57 & $12 / 2 / 2007$ & $14: 45$ & $\mathrm{EHC}$ & 40 & 100 & $400-500$ & $\begin{array}{l}\text { Skipped } 55 \text { owing } \\
\text { to tight formation, } \\
\text { multiple daylights }\end{array}$ \\
\hline PT-10 & 59 & $12 / 2 / 2007$ & 15:06 & $\mathrm{EHC}$ & & & $400-550$ & $\begin{array}{l}\text { Very minimal } \\
\text { Injection but } \\
\text { largely lost to } \\
\text { surface. }\end{array}$ \\
\hline PT-4 & 51 & $12 / 3 / 2007$ & $8: 55$ & $\mathrm{EHC}$ & 40 & 100 & $250-350$ & \\
\hline PT-4 & 53 & $12 / 3 / 2007$ & $9: 15$ & $\mathrm{EHC}$ & 40 & 100 & $300-350$ & \\
\hline PT-4 & 55 & $12 / 3 / 2007$ & $9: 30$ & $\mathrm{EHC}$ & 40 & 100 & $250-350$ & \\
\hline PT-4 & 57 & $12 / 3 / 2007$ & $9: 40$ & $\mathrm{EHC}$ & 40 & 100 & $250-350$ & \\
\hline PT-4 & 59 & $12 / 3 / 2007$ & 9:55 & $\mathrm{EHC}$ & 40 & 100 & $300-500$ & \\
\hline PT-14 & 40 & $12 / 3 / 2007$ & $12: 40$ & $\mathrm{EHC}$ & 40 & 100 & $150-250$ & $\begin{array}{c}\text { EHC Injected at } 40 \\
\mathrm{ft} \text { by mistake }\end{array}$ \\
\hline PT-14 & 51 & $12 / 3 / 2007$ & $12: 55$ & $\mathrm{EHC}$ & 40 & 100 & $200-300$ & \\
\hline PT-14 & 53 & $12 / 3 / 2007$ & $13: 03$ & $\mathrm{EHC}$ & 40 & 100 & $200-300$ & \\
\hline PT-14 & 55 & $12 / 3 / 2007$ & $13: 18$ & $\mathrm{EHC}$ & 40 & 100 & $300-600$ & $\begin{array}{c}\text { High fluctuations in } \\
\text { pressure }\end{array}$ \\
\hline PT-14 & 57 & $12 / 3 / 2007$ & 13:33 & $\mathrm{EHC}$ & 40 & 100 & $300-400$ & $\begin{array}{c}\text { Slight surfical } \\
\text { daylighting - } \\
\text { multiple points + at } \\
\text { MW-02 } \\
\end{array}$ \\
\hline PT-14 & 59 & $12 / 3 / 2007$ & $13: 51$ & $\mathrm{EHC}$ & 40 & $<100$ & $250-300$ & \begin{tabular}{|c|}
$\begin{array}{c}\text { Slight Daylighting } \\
\text { Continues }\end{array}$ \\
\end{tabular} \\
\hline $\begin{array}{l}\text { PT-2 } \\
\end{array}$ & 42 & $12 / 3 / 2007$ & $14: 30$ & $\mathrm{EHC}$ & 54 & 50 & $200-300$ & \\
\hline PT-2 & 45 & $12 / 3 / 2007$ & & $\mathrm{EHC}$ & & & & $\begin{array}{l}\text { Skipped due to } \\
\text { tight formations - } \\
\text { attempted } \\
\text { injection, but } \\
\text { daylighting } \\
\text { occurred around } \\
\text { rods. }\end{array}$ \\
\hline PT-2 & 48 & $12 / 3 / 2007$ & $14: 50$ & $\mathrm{EHC}$ & 40 & 50 & $300-400$ & \\
\hline PT-2 & 52 & $12 / 3 / 2007$ & $15: 05$ & $\mathrm{EHC}$ & 40 & 100 & $150-450$ & $\begin{array}{l}\text { Variations in } \\
\text { pressure }\end{array}$ \\
\hline PT-2 & 55 & $12 / 3 / 2007$ & $5: 16$ & $\mathrm{EHC}$ & 40 & 100 & $300-600$ & 50\# daylighted \\
\hline PT-2 & 59 & $12 / 3 / 2007$ & $15: 40$ & $\mathrm{EHC}$ & 67 & 100 & & $\begin{array}{l}\text { Skipped } 57 \text { due to } \\
\text { daylighting issues. } \\
\text { Injected } 250 \mathrm{lbs} \\
\text { out of } 350 \mathrm{lbs}\end{array}$ \\
\hline
\end{tabular}




\section{Appendix C. Field Injection Log \\ USDA/CCC Argonne Labs Site \\ Centralia, Kansas}

\begin{tabular}{|c|c|c|c|c|c|c|c|c|}
\hline $\begin{array}{c}\text { Boring } \\
\text { ID }\end{array}$ & $\begin{array}{l}\text { Injection } \\
\text { Depth (ft) }\end{array}$ & Date & $\begin{array}{l}\text { Time } \\
\text { (CST) }\end{array}$ & $\begin{array}{l}\text { EHC or } \\
\text { EHC-A }\end{array}$ & $\begin{array}{c}\text { Water } \\
\text { (gallons) }\end{array}$ & $\begin{array}{c}\text { EHC or } \\
\text { EHC-A } \\
\text { (lbs) }\end{array}$ & $\begin{array}{l}\text { Pressure } \\
\text { (psi) }\end{array}$ & Comments \\
\hline PT-11 & 50.5 & $12 / 4 / 2007$ & $8: 40$ & $\mathrm{EHC}$ & 54 & 50 & $400-600$ & \\
\hline PT-11 & 53 & $12 / 4 / 2007$ & $8: 59$ & $\mathrm{EHC}$ & 40 & 100 & $400-600$ & Slow pumping \\
\hline PT-11 & 55 & $12 / 4 / 2007$ & $9: 15$ & $\mathrm{EHC}$ & 54 & 50 & $300-500$ & \\
\hline PT-11 & 57 & $12 / 4 / 2007$ & $9: 28$ & $\mathrm{EHC}$ & 40 & 100 & $300-500$ & Slow pumping \\
\hline PT-11 & 59 & $12 / 4 / 2007$ & $9: 35$ & $\mathrm{EHC}$ & 54 & 50 & $200-300$ & \\
\hline & & & & & & & & \\
\hline PT-1-B & 53 & $12 / 4 / 2007$ & $10: 20$ & $\mathrm{EHC}$ & 0 & 0 & $\bar{N} / \mathrm{A}$ & $\begin{array}{c}\text { Skipped } 51 \text { due to } \\
\text { no flow }\end{array}$ \\
\hline PT-1-B & 55 & $12 / 4 / 2007$ & $10: 40$ & $\mathrm{EHC}$ & 60 & 150 & $300-450$ & $\begin{array}{c}\text { Injected } 150 \text { lbs of } \\
\text { EHC }\end{array}$ \\
\hline PT-1-B & 57 & $12 / 4 / 2007$ & $10: 20$ & $\mathrm{EHC}$ & 54 & 50 & $300-450$ & $250-350$ \\
\hline PT-1-B & 59 & $12 / 4 / 2007$ & $11: 01$ & $\mathrm{EHC}$ & 108 & 100 & $450-600$ & $\begin{array}{c}\text { Max } 900 \text { psi } \\
\text { Recorded for a } \\
\text { very short period } \\
\text { probably due to } \\
\text { some clogging }\end{array}$ \\
\hline PT-12-A & 37 & $12 / 4 / 2007$ & $13: 24$ & EHC-A & 54 & 50 & $300-450$ & \\
\hline PT-12-A & 39 & $12 / 4 / 2007$ & $13: 55$ & EHC-A & 54 & 50 & $300-400$ & \\
\hline PT-12-A & 42 & $12 / 4 / 2007$ & $14: 01$ & $\mathrm{EHC}$ & 54 & 50 & $150-300$ & $\begin{array}{c}\text { Daylighting near } \\
\text { PT-13 }\end{array}$ \\
\hline PT-12-A & 45 & $12 / 4 / 2007$ & $14: 08$ & $\mathrm{EHC}$ & 54 & 50 & $150-300$ & \\
\hline PT-12-A & 48 & $12 / 4 / 2007$ & $14: 15$ & $\mathrm{EHC}$ & 54 & 50 & $150-300$ & \\
\hline PT-12-A & 51 & $12 / 4 / 2007$ & $14: 22$ & $\mathrm{EHC}$ & 54 & 50 & $150-300$ & \\
\hline PT-12-A & 53 & $12 / 4 / 2007$ & $14: 29$ & $\mathrm{EHC}$ & 40 & 100 & $150-300$ & \\
\hline PT-12-A & 55 & $12 / 4 / 2007$ & $14: 36$ & $\mathrm{EHC}$ & 54 & 50 & $300-450$ & \\
\hline PT-12-A & 57 & $12 / 4 / 2007$ & $14: 49$ & $\mathrm{EHC}$ & 40 & 100 & $250-300$ & \\
\hline PT-12-A & 59 & $12 / 4 / 2007$ & $14: 58$ & $\mathrm{EHC}$ & 54 & 50 & $\sim 450$ & \\
\hline PT-9 & 53 & $12 / 4 / 2007$ & $15: 35$ & & & & $300-500$ & $\begin{array}{l}\text { Skipped } 51 \text { due to } \\
\text { very tight } \\
\text { formation - } \\
\text { attempted } 53 \text {, but } \\
\text { had daylighting } \\
\text { around probe hole. }\end{array}$ \\
\hline PT-9 & 55 & $12 / 4 / 2007$ & $16: 20$ & $\mathrm{EHC}$ & 100 & 250 & $300-500$ & $250 \mathrm{lbs}$ EHC \\
\hline PT-9 & 57 & $12 / 4 / 2007$ & $16: 31$ & $\mathrm{EHC}$ & 60 & 150 & $300-500$ & $150 \mathrm{lbs} \mathrm{EHC}$ \\
\hline PT-9 & 59 & $12 / 4 / 2007$ & $16: 48$ & $\mathrm{EHC}$ & 40 & 100 & $300-500$ & \\
\hline PT-11-A & 21 & $12 / 5 / 2007$ & $8: 20$ & EHC-A & 54 & 50 & $150-200$ & \\
\hline PT-11-A & 23 & $12 / 5 / 2007$ & $8: 26$ & EHC-A & 0 & 0 & $100-200$ & $\begin{array}{c}\text { Multiple surficial } \\
\text { daylights }\end{array}$ \\
\hline PT-11-A & 25 & $12 / 5 / 2007$ & $8: 32$ & EHC-A & 80 & 100 & $100-200$ & \\
\hline
\end{tabular}




\section{Appendix C. Field Injection Log \\ USDA/CCC Argonne Labs Site \\ Centralia, Kansas}

\begin{tabular}{|c|c|c|c|c|c|c|c|c|}
\hline $\begin{array}{l}\text { Boring } \\
\text { ID }\end{array}$ & $\begin{array}{l}\text { Injection } \\
\text { Depth (ft) }\end{array}$ & Date & $\begin{array}{l}\text { Time } \\
\text { (CST) }\end{array}$ & $\begin{array}{l}\text { EHC or } \\
\text { EHC-A }\end{array}$ & $\begin{array}{c}\text { Water } \\
\text { (gallons) }\end{array}$ & $\begin{array}{c}\text { EHC or } \\
\text { EHC-A } \\
\text { (lbs) }\end{array}$ & $\begin{array}{l}\text { Pressure } \\
\quad \text { (psi) }\end{array}$ & Comments \\
\hline PT-11-A & 27 & $12 / 5 / 2007$ & $8: 40$ & EHC-A & 40 & 50 & $100-200$ & $\begin{array}{l}\text { EHC-A application } \\
\text { rate was doubled } \\
\text { starting from the } \\
\text { depth of } 27 \text { feet }\end{array}$ \\
\hline PT-11-A & 29 & $12 / 5 / 2007$ & $8: 46$ & EHC-A & 40 & 100 & $100-200$ & \\
\hline PT-11-A & 31 & $12 / 5 / 2007$ & $8: 50$ & EHC-A & 40 & 0 & $100-200$ & $\begin{array}{c}\text { Daylighting around } \\
\text { probe. }\end{array}$ \\
\hline PT-11-A & 33 & $12 / 5 / 2007$ & $9: 00$ & EHC-A & 32 & $\sim 80$ & $100-300$ & $\begin{array}{l}\text { Injected but } \\
\text { daylights }\end{array}$ \\
\hline PT-11-A & 35 & $12 / 5 / 2007$ & $9: 08$ & EHC-A & 20 & $\sim 50$ & $100-200$ & Daylights \\
\hline PT-11-A & 37 & $12 / 5 / 2007$ & $9: 17$ & EHC-A & 40 & $\sim 50$ & $150-200$ & Daylights \\
\hline PT-11-A & 39 & $12 / 5 / 2007$ & $9: 25$ & EHC-A & 40 & 170 & $100-200$ & \\
\hline PT-11-A & 42 & $12 / 5 / 2007$ & $9: 30$ & $\mathrm{EHC}$ & 40 & 100 & $100-200$ & \\
\hline PT-11-A & 45 & $12 / 5 / 2007$ & $9: 40$ & $\mathrm{EHC}$ & 40 & 100 & $100-200$ & \\
\hline PT-11-A & 48 & $12 / 5 / 2007$ & 9.48 & $\mathrm{EHC}$ & 40 & 100 & $100-200$ & \\
\hline PT-6-A & 55 & $12 / 5 / 2007$ & $11: 05$ & $\mathrm{EHC}$ & 40 & 100 & $500-900$ & $\begin{array}{l}\text { High pressures, } \\
\text { skipped } 51 \text { and } 53\end{array}$ \\
\hline PT-6-A & 56.5 & $12 / 5 / 2007$ & $11: 22$ & $\mathrm{EHC}$ & 40 & 100 & $400-600$ & \\
\hline PT-6-A & 58.5 & $12 / 5 / 2007$ & $11: 44$ & $\mathrm{EHC}$ & 40 & 100 & $600-750$ & \\
\hline
\end{tabular}

Total water usage for injection of $\mathbf{8 6 5 0}$ gallons 


\section{Supplement 4:}

Analytical Results for Groundwater and Soil Samples Collected during the Extended Post-Injection Monitoring Program 
TABLE S4.1 Post-injection monitoring: Field measurements made prior to collection of groundwater samples within and adjacent to the treatment area.

\begin{tabular}{|c|c|c|c|c|c|c|c|c|c|c|}
\hline Location & Sample & $\begin{array}{l}\text { Sample } \\
\text { Date }\end{array}$ & $\begin{array}{l}\text { Depth } \\
\text { (ft BGL) }\end{array}$ & $\begin{array}{c}\text { Temperature } \\
\left({ }^{\circ} \mathrm{C}\right)\end{array}$ & $\mathrm{pH}$ & $\begin{array}{l}\text { Conductivity } \\
(\mu \mathrm{S} / \mathrm{cm})\end{array}$ & $\begin{array}{l}\text { Dissolved } \\
\text { Oxygen }\end{array}$ & Iron(II) & $\begin{array}{l}\text { Carbon } \\
\text { Dioxide }\end{array}$ & $\begin{array}{l}\text { ORP } \\
(\mathrm{mV})\end{array}$ \\
\hline MW01 & CNMW01-W-26023 & 3/19/08 & $54.5-64.5$ & 9.5 & 7.31 & 613 & 3.34 & - & - & 122 \\
\hline MW01 & CNMW01-W-26673 & 9/9/08 & $54.5-64.5$ & 13.9 & 7.28 & 595 & 5.18 & 0.03 & 20 & 28 \\
\hline MW02 & CNMW02-W-26074 & $1 / 9 / 08$ & $49.5-59.5$ & 17.0 & 5.81 & 2280 & 0.17 & $3.3+$ & - & -218 \\
\hline MW02 & CNMW02-W-26097 & 1/24/08 & $49.5-59.5$ & 8.6 & 5.81 & 3910 & 0.96 & $3.3+$ & - & 89 \\
\hline MW02 & CNMW02-W-26099 & $2 / 23 / 08$ & $49.5-59.5$ & 9.0 & 5.64 & 8501 & 2.40 & $3.3+$ & - & 45 \\
\hline MW02 & CNMW02-W-26000 & $3 / 12 / 08$ & $49.5-59.5$ & 11.1 & 5.58 & 10174 & 0.28 & $3.3+$ & - & -42 \\
\hline MW02 & CNMW02-W-26045 & $4 / 24 / 08$ & $49.5-59.5$ & 16.9 & 5.68 & 8264 & 0.11 & $3.3+$ & - & -54 \\
\hline MW02 & CNMW02-W-26620 & 6/4/08 & $49.5-59.5$ & 17.4 & 6.04 & 10065 & 0.09 & $3.3+$ & - & -119 \\
\hline MW02 & CNMW02-W-26639 & $7 / 8 / 08$ & $49.5-59.5$ & 15.3 & 6.12 & 8934 & 1.63 & $3.3+$ & - & -49 \\
\hline MW02 & CNMW02-W-26658 & 8/6/08 & $49.5-59.5$ & 16.6 & 5.91 & 8654 & 0.33 & $3.3+$ & - & -75 \\
\hline MW02 & CNMW02-W-26674 & $9 / 8 / 08$ & $49.5-59.5$ & 13.1 & 6.12 & 6821 & 0.40 & $3.3+$ & 50 & -74 \\
\hline MW03 & CNMW03-W-26078 & 1/10/08 & $50.5-60.5$ & 13.0 & 6.94 & 719 & 10.31 & 0.38 & - & 113 \\
\hline MW03 & CNMW03-W-26115 & 2/23/08 & $50.5-60.5$ & 9.5 & 7.10 & 741 & 7.19 & 0.10 & - & 246 \\
\hline MW03 & CNMW03-W-26001 & $3 / 12 / 08$ & $50.5-60.5$ & 14.6 & 7.12 & 777 & 7.90 & 3.13 & - & 89 \\
\hline MW03 & CNMW03-W-26046 & $4 / 23 / 08$ & $50.5-60.5$ & 13.8 & 7.11 & 733 & 5.46 & 0.07 & - & 177 \\
\hline MW03 & CNMW03-W-26621 & 6/4/08 & $50.5-60.5$ & 15.9 & 7.16 & 807 & 5.61 & 0.07 & - & 123 \\
\hline MW03 & CNMW03-W-26640 & $7 / 7 / 08$ & $50.5-60.5$ & 16.1 & 7.07 & 839 & 6.23 & 0.08 & - & 149 \\
\hline MW03 & CNMW03-W-26659 & 8/6/08 & $50.5-60.5$ & 15.0 & 6.34 & 843 & 1.98 & 0.15 & - & 208 \\
\hline MW03 & CNMW03-W-26675 & $9 / 9 / 08$ & $50.5-60.5$ & 14.9 & 7.13 & 763 & 9.60 & 0.12 & 110 & 66 \\
\hline MW04 & CNMW04-W-26024 & 3/19/08 & $37.5-47.5$ & 11.2 & 7.32 & 636 & 7.55 & - & - & 164 \\
\hline MW04 & CNMW04-W-26676 & $9 / 9 / 08$ & $37.5-47.5$ & 14.2 & 7.14 & 648 & 8.68 & 0 & 100 & 72 \\
\hline MW05 & CNMW05-W-26025 & 3/19/08 & $34.5-44.5$ & 12.9 & 7.42 & 642 & 5.42 & - & - & 177 \\
\hline MW05 & CNMW05-W-26677 & $9 / 10 / 08$ & $34.5-44.5$ & 13.9 & 7.11 & 663 & 7.14 & 0 & 95 & 130 \\
\hline MW06 & CNMW06-W-26026 & 3/19/08 & $46.5-56.5$ & 14.1 & 7.01 & 552 & 7.00 & - & - & 172 \\
\hline MW06 & CNMW06-W-26678 & $9 / 9 / 08$ & $46.5-56.5$ & 14.4 & 7.20 & 437 & 0.36 & 0.07 & 105 & -96 \\
\hline
\end{tabular}


TABLE S4.1 (Cont.)

\begin{tabular}{|c|c|c|c|c|c|c|c|c|c|c|}
\hline Location & Sample & $\begin{array}{l}\text { Sample } \\
\text { Date }\end{array}$ & $\begin{array}{c}\text { Depth } \\
\text { (ft BGL) }\end{array}$ & $\begin{array}{l}\text { Temperature } \\
\left({ }^{\circ} \mathrm{C}\right)\end{array}$ & $\mathrm{pH}$ & $\begin{array}{l}\text { Conductivity } \\
(\mu \mathrm{S} / \mathrm{cm})\end{array}$ & $\begin{array}{l}\text { Dissolved } \\
\text { Oxygen }\end{array}$ & Iron(II) & $\begin{array}{l}\text { Carbon } \\
\text { Dioxide }\end{array}$ & $\begin{array}{l}\text { ORP } \\
(\mathrm{mV})\end{array}$ \\
\hline MW07 & CNMW07-W-26027 & $3 / 19 / 08$ & $45-55$ & 12.5 & 7.29 & 647 & 2.70 & - & - & 215 \\
\hline MW07 & CNMW07-W-26679 & 9/9/08 & $45-55$ & 15.6 & 7.10 & 629 & 1.41 & 0 & 68 & 16 \\
\hline MW08 & CNMW08-W-26028 & $3 / 20 / 08$ & $38-53$ & 13.5 & 7.19 & 869 & 2.11 & - & - & 185 \\
\hline MW08 & CNMW08-W-26680 & 9/10/08 & $38-53$ & 16.3 & 7.03 & 864 & 1.17 & 0.03 & 100 & 117 \\
\hline MW09 & CNMW09-W-26029 & $3 / 20 / 08$ & $25-35$ & 13.5 & 7.17 & 720 & 4.70 & - & - & 173 \\
\hline MW09 & CNMW09-W-26681 & $9 / 10 / 08$ & $25-35$ & 14.7 & 7.02 & 706 & 3.68 & 0.07 & 110 & 120 \\
\hline MW10 & CNMW10-W-26030 & $3 / 20 / 08$ & $30-45$ & 10.9 & 7.18 & 898 & 6.12 & - & - & 187 \\
\hline MW10 & CNMW10-W-26682 & 9/9/08 & $30-45$ & 14.8 & 7.05 & 879 & 7.18 & 0.06 & 100 & 94 \\
\hline PMP1 & CNPMP1-W-26079 & 1/10/08 & $50-60$ & 17.7 & 6.26 & 1140 & 3.11 & 0.33 & - & -222 \\
\hline PMP1 & CNPMP1-W-26083 & $1 / 24 / 08$ & $50-60$ & 7.8 & 6.25 & 1446 & 1.01 & $3.3+$ & - & -357 \\
\hline PMP1 & CNPMP1-W-25985 & $2 / 24 / 08$ & $50-60$ & 12.3 & 6.68 & 924 & 3.24 & 0.66 & - & 26 \\
\hline PMP1 & CNPMP1-W-26005 & $3 / 13 / 08$ & $50-60$ & - & - & - & - & 1.32 & - & - \\
\hline PMP1 & CNPMP1-W-26050 & $4 / 24 / 08$ & $50-60$ & 14.9 & 7.09 & 782 & 0.21 & 0 & - & -95 \\
\hline PMP1 & CNPMP1-W-26625 & 6/5/08 & $50-60$ & 15.7 & 7.30 & 735 & 0.46 & 0.08 & - & -113 \\
\hline PMP1 & CNPMP1-W-26644 & $7 / 8 / 08$ & $50-60$ & 15.5 & 7.06 & 756 & 0.47 & 0.15 & - & -53 \\
\hline PMP1 & CNPMP1-W-26663 & $8 / 6 / 08$ & $50-60$ & 15.0 & 7.23 & 714 & 3.52 & 0 & - & -48 \\
\hline PMP1 & CNPMP1-W-26689 & 9/9/08 & $50-60$ & 14.4 & 5.54 & 700 & 1.37 & 0.23 & 115 & 40 \\
\hline PMP2 & CNPMP2-W-26081 & $1 / 10 / 08$ & $50-60$ & 19.9 & 6.55 & 703 & 0.18 & 0.50 & - & -556 \\
\hline PMP2 & CNPMP2-W-26084 & $1 / 24 / 08$ & $50-60$ & 7.2 & 6.74 & 1058 & 1.40 & $3.3+$ & - & -266 \\
\hline PMP2 & CNPMP2-W-25986 & $2 / 24 / 08$ & $50-60$ & 9.7 & 6.35 & 991 & 2.05 & 2.13 & - & -87 \\
\hline PMP2 & CNPMP2-W-26006 & $3 / 13 / 08$ & $50-60$ & - & - & - & - & 1.95 & - & - \\
\hline PMP2 & CNPMP2-W-26051 & $4 / 24 / 08$ & $50-60$ & 14.5 & 6.21 & 1093 & 0.28 & 1.37 & - & -98 \\
\hline PMP2 & CNPMP2-W-26626 & $6 / 5 / 08$ & $50-60$ & 15.6 & 6.88 & 1080 & 0.34 & 1.00 & - & -101 \\
\hline PMP2 & CNPMP2-W-26645 & $7 / 8 / 08$ & $50-60$ & 15.5 & 6.70 & 1100 & 0.16 & 1.26 & - & -66 \\
\hline PMP2 & CNPMP2-W-26664 & $8 / 6 / 08$ & $50-60$ & 15.3 & 6.86 & 1051 & 2.87 & 0.5 & - & -66 \\
\hline PMP2 & CNPMP2-W-26690 & 9/9/08 & $50-60$ & 14.4 & 7.09 & 997 & 0.05 & 1.68 & 180 & -41 \\
\hline PMP3 & CNPMP3-W-26071 & $1 / 9 / 08$ & $50-60$ & 18.3 & 6.56 & 1368 & 0.15 & 0.62 & - & -550 \\
\hline PMP3 & CNPMP3-W-26086 & $1 / 24 / 08$ & $50-60$ & 9.5 & 6.34 & 2420 & 0.26 & $3.3+$ & - & -344 \\
\hline PMP3 & CNPMP3-W-25990 & $2 / 25 / 08$ & $50-60$ & 10.6 & 6.44 & 1901 & 0.62 & 3.16 & - & -17 \\
\hline PMP3 & CNPMP3-W-26007 & 3/13/08 & $50-60$ & 12.5 & 6.73 & 1908 & 0.33 & 0.20 & - & -88 \\
\hline
\end{tabular}


PMP3

PMP3

PMP3

PMP3

PMP4

PMP4

PMP4

PMP4

PMP4

PMP

PMP4

PMP4

PMP4

PMP5

PMP5

PMP5

PMP5

PMP5

PMP5

PMP5

PMP5

PMP6

PMP6

PMP6

PMP6

PMP6

PMP6

PMP6

PMP6

PMP6

Concentration (mg/L)

\begin{tabular}{|c|c|c|c|c|c|c|c|c|}
\hline $\begin{array}{l}\text { Sample } \\
\text { Date }\end{array}$ & $\begin{array}{l}\text { Depth } \\
\text { (ft BGL) }\end{array}$ & $\begin{array}{c}\text { Temperature } \\
\left({ }^{\circ} \mathrm{C}\right)\end{array}$ & $\mathrm{pH}$ & $\begin{array}{l}\text { Conductivity } \\
(\mu \mathrm{S} / \mathrm{cm})\end{array}$ & $\begin{array}{l}\text { Dissolved } \\
\text { Oxygen }\end{array}$ & Iron(II) & $\begin{array}{l}\text { Carbon } \\
\text { Dioxide }\end{array}$ & $\begin{array}{l}\text { ORP } \\
(\mathrm{mV})\end{array}$ \\
\hline 4/24/08 & $50-60$ & 15.1 & 6.99 & 1813 & 0.18 & 2.58 & - & -176 \\
\hline 6/5/08 & $50-60$ & 15.7 & 7.18 & 1678 & 0.03 & 2.15 & - & -193 \\
\hline $7 / 8 / 08$ & $50-60$ & 15.1 & 6.32 & 1646 & 0.21 & 1.46 & - & -119 \\
\hline 8/6/08 & $50-60$ & 14.9 & 7.38 & 1505 & 1.43 & 0.23 & - & -138 \\
\hline 9/9/08 & $50-60$ & 14.5 & 6.98 & 1301 & 0.03 & $3.3+$ & 150 & -150 \\
\hline 1/9/08 & $48.75-58.75$ & 15.8 & 7.13 & 736 & 2.01 & 0.50 & - & -213 \\
\hline 1/24/08 & $48.75-58.75$ & 7.7 & 7.76 & 669 & 2.35 & $3.3+$ & - & -306 \\
\hline 2/25/08 & $48.75-58.75$ & 8.5 & 7.16 & 697 & 2.36 & 0.36 & - & -209 \\
\hline 3/13/08 & $48.75-58.75$ & - & - & - & - & - & - & - \\
\hline 4/24/08 & $48.75-58.75$ & 15.2 & 7.15 & 677 & 2.91 & 0 & - & 66 \\
\hline 6/5/08 & $48.75-58.75$ & 14.6 & 7.46 & 733 & 3.82 & 0.09 & - & 12 \\
\hline 7/7/08 & $48.75-58.75$ & 15.5 & 7.04 & 753 & 12.13 & 0.04 & - & 97 \\
\hline 8/6/08 & $48.75-58.75$ & 14.9 & 7.04 & 761 & 11.61 & 0 & - & 66 \\
\hline 9/9/08 & $48.75-58.75$ & 14.3 & 4.97 & 738 & 4.87 & 0.49 & 100 & 134 \\
\hline $1 / 9 / 08$ & $50-60$ & 13.2 & 7.25 & 743 & 2.45 & 0.31 & - & -189 \\
\hline $1 / 24 / 08$ & $50-60$ & 8.2 & 7.43 & 805 & 2.23 & $3.3+$ & - & 33 \\
\hline $2 / 25 / 08$ & $50-60$ & 9.0 & 7.25 & 831 & 2.02 & 0.15 & - & 150 \\
\hline 3/13/08 & $50-60$ & 10.7 & 7.28 & 828 & 1.83 & 0.14 & - & 221 \\
\hline 4/24/08 & $50-60$ & 16.4 & 7.35 & 858 & 1.04 & 0.11 & - & 154 \\
\hline 6/4/08 & $50-60$ & 22.0 & 7.37 & 927 & 1.25 & 0.12 & - & -107 \\
\hline $7 / 8 / 08$ & $50-60$ & 19.0 & 7.21 & 915 & 3.93 & 0.31 & - & 111 \\
\hline 8/6/08 & $50-60$ & 18.4 & 6.25 & 970 & 0.43 & 0.09 & - & 163 \\
\hline 9/10/08 & $50-60$ & 16.9 & 7.20 & 875 & 2.51 & 0.18 & 105 & 117 \\
\hline 1/8/08 & $50-60$ & 15.5 & 7.63 & 826 & 0.96 & 0.45 & - & -276 \\
\hline $1 / 24 / 08$ & $50-60$ & 8.6 & 7.65 & 547 & 3.36 & $3.3+$ & - & -155 \\
\hline $2 / 25 / 08$ & $50-60$ & 9.1 & 7.22 & 755 & 3.18 & 0.26 & - & 133 \\
\hline 3/13/08 & $50-60$ & 10.3 & 7.32 & 750 & 3.31 & 0.27 & - & 231 \\
\hline 4/24/08 & $50-60$ & 14.9 & 7.07 & 761 & 2.67 & $3.3+$ & - & 197 \\
\hline 6/5/08 & $50-60$ & 15.7 & 7.32 & 783 & 2.94 & 0.08 & - & 100 \\
\hline 7/7/08 & $50-60$ & 15.5 & 7.17 & 806 & 9.96 & 0.11 & - & 108 \\
\hline 8/6/08 & $50-60$ & 15.1 & 7.02 & 816 & 9.13 & 0.08 & - & 89 \\
\hline 9/8/08 & $50-60$ & 13.2 & 6.87 & 787 & 3.32 & 0.09 & 75 & 173 \\
\hline
\end{tabular}


Concentration (mg/L)

\begin{tabular}{|c|c|c|c|c|c|c|c|c|c|c|}
\hline Location & Sample & $\begin{array}{l}\text { Sample } \\
\text { Date }\end{array}$ & $\begin{array}{l}\text { Depth } \\
\text { (ft BGL) }\end{array}$ & $\begin{array}{c}\text { Temperature } \\
\left({ }^{\circ} \mathrm{C}\right)\end{array}$ & $\mathrm{pH}$ & $\begin{array}{l}\text { Conductivity } \\
(\mu \mathrm{S} / \mathrm{cm})\end{array}$ & $\begin{array}{l}\text { Dissolved } \\
\text { Oxygen }\end{array}$ & Iron(II) & $\begin{array}{l}\text { Carbon } \\
\text { Dioxide }\end{array}$ & $\begin{array}{l}\text { ORP } \\
(\mathrm{mV})\end{array}$ \\
\hline PMP7 & CNPMP7-W-26066 & $1 / 8 / 08$ & $50-60$ & 18.0 & 7.14 & 803 & 1.01 & 0.3 & - & -381 \\
\hline PMP7 & CNPMP7-W-26091 & $1 / 24 / 08$ & $50-60$ & 9.5 & 7.55 & 629 & 2.90 & $3.3+$ & - & -201 \\
\hline PMP7 & CNPMP7-W-25996 & $2 / 25 / 08$ & $50-60$ & 6.4 & 7.27 & 782 & 2.73 & 0.32 & - & 129 \\
\hline PMP7 & CNPMP7-W-26011 & 3/13/08 & $50-60$ & 11.3 & 7.29 & 789 & 3.20 & 0.16 & - & 218 \\
\hline PMP7 & CNPMP7-W-26056 & 4/24/08 & $50-60$ & 15.6 & 6.77 & 814 & 3.32 & 0.36 & - & 198 \\
\hline PMP7 & CNPMP7-W-26631 & $6 / 5 / 08$ & $50-60$ & 14.2 & 7.39 & 836 & 3.77 & 0.08 & - & 95 \\
\hline PMP7 & CNPMP7-W-26650 & $7 / 7 / 08$ & $50-60$ & 15.0 & 7.18 & 842 & 12.48 & 0.02 & - & 106 \\
\hline PMP7 & CNPMP7-W-26669 & $8 / 6 / 08$ & $50-60$ & 14.7 & 6.95 & 844 & 9.67 & 0 & - & 85 \\
\hline PMP7 & CNPMP7-W-26695 & 9/9/08 & $50-60$ & 14.2 & 6.30 & 807 & 2.18 & 0.18 & 70 & 15 \\
\hline PMP8 & CNPMP8-W-26075 & $1 / 9 / 08$ & $50-60$ & 18.4 & 6.84 & 1310 & 0.08 & 0.51 & - & -548 \\
\hline PMP8 & CNPMP8-W-26092 & $1 / 24 / 08$ & $50-60$ & 7.7 & 7.15 & 1322 & 1.01 & $3.3+$ & - & -201 \\
\hline PMP8 & CNPMP8-W-25994 & $2 / 25 / 08$ & $50-60$ & 8.8 & 7.07 & 1055 & 0.31 & 1.28 & - & -122 \\
\hline PMP8 & CNPMP8-W-26012 & 3/13/08 & $50-60$ & - & - & - & - & $3.3+$ & - & - \\
\hline PMP8 & CNPMP8-W-26057 & 4/24/08 & $50-60$ & 14.8 & 6.94 & 1385 & 0.18 & $3.3+$ & - & -186 \\
\hline PMP8 & CNPMP8-W-26632 & $6 / 5 / 08$ & $50-60$ & 15.6 & 7.44 & 1304 & 0.06 & 1.78 & - & -190 \\
\hline PMP8 & CNPMP8-W-26651 & $7 / 8 / 08$ & $50-60$ & 15.1 & 6.96 & 1435 & 0.08 & 1.15 & - & -135 \\
\hline PMP8 & CNPMP8-W-26670 & $8 / 6 / 08$ & $50-60$ & 14.9 & 7.56 & 1433 & 1.30 & 0.52 & - & -158 \\
\hline PMP8 & CNPMP8-W-26696 & 9/9/08 & $50-60$ & 14.4 & 7.05 & 1388 & 0.03 & 2.72 & 60 & -129 \\
\hline PMP9 & CNPMP9-W-26077 & $1 / 10 / 08$ & $50-60$ & 14.9 & 7.19 & 656 & 0.59 & 0.42 & - & -323 \\
\hline PMP9 & CNPMP9-W-26109 & $1 / 24 / 08$ & $50-60$ & 8.7 & 7.47 & 598 & 2.64 & 0.47 & - & -244 \\
\hline PMP9 & CNPMP9-W-25989 & $2 / 24 / 08$ & $50-60$ & 9.2 & 7.24 & 603 & 3.53 & 0.2 & - & -23 \\
\hline PMP9 & CNPMP9-W-26013 & $3 / 13 / 08$ & $50-60$ & 13.3 & 7.47 & 595 & 4.18 & 0.04 & - & 75 \\
\hline PMP9 & CNPMP9-W-26058 & $4 / 24 / 08$ & $50-60$ & 14.4 & 7.23 & 618 & 5.32 & 0.17 & - & 41 \\
\hline PMP9 & CNPMP9-W-26633 & 6/5/08 & $50-60$ & 14.6 & 7.41 & 634 & 5.60 & 0.03 & - & 1 \\
\hline PMP9 & CNPMP9-W-26652 & $7 / 8 / 08$ & $50-60$ & 14.5 & 7.27 & 656 & 5.67 & 0.16 & - & 34 \\
\hline PMP9 & CNPMP9-W-26671 & $8 / 6 / 08$ & $50-60$ & 14.9 & 7.24 & 640 & 18.65 & 0 & - & -10 \\
\hline PMP9 & CNPMP9-W-26697 & 9/9/08 & $50-60$ & 14.0 & 6.36 & 606 & 7.78 & 0.10 & 120 & 45 \\
\hline SB01 & CNSB01-W-26031 & $3 / 20 / 08$ & $40-50$ & 15.6 & 7.29 & 783 & 8.02 & - & - & 182 \\
\hline SB01 & CNSB01-W-26683 & 9/10/08 & $40-50$ & 16.5 & 7.10 & 676 & 2.89 & 0.17 & 100 & 100 \\
\hline SB04 & CNSB04-W-26076 & $1 / 10 / 08$ & $51-50$ & 9.0 & 6.82 & 866 & 8.73 & 0.10 & - & 89 \\
\hline SB04 & CNSB04-W-26094 & $1 / 24 / 08$ & $51-50$ & 8.7 & 7.19 & 778 & 6.32 & 0.40 & - & 184 \\
\hline SBO4 & CNSB04-W-26117 & $2 / 23 / 08$ & $51-50$ & 9.6 & 7.08 & 804 & 5.53 & 0 & - & 266 \\
\hline
\end{tabular}


TABLE S4.1 (Cont.)

\begin{tabular}{|c|c|c|c|c|c|c|c|c|c|c|}
\hline \multirow[b]{2}{*}{ Location } & \multirow[b]{2}{*}{ Sample } & \multirow[b]{2}{*}{$\begin{array}{l}\text { Sample } \\
\text { Date }\end{array}$} & \multirow[b]{2}{*}{$\begin{array}{c}\text { Depth } \\
\text { (ft BGL) }\end{array}$} & \multirow[b]{2}{*}{$\begin{array}{c}\text { Temperature } \\
\left({ }^{\circ} \mathrm{C}\right)\end{array}$} & \multirow[b]{2}{*}{$\mathrm{pH}$} & \multirow[b]{2}{*}{$\begin{array}{l}\text { Conductivity } \\
(\mu \mathrm{S} / \mathrm{cm})\end{array}$} & \multicolumn{3}{|c|}{ Concentration (mg/L) } & \multirow[b]{2}{*}{$\begin{array}{l}\text { ORP } \\
(\mathrm{mV})\end{array}$} \\
\hline & & & & & & & $\begin{array}{l}\text { Dissolved } \\
\text { Oxygen }\end{array}$ & Iron(II) & $\begin{array}{l}\text { Carbon } \\
\text { Dioxide }\end{array}$ & \\
\hline SB04 & CNSB04-W-26002 & $3 / 12 / 08$ & $51-50$ & 15.5 & 7.04 & 819 & 6.16 & 0.09 & - & 154 \\
\hline SB04 & CNSB04-W-26047 & 4/24/08 & $51-50$ & 15.4 & 7.18 & 817 & 3.72 & 0.07 & - & 147 \\
\hline SB04 & CNSB04-W-26622 & $6 / 4 / 08$ & $51-50$ & 21.7 & 7.24 & 857 & 4.73 & 0.08 & - & 128 \\
\hline SB04 & CNSB04-W-26641 & $7 / 7 / 08$ & $51-50$ & 24.2 & 7.04 & 896 & 3.88 & 0.08 & - & 164 \\
\hline SB04 & CNSB04-W-26660 & 8/6/08 & $51-50$ & 20.6 & 7.00 & 892 & 0.90 & 0.06 & - & 127 \\
\hline SB04 & CNSB04-W-26684 & $9 / 9 / 08$ & $51-50$ & 16.5 & 7.11 & 802 & 6.48 & 0.02 & 100 & 70 \\
\hline SB05 & CNSB05-W-26032 & $3 / 20 / 08$ & $32-42$ & 14.5 & 7.11 & 870 & 5.56 & - & - & 206 \\
\hline SB05 & CNSB05-W-26685 & 9/9/08 & $32-42$ & 13.7 & 6.79 & 890 & 7.60 & 0.09 & 90 & 56 \\
\hline SB07R & CNSB07R-W-26073 & $1 / 9 / 08$ & $45-60$ & 17.4 & 7.05 & 565 & 6.64 & 0.07 & - & 130 \\
\hline SB07R & CNSB07R-W-26111 & $1 / 24 / 08$ & $45-60$ & 8.4 & 7.29 & 624 & 5.97 & 2.90 & - & 167 \\
\hline SB07R & CNSB07R-W-25984 & $2 / 24 / 08$ & $45-60$ & 14.2 & 7.18 & 629 & 4.64 & 0 & - & 277 \\
\hline SB07R & CNSB07R-W-26003 & 3/12/08 & $45-60$ & 17.3 & 7.18 & 639 & 5.33 & 0 & - & 108 \\
\hline SB07R & CNSB07R-W-26048 & 4/24/08 & $45-60$ & 16.7 & 7.19 & 631 & 1.93 & 0 & - & 158 \\
\hline SB07R & CNSB07R-W-26623 & 6/4/08 & $45-60$ & 16.6 & 7.37 & 651 & 4.06 & 0.05 & - & 111 \\
\hline SB07R & CNSB07R-W-26642 & $7 / 7 / 08$ & $45-60$ & 18.3 & 7.11 & 676 & 4.03 & 0.02 & - & 128 \\
\hline SB07R & CNSB07R-W-26661 & 8/6/08 & $45-60$ & 15.9 & 6.01 & 682 & 0.78 & 0 & - & 154 \\
\hline SB07R & CNSB07R-W-26686 & $9 / 9 / 08$ & $45-60$ & 14.1 & 7.06 & 631 & 5.08 & 0.07 & 100 & 55 \\
\hline SB08 & CNSB08-W-26070 & $1 / 9 / 08$ & $52-62$ & 14.7 & 7.01 & 587 & 4.58 & 0 & - & 118 \\
\hline SB08 & CNSB08-W-26112 & $1 / 24 / 08$ & $52-62$ & 8.2 & 7.27 & 622 & 2.84 & 0.39 & - & 175 \\
\hline SB08 & CNSB08-W-25978 & 2/24/08 & $52-62$ & 9.4 & 7.23 & 628 & 3.30 & 0.03 & - & 255 \\
\hline SB08 & CNSB08-W-26004 & 3/12/08 & $52-62$ & 17.1 & 7.17 & 642 & 3.63 & 0.14 & - & 102 \\
\hline SB08 & CNSB08-W-26049 & $4 / 23 / 08$ & $52-62$ & 15.7 & 7.07 & 642 & 2.55 & 0 & - & 168 \\
\hline SB08 & CNSB08-W-26624 & $6 / 4 / 08$ & $52-62$ & 21.7 & 7.18 & 673 & 2.57 & 0.07 & - & 131 \\
\hline SB08 & CNSB08-W-26643 & $7 / 7 / 08$ & $52-62$ & 21.2 & 6.83 & 700 & 2.04 & 0.07 & - & 176 \\
\hline SB08 & CNSB08-W-26662 & 8/6/08 & $52-62$ & 19.9 & 6.80 & 701 & 0.64 & 0 & - & 102 \\
\hline SB08 & CNSB08-W-26687 & 9/8/08 & $52-62$ & 13.6 & 7.14 & 626 & 2.70 & 0 & 90 & 230 \\
\hline SB09 & CNSB09-W-26033 & 3/20/08 & $32-42$ & 10.1 & 6.94 & 1000 & 1.57 & - & - & 221 \\
\hline SB09 & CNSB09-W-26688 & 9/10/08 & $32-42$ & 18.4 & 6.87 & 977 & 0.56 & 0.11 & 160 & 109 \\
\hline
\end{tabular}


TABLE S4.2 Post-injection monitoring: Results for organic analyses of groundwater samples collected within and adjacent to the pilot test area

\begin{tabular}{|c|c|c|c|c|c|c|c|}
\hline \multirow[b]{2}{*}{ Location } & \multirow[b]{2}{*}{ Sample } & \multirow[b]{2}{*}{$\begin{array}{l}\text { Sample } \\
\text { Date }\end{array}$} & \multirow[b]{2}{*}{$\begin{array}{l}\text { Depth } \\
\text { (ft BGL) }\end{array}$} & \multicolumn{3}{|c|}{ Concentration $(\mu \mathrm{g} / \mathrm{L})$} & \multirow[b]{2}{*}{ Comment } \\
\hline & & & & $\begin{array}{c}\text { Carbon } \\
\text { Tetrachloride }\end{array}$ & Chloroform & $\begin{array}{l}\text { Methylene } \\
\text { Chloride }\end{array}$ & \\
\hline MW01 & CNMW01-W-26023 & 3/19/08 & $54.5-64.5$ & $N D^{a}$ & ND & ND & \\
\hline MW01 & CNMW01-W-26673 & 9/9/08 & $54.5-64.5$ & ND & ND & ND & \\
\hline MW02 & CNMW02-W-26074 & $1 / 9 / 08$ & $49.5-59.5$ & $0.3 \mathrm{~J}^{\mathrm{b}}$ & 23 & ND & No purge \\
\hline MW02 & CNMW02-W-26097 & $1 / 24 / 08$ & $49.5-59.5$ & ND & 11 & 1.8 & No purge \\
\hline MW02 & CNMW02-W-26099 & $2 / 23 / 08$ & $49.5-59.5$ & ND & 1.6 & 1.3 & \\
\hline MW02 & CNMW02-W-26000 & $3 / 12 / 08$ & $49.5-59.5$ & ND & 1.2 & 1.9 & \\
\hline MW02 & CNMW02-W-26045 & $4 / 24 / 08$ & $49.5-59.5$ & ND & 6.6 & 2.4 & \\
\hline MW02 & CNMW02-W-26620 & $6 / 4 / 08$ & $49.5-59.5$ & 9.8 & 22 & 5.6 & \\
\hline MW02 & CNMWO2-W-26639 & $7 / 8 / 08$ & $49.5-59.5$ & 6.8 & 69 & 13 & \\
\hline MW02 & CNMW02-W-26658 & 8/6/08 & $49.5-59.5$ & 21 & 72 & 12 & \\
\hline MW02 & CNMW02-W-26674 & 9/8/08 & $49.5-59.5$ & 18 & 57 & 11 & \\
\hline MW03 & CNMW03-W-26078 & 1/10/08 & $50.5-60.5$ & 2.9 & ND & ND & No purge \\
\hline MW03 & CNMW03-W-26115 & 2/23/08 & $50.5-60.5$ & 2.1 & ND & ND & \\
\hline MW03 & CNMW03-W-26001 & $3 / 12 / 08$ & $50.5-60.5$ & 2.3 & ND & ND & \\
\hline MW03 & CNMW03-W-26046 & $4 / 23 / 08$ & $50.5-60.5$ & 2.4 & ND & ND & \\
\hline MW03 & CNMW03-W-26621 & 6/4/08 & $50.5-60.5$ & 2.7 & $0.2 \mathrm{~J}$ & ND & \\
\hline MW03 & CNMW03-W-26640 & $7 / 7 / 08$ & $50.5-60.5$ & 3.3 & ND & ND & \\
\hline MW03 & CNMW03-W-26659 & $8 / 6 / 08$ & $50.5-60.5$ & 3.2 & $0.5 \mathrm{~J}$ & ND & \\
\hline MW03 & CNMW03-W-26675 & 9/9/08 & $50.5-60.5$ & 3.2 & $0.3 \mathrm{~J}$ & ND & \\
\hline MW04 & CNMW04-W-26024 & 3/19/08 & $37.5-47.5$ & 1.3 & ND & ND & \\
\hline MW04 & CNMW04-W-26676 & 9/9/08 & $37.5-47.5$ & 2.0 & ND & ND & \\
\hline MW05 & CNMW05-W-26025 & 3/19/08 & $34.5-44.5$ & 1.9 & ND & ND & \\
\hline MW05 & CNMW05-W-26677 & $9 / 10 / 08$ & $34.5-44.5$ & 13 & $0.7 \mathrm{~J}$ & ND & Primary sample \\
\hline MW05 & CNMW05DUP-W-26698 & $9 / 10 / 08$ & $34.5-44.5$ & 12 & $0.6 \mathrm{~J}$ & ND & Replicate \\
\hline MW06 & CNMW06-W-26026 & 3/19/08 & $46.5-56.5$ & ND & ND & ND & \\
\hline MW06 & CNMW06-W-26678 & 9/9/08 & $46.5-56.5$ & ND & ND & ND & \\
\hline MW07 & CNMW07-W-26027 & 3/19/08 & $45-55$ & 3.0 & ND & ND & \\
\hline MW07 & CNMW07-W-26679 & $9 / 9 / 08$ & $45-55$ & 4.0 & $0.2 \mathrm{~J}$ & ND & \\
\hline MW08 & CNMW08-W-26028 & 3/20/08 & $38-53$ & ND & ND & ND & \\
\hline MW08 & CNMW08-W-26680 & 9/10/08 & $38-53$ & ND & ND & ND & Primary sample \\
\hline MW08 & CNMW08DUP-W-26699 & 9/10/08 & $38-53$ & ND & ND & ND & Replicate \\
\hline MW09 & CNMW09-W-26029 & 3/20/08 & $25-35$ & ND & ND & ND & \\
\hline MW09 & CNMW09-W-26681 & $9 / 10 / 08$ & $25-35$ & ND & ND & ND & \\
\hline MW10 & CNMW10-W-26030 & $3 / 20 / 08$ & $30-45$ & ND & ND & ND & \\
\hline MW10 & CNMW10-W-26682 & 9/9/08 & $30-45$ & ND & ND & ND & \\
\hline PMP1 & CNPMP1-W-26079 & 1/10/08 & $50-60$ & 158 & 22 & ND & No purge \\
\hline PMP1 & CNPMP1-W-26083 & $1 / 24 / 08$ & $50-60$ & $0.5 \mathrm{~J}$ & 92 & 6.8 & No purge \\
\hline PMP1 & CNPMP1-W-25985 & 2/24/08 & $50-60$ & 71 & 87 & 6.0 & \\
\hline PMP1 & CNPMP1-W-26005 & $3 / 13 / 08$ & $50-60$ & 131 & 49 & ND & \\
\hline PMP1 & CNPMP1-W-26050 & $4 / 24 / 08$ & $50-60$ & 113 & 41 & 1.2 & \\
\hline PMP1 & CNPMP1-W-26625 & $6 / 5 / 08$ & $50-60$ & 150 & 39 & $0.7 \mathrm{~J}$ & \\
\hline PMP1 & CNPMP1-W-26644 & $7 / 8 / 08$ & $50-60$ & 145 & 33 & ND & \\
\hline PMP1 & CNPMP1-W-26663 & $8 / 6 / 08$ & $50-60$ & 142 & 29 & ND & \\
\hline PMP1 & CNPMP1-W-26689 & 9/9/08 & $50-60$ & 136 & 30 & ND & \\
\hline PMP2 & CNPMP2-W-26081 & 1/10/08 & $50-60$ & 980 & 951 & 4.2 & No purge \\
\hline PMP2 & CNPMP2-W-26084 & $1 / 24 / 08$ & $50-60$ & 265 & 875 & 17 & No purge \\
\hline PMP2 & CNPMP2-W-25986 & 2/24/08 & $50-60$ & 1249 & 715 & 24 & \\
\hline PMP2 & CNPMP2-W-26006 & $3 / 13 / 08$ & $50-60$ & 1550 & 456 & Diluted & \\
\hline
\end{tabular}


TABLE S4.2 (Cont.)

\begin{tabular}{|c|c|c|c|c|c|c|c|}
\hline \multirow[b]{2}{*}{ Location } & \multirow[b]{2}{*}{ Sample } & \multirow[b]{2}{*}{$\begin{array}{l}\text { Sample } \\
\text { Date }\end{array}$} & \multirow[b]{2}{*}{$\begin{array}{c}\text { Depth } \\
\text { (ft BGL) }\end{array}$} & \multicolumn{3}{|c|}{ Concentration $(\mu \mathrm{g} / \mathrm{L})$} & \multirow[b]{2}{*}{ Commen } \\
\hline & & & & $\begin{array}{c}\text { Carbon } \\
\text { Tetrachloride }\end{array}$ & Chloroform & $\begin{array}{l}\text { Methylene } \\
\text { Chloride }\end{array}$ & \\
\hline PMP2 & CNPMP2-W-26051 & 4/24/08 & $50-60$ & 2254 & 476 & 8.7 & \\
\hline PMP2 & CNPMP2-W-26626 & $6 / 5 / 08$ & $50-60$ & 2873 & 340 & 6.6 & \\
\hline PMP2 & CNPMP2-W-26645 & $7 / 8 / 08$ & $50-60$ & 1831 & 282 & 6.1 & \\
\hline PMP2 & CNPMP2-W-26664 & $8 / 6 / 08$ & $50-60$ & 1315 & 246 & 5.2 & \\
\hline PMP2 & CNPMP2-W-26690 & 9/9/08 & $50-60$ & 1854 & 318 & 5.6 & \\
\hline PMP3 & CNPMP3-W-26071 & $1 / 9 / 08$ & $50-60$ & 112 & 116 & 1.0 & No purge \\
\hline PMP3 & CNPMP3-W-26086 & $1 / 24 / 08$ & $50-60$ & 4.2 & 79 & 3.4 & No purge \\
\hline PMP3 & CNPMP3-W-25990 & $2 / 25 / 08$ & $50-60$ & 3.1 & 147 & 6.5 & \\
\hline PMP3 & CNPMP3-W-26007 & $3 / 13 / 08$ & $50-60$ & 3.9 & 110 & 10 & \\
\hline PMP3 & CNPMP3-W-26052 & $4 / 24 / 08$ & $50-60$ & 16 & 89 & 28 & \\
\hline PMP3 & CNPMP3-W-26627 & 6/5/08 & $50-60$ & 46 & 129 & 32 & \\
\hline PMP3 & CNPMP3-W-26646 & $7 / 8 / 08$ & $50-60$ & 42 & 90 & 20 & \\
\hline PMP3 & CNPMP3-W-26665 & $8 / 6 / 08$ & $50-60$ & 40 & 67 & 8.9 & \\
\hline PMP3 & CNPMP3-W-26691 & 9/9/08 & $50-60$ & 21 & 57 & 6.2 & \\
\hline PMP4 & CNPMP4-W-26068 & $1 / 9 / 08$ & $48.75-58.75$ & 36 & 12 & ND & No purge \\
\hline PMP4 & CNPMP4-W-26087 & $1 / 24 / 08$ & $48.75-58.75$ & 10 & 6.9 & 1.1 & No purge \\
\hline PMP4 & CNPMP4-W-25992 & $2 / 25 / 08$ & $48.75-58.75$ & 42 & 5.8 & $0.6 \mathrm{~J}$ & \\
\hline PMP4 & CNPMP4-W-26008 & 3/13/08 & $48.75-58.75$ & 31 & 4.4 & ND & \\
\hline PMP4 & CNPMP4-W-26053 & $4 / 24 / 08$ & $48.75-58.75$ & 33 & 6.8 & $0.8 \mathrm{~J}$ & \\
\hline PMP4 & CNPMP4-W-26628 & $6 / 5 / 08$ & $48.75-58.75$ & 60 & 6.3 & ND & \\
\hline PMP4 & CNPMP4-W-26647 & 7/7/08 & $48.75-58.75$ & 52 & 4.9 & ND & \\
\hline PMP4 & CNPMP4-W-26666 & $8 / 6 / 08$ & $48.75-58.75$ & 50 & 4.8 & ND & \\
\hline PMP4 & CNPMP4-W-26692 & 9/9/08 & $48.75-58.75$ & 49 & 4.2 & ND & \\
\hline PMP5 & CNPMP5-W-26069 & $1 / 9 / 08$ & $50-60$ & 321 & 97 & 2.6 & No purge \\
\hline PMP5 & CNPMP5-W-26088 & $1 / 24 / 08$ & $50-60$ & 265 & 121 & 4.6 & No purge \\
\hline PMP5 & CNPMP5-W-25997 & $2 / 25 / 08$ & $50-60$ & 421 & 62 & $0.7 \mathrm{~J}$ & \\
\hline PMP5 & CNPMP5-W-26009 & 3/13/08 & $50-60$ & 70 & 13 & $0.7 \mathrm{~J}$ & \\
\hline PMP5 & CNPMP5-W-26054 & $4 / 24 / 08$ & $50-60$ & 357 & 101 & 2.9 & \\
\hline PMP5 & CNPMP5-W-26629 & 6/4/08 & $50-60$ & 365 & 91 & 2.7 & \\
\hline PMP5 & CNPMP5-W-26648 & 7/8/08 & $50-60$ & 397 & 71 & 2.5 & \\
\hline PMP5 & CNPMP5-W-26667 & $8 / 6 / 08$ & $50-60$ & 476 & 55 & 1.7 & \\
\hline PMP5 & CNPMP5-W-26693 & 9/10/08 & $50-60$ & 418 & 46 & $1.6 \mathrm{~J}$ & \\
\hline PMP6 & CNPMP6-W-26065 & 1/8/08 & $50-60$ & 37 & 18 & ND & No purge \\
\hline PMP6 & CNPMP6-W-26090 & $1 / 24 / 08$ & $50-60$ & 26 & 31 & 2.9 & No purge \\
\hline PMP6 & CNPMP6-W-25993 & $2 / 25 / 08$ & $50-60$ & 111 & 8.8 & ND & \\
\hline PMP6 & CNPMP6-W-26010 & $3 / 13 / 08$ & $50-60$ & 94 & 10 & ND & \\
\hline PMP6 & CNPMP6-W-26055 & $4 / 24 / 08$ & $50-60$ & 103 & 13 & ND & \\
\hline PMP6 & CNPMP6-W-26630 & $6 / 5 / 08$ & $50-60$ & 101 & 9.8 & ND & \\
\hline PMP6 & CNPMP6-W-26649 & 7/7/08 & $50-60$ & 96 & 8.5 & ND & \\
\hline PMP6 & CNPMP6-W-26668 & $8 / 6 / 08$ & $50-60$ & 108 & 8.2 & ND & \\
\hline PMP6 & CNPMP6-W-26694 & 9/8/08 & $50-60$ & 110 & 7.8 & ND & \\
\hline PMP7 & CNPMP7-W-26066 & $1 / 8 / 08$ & $50-60$ & 119 & 26 & ND & No purqe \\
\hline PMP7 & CNPMP7-W-26091 & $1 / 24 / 08$ & $50-60$ & 62 & 40 & 1.6 & No purge \\
\hline PMP7 & CNPMP7-W-25996 & $2 / 25 / 08$ & $50-60$ & 123 & 17 & $0.7 \mathrm{~J}$ & \\
\hline PMP7 & CNPMP7-W-26011 & $3 / 13 / 08$ & $50-60$ & 121 & 14 & $0.6 \mathrm{~J}$ & \\
\hline PMP7 & CNPMP7-W-26056 & $4 / 24 / 08$ & $50-60$ & 134 & 13 & ND & \\
\hline PMP7 & CNPMP7-W-26631 & $6 / 5 / 08$ & $50-60$ & 120 & 8.8 & ND & \\
\hline PMP7 & CNPMP7-W-26650 & $7 / 7 / 08$ & $50-60$ & 120 & 7.6 & ND & \\
\hline PMP7 & CNPMP7-W-26669 & $8 / 6 / 08$ & $50-60$ & 154 & 9.2 & ND & \\
\hline PMP7 & CNPMP7-W-26695 & 9/9/08 & $50-60$ & 119 & 13 & ND & \\
\hline PMP8 & CNPMP8-W-26075 & $1 / 9 / 08$ & $50-60$ & 30 & 606 & 3.4 & No purge \\
\hline PMP8 & CNPMP8-W-26092 & $1 / 24 / 08$ & $50-60$ & 31 & 430 & 28 & No purge \\
\hline PMP8 & CNPMP8-W-25994 & $2 / 25 / 08$ & $50-60$ & 287 & 374 & 25 & \\
\hline PMP8 & CNPMP8-W-26012 & $3 / 13 / 08$ & $50-60$ & 122 & 292 & 20 & \\
\hline PMP8 & CNPMP8-W-26057 & $4 / 24 / 08$ & $50-60$ & 72 & 553 & 41 & \\
\hline
\end{tabular}


TABLE S4.2 (Cont.)

\begin{tabular}{|c|c|c|c|c|c|c|c|}
\hline \multirow[b]{2}{*}{ Location } & \multirow[b]{2}{*}{ Sample } & \multirow[b]{2}{*}{$\begin{array}{l}\text { Sample } \\
\text { Date }\end{array}$} & \multirow[b]{2}{*}{$\begin{array}{l}\text { Depth } \\
\text { (ft BGL) }\end{array}$} & \multicolumn{3}{|c|}{ Concentration $(\mu \mathrm{g} / \mathrm{L})$} & \multirow[b]{2}{*}{ Commen } \\
\hline & & & & $\begin{array}{c}\text { Carbon } \\
\text { Tetrachloride }\end{array}$ & Chloroform & $\begin{array}{l}\text { Methylene } \\
\text { Chloride }\end{array}$ & \\
\hline PMP8 & CNPMP8-W-26632 & $6 / 5 / 08$ & $50-60$ & 73 & 364 & 24 & \\
\hline PMP8 & CNPMP8-W-26651 & $7 / 8 / 08$ & $50-60$ & 67 & 339 & 23 & \\
\hline PMP8 & CNPMP8-W-26670 & $8 / 6 / 08$ & $50-60$ & 105 & 317 & 18 & \\
\hline PMP8 & CNPMP8-W-26696 & 9/9/08 & $50-60$ & 72 & 125 & 3.4 & \\
\hline PMP9 & CNPMP9-W-26077 & 1/10/08 & $50-60$ & 1.9 & $0.9 \mathrm{~J}$ & ND & No purge \\
\hline PMP9 & CNPMP9-W-26109 & $1 / 24 / 08$ & $50-60$ & 3.1 & 1.1 & ND & No purge \\
\hline PMP9 & CNPMP9-W-25989 & $2 / 24 / 08$ & $50-60$ & 4.7 & $0.8 \mathrm{~J}$ & ND & \\
\hline PMP9 & CNPMP9-W-26013 & $3 / 13 / 08$ & $50-60$ & 4.0 & $0.5 \mathrm{~J}$ & ND & \\
\hline PMP9 & CNPMP9-W-26058 & $4 / 24 / 08$ & $50-60$ & 5.7 & 1.8 & ND & \\
\hline PMP9 & CNPMP9-W-26633 & 6/5/08 & $50-60$ & 6.3 & $0.6 \mathrm{~J}$ & ND & \\
\hline PMP9 & CNPMP9-W-26652 & $7 / 8 / 08$ & $50-60$ & 7.2 & 1.4 & ND & \\
\hline PMP9 & CNPMP9-W-26671 & $8 / 6 / 08$ & $50-60$ & 12 & 1.1 & ND & \\
\hline PMP9 & CNPMP9-W-26697 & 9/9/08 & $50-60$ & 7.6 & $0.4 \mathrm{~J}$ & ND & \\
\hline SB01 & CNSB01-W-26031 & $3 / 20 / 08$ & $40-50$ & 325 & 4.8 & ND & \\
\hline SB01 & CNSB01-W-26683 & 9/10/08 & $40-50$ & 378 & 4.1 & ND & \\
\hline SB04 & CNSB04-W-26076 & 1/10/08 & $51-61$ & 10 & 2.1 & ND & No purge \\
\hline SB04 & CNSB04-W-26094 & $1 / 24 / 08$ & $51-61$ & 15 & $0.2 \mathrm{~J}$ & ND & No purge \\
\hline SB04 & CNSB04-W-26117 & 2/23/08 & $51-61$ & 42 & $0.4 \mathrm{~J}$ & ND & \\
\hline SB04 & CNSB04-W-26002 & 3/12/08 & $51-61$ & 30 & $0.3 \mathrm{~J}$ & ND & \\
\hline SB04 & CNSB04-W-26047 & $4 / 24 / 08$ & $51-61$ & 16 & $0.3 \mathrm{~J}$ & ND & \\
\hline SB04 & CNSB04-W-26622 & 6/4/08 & $51-61$ & 25 & $0.4 \mathrm{~J}$ & ND & \\
\hline SB04 & CNSB04-W-26641 & 7/7/08 & $51-61$ & 14 & $0.2 \mathrm{~J}$ & ND & \\
\hline SB04 & CNSB04-W-26660 & $8 / 6 / 08$ & $51-61$ & 11 & $0.2 \mathrm{~J}$ & ND & \\
\hline SB04 & CNSB04-W-26684 & 9/9/08 & $51-61$ & 15 & $0.3 \mathrm{~J}$ & ND & \\
\hline SB05 & CNSB05-W-26032 & $3 / 20 / 08$ & $32-42$ & 224 & 17 & ND & \\
\hline SB05 & CNSB05-W-26685 & 9/9/08 & $32-42$ & 256 & 20 & ND & \\
\hline SB07R & CNSB07R-W-26073 & $1 / 9 / 08$ & $45-60$ & 30 & 1.8 & ND & No purge \\
\hline SB07R & CNSB07R-W-26111 & $1 / 24 / 08$ & $45-60$ & 19 & $0.9 \mathrm{~J}$ & ND & No purge \\
\hline SB07R & CNSB07R-W-25984 & 2/24/08 & $45-60$ & 24 & 1.4 & ND & \\
\hline SB07R & CNSB07R-W-26003 & 3/12/08 & $45-60$ & 13 & $0.9 \mathrm{~J}$ & ND & \\
\hline SB07R & CNSB07R-W-26048 & 4/24/08 & $45-60$ & 10 & $0.8 \mathrm{~J}$ & ND & \\
\hline SB07R & CNSB07R-W-26623 & $6 / 4 / 08$ & $45-60$ & 17 & 1.0 & ND & \\
\hline SB07R & CNSB07R-W-26642 & $7 / 7 / 08$ & $45-60$ & 15 & $0.9 \mathrm{~J}$ & ND & \\
\hline SB07R & CNSB07R-W-26661 & $8 / 6 / 08$ & $45-60$ & 15 & $0.9 \mathrm{~J}$ & ND & \\
\hline SB07R & CNSB07R-W-26686 & 9/9/08 & $45-60$ & 21 & $1.4 \mathrm{~J}$ & ND & \\
\hline SB08 & CNSB08-W-26070 & $1 / 9 / 08$ & $52-62$ & 32 & 1.5 & ND & No purge \\
\hline SB08 & CNSB08-W-26112 & $1 / 24 / 08$ & $52-62$ & 36 & 1.5 & ND & No purge \\
\hline SB08 & CNSB08-W-25978 & 2/24/08 & $52-62$ & 44 & 1.5 & ND & \\
\hline SB08 & CNSB08-W-26004 & $3 / 12 / 08$ & $52-62$ & 28 & 1.1 & ND & \\
\hline SB08 & CNSB08-W-26049 & 4/23/08 & $52-62$ & 23 & 1.2 & ND & \\
\hline SB08 & CNSB08-W-26624 & $6 / 4 / 08$ & $52-62$ & 24 & 1.4 & ND & \\
\hline SB08 & CNSB08-W-26643 & $7 / 7 / 08$ & $52-62$ & 17 & 1.0 & ND & \\
\hline SB08 & CNSB08-W-26662 & $8 / 6 / 08$ & $52-62$ & 20 & 1.1 & ND & \\
\hline SB08 & CNSB08-W-26687 & $9 / 8 / 08$ & $52-62$ & 22 & $1.2 \mathrm{~J}$ & ND & \\
\hline SB09 & CNSB09-W-26033 & $3 / 20 / 08$ & $32-42$ & ND & ND & ND & \\
\hline SB09 & CNSB09-W-26688 & 9/10/08 & $32-42$ & ND & ND & ND & \\
\hline
\end{tabular}

a ND, not detected at instrument detection limit of $0.1 \mu \mathrm{g} / \mathrm{L}$.

b Qualifier J indicates an estimated concentration below the purge-and-trap method quantitation limit of $1.0 \mu \mathrm{g} / \mathrm{L}$. 
TABLE S4.3 Post-injection monitoring: Results for geochemical and attenuation parameter analyses of groundwater samples collected within and adjacent to the pilot test area.

\begin{tabular}{|c|c|c|c|c|c|c|c|c|c|c|c|c|c|}
\hline \multirow[b]{2}{*}{ Location } & \multirow[b]{2}{*}{ Sample } & \multirow[b]{2}{*}{$\begin{array}{l}\text { Sample } \\
\text { Date }\end{array}$} & \multirow[b]{2}{*}{$\begin{array}{l}\text { Depth } \\
\text { (ft BGL) }\end{array}$} & \multicolumn{10}{|c|}{ Concentration (mg/L) } \\
\hline & & & & Alkalinity & Aluminum & Bromide & Calcium & Chloride & Iron & Magnesium & Manganese & $\begin{array}{c}\text { Nitrate } \\
\text { as N }\end{array}$ & Nitrite \\
\hline MW02 & CNMW02-W-26074 & $1 / 9 / 08$ & $49.5-59.5$ & - & - & 4.9 & - & - & - & - & - & _- & - \\
\hline MW02 & CNMW02-W-26099 & $2 / 23 / 08$ & $49.5-59.5$ & - & - & Int. $^{\mathrm{b}}$ & - & 32 & - & - & - & $5 U$ & - \\
\hline MW02 & CNMW02-W-26045 & $4 / 24 / 08$ & $49.5-59.5$ & - & - & 350 & - & - & - & - & - & - & - \\
\hline MW02 & CNMW02-W-26620 & $6 / 4 / 08$ & $49.5-59.5$ & - & - & 490 & - & 26 & - & - & - & $5 \mathrm{U}$ & - \\
\hline MW02 & CNMW02-W-26639 & $7 / 8 / 08$ & $49.5-59.5$ & - & $0.14 \mathrm{~J} \mathrm{~B}$ & Int. $^{\text {b }}$ & 170 & 17 & 190 & 46 & 14 & $1 \mathrm{U}$ & $1 \mathrm{U}$ \\
\hline MW02 & CNMW02-W-26658 & $8 / 6 / 08$ & $49.5-59.5$ & - & 0.54 & 250 & 870 & 14 & 1000 & 240 & 69 & $5 \mathrm{U}$ & $5 \mathrm{U}$ \\
\hline MW03 & CNMW03-W-26078 & $1 / 10 / 08$ & $50.5-60.5$ & - & - & 0.27 & - & - & - & - & - & _- & - \\
\hline MW03 & CNMW03-W-26115 & $2 / 23 / 08$ & $50.5-60.5$ & - & - & 0.11 & - & 20 & - & - & - & 6.8 & - \\
\hline MW03 & CNMW03-W-26046 & $4 / 23 / 08$ & $50.5-60.5$ & - & - & 0.27 & - & - & - & - & - & - & - \\
\hline MW03 & CNMW03-W-26659 & $8 / 6 / 08$ & $50.5-60.5$ & - & $0.2 \mathrm{U}$ & 0.27 & 80 & 22 & $0.1 \mathrm{U}$ & 29 & $0.010 \mathrm{U}$ & 7.9 & $1 U$ \\
\hline PMP1 & CNPMP1-W-26079 & $1 / 10 / 08$ & $50-60$ & - & - & 0.43 & - & - & - & - & - & _- & - \\
\hline PMP1 & CNPMP1-W-25985 & $2 / 24 / 08$ & $50-60$ & - & - & 0.32 & - & 16 & - & - & - & 0.51 & - \\
\hline PMP1 & CNPMP1-W-26050 & $4 / 24 / 08$ & $50-60$ & - & - & 0.36 & - & - & - & - & - & - & - \\
\hline PMP1 & CNPMP1-W-26663 & $8 / 6 / 08$ & $50-60$ & - & 0.35 & 0.52 & 66 & 14 & 0.54 & 25 & 0.75 & 1.4 & $0.1 \mathrm{U}$ \\
\hline PMP2 & CNPMP2-W-26081 & $1 / 10 / 08$ & $50-60$ & - & - & 1.4 & - & - & - & - & - & - & - \\
\hline PMP2 & CNPMP2-W-25986 & $2 / 24 / 08$ & $50-60$ & - & - & 1.1 & - & 20 & - & - & - & 0.12 & - \\
\hline PMP2 & CNPMP2-W-26051 & $4 / 24 / 08$ & $50-60$ & - & - & 1.2 & - & - & - & - & - & - & - \\
\hline PMP2 & CNPMP2-W-26664 & $8 / 6 / 08$ & $50-60$ & - & $0.2 \mathrm{U}$ & 0.84 & 94 & 15 & 0.27 & 29 & 2.0 & 0.12 & $1 U$ \\
\hline PMP3 & CNPMP3-W-26071 & $1 / 9 / 08$ & $50-60$ & - & - & 32 & - & - & - & - & - & - & - \\
\hline PMP3 & CNPMP3-W-25990 & $2 / 25 / 08$ & $50-60$ & - & - & 27 & - & 7.3 & - & - & - & 0.83 & - \\
\hline PMP3 & CNPMP3-W-26052 & $4 / 24 / 08$ & $50-60$ & - & - & 2.1 & - & - & - & - & - & - & - \\
\hline PMP3 & CNPMP3-W-26665 & $8 / 6 / 08$ & $50-60$ & - & $0.2 \mathrm{U}$ & 1.1 & 120 & 6.5 & 7.6 & 39 & 4.5 & 0.78 & 0.95 \\
\hline PMP4 & CNPMP4-W-26068 & $1 / 9 / 08$ & $48.75-58.75$ & - & - & 0.27 & - & - & - & - & - & - & - \\
\hline PMP4 & CNPMP4-W-25992 & $2 / 25 / 08$ & $48.75-58.75$ & - & - & 0.3 & - & 36 & - & - & - & 0.25 & - \\
\hline PMP4 & CNPMP4-W-26053 & $4 / 24 / 08$ & $48.75-58.75$ & - & - & 0.37 & - & - & - & - & - & - & - \\
\hline PMP4 & CNPMP4-W-26666 & $8 / 6 / 08$ & $48.75-58.75$ & - & $0.2 \mathrm{U}$ & 0.36 & 66 & 34 & $0.1 \cup$ & 23 & 0.21 & 0.73 & $1 U$ \\
\hline PMP5 & CNPMP5-W-26069 & $1 / 9 / 08$ & $50-60$ & - & - & 0.3 & - & - & - & - & - & - & - \\
\hline PMP5 & CNPMP5-W-25997 & $2 / 25 / 08$ & $50-60$ & - & - & 0.4 & - & 81 & - & - & - & 1.5 & - \\
\hline PMP5 & CNPMP5-W-26054 & $4 / 24 / 08$ & $50-60$ & - & - & 0.54 & - & - & - & - & - & - & - \\
\hline PMP5 & CNPMP5-W-26667 & $8 / 6 / 08$ & $50-60$ & - & $0.2 \mathrm{U}$ & 0.61 & 73 & 82 & 0.21 & 26 & 0.19 & 1.6 & $1 U$ \\
\hline PMP6 & CNPMP6-W-26065 & $1 / 8 / 08$ & $50-60$ & - & - & 0.28 & - & - & - & - & - & - & - \\
\hline PMP6 & CNPMP6-W-25993 & $2 / 25 / 08$ & $50-60$ & - & - & 0.17 & - & 36 & - & - & - & 6.7 & - \\
\hline PMP6 & CNPMP6-W-26055 & $4 / 24 / 08$ & $50-60$ & - & - & 0.32 & - & - & - & - & - & - & - \\
\hline PMP6 & CNPMP6-W-26668 & $8 / 6 / 08$ & $50-60$ & - & $0.2 \mathrm{U}$ & 0.28 & 75 & 28 & 0.11 & 28 & 0.17 & 8.2 & $1 U$ \\
\hline PMP7 & CNPMP7-W-26066 & $1 / 8 / 08$ & $50-60$ & - & - & 0.59 & - & - & - & - & - & _- & - \\
\hline PMP7 & CNPMP7-W-25996 & $2 / 25 / 08$ & $50-60$ & - & - & 0.49 & - & 20 & - & - & - & 6.1 & - \\
\hline PMP7 & CNPMP7-W-26056 & $4 / 24 / 08$ & $50-60$ & - & - & 0.52 & - & - & - & - & - & - & - \\
\hline PMP7 & CNPMP7-W-26669 & $8 / 6 / 08$ & $50-60$ & - & $0.2 \mathrm{U}$ & 0.55 & 70 & 16 & $0.1 \mathrm{U}$ & 25 & 0.12 & 6.9 & $0.1 \mathrm{U}$ \\
\hline
\end{tabular}


TABLE S4.3 (Cont.)

\begin{tabular}{|c|c|c|c|c|c|c|c|c|c|c|c|c|c|}
\hline \multirow[b]{2}{*}{ Location } & \multirow[b]{2}{*}{ Sample } & \multirow[b]{2}{*}{$\begin{array}{l}\text { Sample } \\
\text { Date }\end{array}$} & \multirow[b]{2}{*}{$\begin{array}{c}\text { Depth } \\
\text { (ft BGL) }\end{array}$} & \multicolumn{10}{|c|}{ Concentration (mg/L) } \\
\hline & & & & Alkalinity & Aluminum & Bromide & Calcium & Chloride & Iron & Magnesium & Manganese & $\begin{array}{c}\text { Nitrate } \\
\text { as N }\end{array}$ & Nitrite \\
\hline PMP8 & CNPMP8-W-26075 & $1 / 9 / 08$ & $50-60$ & - & - & 4.7 & - & - & - & _- & _- & _- & _- \\
\hline PMP8 & CNPMP8-W-25994 & $2 / 25 / 08$ & $50-60$ & - & - & 1.3 & - & 28 & - & - & - & 1.9 & - \\
\hline PMP8 & CNPMP8-W-26057 & $4 / 24 / 08$ & $50-60$ & - & - & 11 & - & - & - & - & - & - & - \\
\hline PMP8 & CNPMP8-W-26670 & $8 / 6 / 08$ & $50-60$ & - & $0.2 \mathrm{U}$ & 9.7 & 120 & 25 & 0.42 & 65 & 2.2 & $0.1 \mathrm{U}$ & $1 U$ \\
\hline PMP9 & CNPMP9-W-26077 & $1 / 10 / 08$ & $50-60$ & - & - & 0.14 & - & - & - & - & - & - & - \\
\hline PMP9 & CNPMP9-W-25989 & $2 / 24 / 08$ & $50-60$ & - & - & $0.1 \mathrm{U}$ & - & 11 & - & - & - & $<0.1$ & - \\
\hline PMP9 & CNPMP9-W-26058 & $4 / 24 / 08$ & $50-60$ & - & - & $0.1 \mathrm{U}$ & - & - & - & - & - & - & - \\
\hline PMP9 & CNPMP9-W-26671 & $8 / 6 / 08$ & $50-60$ & - & $0.2 \mathrm{U}$ & 0.13 & 60 & 8.7 & $0.1 \mathrm{U}$ & 24 & $0.010 \mathrm{U}$ & 0.26 & $0.1 \mathrm{U}$ \\
\hline SB04 & CNSB04-W-26076 & $1 / 10 / 08$ & $51-61$ & _- & _- & 0.26 & - & _- & - & _- & - & _- & - \\
\hline SB04 & CNSB04-W-26117 & $2 / 23 / 08$ & $51-61$ & _- & _- & $0.1 \mathrm{U}$ & _- & 41 & _- & - & - & 2 & - \\
\hline SB04 & CNSB04-W-26047 & $4 / 24 / 08$ & $51-61$ & _- & - & $0.1 \mathrm{U}$ & - & - & - & _- & - & - & - \\
\hline SB04 & CNSB04-W-26660 & $8 / 6 / 08$ & $51-61$ & - & $0.2 \mathrm{U}$ & $0.2 \mathrm{U}$ & 73 & 37 & $0.1 \mathrm{U}$ & 27 & $0.010 \mathrm{U}$ & 1.5 & $1 \mathrm{U}$ \\
\hline SB07R & CNSB07R-W-26073 & $1 / 9 / 08$ & $45-60$ & _- & _- & $0.1 \mathrm{U}$ & _- & _- & _- & _- & _- & _- & - \\
\hline SB07R & CNSB07R-W-25984 & $2 / 24 / 08$ & $45-60$ & _- & - & $0.1 \mathrm{U}$ & - & 20 & _- & _- & - & 0.66 & _- \\
\hline SB07R & CNSB07R-W-26048 & $4 / 24 / 08$ & $45-60$ & - & - & $0.1 \mathrm{U}$ & - & - & - & - & - & - & - \\
\hline SB07R & CNSB07R-W-26661 & $8 / 6 / 08$ & $45-60$ & - & $0.2 \mathrm{U}$ & $0.2 \mathrm{U}$ & 61 & 16 & $0.1 \mathrm{U}$ & 23 & $0.010 \mathrm{U}$ & 0.53 & $0.1 \mathrm{U}$ \\
\hline SB08 & CNSB08-W-26070 & $1 / 9 / 08$ & $52-62$ & _- & _- & $0.1 \mathrm{U}$ & _- & _- & _- & _- & _- & _- & _- \\
\hline SB08 & CNSB08-W-25978 & $2 / 24 / 08$ & $52-62$ & - & _- & $0.1 \mathrm{U}$ & - & 15 & - & - & - & 0.85 & - \\
\hline SB08 & CNSB08-W-26049 & $4 / 23 / 08$ & $52-62$ & - & - & $0.1 \mathrm{U}$ & - & - & - & - & - & - & - \\
\hline SB08 & CNSB08-W-26662 & $8 / 6 / 08$ & $52-62$ & - & $0.2 \mathrm{U}$ & $0.2 \mathrm{U}$ & 61 & 13 & $0.1 \mathrm{U}$ & 24 & $0.010 \mathrm{U}$ & 0.76 & $0.1 \mathrm{U}$ \\
\hline
\end{tabular}

\begin{tabular}{|c|c|c|c|c|c|c|c|c|c|c|c|c|}
\hline \multirow[b]{2}{*}{ Location } & \multirow[b]{2}{*}{ Sample } & \multirow{2}{*}{$\begin{array}{l}\text { Sample } \\
\text { Date }\end{array}$} & \multirow{2}{*}{$\begin{array}{l}\text { Depth } \\
\text { (ft BGL) }\end{array}$} & \multicolumn{8}{|c|}{ Concentration $^{\mathrm{a}}(\mathrm{mg} / \mathrm{L})$} & \multirow{2}{*}{$\begin{array}{c}\text { Methane } \\
(\mu \mathrm{g} / \mathrm{l})\end{array}$} \\
\hline & & & & Phosphate & Potassium & Silicon & Sodium & Sulfate & Sulfide & Zinc & TOC $^{\mathrm{C}}$ & \\
\hline MW02 & CNMW02-W-26074 & $1 / 9 / 08$ & $49.5-59.5$ & - & - & - & - & - & - & - & - & - \\
\hline MW02 & CNMW02-W-26099 & $2 / 23 / 08$ & $49.5-59.5$ & $10 U$ & - & - & - & Int. & - & - & - & - \\
\hline MW02 & CNMW02-W-26045 & $4 / 24 / 08$ & $49.5-59.5$ & - & - & - & - & - & - & - & - & - \\
\hline MW02 & CNMW02-W-26620 & $6 / 4 / 08$ & $49.5-59.5$ & $10 \mathrm{U}$ & - & - & - & 23 & - & - & - & - \\
\hline MW02 & CNMW02-W-26639 & $7 / 8 / 08$ & $49.5-59.5$ & $1.4 \mathrm{~J}$ & 11 & 3.3 & 41 & Int. & - & 0.031 & - & - \\
\hline MW02 & CNMW02-W-26658 & $8 / 6 / 08$ & $49.5-59.5$ & $10 \mathrm{U}$ & 68 & 22 & 220 & 25 & - & 0.26 & - & - \\
\hline MW03 & CNMW03-W-26078 & $1 / 10 / 08$ & $50.5-60.5$ & - & - & - & - & - & - & - & - & - \\
\hline MW03 & CNMW03-W-26115 & $2 / 23 / 08$ & $50.5-60.5$ & $0.2 \mathrm{U}$ & - & - & - & 7.3 & - & - & - & - \\
\hline MW03 & CNMW03-W-26046 & $4 / 23 / 08$ & $50.5-60.5$ & - & - & - & - & - & - & - & - & - \\
\hline MW03 & CNMW03-W-26659 & $8 / 6 / 08$ & $50.5-60.5$ & $0.2 \mathrm{U}$ & 1.7 & 16 & 41 & 7.7 & - & $0.02 \mathrm{U}$ & - & - \\
\hline PMP1 & CNPMP1-W-26079 & $1 / 10 / 08$ & $50-60$ & - & - & - & - & - & - & - & - & - \\
\hline PMP1 & CNPMP1-W-25985 & $2 / 24 / 08$ & $50-60$ & $0.2 \mathrm{U}$ & - & - & - & 8.9 & - & - & - & - \\
\hline PMP1 & CNPMP1-W-26050 & $4 / 24 / 08$ & $50-60$ & - & - & - & - & - & - & - & - & - \\
\hline PMP1 & CNPMP1-W-26663 & $8 / 6 / 08$ & $50-60$ & $0.2 \mathrm{U}$ & 2.6 & 13 & 32 & 7.3 & - & $0.02 \mathrm{U}$ & - & - \\
\hline
\end{tabular}


TABLE S4.3 (Cont.)

\begin{tabular}{|c|c|c|c|c|c|c|c|c|c|c|c|c|}
\hline \multirow[b]{2}{*}{ Location } & \multirow[b]{2}{*}{ Sample } & \multirow[b]{2}{*}{$\begin{array}{l}\text { Sample } \\
\text { Date }\end{array}$} & \multirow[b]{2}{*}{$\begin{array}{l}\text { Depth } \\
\text { (ft BGL) }\end{array}$} & \multicolumn{8}{|c|}{ Concentration $^{\mathrm{a}}(\mathrm{mg} / \mathrm{L})$} & \multirow[b]{2}{*}{$\begin{array}{c}\text { Methane } \\
(\mu \mathrm{g} / \mathrm{l})\end{array}$} \\
\hline & & & & Phosphate & Potassium & Silicon & Sodium & Sulfate & Sulfide & Zinc & TOC $^{\mathrm{C}}$ & \\
\hline PMP2 & CNPMP2-W-26081 & $1 / 10 / 08$ & $50-60$ & - & - & - & - & - & - & - & - & - \\
\hline PMP2 & CNPMP2-W-25986 & $2 / 24 / 08$ & $50-60$ & $0.2 \mathrm{U}$ & - & - & - & 4.0 & - & - & - & - \\
\hline PMP2 & CNPMP2-W-26051 & $4 / 24 / 08$ & $50-60$ & - & - & - & - & - & - & - & - & - \\
\hline PMP2 & CNPMP2-W-26664 & $8 / 6 / 08$ & $50-60$ & $0.2 \mathrm{U}$ & 3.7 & 14 & 44 & 6.9 & - & $0.02 \mathrm{U}$ & - & - \\
\hline PMP3 & CNPMP3-W-26071 & $1 / 9 / 08$ & $50-60$ & - & - & - & - & - & - & - & - & - \\
\hline PMP3 & CNPMP3-W-25990 & $2 / 25 / 08$ & $50-60$ & $0.2 \mathrm{U}$ & - & - & - & 11 & - & - & - & - \\
\hline PMP3 & CNPMP3-W-26052 & $4 / 24 / 08$ & $50-60$ & - & - & - & - & - & - & - & - & - \\
\hline PMP3 & CNPMP3-W-26665 & $8 / 6 / 08$ & $50-60$ & $2 U$ & 14 & 12 & 45 & 8.8 & - & $0.02 \mathrm{U}$ & - & - \\
\hline PMP4 & CNPMP4-W-26068 & $1 / 9 / 08$ & $48.75-58.75$ & - & - & - & - & - & - & - & - & - \\
\hline PMP4 & CNPMP4-W-25992 & $2 / 25 / 08$ & $48.75-58.75$ & $0.2 \mathrm{U}$ & - & - & - & 7.7 & - & - & - & - \\
\hline PMP4 & CNPMP4-W-26053 & $4 / 24 / 08$ & $48.75-58.75$ & - & - & - & - & - & - & - & - & - \\
\hline PMP4 & CNPMP4-W-26666 & $8 / 6 / 08$ & $48.75-58.75$ & $0.2 \mathrm{U}$ & 2.0 & 13 & 35 & 7.2 & - & $0.02 U$ & - & - \\
\hline PMP5 & CNPMP5-W-26069 & $1 / 9 / 08$ & $50-60$ & - & - & - & - & - & - & - & - & - \\
\hline PMP5 & CNPMP5-W-25997 & $2 / 25 / 08$ & $50-60$ & $0.2 \mathrm{U}$ & - & - & - & 8.7 & - & - & - & - \\
\hline PMP5 & CNPMP5-W-26054 & $4 / 24 / 08$ & $50-60$ & - & - & - & - & - & - & - & - & - \\
\hline PMP5 & CNPMP5-W-26667 & 8/6/08 & $50-60$ & $0.2 \mathrm{U}$ & 3.1 & 14 & 68 & 9.2 & - & $0.02 \mathrm{U}$ & - & - \\
\hline PMP6 & CNPMP6-W-26065 & $1 / 8 / 08$ & $50-60$ & - & - & - & - & - & - & - & - & - \\
\hline PMP6 & CNPMP6-W-25993 & $2 / 25 / 08$ & $50-60$ & $0.2 \mathrm{U}$ & - & - & - & 12 & - & - & - & - \\
\hline PMP6 & CNPMP6-W-26055 & $4 / 24 / 08$ & $50-60$ & - & - & - & - & - & - & - & - & - \\
\hline PMP6 & CNPMP6-W-26668 & 8/6/08 & $50-60$ & $0.2 \mathrm{U}$ & 2.4 & 14 & 35 & 14 & - & $0.02 \mathrm{U}$ & - & - \\
\hline PMP7 & CNPMP7-W-26066 & $1 / 8 / 08$ & $50-60$ & - & - & - & - & - & - & - & - & - \\
\hline PMP7 & CNPMP7-W-25996 & $2 / 25 / 08$ & $50-60$ & $0.2 \mathrm{U}$ & - & - & - & 14 & - & - & - & - \\
\hline PMP7 & CNPMP7-W-26056 & $4 / 24 / 08$ & $50-60$ & - & - & - & - & - & - & - & - & - \\
\hline PMP7 & CNPMP7-W-26669 & 8/6/08 & $50-60$ & $0.2 \mathrm{U}$ & 2.5 & 14 & 66 & 15 & - & $0.02 \mathrm{U}$ & - & - \\
\hline PMP8 & CNPMP8-W-26075 & $1 / 9 / 08$ & $50-60$ & - & - & - & - & - & - & - & - & - \\
\hline PMP8 & CNPMP8-W-25994 & $2 / 25 / 08$ & $50-60$ & $<0.2$ & - & - & - & 28 & - & - & - & - \\
\hline PMP8 & CNPMP8-W-26057 & $4 / 24 / 08$ & $50-60$ & - & - & - & - & - & - & - & - & - \\
\hline PMP8 & CNPMP8-W-26670 & $8 / 6 / 08$ & $50-60$ & $0.2 \mathrm{U}$ & 2.9 & 12 & 62 & 2.7 & - & $0.02 \mathrm{U}$ & - & - \\
\hline PMP9 & CNPMP9-W-26077 & 1/10/08 & $50-60$ & - & - & - & - & - & - & - & - & - \\
\hline PMP9 & CNPMP9-W-25989 & $2 / 24 / 08$ & $50-60$ & $0.2 \mathrm{U}$ & - & - & - & 2.0 & - & - & - & - \\
\hline PMP9 & CNPMP9-W-26058 & $4 / 24 / 08$ & $50-60$ & - & - & - & - & - & - & - & - & - \\
\hline PMP9 & CNPMP9-W-26671 & 8/6/08 & $50-60$ & $0.2 \mathrm{U}$ & 1.8 & 14 & 25 & 1.7 & - & $0.02 \mathrm{U}$ & - & - \\
\hline SB04 & CNSB04-W-26076 & $1 / 10 / 08$ & $51-61$ & - & - & - & - & - & - & - & - & - \\
\hline SB04 & CNSB04-W-26117 & 2/23/08 & $51-61$ & $0.2 \mathrm{U}$ & - & - & - & 7.1 & - & - & - & - \\
\hline SB04 & CNSB04-W-26047 & $4 / 24 / 08$ & $51-61$ & - & - & - & - & - & - & - & - & - \\
\hline SB04 & CNSB04-W-26660 & 8/6/08 & $51-61$ & $0.2 \mathrm{U}$ & 2.0 & 14 & 46 & 6.0 & - & $0.02 \mathrm{U}$ & - & - \\
\hline SB07R & CNSB07R-W-26073 & $1 / 9 / 08$ & $45-60$ & - & - & - & - & - & - & - & - & - \\
\hline SB07R & CNSB07R-W-25984 & $2 / 24 / 08$ & $45-60$ & $0.2 \mathrm{U}$ & - & - & - & 6.3 & - & - & - & - \\
\hline SB07R & CNSB07R-W-26048 & $4 / 24 / 08$ & $45-60$ & - & - & - & - & - & - & - & - & - \\
\hline SB07R & CNSB07R-W-26661 & $8 / 6 / 08$ & $45-60$ & $0.2 \mathrm{U}$ & 1.7 & 13 & 27 & 3.5 & - & $0.02 \mathrm{U}$ & - & - \\
\hline
\end{tabular}


TABLE S4.3 (Cont.)

\begin{tabular}{|c|c|c|c|c|c|c|c|c|c|c|c|c|}
\hline \multirow[b]{2}{*}{ Location } & \multirow[b]{2}{*}{ Sample } & \multirow[b]{2}{*}{$\begin{array}{l}\text { Sample } \\
\text { Date }\end{array}$} & \multirow[b]{2}{*}{$\begin{array}{c}\text { Depth } \\
\text { (ft BGL) }\end{array}$} & \multicolumn{8}{|c|}{ Concentration $^{\mathrm{a}}(\mathrm{mg} / \mathrm{L})$} & \multirow[b]{2}{*}{$\begin{array}{c}\text { Methane } \\
(\mu \mathrm{g} / \mathrm{l})\end{array}$} \\
\hline & & & & Phosphate & Potassium & Silicon & Sodium & Sulfate & Sulfide & Zinc & TOC $^{\mathrm{C}}$ & \\
\hline SB08 & CNSB08-W-26070 & $1 / 9 / 08$ & $52-62$ & - & - & - & - & - & - & - & - & - \\
\hline SB08 & CNSB08-W-25978 & $2 / 24 / 08$ & $52-62$ & $0.2 \mathrm{U}$ & - & - & - & 6.8 & - & - & - & - \\
\hline SB08 & CNSB08-W-26049 & $4 / 23 / 08$ & $52-62$ & - & - & - & - & - & - & - & - & - \\
\hline SB08 & CNSB08-W-26662 & 8/6/08 & $52-62$ & $0.2 \mathrm{U}$ & 1.8 & 14 & 21 & 6.1 & - & $0.02 \mathrm{U}$ & - & - \\
\hline
\end{tabular}

a Qualifiers:

B, constituent detected in the associated blank.

$\mathrm{U}$, constituent not detected at the indicated reporting limit.

b Interfering peaks in the analysis prevented quantitation of the concentration.

c TOC, total organic carbon. 
TABLE S4.4 Results of purge-and-trap organic analyses at the AGEM Laboratory for soil samples collected at PSB13 during the August 2008 post-injection sampling event.

\begin{tabular}{|c|c|c|c|c|c|c|}
\hline \multirow[b]{2}{*}{ Location } & \multirow[b]{2}{*}{ Sample } & \multirow[b]{2}{*}{$\begin{array}{l}\text { Sample } \\
\text { Date }\end{array}$} & \multirow[b]{2}{*}{$\begin{array}{l}\text { Depth } \\
\text { (ft BGL) }\end{array}$} & \multicolumn{3}{|c|}{ Concentration $(\mu \mathrm{g} / \mathrm{kg})$} \\
\hline & & & & $\begin{array}{c}\text { Carbon } \\
\text { Tetrachloride }\end{array}$ & Chloroform & $\begin{array}{c}\text { Methylene } \\
\text { Chloride }\end{array}$ \\
\hline \multirow[t]{20}{*}{ PSB13 } & CNPSB13-S-27120 & $8 / 21 / 08$ & 2 & $N D^{a}$ & ND & ND \\
\hline & CNPSB13-S-27121 & $8 / 21 / 08$ & 4 & ND & ND & ND \\
\hline & CNPSB13-S-27122 & $8 / 21 / 08$ & 6 & ND & ND & ND \\
\hline & CNPSB13-S-27123 & 8/21/08 & 8 & ND & ND & ND \\
\hline & CNPSB13-S-27124 & $8 / 21 / 08$ & 10 & ND & $5.1 \mathrm{~J}^{\mathrm{b}}$ & ND \\
\hline & CNPSB13-S-27125 & 8/21/08 & 12 & ND & $3 \mathrm{~J}$ & ND \\
\hline & CNPSB13-S-27126 & $8 / 21 / 08$ & 14 & ND & 13 & ND \\
\hline & CNPSB13-S-27127 & $8 / 21 / 08$ & 16 & $6.5 \mathrm{~J}$ & $8.3 \mathrm{~J}$ & ND \\
\hline & CNPSB13-S-27128 & $8 / 21 / 08$ & 18 & ND & $3.4 \mathrm{~J}$ & 11 \\
\hline & CNPSB13-S-27129 & $8 / 21 / 08$ & 20 & ND & 13 & ND \\
\hline & CNPSB13-S-27130 & 8/21/08 & 22 & $6.2 \mathrm{~J}$ & $6.9 \mathrm{~J}$ & ND \\
\hline & CNPSB13-S-27131 & $8 / 21 / 08$ & 24 & ND & $6.7 \mathrm{~J}$ & ND \\
\hline & CNPSB13-S-27132 & $8 / 21 / 08$ & 26 & $3 \mathrm{~J}$ & 16 & ND \\
\hline & CNPSB13-S-27133 & 8/21/08 & 28 & $4 \mathrm{~J}$ & 15 & ND \\
\hline & CNPSB13-S-27134 & $8 / 21 / 08$ & 30 & ND & 13 & ND \\
\hline & CNPSB13-S-27135 & 8/21/08 & 32 & ND & 17 & ND \\
\hline & CNPSB13-S-27136 & $8 / 21 / 08$ & 34 & 46 & $4.8 \mathrm{~J}$ & ND \\
\hline & CNPSB13-S-27137 & $8 / 21 / 08$ & 36 & 61 & $9.6 \mathrm{~J}$ & ND \\
\hline & CNPSB13-S-27138 & $8 / 21 / 08$ & 38 & 32 & $7 \mathrm{~J}$ & ND \\
\hline & CNPSB13-S-27139 & $8 / 21 / 08$ & 40 & 10 & $3.6 \mathrm{~J}$ & ND \\
\hline
\end{tabular}

a ND, not detected at an instrument detection limit of $1 \mu \mathrm{g} / \mathrm{kg}$.

b Qualifier $\mathrm{J}$ indicates an estimated concentration below the purge-and-trap method quantitation limit of $10 \mu \mathrm{g} / \mathrm{kg}$. 


\section{Supplement 5:}

Outside Laboratory Data for Verification Organic Analyses 


\section{Contents}

Envirosystems Report R070315 …………………............................................ 3 of 194

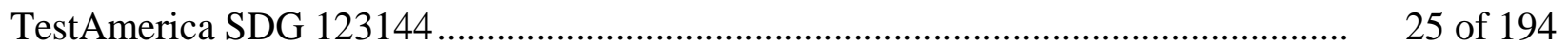

Envirosystems Report R070318 ……………………............................................ 44 of 194

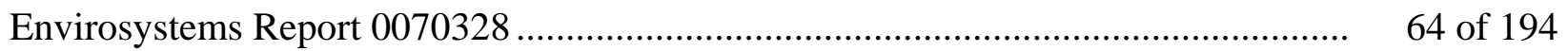

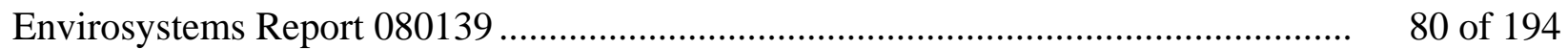

Envirosystems Report 080158 ............................................................................. 95 of 194

Envirosystems Report 080163 ................................................................................. 113 of 194

Envirosystems Report 080190 ............................................................................... 126 of 194

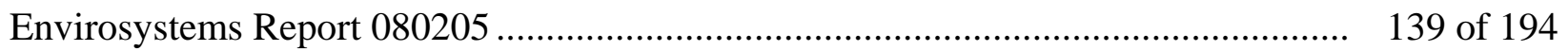

TestAmerica SDG 126456................................................................................... 151 of 194

TestAmerica SDG 126998_............................................................................. 165 of 194

TestAmerica SDG 127561 .................................................................................... 179 of 194 
January 16,2007

Jorge S. Alvarado, PH. D

Argonne National Laboratory

Environmental Research Division

Applied Geosciences and Environmental

Management Section

9700 South Cass Avenue, ER-203

Argonne, Illinois 60439

RE: Report \#070315

Dear Jorge,

Enclosed is the Analytical Data Package for Organics Analysis for the samples received on November 15,2007. These samples were analyzed by using method SW-846 $8260 \mathrm{~B}$ and USEPA CLP SOWOLM04.3 and the chain of custody instructions.

Please do not hesitate to call if you have any questions, comments, or require additional information.

Sincerely,

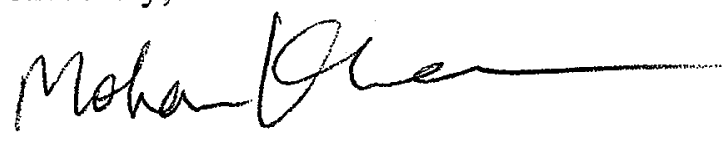

Mohan Khare Ph.D.

President/CEO

Enclosure (1)

$\mathrm{MK} / \mathrm{ncc}$ 
1. Narrative 


\section{SDG NARRATIVE \\ VOLATILE ORGANICS (VOC)}

Envirosystems, Inc.

Contract: N/A

Client: Argonne National Laboratory

Case: N/A

SDG: ARG71104, ARG71105

\section{SAMPLE RECIEPT}

Date received: 11-15-2007

Cooler Temperature: 2

\section{Sample Summary}

\begin{tabular}{|c|c|c|c|}
\hline Client ID & Laboratory ID & Matrix & $\mathbf{p H}$ \\
\hline CNPSB1-W-16246 & $0071104-01$ & WATER & 7 \\
\hline CNPSB1-W-16246DL & $0071104-01 D L$ & WATER & 7 \\
\hline CNQCTB-W-19961 & $0071104-02$ & WATER & 7 \\
\hline CNPSB3-W-16250 & $0071104-03$ & WATER & 7 \\
\hline CNPSB3-W-16250DL & $0071104-03$ DL & WATER & 7 \\
\hline CNPSB6-W-16254 & $0071104-04$ & WATER & 7 \\
\hline CNPUBL-W-26062 & $0071105-01$ & WATER & 7 \\
\hline
\end{tabular}

\section{HOLDING TIMES}

A. Sample Preparation: All holding times were met.

B. Sample Analysis: All holding times were met

\section{METHODS}

The samples were analyzed and reported by using method SW-846 8260B and USEPA CLP SOW OLM04.3 for target compound list.

\section{INSTRUMENT AND CHROMATOGRAPHIC CONDITIONS}

A Hewlett Packard 6890 gas chromatograph equipped with a Hewlett Packard 5975 MSD was used for sample analysis. The capillary column used was a Restek $20 \mathrm{~m}$ by $0.18 \mathrm{~mm}$ ID by $1.0 \mu \mathrm{m}$ film thickness (Restek Cat. \# RTX-624). The trap used with the sample concentrator is an OI Analytical Trap \#10,30cm packed with Tenax/silica gel/cms (PN\#228122).

\section{PREPARATION}

The submitted samples were analyzed as received.

6. ANALYSIS

A. Calibration:

I. Initial calibration 


\section{SDG NARRATIVE VOLATILE ORGANICS (VOC)}

All acceptance criteria as stipulated by SW- $8468260 \mathrm{~b}$ were met for all SPCC's and CCC's. All target compounds met the required percent RSD.

\section{Blanks:}

All acceptance criteria were met.

\section{Surrogates:}

All acceptance criteria were met.

B. Spikes:

\section{Laboratory Control Spikes (LCS)}

LCS and LCSD samples were analyzed.

II. Matrix Spike/Matrix Spike Duplicate (MS/MSD)

The client did not request a MS/MSD.

C. Internal Standards:

All acceptance criteria were met.

\section{Samples}

Sample analysis proceeded normally.

I certify that this Sample Data Package is in compliance with the terms and conditions of the contract, both technically and for completeness, for other than the conditions detailed above. Release of the data contained in the hard copy Sample Data Package and in the Electronic Data Deliverables has been authorized by the laboratory manager or the manager's designee, as verified by the following signatures.

Laboratory Manager

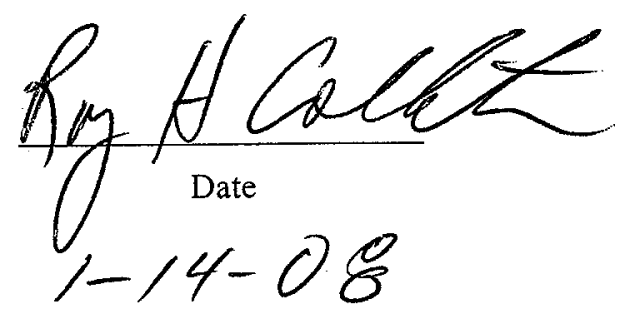


2. SGD Cover Sheet/Traffic Reports 


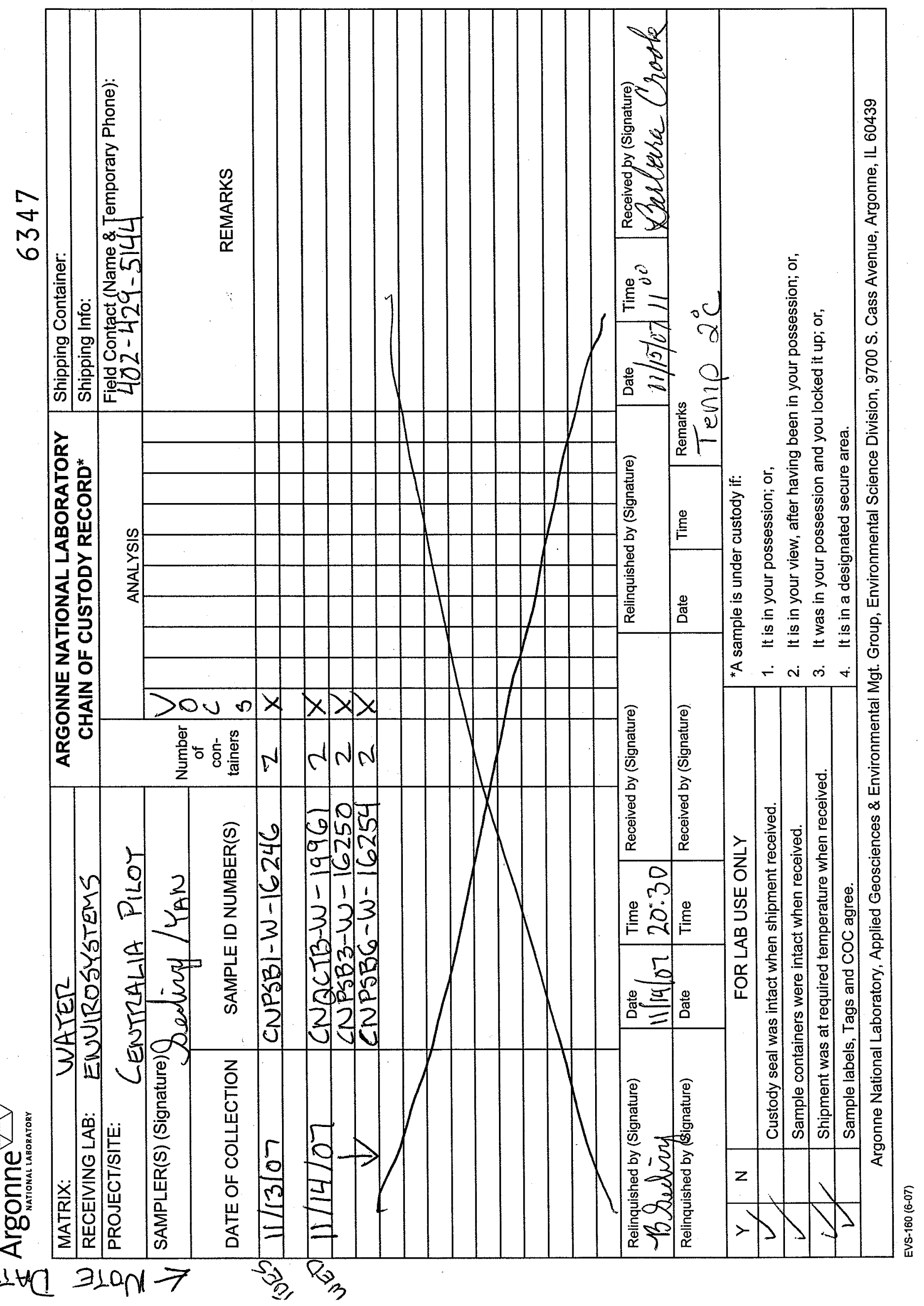




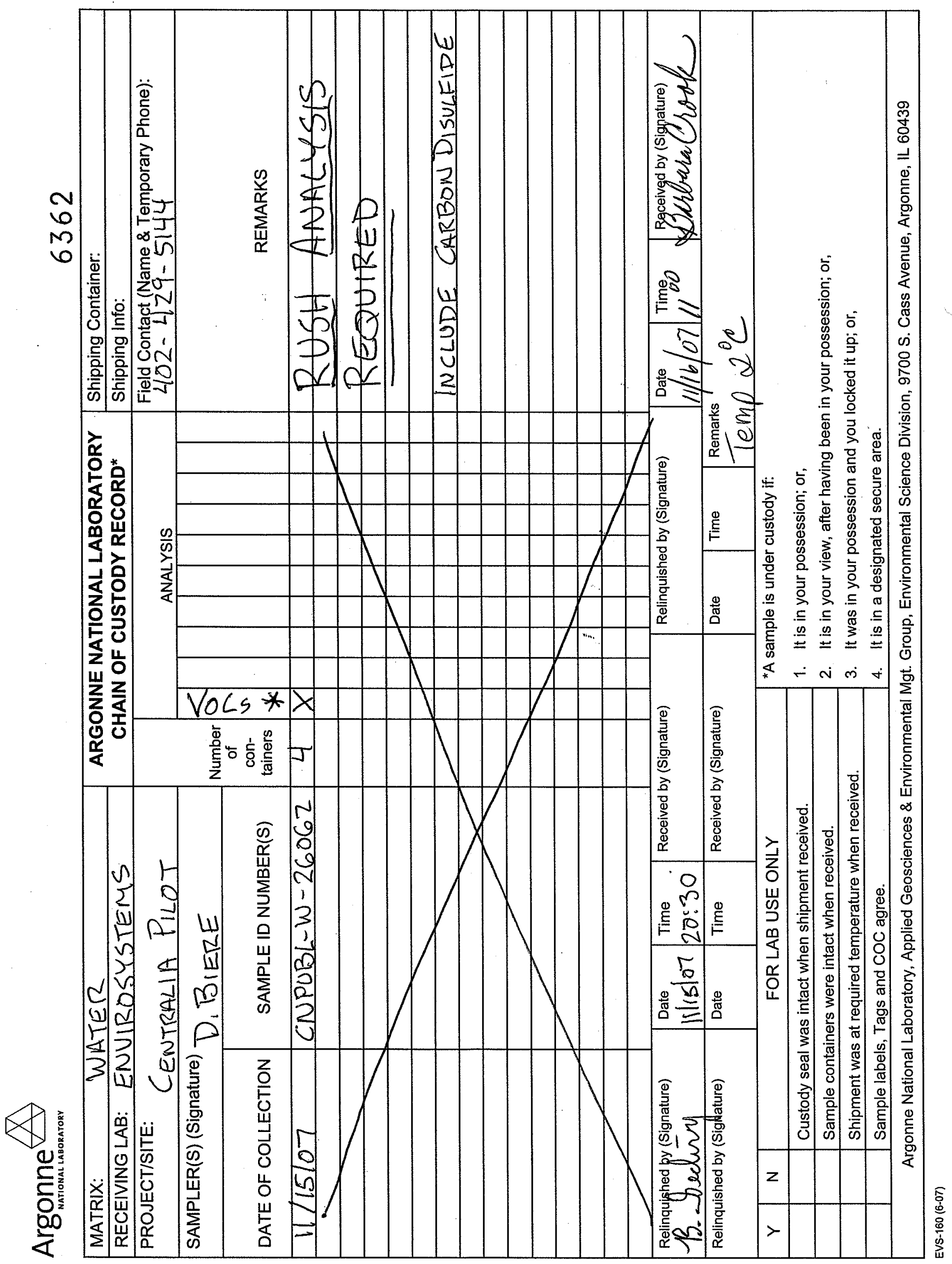


VOLATILE SAMPLE DATA 
$1 A$ - EORM I VOA-1

VOLATILE ORGANICS ANALYSIS DATA SHEET
EPA SAMPLE NO.

CNPSB1-W-16246
Lab Name: Envirosystems, Inc.

Lab Code: ENVSYS Case No.:

Matrix: (SOIL/SED/WATER)

WATER

Sample wt/vol: $5.00(\mathrm{~g} / \mathrm{mL}) \quad \mathrm{ML}$

Level: (TRACE/LOW/MED)

LOW

\% Moisture: not dec.

GC Column: $\quad$ RTX-624 ID: 0.18 (mm)

Soil Extract Volume:

Purge Volume:

5.00 (mL)

Contract:

Mod. Ref No.:

SDG No.: $\quad$ ARG71104

Lab Sample ID:

0071104-01

Lab File ID:

H73FA237.D

Date Received:

Date Analyzed:

$11 / 15 / 2007$

Dilution Factor:

$11 / 16 / 2007$

Soil Aliquot volume: (UL)

\begin{tabular}{|c|c|c|c|}
\hline CAS NO. & COMPOUND & $\begin{array}{l}\text { CONCENTRATION UNITS: } \\
(\mathrm{ug} / \mathrm{L} \text { or } \mathrm{ug} / \mathrm{kg}) \mathrm{UG} / \mathrm{L}\end{array}$ & $Q$ \\
\hline $75-71-8$ & Dichlorodifluoromethane & 5.0 & U \\
\hline $74-87-3$ & Chloromethane & 5.0 & $\mathrm{U}$ \\
\hline $75-01-4$ & Vinyl chloride & 5.0 & $\mathrm{U}$ \\
\hline $74-83-9$ & Bromomethane & 5.0 & $\mathrm{U}$ \\
\hline $75-00-3$ & Chloroethane & 5.0 & $\mathrm{U}$ \\
\hline $75-69-4$ & Trichlorofluoromethane & 5.0 & U \\
\hline $75-35-4$ & 1,1-Dichloroethene & 5.0 & $\mathrm{U}$ \\
\hline $76-13-1$ & $1,1,2$-Trichloro-1,2,2-trifluoroethane & 5.0 & $\mathrm{U}$ \\
\hline $67-64-1$ & Acetone & 5.0 & $\mathrm{U}$ \\
\hline $75-15-0$ & Carbon disulfide & 5.0 & $\mathrm{U}$ \\
\hline $79-20-9$ & Methyl acetate & 5.0 & $\mathrm{U}$ \\
\hline $75-09-2$ & Methylene chloride & 5.0 & $\mathrm{U}$ \\
\hline $156-60-5$ & trans-1,2-Dichloroethene & 5.0 & $\mathrm{U}$ \\
\hline $1634-04-4$ & Methyl tert-butyl ether & 5.0 & U \\
\hline $75-34-3$ & 1,1-Dichloroethane & 5.0 & U \\
\hline $156-59-2$ & cis-1,2-Dichloroethene & 5.0 & $\mathrm{U}$ \\
\hline $78-93-3$ & 2-Butanone & 5.0 & U \\
\hline $67-66-3$ & Chloroform & 29 & \\
\hline $71-55-6$ & $1,1,1$-Trichloroethane & 5.0 & U \\
\hline $110-82-7$ & Cyclohexane & 5.0 & $\mathrm{U}$ \\
\hline $56-23-5$ & Carbon Tetrachloride & 460 & $E$ \\
\hline $71-43-2$ & Benzene & 5.0 & $\mathrm{U}$ \\
\hline $107-06-2$ & 1,2-Dichloroethane & 5.0 & $\mathrm{U}$ \\
\hline
\end{tabular}


$1 B$ - EORM I VOA-2

VOLATILE ORGANICS ANALYSIS DATA SHEET
EPA SAMPLE NO.

CNPSB $1-W-16246$
Lab Name:

Envirosystems, Inc.

Lab Code: ENVSYS Case No.:

Matrix: (SOIL/SED/WATER) WATER

Sample wt/vol: $5.00(\mathrm{~g} / \mathrm{mL})$. ML

Level: (TRACE/LOW/MED)

LOW

\% Moisture: not dec.

GC Column: $\quad$ RTX-624 ID: 0.18 (mm)

Soil Extract Volume:

Purge Volume:

5.00

(uI)

$(\mathrm{mL})$

Contract :

Mod. Ref No.:
Lab Sample ID:

Lab File ID:

Date Received:

Date Analyzed:

Dilution Factor:

Soil Aliquot Volume:
SDG No.: $\quad$ ARG71104

0071104-01

H73FA237.D

$11 / 15 / 2007$

$11 / 16 / 2007$

1.0

\begin{tabular}{|c|c|c|c|}
\hline CAS NO. & COMPOUND & $\begin{array}{l}\text { CONCENTRATION UNITS: } \\
(\mathrm{ug} / \mathrm{L} \text { or } \mathrm{ug} / \mathrm{kg}) \mathrm{UG} / \mathrm{L}\end{array}$ & $Q$ \\
\hline $79-01-6$ & Trichloroethene & 5.0 & $\mathrm{U}$ \\
\hline $108-87-2$ & Methylcyclohexane & 5.0 & $\mathrm{U}$ \\
\hline $78-87-5$ & 1,2-Dichloropropane & 5.0 & $\mathrm{U}$ \\
\hline $75-27-4$ & Bromodichloromethane & 5.0 & $\mathrm{U}$ \\
\hline $10061-01-5$ & cis-1,3-Dichloropropene & 5.0 & U \\
\hline $108-10-1$ & 4-Methyl-2-pentanone & 5.0 & U \\
\hline $108-88-3$ & Toluene & 5.0 & $\mathrm{U}$ \\
\hline $10061-02-6$ & trans-1,3-Dichloropropene & 5.0 & $\mathrm{U}$ \\
\hline $79-00-5$ & $1,1,2$-Trichloroethane & 5.0 & $\mathrm{U}$ \\
\hline $127-18-4$ & Tetrachloroethene & 5.0 & U \\
\hline $591-78-6$ & 2-Hexanone & 5.0 & $\mathrm{U}$ \\
\hline $124-48-1$ & Dibromochloromethane & 5.0 & $\mathrm{U}$ \\
\hline $106-93-4$ & 1,2-Dibromoethane & 5.0 & $\mathrm{U}$ \\
\hline $108-90-7$ & Chlorobenzene & 5.0 & $\mathrm{U}$ \\
\hline $100-41-4$ & Ethylbenzene & 5.0 & $\mathrm{U}$ \\
\hline $95-47-6$ & o-Xylene & 5.0 & $\mathrm{U}$ \\
\hline $179601-23-1$ & $\mathrm{~m}, \mathrm{p}$-XYlene & 5.0 & $\mathrm{U}$ \\
\hline $100-42-5$ & Styrene & 5.0 & $\mathrm{U}$ \\
\hline $75-25-2$ & Bromoform & 5.0 & $\mathrm{U}$ \\
\hline $98-82-8$ & Isopropylbenzene & 5.0 & $\mathrm{U}$ \\
\hline $79-34-5$ & $1,1,2,2$-Tetrachloroethane & 5.0 & $\mathrm{U}$ \\
\hline $541-73-1$ & 1,3-Dichlorobenzene & 5.0 & $\mathrm{U}$ \\
\hline $106-46-7$ & 1,4-Dichlorobenzene & 5.0 & $\mathrm{U}$ \\
\hline $95-50-1$ & 1,2-Dichlorobenzene & 5.0 & $\mathrm{U}$ \\
\hline $96-12-8$ & 1,2-Dibromo-3-chloropropane & 5.0 & $\mathrm{U}$ \\
\hline $120-82-1$ & $1,2,4$-Trichlorobenzene & 5.0 & $\mathrm{U}$ \\
\hline $91-20-3$ & Naphthalene & 5.0 & U \\
\hline
\end{tabular}


$1 A$ - FORM I VOA-1

VOLATILE ORGANICS ANALYSIS DATA SHEET
EPA SAMPLE NO.

CNPSB 1 -W-16246DI

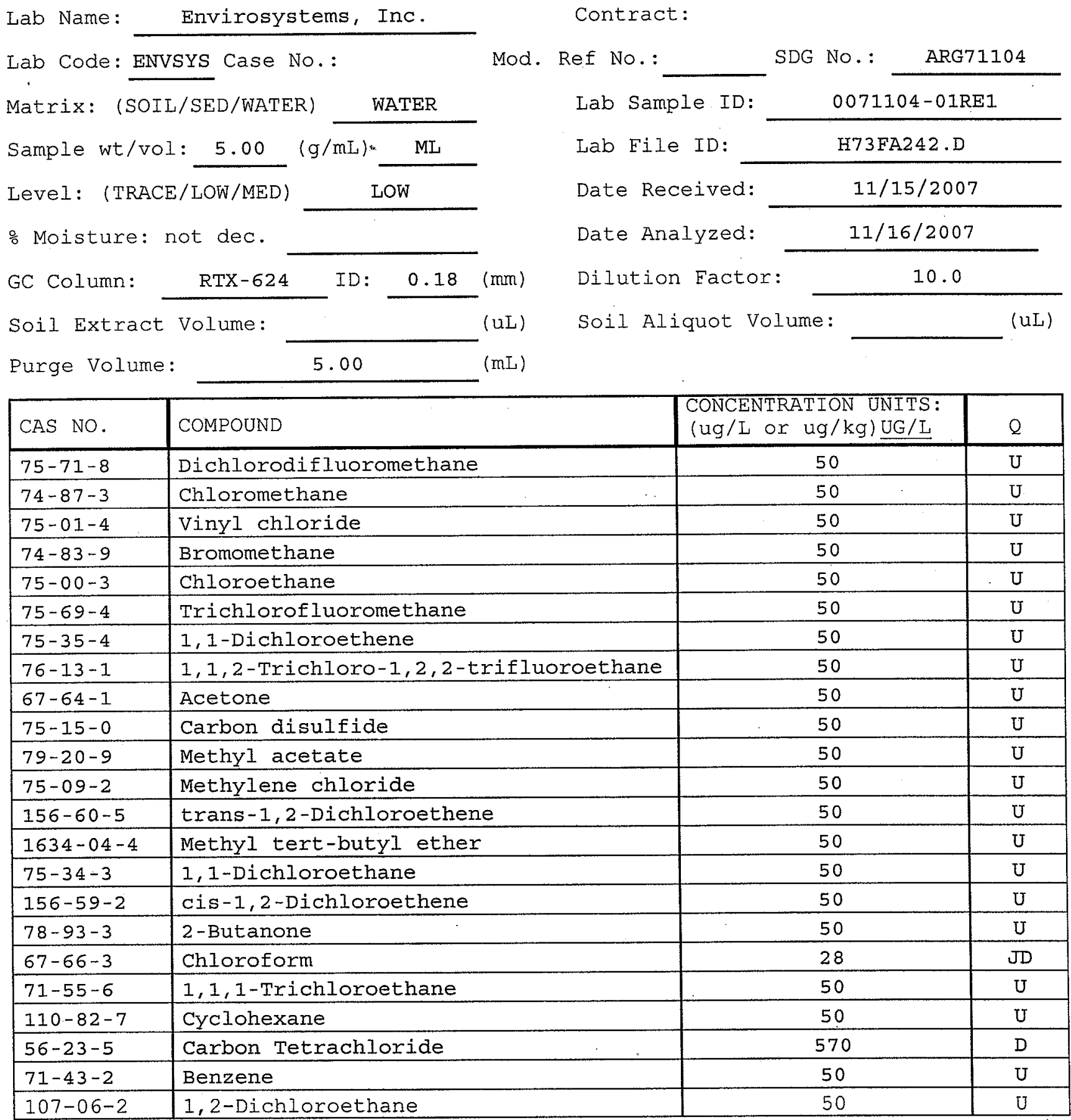


$1 B$ - FORM I VOA-2

VOLATILE ORGANICS ANALYSIS DATA SHEET
EPA SAMPLE NO.

CNPSB $1-W-16246 \mathrm{DL}$
Lab Name: Envirosystems, Inc.

Lab Code: ENvsys Case No.:

Matrix: (SOIL/SED/WATER) WATER

Sample wt/vol: $5.00(\mathrm{~g} / \mathrm{mL}) \quad \mathrm{ML}$

Level: (TRACE/LOW/MED)

LOW

\% Moisture: not dec.

GC Column: $\quad$ RTX-624 ID: 0.18 (mm)

Soil Extract Volume:

Purge Volume:

5.00

(UL)

(mL)

Contract:

Mod. Ref No.:

SDG No.: $\quad$ ARG71104

Lab Sample ID:

0071104-01RE1

Lab File ID:

H73FA242.D

Date Received:

$11 / 15 / 2007$

Date Analyzed:

$11 / 16 / 2007$

Dilution Factor:

10.0

Soil Aliquot Volume: (uL)

\begin{tabular}{|c|c|c|c|}
\hline CAS NO. & COMPOUND & $\begin{array}{l}\text { CONCENTRATION UNITS: } \\
(\mathrm{ug} / \mathrm{L} \text { or } u g / \mathrm{kg}) \mathrm{UG} / \mathrm{L}\end{array}$ & $Q$ \\
\hline $79-01-6$ & Trichloroethene & 50 & $\mathrm{U}$ \\
\hline $108-87-2$ & Methylcyclohexane & 50 & $\mathrm{U}$ \\
\hline $78-87-5$ & 1,2-Dichloropropane & 50 & $\mathrm{U}$ \\
\hline $75-27-4$ & Bromodichloromethane & 50 & $\mathrm{U}$ \\
\hline $10061-01-5$ & cis-1,3-Dichloropropene & 50 & $\mathrm{U}$ \\
\hline $108-10-1$ & 4-Methyl-2-pentanone & 50 & $\mathrm{U}$ \\
\hline $108-88-3$ & Toluene & 50 & $\mathrm{U}$ \\
\hline $10061-02-6$ & trans-1,3-Dichloropropene & 50 & $\mathrm{U}$ \\
\hline $79-00-5$ & $1,1,2$-Trichloroethane & 50 & $\mathrm{U}$ \\
\hline $127-18-4$ & Tetrachloroethene & 50 & $\mathrm{U}$ \\
\hline $591-78-6$ & 2-Hexanone & 50 & $\mathrm{U}$ \\
\hline $124-48-1$ & Dibromochloromethane & 50 & $\mathrm{U}$ \\
\hline $106-93-4$ & 1,2-Dibromoethane & 50 & $\mathrm{U}$ \\
\hline $108-90-7$ & Chlorobenzene & 50 & U \\
\hline $100-41-4$ & Ethylbenzene & 50 & $\mathrm{U}$ \\
\hline $95-47-6$ & o-xylene & 50 & $\mathrm{U}$ \\
\hline $179601-23-1$ & $m, p-X y l e n e$ & 50 & $\mathrm{U}$ \\
\hline $100-42-5$ & styrene & 50 & $\mathrm{U}$ \\
\hline $75-25-2$ & Bromoform & 50 & $\mathrm{U}$ \\
\hline $98-82-8$ & Isopropylbenzene & 50 & $\mathrm{U}$ \\
\hline $79-34-5$ & $1,1,2,2$-Tetrachloroethane & 50 & $\mathrm{U}$ \\
\hline $541-73-1$ & 1,3-Dichlorobenzene & 50 & $\mathrm{U}$ \\
\hline $106-46-7$ & 1,4-Dichlorobenzene & 50 & $\mathrm{U}$ \\
\hline $95-50-1$ & 1,2-Dichlorobenzene & 50 & $\mathrm{U}$ \\
\hline $96-12-8$ & 1,2-Dibromo-3-chloropropane & 50 & $\mathrm{U}$ \\
\hline $120-82-1$ & $1,2,4$-Trichlorobenzene & 50 & $\mathrm{U}$ \\
\hline $91-20-3$ & Naphthalene & 50 & $\mathrm{U}$ \\
\hline
\end{tabular}


$1 A$ - FORM I VOA-1

VOLATILE ORGANICS ANALYSIS DATA SHEET
EPA SAMPLE NO.

CNQCTB -W - 19961
Lab Name: Envirosystems, Inc.

Lab Code: ENVSYS Case No.:

Matrix: (SOIL/SED/WATER) WATER

Sample wt/vol: $5.00(\mathrm{~g} / \mathrm{mL}) \mathrm{ML}$

Level: (TRACE/LOW/MED)

LOW

o Moisture: not dec.

GC Column: $\quad$ RTX-624 ID: 0.18 (mm)

Soil Extract Volume:

Purge Volume:

5.00

uL

$(\mathrm{mL})$

Contract:

Mod. Ref No.: SDG No.: $\quad$ ARG71104

Lab Sample ID:

0071104-02

Lab File ID:

H73FA2 38.D

Date Received:

$11 / 15 / 2007$

Date Analyzed:

$11 / 16 / 2007$

Dilution Eactor:

1.0

Soil Aliquot Volume:

(uL)

\begin{tabular}{|c|c|c|c|}
\hline CAS NO. & COMPOUND & $\begin{array}{l}\text { CONCENTRATION UNITS: } \\
(\mathrm{ug} / \mathrm{L} \text { or } \mathrm{ug} / \mathrm{kg}) \mathrm{UG} / \mathrm{I}\end{array}$ & $Q$ \\
\hline $75-71-8$ & Dichlorodifluoromethane & 5.0 & $\mathrm{U}$ \\
\hline $74-87-3$ & Chloromethane & 5.0 & $\mathrm{U}$ \\
\hline $75-01-4$ & Vinyl chloride & 5.0 & $U$ \\
\hline $74-83-9$ & Bromomethane & 5.0 & $\mathrm{U}$ \\
\hline $75-00-3$ & Chloroethane & 5.0 & $\mathrm{U}$ \\
\hline $75-69-4$ & Trichlorofluoromethane & 5.0 & U \\
\hline $75-35-4$ & 1,1-Dichloroethene & 5.0 & $\mathrm{U}$ \\
\hline $76-13-1$ & $1,1,2$-Trichloro-1,2,2-trifluoroethane & 5.0 & $\mathrm{U}$ \\
\hline $67-64-1$ & Acetone & 5.0 & $\mathrm{U}$ \\
\hline $75-15-0$ & Carbon disulfide & 5.0 & U \\
\hline $79-20-9$ & Methyl acetate & 5.0 & $\mathrm{U}$ \\
\hline $75-09-2$ & Methylene chloride & 5.0 & $\mathrm{U}$ \\
\hline $156-60-5$ & trans-1,2-Dichloroethene & 5.0 & $\mathrm{U}$ \\
\hline $1634-04-4$ & Methyl tert-butyl ether & 5.0 & $\mathrm{U}$ \\
\hline $75-34-3$ & 1,1-Dichloroethane & 5.0 & $\mathrm{U}$ \\
\hline $156-59-2$ & cis-1,2-Dichloroethene & 5.0 & $\mathrm{U}$ \\
\hline $78-93-3$ & 2-Butanone & 5.0 & U \\
\hline $67-66-3$ & Chloroform & 5.0 & $\mathrm{U}$ \\
\hline $71-55-6$ & 1,1,1-Trichloroethane & 5.0 & $\mathrm{U}$ \\
\hline $110-82-7$ & Cyclohexane & 5.0 & $\mathrm{U}$ \\
\hline $56-23-5$ & Carbon Tetrachloride & 5.0 & $\mathrm{U}$ \\
\hline $71-43-2$ & Benzene & 5.0 & $\mathrm{U}$ \\
\hline $107-06-2$ & 1,2-Dichloroethane & 5.0 & $\mathrm{U}$ \\
\hline
\end{tabular}


IB - EORM I VOA-2

VOLATIEE ORGANICS ANALYSIS DATA SHEET
EPA SAMPLE NO.

CNQCTB $-\mathrm{W}-19961$

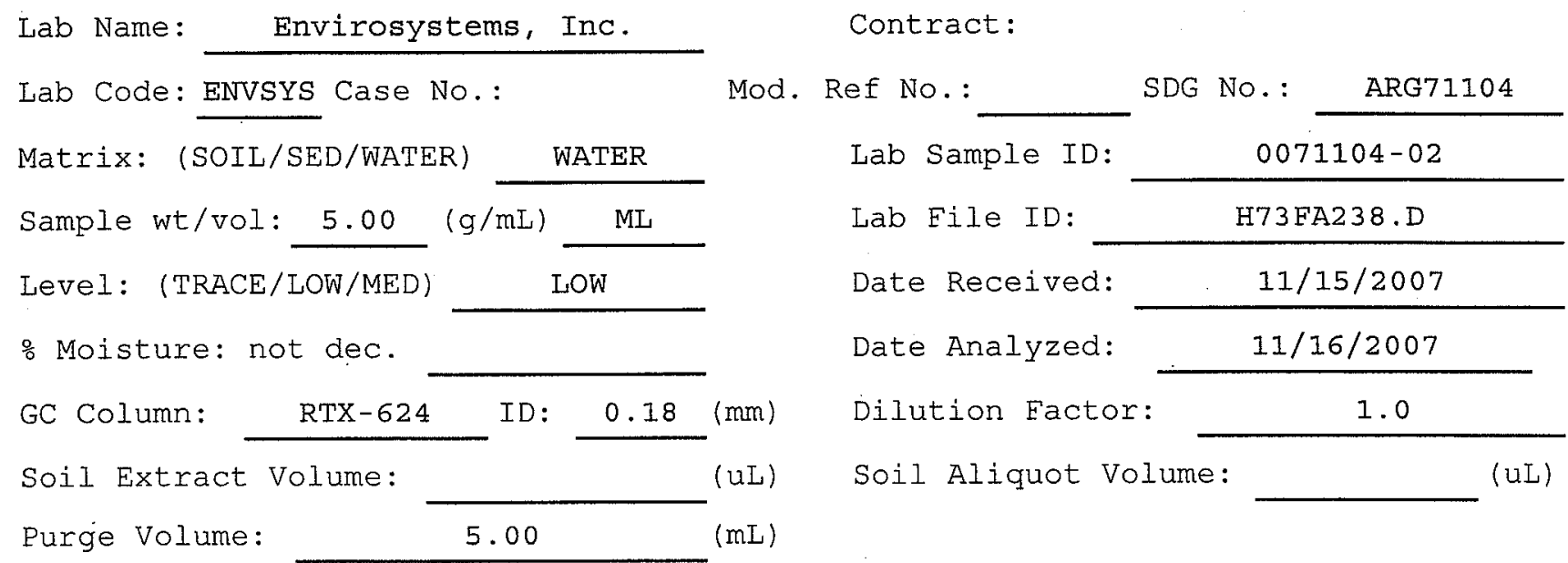

\begin{tabular}{|c|c|c|c|}
\hline CAS NO. & COMPOUND & $\begin{array}{l}\text { CONCENTRATION UNITS: } \\
(\mathrm{ug} / \mathrm{L} \text { or } \mathrm{ug} / \mathrm{kg}) \mathrm{UG} / \mathrm{L}\end{array}$ & $Q$ \\
\hline $79-01-6$ & Trichloroethene & 5.0 & $\mathrm{U}$ \\
\hline $108-87-2$ & Methylcyclohexane & 5.0 & $\mathrm{U}$ \\
\hline $78-87-5$ & 1,2-Dichloropropane & 5.0 & $\mathrm{U}$ \\
\hline $75-27-4$ & Bromodichloromethane & 5.0 & $\mathrm{U}$ \\
\hline $10061-01-5$ & cis-1,3-Dichloropropene & 5.0 & $\mathrm{U}$ \\
\hline $108-10-1$ & 4-Methyl-2-pentanone & 5.0 & $\mathrm{U}$ \\
\hline $108-88-3$ & Toluene & 5.0 & $\mathrm{U}$ \\
\hline $10061-02-6$ & trans-1,3-Dichloropropene & 5.0 & $\mathrm{U}$ \\
\hline $79-00-5$ & 1,1,2-Trichloroethane & 5.0 & $\mathrm{U}$ \\
\hline $127-18-4$ & Tetrachloroethene & 5.0 & $\mathrm{U}$ \\
\hline $591-78-6$ & 2-Hexanone & 5.0 & $\mathrm{U}$ \\
\hline $124-48-1$ & Dibromochloromethane & 5.0 & $\mathrm{U}$ \\
\hline $106-93-4$ & 1,2-Dibromoethane & 5.0 & $\mathrm{U}$ \\
\hline $108-90-7$ & Chlorobenzene & 5.0 & $\mathrm{U}$ \\
\hline $100-41-4$ & Ethylbenzene & 5.0 & $\mathrm{U}$ \\
\hline $95-47-6$ & o-xylene & 5.0 & $\mathrm{U}$ \\
\hline $179601-23-1$ & $\mathrm{~m}, \mathrm{p}$-Xylene & 5.0 & $\mathrm{U}$ \\
\hline $100-42-5$ & styrene & 5.0 & $\mathrm{U}$ \\
\hline $75-25-2$ & Bromoform & 5.0 & $\mathrm{U}$ \\
\hline $98-82-8$ & Isopropylbenzene & 5.0 & $\mathrm{U}$ \\
\hline $79-34-5$ & $1,1,2,2$-Tetrachloroethane & 5.0 & $\mathrm{U}$ \\
\hline $541-73-1$ & 1,3-Dichlorobenzene & 5.0 & $\mathrm{U}$ \\
\hline $106-46-7$ & 1,4-Dichlorobenzene & 5.0 & $\mathrm{U}$ \\
\hline $95-50-1$ & 1,2-Dichlorobenzene & 5.0 & $\mathrm{U}$ \\
\hline $96-12-8$ & 1,2-Dibromo-3-chloropropane & 5.0 & $\mathrm{U}$ \\
\hline $120-82-1$ & $1,2,4-\operatorname{Tr}$ ichlorobenzene & 5.0 & $\mathrm{U}$ \\
\hline $91-20-3$ & Naphthalene & 5.0 & U \\
\hline
\end{tabular}


$1 A$ - FORM I VOA-1

VOLATILE ORGANICS ANALYSIS DATA SHEET
EPA SAMPLE NO.

CNPSB $3-W-16250$
Lab Name:

Envirosystems, Inc.

Lab Code: ENVSYS Case No.:

Matrix: (SOIL/SED/WATER)

Sample wt/vol: $5.00(\mathrm{~g} / \mathrm{mL}) \mathrm{ML}$

Level: (TRACE/LOW/MED)

LOW

\% Moisture: not dec.

GC Column: $\quad$ RTX-624 ID: 0.18 (mm)

Soil Extract Volume:

Purge Volume:

5.00

(UI)

$(\mathrm{mL})$

Contract :

Mod. Ref No.:
Lab Sample ID:

Lab Eile ID:

Date Received:

Date Analyzed:

Dilution Eactor:

Soil Aliquot Volume:

(uL)

\begin{tabular}{|c|c|c|c|}
\hline CAS NO. & COMPOUND & $\begin{array}{l}\text { CONCENTRATION UNITS: } \\
(\mathrm{ug} / \mathrm{L} \text { or } \mathrm{ug} / \mathrm{kg}) \mathrm{UG} / \mathrm{L}\end{array}$ & $Q$ \\
\hline $75-71-8$ & Dichlorodifluoromethane & 5.0 & $\mathrm{U}$ \\
\hline $74-87-3$ & Chloromethane & 5.0 & $\mathrm{U}$ \\
\hline $75-01-4$ & Vinyl chloride & 5.0 & $\mathrm{U}$ \\
\hline $74-83-9$ & Bromomethane & 5.0 & $U$ \\
\hline $75-00-3$ & Chloroethane & 5.0 & $\mathrm{U}$ \\
\hline $75-69-4$ & Trichlorofluoromethane & 5.0 & $\mathrm{U}$ \\
\hline $75-35-4$ & 1,1-Dichloroethene & 5.0 & $\mathrm{U}$ \\
\hline $76-13-1$ & $1,1,2$-Trichloro-1,2,2-trifluoroethane & 5.0 & $\mathrm{U}$ \\
\hline $67-64-1$ & Acetone & 3.2 & $\mathrm{~J}$ \\
\hline $75-15-0$ & Carbon disulfide & 5.0 & $\mathrm{U}$ \\
\hline $79-20-9$ & Methyl acetate & 5.0 & U \\
\hline $75-09-2$ & Methylene chloride & 5.0 & $\mathrm{U}$ \\
\hline $156-60-5$ & trans-1,2-Dichloroethene & 5.0 & $\mathrm{U}$ \\
\hline $1634-04-4$ & Methyl tert-butyl ether & 5.0 & $\mathrm{U}$ \\
\hline $75-34-3$ & 1,1-Dichloroethane & 5.0 & $\mathrm{U}$ \\
\hline $156-59-2$ & Cis-1,2-Dichloroethene & 5.0 & $\mathrm{U}$ \\
\hline $78-93-3$ & 2-Butanone & 5.0 & $\mathrm{U}$ \\
\hline $67-66-3$ & Chloroform & 15 & \\
\hline $71-55-6$ & $1,1,1$-Trichloroethane & 5.0 & $\mathrm{U}$ \\
\hline $110-82-7$ & Cyclohexane & 5.0 & $\mathrm{U}$ \\
\hline $56-23-5$ & Carbon Tetrachloride & 840 & $E$ \\
\hline $71-43-2$ & Benzene & 5.0 & $\mathrm{U}$ \\
\hline $107-06-2$ & 1,2-Dichloroethane & 5.0 & $\mathrm{U}$ \\
\hline
\end{tabular}


$1 B$ - FORM I VOA-2

VOLATILE ORGANICS ANALYSIS DATA SHEET
EPA SAMPLE NO.

CNPSB3 $-W-16250$
Lab Name: Envirosystems, Inc.

Lab Code: ENVSYS Case No.:

Matrix: (SOIL/SED/WATER)

WATER

Sample wt/vol: $5.00(\mathrm{~g} / \mathrm{mL}) \quad \mathrm{ML}$

Level: (TRACE/LOW/MED) LOW

으일 not dec.

GC Column: RTX-624 ID: 0.18 (mm)

Soil Extract Volume:

Purge Volume:

5.00

(mL)

Contract:

(uIs)

Mod. Ref No.:

Lab Sample ID:

Lab File ID:

Date Received:

Date Analyzed:

Dilution Eactor:

Soil Aliquot Volume:

(uL)

\begin{tabular}{|c|c|c|c|}
\hline CAS NO. & COMPOUND & $\begin{array}{l}\text { CONCENTRATION UNITS: } \\
(\mathrm{ug} / \mathrm{L} \text { or } \mathrm{ug} / \mathrm{kg}) \mathrm{UG} / \mathrm{L}\end{array}$ & $Q$ \\
\hline $79-01-6$ & Trichloroethene & 5.0 & $\mathrm{U}$ \\
\hline $108-87-2$ & Methylcyclohexane & 5.0 & U \\
\hline $78-87-5$ & 1,2-Dichloropropane & 5.0 & $\mathrm{U}$ \\
\hline $75-27-4$ & Bromodichloromethane & 5.0 & $U$ \\
\hline $10061-01-5$ & cis-1,3-Dichloropropene & 5.0 & $\mathrm{U}$ \\
\hline $108-10-1$ & 4-Methyl-2-pentanone & 5.0 & $\mathrm{U}$ \\
\hline $108-88-3$ & Toluene & 5.0 & $\mathrm{U}$ \\
\hline $10061-02-6$ & trans $-1,3-$ Dichloropropene & 5.0 & $\mathrm{U}$ \\
\hline $79-00-5$ & $1,1,2$-Trichloroethane & 5.0 & $\mathrm{U}$ \\
\hline $127-18-4$ & Tetrachloroethene & 5.0 & U \\
\hline $591-78-6$ & 2-Hexanone & 5.0 & $\mathrm{U}$ \\
\hline $124-48-1$ & Dibromochloromethane & 5.0 & $\mathrm{U}$ \\
\hline $106-93-4$ & 1,2 -Dibromoethane & 5.0 & $\mathrm{U}$ \\
\hline $108-90-7$ & Chlorobenzene & 5.0 & $\mathrm{U}$ \\
\hline $100-41-4$ & Ethylbenzene & 5.0 & $\mathrm{U}$ \\
\hline $95-47-6$ & o-XYlene & 5.0 & U \\
\hline $179601-23-1$ & $\mathrm{~m}, \mathrm{p}$-Xylene & 5.0 & $\mathrm{U}$ \\
\hline $100-42-5$ & styrene & 5.0 & $\mathrm{U}$ \\
\hline $75-25-2$ & Bromoform & 5.0 & $\mathrm{U}$ \\
\hline $98-82-8$ & Isopropylbenzene & 5.0 & $\mathrm{U}$ \\
\hline $79-34-5$ & $1,1,2,2$-Tetrachloroethane & 5.0 & Uं \\
\hline $541-73-1$ & 1,3-Dichlorobenzene & 5.0 & $\mathrm{U}$ \\
\hline $106-46-7$ & 1,4-Dichlorobenzene & 5.0 & $\mathrm{U}$ \\
\hline $95-50-1$ & 1,2-Dichlorobenzene & 5.0 & $\mathrm{U}$ \\
\hline $96-12-8$ & 1,2-Dibromo-3-chloropropane & 5.0 & $\mathrm{U}$ \\
\hline $120-82-1$ & $1,2,4$-Trichlorobenzene & 5.0 & $\mathrm{U}$ \\
\hline $91-20-3$ & Naphthalene & 5.0 & $\mathrm{U}$ \\
\hline
\end{tabular}


$1 A$ - EORM I VOA-1

VOLATILE ORGANICS ANALYSIS DATA SHEET
EPA SAMPLE NO.

CNPSB $3-\mathrm{W}-16250 \mathrm{DL}$
Lab Name: Envirosystems, Inc.

Lab Code: ENVSYS Case No.:

Matrix: (SOIL/SED/WATER) WATER

Sample wt/vol: $5.00(\mathrm{~g} / \mathrm{mL}) \mathrm{ML}$.

Level: (TRACE/LOW/MED)

LOW

o Moisture: not dec.

GC Column: $\quad$ RTX-624 ID: 0.18 (mm)

Soil Extract Volume:

Purge Volume:

5.00

(uL) (mI)

Contract:

Mod. Ref No.:

SDG No.: $\quad$ ARG71104

Lab Sample ID:

0071104-03RE1

Lab File ID:

H73FA243.D

Date Received:

$11 / 15 / 2007$

Date Analyzed:

$11 / 16 / 2007$

Dilution Eactor:

20.0

Soil Aliquot Volume:

(uL)

\begin{tabular}{|c|c|c|c|}
\hline CAS NO. & COMPOUND & $\begin{array}{l}\text { CONCENTRATION UNITS: } \\
(\mathrm{ug} / \mathrm{L} \text { or } \mathrm{ug} / \mathrm{kg}) \mathrm{UG} / \mathrm{L}\end{array}$ & $Q$ \\
\hline $75-71-8$ & Dichlorodifluoromethane & 100 & $\mathrm{U}$ \\
\hline $74-87-3$ & Chloromethane & 100 & $\mathrm{U}$ \\
\hline $75-01-4$ & Vinyl chloride & 100 & $\mathrm{U}$ \\
\hline $74-83-9$ & Bromomethane & 100 & $\mathrm{U}$ \\
\hline $75-00-3$ & Chloroethane & 100 & $\mathrm{U}$ \\
\hline $75-69-4$ & Trichlorofluoromethane & 100 & $\mathrm{U}$ \\
\hline $75-35-4$ & 1,1-Dichloroethene & 100 & $\mathrm{U}$ \\
\hline $76-13-1$ & $1,1,2$-Trichloro-1,2,2-trifluoroethane & 100 & $\mathrm{U}$ \\
\hline $67-64-1$ & Acetone & 100 & $\mathrm{U}$ \\
\hline $75-15-0$ & Carbon disulfide & 100 & $\mathrm{U}$ \\
\hline $79-20-9$ & Methyl acetate & 100 & $\mathrm{U}$ \\
\hline $75-09-2$ & Methylene chloride & 100 & $\mathrm{U}$ \\
\hline $156-60-5$ & trans $-1,2-$ Dichloroethene & 100 & $\mathrm{U}$ \\
\hline $1634-04-4$ & Methyl tert-butyl ether & 100 & $\mathrm{U}$ \\
\hline $75-34-3$ & 1,1-Dichloroethane & 100 & $\mathrm{U}$ \\
\hline $156-59-2$ & Cis-1,2-Dichloroethene & 100 & $\mathrm{U}$ \\
\hline $78-93-3$ & 2-Butanone & 100 & $\mathrm{U}$ \\
\hline $67-66-3$ & Chloroform & 25 & JD \\
\hline $71-55-6$ & 1,1,1-Trichloroethane & 100 & $\mathrm{U}$ \\
\hline $110-82-7$ & Cyclohexane & 100 & $\mathrm{U}$ \\
\hline $56-23-5$ & Carbon Tetrachloride & 1200 & $\mathrm{D}$ \\
\hline $71-43-2$ & Benzene & 100 & $\mathrm{U}$ \\
\hline $107-06-2$ & 1,2-Dichloroethane & 100 & $\mathrm{U}$ \\
\hline
\end{tabular}


$1 B$ - FORM I VOA-2

VOLATILE ORGANICS ANALYSIS DATA SHEET
EPA SAMPLE NO.

CNPSB $3-W-16250$ DL
Lab Name: Envirosystems, Inc.

Lab Code: ENvṢys Case No.:

Matrix: (SOIL/SED/WATER)

WATER

Sample wt/vol: $5.00(\mathrm{~g} / \mathrm{mL})$ MI

Level: (TRACE/LOW/MED) * LOW

: Moisture: not dec.

GC Column: $\quad$ RTX-624 ID: 0.18 (mm)

Soil Extract Volume:

Purge Volume:

5.00

Contract:

Mod. Ref No.: SDG No.: $\quad$ ARG71104

Lab Sample ID: 0071104-03RE1

Lab File ID:

H73FA243.D

Date Received:

$11 / 15 / 2007$

Date Analyzed:

$11 / 16 / 2007$

Dilution Factor:

20.0

Soil Aliquot Volume:

(uL)

\begin{tabular}{|c|c|c|c|}
\hline CAS NO. & COMPOUND & $\begin{array}{l}\text { CONCENTRATION UNITS: } \\
(\mathrm{ug} / \mathrm{L} \text { or } \mathrm{ug} / \mathrm{kg}) \mathrm{UG} / \mathrm{L}\end{array}$ & $Q$ \\
\hline $79-01-6$ & Trichloroethene & 100 & $\mathrm{U}$ \\
\hline $108-87-2$ & Methylcyclohexane & 100 & $\mathrm{U}$ \\
\hline $78-87-5$ & 1,2-Dichloropropane & 100 & $\mathrm{U}$ \\
\hline $75-27-4$ & Bromodichloromethane & 100 & $\mathrm{U}$ \\
\hline $10061-01-5$ & Cis-1,3-Dichloropropene & 100 & $\mathrm{U}$ \\
\hline $108-10-1$ & 4-Methyl-2-pentanone & 100 & $\mathrm{U}$ \\
\hline $108-88-3$ & Toluene & 100 & $\mathrm{U}$ \\
\hline $10061-02-6$ & trans-1,3-Dichloropropene & 100 & $\mathrm{U}$ \\
\hline $79-00-5$ & 1,1,2-Trichloroethane & 100 & $\mathrm{U}$ \\
\hline $127-18-4$ & Tetrachloroethene & 100 & $\mathrm{U}$ \\
\hline $591-78-6$ & 2-Hexanone & 100 & $\mathrm{U}$ \\
\hline $124-48-1$ & Dibromochloromethane & 100 & $\mathrm{U}$ \\
\hline $106-93-4$ & 1,2-Dibromoethane & 100 & $\mathrm{U}$ \\
\hline $108-90-7$ & Chlorobenzene & 100 & $\mathrm{U}$ \\
\hline $100-41-4$ & Ethylbenzene & 100 & $\mathrm{U}$ \\
\hline $95-47-6$ & o-Xylene & 100 & $\mathrm{U}$ \\
\hline $179601-23-1$ & m,p-xylene & 100 & $\mathrm{U}$ \\
\hline $100-42-5$ & styrene & 100 & $\mathrm{U}$ \\
\hline $75-25-2$ & Bromoform & 100 & $U$ \\
\hline $98-82-8$ & Isopropylbenzene & 100 & $\mathrm{U}$ \\
\hline $79-34-5$ & $1,1,2,2$-Tetrachloroethane & 100 & $\mathrm{U}$ \\
\hline $541-73-1$ & 1,3-Dichlorobenzene & 100 & $\mathrm{U}$ \\
\hline $106-46-7$ & 1,4-Dichlorobenzene & 100 & $\mathrm{U}$ \\
\hline $95-50-1$ & 1,2-Dichlorobenzene & 100 & $\mathrm{U}$ \\
\hline $96-12-8$ & 1,2-Dibromo-3-chloropropane & 100 & $\mathrm{U}$ \\
\hline $120-82-1$ & 1,2,4-Trichlorobenzene & 100 & $\mathrm{U}$ \\
\hline $91-20-3$ & Naphthalene & 100 & $\mathrm{U}$ \\
\hline
\end{tabular}


$1 A$ - FORM I VOA-1

VOLATILE ORGANICS ANALYSIS DATA SHEET
EPA SAMPLE NO.

CNPSB $6-W-16254$
Lab Name: ' Envirosystems, Ino.

Lab Code: ENVSYS Case No.:

Matrix: (SOIL/SED/WATER) WATER

Sample wt/vol:

5.00 $(\mathrm{g} / \mathrm{mL})$

ML

Level: (TRACE/LOW/MED) LOW

\% Moisture: not dec.

GC Column: $\quad$ RTX-624 ID: 0.18 (mm)

Soil Extract Volume:

Purge Volume:

5.00

Contract:

Mod. Ref No.:

SDG No.: $\quad$ ARG71104

Lab Sample ID:

$0071104-04$

Lab File ID:

H7 3FA240.D

Date Received:

$11 / 15 / 2007$

Date Analyzed:

$11 / 16 / 2007$

Dilution Factor:

1.0

Soil Aliquot Volume: (uI)

\begin{tabular}{|c|c|c|c|}
\hline CAS NO. & COMPOUND & $\begin{array}{l}\text { CONCENTRATION UNTTS: } \\
(\mathrm{ug} / \mathrm{L} \text { or } \mathrm{ug} / \mathrm{kg}) \mathrm{UG} / \mathrm{L}\end{array}$ & $Q$ \\
\hline $75-71-8$ & Dichlorodifluoromethane & 5.0 & $\mathrm{U}$ \\
\hline $74-87-3$ & Chloromethane & 5.0 & U \\
\hline $75-01-4$ & Vinyl chloride & 5.0 & $\mathrm{U}$ \\
\hline $74-83-9$ & Bromomethane & 5.0 & $\mathrm{U}$ \\
\hline $75-00-3$ & Chloroethane & 5.0 & $\mathrm{U}$ \\
\hline $75-69-4$ & Trichlorofluoromethane & 5.0 & $\mathrm{U}$ \\
\hline $75-35-4$ & 1,1-Dichloroethene & 5.0 & $\mathrm{U}$ \\
\hline $76-13-1$ & $1,1,2$-Trichloro-1,2,2-trifluoroethane & 5.0 & $\mathrm{U}$ \\
\hline $67-64-1$ & Acetone & 5.0 & $\mathrm{U}$ \\
\hline $75-15-0$ & Carbon disulfide & 5.0 & $\mathrm{U}$ \\
\hline $79-20-9$ & Methyl acetate & 5.0 & $\mathrm{U}$ \\
\hline $75-09-2$ & Methylene chloride & 5.0 & $\mathrm{U}$ \\
\hline $156-60-5$ & trans-1,2-Dichloroethene & 5.0 & $\mathrm{U}$ \\
\hline $1634-04-4$ & Methyl tert-butyl ether & 5.0 & $U$ \\
\hline $75-34-3$ & 1, 1-Dichloroethane & 5.0 & $\mathrm{U}$ \\
\hline $156-59-2$ & cis-1,2-Dichloroethene & 5.0 & U \\
\hline $78-93-3$ & 2-Butanone & 5.0 & $\mathrm{U}$ \\
\hline $67-66-3$ & Chloroform & 5.6 & \\
\hline $71-55-6$ & 1,1,1-Trichloroethane & 5.0 & $\mathrm{U}$ \\
\hline $110-82-7$ & Cyclohexane & 5.0 & $\mathrm{U}$ \\
\hline $56-23-5$ & Carbon Tetrachloride & 57 & \\
\hline $71-43-2$ & Benzene & 5.0 & $\mathrm{U}$ \\
\hline $107-06-2$ & 1,2-Dichloroethane & 5.0 & $\mathrm{U}$ \\
\hline
\end{tabular}


$1 B$ - FORM I VOA-2

VOLATILE ORGANICS ANALYSIS DATA SHEET
EPA SAMPLE NO.

CNPSB 6-W-16254
Lab Name: Envirosystems, Inc.

Lab Code: ENvsys Case No.:

Matrix: (SOIL/SED/WATER) WATER

Sample wt/vol: $5.00(\mathrm{~g} / \mathrm{mL}) \mathrm{ML}$

Level: (TRACE/LOW/MED)

LOW

음 Moisture: not dec.

GC Column: $\quad$ RTX-624 ID: 0.18 (mm)

Soil Extract Volume:

Purge Volume:

5.00

(uL)
Contract:

Mod. Ref No.:

SDG No.: $\quad$ ARG71104

Lab Sample ID:

0071104-04

Lab File ID:

$\mathrm{H} 73 \mathrm{FA} 240 . \mathrm{D}$

Date Received:

$11 / 15 / 2007$

Date Analyzed:

Dilution Factor:

$11 / 16 / 2007$

Soil Aliquot Volume: (uL)

\begin{tabular}{|c|c|c|c|}
\hline CAS NO. & COMPOUND & $\begin{array}{l}\text { CONCENTRATION UNITS: } \\
(\mathrm{ug} / \mathrm{L} \text { or } u \mathrm{~g} / \mathrm{kg}) \text { UG/L }\end{array}$ & $Q$ \\
\hline $79-01-6$ & Trichloroethene & 5.0 & $\mathrm{U}$ \\
\hline $108-87-2$ & Methylcyclohexane & 5.0 & $\mathrm{U}$ \\
\hline $78-87-5$ & 1,2-Dichloropropane & 5.0 & $\mathrm{U}$ \\
\hline $75-27-4$ & Bromodichloromethane & 5.0 & $\mathrm{U}$ \\
\hline $10061-01-5$ & cis-1,3-Dichloropropene & 5.0 & $\mathrm{U}$ \\
\hline $108-10-1$ & 4-Methyl-2-pentanone & 5.0 & $\mathrm{U}$ \\
\hline $108-88-3$ & Toluene & 5.0 & $\mathrm{U}$ \\
\hline $10061-02-6$ & trans-1,3-Dichloropropene & 5.0 & $\mathrm{U}$ \\
\hline $79-00-5$ & 1,1,2-Trichloroethane & 5.0 & $\mathrm{U}$ \\
\hline $127-18-4$ & Tetrachloroethene & 5.0 & $\mathrm{U}$ \\
\hline $591-78-6$ & 2-Hexanone & 5.0 & $\mathrm{U}$ \\
\hline $124-48-1$ & Dibromochloromethane & 5.0 & $\mathrm{U}$ \\
\hline $106-93-4$ & 1,2-Dibromoethane & 5.0 & $\mathrm{U}$ \\
\hline $108-90-7$ & Chlorobenzene & 5.0 & $\mathrm{U}$ \\
\hline $100-41-4$ & Ethylbenzene & 5.0 & $\mathrm{U}$ \\
\hline $95-47-6$ & o-Xylene & 5.0 & $\mathrm{U}$ \\
\hline $179601-23-1$ & $\mathrm{~m}, \mathrm{p}$-Xylene & 5.0 & $\mathrm{U}$ \\
\hline $100-42-5$ & Styrene & 5.0 & $\mathrm{U}$ \\
\hline $75-25-2$ & Bromoform & 5.0 & $\mathrm{U}$ \\
\hline $98-82-8$ & Isopropylbenzene & 5.0 & $\mathrm{U}$ \\
\hline $79-34-5$ & $1,1,2,2$-Tetrachloroethane & 5.0 & $\mathrm{U}$ \\
\hline $541-73-1$ & 1,3-Dichlorobenzene & 5.0 & $\mathrm{U}$ \\
\hline $106-46-7$ & 1,4-Dichlorobenzene & 5.0 & $\mathrm{U}$ \\
\hline $95-50-1$ & 1,2-Dichlorobenzene & 5.0 & $\mathrm{U}$ \\
\hline $96-12-8$ & 1,2-Dibromo-3-chloropropane & 5.0 & $\mathrm{U}$ \\
\hline $120-82-1$ & $1,2,4$-Trichlorobenzene & 5.0 & $\mathrm{U}$ \\
\hline $91-20-3$ & Naphthalene & 5.0 & $\mathrm{U}$ \\
\hline
\end{tabular}


$1 A$ - FORM I VOA-1

VOLATILE ORGANICS ANALYSIS DATA SHEET
EPA SAMPLE NO.

CNPUBL -W-26062
Lab Name: Envirosystems, Inc.

Lab Code: ENVSYS Case No.:

Matrix: (SOIL/SED/WATER) WATER

Sample wt/vol: $5.00(\mathrm{~g} / \mathrm{mL}) \mathrm{ML}$

Level: (TRACE/LOW/MED)

LOW

: Moisture: not dec.

GC Column: $\quad$ RTX-624 ID: $0.18(\mathrm{~mm})$

Soil Extract Volume:

purge volume:

5.00

(uL)
Contract:

Mod. Ref No.: SDG No.: $\quad$ ARG71105

Lab Sample ID:

0071105-01

Lab File ID:

H73FA24 I.D

Date Received:

$11 / 16 / 2007$

Date Analyzed:

$11 / 16 / 2007$

Dilution Factor:

1.0

Soil Aliquot Volume:

(uI)

\begin{tabular}{|c|c|c|c|}
\hline CAS NO. & COMPOUND & $\begin{array}{l}\text { CONCENTRATION UNITS: } \\
(\mathrm{ug} / \mathrm{L} \text { or } \mathrm{ug} / \mathrm{kg}) \mathrm{UG} / \mathrm{I}\end{array}$ & $Q$ \\
\hline $75-71-8$ & Dichlorodifluoromethane & 5.0 & $\mathrm{U}$ \\
\hline $74-87-3$ & Chloromethane & 5.0 & $\mathrm{U}$ \\
\hline $75-01-4$ & Vinyl chloride & 5.0 & $\mathrm{U}$ \\
\hline $74-83-9$ & Bromomethane & 5.0 & $\mathrm{U}$ \\
\hline $75-00-3$ & Chloroethane & 5.0 & $\mathrm{U}$ \\
\hline $75-69-4$ & Trichlorofluoromethane & 5.0 & $\mathrm{U}$ \\
\hline $75-35-4$ & 1,1-Dichloroethene & 5.0 & $\mathrm{U}$ \\
\hline $76-13-1$ & $1,1,2$-Trichloro-1,2,2-trifluoroethane & 5.0 & $\mathrm{U}$ \\
\hline $67-64-1$ & Acetone & 5.0 & $\mathrm{U}$ \\
\hline $75-15-0$ & Carbon disulfide & 5.0 & $\mathrm{U}$ \\
\hline $79-20-9$ & Methyl acetate & 5.0 & $\mathrm{U}$ \\
\hline $75-09-2$ & Methylene chloride & 5.0 & U \\
\hline $156-60-5$ & trans-1,2-Dichloroethene & 5.0 & $\mathrm{U}$ \\
\hline $1634-04-4$ & Methyl tert-butyl ether & 5.0 & U \\
\hline $75-34-3$ & 1,1 -Dichloroethane & 5.0 & $\mathrm{U}$ \\
\hline $156-59-2$ & cis-1,2-Dichloroethene & 5.0 & $\mathrm{U}$ \\
\hline $78-93-3$ & 2-Butanone & 5.0 & $\mathrm{U}$ \\
\hline $67-66-3$ & Chloroform & 2.6 & $\mathrm{~J}$ \\
\hline $71-55-6$ & 1,1,1-Trichloroethane & 5.0 & $\mathrm{U}$ \\
\hline $110-82-7$ & Cyclohexane & 5.0 & $\mathrm{U}$ \\
\hline $56-23-5$ & Carbon Tetrachloride & 5.0 & $\mathrm{U}$ \\
\hline $71-43-2$ & Benzene & 5.0 & $\mathrm{U}$ \\
\hline $107-06-2$ & 1,2-Dichloroethane & 5.0 & $\mathrm{U}$ \\
\hline
\end{tabular}


$1 B$ - FORM I VOA-2

VOLATILE ORGANICS ANALYSIS DATA SHEET
EPA SAMPLE NO.

CNPUBL $-\mathrm{W}-26062$
Lab Name: $\quad$ Envirosystems, Inc.

Lab Code: ENVSYS Case No.:

Matrix: (SOIL/SED/WATER) WATER

Sample wt/vol: $5.00(\mathrm{~g} / \mathrm{mL}) \mathrm{ML}$

Level: (TRACE/LOW/MED)

LOW

c Moisture: not dec.

GC Column: $\quad$ RTX-624 ID: $\underline{0.18}(\mathrm{~mm})$

Soil Extract Volume:

Purge Volume:

5.00

Contract:

Mod. Ref No.:

SDG NO.: $\quad$ ARG71105

Lab Sample ID:

$0071105-01$

Lab File ID:

H73FA241.D

Date Received:

$11 / 16 / 2007$

Date Analyzed:

$11 / 16 / 2007$

Dilution Eactor:

1.0

Soil Aliquot Volume:

(uL)

\begin{tabular}{|c|c|c|c|}
\hline CAS NO. & COMPOUND & $\begin{array}{l}\text { CONCENTRATION UNITS: } \\
(\mathrm{ug} / \mathrm{L} \text { or } \mathrm{ug} / \mathrm{kg}) \mathrm{UG} / \mathrm{L}\end{array}$ & Q \\
\hline $79-01-6$ & Trichloroethene & 5.0 & $\mathrm{U}$ \\
\hline $108-87-2$ & Methylcyclohexane & 5.0 & $\mathrm{U}$ \\
\hline $78-87-5$ & 1,2-Dichloropropane & 5.0 & $\mathrm{U}$ \\
\hline $75-27-4$ & Bromodichloromethane & 5.0 & $\mathrm{U}$ \\
\hline $10061-01-5$ & Cis-1,3-Dichloropropene & 5.0 & $\mathrm{U}$ \\
\hline $108-10-1$ & 4-Methyl-2-pentanone & 5.0 & $\mathrm{U}$ \\
\hline $108-88-3$ & Toluene & 5.0 & $\mathrm{U}$ \\
\hline $10061-02-6$ & trans-1,3-Dichloropropene & 5.0 & $\mathrm{U}$ \\
\hline $79-00-5$ & $1,1,2$-Trichloroethane & 5.0 & $\mathrm{U}$ \\
\hline $127-18-4$ & Tetrachloroethene & 5.0 & $\mathrm{U}$ \\
\hline $591-78-6$ & 2-Hexanone & 5.0 & $\mathrm{U}$ \\
\hline $124-48-1$ & Dibromochloromethane & 5.0 & $\mathrm{U}$ \\
\hline $106-93-4$ & 1,2-Dibromoethane & 5.0 & $\mathrm{U}$ \\
\hline $108-90-7$ & Chlorobenzene & 5.0 & $\mathrm{U}$ \\
\hline $100-41-4$ & Ethylbenzene & 5.0 & $\mathrm{U}$ \\
\hline $95-47-6$ & o-Xylene & 5.0 & $\mathrm{U}$ \\
\hline $179601-23-1$ & $\mathrm{~m}, \mathrm{p}-\mathrm{x} y$ lene & 5.0 & $\mathrm{U}$ \\
\hline $100-42-5$ & Styrene & 5.0 & $\mathrm{U}$ \\
\hline $75-25-2$ & Bromoform & 5.0 & $\mathrm{U}$ \\
\hline $98-82-8$ & Isopropylbenzene & 5.0 & $\mathrm{U}$ \\
\hline $79-34-5$ & $1,1,2,2$-Tetrachloroethane & 5.0 & $\mathrm{U}$ \\
\hline $541-73-1$ & 1,3-Dichlorobenzene & 5.0 & $\mathrm{U}$ \\
\hline $106-46-7$ & 1,4-Dichlorobenzene & 5.0 & $\mathrm{U}$ \\
\hline $95-50-1$ & 1,2-Dichlorobenzene & 5.0 & $\mathrm{U}$ \\
\hline $96-12-8$ & 1,2-Dibromo-3-chloropropane & 5.0 & $\mathrm{U}$ \\
\hline $120-82-1$ & 1,2,4-Trichlorobenzene & 5.0 & $\mathrm{U}$ \\
\hline $91-20-3$ & Naphthalene & 5.0 & $\mathrm{U}$ \\
\hline
\end{tabular}




\section{TestAmerica}

THE LEADER IN ENVIRONMENTAL TESTING

December 11, 2007

Mr. Clyde Dennis

Argonne National Laboratory

9700 S. Cass Avenue, Building 203, Office B149

Argonne, IL 60439

Re: Laboratory Project No. 21005

Case: CENTRALI; SDG: 123144

Dear Mr. Dennis:

Enclosed are analytical results for samples that were received by TestAmerica Burlington on November $28^{\text {th }}, 2007$. Laboratory identification numbers were assigned, and designated as follows:

\begin{tabular}{|c|c|c|}
\hline 21 & $\begin{array}{l}\text { Client } \\
\text { Sample ID }\end{array}$ & $\begin{array}{l}\text { Sample } \\
\text { Date }\end{array}$ \\
\hline
\end{tabular}

Received: 11/28/07 ETR No: 123144

733216

733217

733218

733219

733220

CN-S-19972 (10A)
CN-S-25966D (20A)
CN-S-19950 (20A)
CN-S-16342 (10A)
CN-S-MEOH BLANK

CN-S-19972 (10A)

CN-25960 (20A)

CN-S-MEOH BLANK
$11 / 26 / 07$

$11 / 26 / 07$

$11 / 26 / 07$

$11 / 26 / 07$

$11 / 27 / 07$
LIQUID

LIQUID

LIQUID

LIQUID

LIQUID

Documentation of the condition of the samples at the time of their receipt and any exception to the laboratory's Sample Acceptance Policy is documented in the Sample Handling section of this submittal. In order to accommodate field length limitations in processing the data summary forms, the laboratory did, in certain instances, abbreviate the sample identifier. The electronically formatted data provides for the full sample identifier.

The samples were analyzed by Method $8260 \mathrm{~B}$, using a low-level calibration. In performing the analytical work, 500 microliters of the methanol extract were added to the 5 milliliter purge volume. Each of the analyses associated with the sample set did exhibit an acceptable internal standard performance, and there was an acceptable recovery of the surrogate controls in each analysis. Two types of laboratory control sample analyses were performed in the course of performing the analytical work. One was performed to evaluate method performance, and one was performed with 500 microliters of methanol added to the purge volume in order to characterize the affect on the analytical process. With the exception of that for 1,4-dioxane, there was an acceptable recovery of each target analyte in the laboratory control sample analysis that 
defined method performance. The recovery of 1,4-dioxane was low in that analysis (58 percent). In the laboratory control sample analysis with methanol, several of the earlier eluting compounds did exhibit a lower recovery performance, as did, generally, the ketones and alcohols. Most significantly affected was the recovery performance of acrolein (39 percent) and acetone (46 percent). The recovery of each of the other compounds was greater than 50 percent. Chloroform and carbon tetrachloride were recovered well in each of the laboratory control sample analyses. Matrix spike and matrix spike duplicate analyses were not performed on samples in this sample set. Trace concentrations of chloromethane, bromomethane, methyl iodide, and 2-butanone were identified in the analysis sample CN-S-MEOH BLANK. The laboratory did associate the analysis of CN-S-MEOH BLANK with the analysis of each of the field samples in order to reference the blank association, and accordingly qualify the reported results. A trace concentration of methyl iodide was identified in the analysis of the instrument blank associated with the analytical work. The laboratory did provide for a methanol blank in the analytical sequence in order to properly characterize the results of the laboratory control sample analysis that was performed with 500 microliters of methanol added to the purge volume.

The analytical results associated with the samples presented in this test report were generated under a quality system that adheres to requirements specified in the NELAC standard. Release of the data in this test report and any associated electronic deliverables is authorized by the Laboratory Director's designee as verified by the following signature.

If there are any questions regarding this submittal, please contact me at 802 660-1990.

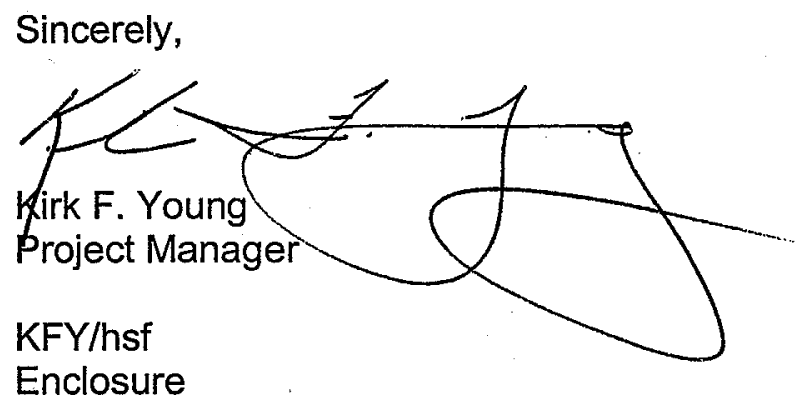




\section{TestAmerica Burlington Data Qualifier Definitions}

\section{Organic}

U: Compound analyzed but not detected at a concentration above the reporting limit.

J: $\quad$ Estimated value.

N: Indicates presumptive evidence of a compound. This flag is used only for tentatively identified compounds (TICs) where the identification of a compound is based on a mass spectral library search.

P: $\quad$ SW-846: Greater than 40\% difference for detected concentrations between two GC columns. Unless otherwise specified the higher of the two values is reported on the Form 1 .

CLP SOW: Greater than 25\% difference for detected concentrations between two GC columns. Unless otherwise specified the lower of the two values is reported on the Form I.

C: Pesticide result whose identification has been confirmed by GC/MS.

B: $\quad$ Analyte is found in the sample and the associated method blank. The flag is used for tentatively identified compounds as well as positively identified compounds.

E: Compounds whose concentrations exceed the upper limit of the calibration range of the instrument for that specific analysis.

D: Concentrations identified from analysis of the sample at a secondary dilution.

A: Tentatively identified compound is a suspected aldol condensation product.

$X, Y, Z$ : Laboratory defined flags that may be used alone or combined, as needed. If used, the description of the flag is defined in the project narrative.

\section{Inorganic/Metals}

E: Reported value is estimated due to the presence of interference.

$\mathrm{N}$ : Matrix spike sample recovery is not within control limits.

* Duplicate sample analysis is not within control limits.

B: The result reported is less than the reporting limit but greater than the instrument detection limit.

U: $\quad$ Analyte was analyzed for but not detected above the reporting limit.

Method Codes:

P ICP-AES

MS ICP-MS

CV Cold Vapor AA

AS Semi-Automated Spectrophotometric 


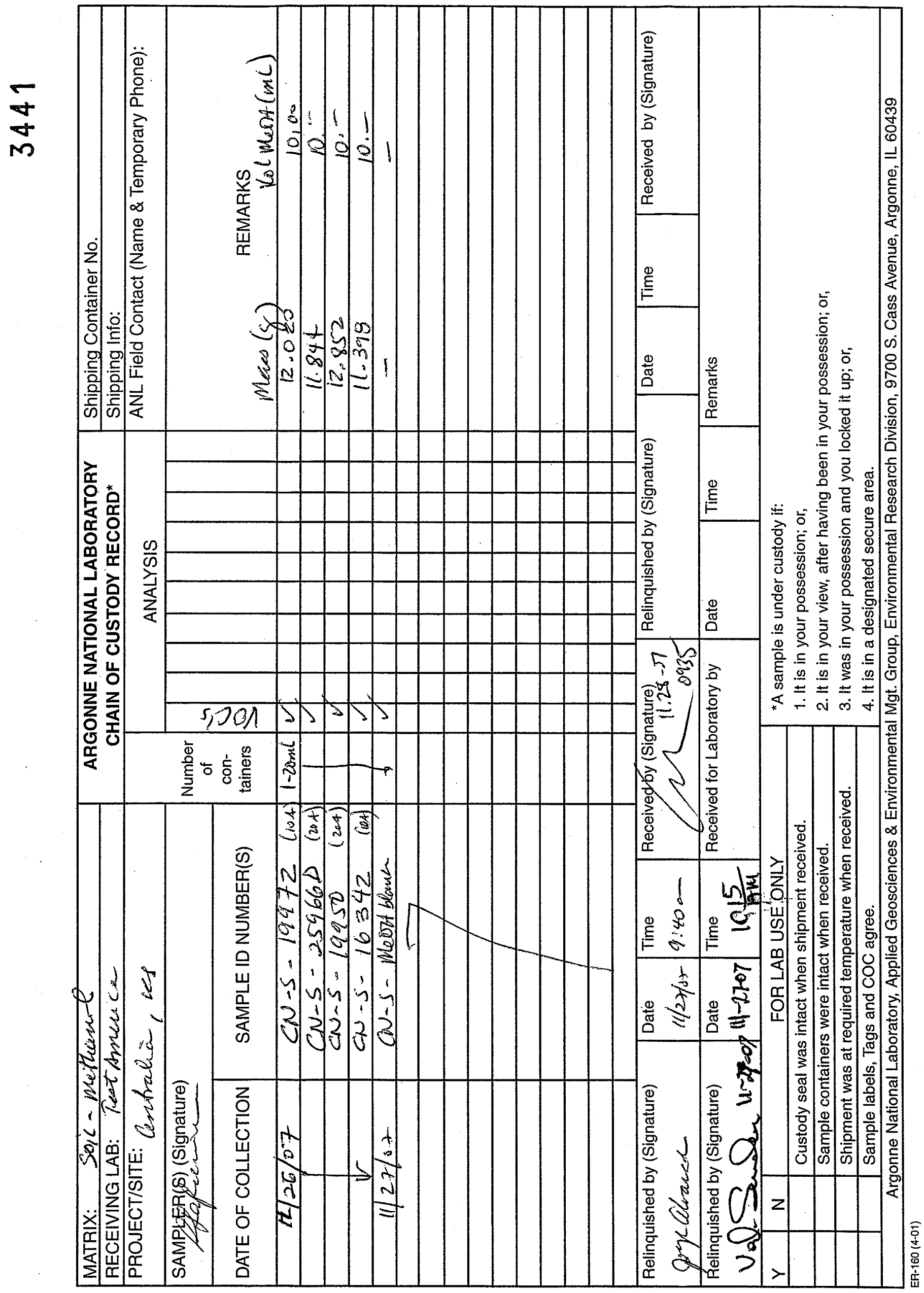


FORM 1

VOLATILE ORGANICS ANALYSIS DATA SHEET

Lab Name: TESTAMERICA BURLINGTON

Contract: 21005

Lab Code: STLV Case No.: CENTRALI SAS No.:

ARGLAB SAMPLE NO.

CN-S-16342 10A

SDG No.: 123144

Matrix: (soil/water) SOIL

Lab Sample ID: 733219

Sample wt/vol: $\quad 11.4(\mathrm{~g} / \mathrm{mL})$ G

Lab File ID: 733219

Level: (low/med) MED

Date Received: 11/28/07

\% Moisture: not dec.

GC Column: CAP ID: 0.53 (mm)

Date Analyzed: 12/03/07

Soil Extract Volume: 10000 (uL)

Dilution Factor: 1.0

Soil Aliquot Volume:

500 (uL)

CONCENTRATION UNITS:

CAS NO.

COMPOUND

(ug/L or ug/Kg) UG/KG

$Q$

\begin{tabular}{r|l|}
8.8 & $\mathrm{U}$ \\
2.6 & $\mathrm{JB}$ \\
8.8 & $\mathrm{U}$ \\
6.2 & $\mathrm{JB}$ \\
8.8 & $\mathrm{U}$ \\
8.8 & $\mathrm{U}$ \\
44 & $\mathrm{U}$ \\
8.8 & $\mathrm{U}$ \\
8.8 & $\mathrm{U}$ \\
44 & $\mathrm{U}$ \\
6.5 & $\mathrm{UB}$ \\
8.8 & $\mathrm{U}$ \\
8.8 & $\mathrm{U}$ \\
8.8 & $\mathrm{U}$ \\
8.8 & $\mathrm{U}$ \\
8.8 & $\mathrm{U}$ \\
8.8 & $\mathrm{U}$ \\
8.8 & $\mathrm{U}$ \\
8.8 & $\mathrm{U}$ \\
8.8 & $\mathrm{U}$ \\
8.8 & $\mathrm{U}$ \\
8.8 & $\mathrm{U}$ \\
8.8 & $\mathrm{U}$ \\
17 & $\mathrm{JB}$ \\
35 & $\mathrm{U}$ \\
8.8 & $\mathrm{U}$ \\
8.8 & $\mathrm{U}$ \\
120 & $\mathrm{U}$ \\
7.0 & $\mathrm{~J}$ \\
8.8 & $\mathrm{U}$ \\
180 & \\
8.8 & $\mathrm{U}$ \\
8.8 & $\mathrm{U}$ \\
\hline & \\
\hline & \\
\hline
\end{tabular}


FORM 1

VOLATILE ORGANICS ANALYSIS DATA SHEET
ARGLAB SAMPLE NO.

$\mathrm{CN}-\mathrm{S}-16342 \quad 10 \mathrm{~A}$

Lab Name: TESTAMERICA BURLINGTON Contract: 21005

Lab Code: STLV Case No.: CENTRALI SAS No.:

SDG No.: 123144

Matrix: (soil/water) SoIL

Sample wt/vol: $\quad 11.4(\mathrm{~g} / \mathrm{mL}) \mathrm{G}$

Level: (low/med) MED

$\%$ Moisture: not dec.

GC Column: CAP ID: 0.53 (mm)

Soil Extract Volume: 10000 (uL)
Lab Sample ID: 733219

Lab File ID: 733219

Date Received: 11/28/07

Date Analyzed: 12/03/07

Dilution Factor: 1.0

Soil Aliquot Volume:

500 (uL)

CAS NO.

COMPOUND

CONCENTRATION UNITS:

(ug/I or $\mathrm{ug} / \mathrm{Kg}$ ) UG/KG

$440 \mathrm{U}$

$8.8 \mathrm{U}$

$8.8 \mathrm{U}$

$8.8 \mathrm{U}$

$8.8 \mathrm{U}$

$8.8 \mathrm{U}$

$440 \mathrm{U}$

$8.8 \mathrm{U}$

$8.8 \mathrm{U}$

$8.8 \mathrm{U}$

$44 \mathrm{U}$

$8.8 \mathrm{U}$

$8.8 \mathrm{U}$

$8.8 \mathrm{U}$

$8.8 \mathrm{U}$

$8.8 \mathrm{U}$

$8.8 \mathrm{U}$

$44 \mathrm{U}$

$8.8 \mathrm{U}$

$8.8 \mathrm{U}$

$8.8 \mathrm{U}$

$8.8 \mathrm{U}$

$8.8 \mathrm{U}$

$8.8 \mathrm{U}$

$8.8 \mathrm{U}$

$8.8 \mathrm{U}$

$8.8 \mathrm{U}$

$8.8 \mathrm{U}$

$8.8 \mathrm{U}$

$8.8 \mathrm{U}$

$8.8 \mathrm{U}$

$8.8 \mathrm{U}$

$8.8 \mathrm{U}$ 
FORM 1

VOLATILE ORGANICS ANALYSIS DATA SHEET

Lab Name: TESTAMERICA BURLINGTON

Case No.: CENTRALI SAS NO.:

Iab code: STLV
Contract: 21005
ARGLAB SAMPLE NO.

$\mathrm{CN}-\mathrm{S}-16342 \quad$ 10A
Matrix: (soil/water) soIL

Sample wt/vol:

$11.4(\mathrm{~g} / \mathrm{mL}) \mathrm{G}$

Level: (low $/ \mathrm{med}$ ) MED

\% Moisture: not dec.

GC Column: CAP ID: 0.53 (mm)

Soil Extract Volume: 10000 (uL)
SDG NO.: 123144
Lab Sample ID: 733219

Lab File ID: 733219

Date Received: 11/28/07

Date Analyzed: 12/03/07

Dilution Factor: 1.0

Soil Aliquot Volume:

$500(\mathrm{uL})$

CAS NO. COMPOUND (ug/L or ug/Kg) UG/KG

Q

110-57-6---.---trans-1,4-Dichloro-2-butene 103-65-1------n-Propylbenzene

95-49-8--.----2-Chlorotoluene 106-43-4-....-4-Chlorotoluene

$108-67-8-\ldots-\ldots-1,3,5-$ Trimethyl benzene

98-06-6--.----tert-Butylbenzene

95-63-6-.-----1, 2, 4-Trimethylbenzene

135-98-8------sec-Butylbenzene

541-73-1-..--1, 3-Dichlorobenzene

99-87-6-------4-Isopropyl toluene

106-46-7------1,4-Dichlorobenzene

95-50-1-....--1,2-Dichlorobenzene

104-51-8--.---n-Butylbenzene

96-12-8------ 1, 2-Dibromo-3-Chloropropane

120-82-1------1,2,4-Trichlorobenzene

87-68-3--..---Hexachlorobutadiene.

91-20-3--.---Naphthalene

87-61-6-...---1, 2, 3-Trichlorobenzene
$8.8 \mathrm{U}$

$8.8 \mathrm{U}$

$8.8 \mathrm{U}$

$8.8 \mathrm{U}$

$8.8 \mathrm{U}$

$8.8 \mathrm{U}$

$8.8 \mathrm{U}$

$8.8 \mathrm{U}$

$8.8 \mathrm{U}$

$8.8 \mathrm{U}$

$8.8 \mathrm{U}$

$8.8 \mathrm{U}$

$8.8 \mathrm{U}$

$8.8 \mathrm{U}$

$8.8 \mathrm{U}$

$8.8 \mathrm{U}$

$8.8 \mathrm{U}$

$8.8 \mathrm{U}$ 
FORM 1

VOLATILE ORGANICS ANALYSIS DATA SHEET

Lab Name: TESTAMERICA BURLINGTON Contract: 21005

Lab Code: STLV Case No.: CENTRALI SAS No.:

Lab Sample ID: 733218

Matrix: (soil/water) SOIL

Sample wt/vol: $\quad 12.9(\mathrm{~g} / \mathrm{mL}) \mathrm{G}$

Level : (low $/ \mathrm{med}) \quad$ MED

\% Moisture: not dec.

GC Column: CAP ID: 0.53 (mm)

Soil Extract Volume: 10000 (uL)

Lab File ID: 733218

Date Received: 11/28/07

Date Analyzed: 12/03/07

Dilution Factor: 1.0

Soil Aliquot Volume:
COMPOUND
ARGLAB SAMPLE NO.

CN-S-19950 20A

SDG NO.: 123144

CAS NO.

500 (uL)

CONCENTRATION UNITS :

(ug/L or $\mathrm{ug} / \mathrm{Kg}$ ) UG/KG

Q

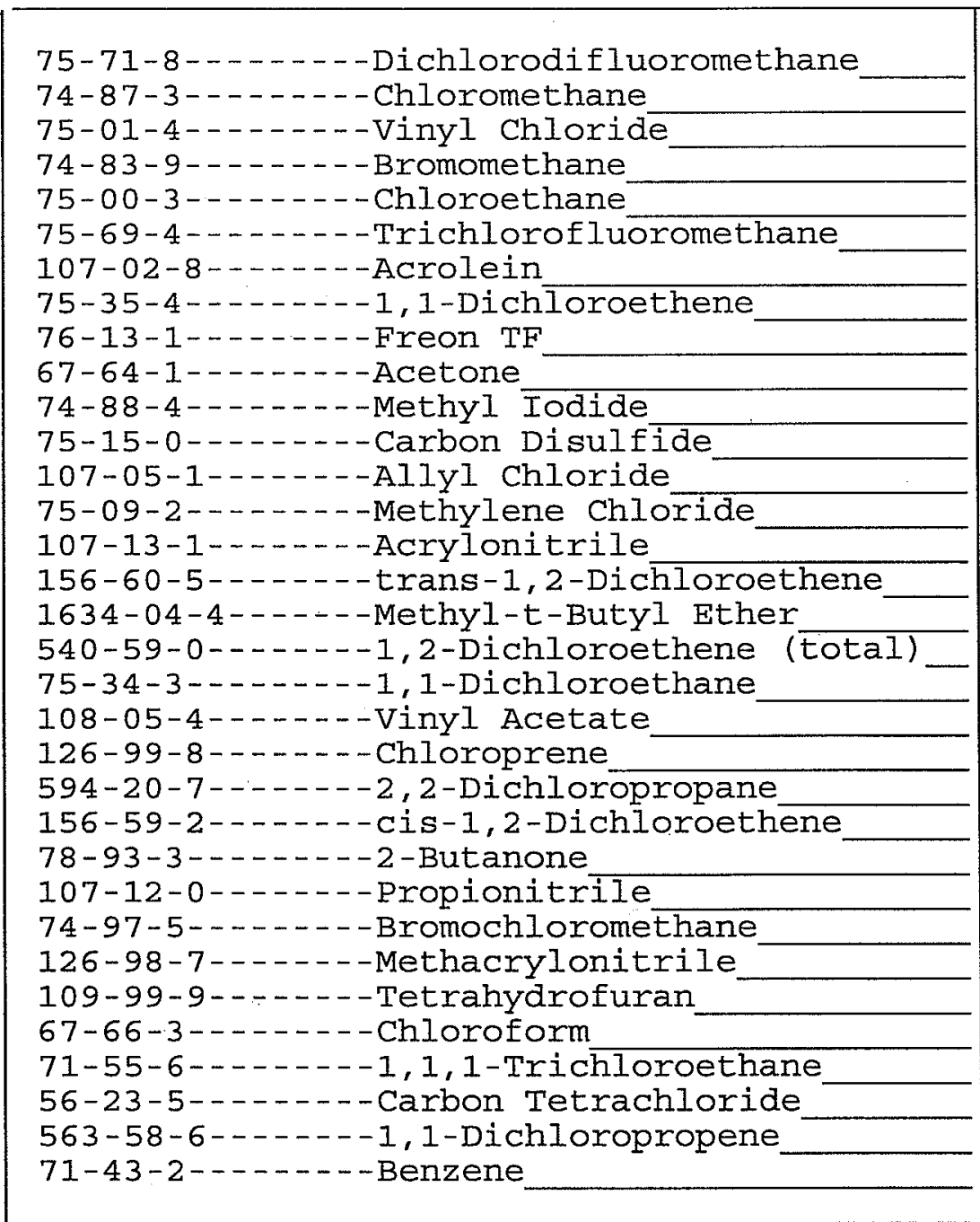

\begin{tabular}{r|l|}
7.8 & $\mathrm{U}$ \\
2.5 & $\mathrm{JB}$ \\
7.8 & $\mathrm{U}$ \\
8.9 & $\mathrm{~B}$ \\
7.8 & $\mathrm{U}$ \\
7.8 & $\mathrm{U}$ \\
39 & $\mathrm{U}$ \\
7.8 & $\mathrm{U}$ \\
7.8 & $\mathrm{U}$ \\
39 & $\mathrm{U}$ \\
7.8 & $\mathrm{~B}$ \\
7.8 & $\mathrm{U}$ \\
7.8 & $\mathrm{U}$ \\
23 & \\
7.8 & $\mathrm{U}$ \\
7.8 & $\mathrm{U}$ \\
7.8 & $\mathrm{U}$ \\
7.8 & $\mathrm{U}$ \\
7.8 & $\mathrm{U}$ \\
7.8 & $\mathrm{U}$ \\
7.8 & $\mathrm{U}$ \\
7.8 & $\mathrm{U}$ \\
7.8 & $\mathrm{U}$ \\
16 & $\mathrm{JB}$ \\
31 & $\mathrm{U}$ \\
7.8 & $\mathrm{U}$ \\
7.8 & $\mathrm{U}$ \\
110 & $\mathrm{U}$ \\
2.8 & $\mathrm{~J}$ \\
7.8 & $\mathrm{U}$ \\
2.2 & $\mathrm{~J}$ \\
7.8 & $\mathrm{U}$ \\
7.8 & $\mathrm{U}$ \\
& \\
\hline 7
\end{tabular}


FORM 1

VOLATILE ORGANICS ANALYSIS DATA SHEET

Lab Name: TESTAMERICA BURLINGTON Contract: 21005

Lab Code: STLV

Case No.: CENTRALI SAS No.:

\section{ARGLAB SAMPLE NO.}

CN-S-19950 20A
Matrix: (soil/water) SoIL

Sample wt/vol:

$12.9(\mathrm{~g} / \mathrm{mL}) \mathrm{G}$

Level: (low/med) MED

\% Moisture: not dec.

GC Column: CAP ID: $0.53 \quad(\mathrm{~mm})$

Soil Extract Volume: 10000 (uL)
Lab Sample ID: 733218

Lab File ID: $\quad 733218$

Date Received: 11/28/07

Date Analyzed: 12/03/07

Dilution Factor: 1.0

Soil Aliquot Volume:

500 (UL)

CAS NO.

COMPOUND

CONCENTRATION UNITS :

$(\mathrm{ug} / \mathrm{L}$ or $\mathrm{ug} / \mathrm{Kg}) \mathrm{UG} / \mathrm{KG}$

$Q$

78-83-1-...---Isobutyl Alcohol

107-06-2--.-.-1,2-Dichloroethane

79-01-6-------Trichloroethene

78-87-5-...--1, 2-Dichloropropane

74-95-3-...--Dibromomethane

80-62-6--..--Methyl Methacrylate

123-91-1--.--1, 4-Dioxane

75-27-4-...--.-Bromodichloromethane

110-75-8-..--2-Chloroethyl Vinyl Ether

10061-01-5--.--cis-1,3-Dichloropropene

108-10-1------4-Methyl-2-pentanone

108-88-3-...--Toluene

10061-02-6-----trans-1,3-Dichloropropene

97-63-2-------Ethyl Methacrylate

79-00-5------1, 1,2-Trichloroethane

127-18-4-_.--Tetrachloroethene

142-28-9--.--1, 3-Dichloropropane

$591-78-6-\cdots---2-$ Hexanone

124-48-1-....-Dibromochloromethane

106-93-4-----1,2-Dibromoethane

108-90-7-..---Chlorobenzene

630-20-6-...-1, 1, 1,2-Tetrachloroethane

100-41-4--.-.-Ethylbenzene

1330-20-7-...-Xylene $(\mathrm{m}, \mathrm{p})$

95-47-6--.-.-Xylene (o)

1330-20-7-..--Xylene (total)

100-42-5------styrene

75-25-2-...---Bromoform

98-82-8-1-..--Isopropyl benzene

1476-11-5-...-.-cis-1,4-Dichloro-2-butene

108-86-1--.---Bromobenzene

79-34-5-..--1, 1,2, 2-Tetrachloroethane

96-18-4-...--1,2,3-Trichloropropane

$390 \mathrm{U}$

$7.8 \mathrm{U}$

$7.8 \mathrm{U}$

$7.8 \mathrm{U}$

$7.8 \mathrm{U}$

$7.8 \mathrm{U}$

$390 \mathrm{U}$

$7.8 \mathrm{U}$

$7.8 \mathrm{U}$

$7.8 \mathrm{U}$

$39 \mathrm{U}$

$6.8 \mathrm{~J}$

$7.8 \mathrm{U}$

$7.8 \mathrm{U}$

$7.8 \mathrm{U}$

$7.8 \mathrm{U}$

$7.8 \mathrm{U}$

$39 \mathrm{U}$

$7.8 \mathrm{U}$

$7.8 \mathrm{U}$

$7.8 \mathrm{U}$

$7.8 \mathrm{U}$

$7.8 \mathrm{U}$

$7.8 \mathrm{U}$

$7.8 \mathrm{U}$

$7.8 \mathrm{U}$

$7.8 \mathrm{U}$

$7.8 \mathrm{U}$

$7.8 \mathrm{U}$

$7.8 \mathrm{U}$

$7.8 \mathrm{U}$

$7.8 \mathrm{U}$

$7.8 \mathrm{U}$ 
FORM 1

VOLATILE ORGANICS ANALYSIS DATA SHEET

Lab Name: TESTAMERICA BURLINGTON Contract: 21005

Lab Code: STLV Case No.: CENTRALI SAS No.:

Lab Sample ID: 733218

Matrix: (soil/water) SOIL

Sample wt/vol:

$12.9(\mathrm{~g} / \mathrm{mL}) \mathrm{G}$

Level: (low/med) MED

\% Moisture: not dec.

GC Column: CAP ID: 0.53 (mm)

Soil Extract Volume: 10000 (uL)

Lab File ID: $\quad 733218$

Date Received: 11/28/07

Date Analyzed: 12/03/07

Dilution Factor: 1.0

Soil Aliquot Volume:
ARGLAB SAMPLE NO.

$\mathrm{CN}-\mathrm{S}-19950 \quad 20 \mathrm{~A}$

SDG No.: 123144

500 (uL)

CONCENTRATION UNITS :

CAS NO.

COMPOUND

(ug/L or $\mathrm{ug} / \mathrm{Kg}$ ) UG/KG

Q

110-57-6------trans-1, 4-Dichloro-2-butene

103-65-1-...-n-Propylbenzene

95-49-8-..-.--2-Chlorotoluene

106-43-4--..--4-Chlorotoluene

108-67-8------1,3,5-Trimethylbenzene

98-06-6--..---tert-Butylbenzene

95-63-6-...-.-1, 2, 4-Trimethylbenzene

135-98-8-...--sec-Butylbenzene

541-73-1------1, 3-Dichlorobenzene

99-87-6-- - - --4-Isopropyltoluene

106-46-7-..---1,4-Dichlorobenzene

95-50-1-...-.-1, 2-Dichlorobenzene

104-51-8--.---n-Butylbenzene

96-12-8-- - - - 1, 2-Dibromo-3-Chloropropane

120-82-1-...--1, 2,4-Trichlorobenzene

87-68-3--.----Hexachlorobutadiene

91-20-3-...--Naphthalene

87-61-6-...-.-1,2,3-Trichlorobenzene

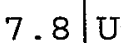

$7.8 \mathrm{U}$

$7.8 \mathrm{U}$

$7.8 \mathrm{U}$

$7.8 \mathrm{U}$

$7.8 \mathrm{U}$

$7.8 \mathrm{U}$

$7.8 \mathrm{U}$

$7.8 \mathrm{U}$

$7.8 \mathrm{U}$

$7.8 \mathrm{U}$

$7.8 \mathrm{U}$

$7.8 \mathrm{U}$

$7.8 \mathrm{U}$

$7.8 \mathrm{U}$

$7.8 \mathrm{U}$

$7.8 \mathrm{U}$

$7.8 \mathrm{U}$ 
FORM 1

VOLATILE ORGANICS ANALYSIS DATA SHEET
ARGLAB SAMPLE NO. CN-S-19972 10A
Lab Name: TESTAMERICA BURLINGTON Contract: 21005
SDG NO.: 123144

Lab Code: STLV Case No.: CENTRALI SAS No.:

Lab Sample ID: 733216

Matrix: (soil/water) soIL

Lab File ID: 733216

sample wt/vol:

$12.0(\mathrm{~g} / \mathrm{mL}) \mathrm{G}$

Date Received: 11/28/07

Level: (low/med) MED

Date Analyzed: 12/03/07

$\div$ Moisture: not dec.

GC Column: CAP ID: 0.53 (mm)

Dilution Factor: 1.0

Soil Extract Volume: 10000 (uL)

Soil Aliquot Volume:

500 (uL)

CONCENTRATION UNITS :

CAS NO.

COMPOUND

(ug/L or $\mathrm{ug} / \mathrm{Kg}$ ) UG/KG

Q

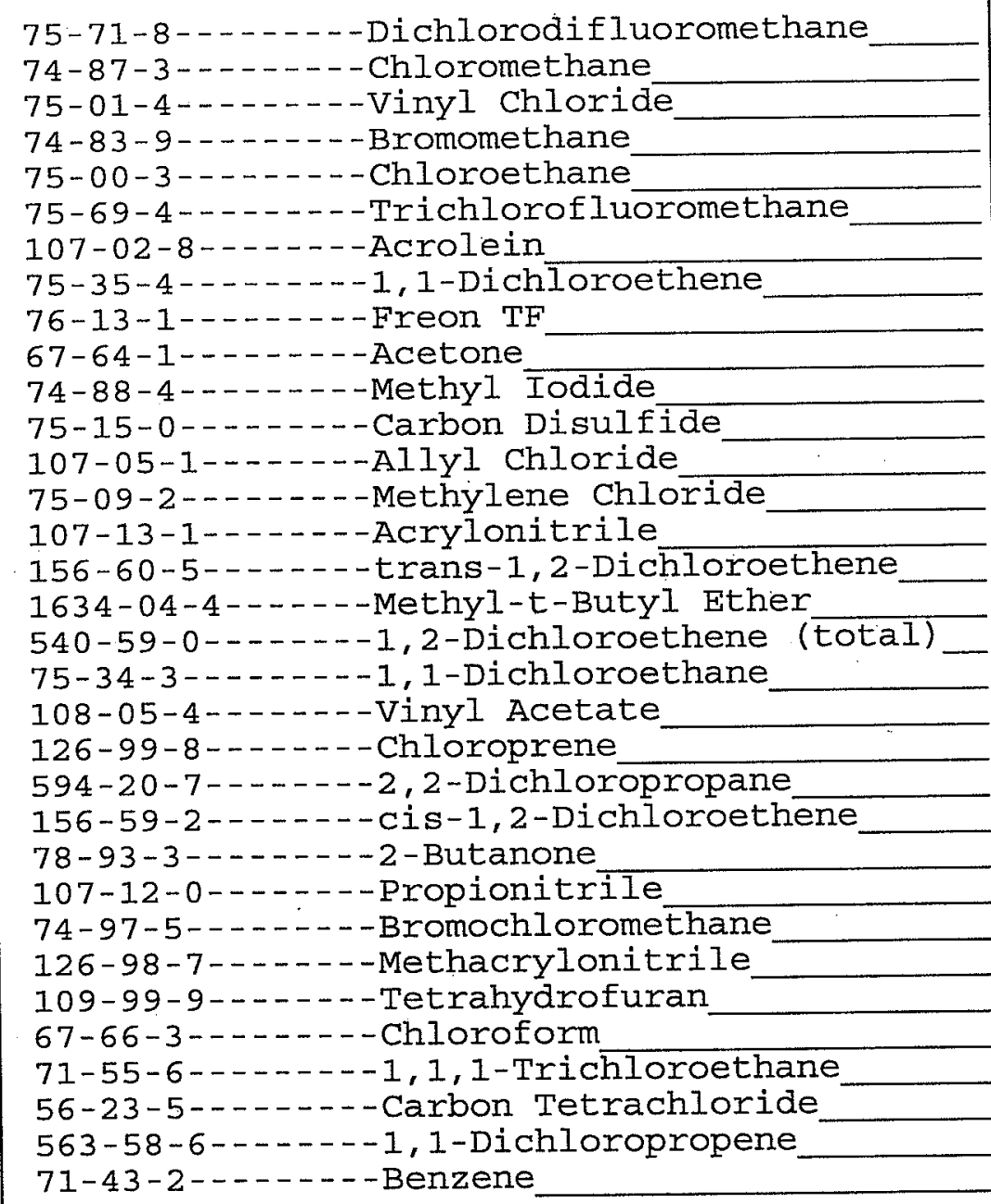

$8.3 \mathrm{U}$

$3.1 \mathrm{JB}$

$8.3 \mathrm{U}$

$5.9 \mathrm{JB}$

$8.3 \mathrm{U}$

$8.3 \mathrm{U}$

$42 \mathrm{U}$

$8.3 \mathrm{U}$

$8.3 \mathrm{U}$

$42 \mathrm{U}$

$6.0 \mathrm{JB}$

$8.3 \mathrm{U}$

$8.3 \mathrm{U}$

$8.3 \mathrm{U}$

$8.3 \mathrm{U}$

$8.3 \mathrm{U}$

$8.3 \mathrm{U}$

$8.3 \mathrm{U}$

$8.3 \mathrm{U}$

$8.3 \mathrm{U}$

$8.3 \mathrm{U}$

$8.3 \mathrm{U}$

$8.3 \mathrm{U}$

$13 \mathrm{JB}$

$33 \mathrm{U}$

$8.3 \mathrm{U}$

$8.3 \mathrm{U}$

$120 \mathrm{U}$

$8.3 \mathrm{U}$

$8.3 \mathrm{U}$

$4.2 \mathrm{~J}$

$8.3 \mathrm{U}$

$8.3 \mathrm{U}$ 
FORM 1

VOLATILE ORGANICS ANALYSIS DATA SHEET

Lab Name: TESTAMERICA BURLINGTON Contract: 21005

Lab Code: STLV Case No.: CENTRALI SAS No.:

Lab Sample ID: 733216

Matrix: (soil/water) SOIL

Lab File ID: 733216

Sample wt/vol:

$12.0(\mathrm{~g} / \mathrm{mL}) \mathrm{G}$

Level: (low/med) MED

$\%$ Moisture: not dec.

GC Column: CAP ID: 0.53 (mm)

Soil Extract Volume: 10000 (uL)

CAS NO.
ARGLAB SAMPLE NO.

$\mathrm{CN}-\mathrm{S}-19972 \quad 10 \mathrm{~A}$

SDG No.: 123144
Date Received: 11/28/07

Date Analyzed: 12/03/07

Dilution Factor: 1.0

Soil Aliquot Volume:

500 (uL)
$420 \mathrm{U}$

$8.3 \mathrm{U}$

$8.3 \mathrm{U}$

$8.3 \mathrm{U}$

$8.3 \mathrm{U}$

$8.3 \mathrm{U}$

$420 \mathrm{U}$

$8.3 \mathrm{U}$

$8.3 \mathrm{U}$

$8.3 \mathrm{U}$

$42 \mathrm{U}$

$8.3 \mathrm{U}$

$8.3 \mathrm{U}$

$8.3 \mathrm{U}$

$8.3 \mathrm{U}$

$8.3 \mathrm{U}$

$8.3 \mathrm{U}$

$42 \mathrm{U}$

$8.3 \mathrm{U}$

$8.3 \mathrm{U}$

$8.3 \mathrm{U}$

$8.3 \mathrm{U}$

$8.3 \mathrm{U}$

$8.3 \mathrm{U}$

$8.3 \mathrm{U}$

$8.3 \mathrm{U}$

$8.3 \mathrm{U}$

$8.3 \mathrm{U}$

$8.3 \mathrm{U}$

$8.3 \mathrm{U}$

$8.3 \mathrm{U}$

$8.3 \mathrm{U}$

$8.3 \mathrm{U}$ 
FORM 1

VOLATILE ORGANICS ANALYSIS DATA SHEET

Lab Name: TESTAMERICA BURLINGTON Contract: 21005

Lab Code: STLV Case No.: CENTRALI SAAS No.:

Lab Sample ID: 733216

Matrix: (soil/water) soIL

$12.0(\mathrm{~g} / \mathrm{mL}) \mathrm{G}$

Sample wt/vol:

Level: (low/med) MED

$\div$ Moisture: not dec.

GC Column: CAP ID: 0.53 (mm)

Soil Extract Volume: 10000 (uL)

Lab File ID: 733216

Date Received: 11/28/07

Date Analyzed: 12/03/07

Dilution Factor: 1.0

Soil Aliquot Volume:
ARGLAB SAMPLE NO.

CN-S-19972 10A

500 (uL)

CONCENTRATION UNITS :

CAS NO.

COMPOUND

(ug/L or $\mathrm{ug} / \mathrm{Kg}) \quad \mathrm{UG} / \mathrm{KG}$

110-57-6-...-trans-1,4-Dichloro-2-butene 103-65-1-.----n-Propylbenzene 95-49-8-------2-Chlorotoluene 106-43-4-...-4-Chlorotoluene 108-67-8-...-1, 3,5-Trimethyl benzene 98-06-6--.----tert-Butylbenzene 95-63-6--.---1, 2,4-Trimethylbenzene 135-98-8--.-.-sec-Butylbenzene 541-73-1--.--1, 3-Dichlorobenzene 99-87-6-1.---4-Isopropyltoluene 106-46-7-...--1,4-Dichlorobenzene 95-50-1-.-.-.-1,2-Dichlorobenzene 104-51-8--.--n-Butylbenzene 96-12-8--..--1, 2-Dibromo-3-Chloropropane 120-82-1-..---1,2,4-Trichlorobenzene 87-68-3-...-.-Hexachlorobutadiene 91-20-3--.---Naphthalene 87-61-6-...-.-1, 2,3-Trichlorobenzene
$8.3 \mathrm{U}$

$8.3 \mathrm{U}$

$8.3 \mathrm{U}$

$8.3 \mathrm{U}$

$8.3 \mathrm{U}$

$8.3 \mathrm{U}$

$8.3 \mathrm{U}$

$8.3 \mathrm{U}$

$8.3 \mathrm{U}$

$8.3 \mathrm{U}$

$8.3 \mathrm{U}$

$8.3 \mathrm{U}$

$8.3 \mathrm{U}$

$8.3 \mathrm{U}$

$8.3 \mathrm{U}$

$8.3 \mathrm{U}$

$8.3 \mathrm{U}$

$8.3 \mathrm{U}$ 
FORM 1

VOLATILE ORGANICS ANALYSIS DATA SHEET

Lab Name: TESTAMERICA BURLINGTON Contract: 21005

Lab Code: STLV Case No.: CENTRALI SAS No.:
ARGLAB SAMPLE NO.

$\mathrm{CN}-\mathrm{S}-25966 \mathrm{D} 20 \mathrm{~A}$
Matrix: (soil/water) soIL

Sample wt/vol: $\quad 11.8(\mathrm{~g} / \mathrm{mL}) \mathrm{G}$

Level: (low/med) MED

$\div$ Moisture: not dec.

GC Column: CAP ID: 0.53 (mm)

Soil Extract Volume: 10000 (uL)
Lab Sample ID: 733217

Lab File ID: $\quad 733217$

Date Received: 11/28/07

Date Analyzed: 12/03/07

Dilution Factor: 1.0

Soil Aliquot Volume:

500 (UL)
CAS NO.
COMPOUND
CONCENTRATION UNITS:

(ug/L or ug/Kg) UG/KG

Q

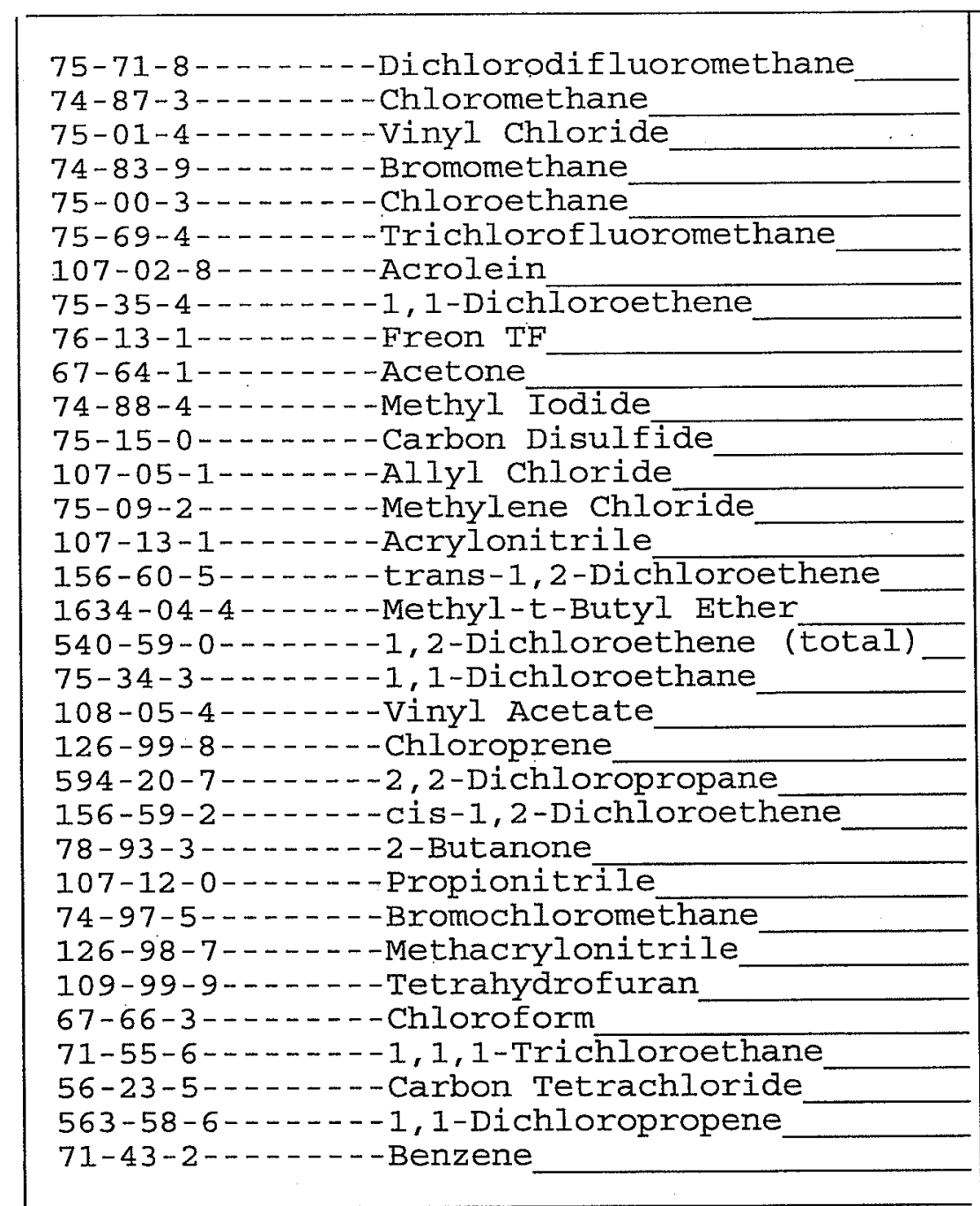

\begin{tabular}{|l|l|}
8.4 & $\mathrm{U}$ \\
3.8 & $\mathrm{JB}$ \\
8.4 & $\mathrm{U}$ \\
8.3 & $\mathrm{JB}$ \\
8.4 & $\mathrm{U}$ \\
8.4 & $\mathrm{U}$ \\
42 & $\mathrm{U}$ \\
8.4 & $\mathrm{U}$ \\
8.4 & $\mathrm{U}$ \\
42 & $\mathrm{U}$ \\
7.1 & $\mathrm{JB}$ \\
8.4 & $\mathrm{U}$ \\
8.4 & $\mathrm{U}$ \\
8.4 & $\mathrm{U}$ \\
8.4 & $\mathrm{U}$ \\
8.4 & $\mathrm{U}$ \\
8.4 & $\mathrm{U}$ \\
8.4 & $\mathrm{U}$ \\
8.4 & $\mathrm{U}$ \\
8.4 & $\mathrm{U}$ \\
8.4 & $\mathrm{U}$ \\
8.4 & $\mathrm{U}$ \\
8.4 & $\mathrm{U}$ \\
13 & $\mathrm{JB}$ \\
34 & $\mathrm{U}$ \\
8.4 & $\mathrm{U}$ \\
8.4 & $\mathrm{U}$ \\
120 & $\mathrm{U}$ \\
8.4 & $\mathrm{U}$ \\
8.4 & $\mathrm{U}$ \\
8.2 & $\mathrm{~J}$ \\
8.4 & $\mathrm{U}$ \\
8.4 & $\mathrm{U}$ \\
& \\
\hline
\end{tabular}


FORM 1

VOLATILE ORGANICS ANALYSIS DATA SHEET

Lab Name: TESTAMERICA BURLINGTON Contract: 21005

Lab Code: STLV

Case No.: CENTRALI SAS NO.:

Lab Sample ID: 733217

Matrix: (soil/water) SOIL

Lab File ID: $\quad 733217$

Sample wt/vol:

$11.8(\mathrm{~g} / \mathrm{mL}) \mathrm{G}$

Level: (low/med) MED

\% Moisture: not dec.

GC Column: CAP ID: $0.53 \quad(\mathrm{~mm})$

Soil Extract Volume: 10000 (uL)

Date Received: 11/28/07

Date Analyzed: 12/03/07

Dilution Factor: 1.0

Soil Aliquot volume:
ARGLAB SAMPLE NO.

CN-S-25966D 20A

SDG No.: 123144

500 (UL)

CONCENTRATION UNITS :

CAS NO. COMPOUND $\quad(\mathrm{ug} / \mathrm{L}$ or $\mathrm{ug} / \mathrm{Kg}) \quad$ UG/KG

Q

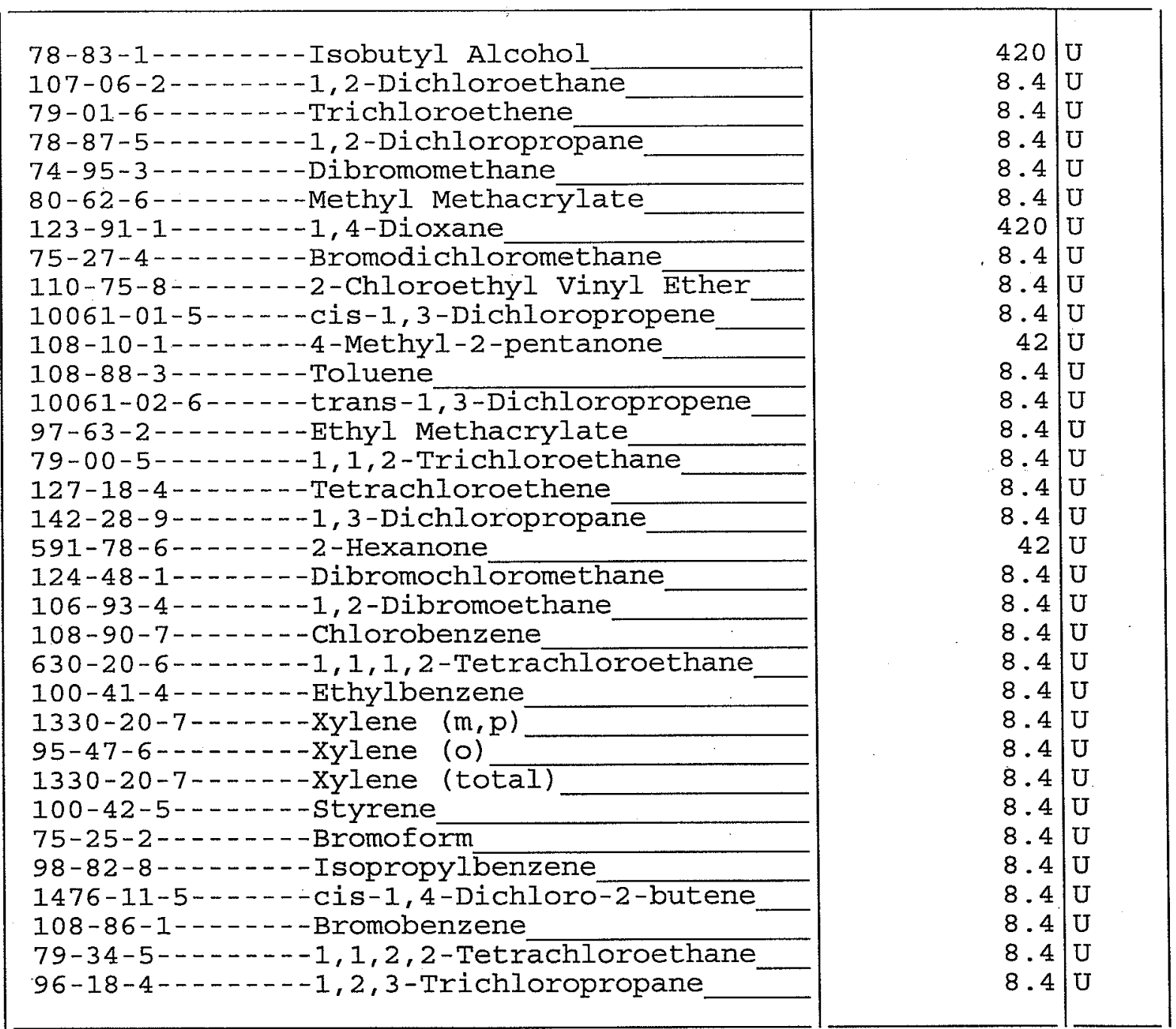


FORM 1

VOLATILE ORGANICS ANALYSIS DATA SHEET

Lab Name: TESTAMERICA BURLINGTON

Contract: 21005

Lab Code: STLV

Case No:: CENTRALI SAS No.:
ARGLAB SAMPLE NO.

CN-S-25966D 20A

SDG No.: 123144
Matrix: (soil/water) SOIL

Sample wt/vol:

Level: (low/med) MED

$\because$ Moisture: not dec.

GC Column: CAP

ID $: 0.53 \quad(\mathrm{~mm})$

Soil Extract Volume: 10000 (uL)
Lab Sample ID: 733217

Lab File ID: $\quad 733217$

Date Received: 11/28/07

Date Analyzed: 12/03/07

Dilution Factor: 1.0

Soil Aliquot Volume:

500 (uL)

CAS NO.

COMPOUND

CONCENTRATION UNITS:

(ug/L or ug/Kg) UG/KG

Q

$8.4 \mathrm{U}$

$8.4 \mathrm{U}$

$8.4 \mathrm{U}$

$8.4 \mathrm{U}$

$8.4 \mathrm{U}$

$8.4 \mathrm{U}$

$8.4 \mathrm{U}$

$8.4 \mathrm{U}$

$8.4 \mathrm{U}$

$8.4 \mathrm{U}$

$8.4 \mathrm{U}$

$8.4 \mathrm{U}$

$8.4 \mathrm{U}$

$8.4 \mathrm{U}$

$8.4 \mathrm{U}$

$8.4 \mathrm{U}$

$8.4 \mathrm{U}$

$8.4 \mathrm{U}$

91-20-3--.---Naphthalene

87-61-6-..--- - 1, 2, 3-Trichlorobenzene 
FORM 1

VOLATILE ORGANICS ANALYSIS DATA SHEET

Lab Name: TESTAMERICA BURLINGTON Contract: 21005
ARGLAB SAMPLE NO.

CN-S-MEOH BIANK

Lab Code: STLV Case No.: CENTRALI SAS No.:

SDG No.: 123144

Matrix: (soil/water) SOIL

Sample wt/vol:

$10.0(\mathrm{~g} / \mathrm{mL}) \mathrm{G}$

Level: (low/med) MED

$\%$ Moisture: not dec.

GC Column: CAP

Soil Extract Volume: 10000 (uL)
Lab Sample ID: 733220

Lab File ID: $\quad 733220$

Date Received: 11/28/07

Date Analyzed: 12/03/07

Dilution Factor: 1.0

Soil Aliquot Volume:

500 (UL)

- CONCENTRATION UNITS:

CAS NO. COMPOUND (ug/L or ug/Kg) UG/KG

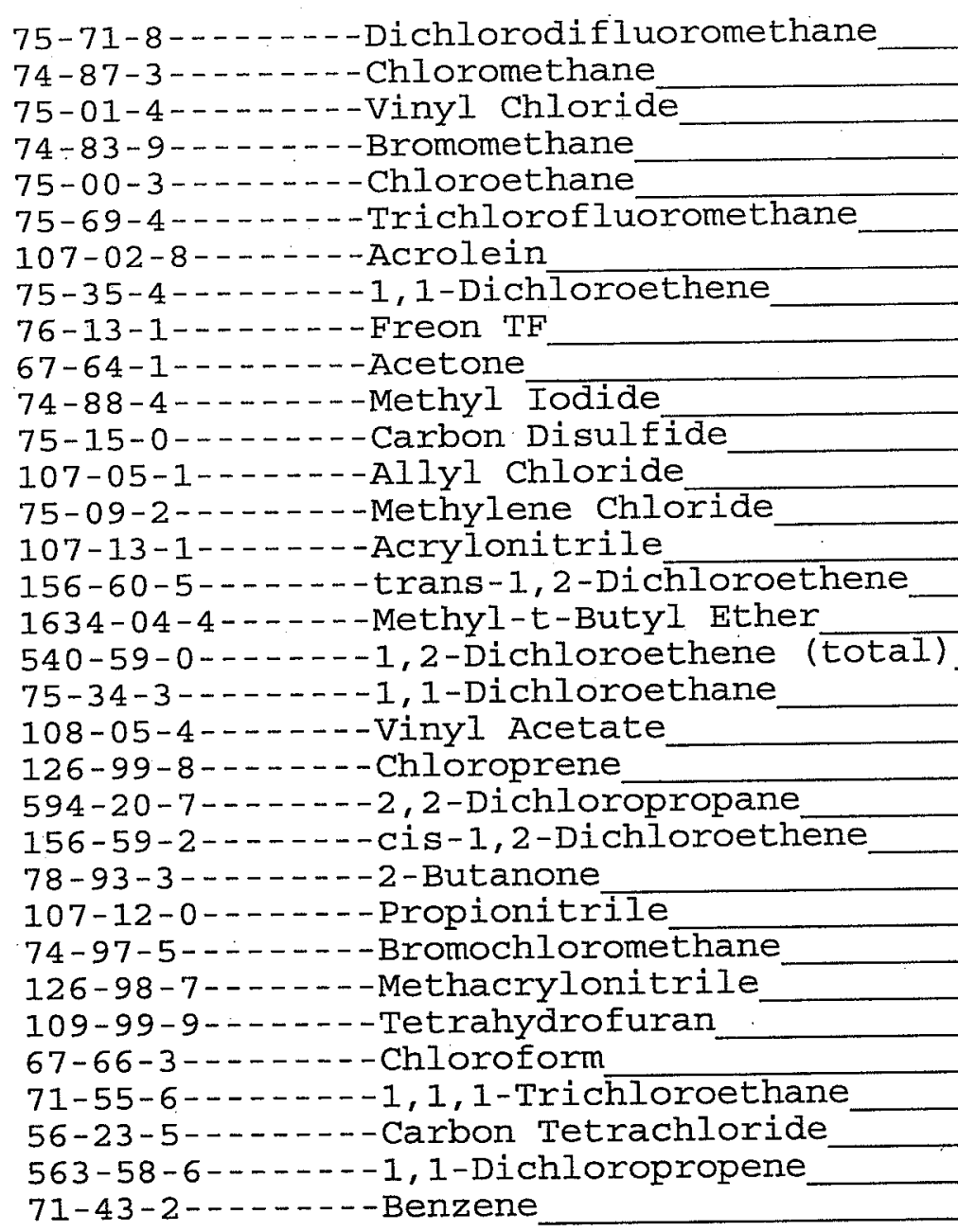

\begin{tabular}{|r|r|l|}
10 & $\mathrm{U}$ \\
4.4 & $\mathrm{~J}$ \\
10 & $\mathrm{U}$ \\
8.7 & $\mathrm{~J}$ \\
10 & $\mathrm{U}$ \\
10 & $\mathrm{U}$ \\
50 & $\mathrm{U}$ \\
10 & $\mathrm{U}$ \\
10 & $\mathrm{U}$ \\
50 & $\mathrm{U}$ \\
9.8 & $\mathrm{~J}$ \\
10 & $\mathrm{U}$ \\
10 & $\mathrm{U}$ \\
10 & $\mathrm{U}$ \\
10 & $\mathrm{U}$ \\
10 & $\mathrm{U}$ \\
10 & $\mathrm{U}$ \\
10 & $\mathrm{U}$ \\
10 & $\mathrm{U}$ \\
10 & $\mathrm{U}$ \\
10 & $\mathrm{U}$ \\
10 & $\mathrm{U}$ \\
10 & $\mathrm{U}$ \\
17 & $\mathrm{~J}$ \\
40 & $\mathrm{U}$ \\
10 & $\mathrm{U}$ \\
10 & $\mathrm{U}$ \\
140 & $\mathrm{U}$ \\
10 & $\mathrm{U}$ \\
10 & $\mathrm{U}$ \\
10 & $\mathrm{U}$ \\
10 & $\mathrm{U}$ \\
10 & $\mathrm{U}$ \\
& & \\
\hline & & \\
\hline
\end{tabular}


FORM 1

VOLATILE ORGANICS ANALYSIS DATA SHEET

Lab Name: TESTAMERICA BURLINGTON Contract: 21005

Lab Code: STLV Case No.: CENTRALI SAS No.:

Lab Sample ID: 733220

Matrix: (soil/water) SoIL

Sample wt/vol: $\quad 10.0(\mathrm{~g} / \mathrm{mL}) \mathrm{G}$

Lab File ID: 733220

Level: (low/med) MED

\% Moisture: not dec.

GC Column: CAP ID: 0.53 (mm)

Soil Extract Volume: 10000 (uL)

CAS NO.

COMPOUND
CONCENTRATION UNITS:

$\mathrm{ug} / \mathrm{L}$ or $\mathrm{ug} / \mathrm{Kg}$ ) UG/KG
ARGLAB SAMPLE NO.

CN-S-MEOH BLANK

SDG No.: 123144
Date Received: 11/28/07

Date Analyzed: 12/03/07

Dilution Factor: 1.0

Soil Aliquot Volume:

500 (uL)
78-83-1-..-.-.-Isobutyl Alcohol

107-06-2-...--1,2-Dichloroethane

79-01-6-1.---Trichloroethene

78-87-5--.---1, 2-Dichloropropane

74-95-3-..---Dibromomethane

80-62-6....--Methyl Methacrylate

123-91-1--..-1, 4-Dioxane

75-27-4-1..---Bromodichloromethane

110-75-8-..--2-Chloroethyl Vinyl Ether

10061-01-5--.--cis-1,3-Dichloropropene

108-10-1--..-4-Methyl-2-pentanone

108-88-3-..---Toluene

10061-02-6-----trans-1,3-Dichloropropene

97-63-2-------Ethyl Methacrylate

79-00-5-....-1, 1,2-Trichloroethane

127-18-4--..---Tetrachloroethene

142-28-9-...--1, 3-Dichloropropane

591-78-6------2-Hexanone

124-48-1-...--Dibromochloromethane

106-93-4--.---1, 2-Dibromoethane

108-90-7-..-.-Chlorobenzene

630-20-6-- - - 1, 1, 1,2-Tetrachloroethane

100-41-4--..---Ethylbenzene

1330-20-7-...- Xylene $(\mathrm{m}, \mathrm{p})$

95-47-6--.-.--Xylene (o)

1330-20-7--.--xylene (total)

100-42-5------ styrene

75-25-2---.---Bromoform

98-82-8---- - - - Isopropyl benzene

1476-11-5-..--cis-1, 4-Dichloro-2-butene

108-86-1-------Bromobenzene

79-34-5-------1, 1, 2, 2-Tetrachloroethane

96-18-4-- - - - 1, 2, 3-Trichloropropane

\begin{tabular}{|r|r|l|}
500 & $\mathrm{U}$ \\
10 & $\mathrm{U}$ \\
10 & $\mathrm{U}$ \\
10 & $\mathrm{U}$ \\
10 & $\mathrm{U}$ \\
10 & $\mathrm{U}$ \\
500 & $\mathrm{U}$ \\
10 & $\mathrm{U}$ \\
10 & $\mathrm{U}$ \\
10 & $\mathrm{U}$ \\
50 & $\mathrm{U}$ \\
10 & $\mathrm{U}$ \\
10 & $\mathrm{U}$ \\
10 & $\mathrm{U}$ \\
10 & $\mathrm{U}$ \\
10 & $\mathrm{U}$ \\
10 & $\mathrm{U}$ \\
50 & $\mathrm{U}$ \\
10 & $\mathrm{U}$ \\
10 & $\mathrm{U}$ \\
10 & $\mathrm{U}$ \\
10 & $\mathrm{U}$ \\
10 & $\mathrm{U}$ \\
10 & $\mathrm{U}$ \\
10 & $\mathrm{U}$ \\
10 & $\mathrm{U}$ \\
10 & $\mathrm{U}$ \\
10 & $\mathrm{U}$ \\
10 & $\mathrm{U}$ \\
10 & $\mathrm{U}$ \\
10 & $\mathrm{U}$ \\
10 & $\mathrm{U}$ \\
10 & $\mathrm{U}$ \\
& & \\
\hline
\end{tabular}


FORM 1

VOLATILE ORGANICS ANALYSIS DATA SHEET

Lab Name: TESTAMERICA BURLINGTON Contract: 21005
ARGLAB SAMPLE NO.

CN-S-MEOH BLANK

Lab Code: STLV Case No.: CENTRALI SAS No.:

SDG No.: 123144

Matrix: (soil/water) SOIL

Sample wt/vol: $\quad 10.0(\mathrm{~g} / \mathrm{mL}) \mathrm{G}$

Level: (low/med) MED

\% Moisture: not dec.

GC Column: CAP

Soil Extract Volume: 10000 (uL)
Lab Sample ID: 733220

Lab File ID: $\quad 733220$

Date Received: 11/28/07

Date Analyzed: 12/03/07

Dilution Factor: 1.0

Soil Aliquot Volume: 500 (uL)

CAS NO.

COMPOUND

CONCENTRATION UNITS :

(ug/L or $\mathrm{ug} / \mathrm{Kg}$ ) UG/KG

Q

110-57-6------trans-1, 4-Dichloro-2-butene

103-65-1-..-.-n-Propylbenzene

95-49-8--...--2-Chlorotoluene

106-43-4-..---4-Chlorotoluene

108-67-8-..----1,3,5-Trimethyl benzene

98-06-6---.-.-tert-Butylbenzene

95-63-6-------1, 2, 4-Trimethylbenzene

135-98-8------sec-Butylbenzene

541-73-1_...--1, 3-Dichlorobenzene

99-87-6-...--4-Isopropyltoluene

106-46-7--..--1,4-Dichlorobenzene

95-50-1-.----1, 2-Dichlorobenzene

104-51-8-.----n-Butylbenzene

96-12-8-- - - - 1, 2-Dibromo-3-Chloropropane

120-82-1------1, 2, 4-Trichlorobenzene

87-68-3--..-- Hexachlorobutadiene

91-20-3-..-.--Naphthalene

87-61-6-..-.-- 1, 2, 3-Trich lorobenzene
$10 \mathrm{U}$

$10 \mathrm{U}$

$10 \mathrm{U}$

$10 \mathrm{U}$

$10 \mathrm{U}$

$10 \mathrm{U}$

$10 \mathrm{U}$

$10 \mathrm{U}$

$10 \mathrm{U}$

$10 \mathrm{U}$

$10 \mathrm{U}$

$10 \mathrm{U}$

$10 \mathrm{U}$

$10 \mathrm{U}$

$10 \mathrm{U}$

$10 \mathrm{U}$

$10 \mathrm{U}$

$10 \mathrm{U}$ 


\section{ENVIROSYSTEMS, INC.}

9200 Rumsey Road • Suite B102 • Columbia, Maryland 21045-1934

Phone (410) 964-0330 - Fax (410) 740-9306

Email: info@envsystems.com - Webpage: www.envsystems.com/envsys

January 21,2007

Jorge S. Alvarado, PH. D

Argonne National Laboratory

Environmental Research Division

Applied Geosciences and Environmental

Management Section

9700 South Cass Avenue, ER-203

Argonne, Illinois 60439

RE: Report \#070318

Dear Jorge,

Enclosed is the Analytical Data Package for Organics Analysis for the samples received on January $11 \& 2008$. These samples were analyzed by using method SW-846 8260B and USEPA CLP SOWOLM04.3 and the chain of custody instructions.

Please do not hesitate to call if you have any questions, comments, or require additional information.

Sincerely,

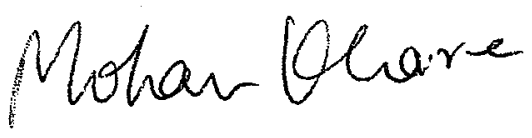

Mohan Khare Ph.D.

President/CEO

Enclosure (1)

$\mathrm{MK} / \mathrm{ncc}$ 
Table of Contents

1. Narrative

2. Chain of Custody Documents

3. Volatiles Data 
1. SDG Case Narrative 


\section{SDG NARRATIVE \\ VOLATILE ORGANICS (VOC)}

Envirosystems, Inc.

Contract: N/A

Client: Argonne National Laboratory

Case: N/A

SDG: ARG80111

1. SAMPLE RECIEPT

Date received: 01-18-2008

Cooler Temperature: 2

Sample Summary

\begin{tabular}{|c|c|c|c|}
\hline Client ID & Laboratory ID & Matrix & pH \\
\hline CNQCTB-W-25976 & $0080111-01$ & WATER & 7 \\
\hline CNPMP4-W-26068 & $0080111-02$ & WATER & 7 \\
\hline CNPMP3-W-26071 & $0080111-03$ & WATER & 7 \\
\hline CNPMP8-W-26075 & $0080111-04$ & WATER & 7 \\
\hline
\end{tabular}

\section{HOLDING TIMES}

A. Sample Preparation: All holding times were met.

B. Sample Analysis: All holding times were met

\section{METHODS}

The samples were analyzed and reported by using method SW-846 8260B and USEPA CLP SOW OLM04.3 for target compound list.

\section{INSTRUMENT AND CHROMATOGRAPHIC CONDITIONS}

A Hewlett Packard 6890 gas chromatograph equipped with a Hewlett Packard 5973 MSD was used for sample analysis. The capillary column used was a Restek $30 \mathrm{~m}$ by $0.25 \mathrm{~mm}$ ID by $1.0 \mu \mathrm{m}$ film thickness (Restek Cat. \# RTX-Volatiles). The trap used with the sample concentrator is an Ol Analytical Trap \#10, $30 \mathrm{~cm}$ packed with Tenax/silica gel/cms (PN\#228122).

\section{PREPARATION}

The submitted samples were analyzed as received.

6. ANALYSIS

A. Calibration:

\section{Initial calibration}

All acceptance criteria as stipulated by SW- $8468260 \mathrm{~b}$ were met for all SPCC's and CCC's. All target compounds met the required percent RSD. 


\section{SDG NARRATIVE \\ VOLATILE ORGANICS (VOC)}

\section{Blanks:}

All acceptance criteria were met.

\section{Surrogates:}

All acceptance criteria were met.

B. Spikes:

I. Laboratory Control Spikes (LCS)

LCS samples were analyzed.

II. Matrix Spike/Matrix Spike Duplicate (MS/MSD)

The client did not request a MS/MSD.

C. Internal Standards:

All acceptance criteria were met.

D. Samples

Sample analysis proceeded normally. Samples CNPMP3-W-26071 required a dilution of $2 \mathrm{X}$ and sample CNPMP8-W-26075 required a dilution of 10X.

I certify that this Sample Data Package is in compliance with the terms and conditions of the contract, both technically and for completeness, for other than the conditions detailed above. Release of the data contained in the hard copy Sample Data Package and in the Electronic Data Deliverables has been authorized by the laboratory manager or the manager's designee, as verified by the following signatures.

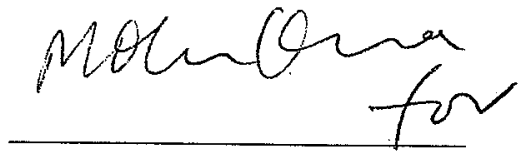

Laboratory Manager

$$
1 / 21 / 08
$$

Date 
2. Chain of Custody Records 


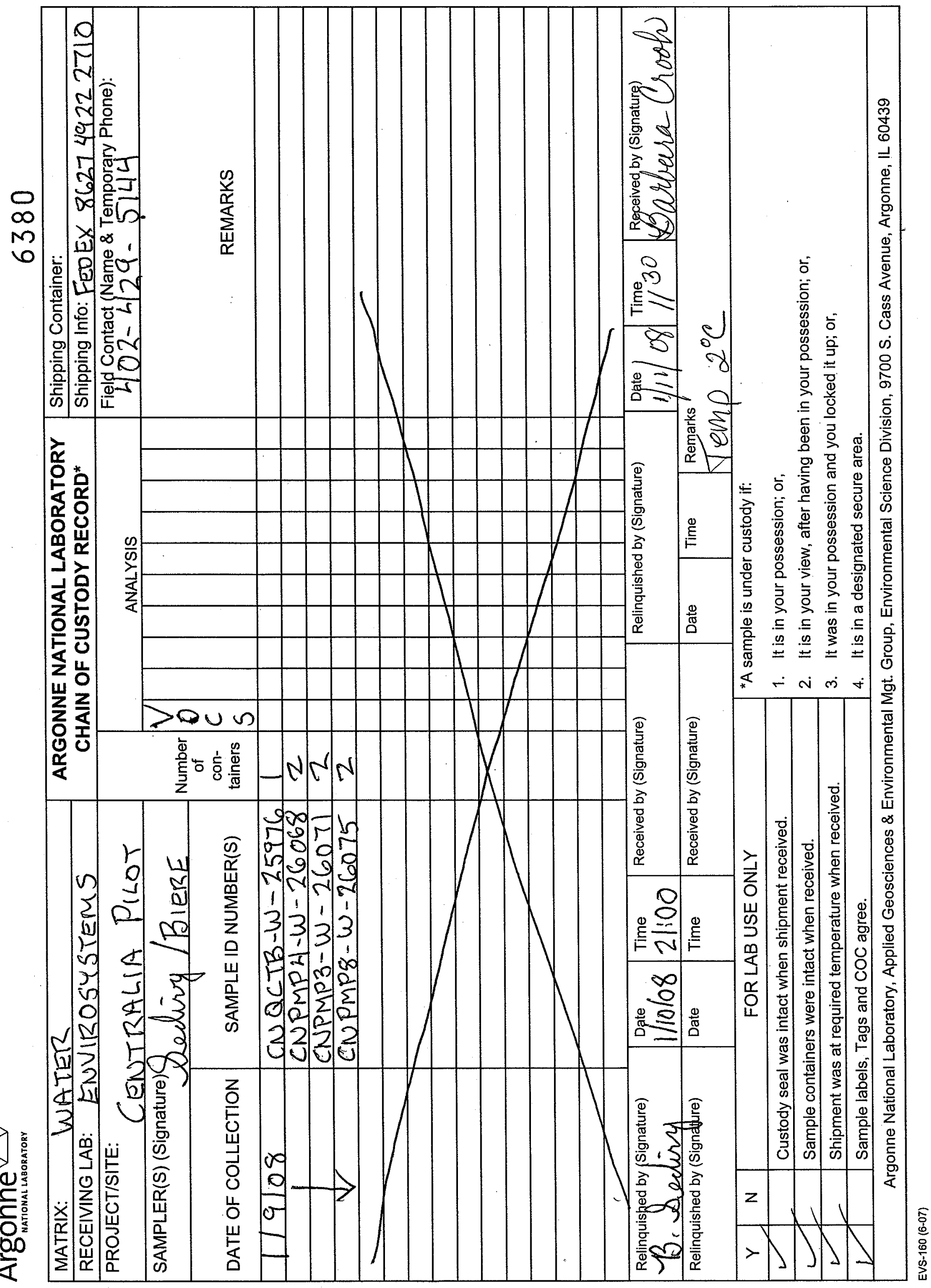


Volatile Sample Results 
$1 A$ - FORM I VOA-1

VOLATILE ORGANICS ANALYSIS DATA SHEET
EPA SAMPLE NO.

CNQCTB-W-25976
Lab Name:

Envirosystems, Inc.

Lab Code: ENVSYS Case No.:

WATER

Matrix: (SOIL/SED/WATER)

WATER

Sample wt/vol: 5.00

$(\mathrm{g} / \mathrm{mL})$

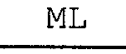

Level: (TRACE/LOW/MED)

LOW

\% Moisture: not dec.

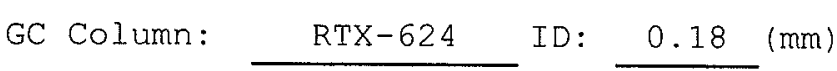

Soil Extract Volume:

Purge Volume:

5.00

(uL) (mL)

Contract:

Mod. Ref No. : SDG NO.:

ARG80111

Lab Sample ID:

0080111-01

Lab File ID:

E000219.D

Date Received:

$01 / 11 / 2008$

Date Analyzed:

$01 / 16 / 2008$

Dilution Factor:

1.0

Soil Aliquot Volume:

(uL)

\begin{tabular}{|c|c|c|c|}
\hline CAS NO. & COMPOUND & $\begin{array}{l}\text { CONCENTRATION UNTTS: } \\
(\mathrm{ug} / \mathrm{L} \text { or } \mathrm{ug} / \mathrm{kg}) \mathrm{UG} / \mathrm{L}\end{array}$ & $Q$ \\
\hline $75-71-8$ & Dichlorodifluoromethane & 5.0 & $\mathrm{U}$ \\
\hline $74-87-3$ & Chloromethane & 5.0 & $\mathrm{U}$ \\
\hline $75-01-4$ & Vinyl chloride & 5.0 & $\mathrm{U}$ \\
\hline $74-83-9$ & Bromomethane & 5.0 & $\mathrm{U}$ \\
\hline $75-00-3$ & Chloroethane & 5.0 & $\mathrm{U}$ \\
\hline $75-69-4$ & Trichlorofluoromethane & 5.0 & $\mathrm{U}$ \\
\hline $75-35-4$ & 1,1-Dichloroethene & 5.0 & $\mathrm{U}$ \\
\hline $76-13-1$ & 1,1,2-Trichloro-1,2,2-trifluoroethane & 5.0 & $\mathrm{U}$ \\
\hline $67-64-1$ & Acetone & 3.1 & $\mathrm{~J}$ \\
\hline $75-15-0$ & Carbon disulfide & 5.0 & $\mathrm{U}$ \\
\hline $79-20-9$ & Methyl acetate & 5.0 & $\mathrm{U}$ \\
\hline $75-09-2$ & Methylene chloride & 5.0 & $\mathrm{U}$ \\
\hline $156-60-5$ & trans-1,2-Dichloroethene & 5.0 & $\mathrm{U}$ \\
\hline $1634-04-4$ & Methyl tert-butyl ether & 5.0 & $\mathrm{U}$ \\
\hline $75-34-3$ & 1,1-Dichloroethane & 5.0 & $\mathrm{U}$ \\
\hline $156-59-2$ & cis-1,2-Dichloroethene & 5.0 & $\mathrm{U}$ \\
\hline $78-93-3$ & 2-Butanone & 5.0 & $\mathrm{U}$ \\
\hline $67-66-3$ & Chloroform & 5.0 & $\mathrm{U}$ \\
\hline $71-55-6$ & $1,1,1$-Trichloroethane & 5.0 & $\mathrm{U}$ \\
\hline $110-82-7$ & Cyclohexane & 5.0 & $\mathrm{U}$ \\
\hline $56-23-5$ & Carbon Tetrachloride & 5.0 & $\mathrm{U}$ \\
\hline $71-43-2$ & Benzene & 5.0 & $\mathrm{U}$ \\
\hline $107-06-2$ & 1,2-Dichloroethane & 5.0 & $\mathrm{U}$ \\
\hline
\end{tabular}


$1 B$ - FORM I VOA-2

VOLATILE ORGANICS ANALYSIS DATA SHEET
EPA SAMPLE NO.

$\mathrm{CNQCTB}-\mathrm{W}-25976$
Lab Name: Envirosystems, Inc.

Lab Code: ENVSYS Case No.:

Matrix: (SOIL/SED/WATER)

WATER

Sample wt/vol: 5.00

$(\mathrm{g} / \mathrm{mL}) \quad \mathrm{ML}$

Level: (TRACE/LOW/MED)

LOW

응 Moisture: not dec.

GC Column: $\quad$ RTX-624 ID: 0.18 (mm)

Soil Extract Volume:

Purge Volume:

5.00

(uL) (mL)

Contract:

Mod. Ref No.: SDG NO.:

ARG80111

Lab Sample ID:

$0080111-01$

Lab File ID:

F000219.D

Date Received:

$01 / 11 / 2008$

Date Analyzed:

$01 / 16 / 2008$

Dilution Factor:

1.0

Soil Aliquot Volume:

(uL)

\begin{tabular}{|c|c|c|c|}
\hline CAS NO. & COMPOUND & $\begin{array}{l}\text { CONCENTRATION DNITS: } \\
(\mathrm{ug} / \mathrm{L} \text { or } u \mathrm{~g} / \mathrm{kg}) \mathrm{UG} / \mathrm{L}\end{array}$ & $Q$ \\
\hline $79-01-6$ & Trichloroethene & 5.0 & $\mathrm{U}$ \\
\hline $108-87-2$ & Methylcyclohexane & 5.0 & $\mathrm{U}$ \\
\hline $78-87-5$ & 1,2-Dichloropropane & 5.0 & $\mathrm{U}$ \\
\hline $75-27-4$ & Bromodichloromethane & 5.0 & $\mathrm{U}$ \\
\hline $10061-01-5$ & cis-1,3-Dichloropropene & 5.0 & $\mathrm{U}$ \\
\hline $108-10-1$ & 4-Methyl-2-pentanone & 5.0 & $\mathrm{U}$ \\
\hline $108-88-3$ & Toluene & 5.0 & $\mathrm{U}$ \\
\hline $10061-02-6$ & trans-1,3-Dichloropropene & 5.0 & $\underline{U}$ \\
\hline $79-00-5$ & $1,1,2$-Trichloroethane & 5.0 & $\mathrm{U}$ \\
\hline $127-18-4$ & Tetrachloroethene & 5.0 & $\mathrm{U}$ \\
\hline $591-78-6$ & 2-Hexanone & 5.0 & U \\
\hline $124-48-1$ & Dibromochloromethane & 5.0 & U \\
\hline $106-93-4$ & 1,2-Dibromoethane & 5.0 & $\mathrm{U}$ \\
\hline $108-90-7$ & Chlorobenzene & 5.0 & $\mathrm{U}$ \\
\hline $100-41-4$ & Ethylbenzene & 5.0 & $\mathrm{U}$ \\
\hline $95-47-6$ & o-Xylene & 5.0 & $U$ \\
\hline $179601-23-1$ & $\mathrm{~m}, \mathrm{p}$-Xylene & 5.0 & $\mathrm{U}$ \\
\hline $100-42-5$ & Styrene & 5.0 & $\mathrm{U}$ \\
\hline $75-25-2$ & Bromoform & 5.0 & $\mathrm{U}$ \\
\hline $98-82-8$ & Isopropylbenzene & 5.0 & $\mathrm{U}$ \\
\hline $79-34-5$ & $1,1,2,2$-Tetrachloroethane & 5.0 & $\mathrm{U}$ \\
\hline $541-73-1$ & 1,3-Dichlorobenzene & 5.0 & $\mathrm{U}$ \\
\hline $106-46-7$ & 1,4-Dichlorobenzene & 5.0 & $\mathrm{U}$ \\
\hline $95-50-1$ & 1,2-Dichlorobenzene & 5.0 & $\mathrm{U}$ \\
\hline $96-12-8$ & 1,2-Dibromo-3-chloropropane & 5.0 & $\mathrm{U}$ \\
\hline $120-82-1$ & $1,2,4$-Trichlorobenzene & 5.0 & $\mathrm{U}$ \\
\hline $91-20-3$ & Naphthalene & 5.0 & $\mathrm{U}$ \\
\hline
\end{tabular}


$1 A$ - EORM I VOA-1

VOLATILE ORGANICS ANALYSIS DATA SHEET
EPA SAMPLE NO

CNPMP $4-W-26068$
Lab Name: Envirosystems, Inc.

Lab Code: ENVSYS Case No.:

Matrix: (SOIL/SED/WATER) WATER

Sample wt/vol: $5.00(\mathrm{~g} / \mathrm{mL}) \mathrm{ML}$

Level: (TRACE/LOW/MED)

LOW

응 Moisture: not dec.

GC Column:

$\operatorname{RTX}-624$ ID

Soil Extract Volume:

Purge Volume:

5.00

Contract:

Mod. Ref No.:

Lab Sample ID:

Lab File ID:

Date Received:

Date Analyzed:

Dilution Factor: SDG No.:

ARG80111

0080111-02

F000220.D

$01 / 11 / 2008$

$01 / 16 / 2008$

1.0

\begin{tabular}{|c|c|c|c|}
\hline CAS NO. & COMPOUND & $\begin{array}{l}\text { CONCENTRATION UNITS: } \\
(\mathrm{ug} / \mathrm{L} \text { or } \mathrm{ug} / \mathrm{kg}) \mathrm{UG} / \mathrm{L}\end{array}$ & $Q$ \\
\hline $75-71-8$ & Dichlorodifluoromethane & 5.0 & $\bar{U}$ \\
\hline $74-87-3$ & Chloromethane & 5.0 & $\mathrm{U}$ \\
\hline $75-01-4$ & Vinyl chloride & 5.0 & $\mathrm{U}$ \\
\hline $74-83-9$ & Bromomethane & 5.0 & $\mathrm{U}$ \\
\hline $75-00-3$ & Chloroethane & 5.0 & $\mathrm{U}$ \\
\hline $75-69-4$ & Trichlorofluoromethane & 5.0 & $\mathrm{U}$ \\
\hline $75-35-4$ & 1,1-Dichloroethene & 5.0 & $\mathrm{U}$ \\
\hline $76-13-1$ & $1,1,2$-Trichloro-1,2,2-trifluoroethane & 5.0 & $\mathrm{U}$ \\
\hline $67-64-1$ & Acetone & 5.0 & U \\
\hline $75-15-0$ & Carbon disulfide & 5.0 & U \\
\hline $79-20-9$ & Methyl acetate & 5.0 & U \\
\hline $75-09-2$ & Methylene chloride & 5.0 & $\mathrm{U}$ \\
\hline $156-60-5$ & trans-1,2-Dichloroethene & 5.0 & $\mathrm{U}$ \\
\hline $1634-04-4$ & Methyl tert-butyl ether & 5.0 & $\mathrm{U}$ \\
\hline $75-34-3$ & 1,1-Dichloroethane & 5.0 & $\mathrm{U}$ \\
\hline $156-59-2$ & cis-1,2-Dichloroethene & 5.0 & $\mathrm{U}$ \\
\hline $78-93-3$ & 2-Butanone & 5.0 & $\mathrm{U}$ \\
\hline $67-66-3$ & Chloroform & 22 & \\
\hline $71-55-6$ & 1,1,1-Trichloroethane & 5.0 & $\mathrm{U}$ \\
\hline $110-82-7$ & Cyclohexane & 5.0 & $\mathrm{U}$ \\
\hline $56-23-5$ & Carbon Tetrachloride & 7.9 & \\
\hline $71-43-2$ & Benzene & 5.0 & U \\
\hline $107-06-2$ & 1,2-Dichloroethane & 5.0 & $\mathrm{U}$ \\
\hline
\end{tabular}


$1 B$ - FORM I VOA-2

VOLATILE ORGANICS ANALYSIS DATA SHEET
EPA SAMPLE NO.

CNPMP 4-W-26068
Lab Name: Envirosystems, Inc.

Lab Code: ENVSYS Case No.:

Matrix: (SOIL/SED/WATER) : WATER

Sample wt/vol: 5.00

$(\mathrm{g} / \mathrm{mL}) \quad \mathrm{ML}$

Level: (TRACE/LOW/MED)

LOW

응 Moisture: not dec.

GC Column:

$\mathrm{RTX}-62$ ID

Soil Extract Volume:

Purge volume:

5.00

Contract:

Mod. Ref No.: SDG No.:

ARG80111

Lab Sample ID:

0080111-02

Lab File ID:

E000220.D

Date Received:

$01 / 11 / 2008$

Date Analyzed:

$01 / 16 / 2008$

Dilution Factor:

1.0

(uL) Soil Aliquot Volume:

(uL) (mL)

\begin{tabular}{|c|c|c|c|}
\hline CAS NO. & COMPOUND & $\begin{array}{l}\text { CONCENTRATION UNITS: } \\
(\mathrm{ug} / \mathrm{L} \text { or } \mathrm{ug} / \mathrm{kg}) \text { UG/L }\end{array}$ & Q \\
\hline $79-01-6$ & Trichloroethene & 5.0 & $\mathrm{U}$ \\
\hline $108-87-2$ & Methylcyclohexane & 5.0 & $\mathrm{U}$ \\
\hline $78-87-5$ & 1,2-Dichloropropane & 5.0 & $\mathrm{U}$ \\
\hline $75-27-4$ & Bromodichloromethane & 5.0 & $\mathrm{U}$ \\
\hline $10061-01-5$ & cis-1,3-Dichloropropene & 5.0 & $\mathrm{U}$ \\
\hline $108-10-1$ & 4-Methyl-2-pentanone & 5.0 & $\mathrm{U}$ \\
\hline $108-88-3$ & Toluene & 5.0 & $\mathrm{U}$ \\
\hline $10061-02-6$ & trans-1,3-Dichloropropene & 5.0 & $\mathrm{U}$ \\
\hline $79-00-5$ & 1,1,2-Trichloroethane & 5.0 & $\mathrm{U}$ \\
\hline $127-18-4$ & Tetrachloroethene & 5.0 & U \\
\hline $591-78-6$ & 2-Hexanone & 5.0 & U \\
\hline $124-48-1$ & Dibromochloromethane & 5.0 & $\mathrm{U}$ \\
\hline $106-93-4$ & 1,2-Dibromoethane & 5.0 & $\mathrm{U}$ \\
\hline $108-90-7$ & Chlorobenzene & 5.0 & $\mathrm{U}$ \\
\hline $100-41-4$ & Ethylbenzene & 5.0 & $\mathrm{U}$ \\
\hline $95-47-6$ & o-Xylene & 5.0 & $\mathrm{U}$ \\
\hline $179601-23-1$ & $\mathrm{~m}, \mathrm{p}$-Xylene & 5.0 & $\mathrm{U}$ \\
\hline $100-42-5$ & Styrene & 5.0 & $\mathrm{U}$ \\
\hline $75-25-2$ & Bromoform & 5.0 & $\mathrm{U}$ \\
\hline $98-82-8$ & Isopropylbenzene & 5.0 & U \\
\hline $79-34-5$ & $1,1,2,2$-Tetrachloroethane & 5.0 & $\mathrm{U}$ \\
\hline $541-73-1$ & 1,3-Dichlorobenzene & 5.0 & $\mathrm{U}$ \\
\hline $106-46-7$ & 1,4-Dichlorobenzene & 5.0 & $\mathrm{U}$ \\
\hline $95-50-1$ & 1,2-Dichlorobenzene & 5.0 & $\mathrm{U}$ \\
\hline $96-12-8$ & 1,2-Dibromo-3-chloropropane & 5.0 & $\mathrm{U}$ \\
\hline $120-82-1$ & 1,2,4-Trichlorobenzene & 5.0 & $\mathrm{U}$ \\
\hline $91-20-3$ & Naphthalene & 5.0 & $\mathrm{U}$ \\
\hline
\end{tabular}


EPA SAMPLE NO.

CNPMP $3-W-26071$
Lab Name:

Envirosystems, Inc.

Lab Code: ENVSYS Case No.:

Matrix: (SOIL/SED/WATER)

Sample wt/vol:

5.00

$(g / m L)$

Level: (TRACE/LOW/MED) LOW

\%. Moisture: not dec.

GC Column: RTX-624 ID: 0.18 (mm)

Soil Extract Volume:

(uI)

Purge Volume:

5.00

(mL)
Contract:

Mod. Ref No.: SDG No.:

ARG80111

Lab Sample ID:

Lab File ID:

$0080111-03$

Date Received:

E000221.D

Date Analyzed:

Dilution Factor:

$01 / 17 / 2008$

Soil Aliquot Volume:

1.0

\begin{tabular}{|c|c|c|c|}
\hline CAS NO. & COMPOUND & $\begin{array}{l}\text { CONCENTRATION UNTTS: } \\
(u g / L \text { or } u g / k g) U G / L\end{array}$ & $Q$ \\
\hline $75-71-8$ & Dichlorodifluoromethane & 5.0 & U \\
\hline $74-87-3$ & Chloromethane & 5.0 & $\mathrm{U}$ \\
\hline $75-01-4$ & Vinyl chloride & 5.0 & $\mathrm{U}$ \\
\hline $74-83-9$ & Bromomethane & 5.0 & $\mathrm{U}$ \\
\hline $75-00-3$ & Chloroethane & 5.0 & $\mathrm{U}$ \\
\hline $75-69-4$ & Trichlorofluoromethane & 5.0 & $\mathrm{U}$ \\
\hline $75-35-4$ & 1,1-Dichloroethene & 5.0 & $\mathrm{U}$ \\
\hline $76-13-1$ & $1,1,2-$ Trichloro-1,2,2-trifluoroethane & 5.0 & $\mathrm{U}$ \\
\hline $67-64-1$ & Acetone & 48 & \\
\hline $75-15-0$ & Carbon disulfide & 5.0 & $\mathrm{U}$ \\
\hline $79-20-9$ & Methyl acetate & 5.0 & U \\
\hline $75-09-2$ & Methylene chloride & 5.0 & $\mathrm{U}$ \\
\hline $156-60-5$ & trans-1,2-Dichloroethene & 5.0 & $\mathrm{U}$ \\
\hline $1634-04-4$ & Methyl tert-butyl ether & 5.0 & $\mathrm{U}$ \\
\hline $75-34-3$ & 1,1-Dichloroethane & 5.0 & $\mathrm{U}$ \\
\hline $156-59-2$ & Cis-1,2-Dichloroethene & 5.0 & $\mathrm{U}$ \\
\hline $78-93-3$ & 2-Butanone & 290 & $\mathrm{E}$ \\
\hline $67-66-3$ & Chloroform & 150 & \\
\hline $71-55-6$ & 1,1,1-Trichloroethane & 5.0 & $\mathrm{U}$ \\
\hline $110-82-7$ & Cyclohexane & 5.0 & $\mathrm{U}$ \\
\hline $56-23-5$ & Carbon Tetrachloride & 5.0 & $\mathrm{U}$ \\
\hline $71-43-2$ & Benzene & 5.0 & $\mathrm{U}$ \\
\hline $107-06-2$ & 1,2-Dichloroethane & 5.0 & U \\
\hline
\end{tabular}


$1 B$ - FORM I VOA-2

VOLATILE ORGANICS ANALYSIS DATA SHEET
EPA SAMPLE NO.

CNPMP $3-W-26071$
Lab Name: Envirosystems, Inc.

Lab Code: ENVSYS Case No.:

Matrix: (SOIL/SED/WATER) WATER

Sample wt/vol: $5.00(\mathrm{~g} / \mathrm{mL}) \mathrm{ML}$

Level: (TRACE/LOW/MED) LOW

응 Moisture: not dec.

GC Column:

$\mathrm{RTX}^{-624}$ ID: $0.18(\mathrm{~mm})$

Soil Extract Volume:

Purge Volume:

5.00 (UL)

Contract:

Mod. Ref No.: SDG No.:

ARG80111

Lab Sample ID:

$0080111-03$

Lab File ID:

F000221.D

Date Received:

Date Analyzed:

$\frac{01 / 11 / 2008}{\frac{01 / 27 / 200}{1.0}}$
(uL)

\begin{tabular}{|c|c|c|c|}
\hline CAS NO. & COMPOUND & $\begin{array}{l}\text { CONCENTRATION UNITS: } \\
(\mathrm{ug} / \mathrm{L} \text { or } \mathrm{ug} / \mathrm{kg}) \mathrm{UG} / \mathrm{L}\end{array}$ & $Q$ \\
\hline $79-01-6$ & Trichloroethene & 5.0 & $\mathrm{U}$ \\
\hline $108-87-2$ & Methylcyclohexane & 5.0 & $\mathrm{U}$ \\
\hline $78-87-5$ & 1,2-Dichloropropane & 5.0 & $\mathrm{U}$ \\
\hline $75-27-4$ & Bromodichloromethane & 5.0 & $\mathrm{U}$ \\
\hline $10061-01-5$ & cis-1,3-Dichloropropene & 5.0 & $\mathrm{U}$ \\
\hline $108-10-1$ & 4-Methyl-2-pentanone & 5.0 & $\mathrm{U}$ \\
\hline $108-88-3$ & Toluene & 5.0 & $\mathrm{U}$ \\
\hline $10061-02-6$ & trans-1,3-Dichloropropene & 5.0 & $\mathrm{U}$ \\
\hline $79-00-5$ & 1,1,2-Trichloroethane & 5.0 & $\mathrm{U}$ \\
\hline $127-18-4$ & Tetrachloroethene & 5.0 & $\mathrm{U}$ \\
\hline $591-78-6$ & 2-Hexanone & 5.0 & $\mathrm{U}$ \\
\hline $124-48-1$ & Dibromochloromethane & 5.0 & 0 \\
\hline $106-93-4$ & 1,2-Dibromoethane & 5.0 & $\mathrm{U}$ \\
\hline $108-90-7$ & Chlorobenzene & 5.0 & $\mathrm{U}$ \\
\hline $100-41-4$ & Ethylbenzene & 5.0 & $\mathrm{U}$ \\
\hline $95-47-6$ & o-Xylene & 5.0 & $\mathrm{U}$ \\
\hline $179601-23-1$ & m;p-Xylene & 5.0 & $\mathrm{U}$ \\
\hline $100-42-5$ & Styrene & 5.0 & 0 \\
\hline $75-25-2$ & Bromoform & 5.0 & $\mathrm{U}$ \\
\hline $98-82-8$ & Isopropylbenzene & 5.0 & $\mathrm{U}$ \\
\hline $79-34-5$ & $1,1,2,2$-Tetrachloroethane & 5.0 & $\mathrm{U}$ \\
\hline $541-73-1$ & 1,3-Dichlorobenzene & 5.0 & U \\
\hline $106-46-7$ & 1,4-Dichlorobenzene & 5.0 & $\mathrm{U}$ \\
\hline $95-50-1$ & 1,2-Dichlorobenzene & 5.0 & $\mathrm{U}$ \\
\hline $96-12-8$ & 1,2-Dibromo-3-chloropropane & 5.0 & $\mathrm{U}$ \\
\hline $120-82-1$ & $1,2,4$-Trichlorobenzene & 5.0 & $\mathrm{U}$ \\
\hline $91-20-3$ & Naphthalene & 5.0 & $\mathrm{U}$ \\
\hline
\end{tabular}


$1 A \cdot-$ FORM I VOA-1

VOLATILE ORGANICS ANALYSIS DATA SHEET
EPA SAMPLE NO.

CNPMP3-W-26071DL
Lab Name:

Envirosystems, Inc.

Lab Code: ENVSYS Case No.:

Matrix: (SOIL/SED/WATER)

WATER

Sample wt/vol: 5.0

$(\mathrm{g} / \mathrm{mL})$

C

Level: (TRACE/LOW/MED)

LOW

o Moisture: not dec.

GC Column: $\quad$ RTX-624 ID: 0.18 (mm)

Soil Extract Volume:

(uL)

(mL)

Purge volume:

5.00
Contract:

Mod. Ref No.: SDG NO.:

ARG80111

Lab Sample ID:

Lab File ID:

0080111-03REl

Date Received:

E000230.D

Date Analyzed:

$01 / 11 / 2008$

$01 / 17 / 2008$

Dilution Eactor:

2.0

Soil Aliquot Volume:

\begin{tabular}{|c|c|c|c|}
\hline CAS NO. & COMPOUND & $\begin{array}{l}\text { CONCENTRATION UNITS: } \\
(\mathrm{ug} / \mathrm{L} \text { or } \mathrm{ug} / \mathrm{kg}) \mathrm{UG} / \mathrm{L}\end{array}$ & Q \\
\hline $75-71-8$ & Dichlorodifluoromethane & 10 & $\mathrm{U}$ \\
\hline $74-87-3$ & Chloromethane & 10 & $\mathrm{U}$ \\
\hline $75-01-4$ & Vinyl chloride & 10 & $\mathrm{U}$ \\
\hline $74-83-9$ & Bromomethane & 10 & $\mathrm{U}$ \\
\hline $75-00-3$ & Chloroethane & 10 & $\mathrm{U}$ \\
\hline $75-69-4$ & Trichlorofluoromethane & 10 & $\mathrm{U}$ \\
\hline $75-35-4$ & 1,1-Dichloroethene & 10 & $\mathrm{U}$ \\
\hline $76-13-1$ & 1,1,2-Trichloro-1,2,2-trifluoroethane & 10 & U \\
\hline $67-64-1$ & Acetone & 62 & D \\
\hline $75-15-0$ & Carbon disulfide & 10 & $\mathrm{U}$ \\
\hline $79-20-9$ & Methyl acetate & 10 & $\mathrm{U}$ \\
\hline $75-09-2$ & Methylene chloride & 10 & $\mathrm{U}$ \\
\hline $156-60-5$ & trans-1,2-Dichloroethene & 10 & $\mathrm{U}$ \\
\hline $1634-04-4$ & Methyl tert-butyl ether & 10 & $\mathrm{U}$ \\
\hline $75-34-3$ & 1,1-Dichloroethane & 10 & U \\
\hline $156-59-2$ & cis-1,2-Dichloroethene & 10 & $\mathrm{U}$ \\
\hline $78-93-3$ & 2-Butanone & 270 & D \\
\hline $67-66-3$ & Chloroform & 140 & $\mathrm{D}$ \\
\hline $71-55-6$ & $1,1,1$-Trichloroethane & 10 & $\mathrm{U}$ \\
\hline $110-82-7$ & Cyclohexane & 10 & $\mathrm{U}$ \\
\hline $56-23-5$ & Carbon Tetrachloride & 10 & U \\
\hline $71-43-2$ & Benzene & 10 & $\mathrm{U}$ \\
\hline $107-06-2$ & 1,2-Dichloroethane & 10 & $\mathrm{U}$ \\
\hline
\end{tabular}


$1 B$ - FORM I VOA-2

VOLATILE ORGANICS ANALYSIS DATA SHEET
EPA SAMPLE NO.

CNPMP 3-W-26071DL
Lab Name: Envirosystems, Inc.

Lab Code: ENVSYS Case No.:

Matrix: (SOIL/SED/WATER) WATER

Sample wt/vol: $5.00(\mathrm{~g} / \mathrm{mL}) \quad \mathrm{ML}$

LeVEl: (TRACE/LOW/MED)

LOW

o Moisture: not dec.

GC Column:

$\underline{\mathrm{RTX}-624}$ ID: $0.18(\mathrm{~mm})$

Soil Extract Volume:

Purge Volume:

5.00

Contract:

Mod. Ref No.:

SDG No.: ARG80111

Lab Sample ID:

$0080111-03 R E 1$

Lab File ID:

F000230.D

Date Received:

$01 / 11 / 2008$

Date Analyzed:

$01 / 17 / 2008$

Dilution Factor:

2.0

\begin{tabular}{|c|c|c|c|}
\hline CAS NO. & COMPOUND & $\begin{array}{l}\text { CONCENTRATION UNITS: } \\
(\mathrm{ug} / \mathrm{L} \text { or } \mathrm{ug} / \mathrm{kg}) \mathrm{UG} / \mathrm{L}\end{array}$ & Q \\
\hline $79-01-6$ & Trichloroethene & 10 & $\mathrm{U}$ \\
\hline $108-87-2$ & Methylcyclohexane & 10 & $\mathrm{U}$ \\
\hline $78-87-5$ & 1,2-Dichloropropane & 10 & U \\
\hline $75-27-4$ & Bromodichloromethane & 10 & $\mathrm{U}$ \\
\hline $10061-01-5$ & cis-1,3-Dichloropropene & 10 & $\mathrm{U}$ \\
\hline $108-10-1$ & 4-Methyl-2-pentanone & 10 & $\mathrm{U}$ \\
\hline $108-88-3$ & Toluene & 10 & $\mathrm{U}$ \\
\hline $10061-02-6$ & trans-1,3-Dichloropropene & 10 & $\mathrm{U}$ \\
\hline $79-00-5$ & 1,1,2-Trichloroethane & 10 & $\mathrm{U}$ \\
\hline $127-18-4$ & Tetrachloroethene & 10 & $\mathrm{U}$ \\
\hline $591-78-6$ & 2-Hexanone & 10 & $\mathrm{U}$ \\
\hline $124-48-1$ & Dibromochloromethane & 10 & U \\
\hline $106-93-4$ & 1,2-Dibromoethane & 10 & $\mathrm{~J}$ \\
\hline $108-90-7$ & Chlorobenzene & 10 & $\mathrm{U}$ \\
\hline $100-41-4$ & Ethylbenzene & 10 & $\mathrm{U}$ \\
\hline $95-47-6$ & o-xylene & 10 & U \\
\hline $179601-23-1$ & $\mathrm{~m}, \mathrm{p}$-Xylene & 10 & U \\
\hline $100-42-5$ & Styrene & 10 & $\mathrm{U}$ \\
\hline $75-25-2$ & Bromoform & 10 & $\mathrm{U}$ \\
\hline $98-82-8$ & Isopropylbenzene & 10 & $\mathrm{U}$ \\
\hline $79-34-5$ & $1,1,2,2$-Tetrachloroethane & 10 & $\mathrm{U}$ \\
\hline $541-73-1$ & 1,3-Dichlorobenzene & 10 & $\mathrm{U}$ \\
\hline $106-46-7$ & 1,4-Dichlorobenzene & 10 & $\mathrm{U}$ \\
\hline $95-50-1$ & 1,2-Dichlorobenzene & 10 & $\mathrm{U}$ \\
\hline $96-12-8$ & 1,2-Dibromo-3-chloropropane & 10 & $\mathrm{U}$ \\
\hline $120-82-1$ & $1,2,4$-Trichlorobenzene & 10 & $\mathrm{U}$ \\
\hline $91-20-3$ & Naphthalene & 10 & $\mathrm{U}$ \\
\hline
\end{tabular}


$1 A$ - EORM I VOA-1

VOLATILE ORGANICS ANALYSIS DATA SHEET
EPA SAMPLE NO.

CNPMP8-W-26075
Lab Name:

Envirosystems, Inc.

Lab Code: ENVSYS Case No.:

Matrix: (SOIL/SED/WATER) WATER

Sample wt/vol:

5.00 $(\mathrm{g} / \mathrm{mL})$

ML

Level: (TRACE/LOW/MED)

LOW

\% Moisture: not dec.

GC Column: $\quad$ RTX-624 ID: 0.18 (mm)

Soil Extract Volume:

(uL)

Contract:

Mod. Ref No.: SDG No.:

ARG80111

Lab Sample ID:

Lab File ID:

Date Received:

Date Analyzed:

Dilution Factor:

Soil Aliquot Volume:

(uL)

Purge Volume:

5.00

(mL)

\begin{tabular}{|c|c|c|c|}
\hline CAS NO. & COMPOUND & $\begin{array}{l}\text { CONCENTRATION UNITS: } \\
(\mathrm{ug} / \mathrm{L} \text { or } \mathrm{ug} / \mathrm{kg}) \mathrm{UG} / \mathrm{L}\end{array}$ & Q \\
\hline $75-71-8$ & Dichlorodifluoromethane & 5.0 & $\mathrm{U}$ \\
\hline $74-87-3$ & Chloromethane & 5.0 & $\mathrm{U}$ \\
\hline $75-01-4$ & Vinyl chloride & 5.0 & $\mathrm{U}$ \\
\hline $74-83-9$ & Bromomethane & 5.0 & $\mathrm{U}$ \\
\hline $75-00-3$ & Chloroethane & 5.0 & $\mathrm{U}$ \\
\hline $75-69-4$ & Trichlorofluoromethane & 5.0 & $\mathrm{U}$ \\
\hline $75-35-4$ & 1,1-Dichloroethene & 5.0 & $\mathrm{U}$ \\
\hline $76-13-1$ & 1,1,2-Trichloro-1,2,2-trifluoroethane & 5.0 & $\mathrm{U}$ \\
\hline $67-64-1$ & Acetone & 5.0 & $\mathrm{U}$ \\
\hline $75-15-0$ & Carbon disulfide & 5.0 & $\mathrm{U}$ \\
\hline $79-20-9$ & Methyl acetate & 5.0 & $\mathrm{U}$ \\
\hline $75-09-2$ & Methylene chloride & 4.1 & $\mathrm{~J}$ \\
\hline $156-60-5$ & trans-1;2-Dichloroethene & 5.0 & $\mathrm{U}$ \\
\hline $1634-04-4$ & Methyl tert-butyl ether & 5.0 & $\mathrm{U}$ \\
\hline $75-34-3$ & 1,1-Dichloroethane & 5.0 & $\mathrm{U}$ \\
\hline $156-59-2$ & cis-1,2-Dichloroethene & 5.0 & $\mathrm{U}$ \\
\hline $78-93-3$ & 2-Butanone & 16 & \\
\hline $67-66-3$ & Chloroform & 740 & $E$ \\
\hline $71-55-6$ & 1,1,1-Trichloroethane & 5.0 & $\mathrm{U}$ \\
\hline $110-82-7$ & Cyclohexane & 5.0 & $\mathrm{U}$ \\
\hline $56-23-5$ & Carbon Tetrachloride & 5.1 & \\
\hline $71-43-2$ & Benzene & 5.0 & $\mathrm{U}$ \\
\hline $107-06-2$ & 1,2-Dichloroethane & 5.0 & $\mathrm{U}$ \\
\hline
\end{tabular}


$1 B$ - EORM I VOA-2

VOLATILE ORGANICS ANALYSIS DATA SHEET
EPA SAMPLE NO.

CNPMP8-W-26075
Lab Name:

Envirosystems, Inc.

Lab Code: ENVSYS Case No.:

Matrix: (SOIL/SED/WATER) WATER

Sample wt/vol: $5.00(\mathrm{~g} / \mathrm{mL}) \mathrm{ML}$

Level: (TRACE/LOW/MED)

LOW

\% Moisture: not dec.

GC Column: $\quad$ RTX-624 ID: 0.18 (mm)

Soil Extract Volume:

(uL)

(mL)

5.00

Contract:

Mod. Ref No.:

SDG No.: . ARG80111

Lab Sample ID:

$0080111-04$

Lab File ID:

F000222.D

Date Received:

$01 / 11 / 2008$

Date Analyzed:

$01 / 17 / 2008$

Dilution Factor:

1.0

Soil Aliquot Volume:

(UL)

Purge Volume:

CAS NO.

$79-01-6$

$108-87-2$

$78-87-5$

$75-27-4$

$10061-01-5$

$108-10-1$

$108-88-3$

$10061-02-6$

$79-00-5$

$127-18-4$

$591-78-6$

$124-48-1$

$106-93-4$

$108-90-7$

$100-41-4$

$95-47-6$

179601-23-1

$100-42-5$

$75-25-2$

$98-82-8$

$79-34-5$

541-73-1

$106-46-7$

$95-50-1$

$96-12-8$

$120-82-1$

$91-20-3$

Methylcyclohexane

Toluene

Tetrachloroethene

2-Hexanone

1,2-Dibromoethane

Chlorobenzene

Ethylbenzene

o-xylene

m,p-xylene

Styrene

Bromoform

Isopropylbenzene

Naphthalene

COMPOUND
Trichloroethene

1,2-Dichloropropane

Bromodichloromethane

Cis-1,3-Dichloropropene

4-Methyl-2-pentanone

trans-1,3-Dichloropropene

1,1,2-Trichloroethane

Dibromochloromethane

$1,1,2,2$-Tetrachloroethane

1,3-Dichlorobenzene

1,4-Dichlorobenzene

1,2-Dichlorobenzene

1,2-Dibromo-3-chloropropane

1,2,4-Trichlorobenzene

CONCENTRATION UNITS:

(ug/L or ug/kg) UG/L

5.0

5.0

5.0

5.0

5.0

5.0

5.0

5.0

5.0

5.0

5.0

5.0

5.0

5.0

5.0

5.0

5.0

5.0

5.0

5.0

5.0

5.0

5.0

5.0

5.0

5.0

5.0 
$1 A$ - FORM I VOA-1

VOLATILE ORGANICS ANALYSIS DATA SHEET
EPA SAMPLE NO.

CNPMP8-W-26075DL

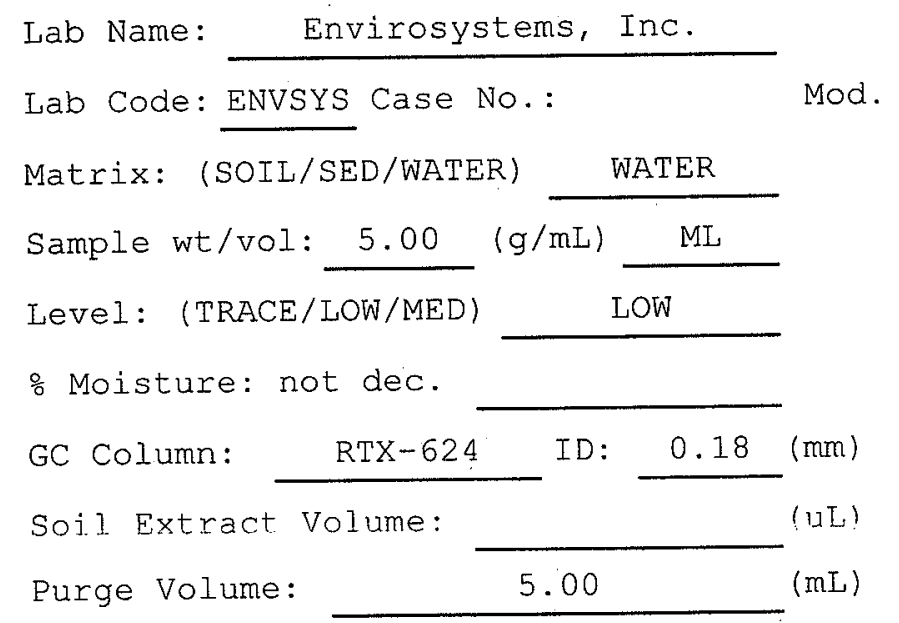

Contract:

Ref No.: SDG No.: $\quad$ ARG80111 Lab Sample ID: Lab File ID: 0080111-04RE1

Date Received: E000231.D

Date Analyzed:

Dilution Eactor: $01 / 11 / 2008$

$01 / 17 / 2008$

10.0 11?T:)

\begin{tabular}{|c|c|c|c|}
\hline CAS NO. & COMPOUND & $\begin{array}{l}\text { CONCENTRATION UNITS: } \\
(\mathrm{ug} / \mathrm{L} \text { or } \mathrm{ug} / \mathrm{kg}) \mathrm{UG} / \mathrm{L}\end{array}$ & $Q$ \\
\hline $75-71-8$ & Dichlorodifluoromethane & 50 & $\mathrm{U}$ \\
\hline $74-87-3$ & Chloromethane & 50 & $\mathrm{U}$ \\
\hline $75-01-4$ & Vinyl chloride & 50 & $\mathrm{U}$ \\
\hline $74-83-9$ & Bromomethane & 50 & $\mathrm{U}$ \\
\hline $75-00-3$ & Chloroethane & 50 & $\mathrm{U}$ \\
\hline $75-69-4$ & Trichlorofluoromethane & 50 & $\mathrm{U}$ \\
\hline $75-35-4$ & 1,1-Dichloroethene & 50 & $\mathrm{U}$ \\
\hline $76-13-1$ & $1,1,2-\operatorname{Trichloro}-1,2,2-t r i f l u o r o e t h a n e$ & 50 & $\mathrm{U}$ \\
\hline $67-64-1$ & Acetone & 50 & $\mathrm{U}$ \\
\hline $75-15-0$ & Carbon disulfide & 50 & $\mathrm{U}$ \\
\hline $79-20-9$ & Methyl acetate & 50 & $\mathrm{U}$ \\
\hline $75-09-2$ & Methylene chloride & 50 & $\mathrm{U}$ \\
\hline $156-60-5$ & trans-1,2-Dichloroethene & 50 & $\mathrm{U}$ \\
\hline $1634-04-4$ & Methyl tert-butyl ether & 50 & $\mathrm{U}$ \\
\hline $75-34-3$ & 1,1-Dichloroethane & 50 & $\mathrm{U}$ \\
\hline $156-59-2$ & Cis-1,2-Dichloroethene & 50 & $\mathrm{U}$ \\
\hline $78-93-3$ & 2-Butanone & 50 & $\mathrm{U}$ \\
\hline $67-66-3$ & Chloroform & 720 & $\mathrm{D}$ \\
\hline $71-55-6$ & $1,1,1$-Trichloroethane & 50 & $\mathrm{U}$ \\
\hline $110-82-7$ & Cyclohexane & 50 & $\mathrm{U}$ \\
\hline $56-23-5$ & Carbon Tetrachloride & 50 & $\mathrm{U}$ \\
\hline $71-43-2$ & Benzene & 50 & $\mathrm{U}$ \\
\hline $107-06-2$ & 1,2-Dichloroethane & 50 & $\mathrm{U}$ \\
\hline
\end{tabular}


$1 B$ - FORM I VOA-2

VOLATILE ORGANICS ANALYSIS DATA SHEET
EPA SAMPLE NO.

CNPMP8 $-W-26075$ DL
Lab Name: Envirosystems, Inc.

Lab Code: ENVSYS Case No.:

Matrix: (SOIL/SED/WATER)

WATER

Sample wt/vol: $5.00(\mathrm{~g} / \mathrm{mL}) \mathrm{ML}$

Level: (TRACE/LOW/MED)

LOW

\% Moisture: not dec.

GC Column: $\quad$ RTX-624 ID: 0.18 (mm)

Soil Extract Volume:

Purge Volume:

5.00

(uL)

(mL)

Contract:

Mod. Ref No.:

SDG No.: $\quad$ ARG80111

Lab Sample ID:

Lab File ID:

Date Received:

Date Analyzed:

Dilution Factor:

Soil Aliquot Volume:

(uL)

\begin{tabular}{|c|c|c|c|}
\hline CAS NO. & COMPOUND & $\begin{array}{l}\text { CONCENTRATION UNTTS: } \\
(\mathrm{ug} / \mathrm{L} \text { or } \mathrm{ug} / \mathrm{kg}) \mathrm{UG} / \mathrm{L}\end{array}$ & $Q$ \\
\hline $79-01-6$ & Trichloroethene & 50 & $\mathrm{U}$ \\
\hline $108-87-2$ & Methylcyclohexane & 50 & $\mathrm{U}$ \\
\hline $78-87-5$ & 1,2-Dichloropropane & 50 & $\mathrm{U}$ \\
\hline $75-27-4$ & Bromodichloromethane & 50 & $\mathrm{U}$ \\
\hline $10061-01-5$ & cis-1,3-Dichloropropene & 50 & U \\
\hline $108-10-1$ & 4-Methyl-2-pentanone & 50 & $\mathrm{U}$ \\
\hline $108-88-3$ & Toluene & 50 & $\mathrm{U}$ \\
\hline $10061-02-6$ & trans-1,3-Dichloropropene & 50 & $\mathrm{U}$ \\
\hline $79-00-5$ & 1,1,2-Trichloroethane & 50 & $\mathrm{U}$ \\
\hline $127-18-4$ & Tetrachloroethene & 50 & $\mathrm{U}$ \\
\hline $591-78-6$ & 2-Hexanone & 50 & $\mathrm{U}$ \\
\hline $124-48-1$ & Dibromochloromethane & 50 & U \\
\hline $106-93-4$ & 1,2-Dibromoethane & 50 & $\mathrm{U}$ \\
\hline $108-90-7$ & Chlorobenzene & 50 & $y$ \\
\hline $100-41-4$ & Ethylbenzene & 50 & U \\
\hline $95-47-6$ & o-xylene & 50 & $\mathrm{U}$ \\
\hline $179601-23-1$ & $\mathrm{~m}, \mathrm{p}-\mathrm{XY}$ lene & 50 & $\mathrm{U}$ \\
\hline $100-42-5$ & Styrene & 50 & $\mathrm{U}$ \\
\hline $75-25-2$ & Bromoform & 50 & $\mathrm{U}$ \\
\hline $98-82-8$ & Isopropylbenzene & 50 & $\mathrm{U}$ \\
\hline $79-34-5$ & $1,1,2,2$-Tetrachloroethane & 50 & $\mathrm{U}$ \\
\hline $541-73-1$ & 1,3-Dichlorobenzene & 50 & $\mathrm{U}$ \\
\hline $106-46-7$ & 1,4-Dichlorobenzene & 50 & $\mathrm{U}$ \\
\hline $95-50-1$ & 1,2-Dichlorobenzene & 50 & i \\
\hline $96-12-8$ & 1,2-Dibromo-3-chloropropane & 50 & $\mathrm{U}$ \\
\hline $120-82-1$ & $1,2,4$-Trichlorobenzene & 50 & $\mathrm{U}$ \\
\hline $91-20-3$ & Naphthalene & 50 & $\mathrm{U}$ \\
\hline
\end{tabular}




\section{ENVIROSYSTEMS, INC.}

9200 Rumsey Road • Suite B102 • Columbia, Maryland 21045-1934

Phone (410) 964-0330 - Fax (410) 740-9306

Email: info@envsystems.com.Webpage: www.envsystems.com/envsys

Date: 02-11-2008

Name: Jorge S. Alvarado Ph.D

Company: Argonne National Laboratory

Address: Enviromental Research Division

Applied Geosciences and Enviromental

Management Section

9700 South Cass Avenue

Bldg: 203, Room \# A137

Lemont, IL 60439

RE: Report\# 0070328

Dear Jorge,

Enclosed are the results of analyses for samples received by the laboratory on Febuary 25,2008 . If you have any questions concerning this report, please feel free to contact me.

Please do not hesitate to call if you have any questions, comments or require additional information

Sincerely,

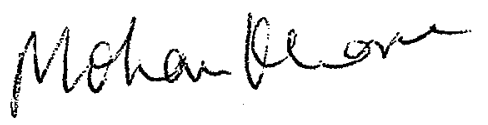

Mohan Khare, Ph.D

President/ CEO

Report \# 0070328 
Table of Contents

1. Narrative

2. Chain of Custody Documents

3. Volatiles Data 
1. Case Narrative 


\section{SDG NARRATIVE \\ VOLATILE ORGANICS (VOC)}

Envirosystems, Inc.

Contract: N/A

Client: Argonne National Laboratory

Case: N/A

SDG: ARG80114

\section{SAMPLE RECIEPT}

Date received: 01-25-2008

Cooler Temperature: 2

Sample Summary

\begin{tabular}{|c|c|c|c|}
\hline Client ID & Laboratory ID & Matrix & pH \\
\hline CNQCTB-W-25981 & $0080114-01$ & WATER & 7 \\
\hline CNPMP2-W-26084 & $0080114-02$ & WATER & 7 \\
\hline CNPMP2-W-26084DL & $0080114-02 R E 1$ & WATER & 7 \\
\hline CNPMP5-W-26088 & $0080114-03$ & WATER & 7 \\
\hline
\end{tabular}

2. HOLDING TIMES

A. Sample Preparation: All holding times were met.

B. Sample Analysis: All holding times were met

\section{METHODS}

The samples were analyzed and reported by using method SW-846 8260B and USEPA CLP SOW OLM04.3 for target compound list.

\section{INSTRUMENT AND CHROMATOGRAPHIC CONDITIONS}

A Hewlett Packard 6890 gas chromatograph equipped with a Hewlett Packard 5973 MSD was used for sample analysis. The capillary column used was a Restek $30 \mathrm{~m}$ by $0.25 \mathrm{~mm}$ ID by $1.0 \mu \mathrm{m}$ film thickness (Restek Cat. \# RTX-Volatiles). The trap used with the sample concentrator is an OI Analytical Trap \#10, $30 \mathrm{~cm}$ packed with Tenax/silica gel/cms (PN\#228122).

\section{PREPARATION}

The submitted samples were analyzed as received.

\section{ANALYSIS}

\section{A. Calibration:}

I. Initial calibration

All acceptance criteria as stipulated by SW-846 $8260 \mathrm{~b}$ were met for all SPCC's and CCC's. All target compounds met the required percent RSD. 


\section{SDG NARRATIVE \\ VOLATILE ORGANICS (VOC)}

II. Blanks:

All acceptance criteria were met.

II. Surrogates:

All acceptance criteria were met.

B. Spikes:

I. Laboratory Control Spikes (LCS)

I.CS samples were analyzed.

II. Matrix Spike/Matrix Spike Duplicate (MS/MSD)

The client did not request a MS/MSD.

C. Internal Standards:

All acceptance criteria were met.

D. Samples

Sample analysis proceeded normally. Samples CNPMP2-W-26084 required a dilution of 10X.

I certify that this Sample Data Package is in compliance with the terms and conditions of the contract, both technically and for completeness, for other than the conditions detailed above. Release of the data contained in the hard copy Sample Data Package and in the Electronic Data Deliverables has been authorized by the laboratory manager or the manager's designee, as verified by the following signatures.
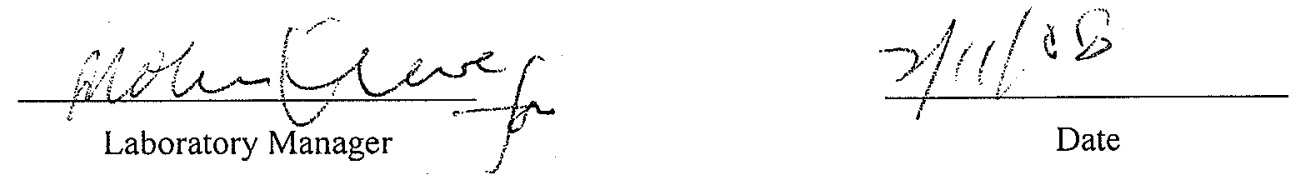
2. Traffic Report and Chain of Custody Records 


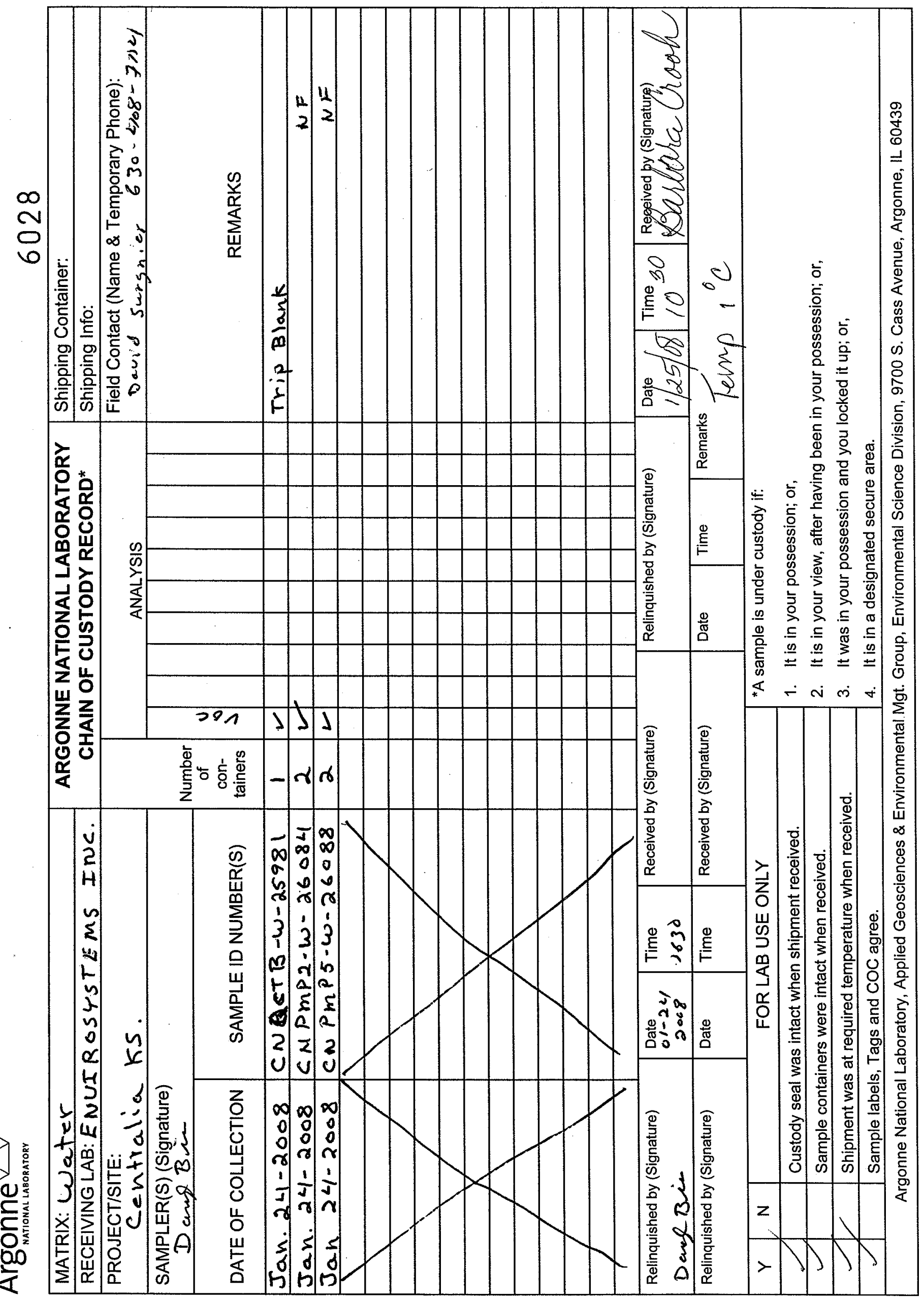


3. Volatiles Sample Data 
FORM 1

VOLATILE ORGANICS ANALYSIS DATA SHEET

Lab Name: ENVIROSYSTEMS, INC.

Contract: $\mathrm{N} / \mathrm{A}$

Lab Code: ENVSYS Case No.:

Matrix: (soil/water) WATER

SAS NO.: N/A

Lab Sample ID: 0080114-01

Sample wt/vol:

$5.000(\mathrm{~g} / \mathrm{mL}) \mathrm{ML}$

Level: (low/med) LOW

\% Moisture: not dec.

GC Column: RTX-624 ID: 0.18 (mm)

Soil Extract Volume: (UIL)
Lab File ID: F000361

Date Received: 01/25/08

Date Analyzed: 02/07/08

Dilution Factor: 1.0

Soil Aliquot Volume:
ARGONNE SAMPLE NO.

CNQCTB-W-25981 (uL)

CONCENTRATION UNITS: (ug/L or ug/Kg) UG/L

\begin{tabular}{|r|l|}
\hline $75-71-8$ & Dichlorodifluoromethane \\
$74-87-3$ & Chloromethane \\
$75-01-4$ & Vinyl Chloride \\
$74-83-9$ & Bromomethane \\
$75-00-3$ & Chloroethane \\
$75-69-4$ & Trichlorofluoromethane \\
$75-35-4$ & $1,1-D i c h l o r o e t h e n e$ \\
$76-13-1$ & $1,1,2-$ Trichloro-1,2,2-trifluoroethane \\
$67-64-1$ & Acetone \\
$75-15-0$ & Carbon Disulfide \\
$79-20-9$ & Methyl Acetate \\
$75-09-2$ & Methylene Chloride \\
$156-60-5$ & trans-1,2-Dichloroethene \\
$1634-04-4$ & Methyl tert-Butyl Ether \\
$75-34-3$ & 1,1-Dichloroethane \\
$156-59-2$ & cis-1,2-Dichloroethene \\
$78-93-3$ & 2-Butanone \\
$67-66-3$ & Chloroform \\
$71-55-6$ & $1,1,1-T r i c h l o r o e t h a n e$ \\
$110-82-7$ & Cyclohexane \\
$56-23-5$ & Carbon Tetrachloride \\
$71-43-2$ & Benzene \\
$107-06-2$ & 1,2-Dichloroethane \\
$79-01-6$ & Trichloroethene \\
$108-87-2$ & Methylcyclohexane \\
$78-87-5$ & $1,2-$ Dichloropropane \\
$75-27-4$ & Bromodichloromethane \\
$10061-01-5$ & Cis-1,3-Dichloropropene \\
$108-10-1$ & $4-$ Methyl-2-Pentanone \\
$108-88-3$ & Toluene \\
$10061-02-6$ & trans-1,3-Dichloropropene \\
$79-00-5$ & 1,1,2-Trichloroethane \\
$127-18-4$ & Tetrachloroethene \\
& \\
\hline
\end{tabular}

$5.0 \quad \mathrm{U}$

$5.0 \quad \mathrm{U}$

$5.0 \mathrm{U}$

$5.0 \mathrm{U}$

$5.0 \quad \mathrm{U}$

$5.0 \mathrm{U}$

$5.0 \mathrm{U}$

$5.0 \mathrm{U}$

$3.5 \mathrm{~J}$

$5.0 \mathrm{U}$

$5.0 \mathrm{U}$

$5.0 \mathrm{U}$

$5.0 \mathrm{U}$

$5.0 \quad \mathrm{U}$

$5.0 \quad \mathrm{U}$

$5.0 \mathrm{U}$

$5.0 \quad \mathrm{U}$

$5.0 \quad \mathrm{U}$

$5.0 \mathrm{U}$

$5.0 \mathrm{U}$

$5.0 \cdot \mathrm{U}$

$5.0 \mathrm{U}$

$5.0 \mathrm{U}$

5.0 U

$5.0 \mathrm{U}$

$5.0^{\circ} \mathrm{U}$

$5.0 \mathrm{U}$

$5.0 \mathrm{U}$

$5.0 \mathrm{U}$

$5.0 \mathrm{U}$

$5.0 \mathrm{U}$

5.0 U

$5.0 \mathrm{U}$ 
FORM 1

VOLATILE ORGANICS ANALYSIS DATA SHEET

Lab Name: ENVIROSYSTEMS, INC.

Contract: $\mathrm{N} / \mathrm{A}$

Lab Code: ENVSYS Case No.:

Matrix: (soil/water) WATER

Sample wt/vol: $\quad 5.000(\mathrm{~g} / \mathrm{mL}) \mathrm{ML}$

Level: (low/med) LOW

\% Moisture: not dec.

GC Column: RTX-624 ID: 0.18 (mm)

Soil Extract Volume: (uL)

SAS NO.: N/A
ARGONNE SAMPLE NO.

CNQCTB-W-25981

SDG No.: ARG0114

Lab Sample ID: 0080114-01

Lab File ID: F000361

Date Received: 01/25/08

Date Analyzed: 02/07/08

Dilution Factor: 1.0

Soil Aliquot Volume: (UL)

CAS NO.

COMPOUND

CONCENTRATION UNITS:

(ug/L or ug/Kg) UG/L

\begin{tabular}{r|l}
$591-78-6$ & 2-Hexanone \\
$124-48-1$ & Dibromochloromethane \\
$106-93-4$ & 1,2-Dibromoethane \\
$108-90-7$ & Chlorobenzene \\
$100-41-4$ & Ethylbenzene \\
$1330-20-7$ & Xylene (Total) \\
$100-42-5$ & Styrene \\
$75-25-2$ & Bromoform \\
$98-82-8$ & Isopropylbenzene \\
$79-34-5$ & $1,1,2,2-$ Tetrachloroethane \\
$541-73-1$ & $1,3-D i c h l o r o b e n z e n e$ \\
$106-46-7$ & $1,4-D i c h l o r o b e n z e n e$ \\
$95-50-1$ & $1,2-$ ichlorobenzene \\
$96-12-8$ & $1,2-$-Dibromo-3-chloropropane \\
$120-82-1$ & $1,2,4-$ Trichlorobenzene \\
$91-20-3$ & Naphthalene \\
$75-65-0$ & tert-Butanol \\
$108-20-3$ & Diisopropyl ether \\
$637-92-3$ & Ethyl-tert-butyl ether \\
$994-05-8$ & tert-Amyl methyl ether \\
$919-94-8$ & tert-Amyl ethyl ether
\end{tabular}

2-Hexanone

$5.0 \quad$ U

$5.0 \quad \mathrm{U}$

$5.0 \quad \mathrm{U}$

$5.0 \mathrm{U}$

$5.0 \quad \mathrm{U}$

$5.0 \mathrm{U}$

$5.0 \mathrm{U}$

$5.0 \mathrm{U}$

$5.0 \mathrm{U}$

$5.0 \mathrm{U}$

$5.0 \mathrm{U}$

$5.0 \mathrm{U}$

$5.0 \mathrm{U}$

$5.0 \mathrm{U}$

$5.0 \mathrm{U}$

$10 \mathrm{U}$

$5.0 \mathrm{U}$

$10 \mathrm{U}$

$10 \mathrm{U}$

$10 \mathrm{U}$

10 
FORM 1

VOLATILE ORGANICS ANALYSIS DATA SHEET

Lab Name: ENVIROSYSTEMS, INC.

Lab Code: ENvSYS Case No.:

Matrix: (soil/water) WATER

Contract: N/A

SAS NO.: N/A
ARGONNE SAMPLE NO.

CNPMP2 $-W-26084$
Sample wt/vol: $\quad 5.000(\mathrm{~g} / \mathrm{mL})$ ML

Level: (low/med) LOW

$\because$ Moisture: not dec.

GC Column: RTX-624 ID: 0.18 (mm)

Soil Extract Volume: (uL)
Lab Sample ID: 0080114-02

Lab File ID: F000362

Date Received: 01/25/08

Date Analyzed: 02/07/08

Dilution Factor: 1.0

Soil Aliquot Volume: (uL)

CAS NO.

COMPOUND

CONCENTRATION UNITS :

(ug/L or $u g / \mathrm{Kg}) \quad \mathrm{UG} / \mathrm{I}$

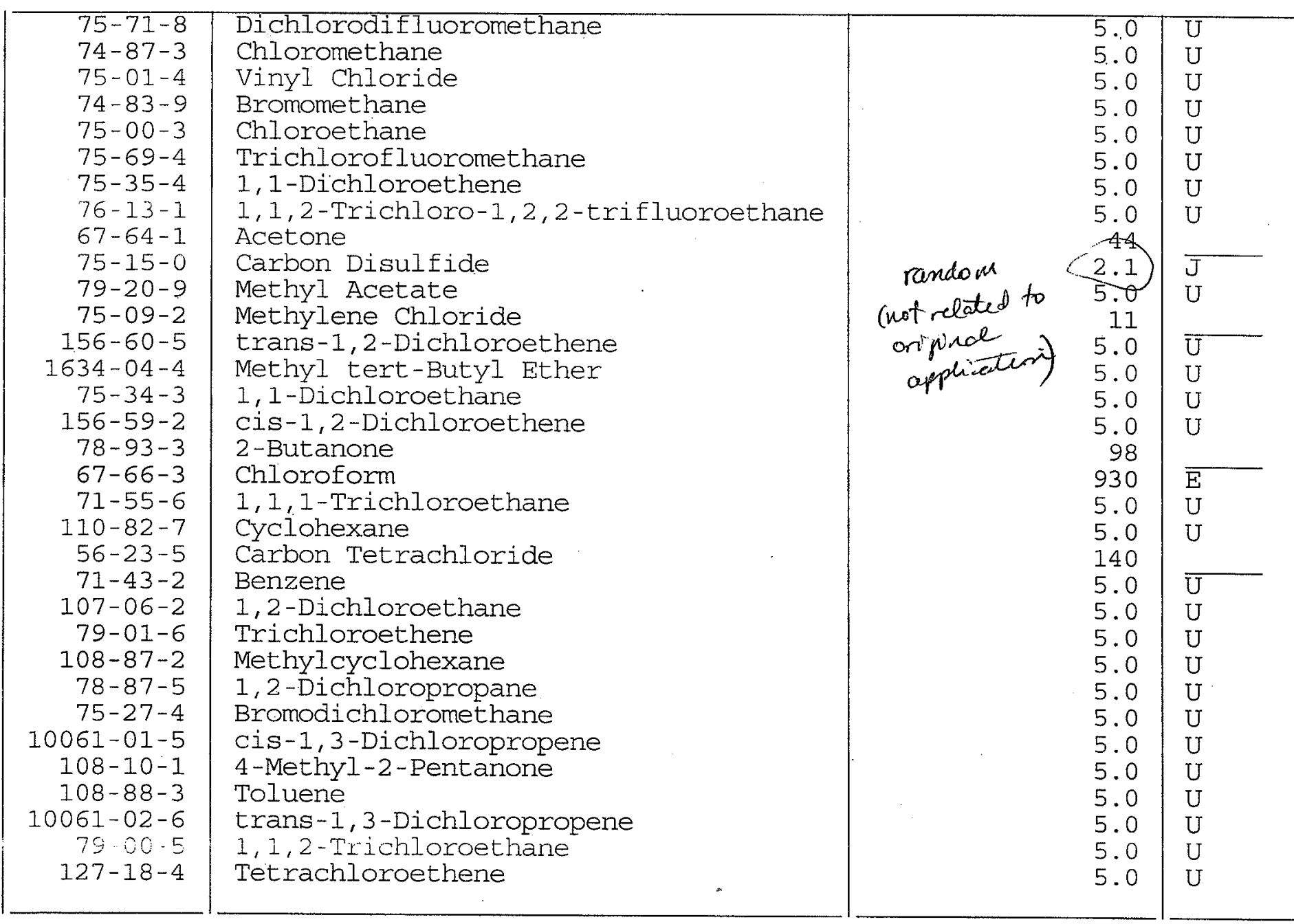


FORM 1

VOLATILE ORGANICS ANALYSIS DATA SHEET

Lab Name: ENVIROSYSTEMS, INC.

Contract: N/A

Lab Code: ENVSYS Case No.:

SAS NO.: N/A
ARGONNE SAMPLE NO.

CNPMP2 -W -26084
Matrix: (soil/water) WATER

Sample wt/vol: $\quad 5.000(\mathrm{~g} / \mathrm{mL}) \mathrm{ML}$

Level: (low/med) LOW

\% Moisture: not dec.

GC Column: RTX-624 ID: 0.18 (mm)

Soil Extract Volume: (uL)
Lab Sample ID: 0080114-02

Lab File ID: F000362

Date Received: 01/25/08

Date Analyzed: 02/07/08

Dilution Factor: 1.0

Soil Aliquot Volume: (UL)

CONCENTRATION UNITS:

CAS NO.

COMPOUND

\section{(ug/T or ug/}

\begin{tabular}{|r|l|r|l}
\hline $591-78-6$ & 2-Hexanone & 5.0 & $\mathrm{U}$ \\
$124-48-1$ & Dibromochloromethane & 5.0 & $\mathrm{U}$ \\
$106-93-4$ & 1,2-Dibromoethane & 5.0 & $\mathrm{U}$ \\
$108-90-7$ & Chlorobenzene & 5.0 & $\mathrm{U}$ \\
$100-41-4$ & Ethylbenzene & 5.0 & $\mathrm{U}$ \\
$1330-20-7$ & Xylene (Total) & 5.0 & $\mathrm{U}$ \\
$100-42-5$ & Styrene & 5.0 & $\mathrm{U}$ \\
$75-25-2$ & Bromoform & 5.0 & $\mathrm{U}$ \\
$98-82-8$ & Isopropylbenzene & 5.0 & $\mathrm{U}$ \\
$79-34-5$ & $1,1,2,2-$ Tetrachloroethane & 5.0 & $\mathrm{U}$ \\
$541-73-1$ & $1,3-$ Dichlorobenzene & 5.0 & $\mathrm{U}$ \\
$106-46-7$ & $1,4-D i c h l o r o b e n z e n e$ & 5.0 & $\mathrm{U}$ \\
$95-50-1$ & $1,2-$ Dichlorobenzene & 5.0 & $\mathrm{U}$ \\
$96-12-8$ & $1,2-$ Dibromo-3-chloropropane & 5.0 & $\mathrm{U}$ \\
$120-82-1$ & $1,2,4-T r i c h l o r o b e n z e n e$ & 5.0 & $\mathrm{U}$ \\
$91-20-3$ & Naphthalene & 10 & $\mathrm{U}$ \\
$75-65-0$ & tert-Butanol & 5.0 & $\mathrm{U}$ \\
$108-20-3$ & Diisopropyl ether & 10 & $\mathrm{U}$ \\
$637-92-3$ & Ethyl-tert-butyl ether & 10 & $\mathrm{U}$ \\
$994-05-8$ & tert-Amyl methyl ether & 10 & $\mathrm{U}$ \\
$919-94-8$ & tert-Amyl ethyl ether & 10 & $\mathrm{U}$ \\
\hline
\end{tabular}


FORM 1

VOLATILE ORGANICS ANALYSIS DATA SHEET

Lab Name: ENVIROSYSTEMS, INC.

Lab Code: ENVSYS Case No.:

Matrix: (soil/water) WATER

Sample wt/vol:

5.000 (g/mL) ML

Level: (low/med) LOW

․oisture: not dec.

GC Column: RTX-624 ID: 0.18 (mm)

Soil Extract Volume: (uI)
Contract: N/A

SAS NO.: N/A
ARGONNE SAMPLE NO.

CNPMP-2-26084DI
SDG No.: NA

Lab Sample ID: 0080114-02RE1

Lab File ID: F000376

Date Received: 01/25/08

Date Analyzed: 02/08/08

Dilution Factor: 10.0

Soil Aliquot. Volume: (UI)

CAS NO.

COMPOUND

CONCENTRATION UNITS :

(ug/L or $u g / \mathrm{Kg}$ ) UG/L Q

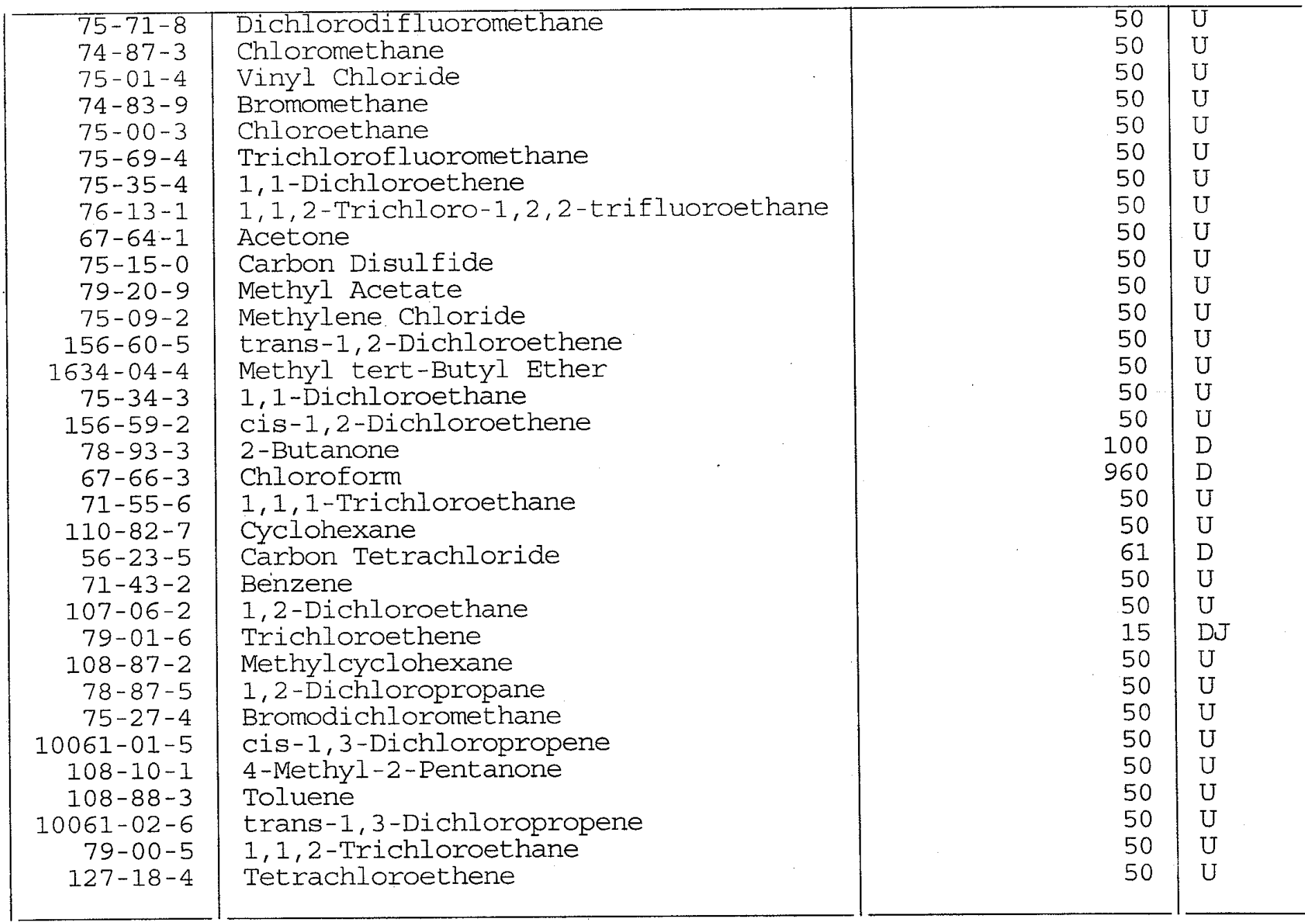


FORM 1

VOLATILE ORGANICS ANALYSIS DATA SHEET

Lab Name: ENVIROSYSTEMS, INC.

Lab Code: ENVSYS Case No.:

Matrix: (soil/water) WATER

Sample wt/vol:

$5.000(\mathrm{~g} / \mathrm{mL}) \mathrm{NL}$

Level: (low/med) LOW

\% Moisture: not dec.

GC Column: RTX-624 ID: 0.18 (mm)

Soil Extract Volume: (UI)
Contract: $\mathrm{N} / \mathrm{A}$

SAS NO.: N/A
ARGONNE SAMPLE NO.

CNPMP - 2-26084DL

Lab Sample ID: 0080114-02REI

Lab File ID: F000376

Date Received: 01/25/08

Date Analyzed: 02/08/08

Dilution Factor: 10.0

Soil Aliquot Volume: (uL)

CAS NO.

COMPOUND

CONCENTRATION UNITS:

$(\mathrm{ug} / \mathrm{L}$ or $\mathrm{ug} / \mathrm{Kg})$ UG/L Q

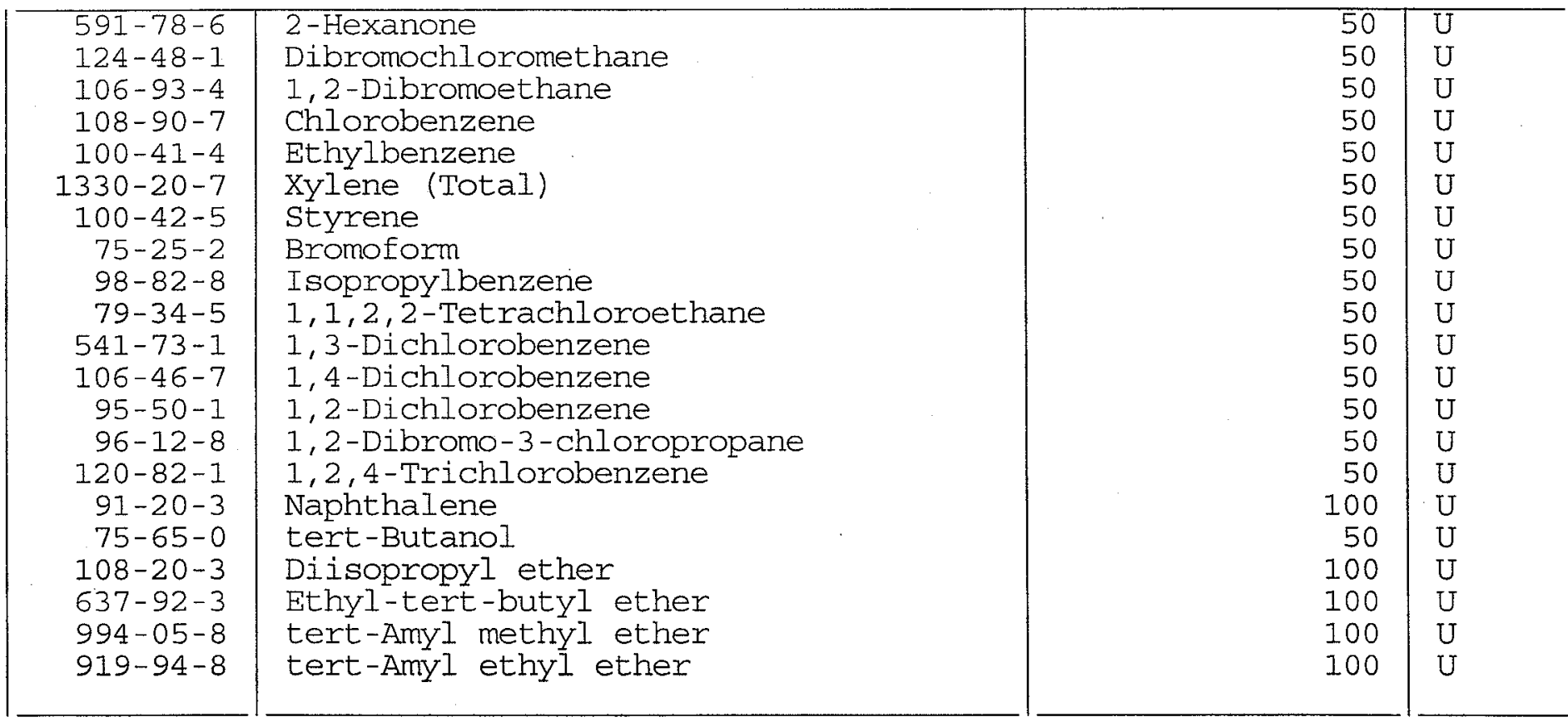


FORM 1

VOLATILE ORGANICS ANALYSIS DATA SHEET

Lab Name: ENVIROSYSTEMS, INC.

Lab Code: ENVSYS Case No.:

Matrix: (soil/water) WATER

Sample wt/vol: $\quad 5.000(\mathrm{~g} / \mathrm{mL}) \mathrm{ML}$

Level: (low/med) LOW

\% Moisture: not dec.

GC Column: RTX-624 ID: 0.18 (mm)

Soil Extract Volume: (UI)
Contract: $\mathrm{N} / \mathrm{A}$

SAS No.: N/A
ARGONNE SAMPLE NO.

CNPMP2 $-W-26088$
Lab Sample ID: 0080114-03

Lab File ID: F000363

Date Received: 01/25/08

Date Analyzed: 02/07/08

Dilution Factor: 1.0

Soil Aliquot Volume:

CAS NO

COMPOUND

(ug/L or $\mathrm{ug} / \mathrm{kg}$ )

$\mathrm{UG} / \mathrm{L} \quad \mathrm{Q}$

\begin{tabular}{|c|c|c|c|c|}
\hline $\begin{array}{r}75-71-8 \\
74-87-3 \\
75-01-4 \\
74-83-9 \\
75-00-3 \\
75-69-4 \\
75-35-4 \\
76-13-1 \\
67-64-1 \\
75-15-0 \\
79-20-9 \\
75-09-2 \\
156-60-5 \\
1634-04-4 \\
75-34-3 \\
156-59-2 \\
78-93-3 \\
67-66-3 \\
71-55-6 \\
110-82-7 \\
56-23-5 \\
71-43-2 \\
107-06-2 \\
79-01-6 \\
108-87-2 \\
78-87-5 \\
75-27-4 \\
10061-01-5 \\
108-10-1 \\
108-88-3 \\
10061-02-6 \\
79-00-5 \\
127-18-4\end{array}$ & $\begin{array}{l}\text { Dichlorodifluoromethane } \\
\text { Chloromethane } \\
\text { Vinyl Chloride } \\
\text { Bromomethane } \\
\text { Chloroethane } \\
\text { Trichlorofluoromethane } \\
\text { 1,1-Dichloroethene } \\
\text { 1,1,2-Trichloro-1,2,2-trifluoroethane } \\
\text { Acetone } \\
\text { Carbon Disulfide } \\
\text { Methyl Acetate } \\
\text { Methylene Chloride } \\
\text { trans-1,2-Dichloroethene } \\
\text { Methyl tert-Butyl Ether } \\
1,1-D i c h l o r o e t h a n e \\
\text { Cis-1,2-Dichloroethene } \\
\text { 2-Butanone } \\
\text { Chloroform } \\
1,1,1-T r i c h l o r o e t h a n e \\
\text { Cyclohexane } \\
\text { Carbon Tetrachloride } \\
\text { Benzene } \\
\text { 1,2-Dichloroethane } \\
\text { Trichloroethene } \\
\text { Methylcyclohexane } \\
\text { 1,2-Dichloropropane } \\
\text { Bromodichloromethane } \\
\text { cis-1,3-Dichloropropene } \\
4-\text { Methyl-2-Pentanone } \\
\text { Toluene } \\
\text { trans-1,3-Dichloropropene } \\
\text { 1,1,2-Trichloroethane } \\
\text { Tetrachloroethene }\end{array}$ & 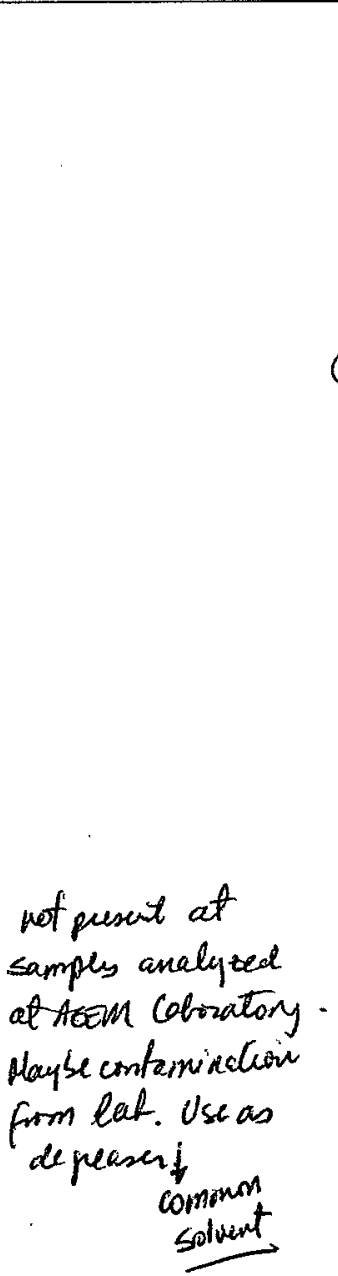 & $\begin{array}{l}5.0 \\
5.0 \\
5.0 \\
5.0 \\
5.0 \\
5.0 \\
5.0 \\
5.0 \\
3.0 \\
5.0 \\
5.0 \\
3.2 \\
5.0 \\
5.0 \\
5.0 \\
5.0 \\
5.0 \\
100 \\
5.0 \\
5.0 \\
150 \\
5.0 \\
5.0 \\
50 \\
5.0 \\
5.0 \\
5.0 \\
5.0 \\
5.0 \\
5.0 \\
5.0 \\
5.0 \\
5.0\end{array}$ & $\begin{array}{l}U \\
U \\
U \\
U \\
U \\
U \\
U \\
U \\
J \\
U \\
U \\
J \\
U \\
U \\
U \\
U \\
U \\
U\end{array}$ \\
\hline
\end{tabular}


FORM 1

VOLATILE ORGANICS ANALYSIS DATA SHEET
Lab Name: ENVIROSYSTEMS, INC.

Lab Code: ENVSYS Case No.:

Matrix: (soil/water) WATER

Sample wt/vol:

$5.000(\mathrm{~g} / \mathrm{mL}) \mathrm{ML}$

Level: (low/med) LOW

o Moisture: not dec.

GC Column: RTX-624 ID: 0.18 (mm)

Soil Extract Volume: (uL)
Contract: N/A

SAS NO.: N/A
ARGONNE SAMPLE NO.

CNPMP2 -W-26088

Lab Sample ID: 0080114-03

Lab File ID: F000363

Date Received: 01/25/08

Date Analyzed: 02/07/08

Dilution Factor: 1.0

Soil Aliquot Volume: (uL)

CONCENTRATION UNITS:

CAS NO.

COMPOUND

(ug/L or $\mathrm{ug} / \mathrm{Kg}$ )

UG/I

\begin{tabular}{|r|l|r|l|}
\hline $591-78-6$ & 2-Hexanone & 5.0 & $\mathrm{U}$ \\
$124-48-1$ & Dibromochloromethane & 5.0 & $\mathrm{U}$ \\
$106-93-4$ & 1,2-Dibromoethane & 5.0 & $\mathrm{U}$ \\
$108-90-7$ & Chlorobenzene & 5.0 & $\mathrm{U}$ \\
$100-41-4$ & Ethylbenzene & 5.0 & $\mathrm{U}$ \\
$1330-20-7$ & Xylene (Total) & 5.0 & $\mathrm{U}$ \\
$100-42-5$ & Styrene & 5.0 & $\mathrm{U}$ \\
$75-25-2$ & Bromoform & 5.0 & $\mathrm{U}$ \\
$98-82-8$ & Isopropylbenzene & 5.0 & $\mathrm{U}$ \\
$79-34-5$ & $1,1,2,2-$ Tetrachloroethane & 5.0 & $\mathrm{U}$ \\
$541-73-1$ & 1,3 -Dichlorobenzene & 5.0 & $\mathrm{U}$ \\
$106-46-7$ & $1,4-$ Dichlorobenzene & 5.0 & $\mathrm{U}$ \\
$95-50-1$ & $1,2-$ Dichlorobenzene & 5.0 & $\mathrm{U}$ \\
$96-12-8$ & $1,2-$ Dibromo-3-chloropropane & 5.0 & $\mathrm{U}$ \\
$120-82-1$ & 1,2,4-Trichlorobenzene & 10 & $\mathrm{U}$ \\
$91-20-3$ & Naphthalene & 5.0 & $\mathrm{U}$ \\
$75-65-0$ & tert-Butanol & 10 & $\mathrm{U}$ \\
$108-20-3$ & Diisopropyl ether & 10 & $\mathrm{U}$ \\
$637-92-3$ & Ethyl-tert-butyl ether & 10 & $\mathrm{U}$ \\
$994-05-8$ & tert-Amyl methyl ether & 10 & $\mathrm{U}$ \\
$919-94-8$ & tert-Amyl ethyl ether & & \\
\hline
\end{tabular}




\section{ENVIROSYSTEMS, INC.}

9200 Rumsey Road - Suite B102 - Columbia, Maryland 21045-1934

Phone (410) 964-0330 - Fax (410) 740-9306

Email: info@envsystems.com - Webpage: www.envsystems.com/envsys

March 14, 2008

Jorge S. Alvarado, Ph.D

Argonne National Laboratory

Environmental Sciences Division

Applied Geoscience and Environmental

Management Section

9700 South Cass Avenue, EV-203-A137

Argonne, Illinois 60439

\section{RE: ENVSYS Report 080139}

Dear Jorge:

Enclosed is the Analytical Data Package for the samples received on February 26, 2008 for volatile organics analysis by US EPA CLP SOW OLM04.3

Please do not hesitate to call me if you have any questions, comments, or require additional information.

Sincerely,

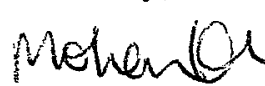

Mohan Khare, Ph.D

President/CEO

$\mathrm{MK} / \mathrm{pl}$ 
Table Of Contents

1.Case Narrative

2. Traffic Reports/Chain Of Custody

3. Volatiles Data

3.1 Volatiles QC Summary

3.2 Volatiles Sample Data

3.3 Volatiles Standard Data

3.4 Volatiles Raw QC Data 
1.Narrative 
Narrative

Three samples were received on February 28, 2008 for VOA analysis and CLP protocols according to the chain of custody instructions. These samples were logged in and distributed for analysis. The results are present in this report along with the back up data and chain of custody records. 
2. Traffic Reports/ Chain of Custody Records 


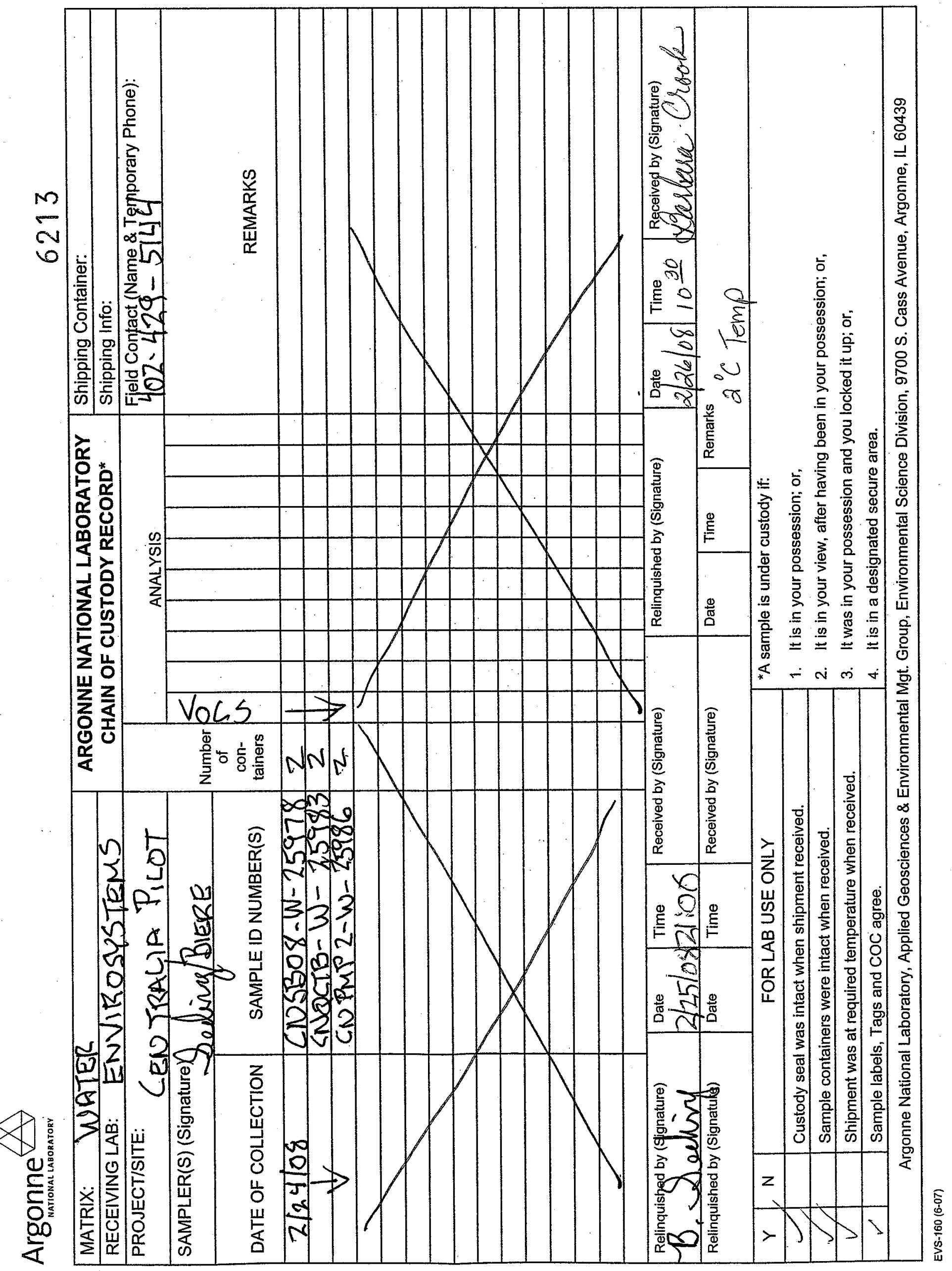


3. Volatiles Data 
FORM 1

VOLATILE ORGANICS ANALYSIS DATA SHEET
Lab Name: ENVIROSYSTEMS, INC.

Lab Code: ENVSYS Case No.:

Matrix: (soil/water) WATER

Sample wt/vol: $\quad 5.000(\mathrm{~g} / \mathrm{mL}) \mathrm{ML}$

Level: (low/med) LOW

\% Moisture: not dec.

GC Column: RTX-624 ID: 0.18 (mm)

Soil Extract Volume:

(uL)
Contract: N/A

SAS NO.: N/A
ARGONNE SAMPLE NO.

CNSB08-W-25978
Lab Sample ID: 0080215-01.

Lab File ID: F000407

Date Received: 01/25/08

Date Analyzed: 02/26/08

Dilution Factor: 1.0

Soil Aliquot Volume: (UI)

CAS NO.

COMPOUND

CONCENTRATION UNITS:

(ug/L or ug/Kg) UG/L

Q

$5.0 \mathrm{U}$

$5.0 \mathrm{U}$

$5.0 \mathrm{U}$

$5.0 \mathrm{U}$

$5.0 \mathrm{U}$

$5.0 \mathrm{U}$

$5.0 \mathrm{U}$

$5.0 \mathrm{U}$

$5.0 \mathrm{U}$

$5.0 \mathrm{U}$

$5.0 \mathrm{U}$

$3.0 \mathrm{~J}$

$5.0 \mathrm{U}$

$5.0 \mathrm{U}$

$5.0 \mathrm{U}$

$5.0 \mathrm{U}$

$5.0 \mathrm{U}$

$5.0 \mathrm{U}$

$5.0 \mathrm{U}$

$5.0 \mathrm{U}$

43

$5.0 \overline{\mathrm{U}}$

$5.0 \mathrm{U}$

$5.0 \mathrm{U}$

$5.0 \mathrm{U}$

$5.0 \mathrm{U}$

$5.0 \mathrm{U}$

$5.0 \mathrm{U}$

$5.0 \mathrm{U}$

$5.0 \mathrm{U}$

$5.0 \mathrm{U}$

$5.0 \mathrm{U}$

$5.0 \mathrm{U}$ 
FORM 1

VOLATILE ORGANICS ANALYSIS DATA SHEET
Lab Name: ENVIROSYSTEMS, INC.

Lab Code: ENVSYS Case No.:

Matrix: (soil/water) WATER

Sample wt/vol: $\quad 5.000(\mathrm{~g} / \mathrm{mL}) \mathrm{ML}$

Level: (low/med) LOW

\% Moisture: not dec.

GC Column: RTX-624 ID: 0.18 (mm)

Soil Extract Volume: (uI)
Contract: $\mathrm{N} / \mathrm{A}$

SAS NO.: N/A
ARGONNE SAMPLE NO.

CNSB08-W-25978

Lab Sample ID: 0080215-01

Lab File ID: F000407

Date Received: 01/25/08

Date Analyzed: 02/26/08

Dilution Factor: 1.0

Soil Aliquot Volume: (uL) CONCENTRATION UNITS : (ug/L or ug/Kg) UG/L

Q

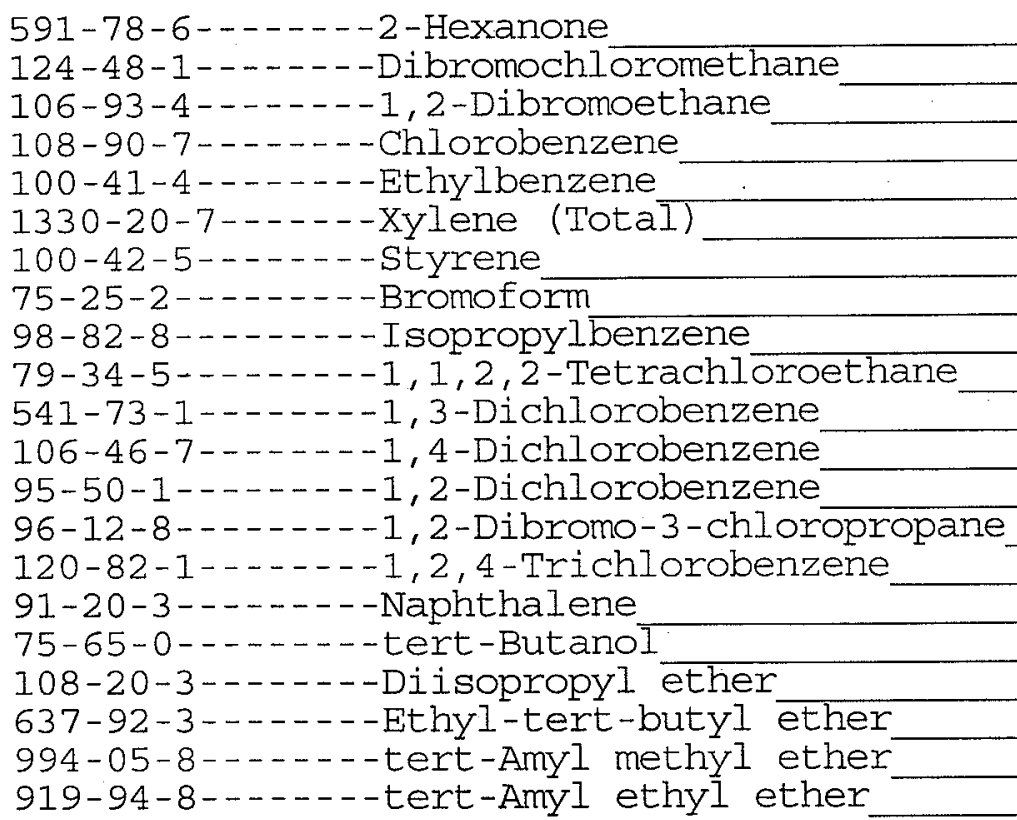

$5.0 \cdot \mathrm{U}$

$5.0 \mathrm{U}$

$5.0 \mathrm{U}$

$5.0 \mathrm{U}$

$5.0 \mathrm{U}$

$5.0 \mathrm{U}$

$5.0 \mathrm{U}$

$5.0 \mathrm{U}$

$5.0 \mathrm{U}$

$5.0 \mathrm{U}$

$5.0 \mathrm{U}$

$5.0 \mathrm{U}$

$5.0 \mathrm{U}$

$5.0 \mathrm{U}$

$5.0 \mathrm{U}$

$4.5 \mathrm{~J}$

$5.0 \mathrm{U}$

$10 \mathrm{U}$

$10 \mathrm{U}$

$10 \mathrm{U}$

$10 \mathrm{U}$ 
FORM 1

VOLATILE ORGANICS ANALYSIS DATA SHEET
Lab Name: ENVIROSYSTEMS, INC.

Lab Code: ENVSYS Case No.:

Matrix: (soil/water) WATER

Sample wt/vol: $\quad 5.000(\mathrm{~g} / \mathrm{mL}) \mathrm{ML}$

Level: . (low/med) LOW

\% Moisture: not dec.

GC Column: RTX-624 ID: 0.18 (mm)

Soil Extract Volume: (uL)
Contract: N/A

SAS No.: N/A
ARGONNE SAMPLE NO.

CNOCTB-W-25983

Lab Sample ID: 0080215-02

Lab File ID: F000408

Date Received: 01/25/08

Date Analyzed: 02/26/08

Dilution Factor: 1.0

Soil Aliquot Volume: (UL)

CONCENTRATION UNITS :

CAS NO.

COMPOUND

(ug/L or $\mathrm{ug} / \mathrm{Kg}$ ) UG/L

Q

$5.0 \mathrm{U}$

$5.0 \mathrm{U}$

$5.0 \mathrm{U}$

$5.0 \mathrm{U}$

$5.0 \mathrm{U}$

$5.0 . \mathrm{U}$

$5.0 \mathrm{U}$

$5.0 \mathrm{U}$

$5.0 \mathrm{U}$

$5.0 \mathrm{U}$

$5.0 \mathrm{U}$

$4.6 \mathrm{~J}$

$5.0 \mathrm{U}$

$5.0 \mathrm{U}$

$5.0 \mathrm{U}$

$5.0 \mathrm{U}$

$5.0 \mathrm{U}$

$5.0 \mathrm{U}$

$5.0 \mathrm{U}$

$5.0 \mathrm{U}$

$5.0 \mathrm{U}$

$5.0 \mathrm{U}$

$5.0 \mathrm{U}$

$5.0 \mathrm{U}$

$5.0 \mathrm{U}$

$5.0 \mathrm{U}$

$5.0 \mathrm{U}$

$5.0 \mathrm{U}$

$5.0 \mathrm{U}$

$5.0 \mathrm{U}$

$5.0 \mathrm{U}$

$5.0 \mathrm{U}$

$5.0 \mathrm{U}$ 
Lab Name: ENVIROSYSTEMS, INC.

Lab Code: ENVSYS Case No.:

Matrix: (soil/water) WATER

Sample wt/vol:

$5.000(\mathrm{~g} / \mathrm{mL})$ ML

Level: (low/med) LOW

\% Moisture: not dec.

GC Column: RTX-624 ID: 0.18 (mm)

Soil Extract Volume: (uT)
Contract: N/A

SAS NO.: N/A
CNOCTB-W-25983
Lab Sample ID: 0080215-02

Lab File ID: F000408

Date Received: 01/25/08

Date Analyzed: 02/26/08

Dilution Factor: 1.0

Soil Aliquot Volume: (uL)

\section{CAS NO.}

COMPOUND

CONCENTRATION UNITS:

(ug/L or ug/Kg) UG/L

$Q$

$5.0 \mathrm{U}$

$5.0 \mathrm{U}$

$5.0 \mathrm{U}$

$5.0 \mathrm{U}$

$5.0 \mathrm{U}$

$5.0 \mathrm{U}$

$5.0 \mathrm{U}$

$5.0 \mathrm{U}$

$5.0 \mathrm{U}$

$5.0 \mathrm{U}$

$5.0 \mathrm{U}$

$5.0 \mathrm{U}$

$5.0 \mathrm{U}$

$5.0 \mathrm{U}$

$5.0 \mathrm{U}$

$3.5 \mathrm{~J}$

$5.0 \mathrm{U}$

$10 \mathrm{U}$

$10 \mathrm{U}$

$10 \mathrm{U}$

$10 . \mathrm{U}$ 
FORM 1

VOLATILE ORGANICS ANALYSIS DATA SHEET
ARGONNE SAMPLE NO.

CNPMP2 -W-25986
Lab Name: ENVIROSYSTEMS, INC.

Lab Code: ENVSYS Case No.:

Matrix: (soil/water) WATER

Sample wt/vol: $\quad 5.000(\mathrm{~g} / \mathrm{mL})$ ML

Level: (low/med) LOW

\% Moisture: not dec.

GC Column: RTX-624 ID: 0.18 (mm)

Soil Extract Volume: (UI)
Contract: $\mathrm{N} / \mathrm{A}$

SAS NO.: N/A
SDG No. : NA
Lab File ID: F000409

Date Received: 01/25/08

Date Analyzed: 02/26/08

Dilution Factor: 1.0

Soil Aliquot Volume: (UL)

CAS NO.

COMPOUND

CONCENTRATION UNITS:

(ug/L or $\mathrm{ug} / \mathrm{Kg}$ ) UG/L

Q

$5.0 \mathrm{U}$

$5.0 \mathrm{U}$

$5.0 \mathrm{U}$

$5.0 \mathrm{U}$

$5.0 \mathrm{U}$

$5.0 \mathrm{U}$

$5.0 \mathrm{U}$

$5.0 \mathrm{U}$

14

$5.0 \overline{\mathrm{U}}$

$5.0 \mathrm{U}$

13

$5.0 \overline{\mathrm{U}}$

$5.0 \mathrm{U}$

$5.0 \mathrm{U}$

$5.0 \mathrm{U}$

9.5

$570 \overline{\mathrm{E}}$

$5.0 \mathrm{U}$

$5.0 \mathrm{U}$

$720 \mathrm{E}$

$5.0 \mathrm{U}$

$5.0 \mathrm{U}$

$5.0 \mathrm{U}$

$5.0 \mathrm{U}$

$5.0 \mathrm{U}$

$5.0 \mathrm{U}$

$5.0 \mathrm{U}$

$5.0 \mathrm{U}$

1.1 J

$5.0 \mathrm{U}$

$5.0 \mathrm{U}$

$5.0 \mathrm{U}$ 
FORM 1

VOLATILE ORGANICS ANALYSIS DATA SHEET

Lab Name: ENVIROSYSTEMS, INC.

Lab Code: ENVSYS Case No.:

Matrix: (soil/water) WATER

Sample wt/vol: $\quad 5.000(\mathrm{~g} / \mathrm{mL}) \mathrm{ML}$

Level: (low/med) LOW

\% Moisture: not dec.

GC Column: RTX-624 ID: 0.18 (mm)

Soil Extract Volume: (uL)
Contract: $\mathrm{N} / \mathrm{A}$

SAS NO.: N/A
ARGONNE SAMPIE NO.

CNPMP2 $-W-25986$

Lab Sample ID: 0080215-03

Lab File ID: F000409

Date Received: 01/25/08

Date Analyzed: 02/26/08

Dilution Factor: 1.0

Soil Aliquot Volume: (uL)

CONCENTRATION UNITS :

(ug/L or $\mathrm{ug} / \mathrm{Kg}$ ) UG/L

Q

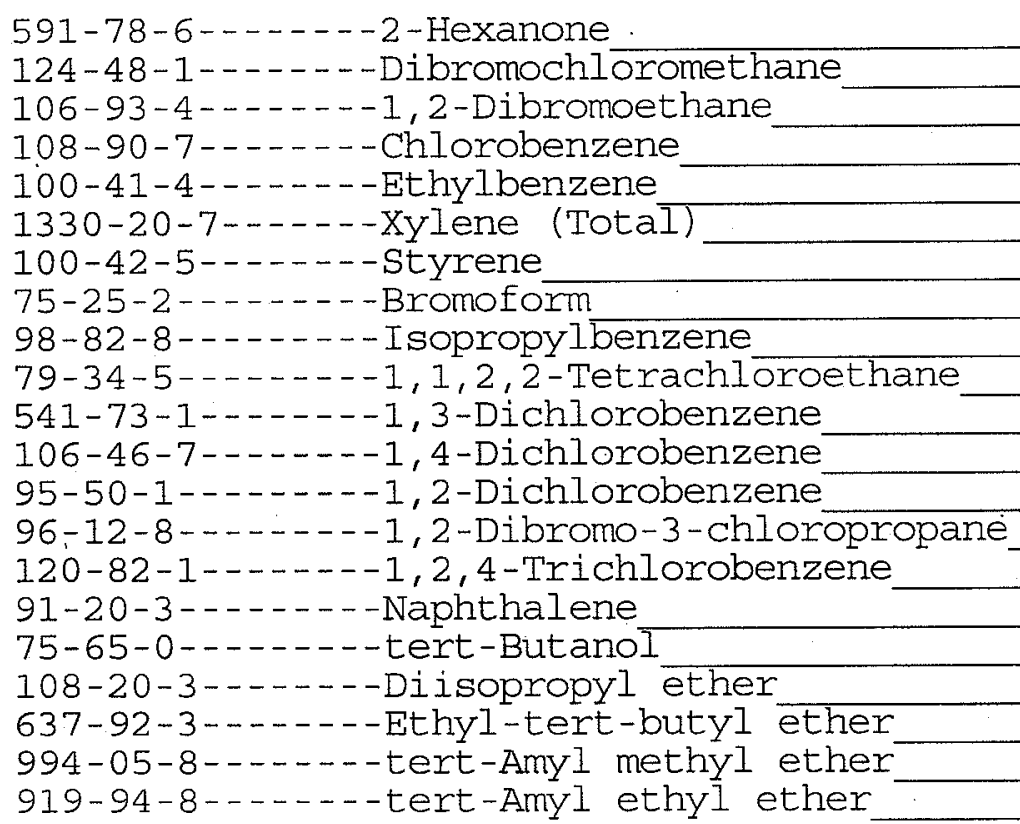

$5.0 \mathrm{U}$

5.0 U

$5.0 \mathrm{U}$

$5.0 \mathrm{U}$

$5.0 \mathrm{U}$

$5.0 \mathrm{U}$

$5.0 \mathrm{U}$

5. $0 \mathrm{U}$

$5.0 \mathrm{U}$

$5.0 \mathrm{U}$

$5.0 \mathrm{U}$

$5.0 \mathrm{U}$

$5.0 \mathrm{U}$

$5.0 \mathrm{U}$

$5.0 \mathrm{U}$

$2.6 \mathrm{~J}$

$5.0 \mathrm{U}$

$10 \mathrm{U}$

$10 \mathrm{U}$

$10 \mathrm{U}$

$10 \mathrm{U}$ 
FORM 1

VOLATILE ORGANICS ANALYSIS DATA SHEET

Lab Name: ENVIROSYSTEMS, INC.

Lab Code: ENVSYS Case No.:

Contract: $\mathrm{N} / \mathrm{A}$

SAS NO.: N/A
ARGONNE · SAMPLE NO.

\begin{tabular}{l}
\hline CNPMP2-W \\
$-25986 \mathrm{DL}$ \\
\hline
\end{tabular}

SDG NO.: NA
Matrix: (soil/water) WATER

Sample wt/vol: $\quad 5.000(\mathrm{~g} / \mathrm{mL}) \mathrm{ML}$

Level: (low/med) LOW

\% Moisture: not dec.

GC Column: RTX-624 ID: 0.18 (mm)

Soil Extract Volume: (uL)
Lab Sample ID: 0080215-03RE1

Lab File ID: F000415

Date Received: 01/25/08

Date Analyzed: 02/26/08

Dilution Factor: 10.0

Soil Aliquot Volume: (uL)

CAS NO. COMPOUND (ug/L or $\mathrm{ug} / \mathrm{Kg})$ UG/L

Q

$50 \mathrm{U}$

$50 \mathrm{U}$

$50 \mathrm{U}$

$50 \mathrm{U}$

$50 \mathrm{U}$

$50 \mathrm{U}$

$50 \mathrm{U}$

$50 \mathrm{U}$

$50 \mathrm{U}$

$50 \mathrm{U}$

$50 \mathrm{U}$

$61 \mathrm{D}$

$50 \mathrm{U}$

$50 \mathrm{U}$

$50 \mathrm{U}$

$50 \mathrm{U}$

$50 \mathrm{U}$

640 D

$50 \mathrm{U}$

$50 \mathrm{U}$

$700 \mathrm{D}$

$50 \mathrm{U}$

$50 \mathrm{U}$

$50 \mathrm{U}$

$50 \mathrm{U}$

$50 \mathrm{U}$

$50 \mathrm{U}$

$50 \mathrm{U}$

$50 \mathrm{U}$

$50 \mathrm{U}$

$50 \mathrm{U}$

$50 \mathrm{U}$

50 
FORM 1

VOLATILE ORGANICS ANALYSIS DATA SHEET
Lab Name: ENVIROSYSTEMS, INC.

Lab Code: ENVSYS Case No.:

Matrix: (soil/water) WATER

Sample wt/vol: $\quad 5.000(\mathrm{~g} / \mathrm{mL}) \mathrm{ML}$

Level: (low/med) LOW

\% Moisture: not dec.

GC Column: RTX-624 ID: 0.18 (mm)

Soil Extract Volume: (UL)
Contract: $\mathrm{N} / \mathrm{A}$

SAS NO.: N/A
ARGONNE SAMPLE NO.

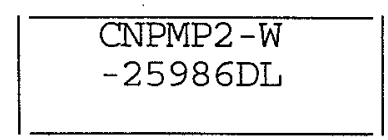

SDG No.: NA

Lab Sample ID: 0080215-03RE1

Lab File ID: F000415

Date Received: 01/25/08

Date Analyzed: 02/26/08

Dilution Factor: 10.0

Soil Aliquot Volume: (uL)

CONCENTRATION UNITS:

CAS NO.

COMPOUND

(ug/L or ug/Kg) UG/L

Q

591-78-6--.---2-Hexanone

124-48-1-.....-Dibromochloromethane

106-93-4--.---1, 2-Dibromoethane

108-90-7-------Chlorobenzene

100-41-4-------Ethylbenzene

1330-20-7--.--Xylene (Total)

100-42-5-..---styrene

75-25-2-...-.-Bromoform

98-82-8--.-.--- Isopropyl benzene

79-34-5-------1, 1, 2, 2-Tetrachloroethane

541-73-1------1,3-Dichlorobenzene

106-46-7--..--1,4-Dichlorobenzene

95-50-1-..----1,2-Dichlorobenzene

96-12-8-...---1, 2-Dibromo-3-chloropropane

120-82-1------1, 2, 4-Trichlorobenzene

91-20-3-....--Naphthalene

75-65-0-.....-tert-Butanol

108-20-3-...--Diisopropyl ether

637-92-3------Ethyl-tert-butyl ether

994-05-8------tert-Amyl methyl ether

919-94-8------tert-Amyl ethyl ether
$50 \mathrm{U}$

$50 \mathrm{U}$

$50 \mathrm{U}$

$50 \mathrm{U}$

$50 \mathrm{U}$

50 U

$50 \mathrm{U}$

$50 \mathrm{U}$

$50 \mathrm{U}$

$50 \mathrm{U}$

$50 \mathrm{U}$

$50 \mathrm{U}$

$50 \mathrm{U}$

$50 \mathrm{U}$

$50 \mathrm{U}$

12 DJ

$50 \mathrm{U}$

$100 \mathrm{U}$

$100 \mathrm{U}$

$100 \mathrm{U}$

100 


\section{ENVIROSYSTEMS, INC.}

9200 Rumsey Road - Suite B102 - Columbia, Maryland 21045-1934

Phone (410) 964-0330 - Fax (410) 740-9306

Email: into@envsystems.com - Webpage: www.envsystems.com/envsys

April 17, 2008

Jorge S. Alvarado, PH. D

Argonne National Laboratory

Environmental Research Division

Applied Geosciences and Environmental

Management Section

9700 South Cass Avenue, ER-203

Argonne, Illinois 60439

RE: Report \#080158

Dear Jorge,

Enclosed is the Analytical Data Package for the samples received on March 14, 2008 for volatile organics analysis by USEPA SW846 method 8260B/CLP SOW OLM04.3 protocols.

Please do not hesitate to call if you have any questions, comments, or require additional information.

Sincerely,

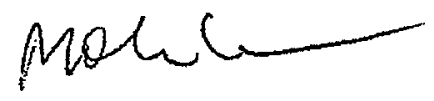

Mohan Khare Ph.D.

President/CEO

Enclosure (1)

$\mathrm{MK} / \mathrm{ncc}$

Envirosystems, Inc.

Report\#080158 


\section{SDG Case Narrative}




\section{SDG NARRATIVE VOLATILE ORGANICS (VOC)}

Envirosystems, Inc.

Contract: N/A

Client: Argonne National Laboratory

Case: N/A

SDG: ARG0080308

\section{SAMPLE RECIEPT}

Date received: 03-14-2008

Cooler Temperature: 2

\section{Sample Summary}

\begin{tabular}{|c|c|c|c|}
\hline Client ID & Laboratory ID & Matrix & pH \\
\hline CNMW03-W-26001 & $0080308-01$ & WATER & 2 \\
\hline CNPMP7-W-26011 & $0080308-02$ & WATER & 2 \\
\hline CNPMP3-W-26007 & $0080308-03$ & WATER & 2 \\
\hline CNQCTB-W-26014 & $0080308-04$ & WATER & 2 \\
\hline
\end{tabular}

2. HOLDING TIMES

3.
A. Sample Preparation: All holding times were met.
B. Sample Analysis: Sample analysis proceeded normally.

\section{METHODS}

5.

The samples were analyzed and reported by using method SW-846 8260B and USEPA CLP SOW OLM04.3 for target compound list.

\section{INSTRUMENT AND CHROMATOGRAPHIC CONDITIONS}

A Hewlett Packard 6890 gas chromalogriph equipped with a Hewleu Packard 5975 MSD was used for sample analysis. The capillary column used was a Restek $20 \mathrm{~m}$ by $0.18 \mathrm{~mm}$ ID by $1.0 \mu \mathrm{m}$ film thickness (Restek.Cat. \# RTX-624). The trap used with the sample concentrator is an OI Analytical Trap \#10,30cm packed with Tenax/silica gel/cms (PN\#228122).

\section{PREPARATION}

The submitted samples were prepared and analyzed using method SW-846 8260B.

8. ANALYSIS

\section{A. Calibration:}

\section{Initial calibration}

All acceptance criteria as stipulated by SW-846 8260 bere met for all SPCC's and CCC's. All target compounds met the required percent RSD.

\section{Blanks:}

All acceptance criteria were net.

II. Surrogates:

All acceptance criteria were met except sample CNPMP3-W-26007 \& CNPMP3-W26007DL each had one surrogate slightly lower than QC limit.

B. Spikes:

1. Laboratory Control Spikes (LCS) 


\section{SDG NARRATIVE \\ VOLATILE ORGANICS (VOC)}

LCS sample was not analyzed with this batch of samples.

\section{Matrix Spike/Matrix Spike Duplicate (MS/MSD)}

MS/MSD were not performed for this batch but shared with work order 0080313.performed for sample CNMW04-W-26024. All QC criteria were met.

\section{Internal Standards:}

All acceptance criteria were met.

\section{Samples}

Sample analysis proceeded normally. Sample CNPMP3-W-26007 was reanalyzed diluted to bring the compound concentration within QC limits of the calibration.

I certify that this Sample Data Package is in compliance with the terms. and conditions of the contract, both technically and for completeness, for other than the conditions detailed above. Release of the data contained in the hard copy Sample Data Package and in the Electronic Data Deliverables has been authorized by the laboratory manager or the manager's designee, as verified by the following signatures.
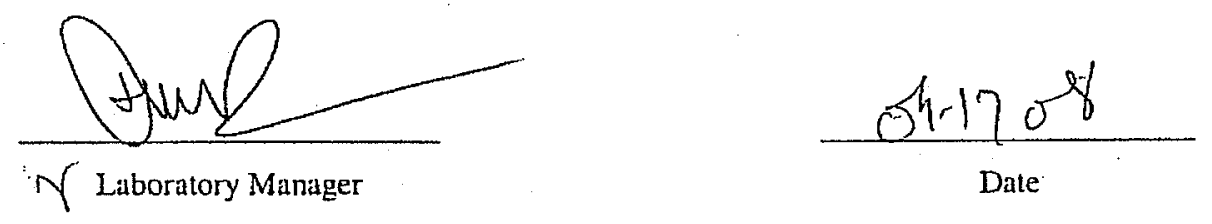


\section{Chain Of Custody}




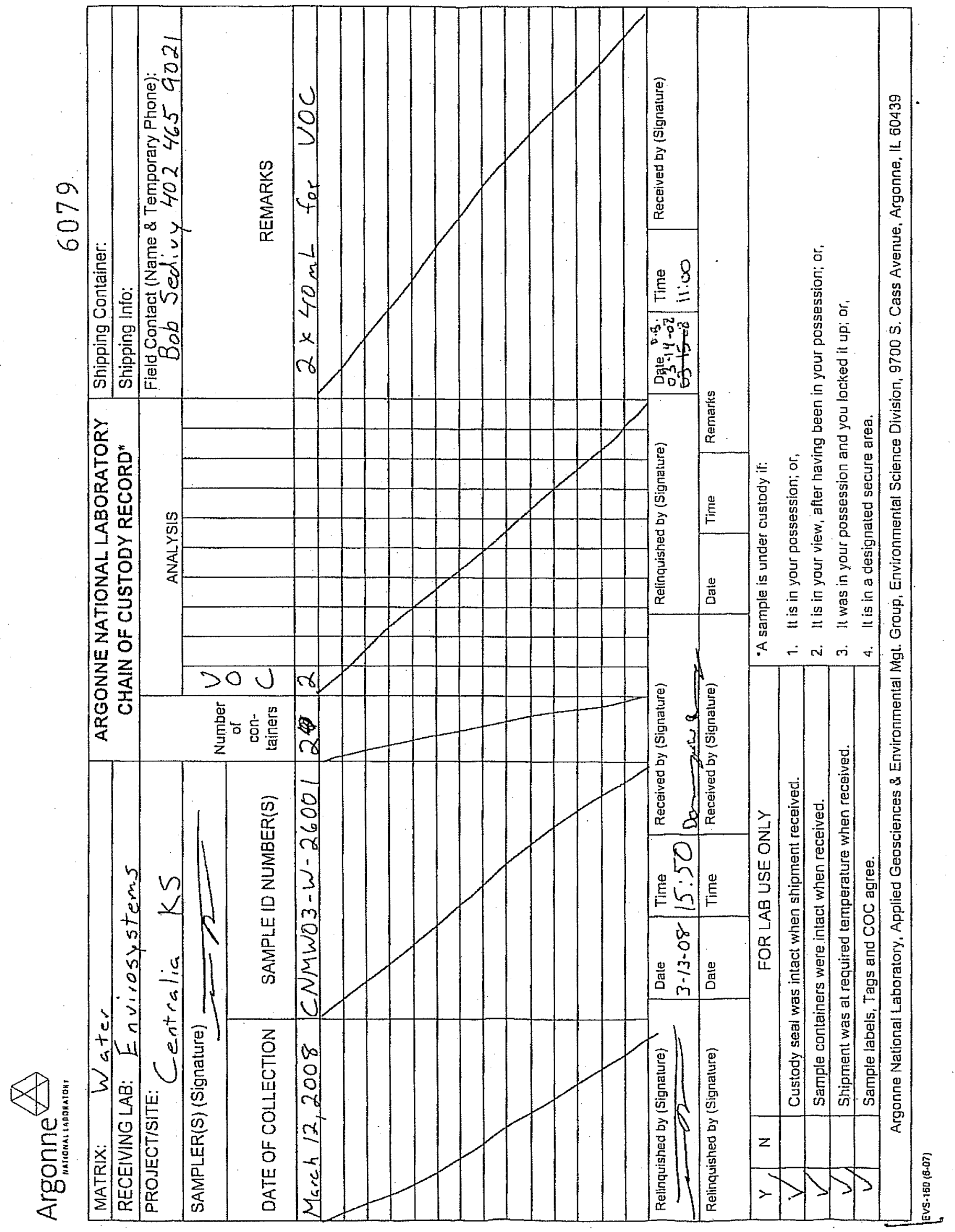




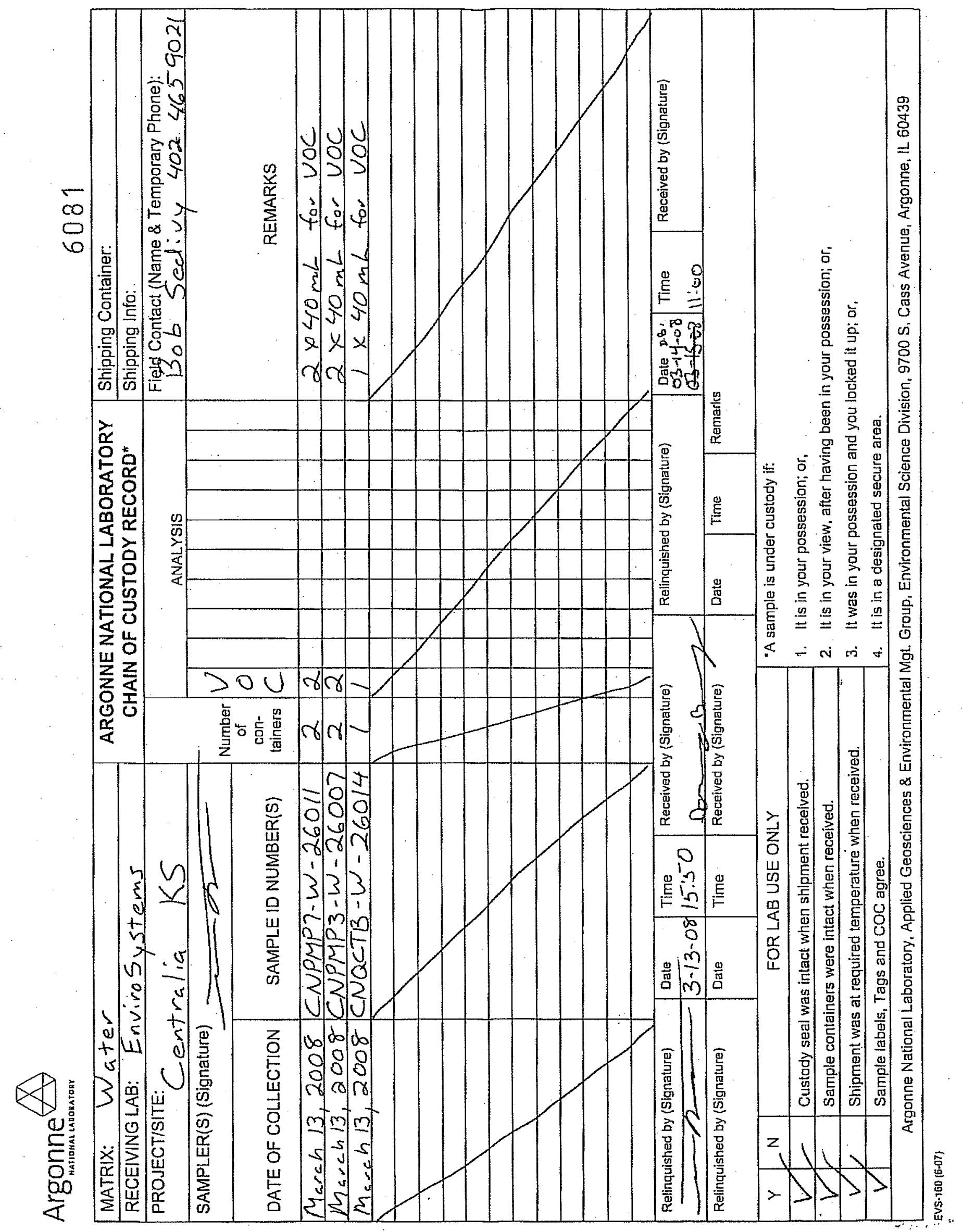




\section{Low/Medium Volatiles Data}


FORM 1

VOLATILE ORGANICS ANALYSIS DATA SHEET

Lab Name: ENVIROSYSTEMS, INC.

Lab Code: ENvSYS Case No.:

Matrix: (soil/water) WATER

Sample wt/vol: $\quad 5.000(\mathrm{~g} / \mathrm{mL}) \mathrm{ML}$

Level: (low/med) Low

: Moisture: not dec.

GC Column: RTX-624 ID: 0.18 (mm)

Soil Extract Volume: (UT)
Contract: $N / A$

SAS NO.: N/A
ARGONNE SAMPIE NO.

CNMW03-W-26001

SDG NO.: NA

Lab Sample ID: 0080308-01

Lab File ID: F000444

Date Received: 03/14/08

Date Analyzed: 03/14/08

Dilution Factor: 1.0

Soil Aliquot Volume:

(UI)

CONCENTRATION UNITS:

$(\mathrm{ug} / \mathrm{L}$ or $\mathrm{ug} / \mathrm{Kg}) \mathrm{UG} / \mathrm{L} \quad Q$

\begin{tabular}{|c|c|c|}
\hline 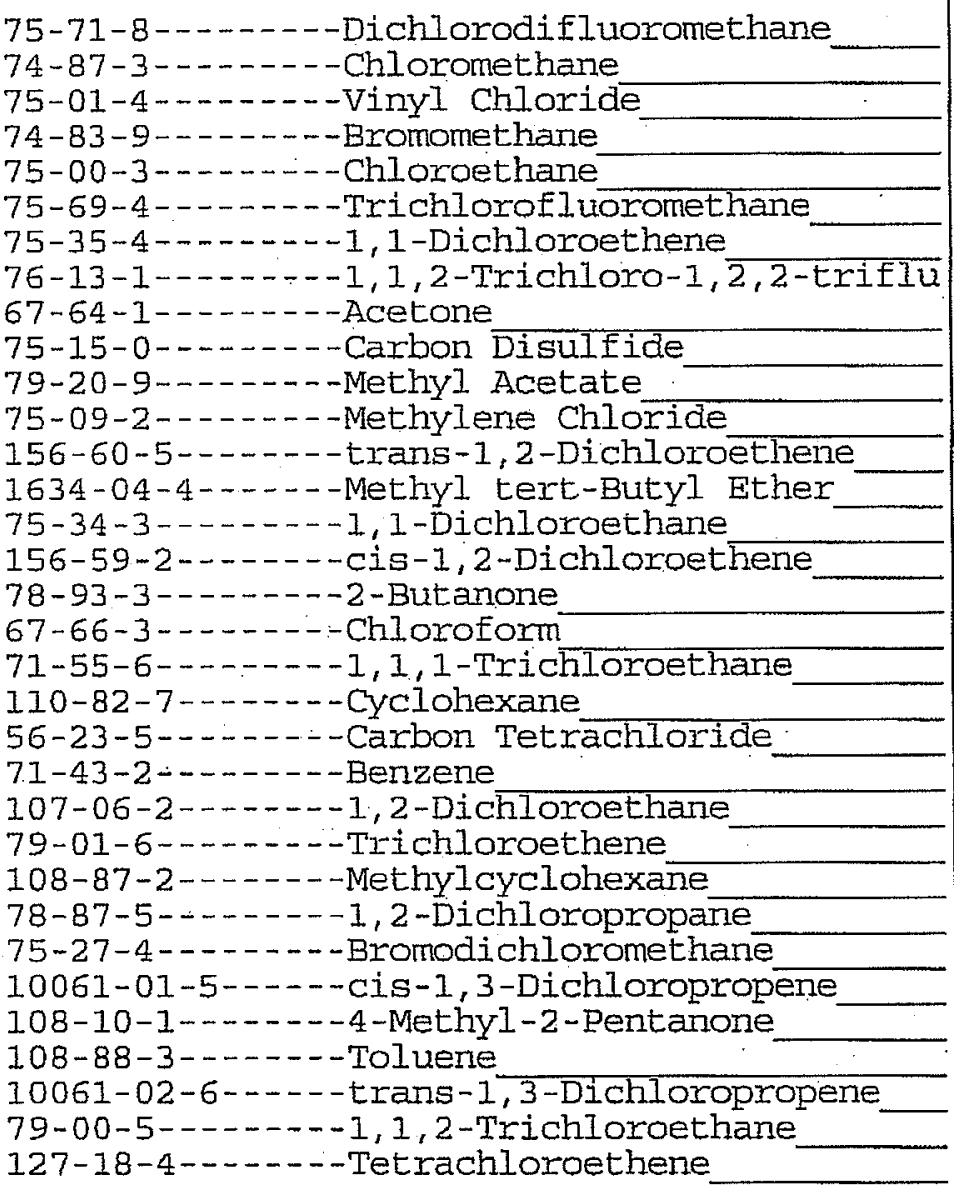 & $\begin{array}{l}5.0 \\
5.0 \\
5.0 \\
5.0 \\
5.0 \\
5.0 \\
5.0 \\
5.0 \\
5.0 \\
5.0 \\
5.0 \\
3.5 \\
5.0 \\
5.0 \\
5.0 \\
5.0 \\
5.0 \\
5.0 \\
5.0 \\
5.0 \\
5.0 \\
5.0 \\
5.0 \\
5.0 \\
5.0 \\
5.0 \\
5.0 \\
5.0 \\
5.0 \\
5.0 \\
5.0 \\
5.0 \\
5.0\end{array}$ & $\begin{array}{l}U \\
U \\
U \\
U \\
U \\
U \\
U \\
U \\
U \\
U \\
U \\
J \\
U \\
U \\
U \\
U \\
U \\
U \\
U \\
U \\
U \\
U \\
U \\
U \\
U \\
U \\
U \\
U \\
U \\
U \\
U \\
U \\
U \\
U\end{array}$ \\
\hline
\end{tabular}


FORM 1

VOLATILE ORGANICS ANALYSIS DATA SHEET

Lab Name: ENVIROSYSTEMS, INC.

Lab Code: ENvSYS Case No.:

Contract: N/A

SAS NO: : N/A
ARGONNE SAMPLE NO.

CNMW03-W-26001
Matrix: (soil/water) WATER

Sample wt/vol:

$5.000(\mathrm{~g} / \mathrm{mL}) \mathrm{ML}$

Level: (low/med) Low

․ Moisture: not dec.

GC Column: RTX-624 ID: 0.18 (mm)

Soil Extract Volume:

Lab Sample ID: 0080308-01

Lab File ID: F000444

Date Received: 03/14/08

Date Analyzed: 03/14/08

Dilution Factor: 1.0

Soil Aliquot Volume: (UL) CONCENTRATION UNITS: CAS NO. COMPOUND (ug/t or ug/Kg) UG/I

$Q$

$5.0 \mathrm{U}$

$5.0 \mathrm{U}$

$5.0 \mathrm{U}$

$5.0 \mathrm{U}$

$5.0 \mathrm{U}$

$5.0 \mathrm{U}$

$5.0 \mathrm{U}$

$5.0 \mathrm{U}$

$5.0 \mathrm{U}$

$5.0 \mathrm{U}$

$5.0 \mathrm{U}$

$5.0 \mathrm{U}$

5.0 U

$5.0 \mathrm{U}$

$5.0 \mathrm{U}$

$10 \mathrm{U}$

$5.0 \mathrm{U}$

$10 \mathrm{U}$

$10 \mathrm{U}$

$10 \mathrm{U}$

$10 \mathrm{U}$ 
FORM 1

\section{VOLATILE ORGANICS ANALYSIS DATA SHEET}

Lab Name: ENVIROSYSTEMS, INC.

Contract: $N / A$

Lab Code: ENVSYS Case No.:

SAS NO.: N/A
ARGONNE SAMPLE NO.

CNPMP7-W-26011
Matrix: (soil/water) WATER

Sample wt/vol:

$5.000(\mathrm{~g} / \mathrm{mL}) \mathrm{ML}$

Level: (low/med) LoW

\% Moisture: not dec.

GC Column: RTX-624 ID: 0.18 (mm)

Soil Extract Volume: (ut)
Lab Sample ID: 0080308-02

Lab File ID: F000445

Date Received: 03/14/08

Date Analyzed: 03/14/08

Dilution Factor: 1.0

Soil Aliquot Volume: (UI)
CAS NO.
CONCENTRATION UNITS:

(ug/L or $\mathrm{ug} / \mathrm{kg}) \mathrm{UG} / \mathrm{L}$

Q

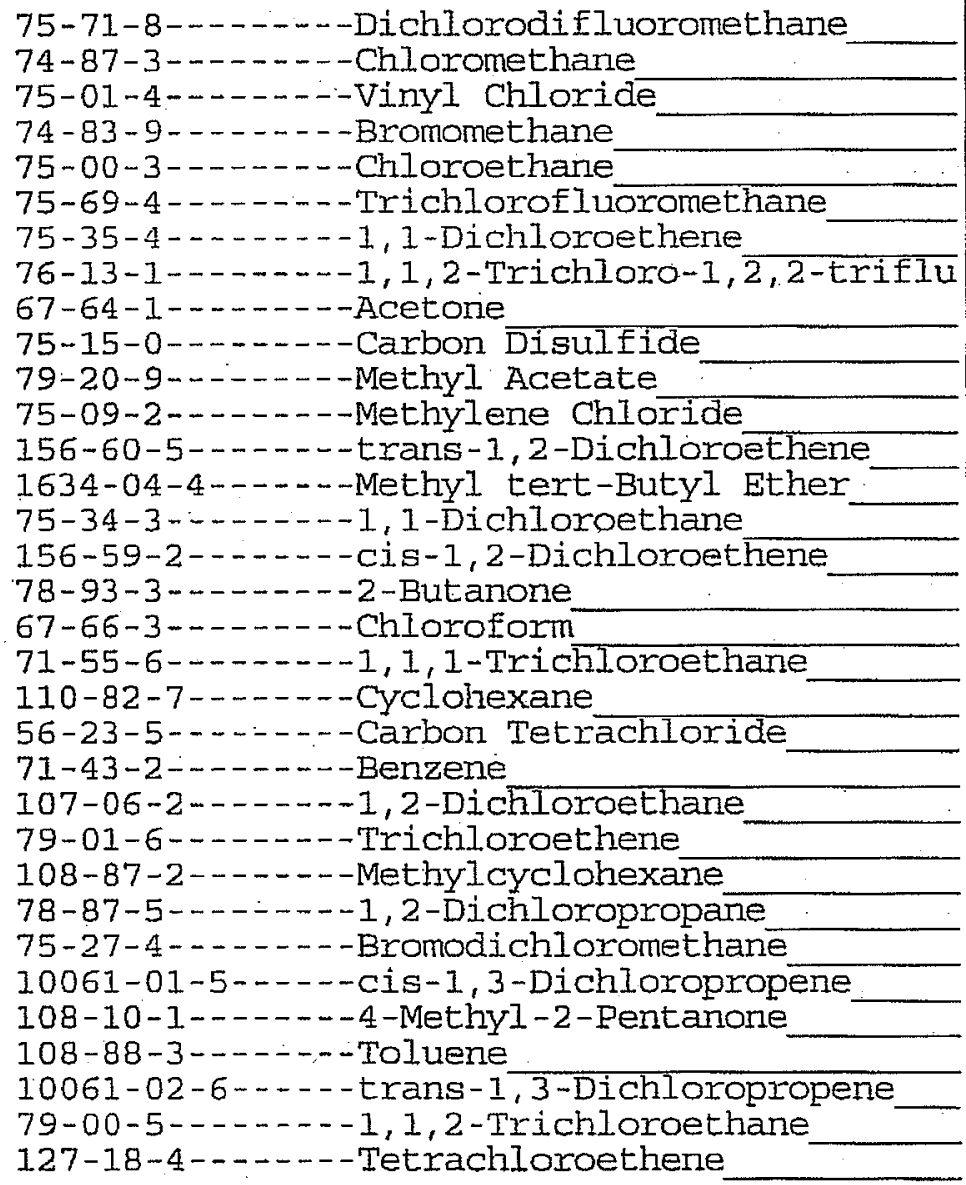

FORM I VOA
$5.0 \mathrm{U}$

$5.0 \mathrm{U}$

$5.0 \mathrm{U}$

$5.0 \mathrm{U}$

$5.0 \mathrm{U}$

$5.0 \mathrm{U}$

$5.0 \mathrm{U}$

$5.0 \mathrm{U}$

$5.0 \mathrm{U}$

5.0 U

$5.0 \mathrm{U}$

$4.4 \mathrm{~J}$

$5.0 \mathrm{U}$

$5.0 \mathrm{U}$

$5.0 \mathrm{U}$

$5.0 \mathrm{U}$

$5.0 \mathrm{U}$

16

$5.0 \mathrm{U}$

$5.0 \mathrm{U}$

67

5.0

$5.0 \mathrm{U}$

$5.0 \mathrm{U}$

$5.0 \mathrm{U}$

$5.0 \mathrm{U}$

$5.0 \mathrm{U}$

$5.0 \mathrm{U}$

$5.0 \mathrm{U}$

$1.2 \mathrm{~J}$

$5.0 \mathrm{U}$

$5.0 \mathrm{U}$

$5.0 \mathrm{U}$ 
FORM 1

VOLATILE ORGANICS ANALYSIS DATA SHEET

Lab Name: ENVIROSYSTEMS, INC.

Lab Code: ENVSYS Case No.:

Matrix: (soil/water) WATER

Sample wt/vol: $\quad 5.000(\mathrm{~g} / \mathrm{mL}) \mathrm{ML}$

Level: (low/med) LOW

․ㅡㅁ Moisture: not dec.

GC Column: RTX-624 ID: 0.18 (mm)

Soil Extract Volume:

(UL)
Contract: $\mathrm{N} / \mathrm{A}$

SAS NO.: N/A
ARGONNE SAMPLE NO.

CNPMP7 $-W-26011$
SDG NO.: NA
Lab Sample ID: 0080308-02

Lab File ID: F000445

Date Received: 03/14/08

Date Analyzed: 03/14/08

Dilution Factor: 1.0 (U士)

CONCENTRATION UNITS:

(ug/L or $\mathrm{ug} / \mathrm{Kg}$ ) UG/L

$\mathrm{Q}$

$5.0 \mathrm{U}$

$5.0 \mathrm{U}$

$5.0 \mathrm{U}$

$5.0 \mathrm{U}$

$5.0 \mathrm{U}$

$5.0 \mathrm{U}$

$5.0 \mathrm{U}$

$5.0 \mathrm{U}$

$5.0 \mathrm{U}$

$5.0 \mathrm{U}$

$5.0 \mathrm{U}$

$5.0 \mathrm{U}$

$5.0 \mathrm{U}$

$5.0 \mathrm{U}$

$5.0 \mathrm{U}$

I0 U

$5.0 \mathrm{U}$

$10 \mathrm{U}$

$10 \mathrm{U}$

$10 \mathrm{U}$

$10 \mathrm{U}$ 
FORM 1

VOLATILE ORGANICS ANALYSIS DATA SHEET

Lab Name: ENVIROSYSTEMS, INC.

Lab Code: ENVSYS Case No.:

Matrix: (soil/water) WATER

Sample wt/vol: $\quad 5.000(\mathrm{~g} / \mathrm{mL}) \mathrm{ML}$

Level : (low/med) LOW

음 Moisture: not dec.

GC Column: RTX-624 ID: 0.18 (nm)

Soil Extract Volume:

(uI)
Contract: N/A

SAS NO.: N/A
ARGONNE SAMPLE NO.

CNPMP $3-W-26007$

Lab Sample ID: 0080308-03

Lab File ID: F000448

Date Received: 03/14/08

Date Analyzed: 03/14/08

Dilution Factor: 1.0

Soil AIiquot Volume: (uI)

CONCENTRATION UNITS :

CAS NO.

COMPOUND

(ug/L or $\mathrm{ug} / \mathrm{Kg}$ ) UG/L

$Q$

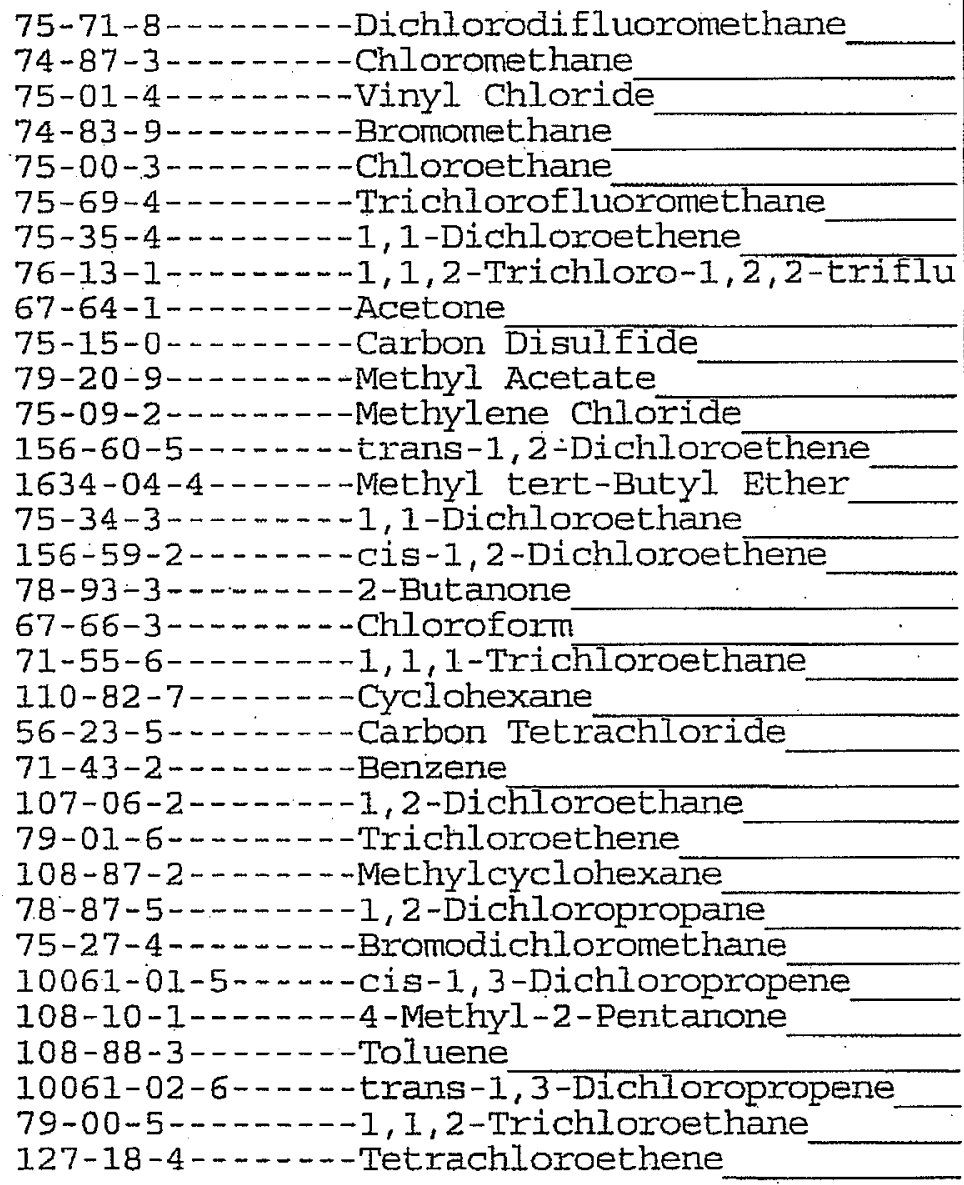


FORM 1

VOLATIIE ORGANICS ANAYYSIS DATA SHEET

Lab Name: ENVIROSYSTEMS, INC.

Lab Code: ENVSYS Case No.:

Matrix: (soil/water) WATER

Sample wt/vol: $\quad 5.000(\mathrm{~g} / \mathrm{mL})$ ML

Level: (low/med) LOW

․ Moisture: not dec.

GC Column: RTX-624 ID: 0.18 (mm)

Soil Extract Volume:

(uL)
Contract: $N / A$

SAS NO.: N/A
ARGONNE SAMPLE NO.

CNPMP3-W-26007

Lab Sample ID: 0080308-03

Lab File ID: F000448

Date Received: 03/14/08

Date Analyzed: 03/14/08

Dilution Factor: 1.0

Soil Aliquot Volume: (uL)

CONCENTRATION UNTTTS:

CAS NO.

COMPOUNND

(ug/L or $\mathrm{ug} / \mathrm{Kg}$ ) UG/L

Q

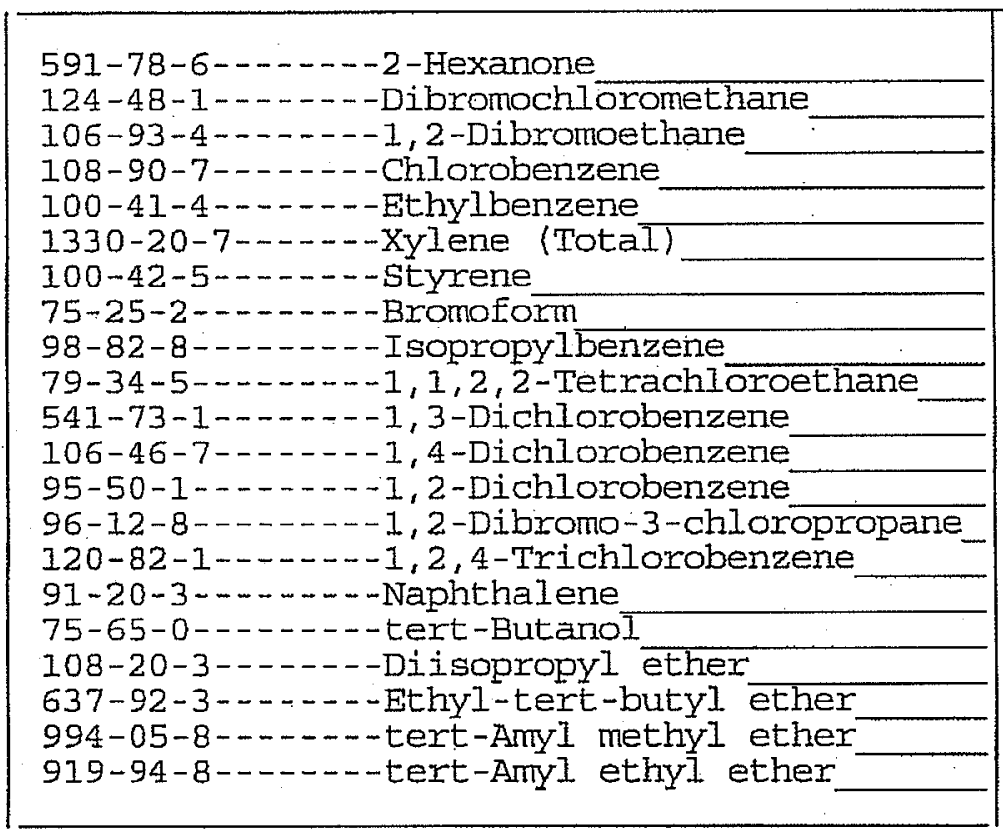

$5.0 \mathrm{U}$

$5.0 \mathrm{U}$

$5.0 \mathrm{U}$

$5.0 \mathrm{U}$

$5.0 \mathrm{U}$

$5.0 \mathrm{U}$

$5.0 \mathrm{U}$

$5.0 \mathrm{U}$

$5.0 \mathrm{U}$

$5.0 \mathrm{U}$

$5.0 \mathrm{U}$

$5.0 \mathrm{U}$

$5.0 \mathrm{U}$

$5.0 \mathrm{U}$

$5.0 \mathrm{U}$

$10 \mathrm{U}$

12

$10 \mathrm{U}$

$10 \mathrm{U}$

$10 \mathrm{U}$

$10 \mathrm{U}$

FORM I VOA 
FORM 1

VOLATILE ORGANICS ANALYSTS DATA SHEET
ARGONNE SAMPLE NO.

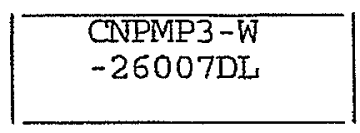

Lab Name: ENVIROSYSTEMS, INC.

Contract: $\mathrm{N} / \mathrm{A}$

Lab Code: ENVSYS Case No.:

SAS NO.: N/A

SDG No. : NA

Lab Sample ID: 0080308-03RE1

Matrix: (soil/water) WATER

Lab File ID: F000447

Sample wt/vol: $\quad 5.000$ (g/mL) ML

Level: (low/med). LoW

: Moisture: not dec.

GC Columi: $\mathrm{RTX}-624$ ID: 0.18 (mm)

Soil Extract Volume:

(uL)
Date Received: 03/14/08

Date Analyzed: 03/14/08

Dilution Factor: 50.0

Soil Aliquot Volume: (uL)

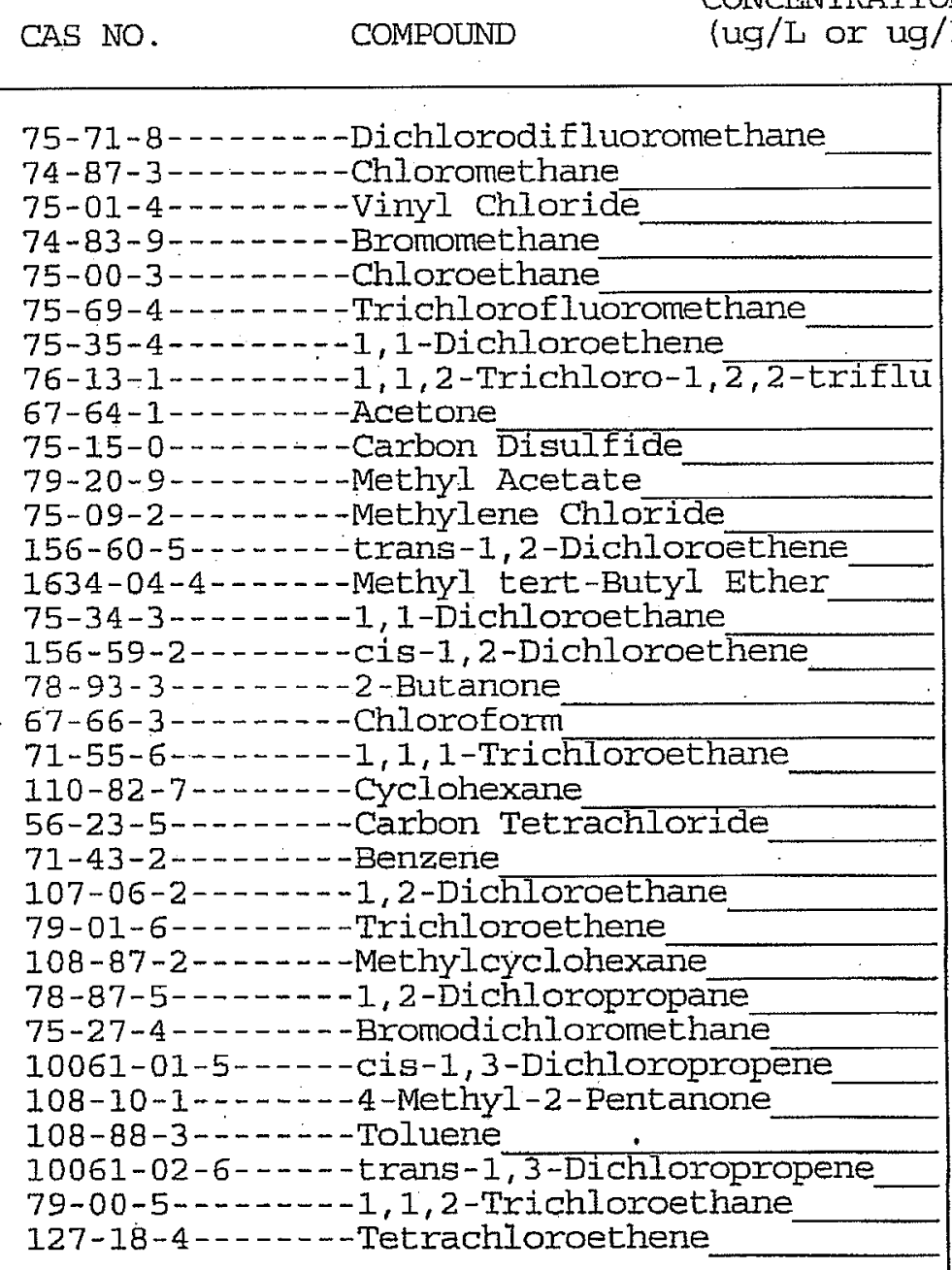

FORM I VOA 
FORM 1

\section{VOLATILE ORGANICS ANALYSIS DATA SHEET}

Lab Name: ENVIROSYSTEMS, INC.

Lab Code: ENVSYS Case No.:

Matrix: (soil/water) WATER

Sample wt/vol: $\quad 5.000(\mathrm{~g} / \mathrm{mL}) \mathrm{ML}$

Level: (low/med) LOW

믐 Moisture: not dec.

GC Column: RTX-624 ID: 0.18 (mm)

Soil Extract Volume:

(uI)
Contract: N/A

SAS NO.: N/A
ARGONNE SAMPLE NO.

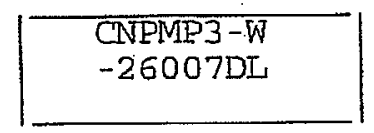

SDG No.: NA

Lab Sample ID: 0080308-03RE1

Lab File ID: F000447

Date Received: 03/14/08

Date Analyzed: 03/14/08

Dilution Factor: 50.0

Soil Aliquot Volume: (UI)

CONCENTRATION UNITS:
$(\mathrm{ug} / \mathrm{L}$ or $\mathrm{ug} / \mathrm{Kg})$

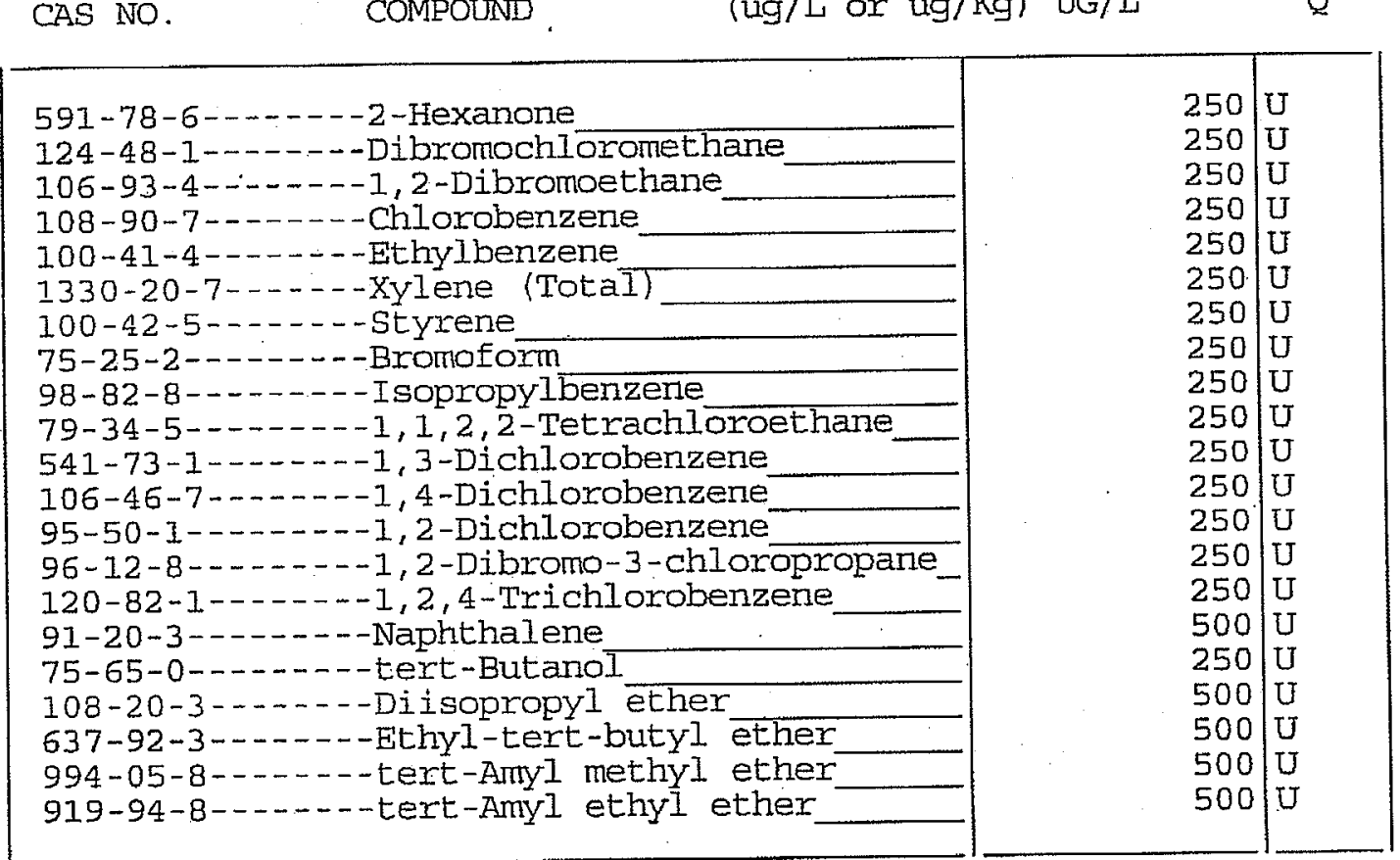

FORM I VOA 
FORM 1

VOLATILE ORGANICS ANALYSIS DATA SHEET

Lab Name: ENVIROSYSTEMS, INC.

Lab Code: ENVSYS Case No.:

Matrix: (soil/water) WATER

Sample wt/vol:

$5.000(\mathrm{~g} / \mathrm{mL}) \mathrm{ML}$

Level: (low/med) LOW

\% Moisture: not dec.

GC Colurn: RTX-624 ID: 0.18 (mm)

Soil Extract Volume: (uI)
Contract: $\mathrm{N} / \mathrm{A}$

SAS NO.: N/A
ARGONNE SAMPLE NO.

CNOCTB-W-26014
Lab Sample ID: 0080308-04

Iab File ID: F000446

Date Received: 03/14/08

Date Analyzed: 03/14/08

Dilution Factor: 1.0

Soil Aliquot volume:

(uI)

CAS NO.

COMPOUND

CONCENTRATION UNITS :

$(\mathrm{ug} / \mathrm{L}$ or $u g / \mathrm{Kg}) \mathrm{UG} / \mathrm{L}$

$Q$

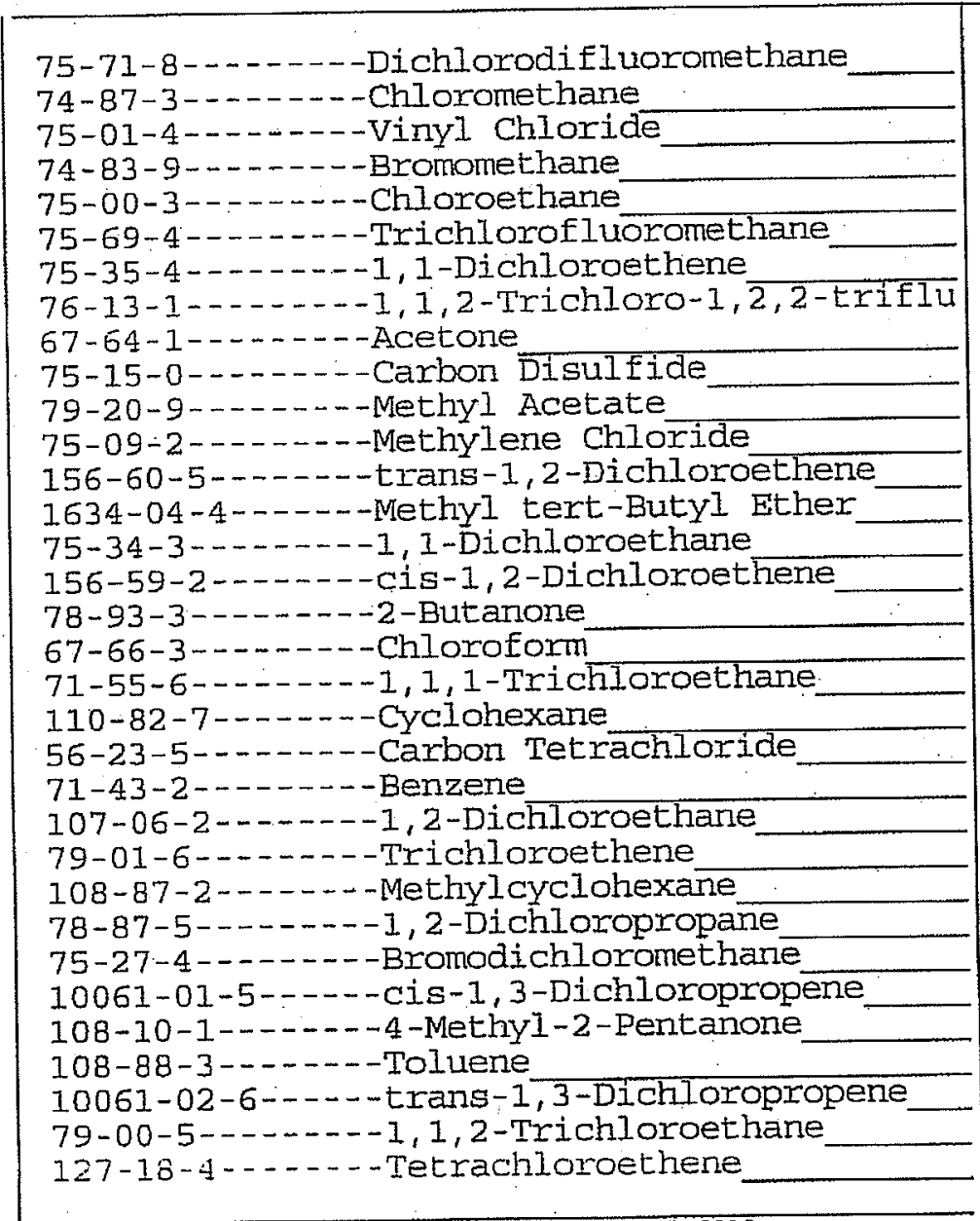

$5.0 \mathrm{U}$

$5.0 \mathrm{U}$

$5.0 \mathrm{U}$

$5.0 \mathrm{U}$

$5.0 \mathrm{U}$

$5.0 \mathrm{U}$

$5.0 \mathrm{U}$

$5.0 \mathrm{U}$

$5.0 \mathrm{U}$

$5.0 \mathrm{U}$

$5.0 \mathrm{U}$

$3.9 \mathrm{~J}$

$5.0 \mathrm{U}$

$5.0 \mathrm{U}$

$5.0 \mathrm{U}$

$5.0 \mathrm{U}$

$5.0 \mathrm{U}$

$5.0 \mathrm{U}$

$5.0 . \mathrm{U}$

$5.0 \mathrm{U}$

$5.0 \mathrm{U}$

$5.0 \mathrm{U}$

$5.0 \mathrm{U}$

$5.0 \mathrm{U}$

$5.0 \mathrm{U}$

$5.0 \mathrm{U}$

$5.0 \mathrm{U}$

$5.0 \mathrm{U}$

$5.0 \mathrm{U}$

$5.0 \mathrm{U}$

$5.0 \mathrm{U}$

$5.0 \mathrm{U}$

$5.0 \mathrm{U}$ 
FORM 1

VOLATILE ORGANICS ANALYSIS DATA SHEET

Lab Name: ENVIROSYSTEMS, INC.

Lab Code: ENVSYSS Case No.:

Matrix: (soil/water) WATER

Sample wt/vol: $\quad 5.000(\mathrm{~g} / \mathrm{mL}) \mathrm{ML}$

Level: (low/med) LOW

- Moisture: not dec.

GC Column: RTX-624 ID: 0.18 (mm)

Soil Extract Volume:

(UI.)
Contract: $N / A$

SAS NO.: N/A
ARGONNE SAMPLE NO.

\section{CNQCTB-W-26014}

CAS NO.
SDG No.: NA
Lab Sample ID: 0080308-04

Lab File ID: F000446

Date Received: 03/14/08

Date Analyzed: 03/14/08

Dilution Factor: 1.0

Soil Aliquot Volume: (uL)

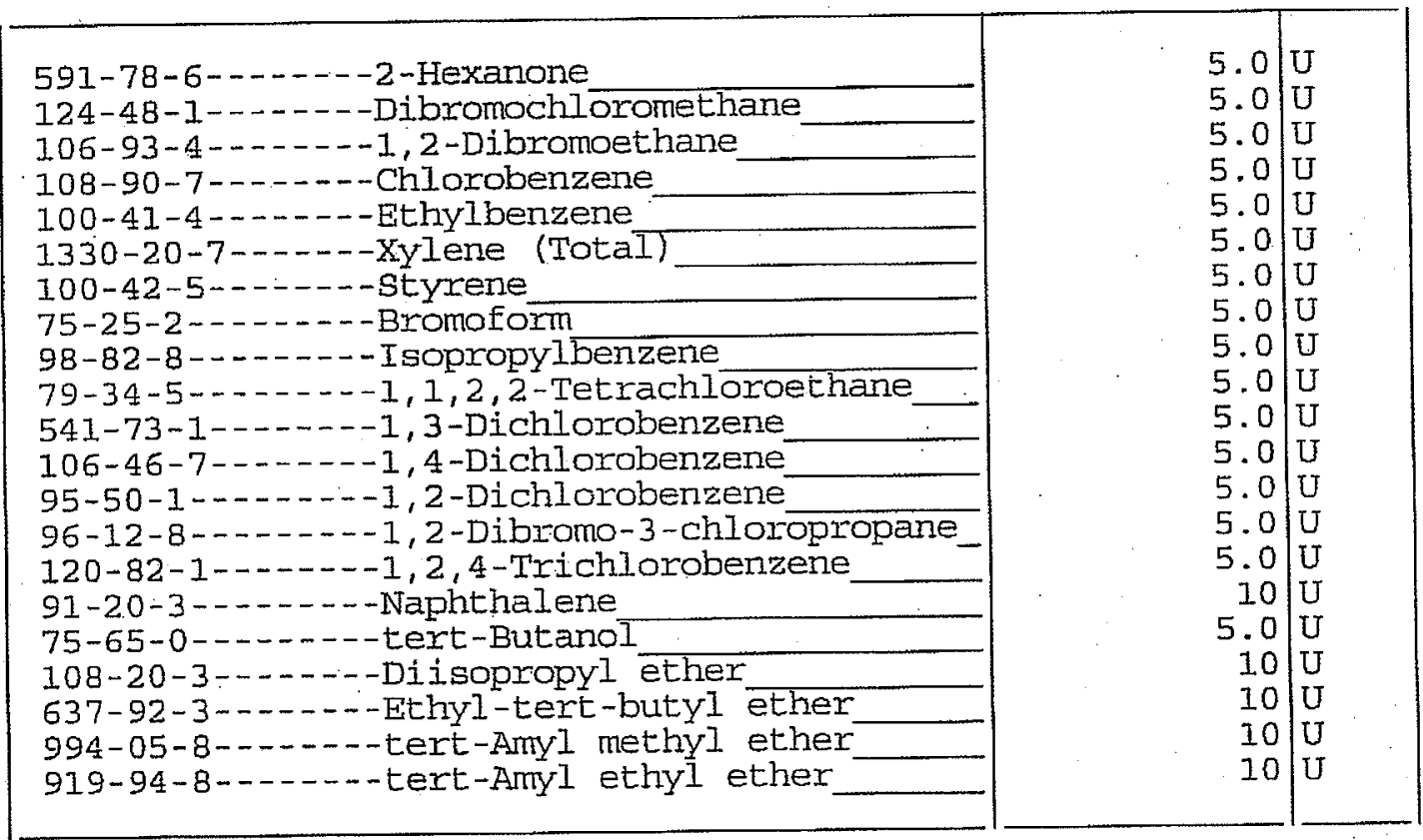

FORM I VOA

CONCENTRATION UNITS:

$(\mathrm{ug} / \mathrm{L}$ or $\mathrm{ug} / \mathrm{Kg})$ UG/L $\quad Q$ 


\section{ENVIROSYSTEMS, INC.}

9200 Rumsey Road - Suite B102 - Columbia, Maryland 21045-1934

Phone (410) 964-0330 - Fax (410) 740-9306

Email: info@envsystems.com - Webpage: www.envsystems.com/envsys

April 17, 2008

Jorge S. Alvarado, PH. D

Argonne National Laboratory

Environmental Research Division

Applied Geosciences and Environmental

Management Section

9700 South Cass Avenue, ER-203

Argonne, Illinois 60439

RE: Report \#080163

Dear Jorge,

Enclosed is the Analytical Data Package for the samples received on March 20, 2008 for volatile organics analysis by USEPA SW846 method $8260 \mathrm{~B} / \mathrm{CL}$.P SOW OLM04.3 protocols.

Please do not hesitate to call if you have any questions, comments, or require additional information.

Sincerely;<smiles>CCCCCc1ccc(Br)cc1</smiles>

Mohan Khare Ph.D.

President/CEO

Enclosure (1)

MK/ncc

Envirosystems, Inc.

Report\#080163 


\section{SDG Case Narrative}




\section{SDG NARRATIVE \\ VOLATILE ORGANICS (VOC)}

Envirosystems, Inc.

Contract: N/A

Client: Argonne National Laboratory

Case: N/A

SDG: ARG032008

\section{SAMPLE RECIEPT}

Date received: 03-20-2008

Cooler Temperature: 2

\section{Sample Summary}

\begin{tabular}{|c|c|c|c|}
\hline Client ID & Laboratory ID & Matrix & $\mathbf{p H}$ \\
\hline CNMW04-W-26024 & $0080313-01$ & WATER & 2 \\
\hline CNMW04W-26026 & $0080313-02$ & WATER & 2 \\
\hline CNQCTB-W26035 & $0080313-03$ & WATER & 2 \\
\hline
\end{tabular}

\section{HOLDING TIMES}

3.

A. Sample Preparation: All holding times were met.

B. Sample Analysis: Sample analysis proceeded normally.

4. METHODS

5.

The samples were analyzed and reported by using method SW-846 $8260 \mathrm{~B}$ and USEPA CLP SOW OLM04.3 for target compound list.

\section{INSTRUMENT AND CFROMATOGRAPHIC CONDITIONS}

A Hewlett Packard 6890 gas chromatograph equipped with a Hewlett Packard 5975 MSD was used for sample analysis. The capillary column used was a Restek $20 \mathrm{~m}$ by $0.18 \mathrm{~mm} \mathrm{ID}$ by $1.0 \mu \mathrm{m}$ film thickness (Restek Cat. \# RTX-624). The trap used with the sample concentrator is an O1 Analytical Trap \#10,30cm packed with Tenax/silica gel/cms (PN\#228122).

\section{PREPARATION}

The submitted samples were prepared and analyzed using method SW-846 8260B.

8. ANALYSIS

A. Calibration:

1. Initial calibration

All acceptance criteria as stipulated by SW-846 $8260 \mathrm{~b}$ were met for all SPCC's and $\mathrm{CCC}^{\prime} s$. All target compounds met the required percent RSD.

II. Blanks:

All acceptance criteria were met.

II. Surrogates:

All acceptance criteria were met.

B. Spikes:

I. Laboratory Control Spikes (LCS)

II. LCS sample was analyzed which met all the QC criteria. 


\section{SDG NARRATIVE \\ VOLATILE ORGANICS (VOC)}

\section{Matrix Spike/Matrix Spike Duplicate (MS/MSD)}

MS/MSD were performed for sample CNMW04-W-26024. All QC criteria were met.

\section{Internal Standards:}

All acceptance criteria were met.

D. Samples

Sample analysis proceeded normally.

I certify that this Sample Data Package is in compliance with the terms and conditions of the contract, both technically and for completeness, for other than the conditions detailed above. Release of the data contained in the hard copy Sample Data Package and in the Electronic Data Deliverables has been authorized by the laboratory manager or the manager's designee, as verified by the following signatures.
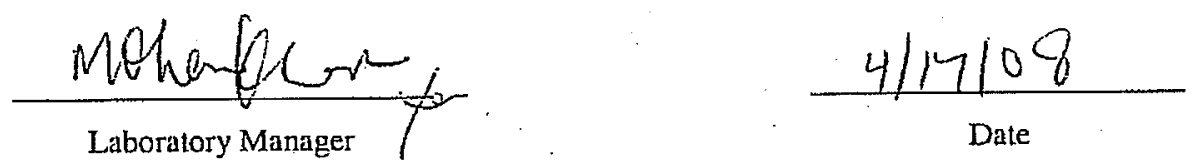


\section{CHAIN OF CUSTODY SDG-Case-Sheet/Traffic Report}




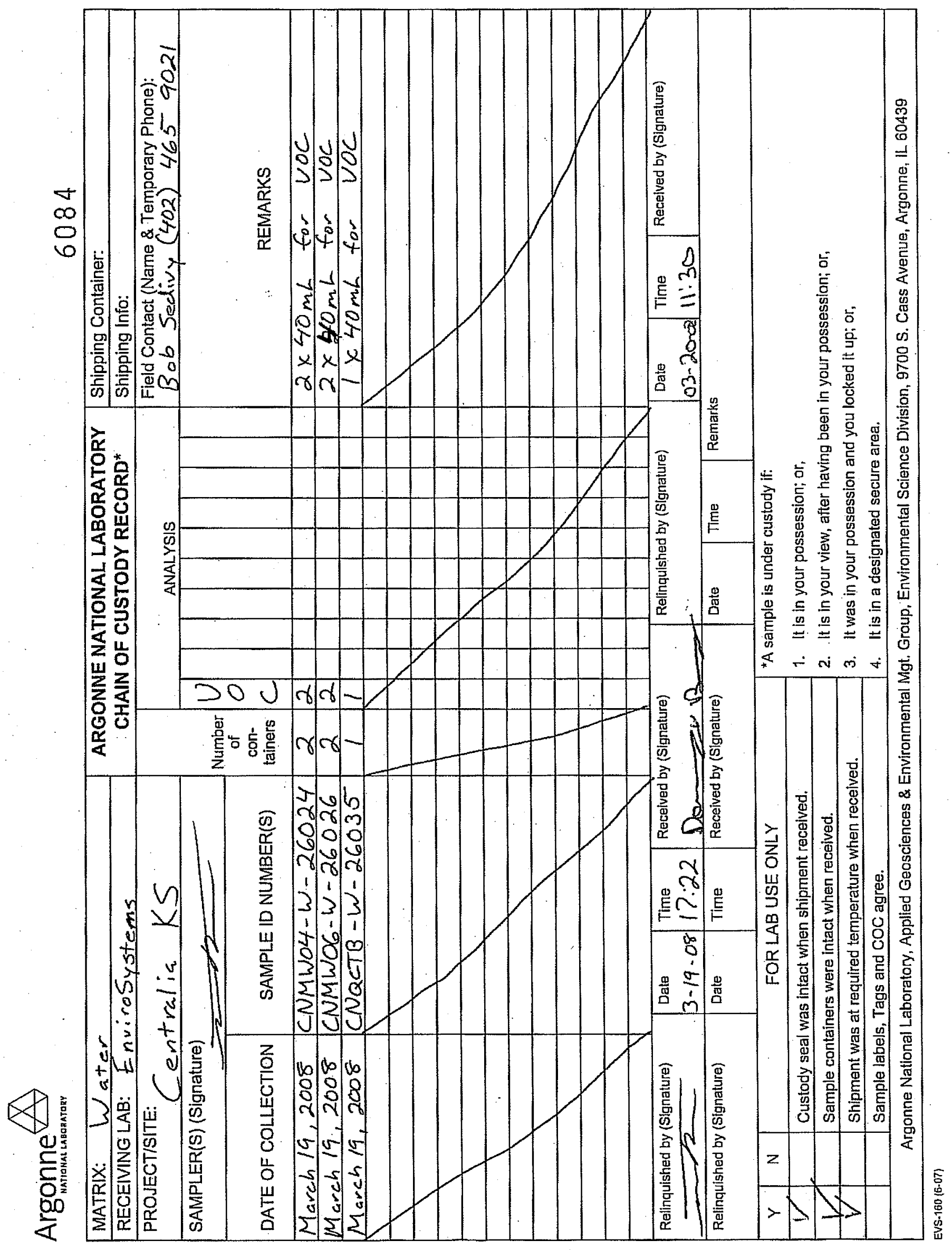




\section{Low/Medium Volatiles Data}


FORM 1

VOLATILE ORGANICS ANALYSIS DATA SHEET
ARGONNE SAMPLE NO.

CNNWO4-W-26024
Lab Name: ENVIROSYSTEMS, INC.

Lab Code: ENVSYS Case No.:

Matrix: (soil/water) WATER

Sample wt/vol: $\quad 5.000(\mathrm{~g} / \mathrm{mL}) \mathrm{ML}$

Level: (low/med) LOW

$\because$ Moisture: not dec.

GC Column: RTX-624 ID: 0.18 (mm)

Soil Extract Volume:

(uL)
Contract: $\mathrm{N} / \mathrm{A}$

SAS NO.: N/A
SDG No.: NA
Lab File ID: F000458

Date Received: 03/20/08

Date Analyzed: 03/21/08

Dilution Factor: 1.0

Soil Aliquot Volume: (LII)

CAS NO.

COMPOUND

CONCENTRATION UNITS:

(ug/L or ug/Kg) UG/L

$Q$

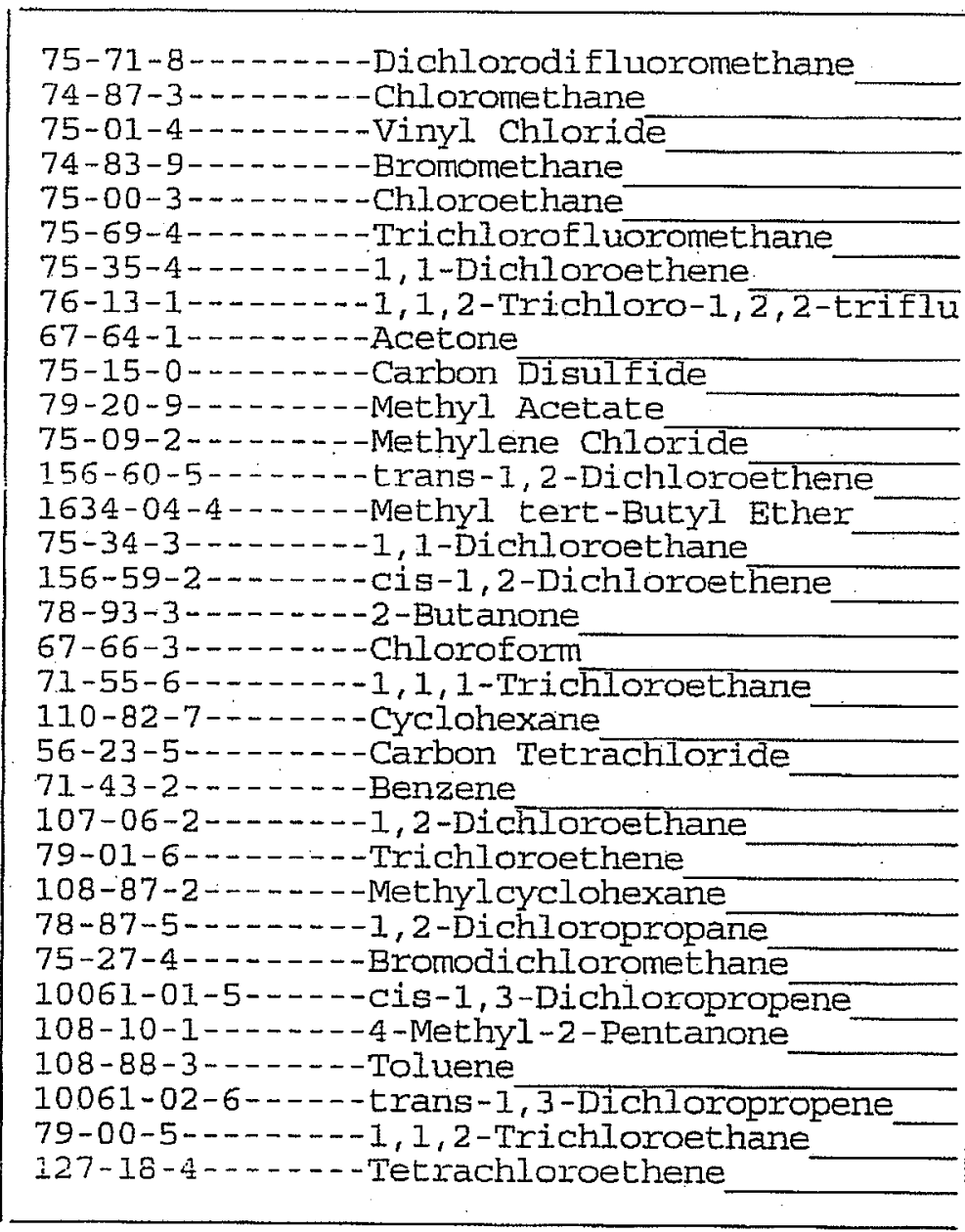

$5.0 \mathrm{U}$

5.00

$5.0 \mathrm{U}$

$5.0 \mathrm{U}$

$5.0 \mathrm{U}$

$5.0 \mathrm{U}$

$5.0 \mathrm{U}$

$5.0 \mathrm{U}$

$5.0 \mathrm{U}$

$5: 0 \mathrm{U}$

$5.0 \mathrm{U}$

6.8

$5.0 \mathrm{U}$

$5.0 \mathrm{U}$

$5.0 \mathrm{U}$

$5.0 \mathrm{U}$

$5.0 \mathrm{U}$

$5.0 \mathrm{U}$

$5.0 \mathrm{U}$

$5.0 \mathrm{U}$

$5.0 \mathrm{U}$

$5.0 \mathrm{U}$

$5.0 \mathrm{U}$

$5.0 \mathrm{U}$

$5.0 \mathrm{U}$

$5.0 \mathrm{U}$

$5.0 \mathrm{U}$

$5.0 \mathrm{U}$

$5.0 \mathrm{U}$

1.2 $\mathrm{J}$

$5.0 \mathrm{U}$

$5.0 \mathrm{U}$

$5.0 \mathrm{U}$ 
FORM 1

VOLATILE ORGANICS ANALYSIS DATA SHEET

Lab Name: ENVIROSYSTEMS, INC.

Lab Code: ENVSYS Case No.:

Matrix: (soil/water) WATER

Sample wt/vol: $\quad 5.000(g / \mathrm{mL}) \mathrm{ML}$

Level: (Low/med) LOW

\% Moisture: not dec.

GC Column: RTX-624 ID: 0.18 (mm)

Soil Extract Volume: (uบ)
Contract: $N / A$

SAS NO.: N/A
ARGONNE SAMPLE NO.

CNMW04-W-26024
SDG NO. : NA

Lab Sample ID: 0080313-0I

Iab File ID: Fo00458

Date Received: 03/20/08

Date Analyzed: 03/21/08

Dilution Factor: 1.0

Soil Aliquot volume: (u山)

CAS NO.

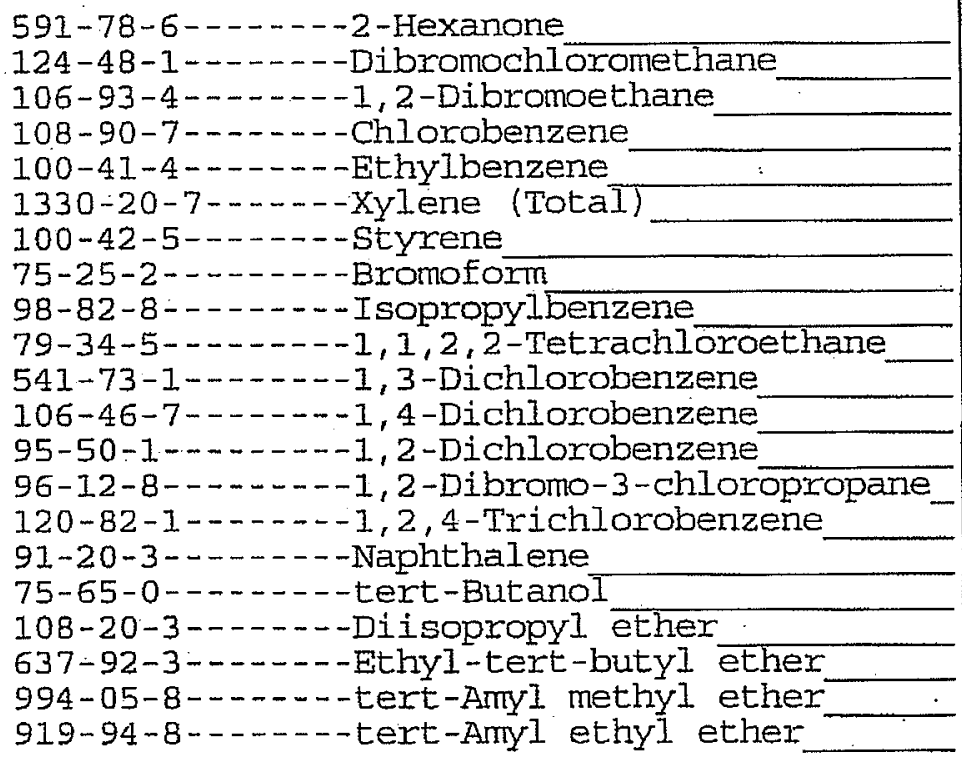

$5.0 \mathrm{U}$

$5.0 \mathrm{U}$

$5.0 \mathrm{U}$

$5.0 \mathrm{U}$

$5.0 \mathrm{U}$

$5.0 \mathrm{U}$

5.0. U

$5.0 \mathrm{U}$

5..0 U

$5.0 \mathrm{U}$

$5.0 \mathrm{U}$

$5.0 \mathrm{U}$

$5.0 \mathrm{U}$

5.0 U

$5.0 \mathrm{U}$

$10 \mathrm{U}$

$5.0 \mathrm{U}$

$10 \mathrm{U}$

$10 \mathrm{U}$

$10 \mathrm{U}$

$10 \mathrm{U}$ 
FORM 1

VOLATILE ORGANICS ANALYSIS DATA SHEET

Lab Name: ENVIROSYSTEMS, INC.

Lab Code: ENVSYS Case No.:

Matrix: (soil/water) WATER

Sample wt/vol: $\quad 5.000\{\mathrm{~g} / \mathrm{mL}\rangle \mathrm{ML}$,

Level: (low/med) Low

\% Moisture: not dec.

GC Column: RTX-624 ID: 0.18 (mm)

Soil Extract Volume:

(uL)
Contract: N/A

SAS NO.: $N / A$
ARGONNE SAMPLE NO.

CNNWW06-W-26026
SDG No. : NA

Lab Sample ID: 0080313-02

Lab File ID: F000461

Date Received: 03/20/08

Date Analyzed: 03/21/08

Dilution Factor: 1.0

Soil Aliquot Volume:

(uts)

CAS NO.

COMPOUND

CONCENTRATION UNITS:

(ug/L or ug/Kg) UG/L

$Q$

$5.0 \mathrm{U}$

$5.0 \mathrm{U}$

$5.0 \mathrm{U}$

$5.0 \mathrm{U}$

$5.0 \mathrm{U}$

$5.0 \mathrm{U}$

$5.0 \mathrm{U}$

$5.0 \mathrm{U}$

$5.0 . \mathrm{U}$

$5.0 \mathrm{U}$

$5.0 \mathrm{U}$

7.0

$5.0 \mathrm{U}$

$5.0 \mathrm{U}$

$5.0 \mathrm{U}$

$5.0 \mathrm{U}$

$5.0 \mathrm{U}$

$5.0 \mathrm{U}$

$5.0 \mathrm{U}$

$5.0 \mathrm{U}$

$5.0 \mathrm{U}$

$5.0 \mathrm{U}$

$5.0 \mathrm{U}$

$5.0 \mathrm{U}$

$5.0 \mathrm{U}$

$5.0 \mathrm{U}$

$5.0 \mathrm{U}$

$5.0 \mathrm{U}$

$5.0 \mathrm{U}$

$1.4 \mathrm{~J}$

$5.0 \mathrm{U}$

$5.0 \mathrm{U}$

$5.0 \mathrm{U}$ 
FORM 1

VOLATILE ORGANICS ANAIYSIS DATA SHEET

Lab Name: ENVIROSYSTEMS, INC.

Lab Code: ENVSYS Case No.:

Märix: (soil/water) WATER

Sample wt/vol: $\quad 5.000(\mathrm{~g} / \mathrm{mL}) \mathrm{ML}$

Level: (low/med) Low

음 Moisture: not dec.

GC Column: RTX-624 ID: 0.18 (mm)

Soil Extract Volume:

(UT)
Contract: N/A

SAS NO.: N/A
ARGONNE SAMPLE NO.

CNNWWO $-W-26026$

Lab Sample ID: 0080313-02

Lab File ID: F000461

Date Received: 03/20/08

Date Anal.yzed: 03/2I/08

Dilution Factor: 1.0

Soil Aliquot Volume: (UI)

CONCENTRATION UNITS :

CAS NO. COMPOUND (ug/L or ug/Kg) UG/L

$Q$

591-78-6-------2-Hexanone

124-48-1 .....- Dibromochloromethane

$106-93-4 \ldots-\ldots-1$, 2-Dibromoethane

108-90-7--.-.-Chlorobenzene

I00-41-4-...-.-Ethylbenzene

1330-20-7-...-Xylene (TotaI)

100-42-5-..---styrene.

$75-25-2 \ldots \ldots$ - . . Bromoform

98-82-8----.-- Isopropyl benzene

79-34-5--.---1, 1,2,2-Tetrachloroethane

541-73-1-...-.1, 3-Dichlorobenzene

106-46-7--.----1, 4-Dichlorobenzene

95-50-1-....-. 2, 2-Dichlorobenzene

96-12-8 -....- 1, 2-Dibromo-3-chloropropane

120-82-1-.----1, 2, 4-Trichlorobenzene

91-20-3-...... Naphthalene

75-65-0-..---tert-Butanol

108-20-3-...-Disopropyl ether

637-92-3-....-.-Ethyl-tert-butyl ether

994-05-8---n-tert-AmyI methyl ether

919-94-8------tert-Anyl ethyl ether
$5.0 \mathrm{U}$

$5.0 \mathrm{U}$

$5.01 \mathrm{U}$

$5.0 \mathrm{U}$

$5.0 \mathrm{U}$

5.0. U

$5.0 \mathrm{U}$

$5.0 \mathrm{U}$

$5.0 \mathrm{U}$

$5.0 \mathrm{U}$

$5.0 \mathrm{U}$

$5.0 \mathrm{U}$

$5.0 \mathrm{U}$

$5.0 \mathrm{U}$

$5.0 \mathrm{U}$

$10 \mathrm{U}$

$5.0 \mathrm{U}$

$10 \mathrm{U}$

$10 \mathrm{U}$

$10 \mathrm{U}$

$10 \mathrm{U}$ 
FORM 1

VOLATILE ORGANICS ANATYSIS DATA SHEET

Lab Name: ENVIROSYSTEMS, INC.

Iuab Code: ENVSYS Case No.:

Matrix: (soil/water) WATER

Sample wt/vol: $\quad 5.000(\mathrm{~g} / \mathrm{mL}) \mathrm{ML}$

Level: (low/med) LOW

\% Moisture: not dec.

GC CoIumn: RTX-624 ID: 0.18 (mm)

Soil Extract Volume:

(uI)
Contract: N/A

SAS NO.: N/A SDG NO.: NA
ARGONNE SAMPLE NO.

CNQCTB-W-26035
Lab Sarmple ID: 0080313-03

Lab File ID: F000462

Date Received: 03/20/08

Date Analyzed: 03/21/08

Dilution Factor: 1.0

Soil Aliquot Volume: (uT)

CAS NO.

COMPOUND

CONCENTRATION UNITS:

(ug/L or $\mathrm{ug} / \mathrm{Kg}$ ) UG/L

Q

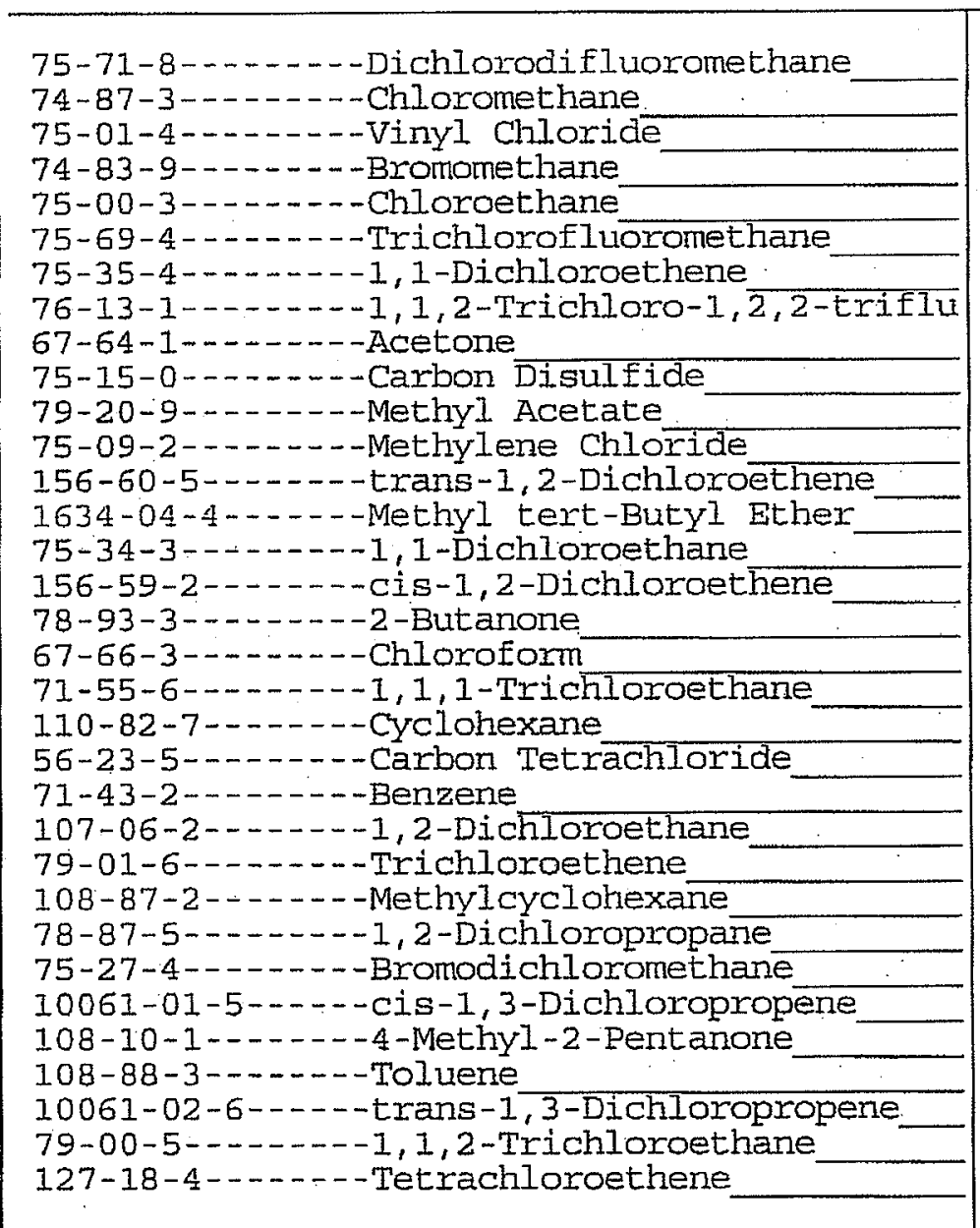

$5.0 \mathrm{U}$

$5.0 \mathrm{U}$

$5.0 \mathrm{U}$

$5.0 \mathrm{U}$

$5.0 \mathrm{U}$

$5.0 \mathrm{U}$

$5.0 \mathrm{U}$

$5.0 \mathrm{U}$

$5.0 \mathrm{U}$

$5.0 \mathrm{U}$

$5.0 \mathrm{U}$

6.6

$5.0 \mathrm{U}$

$5: 0 \mathrm{U}$

$5.0 \mathrm{U}$

$5.0 \mathrm{U}$

$5.0 \mathrm{U}$

$5.0 \mathrm{U}$

$5.0 \mathrm{U}$

$5.0 \mathrm{U}$

$5.0 \mathrm{U}$

$5.0 \mathrm{U}$

$5.0 \mathrm{U}$

$5.0 \mathrm{U}$

$5.0 \mathrm{U}$

$5.0 \mathrm{U}$

$5.0 \mathrm{U}$

$5.0 \mathrm{U}$

$5.0 \mathrm{U}$

$5.0 \mathrm{U}$

$5.0 \mathrm{U}$

$5.0 \mathrm{U}$

$5.0 \mathrm{U}$

FORM I VOA 
FORM 1

VOLATILE ORGANICS ANALYSIS DATA SHEET

Lab Name: ENVIROSYSTEMS, INC.

Lab Code: ENVSYS Case No.:

Matrix: (soil/water) WATER.

Sample wt/vol: $\quad 5.000(\mathrm{~g} / \mathrm{mL}) \mathrm{ML}$

Level: (low/med) LOW

\% Moisture: not dec.

GC Column: RTX-624 ID: 0.18 (mm)

Soil Extract Volume: (UIS)
Contract: $N / A$

SAS NO.: N/A
ARGONNE SAMPLE NO.

CNQCTB $-W-26035$

Lab Sample ID: 0080313-03

Lab File ID: F000462

Date Received: 03/20/08

Date Analyzed: 03/21/08

Dilution Factor: 1.0

Soil Aliquot Volume: (UL) CONCENTRATION UNITS: (ug/L or $\mathrm{ug} / \mathrm{Kg})$ UG/L

$Q$

COMPOUND

CAS NO.

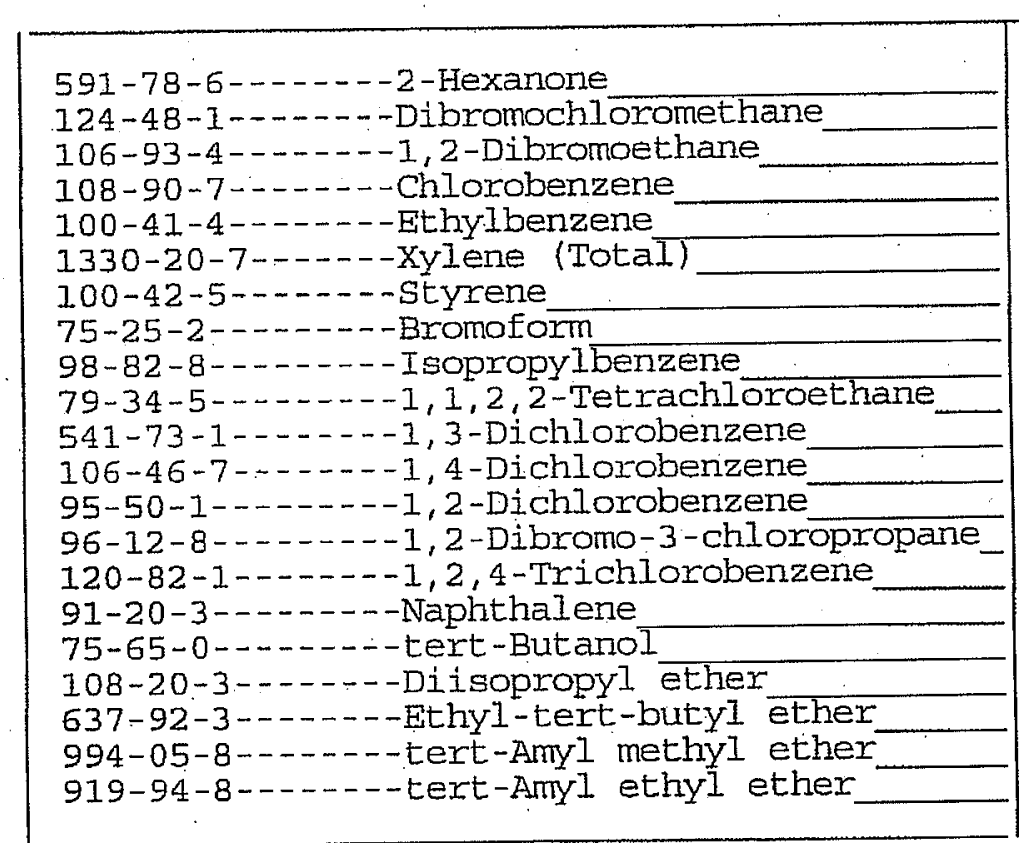

$-$\begin{tabular}{rr|l|}
5.0 & $\mathrm{U}$ \\
5.0 & $\mathrm{U}$ \\
5.0 & $\mathrm{U}$ \\
5.0 & $\mathrm{U}$ \\
5.0 & $\mathrm{U}$ \\
5.0 & $\mathrm{U}$ \\
5.0 & $\mathrm{U}$ \\
5.0 & $\mathrm{U}$ \\
5.0 & $\mathrm{U}$ \\
5.0 & $\mathrm{U}$ \\
5.0 & $\mathrm{U}$ \\
5.0 & $\mathrm{U}$ \\
5.0 & $\mathrm{U}$ \\
5.0 & $\mathrm{U}$ \\
5.0 & $\mathrm{U}$ \\
10 & $\mathrm{U}$ \\
5.0 & $\mathrm{U}$ \\
10 & $\mathrm{U}$ \\
10 & $\mathrm{U}$ \\
10 & $\mathrm{U}$ \\
10 & $\mathrm{U}$ \\
\hline
\end{tabular}

FORM I VOA 


\section{ENVIROSYSTEMS, INC.}

9200 Rumsey Road • Suite B102 • Columbia, Maryland 21045-1934

Phone (410) 964-0330 - Fax (410) 740-9306

Email: info@envsystems.com -Webpage: www.envsystems.com/envsys

Date: June-1 1-2008

Name: Jorge S. Alvarado Ph.D

Company: Argonne National Laboratory

Address: Enviromental Research Division

Applied Geosciences and Enviromental

Management Section

9700 South Cass Avenue

Bldg: 203, Room \# A137

Lemont, IL 60439

RE: Report\# 160.0

Dear Jorge,

Enclosed are the results of analyses for samples received by the laboratory on $4-25$. B. 2008 . If you have any questions concerning this report, please feel free to contact me.

Please do not hesitate to call if you have any questions, comments or require additional information

Sincerely,

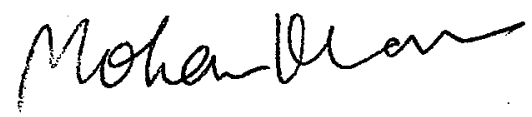

Mohan Khare, Ph.D

President/ CEO

Report \# 080190 


\section{Narrative}

This analytical data package contains the volatile organic analysis by USEPA SW-846 Method 8260B and CLP protocols for samples received April 25, 2008

The chain of custody document for this report is in section 2, the analytica] data summary, sample data, and standard data is in section 3 . 
2. Traffic Repoits/ Chain of Custody Records 


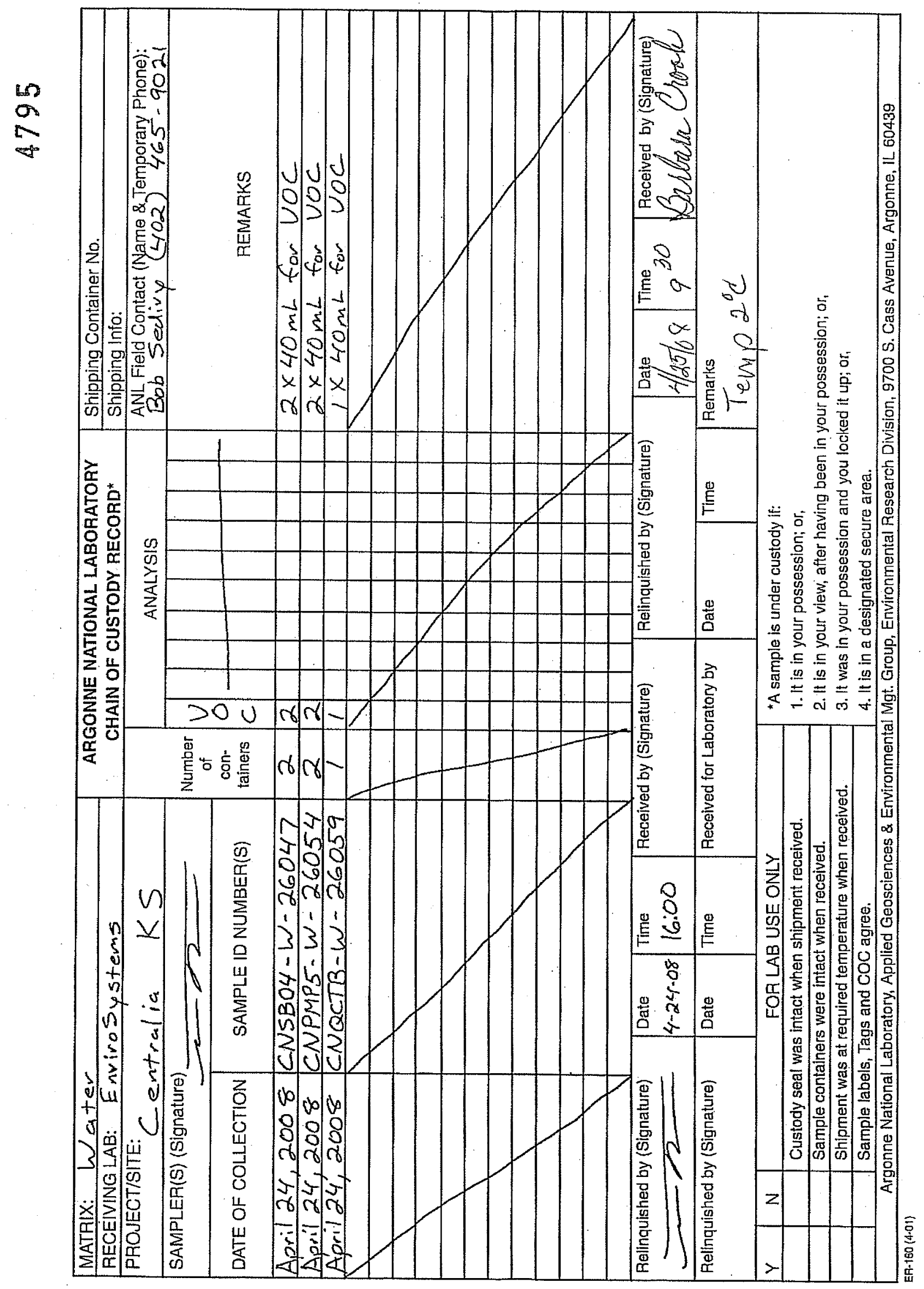


3. VOA Data 
FORM 1

VOLATILE ORGANICS ANALYSIS DATA SHEET

Lab Name: ENVIROSYSTEMS, INC.

Lab Code: ENVSYS Case No.:

Matrix: (soil/water) WATER

Sample wt/vol: $\quad 5.000(\mathrm{~g} / \mathrm{mL}) \mathrm{ML}$

Contract: $\mathrm{N} / \mathrm{A}$

SAS NO.: N/A

Lab Sample ID: 0080416-0I

Lab File ID: $\quad$ H001287

Level: (low/med) LOW

믐 Moisture: not dec.

GC Column: RTX-624 ID: 0.18 (mm)

Soil Extract Volume:

(uI)

COMPOUND

CAS NO.

CONCENTRATION UNITS:

(ug/L or $\mathrm{ug} / \mathrm{Kg}$ ) UG/L

\section{Q}

ARGONNE SAMPLE NO.

CNSB04-W-26047
Date Received: 04/25/08

Date Analyzed: 05/05/08

Dilution Factor: 1.0

Soil Aliquot Volume: (uI)

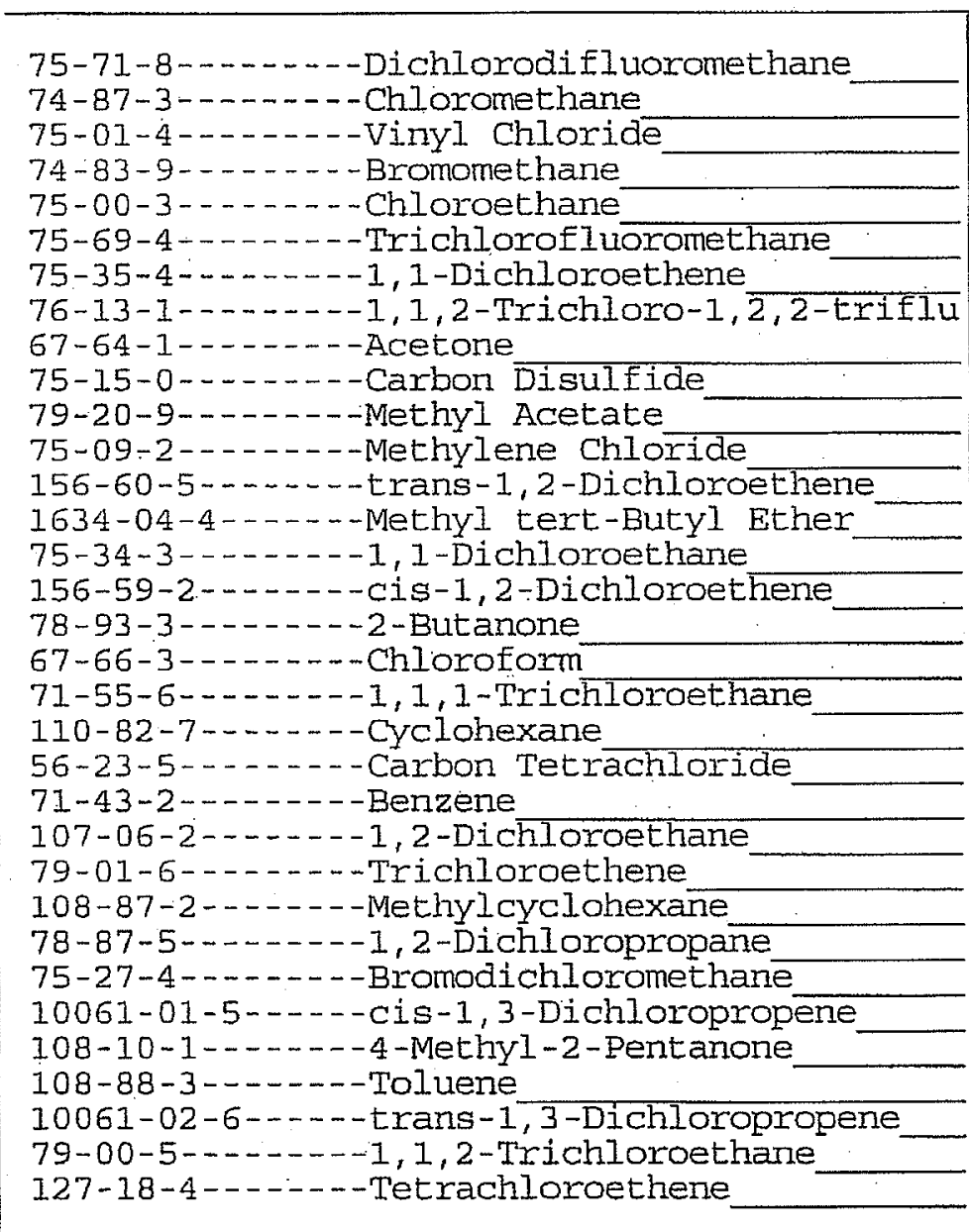

\begin{tabular}{|l|l|}
5.0 & $\mathrm{U}$ \\
5.0 & $\mathrm{U}$ \\
5.0 & $\mathrm{U}$ \\
5.0 & $\mathrm{U}$ \\
5.0 & $\mathrm{U}$ \\
5.0 & $\mathrm{U}$ \\
5.0 & $\mathrm{U}$ \\
5.0 & $\mathrm{U}$ \\
11 & \\
5.0 & $\mathrm{U}$ \\
5.0 & $\mathrm{U}$ \\
9.0 & \\
5.0 & $\mathrm{U}$ \\
5.0 & $\mathrm{U}$ \\
5.0 & $\mathrm{U}$ \\
5.0 & $\mathrm{U}$ \\
5.0 & $\mathrm{U}$ \\
5.0 & $\mathrm{U}$ \\
5.0 & $\mathrm{U}$ \\
5.0 & $\mathrm{U}$ \\
5.0 & $\mathrm{U}$ \\
5.0 & $\mathrm{U}$ \\
5.0 & $\mathrm{U}$ \\
5.0 & $\mathrm{U}$ \\
5.0 & $\mathrm{U}$ \\
5.0 & $\mathrm{U}$ \\
5.0 & $\mathrm{U}$ \\
5.0 & $\mathrm{U}$ \\
1.4 & $\mathrm{~J}$ \\
5.0 & $\mathrm{U}$ \\
5.0 & $\mathrm{U}$ \\
5.0 & $\mathrm{U}$ \\
5.0 & $\mathrm{U}$ \\
& \\
\hline
\end{tabular}

FORM I VOA 
FORM 1

VOLATILE ORGANICS ANATYSIS DATA SHEET

Lab Name: ENVIROSYSTEMS, INC.

Lab Code: ENVSYS Case No.:

Matrix: (soil/water) WATER

Sample wt/vol: $\quad 5.000(\mathrm{~g} / \mathrm{mL})$ MI

Level: (low/med) Low

¿ Moisture: not dec.

GC Column: RTX-624 ID: 0.18 (mm)

Soil Extract Volume:

(UI)
Contract: $\mathrm{N} / \mathrm{A}$

SAS NO.: N/A
ARGONNE SAMPLE NO.

CNSBO4-W-26047
SDG NO. : NA

Lab Sample ID: 0080416-01

Lab File ID: $\quad H 001287$

Date Received: 04/25/08

Date Analyzed: 05/05/08

Dilution Factor: 1.0

Soil Aliquot Volume:

(ut)

CAS NO. COMPOUND $(\mathrm{ug} / \mathrm{L}$ or $\mathrm{ug} / \mathrm{Kg}) \mathrm{UG} / \mathrm{I} \quad \mathrm{Q}$

\begin{tabular}{|c|}
\hline 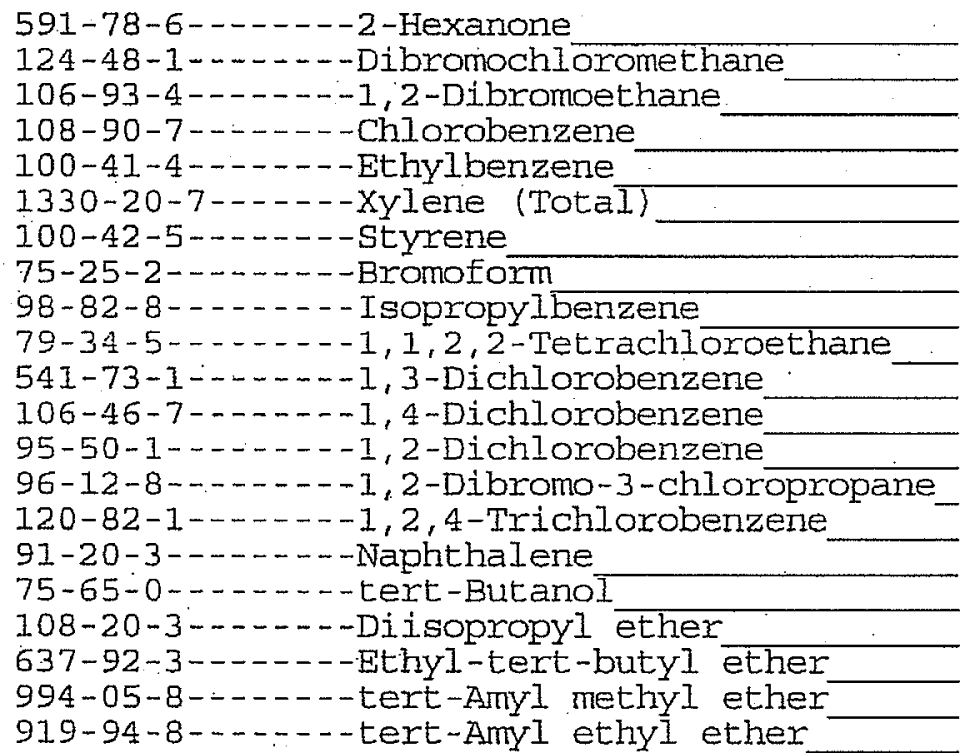 \\
\hline
\end{tabular}

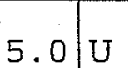

5. $0 \mathrm{U}$

$5.0 \mathrm{U}$

$5.0 \mathrm{U}$

$5.0 \mathrm{U}$

$5.0 \mathrm{U}$

$5.0 \mathrm{U}$

$5.0 \mathrm{U}$

$5.0 \mathrm{U}$

$5.0 \mathrm{U}$

$5.0 \mathrm{U}$

$5.0 \mathrm{U}$

$5.0 \mathrm{U}$

$5.0 \mathrm{U}$

$5.0 \mathrm{U}$

$10 \mathrm{U}$

$5.0 \mathrm{U}$

$10 \mathrm{U}$

$10 \mathrm{U}$

$10 \mathrm{U}$

$10 \mathrm{U}$ 
FORM I

VOL.ATILE ORGANICS ANALYSIS DATA SHEET

Lab Name: ENVIROSYSTEMS, INC.

Contract: $\mathrm{N} / \mathrm{A}$

Lab Code: ENVSYS Case No.:

Matrix: (soil/water) WATER

Sample wt/vol:

$5.000(\mathrm{~g} / \mathrm{mL}) \mathrm{ML}$

Level: (low/med) LOW

\% Moisture: not dec.

GC Column: RTX-624 ID: $0.18(\mathrm{~mm})$

Soil Extract Volume:

(US)
ARGONNE SAMPLE NO.

CNPMP5-W-26054

SAS NO.: N/A SDG NO.: NA

Lab Sample ID: 0080416-02

Lab File ID: Ho01288

Date Received: 04/25/08

Date Analyzed: 05/05/08

Dilution Factor: 1.0

Soil Aliquot Volume:

(uI)

CONCENTRATION UNITS:

CAS NO.

COMPOUND

(ug/I or $\mathrm{ug} / \mathrm{Kg}$ ) UG/I

Q

5.00

$5.0 \mathrm{U}$

$5.0 \mathrm{U}$

$5.0 \mathrm{U}$

$5.0 \mathrm{U}$

$5.0 \mathrm{U}$

$5.0 \mathrm{U}$

$5.0 \mathrm{U}$

14

$5.0 \overline{\mathrm{U}}$

$5.0 \mathrm{U}$

10

$5.0 \overline{\mathrm{U}}$

$5.0 \mathrm{U}$

$5.0 \mathrm{U}$

$5.0 \mathrm{U}$

$5.0 \mathrm{U}$

72

$5.0 \mathrm{U}$

$5.0 \mathrm{U}$

$240 \mathrm{E}$

$5.0 \mathrm{U}$

$5.0 \mathrm{U}$

$5.0 \mathrm{U}$

$5.0 \mathrm{U}$

$5.0 \mathrm{U}$

$5.0 \mathrm{U}$

$5.0 \mathrm{U}$

$5.0 \mathrm{U}$

$5.0 \mathrm{U}$

$5.0 \mathrm{U}$

$5.0 \mathrm{U}$

$5.0 \mathrm{U}$ 
FORM 1

VOLATILE ORGANICS ANALYSIS DATA SHEET

Lab Name: ENVIROSYSTEMS, INC.

Lab Code: ENVSYS Case No.:

Matrix: (soil/water) WATER

Sample wt/vol: $\quad 5.000(\mathrm{~g} / \mathrm{mL}) \mathrm{ML}$

Level: (low/med) Low

- $\frac{0}{5}$ Moisture: not dec.

GC Column: RTX-624 ID: 0.18 (mm)

Soil Extract Volume:

(UL)
Contract: N/A

SAS NO.: N/A
ARGONNE SAMPLE NO.

CNPMP5-W-26054
SDG NO. : NA
Lab Sample ID: 0080416-02

Lab File ID: H001288

Date Received: 04/25/08

Date Analyzed: 05/05/08

Dilution Factor: 1.0

Soil Aliquot Volume:

(uL)

CAS NO.

COMPOUND

CONCENTRATION UNITS:

(ug/L or $u g / \mathrm{kg}$ ) UG/L

Q

$5.0 \mathrm{U}$

$5.0 \mathrm{U}$

$5.0 . \mathrm{U}$

$5.0 \mathrm{U}$

$5.0 \mathrm{U}$

$5.0 \mathrm{U}$

$5.01 \mathrm{U}$

$5.0 \mathrm{U}$

$5.0 \mathrm{U}$

$5.0 \mathrm{U}$

$5.0 \mathrm{U}$

$5.0 \mathrm{U}$

$5.0 \mathrm{U}$

$5.0 \mathrm{U}$

$5.0 \mathrm{U}$

$10 \mathrm{U}$

$5.0 \mathrm{U}$

$10 \mathrm{U}$

$10 \mathrm{U}$

$10 \mathrm{U}$

$10 \mathrm{U}$ 
FORM 1

VOLATILE ORGANICS ANALYSIS DATA SHEET

Lab Name: ENVIROSYSTEMS, INC.

Lab Code: ENvsys Case No.:

Matrix: (soil/water). WATER

Sample wt/vol: $\quad 5.000(\mathrm{~g} / \mathrm{mL}) \mathrm{ML}$

Level: (low/med) LoW

. Moisture: not dec.

GC Column: RTX-624 ID: 0.18 (mm)

Soil Extract Volume:

(UI)
Contract :

SAS NO.:
ARGONNE SAMPLE NO.

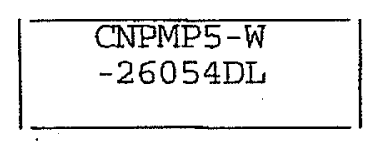

SDG No.: NA

Lab sample ID: 0080416-02REI

Lab File ID: $\quad$ H001307

Date Received: 04/25/08

Date Analyzed: 05/06/08

Dilution Factor: 5.0

Soil Aliquot Volume:

(ut)

CAS NO. COMPOUND $(\mathrm{ug} / \mathrm{L}$ or $\mathrm{ug} / \mathrm{Kg}$ ) UG/I

Q

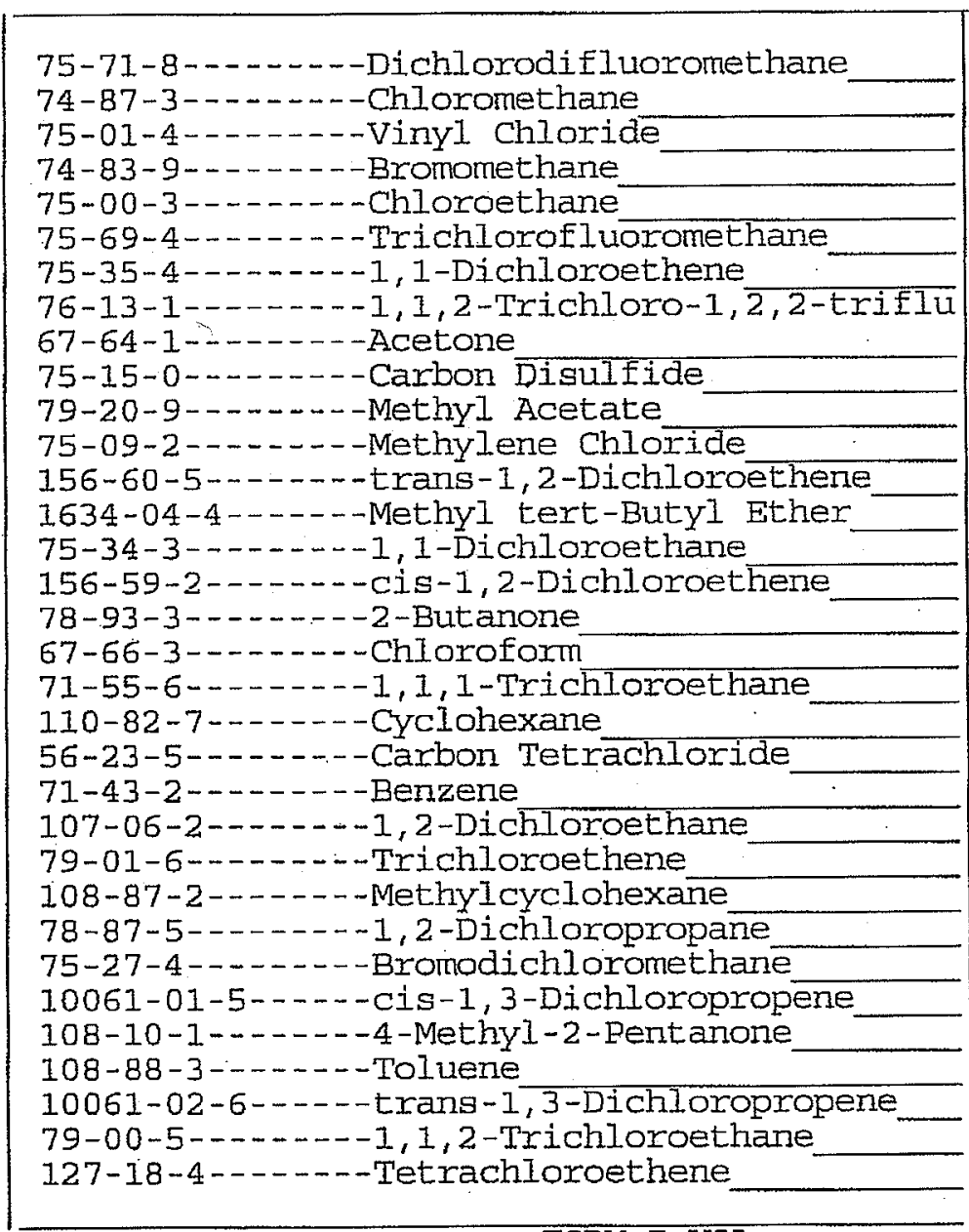

FORM I VOA

\begin{tabular}{|r|r|}
25 & $\mathrm{U}$ \\
25 & $\mathrm{U}$ \\
25 & $\mathrm{U}$ \\
25 & $\mathrm{U}$ \\
25 & $\mathrm{U}$ \\
25 & $\mathrm{U}$ \\
25 & $\mathrm{U}$ \\
25 & $\mathrm{U}$ \\
25 & $\mathrm{U}$ \\
25 & $\mathrm{U}$ \\
25 & $\mathrm{U}$ \\
20 & $\mathrm{D}$ \\
25 & $\mathrm{U}$ \\
25 & $\mathrm{U}$ \\
25 & $\mathrm{U}$ \\
25 & $\mathrm{U}$ \\
25 & $\mathrm{U}$ \\
$8 \mathrm{D}$ & $\mathrm{D}$ \\
25 & $\mathrm{U}$ \\
25 & $\mathrm{U}$ \\
280 & $\mathrm{D}$ \\
25 & $\mathrm{U}$ \\
25 & $\mathrm{U}$ \\
25 & $\mathrm{U}$ \\
25 & $\mathrm{U}$ \\
25 & $\mathrm{U}$ \\
25 & $\mathrm{U}$ \\
25 & $\mathrm{U}$ \\
25 & $\mathrm{U}$ \\
25 & $\mathrm{U}$ \\
25 & $\mathrm{U}$ \\
25 & $\mathrm{U}$ \\
25 & $\mathrm{U}$ \\
& \\
\hline
\end{tabular}


FORM 1

VOLATILE ORGANICS ANALYSIS DATA SHEET

Lab Name: ENVIROSYSTEMS, INC.

Lab Code: ENVSYS Case No.:

Matrix: (soil/water) WATER

Sample wt/vol: $\quad 5.000(\mathrm{~g} / \mathrm{mL}) \mathrm{ML}$

Level: (low $/ \mathrm{med}$ ) LOW

: Moisture: not dec.

GC Column: RTX-624 ID: 0.18 (mun)

Soil Extract Volume: (UL)

Contract :

SAS NO. :

ARGONNNE SAMPLE NO.

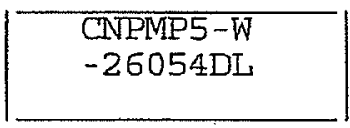

SDG No.: NA

Lab Sample ID: 0080416-02RE1

Lab File ID: H001307

Date Received: 04/25/08

Date Analyzed: 05/06/08

Dilution Factor: 5.0

Soil Aliquot Volume: (uL)

CAS NO.

COMPOUND

CONCENTRATION UNITS:

(ug/L or $\mathrm{ug} / \mathrm{Kg}$ ) UG/L

Q

\begin{tabular}{|c|}
\hline 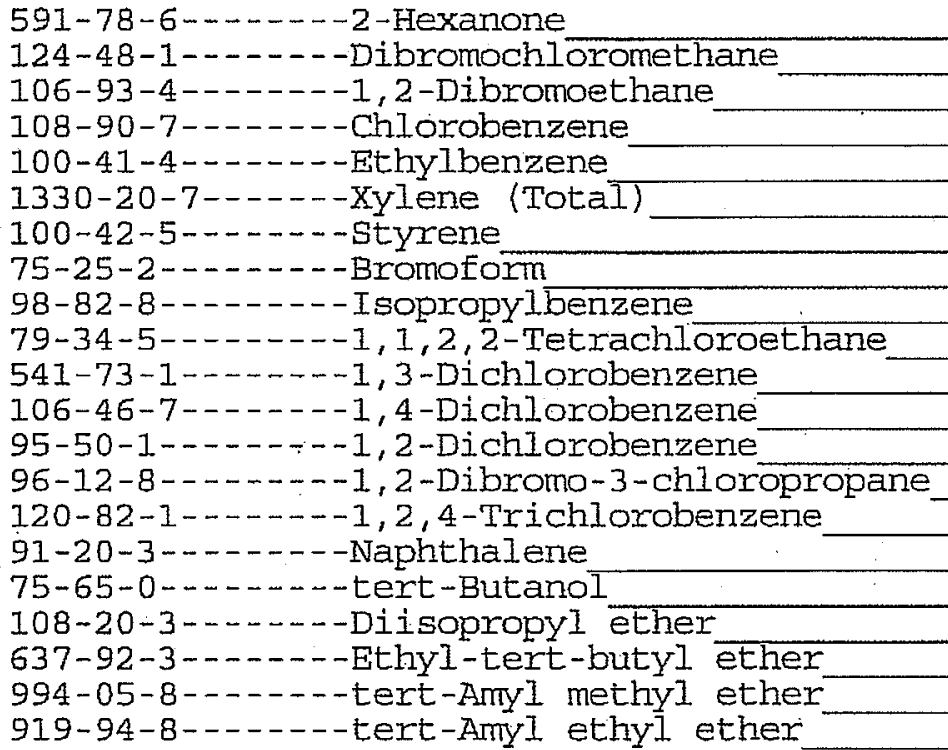 \\
\hline
\end{tabular}

\begin{tabular}{|l|l|l|}
25 & $\mathrm{U}$ \\
25 & $\mathrm{U}$ \\
25 & $\mathrm{U}$ \\
25 & $\mathrm{U}$ \\
25 & $\mathrm{U}$ \\
25 & $\mathrm{U}$ \\
25 & $\mathrm{U}$ \\
25 & $\mathrm{U}$ \\
25 & $\mathrm{U}$ \\
25 & $\mathrm{U}$ \\
25 & $\mathrm{U}$ \\
25 & $\mathrm{U}$ \\
25 & $\mathrm{U}$ \\
25 & $\mathrm{U}$ \\
25 & $\mathrm{U}$ \\
50 & $\mathrm{U}$ \\
25 & $\mathrm{U}$ \\
50 & $\mathrm{U}$ \\
50 & $\mathrm{U}$ \\
50 & $\mathrm{U}$ \\
50 & $\mathrm{U}$ \\
& \\
\hline
\end{tabular}

FORM I VOA 
FORM 1

\section{VOLATILE ORGANICS ANALYSIS DATA SHEET}

Lab Name: ENVIROSYSTEMS, INC.

Lab Code: ENVSYS Case No: :

Matrix: (soil/water) WATER

Sample wt/vol: $\quad 5.000(\mathrm{~g} / \mathrm{mL}) \mathrm{ML}$

Level : (low/med) LOW

\% Moisture: not dec.

GC Column: RTX-624 ID: 0.18 (mm)

Soil Extract Volume: (uI)
Contract: N/A

SAS NO.: $N / A$
ARGONNE SAMPLE NO.

CNQCTB-W-26059

Lab Sample ID: 0080416-03

Lab File ID: Ho01289

Date Received: 04/25/08

Date Analyzed: 05/05/08

Dilution Factor: 1.0

Soil Aliquot volume: (uI)

CONCENTRATION UNITS:

(ug/L or $\mathrm{ug} / \mathrm{Kg}$ ) UG/L

Q

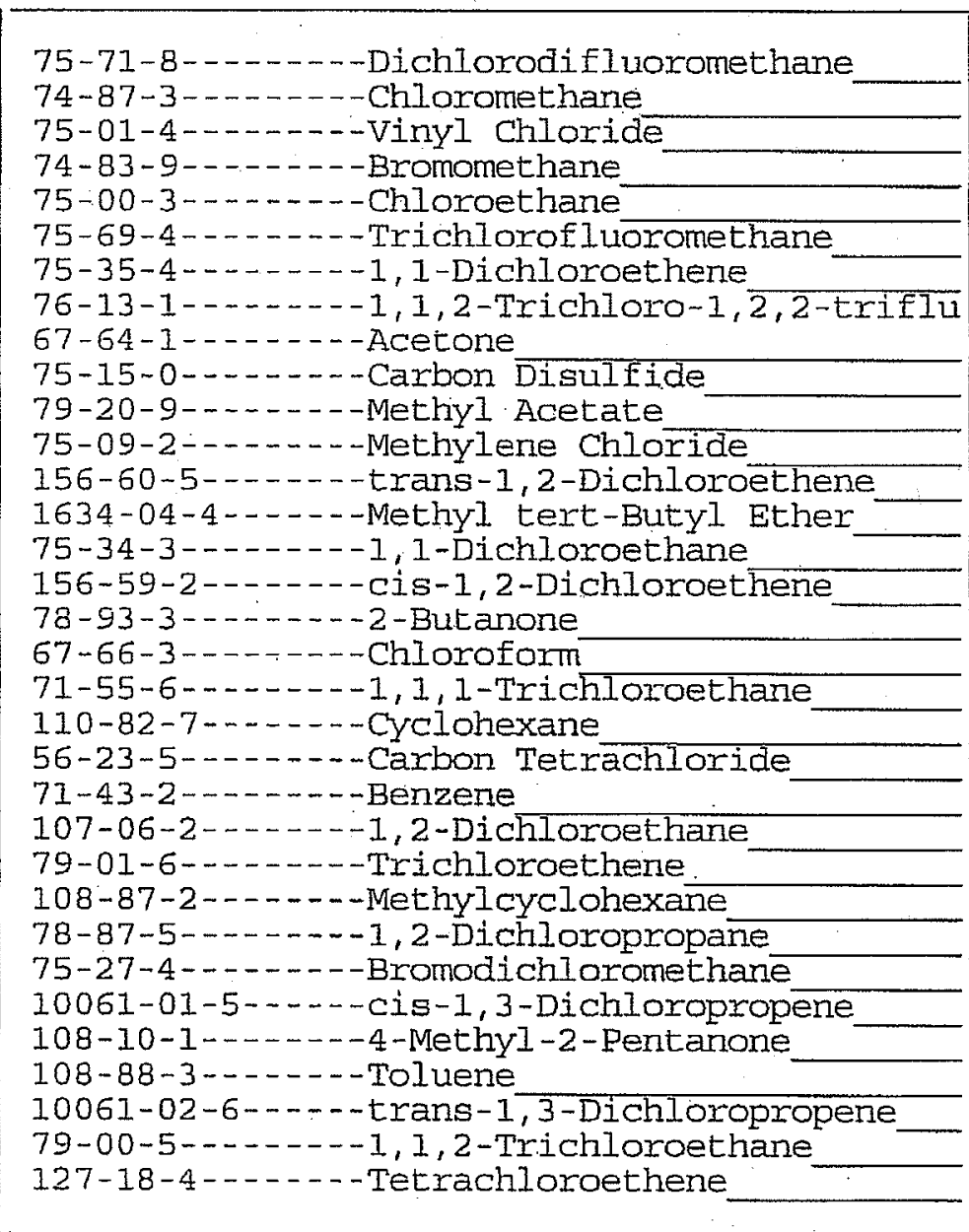

$5.0 \mathrm{U}$

$5.0 \mathrm{U}$

5.0 U

$5.0 \mathrm{U}$

$5.0 \mathrm{U}$

5.0 U

$5.0 \mathrm{U}$

$5.0 \mathrm{U}$

14

$5.0 \mathrm{U}$

$5.0 \mathrm{U}$

7.7

$5.0 \overline{\mathrm{U}}$

5. $0 \mathrm{U}$

$5.0 \mathrm{U}$

$5.0 \mathrm{U}$

$5.0 \mathrm{U}$

$5.0 \mathrm{U}$

$5.0 \mathrm{U}$

$5.0 \mathrm{U}$

$5.0 \mathrm{U}$

$5.0 \mathrm{U}$

$5.0 \mathrm{U}$

$5.0 \mathrm{U}$

$5.0 \mathrm{U}$

$5.0 \mathrm{U}$

$5.0 \mathrm{U}$

$5.0 \mathrm{U}$

$5.0 \mathrm{U}$

$5.0 \mathrm{U}$

$5.0 \mathrm{U}$

$5.0 \mathrm{U}$

$5.0 \mathrm{U}$

FORM I VOA 
FORM 1

VOLATILE ORGANICS ANALYSIS DATA SHEET

Lab Name: ENVIROSYSTEMS, INC.

Lab Code: ENVSYS Case No.:

Matrix: (soil/water) WATER

Sample wt/vol: $\quad 5.000(\mathrm{~g} / \mathrm{mL}) \mathrm{ML}$

Level: (low/med) LOW

\% Moisture: not dec.

GC Column: $\operatorname{RTX}-624$ ID: 0.18 (mm)

Soil Extract Volume:

(uI)
Contract: $N / A$

SAS NO.: N/A
ARGONNE SAMPLE NO.

CNQCTB-W-26059

Lab Sample ID: 0080416-03

Lab File ID: H001289

Date Received: 04/25/08

Date Analyzed: 05/05/08

Dilution Factor: 1.0

Soil Aliquot Volume:

CAS NO.

COMPOUND

CONCENTRATION UNITS:

(ug/L or $\mathrm{ug} / \mathrm{Kg}$ ) UG/L

$Q$

$5.0 \mathrm{U}$

$5.0 \mathrm{U}$

$5.0 \mathrm{U}$

$5.0 \mathrm{U}$

$5.0 \mathrm{U}$

5.0. U

$5.0 \mathrm{U}$

$5.0 \mathrm{U}$

$5.0 \mathrm{U}$

$5.0 \mathrm{U}$

$5.0 \mathrm{U}$

$5.0 \mathrm{U}$

$5.0 \mathrm{U}$

$5.0 \mathrm{U}$

$5.0 \mathrm{U}$

$10 \mathrm{U}$

$5.0 \mathrm{U}$

$10 \mathrm{U}$

$10 \mathrm{U}$

$10 \mathrm{U}$

$10 \mathrm{U}$

994-05-8-....-tert-Amyl methyl ether

919-94-8-.-.--tert-Amyl ethyl ether 


\section{ENVIROSYSTEMS, INC.}

9200 Rumsey Road - Suite B102 - Columbia, Maryland 21045-1934

Phone (410) 964-0330 - Fax (410) 740-9306

Email: info@envsystems.com - Webpage: www.envsystems.com/envsys

Jorge S. Alvarado, PH. D

Argonne National Laboratory

Environmental Research Division

Applied Geosciences and Environmental

Management Section

9700 South Cass Avenue, ER-203

Argonne, Illinois 60439

RE: Report \#080205

Dear Jorge,

Enclosed is the Analytical Data Package for the samples received on June 6, 2.008 for volatile organics analysis by USEPA SW846 method 8260B/CLP SOW OLM04.3 protocols.

Please do not hesitate to call if you have any questions, comments, or require additional information.

Sincerely,

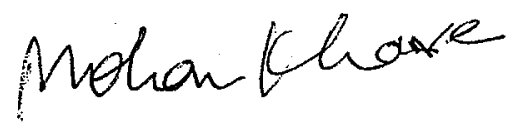

Mohan Khare Ph.D.

President/CEO

Enclosure (1)

$\mathrm{MK} / \mathrm{ncc}$

Envirosystems, Inc.

Report\#080205 
1. Narrative

$-3-$ 


\section{Narrative}

This analytical data package contains Volatile analysis by US EPA SW-846 and CLP protocols for samples received June 6, 2008.

The Chain of Custody document for report is in section 2, the analytical data, QC summary, Sample data, Standards data and Raw QC data is present in section 3.

Please note that these samples were inadvertently analyzed out of holding time due to analyst's oversight. All the data Quality Control criteria for Surrogate Recovery, Matrix Spike/ Matrix Spike Duplicate, Initial and Continuing Calibration, BFB Tuning and Method Blanks are compliant for this case.

Laboratory regrets very much the noncompliance for analytical holding time and late data and apologizes for any inconvenience it may have caused. 
2. Traffic Reports/ Chain of Custody Records 


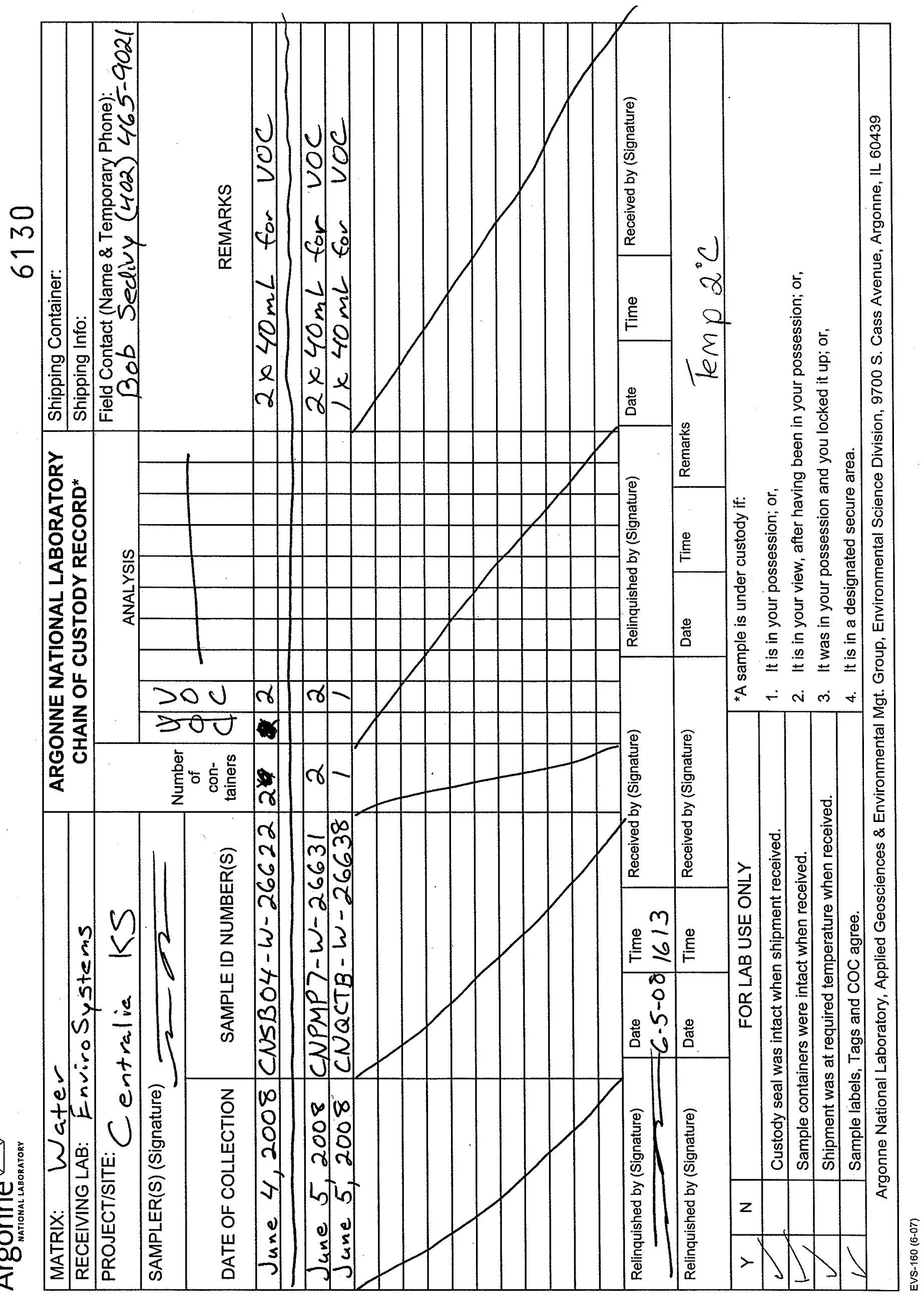


3. VOA Data

$-8-$ 
FORM 1

VOLATILE ORGANICS ANALYSIS DATA SHEET

Lab Name: ENVIROSYSTEMS, INC.

Lab Code: ENVSYS Case No.:

Matrix: (soil/water) WATER

Sample wt/vol: $\quad 5.000(\mathrm{~g} / \mathrm{mL}) \mathrm{ML}$

Level: (low/med) LOW

\% Moisture: not dec.

GC Column: RTX-624 ID: 0.18 (mm)

Soil Extrąct Volume:

(UL)
Contract:

SAS NO. :
Argonne. SAMPLE NO.

CNSB04 -W-26622

SDG No.: NA

Lab Sample ID: 0080607-01

Lab File ID: H002490

Date Received: 06/06/08

Date Analyzed: 07/11/08

Dilution Factor: 1.0

Soil Aliquot Volume: (U工) CONCENTRATION UNITS: (ug/L or $\mathrm{ug} / \mathrm{Kg}$ ) UG/L

Q

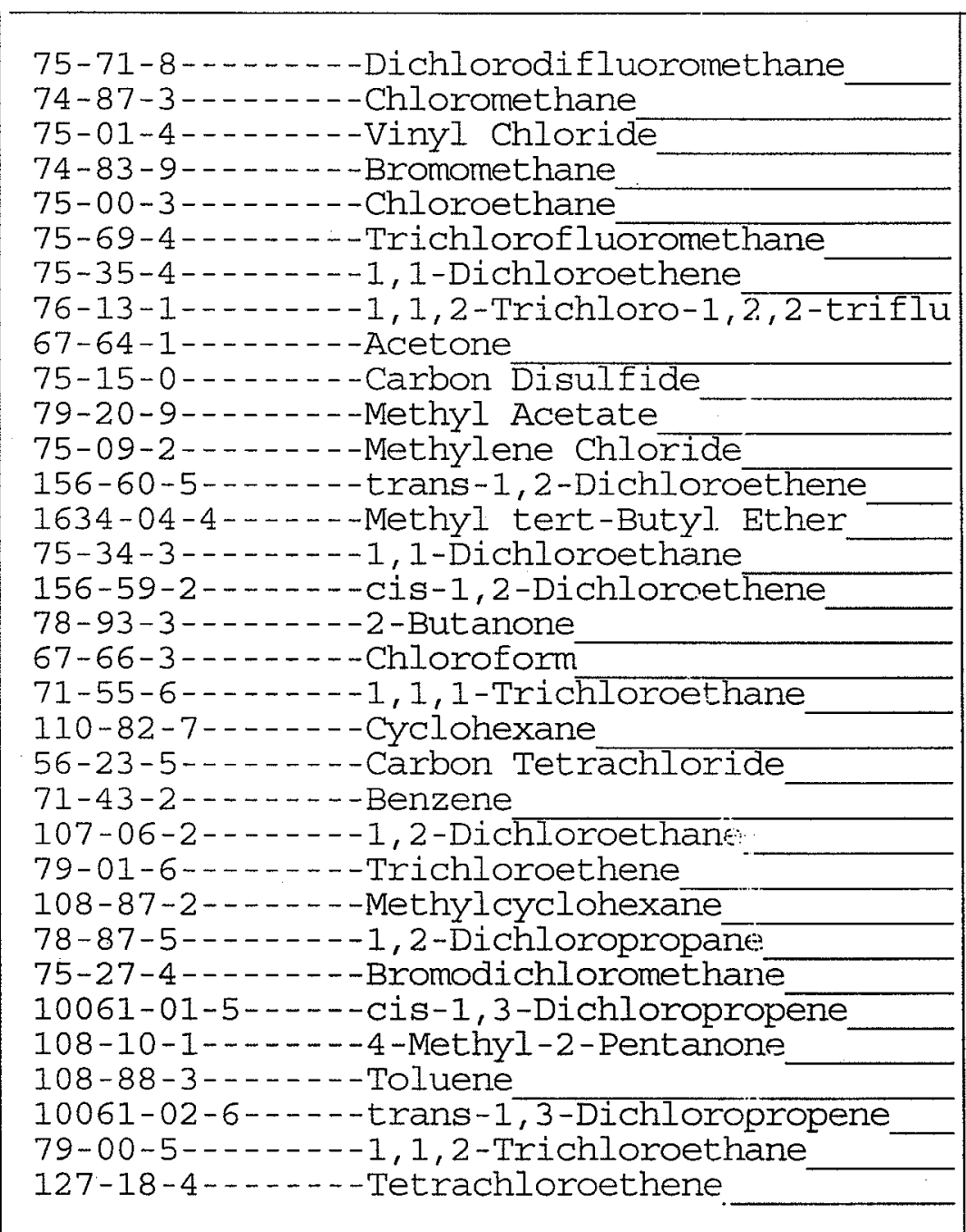

FORM I. VOA

\begin{tabular}{|l|l|}
5.0 & $\mathrm{U}$ \\
5.0 & $\mathrm{U}$ \\
5.0 & $\mathrm{U}$ \\
5.0 & $\mathrm{U}$ \\
5.0 & $\mathrm{U}$ \\
5.0 & $\mathrm{U}$ \\
5.0 & $\mathrm{U}$ \\
5.0 & $\mathrm{U}$ \\
5.0 & $\mathrm{U}$ \\
5.0 & $\mathrm{U}$ \\
5.0 & $\mathrm{U}$ \\
5.0 & $\mathrm{U}$ \\
5.0 & $\mathrm{U}$ \\
5.0 & $\mathrm{U}$ \\
5.0 & $\mathrm{U}$ \\
5.0 & $\mathrm{U}$ \\
5.0 & $\mathrm{U}$ \\
5.0 & $\mathrm{U}$ \\
5.0 & $\mathrm{U}$ \\
5.0 & $\mathrm{U}$ \\
16 & \\
5.0 & $\mathrm{U}$ \\
5.0 & $\mathrm{U}$ \\
5.0 & $\mathrm{U}$ \\
5.0 & $\mathrm{U}$ \\
5.0 & $\mathrm{U}$ \\
5.0 & $\mathrm{U}$ \\
5.0 & $\mathrm{U}$ \\
5.0 & $\mathrm{U}$ \\
5.0 & $\mathrm{U}$ \\
5.0 & $\mathrm{U}$ \\
5.0 & $\mathrm{U}$ \\
5.0 & $\mathrm{U}$ \\
& \\
\hline 5 & \\
\hline 5 \\
5.0
\end{tabular}


FORM 1

VOLATILE ORGANICS ANALYSIS DATA SHEET

Lab Name: ENVIROSYSTEMS, INC.

Lab Code: ENVSYS Case No.:

Matrix: (soil/water) WATER

Sample wt/vol:

$5.000(\mathrm{~g} / \mathrm{mL}) \mathrm{ML}$

Level : (low/med) LOW

\% Moisture: not dec.

GC Column: RTX-624 ID: 0.18 (mm)

Soil Extract Volume:

(UI)
Argonne. SAMPLE NO.

Contract:

SAS NO. :

SDG No.: NA

CNSB0 $4-\mathrm{W}-26622$

Lab Sample ID: 0080607-01

Lab File ID: H002490

Date Received: 06/06/08

Date Analyzed: 07/11/08

Dilution Factor: 1.0

Soil Aliquot Volume: (uL)

CONCENTRATION UNITS :

CAS NO. COMPOUND (ug/L or ug/Kg) UG/L

Q

$5.0 \mathrm{U}$

$5.0 \mathrm{U}$

$5.0 \mathrm{U}$

$5.0 \mathrm{U}$

$5.0 \mathrm{U}$

$5.0 \mathrm{U}$

$5.0 \mathrm{U}$

$5.0 \mathrm{U}$

$5.0 \mathrm{U}$

$5.0 \mathrm{U}$

$5.0 \mathrm{U}$

$5.0 \mathrm{U}$

$5.0 \mathrm{U}$

$5.0 \mathrm{U}$

$5.0 \mathrm{U}$

$10 \mathrm{U}$

$5.0 \mathrm{U}$

$10 \mathrm{U}$

$10 \mathrm{U}$

$10 \mathrm{U}$

$10 \mathrm{U}$

-tert-Amyl methyl ether

919-94-8-.----tert-Amyl ethyl ether 
FORM 1

VOLATILE ORGANICS ANALYSIS DATA SHEET

Lab Name: ENVIROSYSTEMS, INC.

Contract:

Lab Code: ENVSYS Case No.:

Matrix: (soil/water) WATER

Sample wt/vol:

$5.000(\mathrm{~g} / \mathrm{mL}) \mathrm{ML}$

Level : (low/med) LOW

\% Moisture: not dec.

GC Column: RTX-624 ID: 0.18 (mm)

Soil Extract Volume:

(UL)

SAS NO. :
Argonne. SAMPLE NO.

CNPMP7-W-26631

SDG NO. : NA

Lab Sample ID: 0080607-02

Lab File ID: H002491

Date Received: 06/06/08

Date Analyzed: 07/11/08

Dilution Factor: 1.0

Soil Aliquot Volume: (uL) CONCENTRATION UNITS :

CAS NO. COMPOUND $(\mathrm{ug} / \mathrm{L}$ or $\mathrm{ug} / \mathrm{Kg}) \mathrm{UG} / \mathrm{L}$

$5.0 \mathrm{U}$

$5.0 \mathrm{U}$

$5.0 \mathrm{U}$

$5.0 \mathrm{U}$

$5.0 \mathrm{U}$

$5.0 \mathrm{U}$

$5.0 \mathrm{U}$

$5.0 \mathrm{U}$

$5.0 \mathrm{U}$

$5.0 \mathrm{U}$

$5.0 \mathrm{U}$

$5.0 \mathrm{U}$

$5.0 \mathrm{U}$

$5.0 \mathrm{U}$

$5.0 \mathrm{U}$

$5.0 \mathrm{U}$

$5.0 \mathrm{U}$

9.1

$5.0 \mathrm{U}$

$5.0 \mathrm{U}$

130

$5.0 \overline{\mathrm{U}}$

$5.0 \mathrm{U}$

$5.0 \mathrm{U}$

$5.0 \mathrm{U}$

$5.0 \mathrm{U}$

$5.0 \mathrm{U}$

$5.0 \mathrm{U}$

$5.0 \mathrm{U}$

$5.0 \mathrm{U}$

$5.0 \mathrm{U}$

$5.0 \mathrm{U}$

$5.0 \mathrm{U}$

79-00-5-..---1, 1, 2-Trichloroethane

127-18-4-----. Tetrachloroethene 
FORM 1

VOLATILE ORGANICS ANALYSIS DATA SHEET

Lab Name: ENVIROSYSTEMS, INC.

Lab Code: ENVSYS Case No.:

Matrix: (soil/water) WATER

Sample wt/vol:

$5.000(\mathrm{~g} / \mathrm{mL}) \mathrm{ML}$

Level : (low/med) LOW

\% Moisture: not dec.

GC Column: RTX-624 ID: 0.18 (mm)

Soil Extract Volume:

(uL)
Argonne. SAMPLE NO.

Contract:

SAS No. :

SDG No.: NA

Lab Sample ID: 0080607-02

Lab File ID: H002491

Date Received: 06/06/08

Date Analyzed: 07/11/08

Dilution Factor: 1.0

Soil Aliquot Volume: (uL) CONCENIRATION UNITS:

CAS NO.

COMPOUND

(ug/L or ug/Kg) UG/L

Q

591-78-6------2-Hexanone

124-48-1-...--Dibromochloromethane

106-93-4--..-1, 2-Dibromoethane

108-90-7------Chlorobenzene

100-41-4-...--Ethylbenzene

1330-20-7-..--XYlene (Total)

100-42-5------Styrene

75-25-2 - ...--Bromoform

98-82-8--- - - - Isopropylbenzene

79-34-5-...-1, 1,2,2-Tetrachloroethane

541-73-1-...-1,3-Dichlorobenzene

106-46-7-_...-1,4-Dichlorobenzene

95-50-1-...-1, 2-Dichlorobenzene

96-12-8-..---1,2-Dibromo-3-chloropropane

120-82-1-----1, 2,4-Trichlorobenzene

91-20-3-..----Naphthalene

75-65-0-.-.-.-tert-Butanol

108-20-3 -...-Diisopropyl ether

637-92-3-...--Ethyl-tert-butyl ether

994-05-8-..---tert-Amyl methyl ether

919-94-8-...--tert-Amyl ethyl ether
$5.0 \mathrm{U}$

$5.0 \mathrm{U}$

$5.0 \mathrm{U}$

$5.0 \mathrm{U}$

$5.0 \mathrm{U}$

$5.0 \mathrm{U}$

$5.0 \mathrm{U}$

$5.0 \mathrm{U}$

$5.0 \mathrm{U}$

$5.0 \mathrm{U}$

$5.0 \mathrm{U}$

$5.0 \mathrm{U}$

$5.0 \mathrm{U}$

$5.0 \mathrm{U}$

$5.0 \mathrm{U}$

$10 \mathrm{U}$

$5.0 \mathrm{U}$

$10 \mathrm{U}$

$10 \mathrm{U}$

$10 \mathrm{U}$

$10 \mathrm{U}$ 
FORM 1

VOLATILE ORGANICS ANALYSIS DATA SHEET

Lab Name: ENVIROSYSTEMS, INC.

Lab Code: ENVSYS Case No.:

Matrix: (soil/water) WATER

Sample wt/vol:

$5.000(\mathrm{~g} / \mathrm{mL}) \mathrm{ML}$

Level: (low/med) LOW

\% Moisture: not dec.

GC Column: RTX-624 ID: 0.18 (mm)

Soil Extract Volume:

(UL)

CAS NO.

COMPOUND
Contract:

SAS NO. :

SDG No.: NA

Lab Sample ID: 0080607-03

Lab File ID: H002494

Date Received: 06/06/08

Date Analyzed: 07/11/08

Dilution Factor: 1.0

Soil Aliquot Volume: (UL)

CONCENTRATION UNITS :

$(\mathrm{ug} / \mathrm{L}$ or $\mathrm{ug} / \mathrm{Kg}) \mathrm{UG} / \mathrm{L}$

$Q$

$5.0 \mathrm{U}$

$5.0 \mathrm{U}$

$5.0 \mathrm{U}$

$5.0 \mathrm{U}$

$5.0 \mathrm{U}$

$5.0 \mathrm{U}$

$5.0 \mathrm{U}$

$5.0 \mathrm{U}$

$5.0 \mathrm{U}$

$5.0 \mathrm{U}$

$5.0 \mathrm{U}$

$5.0 \mathrm{U}$

$5.0 \mathrm{U}$

$5.0 \mathrm{U}$

$5.0 \mathrm{U}$

$5.0 \mathrm{U}$

$5.0 \mathrm{U}$

$5.0 \mathrm{U}$

$5.0 \mathrm{U}$

$5.0 \mathrm{U}$

$5.0 \mathrm{U}$

$5.0 \mathrm{U}$

$5.0 \mathrm{U}$

$5.0 \mathrm{U}$

$5.0 \mathrm{U}$

$5.0 \mathrm{U}$

$5.0 \mathrm{U}$

$5.0 \mathrm{U}$

$5.0 \mathrm{U}$

$5.0 \mathrm{U}$

$5.0 \mathrm{U}$

$5.0 \mathrm{U}$

$5.0 \mathrm{U}$ 
FORM 1

VOLATILE ORGANICS ANALYSIS DATA SHEET

Lab Name: ENVIROSYSTEMS, INC.

Lab Code: ENVSYS Case No.:

Matrix: (soil/water) WATER

Sample wt/vol: $\quad 5.000(\mathrm{~g} / \mathrm{mL}) \mathrm{ML}$

Level: (low/med) LOW

\% Moisture: not dec.

GC Column: RTX-624 ID: 0.18 (mm)

Soil Extract Volume:

(uL)
Argonne. SAMPLE NO.

Contract :

SAS NO. :
CNQCTB-W-26638
SDG No.: NA

Lab Sample ID: 0080607-03

Lab File ID: H002494

Date Received: 06/06/08

Date Analyzed: 07/11/08

Dilution Factor: 1.0

Soil Aliquot Volume: (UL)

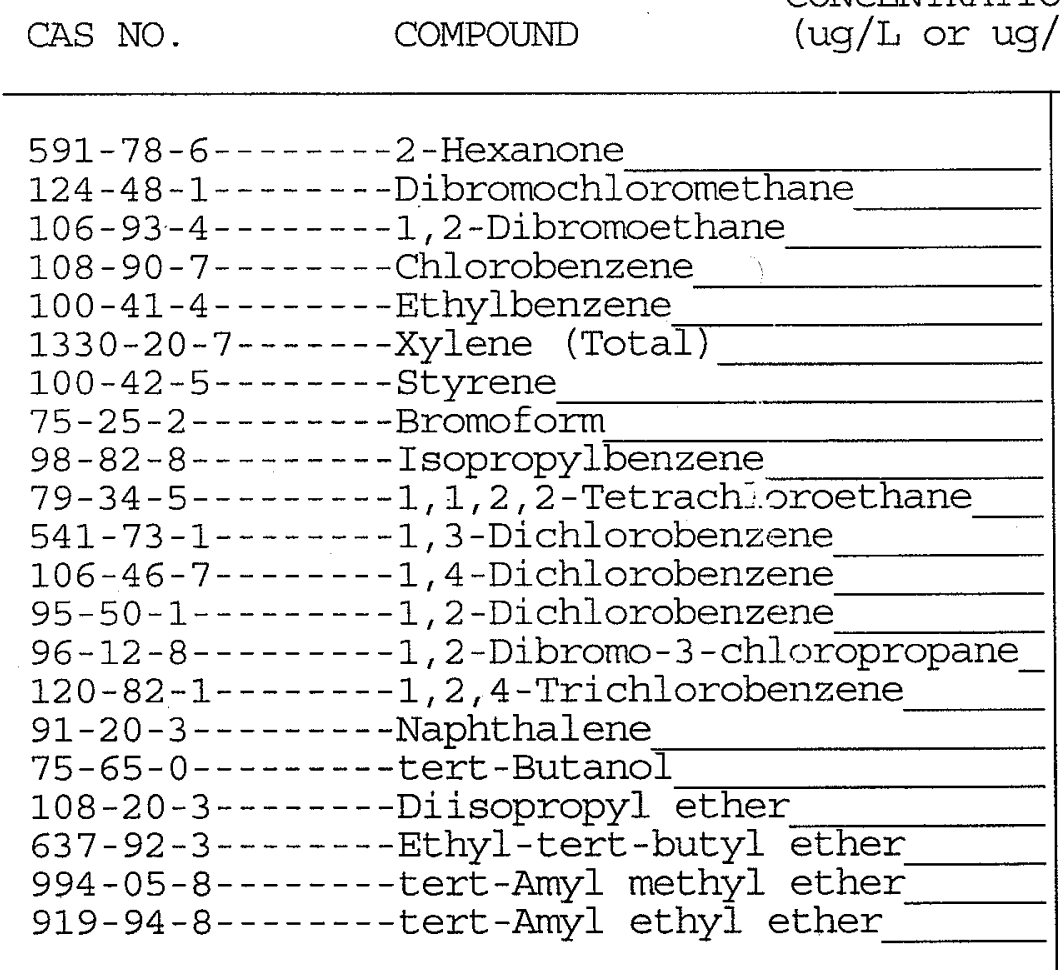
CONCENTRATION UNITS : (ug/L or $\mathrm{ug} / \mathrm{Kg}$ ) UG/L

Q 


\section{TestAmerica}

THE LEADER IN ENVIRONMENTAL TESTING

July 29, 2008

TestAmerica Laboratories, Inc.

Mr. Clyde Dennis

Argonne National Laboratory

9700 S. Cass Avenue

Building 203, Office B149

Argonne, IL 60439

Re: Laboratory Project No. 21005

Case: CENTRALI; SDG: 126456

Dear Mr. Dennis:

Enclosed are analytical results for samples that were received by TestAmerica Burlington on July $10^{\text {th }}, 2008$. Laboratory identification numbers were assigned, and designated as follows:

\begin{tabular}{|c|c|c|}
\hline Lab ID & $\begin{array}{l}\text { Client } \\
\text { Sample ID }\end{array}$ & $\begin{array}{l}\text { Sample } \\
\text { Date }\end{array}$ \\
\hline
\end{tabular}

Received: 07/10/08 ETR No: 126456

$\begin{array}{llll}759110 & \text { CNMW03-W-26640 } & 07 / 07 / 08 & \text { WATER } \\ 759111 & \text { CNPMP3-W-26646 } & 07 / 08 / 08 & \text { WATER } \\ 759112 & \text { CNQCTB-W-26657 } & 07 / 08 / 08 & \text { WATER } \\ 759113 & \text { VHBLK01 } & 07 / 10 / 08 & \text { WATER }\end{array}$

Documentation of the condition of the samples at the time of their receipt and any exception to the laboratory's Sample Acceptance Policy is documented in the Sample Handling section of this submittal. The samples, as received, were not acid preserved. On that basis the laboratory did provide for the analytical work to be performed within seven days of sample collection.

In order to accommodate field length limitations in processing the data summary forms, the laboratory did, in certain instances, abbreviate the sample identifier. The electronically formatted data provides for the full sample identifier.

\section{SOM01.2 Volatile Organics (Trace Level Water)}

A storage blank was prepared for volatile organics analysis, and stored in association with the storage of the sample. That storage blank, identified as VHBLK01, was carried through the holding period with the samples, and analyzed.

Sample CNPMP3-W-26646 was analyzed at a 6.3-fold dilution, based on the results of preliminary screening and the need to provide for a lower level of reporting. An additional, 
dilution analysis was performed on sample CNPMP3-W-26646 in order to provide for the quantification of acetone and 2-butanone within the range of calibrated instrument response. That analysis was performed at a 110-fold dilution. Both sets of results for the analysis of sample CNPMP3-W-26646 are included in this submittal. Each of the analyses associated with the sample set exhibited an acceptable internal standard performance. There was an acceptable recovery of each deuterated monitoring compound (DMC) in the analysis of the method blank and the analysis of the storage blank. The analysis of the samples in this sample set did meet the technical acceptance criteria specific to DMC recoveries, although not all DMC recoveries were within the control range in each analysis. The technical acceptance criteria does provide for the recovery of up to three DMCs to fall outside of the control range in the analysis of field samples. The recoveries of 2-butanone- $d_{5}$ and 2 -hexanone- $d_{5}$ were elevated in the analysis of sample CNMW03-W-26640, and the dilution analysis that was performed on sample CNPMP3W-26646. Matrix spike and matrix spike duplicate analyses were not performed on the samples in this sample set. The analysis of the method blank associated with the analytical work was free of target analyte contamination, as was the analysis of the storage blank. Present in the storage blank and method blank analyses was a non-target constituent that represented a compound that is related to either the DMC formulation or to column bleed. The fact that the presence of this compound is not within the laboratory's control is at issue. The derived results for that compound have been qualified with an "X" qualifier to reflect the source of the contamination. An instrument blank was analyzed following the more concentrated analysis of sample CNPMP3-W-26646. That analysis was performed without the introduction of the DMCs, and that is apparent in the DMC recovery summary. The analysis of the instrument blank was free of target analyte contamination.

The responses for each target analyte met the relative standard deviation criterion in the initial calibration. The response for each target analyte met the percent difference criterion in the continuing calibration check acquisition. The response for each target analyte met the 50.0 percent difference criterion in the closing calibration check acquisition. In the initial calibration and the calibration check acquisitions, the response for acetone did not meet the minimum relative response criterion of 0.010 in each instance. The relative response for acetone was above 0.009 in all instances.

The primary quantitation mass for methylcyclohexane that is specified in the Statement of Work is mass 83 . The laboratory did identify a contribution to mass 83 from 1,2-dichloropropane- $d_{6}$, one of the deuterated monitoring compounds (DMCs). The laboratory did change the primary quantitation mass assignment to mass 55 for the quantification of methylcyclohexane.

Manual integration was employed in deriving certain of the analytical results. The values that have been derived from manual integration are qualified on the quantitation reports. Extracted ion current profiles for each manual integration are included in the data package, and further documented in the Sample Preparation section of this submittal.

Any reference within this report to Severn Trent Laboratories, Inc. or STL, should be understood to refer to TestAmerica Laboratories, Inc. (formerly known as Severn Trent Laboratories, Inc.) The analytical results associated with the samples presented in this test report were generated under a quality system that adheres to requirements specified in the NELAC standard. Release of the data in this test report and any associated electronic deliverables is authorized by the Laboratory Director's designee as verified by the following signature. 


\section{TestAmerica}

July 29,2008

THE LEADER IN ENVIRONMENTAL TESTING

Mr. Clyde Dennis

Page 3 of 3

If there are any questions regarding this submittal, please contact me at 802 660-1990.

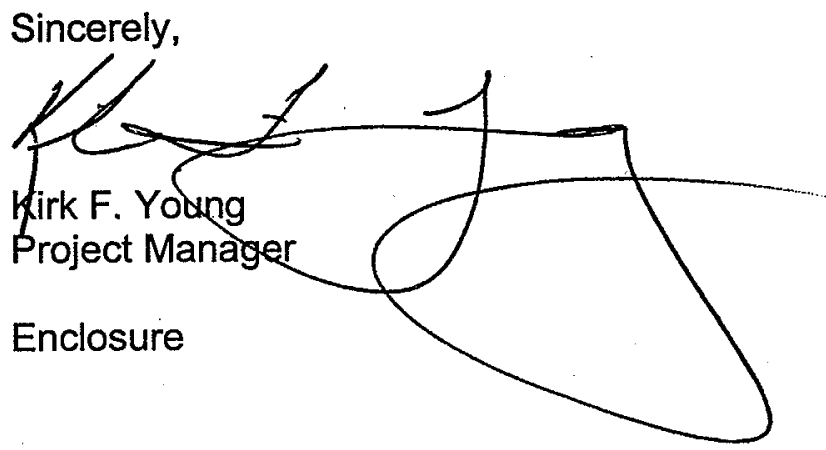




\section{TestAmerica Burlington Data Qualifier Definitions}

\section{Organic}

U: Compound analyzed but not detected at a concentration above the reporting limit.

J: $\quad$ Estimated value.

N: Indicates presumptive evidence of a compound. This flag is used only for tentatively identified compounds (TICs) where the identification of a compound is based on a mass spectral library search.

P: $\quad$ SW-846: The relative percent difference for detected concentrations between two GC columns is greater than $40 \%$. Unless otherwise specified the higher of the two values is reported on the Form I.

CLP SOW: Greater than 25\% difference for detected concentrations between two GC columns. Unless otherwise specified the lower of the two values is reported on the Form 1 .

C: Pesticide result whose identification has been confirmed by GC/MS.

B: $\quad$ Analyte is found in the sample and the associated method blank. The flag is used for tentatively identified compounds as well as positively identified compounds.

E: Compounds whose concentrations exceed the upper limit of the calibration range of the instrument for that specific analysis.

D: Concentrations identified from analysis of the sample at a secondary dilution.

A: Tentatively identified compound is a suspected aldol conden sation product.

$X, Y, Z$ : Laboratory defined flags that may be used alone or combined, as needed. If used, the description of the flag is defined in the project narrative.

\section{Inorganic/Metals}

E: Reported value is estimated due to the presence of interference.

$\mathrm{N}$ : Matrix spike sample recovery is not within control limits.

* Duplicate sample analysis is not within control limits.

B: The result reported is less than the reporting limit but greater than the instrument detection limit.

U: $\quad$ Analyte was analyzed for but not detected above the reporting limit.

Method Codes:

P ICP-AES

MS ICP-MS

CV Cold Vapor AA

AS Semi-Automated Spectrophotometric 


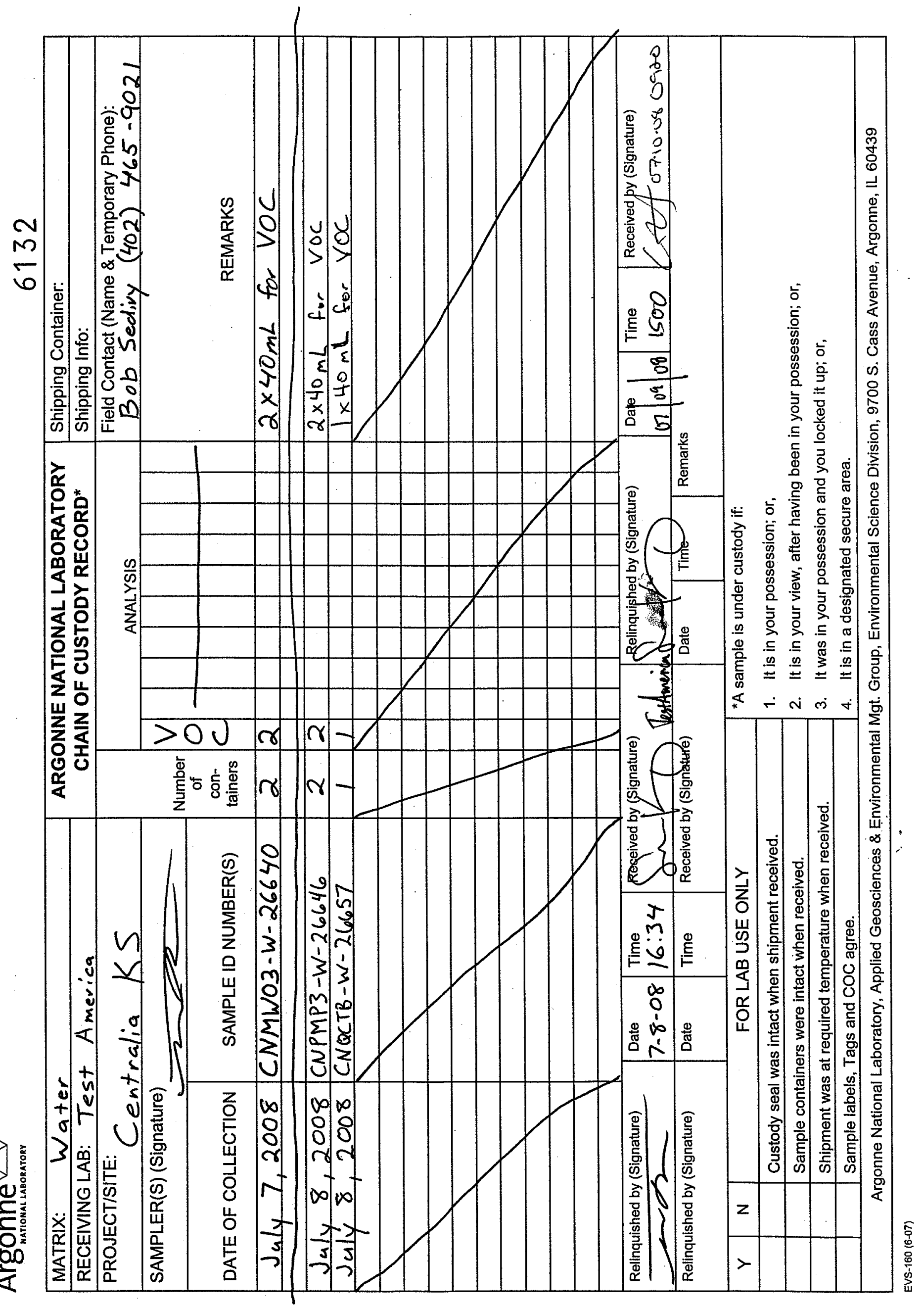




\section{TestAmerica}

THE LEADER IN ENVIRONMENTAL TESTING

\section{Sample Data Summary - SOM01.2 Volatiles - Trace}


Lab Name: TestAmerica BURLINGTON

Contract: $8 \mathrm{E}-00302$

Lab Code: STLV Case No.: CENTRALI Mod. Ref No.:

SDG NO.: 126456

Matrix: (SOIL/SED/WATER) Water

Sample wt/vol: 25.0

$(\mathrm{g} / \mathrm{mL}) \mathrm{mL}$

Level: (TRACE/LOW/MED) TRACE

$\div$ Moisture: not dec.

GC Column: DB-624

ID $: 0.53$

Soil Extract Volume:

Purge Volume: 25.0
Lab Sample ID: 759110

Lab File ID: 759110

Date Received: 07/10/2008

Date Analyzed: 07/14/2008

Dilution Factor: 1.0

Soil Aliquot Volume:

(uL)

(mL)

\begin{tabular}{|c|c|}
\hline CAS NO. & COMPOUND \\
\hline 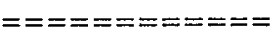 & 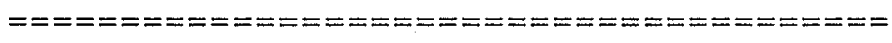 \\
\hline $75-71-8$ & Dichlorodifluoromethane \\
\hline $74-87-3$ & Chloromethane \\
\hline $75-01-4$ & Vinyl chloride \\
\hline $74-83-9$ & Bromomethane \\
\hline $75-00-3$ & Chloroethane \\
\hline $75-69-4$ & Trichlorofluoromethane \\
\hline $75-35-4$ & 1,1-Dichloroethene \\
\hline $76-13-1$ & 1,1,2-Trichloro-1,2,2-trifluoroethane \\
\hline $67-64-1$ & Acetone \\
\hline $75-15-0$ & Carbon disulfide \\
\hline $79-20-9$ & Methyl acetate \\
\hline $75-09-2$ & Methylene chloride \\
\hline $156-60-5$ & trans-1,2-Dichloroethene \\
\hline $1634-04-4$ & Methyl tert-butyl ether \\
\hline $75-34-3$ & 1,1-Dichloroethane \\
\hline $156-59-2$ & cis-1,2-Dichloroethene. \\
\hline $78-93-3$ & 2-Butanone \\
\hline $74-97-5$ & Bromochloromethane \\
\hline $67-66-3$ & Chloroform \\
\hline $71-55-6$ & $1,1,1$-Trichloroethane \\
\hline $110-82-7$ & Cyclohexane \\
\hline $56-23-5$ & Carbon tetrachloride \\
\hline $71-43-2$ & Benzene \\
\hline $107-06-2$ & 1,2-Dichloroethane \\
\hline
\end{tabular}

\begin{tabular}{|cc|c|} 
CONCENTRATION UNITS: & \\
(ug/ $/ \mathrm{L}$ or $\mathrm{ug} / \mathrm{kg}$ ) & $\mathrm{ug} / \mathrm{L}$ & $\mathrm{Q}$ \\
$=================$ & $\mathrm{Q}$ \\
0.50 & $\mathrm{U}$ \\
0.50 & $\mathrm{U}$ \\
0.50 & $\mathrm{U}$ \\
0.50 & $\mathrm{U}$ \\
0.50 & $\mathrm{U}$ \\
0.50 & $\mathrm{U}$ \\
0.50 & $\mathrm{U}$ \\
0.50 & $\mathrm{U}$ \\
5.0 & $\mathrm{U}$ \\
0.50 & $\mathrm{U}$ \\
0.50 & $\mathrm{U}$ \\
0.50 & $\mathrm{U}$ \\
0.50 & $\mathrm{U}$ \\
0.50 & $\mathrm{U}$ \\
0.50 & $\mathrm{U}$ \\
0.50 & $\mathrm{U}$ \\
5.0 & $\mathrm{U}$ \\
0.50 & $\mathrm{U}$ \\
0.50 & $\mathrm{U}$ \\
0.50 & $\mathrm{U}$ \\
0.50 & $\mathrm{U}$ \\
2.5 & \\
0.50 & $\mathrm{U}$ \\
0.50 & $\mathrm{U}$ \\
&
\end{tabular}

Report 1,4-Dioxane for Low-Medium VOA analysis only 
Lab Name: TestAmerica BURLINGTON

Contract: $8 \mathrm{E}-00302$

Lab Code: STLV Case No.: CENTRALI Mod. Ref No.:

SDG No.: 126456

Matrix: (SOIL/SED/WATER) Water

Sample wt/vol: 25.0

$(\mathrm{g} / \mathrm{mL}) \mathrm{mL}$

Level: (TRACE/LOW/MED) TRACE

\% Moisture: not dec.

GC Column: DB-624

Soil Extract Volume:

ID $: 0.53$

(mm)

(uL)

(mL)

Purge Volume: 25.0
Lab Sample ID: 759110

Lab File ID: 759110

Date Received: 07/10/2008

Date Analyzed: 07/14/2008

Dilution Factor: 1.0

Soil Aliquot Volume:

(uL)

\begin{tabular}{|c|c|c|c|}
\hline CAS NO. & COMPOUND & $\begin{array}{l}\text { CONCENTRATION UNITS: } \\
(\mathrm{ug} / \mathrm{L} \text { or } \mathrm{ug} / \mathrm{kg}) \mathrm{ug} / \mathrm{L}\end{array}$ & Q \\
\hline$==========$ & 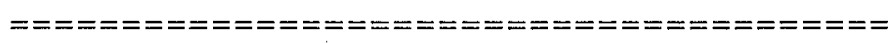 & 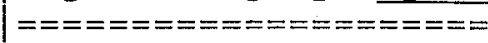 & $=======$ \\
\hline $79-01-6$ & Trichloroethene & 0.50 & $\mathrm{U}$ \\
\hline $108-87-2$ & Methylcyclohexane & 0.50 & $\mathrm{U}$ \\
\hline $78-87-5$ & 1,2-Dichloropropane & 0.50 & $\mathrm{U}$ \\
\hline $75-27-4$ & Bromodichloromethane & 0.50 & $\mathrm{U}$ \\
\hline $10061-01-5$ & cis-1,3-Dichloropropene & 0.50 & $\mathrm{U}$ \\
\hline $108-10-1$ & 4-Methyl-2-pentanone & 5.0 & $\mathrm{U}$ \\
\hline $108-88-3$ & Toluene & 0.50 & $\mathrm{U}$ \\
\hline $10061-02-6$ & trans-1,3-Dichloropropene & 0.50 & $\mathrm{U}$ \\
\hline $79-00-5$ & 1,1,2-Trichloroethane & 0.50 & $\mathrm{U}$ \\
\hline $127-18-4$ & Tetrachloroethene & 0.50 & $\mathrm{U}$ \\
\hline $591-78-6$ & 2-Hexanone & 5.0 & $\mathrm{U}$ \\
\hline $124-48-1$ & Dibromochloromethane & 0.50 & $\mathrm{U}$ \\
\hline $106-93-4$ & 1,2-Dibromoethane & 0.50 & $\mathrm{U}$ \\
\hline $108-90-7$ & Chlorobenzene & 0.50 & $\mathrm{U}$ \\
\hline $100-41-4$ & Ethylbenzene & 0.50 & $\mathrm{U}$ \\
\hline $95-47-6$ & o-Xylene & 0.50 & $\mathrm{U}$ \\
\hline $179601-23-1$ & $\mathrm{~m}, \mathrm{p}-\mathrm{Xyl}$ lene & 0.50 & $\mathrm{U}$ \\
\hline $100-42-5$ & styrene & 0.50 & $\mathrm{U}$ \\
\hline $75-25-2$ & Bromoform & 0.50 & $\mathrm{U}$ \\
\hline $98-82-8$ & Isopropylbenzene & 0.50 & $\mathrm{U}$ \\
\hline $79-34-5$ & $1,1,2,2$-Tetrachloroethane & 0.50 & $\mathrm{U}$ \\
\hline $541-73-1$ & 1,3-Dichlorobenzene & 0.50 & $\mathrm{U}$ \\
\hline $106-46-7$ & I,4-Dichlorobenzene & 0.50 & $\mathrm{U}$ \\
\hline $95-50-1$ & 1,2-Dichlorobenzene & 0.50 & $\mathrm{U}$ \\
\hline $96-12-8$ & 1,2-Dibromo-3-chloropropane & 0.50 & $\mathrm{U}$ \\
\hline $120-82-1$ & 1,2,4-Trichlorobenzene & 0.50 & $\mathrm{U}$ \\
\hline $87-61-6$ & 1,2,3-Trichlorobenzene & 0.50 & $\mathrm{U}$ \\
\hline
\end{tabular}


Lab Name: TestAmerica BURLINGTON

Contract: $8 \mathrm{E}-00302$

Lab Code: STLV Case No.: CENTRALI Mod. Ref No.:

SDG No.: 126456

Matrix: (SOIL/SED/WATER) Water

Sample wt/vol: $25.0 \quad(\mathrm{~g} / \mathrm{mL}) \mathrm{mL}$

Level: (TRACE/LOW/MED) TRACE

$\%$ Moisture: not dec.

GC Column: DB-624

Soil Extract Volume:

ID $: 0.53$

(mm)

(UL)

(mL)
Lab Sample ID: 759112

Lab File ID: 759112

Date Received: 07/10/2008

Date Analyzed: 07/14/2008

Dilution Factor: 1.0

Soil Aliquot Volume:

(uL)

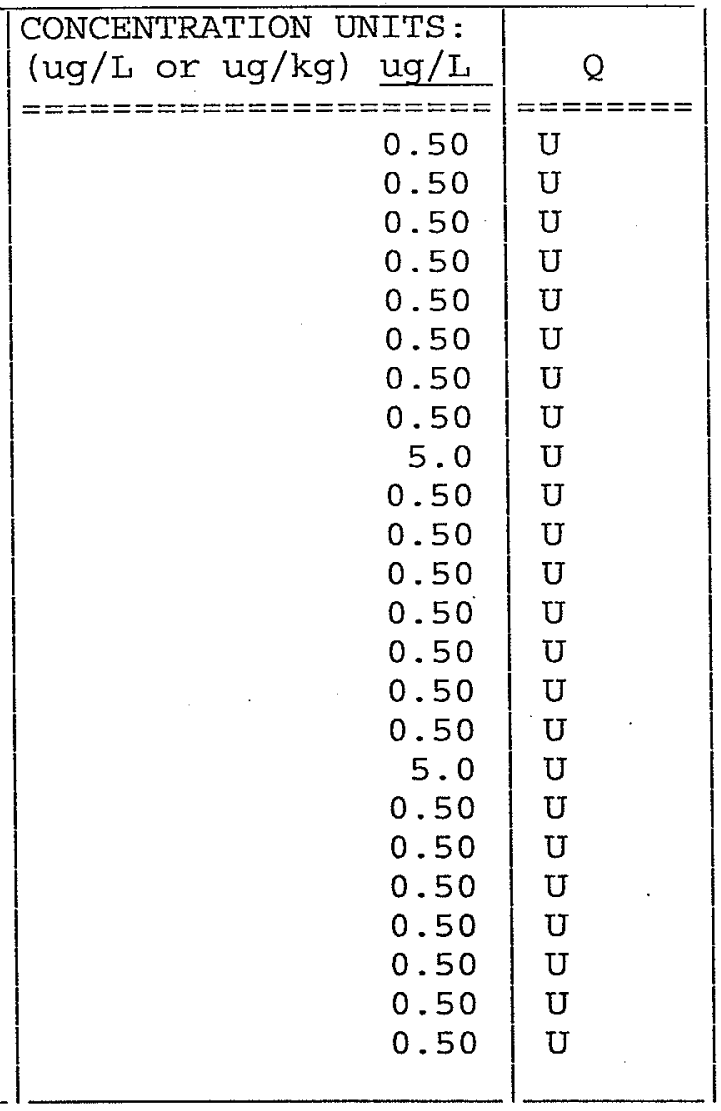

Report 1,4-Dioxane for Low-Medium VOA analysis only 
$1 B$ - FORM I VOA-2

VOLATILE ORGANICS ANALYSIS DATA SHEET
EPA SAMPLE NO.

CNQCTBW26657
Lab Name: TestAmerica BURLINGTON

Lab Code: STLV Case No.: CENTRAII Mod. Ref No.:
Contract: $8 E-00302$
Matrix: (SOIL/SED/WATER) Water

Sample wt/vol: 25.0

$(\mathrm{g} / \mathrm{mL}) \mathrm{mL}$

Level: (TRACE/LOW/MED) TRACE

\% Moisture: not dec.

GC Column: DB-624

ID : 0.53

Soil Extract Volume:

Purge Volume: 25.0
Lab Sample ID: 759112

Lab File ID: 759112

Date Received: 07/10/2008

Date Analyzed: 07/14/2008

Dilution Factor: 1.0

Soil Aliquot Volume:

(UIs)

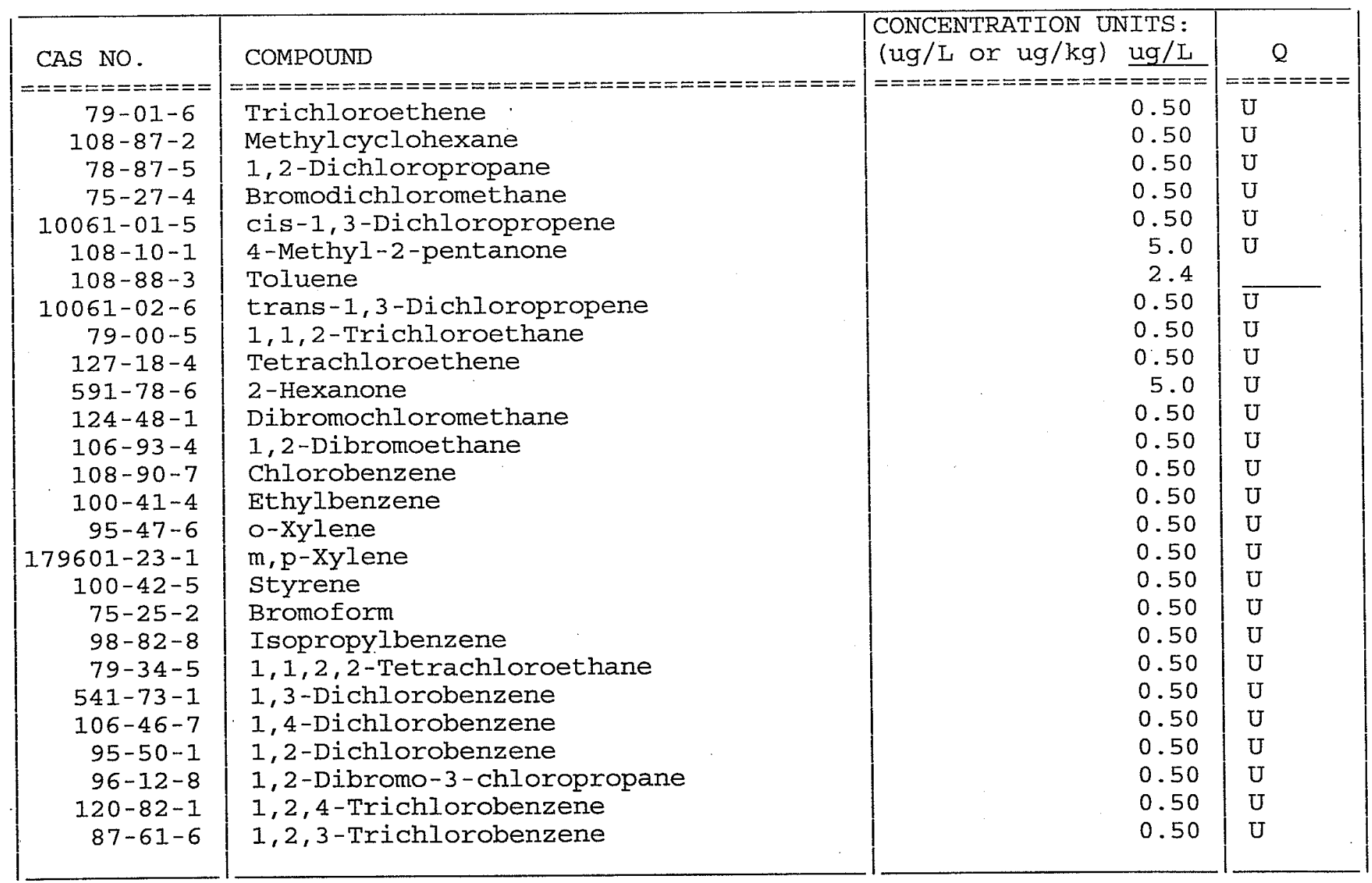


Lab Name: TestAmerica BURLINGTON

Contract: $8 \mathrm{E}-00302$

Lab Code: STLV Case No.: CENTRALI Mod. Ref No.:

SDG No.: 126456

Matrix: (SOIL/SED/WATER) Water

Lab Sample ID: 759111

Sample wt/vol: $25.0 \quad(\mathrm{~g} / \mathrm{mL}) \mathrm{mL}$

Lab File ID: 759111D

Level: (TRACE/LOW/MED) TRACE

Date Received: 07/10/2008

$\div$ Moisture: not dec.

Date Analyzed: 07/14/2008

GC Column: DB-624

ID $: 0.53$

(mm)

Dilution Factor: 6.3

Soil Extract Volume:

(uL) Soil Aliquot Volume:

Purge Volume: 25.0

(mL)

\begin{tabular}{|c|c|c|c|}
\hline CAS NO. & COMPOUND & $\begin{array}{l}\text { CONCENTRATION UNITS: } \\
(\mathrm{ug} / \mathrm{L} \text { or } \mathrm{ug} / \mathrm{kg}) \mathrm{ug} / \mathrm{L}\end{array}$ & Q \\
\hline$===========$ & 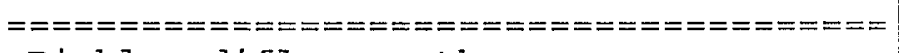 & $==-===========0====0$ & $=======$ \\
\hline $75-71-8$ & Dichlorodifluoromethane & 3.2 & $\mathrm{U}$ \\
\hline $74-87-3$ & Chloromethane & 3.2 & $\mathrm{U}$ \\
\hline $75-01-4$ & Vinyl chloride & 3.2 & $\mathrm{U}$ \\
\hline $74-83-9$ & Bromomethane & 3.2 & $\mathrm{U}$ \\
\hline $75-00-3$ & Chloroethane & 3.2 & $\mathrm{U}$ \\
\hline $75-69-4$ & Trichlorofluoromethane & 3.2 & $\mathrm{U}$ \\
\hline $75-35-4$ & I,1-Dichloroethene & 3.2 & $\mathrm{U}$ \\
\hline $76-13-1$ & $1,1,2$-Trichloro-1,2,2-trifluoroethane & 3.2 & $\mathrm{U}$ \\
\hline $67-64-1$ & Acetone & 1500 & $\mathrm{E}$ \\
\hline $75-15-0$ & Carbon disulfide & 3.2 & $\mathrm{U}$ \\
\hline $79-20-9$ & Methyl acetate & 3.2 & $\mathrm{U}$ \\
\hline $75-09-2$ & Methylene chloride & 17 & \\
\hline $156-60-5$ & trans-1,2-Dichloroethene & 3.2 & $\overline{\mathrm{U}}$ \\
\hline $1634-04-4$ & Methyl tert-butyl ether & 3.2 & $\mathrm{U}$ \\
\hline $75-34-3$ & 1,1-Dichloroethane & 3.2 & $\mathrm{U}$ \\
\hline $156-59-2$ & Cis-1,2-Dichloroethene & 3.2 & $\mathrm{U}$ \\
\hline $78-93-3$ & 2-Butanone & 1300 & $\mathrm{E}$ \\
\hline $74-97-5$ & Bromochloromethane & 3.2 & $\mathrm{U}$ \\
\hline $67-66-3$ & Chloroform & 92 & \\
\hline $71-55-6$ & $1,1,1$-Trichloroethane & 3.2 & $\overline{\mathrm{U}}$ \\
\hline $110-82-7$ & Cyclohexane & 3.2 & $\mathrm{U}$ \\
\hline $56-23-5$ & Carbon tetrachloride & 33 & \\
\hline $71-43-2$ & Benzene & 3.2 & $\overline{\mathrm{U}}$ \\
\hline $107-06-2$ & 1,2-Dichloroethane & 3.2 & $\mathrm{U}$ \\
\hline
\end{tabular}

Report 1,4-Dioxane for Lów-Medium VOA analysis only 
Lab Name: TestAmerica BURLINGTON

Contract: $8 \mathrm{E}-00302$

Lab Code: STLV Case No.: CENTRAII Mod. Ref No.:

SDG No. : 126456

Matrix: (SOIL/SED/WATER) Water

Sample wt/vol: 25.0

$(\mathrm{g} / \mathrm{mL}) \mathrm{mL}$

Level: (TRACE/LOW/MED) TRACE

$\%$ Moisture: not dec.

GC Column: DB-624

ID : 0.53

(mm)

(UL)

Soil Aliquot Volume:

$(\mathrm{mL})$

Lab Sample ID: 759111

Lab File ID: 759111D

Date Received: 07/10/2008

Date Analyzed: 07/14/2008

Dilution Factor: 6.3

Soil Extract Volume:

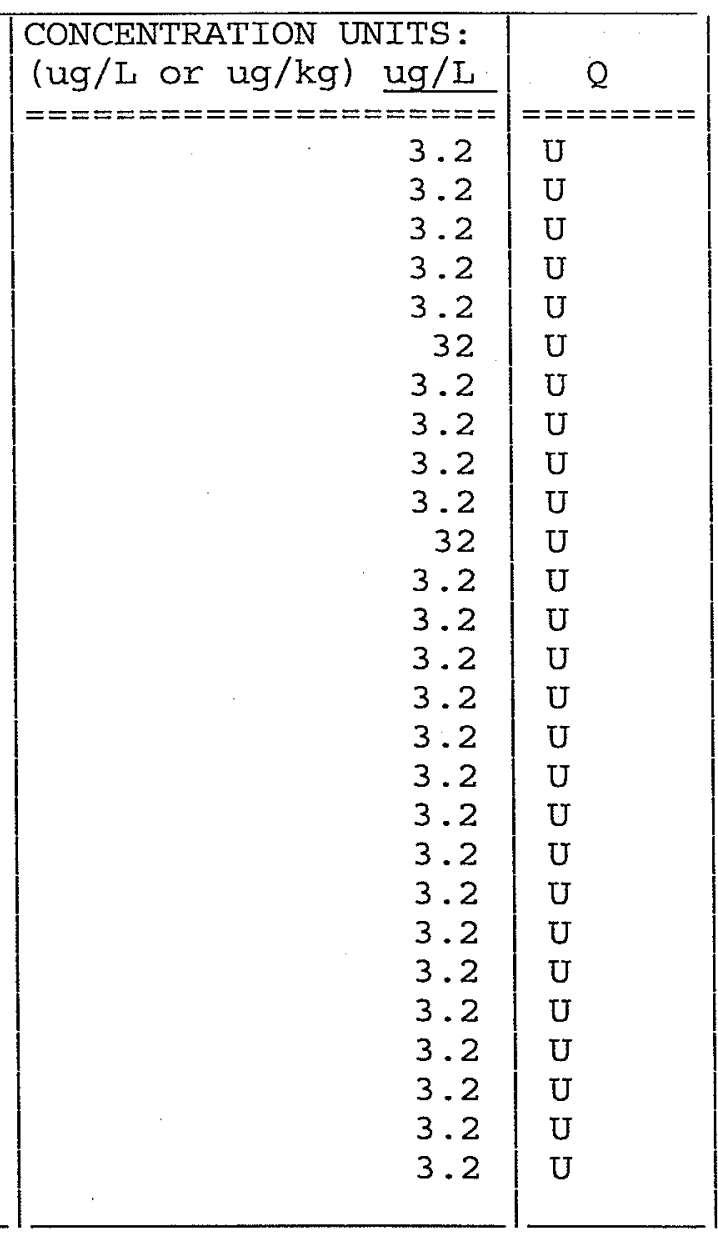


Lab Name: TestAmerica BURLINGTON

Contract: $8 E-00302$

Lab Code: STLV Case No.: CENTRALI Mod. Ref No.:

SDG No.: 126456

Matrix: (SOIL/SED/WATER) Water

Sample wt/vol: 25.0

$(\mathrm{g} / \mathrm{mL}) \mathrm{mL}$

Level: (TRACE/LOW/MED) TRACE

\% Moisture: not dec.

GC Column: DB-624

Soil Extract Volume:

ID: 0.53

Lab Sample ID: 759111D1

Purge Volume: 25.0 (mm)

(UL)

Lab File ID: 75911ID2

Date Received: 07/10/2008

Date Analyzed: 07/14/2008

Dilution Factor: 110.0

Soil Aliquot Volume:

(uL)

(mL)

\begin{tabular}{|c|c|}
\hline CAS NO. & COMPOUND \\
\hline$==========$ & 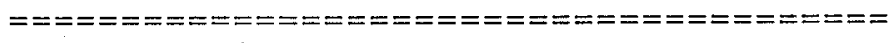 \\
\hline $75-71-8$ & Dichlorodifluoromethane \\
\hline $74-87-3$ & Chloromethane \\
\hline $75-01-4$ & Vinyl chloride \\
\hline $74-83-9$ & Bromomethane \\
\hline $75-00-3$ & Chloroethane \\
\hline $75-69-4$ & Trichlorofluoromethane \\
\hline $75-35-4$ & 1,1-Dichloroethene \\
\hline $76-13-1$ & 1,1,2-Trichloro-1,2,2-trifluoroethane \\
\hline $67-64-1$ & Acetone \\
\hline $75-15-0$ & Carbon disulfide \\
\hline $79-20-9$ & Methyl acetate \\
\hline $75-09-2$ & Methylene chloride \\
\hline $156-60-5$ & trans-1,2-Dichloroethene \\
\hline $1634-04-4$ & Methyl tert-butyl ether \\
\hline $75-34-3$ & 1,1-Dichloroethane \\
\hline $156-59-2$ & cis-1,2-Dichloroethene \\
\hline $78-93-3$ & 2-Butanone \\
\hline $74-97-5$ & Bromochloromethane \\
\hline $67-66-3$ & Chloroform \\
\hline $71-55-6$ & $1,1,1$-Trichloroethane \\
\hline $110-82-7$ & Cyclohexane \\
\hline $56-23-5$ & Carbon tetrachloride \\
\hline $71-43-2$ & Benzene \\
\hline $107-06-2$ & 1,2-Dichloroethane \\
\hline
\end{tabular}

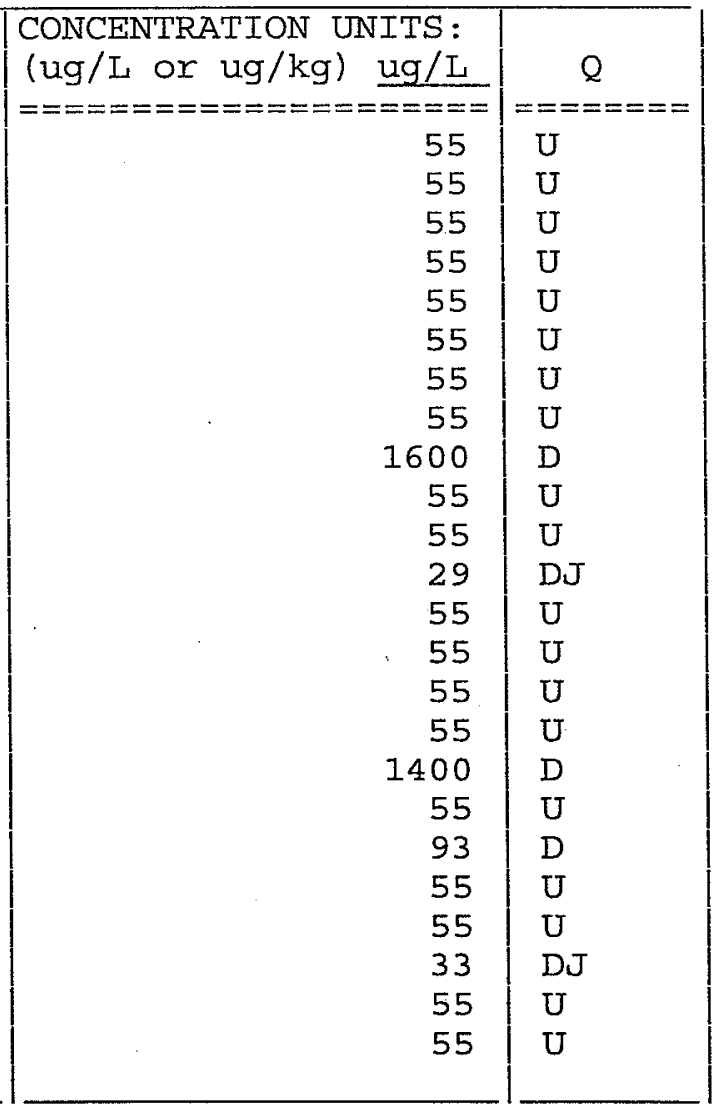

Report 1,4-Dioxane for Low-Medium VOA analysis only 
Lab Name: TestAmerica BURLINGTON

Contract: $8 \mathrm{E}-00302$

Lab Code: STLV Case No.: CENTRALI Mod. Ref No.:

SDG NO.: 126456

Matrix: (SOIL/SED/WATER) Water

Sample wt/vol: $25.0 \quad(\mathrm{~g} / \mathrm{mL}) \mathrm{mL}$

Level: (TRACE/LOW/MED) TRACE

\% Moisture: not dec.

GC Column: DB-624

Soil Extract Volume:

ID $: 0.53 \quad(\mathrm{~mm})$

(uL)

$(\mathrm{mL})$

Purge Volume: 25.0
Lab Sample ID: 759111D1

Lab File ID: 759111D2

Date Received: 07/10/2008

Date Analyzed: 07/14/2008

Dilution Factor: 110.0

Soil Aliquot Volume:

(uL)

\begin{tabular}{|c|c|c|c|}
\hline CAS NO. & COMPOUND & $\begin{array}{l}\text { CONCENTRATION UNITS: } \\
(\mathrm{ug} / \mathrm{L} \text { or } \mathrm{ug} / \mathrm{kg}) \mathrm{ug} / \mathrm{I}\end{array}$ & $Q$ \\
\hline$===========$ & 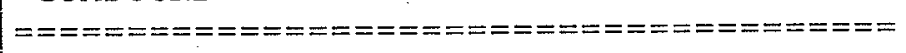 & 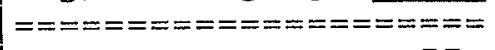 & $=======$ \\
\hline $79-01-6$ & Trichloroethene & 55 & $\mathrm{U}$ \\
\hline $108-87-2$ & Methylcyclohexane & 55 & U \\
\hline $78-87-5$ & 1,2-Dichloropropane & 55 & $\mathrm{U}$ \\
\hline $75-27-4$ & Bromodichloromethane & 55 & $\mathrm{U}$ \\
\hline $10061-01-5$ & cis-1,3-Dichloropropene & 55 & $\mathrm{U}$ \\
\hline $108-10-1$ & 4-Methyl-2-pentanone & 550 & $\mathrm{U}$ \\
\hline $108-88-3$ & Toluene & 55 & $\mathrm{U}$ \\
\hline $10061-02-6$ & trans-1,3-Dichloropropene & 55 & U \\
\hline $79-00-5$ & $1,1,2$-Trichloroethane & 55 & $\mathrm{U}$ \\
\hline $127-18-4$ & Tetrachloroethene & 55 & $\mathrm{U}$ \\
\hline $591-78-6$ & 2-Hexanone & 550 & $\mathrm{U}$ \\
\hline $124-48-1$ & Dibromochloromethane & 55 & $\mathrm{U}$ \\
\hline $106-93-4$ & 1,2-Dibromoethane & 55 & U \\
\hline $108-90-7$ & Chlorobenzene & 55 & $U$ \\
\hline $100-41-4$ & Ethylbenzene & 55 & $\mathrm{U}$ \\
\hline $95-47-6$ & o-Xylene & 55 & U \\
\hline $179601-23-1$ & $\mathrm{~m}, \mathrm{p}$-Xylene & 55 & $\mathrm{U}$ \\
\hline $100-42-5$ & Styrene & 55 & $\mathrm{U}$ \\
\hline $75-25-2$ & Bromoform & 55 & $\mathrm{U}$ \\
\hline $98-82-8$ & Isopropylbenzene & 55 & $\mathrm{U}$ \\
\hline $79-34-5$ & $1,1,2,2$-Tetrachloroethane & 55 & $\mathrm{U}$ \\
\hline $541-73-1$ & 1,3-Dichlorobenzene & 55 & $\mathrm{U}$ \\
\hline $106-46-7$ & 1,4-Dichlorobenzene & 55 & U \\
\hline $95-50-1$ & 1,2-Dichlorobenzene & 55 & U \\
\hline $96-12-8$ & 1,2-Dibromo-3-chloropropane & 55 & $\mathrm{U}$ \\
\hline $120-82-1$ & $1,2,4$-Trichlorobenzene & 55 & $\mathrm{U}$ \\
\hline $87-61-6$ & $1,2,3$-Trichlorobenzene & 55 & $\mathrm{U}$ \\
\hline
\end{tabular}




\section{TestAmerica}

THE LEADER IN ENVIRONMENTAL TESTING

August 27, 2008

TestAmerica Laboratories, Inc.

Mr. Clyde Dennis

Argonne National Laboratory

Chief Financial Officer

9700 S. Cass Avenue, Bldg. 201

Argonne, IL 60439

Re: Laboratory Project No. 21005

Case: CENTRALIA; SDG: 126998

Dear Mr. Dennis:

Enclosed are analytical results for samples that were received by TestAmerica Burlington on August $8^{\text {th }}, 2008$. Laboratory identification numbers were assigned, and designated as follows:

$\begin{array}{llll}\text { Lab ID } & \begin{array}{l}\text { Client } \\ \text { Sample ID }\end{array} & \begin{array}{l}\text { Sample } \\ \text { Date }\end{array} & \begin{array}{l}\text { Sample } \\ \text { Matrix }\end{array} \\ & \text { Received: 08/08/08 ETR No: } & & \\ & & & \\ 762961 & \text { CNSB07R-W-26661 } & 08 / 06 / 08 & \text { WATER } \\ 762962 & \text { CNPMP6-W-26668 } & 08 / 06 / 08 & \text { WATER } \\ 762963 & \text { CNQCTB-W-26672 } & 08 / 06 / 08 & \text { WATER } \\ 762964 & \text { VHBLK01 } & 08 / 08 / 08 & \text { WATER }\end{array}$

Documentation of the condition of the samples at the time of their receipt and any exception to the laboratory's Sample Acceptance Policy is documented in the Sample Handling section of this submittal. The samples, as received, were not acid preserved. On that basis the laboratory did provide for the analytical work to be performed within seven days of sample collection.

In order to accommodate field length limitations in processing the data summary forms, the laboratory did, in certain instances, abbreviate the sample identifier. The electronically formatted data provides for the full sample identifier.

\section{SOM01.2 Volatile Organics (Trace Level Water)}

A storage blank was prepared for volatile organics analysis, and stored in association with the storage of the sample. That storage blank, identified as VHBLK01, was carried through the holding period with the samples, and analyzed.

Sample CNPMP6-W-26668 was analyzed at a dilution, based on the results of preliminary screening. An additional, more concentrated analysis was performed on the sample in order to 


\section{TestAmerica}

THE LEADER IN ENVIRONMENTAL TESTING

provide a lower reporting limit for those target analytes that were not identified as constituents in the primary analysis. Both sets of results for the analysis of sample CNPMP6-W-26668 are included in this submittal. Each of the analyses associated with the sample set exhibited an acceptable internal standard performance. There was an acceptable recovery of each deuterated monitoring compound (DMC) in the analysis of the method blank and the analysis of the storage blank. The analysis of the samples in this sample set did meet the technical acceptance criteria specific to DMC recoveries, although not all DMC recoveries were within the control range in each analysis. The technical acceptance criteria does provide for the recovery of up to three DMCs to fall outside of the control range in the analysis of field samples. With the exception of that performed on sample CNQCTB-W-26672, the recovery of 2-hexanone- $d_{5}$ was elevated in the analysis of each of the field samples, as was the recovery of 2-butanone- $d_{5}$ in the analysis of sample CNSB07R-W-26661 and the more dilute analysis of sample CNPMP6-W-26668. Matrix spike and matrix spike duplicate analyses were not performed on the samples in this sample set. Trace concentrations of 1,2,3-trichlorobenzene were identified in the analysis of each method blank associated with the analytical work. The derived concentration of that compound in each analysis was below the established reporting limit, and each analysis did meet the technical acceptance criteria for a compliant method blank acquisition. A trace concentration of acetone was identified in the analysis of the storage blank associated with the sample set. The derived concentration of acetone in that analysis was below the established reporting limit, and the analysis did meet the technical acceptance criteria for a compliant storage blank acquisition. Present in the storage blank and method blank analyses was a non-target constituent that represented a compound that is related to either the DMC formulation or to column bleed. The fact that the presence of this compound is not within the laboratory's control is at issue. The derived results for that compound have been qualified with an " $X$ " qualifier to reflect the source of the contamination.

The responses for each target analyte met the relative standard deviation criterion in the initial calibration. The response for each target analyte met the percent difference criterion in each continuing calibration check acquisition. The response for each target analyte met the 50.0 percent difference criterion in each closing calibration check acquisition.

The primary quantitation mass for methylcyclohexane that is specified in the Statement of Work is mass 83 . The laboratory did identify a contribution to mass 83 from 1,2-dichloropropane- $d_{6}$, one of the deuterated monitoring compounds (DMCs). The laboratory did change the primary quantitation mass assignment to mass 55 for the quantification of methylcyclohexane.

Manual integration was employed in deriving certain of the analytical results. The values that have been derived from manual integration are qualified on the quantitation reports. Extracted ion current profiles for each manual integration are included in the data package, and further documented in the Sample Preparation section of this submittal.

Any reference within this report to Severn Trent Laboratories, Inc. or STL, should be understood to refer to TestAmerica Laboratories, Inc. (formerly known as Severn Trent Laboratories, Inc.) The analytical results associated with the samples presented in this test report were generated under a quality system that adheres to requirements specified in the NELAC standard. Release of the data in this test report and any associated electronic deliverables is authorized by the Laboratory Director's designee as verified by the following signature. 


\section{TestAmerica}

THE LEADER IN ENVIRONMENTAL TESTING

If there are any questions regarding this submittal, please contact me at 802 660-1990.

Sincerely,

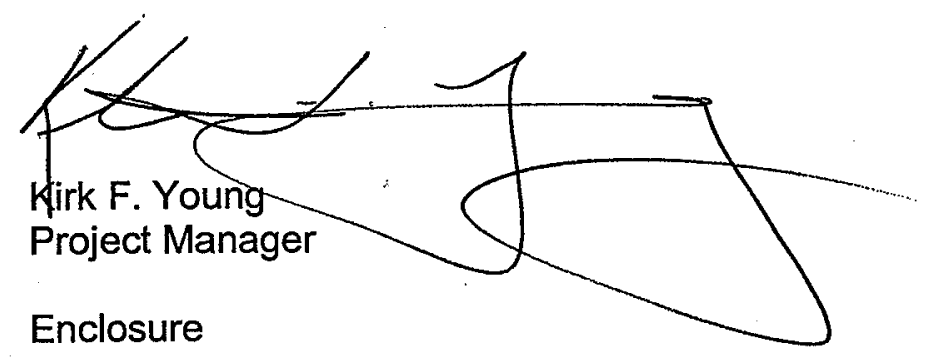




\section{TestAmerica Burlington Data Qualifier Definitions}

\section{Organic}

$\mathrm{U}$ : Compound analyzed but not detected at a concentration above the reporting limit.

$\mathrm{J}$ : Estimated value.

$\mathrm{N}$ : Indicates presumptive evidence of a compound. This flag is used only for tentatively identified compounds (TICs) where the identification of a compound is based on a mass spectral library search.

P: $\quad$ SW-846: The relative percent difference for detected concentrations between two GC columns is greater than $40 \%$. Unless otherwise specified the higher of the two values is reported on the Form $\mathrm{I}$.

CLP SOW: Greater than $25 \%$ difference for detected concentrations between two GC columns. Unless otherwise specified the lower of the two values is reported on the Form I.

C: Pesticide result whose identification has been confirmed by GC/MS.

B: $\quad$ Analyte is found in the sample and the associated method blank. The flag is used for tentatively identified compounds as well as positively identified compounds.

E: Compounds whose concentrations exceed the upper limit of the calibration range of the instrument for that specific analysis.

D: Concentrations identified from analysis of the sample at a secondary dilution.

A: Tentatively identified compound is a suspected aldol condensation product.

$X, Y, Z$ : Laboratory defined flags that may be used alone or combined, as needed. If used, the description of the flag is defined in the project narrative.

\section{Inorganic/Metals}

E: Reported value is estimated due to the presence of interference.

$\mathrm{N}$ : Matrix spike sample recovery is not within control limits.

* Duplicate sample analysis is not within control limits.

B: The result reported is less than the reporting limit but greater than the instrument detection limit.

U: $\quad$ Analyte was analyzed for but not detected above the reporting limit.

Method Codes:

$P \quad$ ICP-AES

MS ICP-MS

CV Cold Vapor AA

AS Semi-Automated Spectrophotometric 


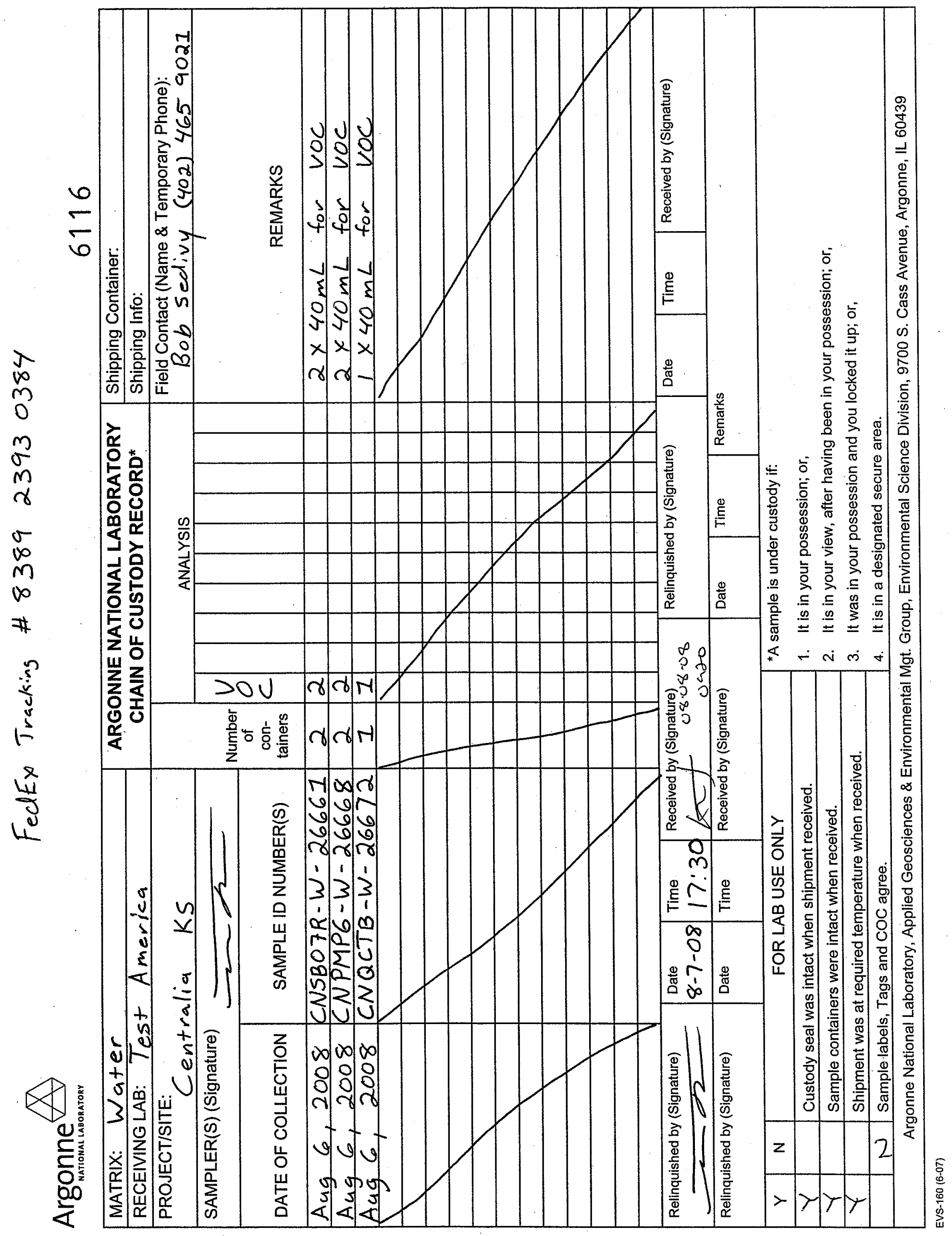




\section{TestAmerica \\ 15. \\ THE LEADER IN ENVIRONMENTAL TESTING}

\section{Sample Data Summary - SOM01.2 Volatiles - Trace}


IA - FORM I VOA-1

VOLATILE ORGANICS ANALYSIS DATA SHEET
EPA SAMPLE NO.

PMP6W26668

Lab Name: TestAmerica BURLINGTON

Contract: $8 \mathrm{E}-00302$

Lab Code: STLV Case No.: CENTRALIA Mod. Ref No.:

SDG No.: 126998

Matrix: (SOIL/SED/WATER) Water

Sample wt/vol: 25.0

$(\mathrm{g} / \mathrm{mL}) \mathrm{mL}$

Level: (TRACE/LOW/MED) TRACE

\% Moisture: not dec.

GC Column: DB-624

Soil Extract Volume:

ID $: 0.53$

(mm)

(UL)

(mL)

Purge Volume: 25.0
Lab Sample ID: 762962

Lab File ID: 762962

Date Received: 08/08/2008

Date Analyzed: 08/11/2008

Dilution Factor: 1.0

Soil Aliquot Volume:

(uL)

\begin{tabular}{|c|c|c|c|}
\hline CAS NO. & COMPOUND & $\begin{array}{l}\text { CONCENTRATION UNITS: } \\
(\mathrm{ug} / \mathrm{L} \text { or } \mathrm{ug} / \mathrm{kg}) \mathrm{ug} / \mathrm{L}\end{array}$ & Q \\
\hline 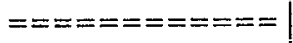 & 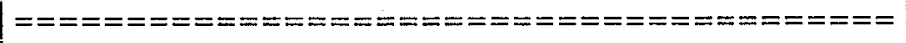 & 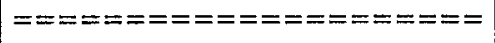 & $===== \pm==$ \\
\hline $75-71-8$ & Dichlorodifluoromethane & 0.50 & $\mathrm{U}$ \\
\hline $74-87-3$ & Chloromethane & 0.50 & $\mathrm{U}$ \\
\hline $75-01-4$ & Vinyl chloride & 0.50 & $\mathrm{U}$ \\
\hline $74-83-9$ & Bromomethane & 0.50 & $\mathrm{U}$ \\
\hline & Chloroethane & 0.50 & $\mathrm{U}$ \\
\hline $75-69-4$ & Trichlorofluoromethane & 0.50 & $\mathrm{U}$ \\
\hline $75-35-4$ & 1,1-Dichloroethene & 0.50 & $\mathrm{U}$ \\
\hline $76-13-1$ & $1,1,2$-Trichloro-1,2,2-trifluoroethane & 0.50 & $\mathrm{U}$ \\
\hline $67-64-1$ & Acetone & 5.0 & $\mathrm{U}$ \\
\hline $75-15-0$ & Carbon disulfide & 0.50 & $\mathrm{U}$ \\
\hline $79-20-9$ & Methyl acetate & 0.50 & $\mathrm{U}$ \\
\hline $75-09-2$ & Methylene chloride & 0.29 & $\mathrm{~J}$ \\
\hline $156-60-5$ & trans-1,2-Dichloroethene & 0.50 & $\mathrm{U}$ \\
\hline $1634-04-4$ & Methyl tert-butyl ether & 0.50 & $\mathrm{U}$ \\
\hline $75-34-3$ & 1,1-Dichloroethane & 0.50 & $\mathrm{U}$ \\
\hline $156-59-2$ & cis-1,2-Dichloroethene & 0.50 & $\mathrm{U}$ \\
\hline $78-93-3$ & 2-Butanone & 5.0 & $\mathrm{U}$ \\
\hline $74-97-5$ & Bromochloromethane & 0.50 & $\mathrm{U}$ \\
\hline $67-66-3$ & Chloroform & 8.5 & \\
\hline $71-55-6$ & 1,1,1-Trichloroethane & 0.50 & $\mathrm{U}$ \\
\hline $110-82-7$ & Cyclohexane & 0.50 & $\mathrm{U}$ \\
\hline $56-23-5$ & Carbon tetrachloride & 95 & $\mathrm{E}$ \\
\hline $71-43-2$ & Benzene & 0.50 & $\mathrm{U}$ \\
\hline $107-06-2$ & 1,2-Dichloroethane & 0.50 & $\mathrm{U}$ \\
\hline
\end{tabular}

Report 1,4-Dioxane for Low-Medium VOA analysis only 
IB - FORM I VOA-2

VOLATILE ORGANICS ANALYSIS DATA SHEET
EPA SAMPLE NO.

PMP6W26668

Lab Name: TestAmerica BURLINGTON

Contract: $8 \mathrm{E}-00302$

Lab Code: STLV Case No.: CENTRALIA Mod. Ref No.:

SDG No.: 126998

Matrix: (SOIL/SED/WATER) Water

Sample wt/vol: $25.0 \quad(\mathrm{~g} / \mathrm{mL}) \mathrm{mL}$

Level: (TRACE/LOW/MED) TRACE

\% Moisture: not dec.

GC Column: DB-624

Soil Extract Volume:

ID $: 0.53$

Purge Volume: 25.0
Lab Sample ID: 762962

Lab File ID: 762962

Date Received: 08/08/2008

Date Analyzed: 08/11/2008

Dilution Factor: 1.0

Soil Aliquot Volume:

(mL)

\begin{tabular}{|c|c|c|c|}
\hline CAS NO. & COMPOUND & $\begin{array}{l}\text { CONCENTRATION UNITS: } \\
(\mathrm{ug} / \mathrm{L} \text { or } \mathrm{ug} / \mathrm{kg}) \text { ug } / \mathrm{L}\end{array}$ & Q \\
\hline 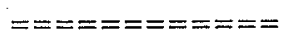 & 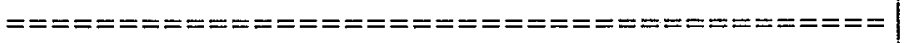 & 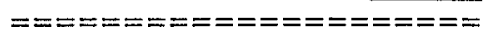 & $=======$ \\
\hline $79-01-6$ & Trichloroethene & 0.50 & $\mathrm{U}$ \\
\hline $108-87-2$ & Methylcyclohexane & 0.50 & $\mathrm{U}$ \\
\hline $78-87-5$ & 1,2-Dichloropropane & 0.50 & $\mathrm{U}$ \\
\hline $75-27-4$ & Bromodichloromethane & 0.50 & $\mathrm{U}$ \\
\hline $10061-01-5$ & Cis-1,3-Dichloropropene & 0.50 & $\mathrm{U}$ \\
\hline $108-10-1$ & 4-Methyl-2-pentanone & 5.0 & $\mathrm{U}$ \\
\hline $108-88-3$ & Toluene & 0.39 & $\mathrm{~J}$ \\
\hline $10061-02-6$ & trans-1,3-Dichloropropene & 0.50 & $\mathrm{U}$ \\
\hline $79-00-5$ & 1,1,2-Trichloroethane & 0.50 & U \\
\hline $127-18-4$ & Tetrachloroethene & 0.50 & $\mathrm{U}$ \\
\hline $591-78-6$ & 2-Hexanone & 5.0 & $\mathrm{U}$ \\
\hline $124-48-1$ & Dibromochloromethane & 0.50 & $\mathrm{U}$ \\
\hline $106-93-4$ & 1,2-Dibromoethane & 0.50 & $\mathrm{U}$ \\
\hline $108-90-7$ & Chlorobenzene & 0.50 & $\mathrm{U}$ \\
\hline $100-41-4$ & Ethylbenzene & 0.50 & $\mathrm{U}$ \\
\hline $95-47-6$ & o-Xylene & 0.50 & $\mathrm{U}$ \\
\hline $179601-23-1$ & $\mathrm{~m}, \mathrm{p}$-xylene & 0.25 & $\mathrm{~J}$ \\
\hline $100-42-5$ & styrene & 0.50 & U \\
\hline $75-25-2$ & Bromoform & 0.50 & $\mathrm{U}$ \\
\hline $98-82-8$ & Isopropylbenzene & 0.50 & $\mathrm{U}$ \\
\hline $79-34-5$ & $1,1,2,2$-Tetrachloroethane & 0.50 & $\mathrm{U}$ \\
\hline $541-73-1$ & 1,3-Dichlorobenzene & 0.50 & $\mathrm{U}$ \\
\hline $106-46-7$ & 1,4-Dichlorobenzene & 0.50 & $\mathrm{U}$ \\
\hline $95-50-1$ & 1,2-Dichlorobenzene & 0.50 & $\mathrm{U}$ \\
\hline $96-12-8$ & 1,2-Dibromo-3-chloropropane & 0.50 & $\mathrm{U}$ \\
\hline $120-82-1$ & 1,2,4-Trichlorobenzene & 0.50 & $\mathrm{U}$ \\
\hline $87-61-6$ & 1,2,3-Trichlorobenzene & 0.50 & $\mathrm{U}$ \\
\hline
\end{tabular}


$1 A$ - FORM I VOA-I

VOLATILE ORGANICS ANALYSIS DATA SHEET
EPA SAMPLE NO.

PMP6W26668DL

Lab Name: TestAmerica BURLINGTON

Contract: $8 \mathrm{E}-00302$

Lab Code: STLV Case No.: CENTRALIA Mod. Ref No.:

SDG No.: 126998

Matrix: (SOIL/SED/WATER) Water

Sample wt/vol: 25.0

$(\mathrm{g} / \mathrm{mL}) \mathrm{mL}$

Level: (TRACE/LOW/MED) TRACE

$\%$ Moisture: not dec.

GC Column: DB-624

ID $: 0.53$

Soil Extract Volume:

Purge Volume: 25.0
Lab Sample ID: 762962D1

Lab File ID: 762962D

Date Received: 08/08/2008

Date Analyzed: 08/11/2008

Dilution Factor: 6.3

Soil Aliquot Volume:

(mL)

\begin{tabular}{|c|c|c|c|}
\hline CAS NO. & COMPOUND & $\begin{array}{l}\text { CONCENTRATION UNITS: } \\
(\mathrm{ug} / \mathrm{L} \text { or } \mathrm{ug} / \mathrm{kg}) \mathrm{ug} / \mathrm{L}\end{array}$ & Q \\
\hline$==========$ & 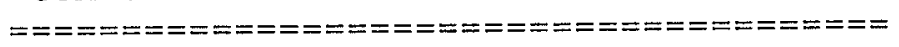 & 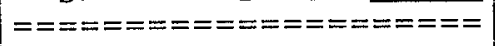 & $========$ \\
\hline $75-71-8$ & Dichlorodifluoromethane & 3.2 & $\mathrm{U}$ \\
\hline $74-87-3$ & Chloromethane & 3.2 & U \\
\hline $75-01-4$ & Vinyl chloride & 3.2 & $\mathrm{U}$ \\
\hline $74-83-9$ & Bromomethane & 3.2 & U \\
\hline & Chloroethane & 3.2 & $\mathrm{U}$ \\
\hline $75-69-4$ & Trichlorofluoromethane & 3.2 & U \\
\hline $75-35-4$ & 1,1-Dichloroethene & 3.2 & $\mathrm{U}$ \\
\hline $76-13-1$ & $1,1,2$-Trichloro-1,2,2-trifluoroethane & 3.2 & $\mathrm{U}$ \\
\hline $67-64-1$ & Acetone & 32 & $\mathrm{U}$ \\
\hline $75-15-0$ & Carbon disulfide & 3.2 & $\mathrm{U}$ \\
\hline $79-20-9$ & Methyl acetate & 3.2 & $\mathrm{U}$ \\
\hline $75-09-2$ & Methylene chloride & 3.2 & U \\
\hline $156-60-5$ & trans-1,2-Dichloroethene & 3.2 & $\mathrm{U}$ \\
\hline $1634-04-4$ & Methyl tert-butyl ether & 3.2 & $\mathrm{U}$ \\
\hline $75-34-3$ & 1,1-Dichloroethane & 3.2 & $\mathrm{U}$ \\
\hline $156-59-2$ & cis-1,2-Dichloroethene & 3.2 & $\mathrm{U}$ \\
\hline $78-93-3$ & 2-Butanone & 32 & $\mathrm{U}$ \\
\hline $74-97-5$ & Bromochloromethane & 3.2 & $\mathrm{U}$ \\
\hline $67-66-3$ & Chloroform & 8.6 & $\mathrm{D}$ \\
\hline $71-55-6$ & $1,1,1$-Trichloroethane & 3.2 & U \\
\hline $110-82-7$ & Cyclohexane & 3.2 & $\mathrm{U}$ \\
\hline $56-23-5$ & Carbon tetrachloride & 110 & D \\
\hline $71-43-2$ & Benzene & 3.2 & $\mathrm{U}$ \\
\hline $107-06-2$ & 1,2-Dichloroethane & 3.2 & $\mathrm{U}$ \\
\hline
\end{tabular}

Report 1,4-Dioxane for Low-Medium VOA analysis only 
$I B$ - FORM I VOA-2

VOLATILE ORGANICS ANALYSIS DATA SHEET
EPA SAMPLE NO.

PMP6W26668DL

Lab Name: TestAmerica BURLINGTON

Contract: $8 \mathrm{E}-00302$

Lab Code: STLV Cáse No.: CENTRALIA Mod. Ref No.:

SDG No.: 126998

Matrix: (SOIL/SED/WATER) Water

Sample wt/vol: $25.0 \quad(\mathrm{~g} / \mathrm{mL}) \mathrm{mL}$

Level : (TRACE/LOW/MED) TRACE

$\%$ Moisture: not dec.

GC Column: DB-624

ID $: 0.53$

Soil Extract Volume:

Purge Volume: 25.0
Lab Sample ID: 762962D1

Lab File ID: 762962D

Date Received: 08/08/2008

Date Analyzed: 08/11/2008

Dilution Factor: 6.3

Soil Aliquot Volume:

(mL)

\begin{tabular}{|c|c|c|c|}
\hline CAS NO. & COMPOUND & $\begin{array}{l}\text { CONCENTRATION UNITS: } \\
(\mathrm{ug} / \mathrm{L} \text { or } \mathrm{ug} / \mathrm{kg}) \mathrm{ug} / \mathrm{L}\end{array}$ & Q \\
\hline$======== \pm==$ & 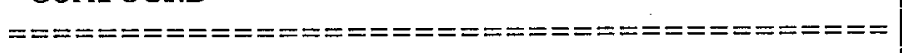 & $==================$ & $=======$ \\
\hline $79-01-6$ & Trichloroethene & 3.2 & $\mathrm{U}$ \\
\hline $108-87-2$ & Methylcyclohexane & 3.2 & $\mathrm{U}$ \\
\hline $78-87-5$ & 1,2-Dichloropropane & 3.2 & $\mathrm{U}$ \\
\hline $75-27-4$ & Bromodichloromethane & 3.2 & $\mathrm{U}$ \\
\hline $10061-01-5$ & cis-1,3-Dichloropropene & 3.2 & $\mathrm{U}$ \\
\hline $108-10-1$ & 4-Methyl-2-pentanone & 32 & U \\
\hline $108-88-3$ & Toluene & 3.2 & $\mathrm{U}$ \\
\hline $10061-02-6$ & trans-1,3-Dichloropropene & 3.2 & $\mathrm{U}$ \\
\hline $79-00-5$ & 1,1,2-Trichloroethane & 3.2 & $\mathrm{U}$ \\
\hline $127-18-4$ & Tetrachloroethene & 3.2 & U \\
\hline $591-78-6$ & 2-Hexanone & 32 & $\mathrm{U}$ \\
\hline $124-48-1$ & Dibromochloromethane & 3.2 & $\mathrm{U}$ \\
\hline $106-93-4$ & 1,2-Dibromoethane & 3.2 & $\mathrm{U}$ \\
\hline $108-90-7$ & Chlorobenzene & 3.2 & $\mathrm{U}$ \\
\hline $100-41-4$ & Ethylbenzene & 3.2 & $\mathrm{U}$ \\
\hline $95-47-6$ & o-Xylene & 3.2 & $\mathrm{U}$ \\
\hline $179601-23-1$ & $\mathrm{~m}, \mathrm{p}$-Xylene & 3.2 & $\mathrm{U}$ \\
\hline $100-42-5$ & styrene & 3.2 & $\mathrm{U}$ \\
\hline $75-25-2$ & Bromoform & 3.2 & $\mathrm{U}$ \\
\hline $98-82-8$ & Isopropylbenzene & 3.2 & $\mathrm{U}$ \\
\hline $79-34-5$ & $1,1,2,2$-Tetrachloroethane & 3.2 & $\mathrm{U}$ \\
\hline $541-73-1$ & 1,3-Dichlorobenzene & 3.2 & $\mathrm{U}$ \\
\hline $106-46-7$ & 1,4-Dichlorobenzene & 3.2 & $\mathrm{U}$ \\
\hline $95-50-1$ & 1,2-Dichlorobenzene & 3.2 & U \\
\hline $96-12-8$ & 1,2-Dibromo-3-chloropropane & 3.2 & $\mathrm{U}$ \\
\hline $120-82-1$ & $1,2,4$-Trichlorobenzene & 3.2 & U \\
\hline $87-61-6$ & 1,2,3-Trichlorobenzene & 3.2 & $\mathrm{U}$ \\
\hline
\end{tabular}


IA - FORM I VOA-I

VOLATILE ORGANICS ANALYSIS DATA SHEET
EPA SAMPLE NO.

QCTBW26672
Lab Name: TestAmerica BURLINGTON

Lab Code: STLV Case No.: CENTRALIA Mod. Ref No.:
Contract: $8 E-00302$
Matrix: (SOIL/SED/WATER) Water

Sample wt/vol: 25.0

$(\mathrm{g} / \mathrm{mL}) \mathrm{mL}$

Level: (TRACE/LOW/MED) TRACE

\% Moisture: not dec.

GC Column: DB-624

ID $: 0.53$

Soil Extract Volume:

Purge Volume: 25.0
Lab Sample ID: 762963

Lab File ID: 762963

Date Received: 08/08/2008

Date Analyzed: 08/11/2008

Dilution Factor: 1.0

Soil Aliquot Volume:

(uL)

\begin{tabular}{|c|c|c|c|}
\hline $\begin{array}{l}\text { CAS NO. } \\
========== \\
75-71-8 \\
74-87-3 \\
75-01-4 \\
74-83-9 \\
75-69-4 \\
75-35-4 \\
76-13-1 \\
67-64-1 \\
75-15-0 \\
79-20-9 \\
75-09-2 \\
156-60-5 \\
1634-04-4 \\
75-34-3 \\
156-59-2 \\
78-93-3 \\
74-97-5 \\
67-66-3 \\
71-55-6 \\
110-82-7 \\
56-23-5 \\
71-43-2 \\
107-06-2\end{array}$ & $\begin{array}{l}\text { COMPOUND } \\
=========================== \\
\text { Dichlorodifluoromethane } \\
\text { Chloromethane } \\
\text { Vinyl chloride } \\
\text { Bromomethane } \\
\text { Chloroethane } \\
\text { Trichlorofluoromethane } \\
1,1-\text { Dichloroethene } \\
1,1,2-\text { Trichloro-1, } 2,2-t r i f l u o r o e t h a n e \\
\text { Acetone } \\
\text { Carbon disulfide } \\
\text { Methyl acetate } \\
\text { Methylene chloride } \\
\text { trans-1,2-Dichloroethene } \\
\text { Methyl tert-butyl ether } \\
1,1-D i c h l o r o e t h a n e \\
\text { cis-1,2-Dichloroethene } \\
2-\text { Butanone } \\
\text { Bromochloromethane } \\
\text { Chloroform } \\
1,1,1-\text { Trichloroethane } \\
\text { Cyclohexane } \\
\text { Carbon tetrachloride } \\
\text { Benzene } \\
1,2-\text { Dichloroethane }\end{array}$ & \begin{tabular}{|cc} 
CONCENTRATION UNITS : \\
(ug/L or ug/kg) & ug/L \\
$=================$ \\
0.50 \\
0.50 \\
0.50 \\
0.50 \\
0.50 \\
0.50 \\
0.50 \\
0.50 \\
4.8 \\
0.50 \\
0.50 \\
0.50 \\
0.50 \\
0.50 \\
0.50 \\
0.50 \\
5.0 \\
0.50 \\
0.50 \\
0.50 \\
0.50 \\
0.50 \\
0.26 \\
0.50
\end{tabular} & $\begin{array}{l}========= \\
U \\
U \\
U \\
U \\
U \\
U \\
U \\
U \\
J \\
U \\
U \\
U \\
U \\
U \\
U \\
U \\
U \\
U \\
U \\
U \\
U \\
U \\
J \\
U\end{array}$ \\
\hline
\end{tabular}

Report 1,4-Dioxane for Low-Medium VOA analysis only 
Lab Name: TestAmerica BURLINGTON

Contract: $8 \mathrm{E}-00302$

Lab Code: STLV Case No.: CENTRALIA Mod. Ref No.:

SDG No.: 126998

Matrix: (SOIL/SED/WATER) Water

Sample wt/vol: $25.0 \quad(\mathrm{~g} / \mathrm{mL}) \mathrm{mL}$

Level: (TRACE/LOW/MED) TRACE

\% Moisture: not dec.

GC Column: DB-624

Soil Extract volume:

ID $: 0.53$

(mm)

(uL)

(mL)

Purge Volume: 25.0
Lab Sample ID: 762963

Lab File ID: 762963

Date Received: $08 / 08 / 2008$

Date Analyzed: 08/11/2008

Dilution Factor: 1.0

Soil Aliquot Volume:

(uL)

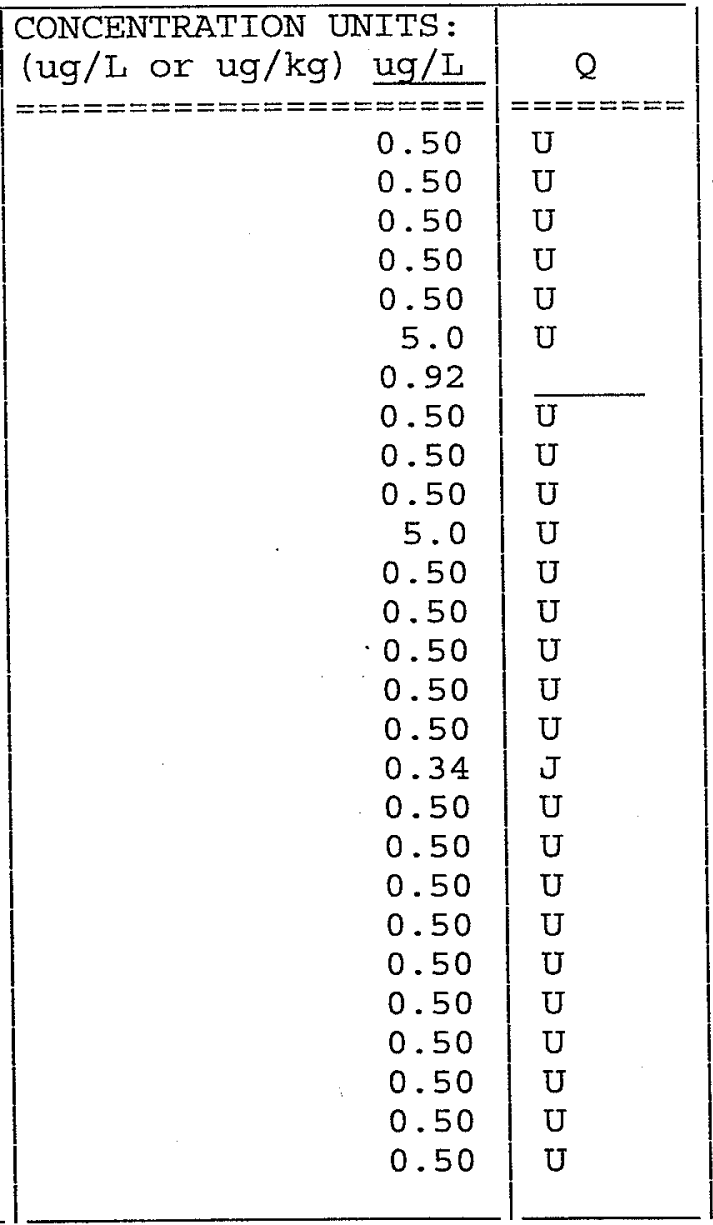


Lab Name: TestAmerica BURLINGTON

Contract: $8 \mathrm{E}-00302$

Lab Code: STLV Case No.: CENTRALIA Mod. Ref No.:

SDG No.: 126998

Matrix: (SOIL/SED/WATER) Water

Sample wt/vol: 25.0

$(\mathrm{g} / \mathrm{mL}) \mathrm{mL}$

Level: (TRACE/LOW/MED) TRACE

$\%$ Moisture: not dec.

GC Column: $\mathrm{DB}-624$

Soil Extract Volume:

ID : 0.53

Purge Volume: 25.0
Lab Sample ID: 762961

Lab File ID: 762961

Date Received: 08/08/2008

Date Analyzed: 08/11/2008

Dilution Factor: 1.0

Soil Aliquot Volume:

(mL)

\begin{tabular}{|c|c|c|c|}
\hline CAS NO. & COMPOUND & $\begin{array}{l}\text { CONCENTRATION UNITS: } \\
(\mathrm{ug} / \mathrm{L} \text { or } \mathrm{ug} / \mathrm{kg}) \mathrm{ug} / \mathrm{L}\end{array}$ & Q \\
\hline$== \pm=======$ & 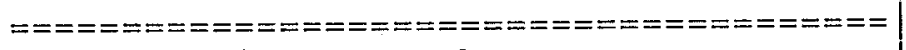 & 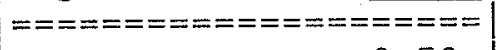 & $======3$ \\
\hline $75-71-8$ & Dichlorodifluoromethane & 0.50 & $\mathrm{U}$ \\
\hline $74-87-3$ & Chloromethane & 0.50 & $U$ \\
\hline $75-01-4$ & Vinyl chloride & 0.50 & $\mathrm{U}$ \\
\hline $74-83-9$ & Bromomethane & 0.50 & $\mathrm{U}$ \\
\hline & Chloroethane & 0.50 & $\mathrm{U}$ \\
\hline $75-69-4$ & Trichlorofluoromethane & 0.50 & $\mathrm{U}$ \\
\hline $75-35-4$ & 1,1-Dichloroethene & 0.50 & $\mathrm{U}$ \\
\hline $76-13-1$ & $1,1,2$-Trichloro-1,2,2-trifluoroethane & 0.50 & $\mathrm{U}$ \\
\hline $67-64-1$ & Acetone & 5.0 & U \\
\hline $75-15-0$ & Carbon disulfide & 0.50 & $\mathrm{U}$ \\
\hline $79-20-9$ & Methyl acetate & 0.50 & U \\
\hline $75-09-2$ & Methylene chloride & 0.50 & U \\
\hline $156-60-5$ & trans-1,2-Dichloroethene & 0.50 & $\mathrm{U}$ \\
\hline $1634-04-4$ & Methyl tert-butyl ether & 0.50 & $\mathrm{U}$ \\
\hline $75-34-3$ & 1,1-Dichloroethane & 0.50 & $\mathrm{U}$ \\
\hline $156-59-2$ & Cis-1,2-Dichloroethene & $0.50^{\circ}$ & $\mathrm{U}$ \\
\hline $78-93-3$ & 2-Butanone & 5.0 & $\mathrm{U}$ \\
\hline $74-97-5$ & Bromochloromethane & 0.50 & $\mathrm{U}$ \\
\hline $67-66-3$ & Chloroform & 1.1 & \\
\hline $71-55-6$ & 1,1,1-Trichloroethane & 0.50 & $\bar{U}$ \\
\hline $110-82-7$ & Cyclohexane & 0.50 & $\mathrm{U}$ \\
\hline $56-23-5$ & Carbon tetrachloride & 15 & \\
\hline $71-43-2$ & Benzene & 0.50 & $\mathrm{U}$ \\
\hline $107-06-2$ & 1,2-Dichloroethane & 0.50 & $\mathrm{U}$ \\
\hline
\end{tabular}

Report 1,4-Dioxane for Low-Medium VOA analysis only 
$1 B$ - FORM I VOA-2

VOLATILE ORGANICS ANALYSIS DATA SHEET
EPA SAMPLE NO.

SB07RW26661
Lab Name: TestAmerica BURLINGTON

Lab Code: STLV Case No.: CENTRALIA Mod, Ref No.:
Contract: $8 \mathrm{E}-00302$

SDG No.: 126998
Matrix: (SOIL/SED/WATER) Water

Sample wt/vol: 25.0

$(\mathrm{g} / \mathrm{mL}) \mathrm{mL}$

Level: (TRACE/LOW/MED) TRACE

\% Moisture: not dec.

GC Column: DB-624

ID $: 0.53$

Soil Extract Volume:

Purge Volume: 25.0
Lab Sample ID: 762961

Lab File ID: 762961

Date Received: 08/08/2008

Date Analyzed: 08/11/2008

Dilution Factor: 1.0

Soil Aliquot Volume:

\begin{tabular}{|c|c|c|c|}
\hline CAS NO. & COMPOUND & $\begin{array}{l}\text { CONCENTRATION UNITS: } \\
(\mathrm{ug} / \mathrm{L} \text { or } \mathrm{ug} / \mathrm{kg}) \mathrm{ug} / \mathrm{L}\end{array}$ & Q \\
\hline$===== \pm=====$ & 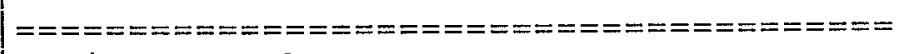 & 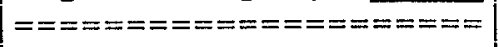 & $========$ \\
\hline $79-01-6$ & Trichloroethene & 0.50 & $\mathrm{U}$ \\
\hline $108-87-2$ & Methylcyclohexane & 0.50 & U \\
\hline $78-87-5$ & 1,2-Dichloropropane & 0.50 & $\mathrm{U}$ \\
\hline $75-27-4$ & Bromodichloromethane & 0.50 & $\mathrm{U}$ \\
\hline $10061-01-5$ & cis-1,3-Dichloropropene & 0.50 & $\mathrm{U}$ \\
\hline $108-10-1$ & 4-Methyl-2-pentanone & 5.0 & $\mathrm{U}$ \\
\hline $108-88-3$ & Toluene & 0.50 & $\mathrm{U}$ \\
\hline $10061-02-6$ & trans-1,3-Dichloropropene & 0.50 & $\mathrm{U}$ \\
\hline $79-00-5$ & $1,1,2$-Trichloroethane & 0.50 & U \\
\hline $127-18-4$ & Tetrachloroethene & 0.50 & $\mathrm{U}$ \\
\hline $591-78-6$ & 2-Hexanone & 5.0 & U \\
\hline $124-48-1$ & Dibromochloromethane & 0.50 & U \\
\hline $106-93-4$ & 1,2-Dibromoethane & 0.50 & U \\
\hline $108-90-7$ & Chlorobenzene & 0.50 & $\mathrm{U}$ \\
\hline $100-41-4$ & Ethylbenzene & 0.50 & $\mathrm{U}$ \\
\hline $95-47-6$ & o-xylene & 0.50 & $\mathrm{U}$ \\
\hline $179601-23-1$ & $\mathrm{~m}, \mathrm{p}$-Xylene & 0.50 & $\mathrm{U}$ \\
\hline $100-42-5$ & Styrene & 0.50 & $\mathrm{U}$ \\
\hline $75-25-2$ & Bromoform & 0.50 & $\mathrm{U}$ \\
\hline $98-82-8$ & Isopropylbenzene & 0.50 & $\mathrm{U}$ \\
\hline $79-34-5$ & $1,1,2,2$-Tetrachloroethane & 0.50 & $\mathrm{U}$ \\
\hline $541-73-1$ & 1,3-Dichlorobenzene & 0.50 & $\mathrm{U}$ \\
\hline $106-46-7$ & 1,4-Dichlorobenzene & 0.50 & $\mathrm{U}$ \\
\hline $95-50-1$ & 1,2-Dichlorobenzene & 0.50 & $\mathrm{U}$ \\
\hline $96-12-8$ & 1,2-Dibromo-3-chloropropane & 0.50 & $\mathrm{U}$ \\
\hline $120-82-1$ & $1,2,4$-Trichlorobenzene & 0.50 & $\mathrm{U}$ \\
\hline $87-61-6$ & $1,2,3$-Trichlorobenzene & 0.50 & $\mathrm{U}$ \\
\hline
\end{tabular}




\section{TestAmerica}

THE LEADER IN ENVIRONMENTAL TESTING

September 29, 2008

TestAmerica Laboratories, Inc.

Mr. Clyde Dennis

Argonne National Laboratory

9700 S. Cass Avenue

Building 203, Office B149

Argonne, IL 60439

Re: Laboratory Project No. 21005

Case: CENTRALIA; SDG: 127561

Dear Mr. Dennis:

Enclosed are analytical results for samples that were received by TestAmerica Burlington on September $11^{\text {th }}, 2008$. Laboratory identification numbers were assigned, and designated as follows:

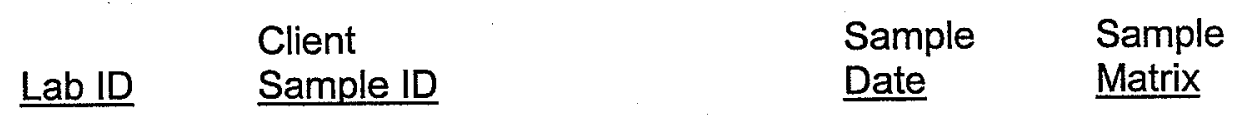

Received: 09/11/08 ETR No: 127561

$\begin{array}{llll}767257 & \text { CNMW01-W-26673 } & 09 / 09 / 08 & \text { WATER } \\ 767258 & \text { CNMW04-W-26676 } & 09 / 09 / 08 & \text { WATER } \\ 767259 & \text { CNPMP1-W-26689 } & 09 / 09 / 08 & \text { WATER } \\ 767260 & \text { CNQCTB-W-26702 } & 09 / 10 / 08 & \text { WATER } \\ 767261 & \text { VHBLK01 } & 09 / 11 / 08 & \text { WATER }\end{array}$

Documentation of the condition of the samples at the time of their receipt and any exception to the laboratory's Sample Acceptance Policy is documented in the Sample Handling section of this submittal. The samples, as received, were not acid preserved. On that basis the laboratory did provide for the analytical work to be performed within seven days of sample collection.

In order to accommodate field length limitations in processing the data summary forms, the laboratory did, in certain instances, abbreviate the sample identifier. The electronically formatted data provides for the full sample identifier.

\section{SOM01.2 Volatile Organics (Trace Level Water)}

A storage blank was prepared for volatile organics analysis, and stored in association with the storage of the sample. That storage blank, identified as VHBLK01, was carried through the holding period with the samples, and analyzed. 


\section{TestAmerica}

THE LEADER IN ENVIRONMENTAL TESTING

Sample CNPMP1-W-26689 was analyzed at a dilution, based on the results of preliminary screening. An additional, more concentrated analysis was performed on the sample in order to provide a lower reporting limit for those target analytes that were not identified as constituents in the primary analysis. Both sets of results for the analysis of sample CNPMP1-W-26689 are included in this submittal. Each of the analyses associated with the sample set exhibited an acceptable internal standard performance. There was an acceptable recovery of each deuterated monitoring compound (DMC) in the analysis of the method blank and the analysis of the storage blank. The analysis of the samples in this sample set did meet the technical acceptance criteria specific to DMC recoveries, although not all DMC recoveries were within the control range in each analysis. The technical acceptance criteria does provide for the recovery of up to three DMCs to fall outside of the control range in the analysis of field samples. With the exception of that performed on sample CNQCTB-W-26702, the derived recovery of 2-hexanone- $d_{5}$ was elevated in the analysis of each of the field samples. Additionally, the recovery of 2-butanone- $d_{5}$ was high in the analysis of sample CNMW01-W-26673. Matrix spike and matrix spike duplicate analyses were not performed on the samples in this sample set. A trace concentration of acetone was identified in the analysis of the method blank associated with the analytical work. The derived concentration of acetone in that analysis was below the established reporting limit, and the analysis did meet the technical acceptance criteria for a compliant method blank acquisition. A trace concentration of acetone was identified in the analysis of the storage blank associated with the sample set. The derived concentration of acetone in that analysis was below the established reporting limit, and the analysis did meet the technical acceptance criteria for a compliant storage blank acquisition. Present in the storage blank and method blank analyses was a non-target constituent that represented a compound that is related to either the DMC formulation or to column bleed. The fact that the presence of this compound is not within the laboratory's control is at issue. The derived results for that compound have been qualified with an " $X$ " qualifier to reflect the source of the contamination. An instrument blank was analyzed following the more concentrated analysis of sample CNPMP1-W-26689. A trace concentration of acetone was identified in that analysis at a concentration below the established reporting limit, and the analysis did meet the technical acceptance criteria for a compliant instrument blank acquisition.

The responses for each target analyte met the relative standard deviation criterion in the initial calibration. The response for each target analyte met the percent difference criterion in the continuing calibration check acquisition. The response for each target analyte met the 50.0 percent difference criterion in the closing calibration check acquisition.

The primary quantitation mass for methylcyclohexane that is specified in the Statement of Work is mass 83 . The laboratory did identify a contribution to mass 83 from 1,2-dichloropropane- $d_{6}$, one of the deuterated monitoring compounds (DMCs). The laboratory did change the primary quantitation mass assignment to mass 55 for the quantification of methylcyclohexane.

Manual integration was employed in deriving certain of the analytical results. The values that have been derived from manual integration are qualified on the quantitation reports. Extracted ion current profiles for each manual integration are included in the data package, and further documented in the Sample Preparation section of this submittal.

Any reference within this report to Severn Trent Laboratories, Inc. or STL, should be understood to refer to TestAmerica Laboratories, Inc. (formerly known as Severn Trent Laboratories, Inc.) 


\section{TestAmerica}

THE LEADER IN ENVIRONMENTAL TESTING

The analytical results associated with the samples presented in this test report were generated under a quality system that adheres to requirements specified in the NELAC standard. Release of the data in this test report and any associated electronic deliverables is authorized by the Laboratory Director's designee as verified by the following signature.

If there are any questions regarding this submittal, please contact me at 802 660-1990.

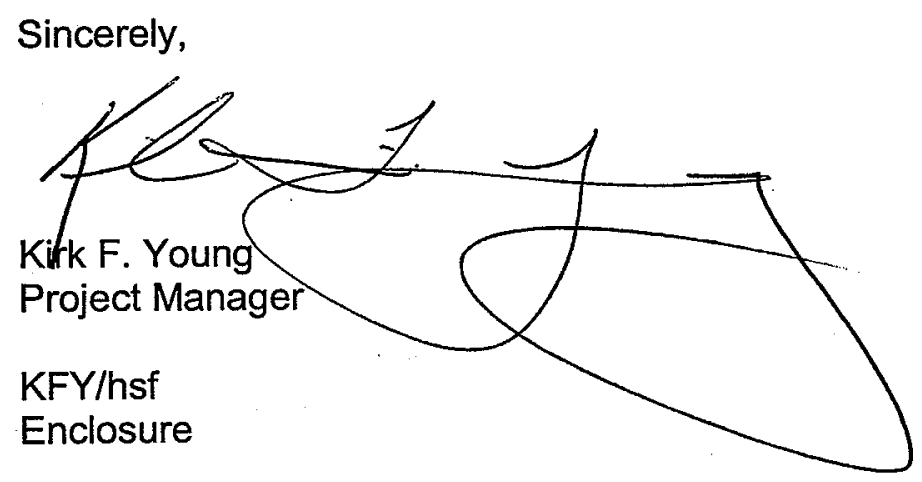




\section{TestAmerica Burlington Data Qualifier Definitions}

\section{Organic}

U: Compound analyzed but not detected at a concentration above the reporting limit.

J: Estimated value.

$\mathrm{N}$ : Indicates presumptive evidence of a compound. This flag is used only for tentatively identified compounds (TICs) where the identification of a compound is based on a mass spectral library search.

P: $\quad$ SW-846: The relative percent difference for detected concentrations between two GC columns is greater than $40 \%$. Unless otherwise specified the higher of the two values is reported on the Form 1 .

CLP SOW: Greater than 25\% difference for detected concentrations between two GC columns. Unless otherwise specified the lower of the two values is reported on the Form I.

C: Pesticide result whose identification has been confirmed by GC/MS.

B: Analyte is found in the sample and the associated method blank. The flag is used for tentatively identified compounds as well as positively identified compounds.

E: Compounds whose concentrations exceed the upper limit of the calibration range of the instrument for that specific analysis.

D: Concentrations identified from analysis of the sample at a secondary dilution.

A: Tentatively identified compound is a suspected aldol condensation product.

$X, Y, Z$ : Laboratory defined flags that may be used alone or combined, as needed. If used, the description of the flag is defined in the project narrative.

\section{Inorganic/Metals}

E: $\quad$ Reported value is estimated due to the presence of interference.

$\mathrm{N}$ : Matrix spike sample recovery is not within control limits.

* Duplicate sample analysis is not within control limits.

B: The result reported is less than the reporting limit but greater than the instrument detection limit.

U: Analyte was analyzed for but not detected above the reporting limit.

Method Codes:

P ICP-AES

MS ICP-MS

CV Cold Vapor AA

AS Semi-Automated Spectrophotometric 


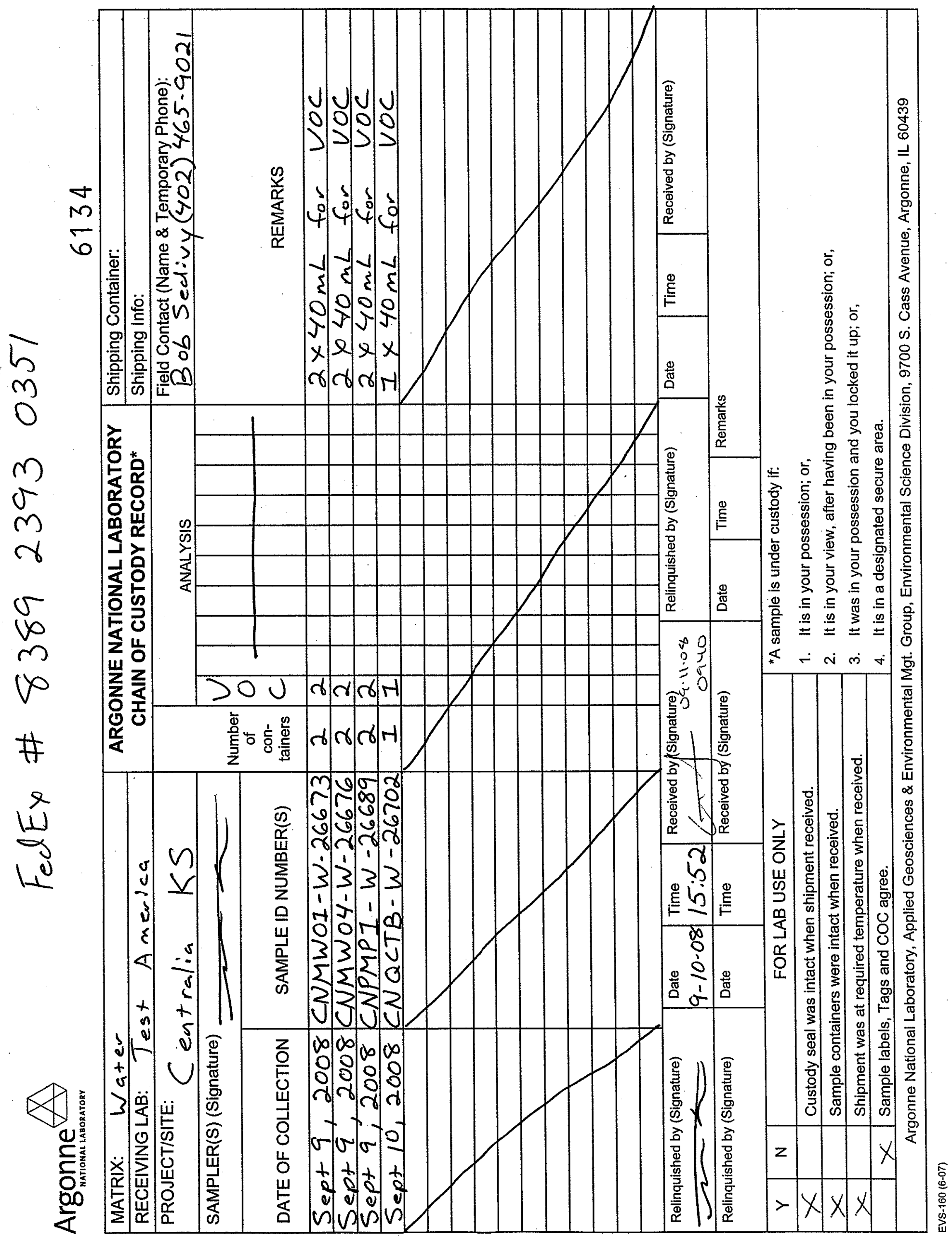




\section{TestAmerica}

THE LEADER IN ENVIRONMENTAL. TESTING

\section{Sample Data Summary - SOM01.2 Volatiles - Trace}


Lab Name: TestAmerica BURLINGTON

Contract: 21005

Lab Code: STLV Case No.: CENTRALIA Mod. Ref No.:

SDG No.: 127561

Matrix: (SOIL/SED/WATER) Water

Sample wt/vol: $25.0 \quad(\mathrm{~g} / \mathrm{mL}) \mathrm{mL}$

Level: (TRACE/LOW/MED) TRACE

\% Moisture: not dec.

GC Column: DB-624

Soil Extract Volume:

ID $: 0.53$

(mm)

(uL)

(mI)

Purge Volume: 25.0
Lab Sample ID: 767257

Lab File ID: 767257

Date Received: 09/11/2008

Date Analyzed: 09/12/2008

Dilution Factor: 1.0

Soil Aliquot Volume:

(uL)

\begin{tabular}{|c|c|c|c|}
\hline CAS NO. & COMPOUND & $\begin{array}{l}\text { CONCENTRATION UNITS: } \\
(\mathrm{ug} / \mathrm{L} \text { or } \mathrm{ug} / \mathrm{kg}) \mathrm{ug} / \mathrm{L}\end{array}$ & $Q$ \\
\hline$=====-=====$ & 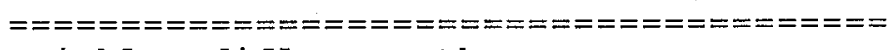 & 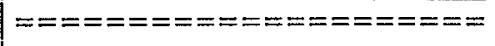 & $=======$ \\
\hline $75-71-8$ & Dichlorodifluoromethane & 0.50 & $\mathrm{U}$ \\
\hline $74-87-3$ & Chloromethane & 0.50 & $\mathrm{U}$ \\
\hline $75-01-4$ & Vinyl chloride & 0.50 & $\mathrm{U}$ \\
\hline $74-83-9$ & Bromomethane & 0.50 & $\mathrm{U}$ \\
\hline $75-00-3$ & Chloroethane & 0.50 & $\mathrm{U}$ \\
\hline $75-69-4$ & Trichlorofluoromethane & 0.50 & $\mathrm{U}$ \\
\hline $75-35-4$ & 1,1-Dichloroethene & 0.50 & $\mathrm{U}$ \\
\hline $76-13-1$ & $1,1,2$-Trichloro-1,2,2-trifluoroethane & 0.50 & $\mathrm{U}$ \\
\hline $67-64-1$ & Acetone & 1.0 & JB \\
\hline $75-15-0$ & Carbon disulfide & 0.50 & $\mathrm{U}$ \\
\hline $79-20-9$ & Methyl acetate. & 0.50 & U \\
\hline $75-09-2$ & Methylene chloride & 0.50 & $\mathrm{U}$ \\
\hline $156-60-5$ & trans-1,2-Dichloroethene & 0.50 & $\mathrm{U}$ \\
\hline $1634-04-4$ & Methyl tert-butyl ether & 0.50 & U \\
\hline $75-34-3$ & 1,1-Dichloroethane & 0.50 & $\mathrm{U}$ \\
\hline $156-5.9-2$ & cis-1,2-Dichloroethene & 0.50 & U \\
\hline $78-93-3$ & 2-Butanone & 5.0 & U \\
\hline $74-97-5$ & Bromochloromethane & 0.50 & $\mathrm{U}$ \\
\hline $67-66-3$ & Chloroform & 0.50 & $\mathrm{U}$ \\
\hline $71-55-6$ & $1,1,1$-Trichloroethane & 0.50 & $\mathrm{U}$ \\
\hline $110-82-7$ & Cyclohexane & 0.50 & $\mathrm{U}$ \\
\hline $56-23-5$ & Carbon tetrachloride & 0.50 & U \\
\hline $71-43-2$ & Benzene & 0.50 & U \\
\hline $107-06-2$ & 1,2-Dichloroethane & 0.50 & $\mathrm{U}$ \\
\hline
\end{tabular}

Report 1,4-Dioxane for Low-Medium VOA analysis only 
IB - FORM I VOA-2

VOLATILE ORGANICS ANALYSIS DATA SHEET
EPA SAMPLE NO.
Lab Name: TestAmerica BURLINGTON

Contract: 21005

Lab Code: STLV Case No.: CENTRALIA Mod. Ref No.:

SDG NO.: 127561

Matrix: (SOIL/SED/WATER) Water

Sample wt/vol: 25.0

(g/mL) $\mathrm{mL}$

Level: (TRACE/LOW/MED) TRACE

\% Moisture: not dec.

GC Column: DB-624

ID $: 0.53$

Soil Extract Volume:

Purge Volume: 25.0
Lab Sample ID: 767257

Lab File ID: 767257

Date Received: 09/11/2008

Date Analyzed: 09/12/2008

Dilution Factor: 1.0

(uL) Soil Aliquot Volume:

(uL)

(mL)

\begin{tabular}{|c|c|c|c|}
\hline CAS NO. & COMPOUND & $\begin{array}{l}\text { CONCENTRATION UNITS: } \\
(\mathrm{ug} / \mathrm{L} \text { or } \mathrm{ug} / \mathrm{kg}) \mathrm{ug} / \mathrm{L}\end{array}$ & Q \\
\hline$==== \pm=====$ & 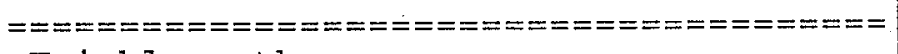 & 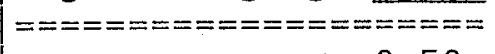 & $=======$ \\
\hline $79-01-6$ & Trichloroethene & 0.50 & $\mathrm{U}$ \\
\hline $108-87-2$ & Methylcyclohexane & 0.50 & $\mathrm{U}$ \\
\hline $78-87-5$ & 1,2-Dichloropropane & 0.50 & $\mathrm{U}$ \\
\hline $75-27-4$ & Bromodichloromethane & 0.50 & $\mathrm{U}$ \\
\hline $10061-01-5$ & cis-1,3-Dichloropropene & 0.50 & $\mathrm{U}$ \\
\hline $108-10-1$ & 4-Methyl-2-pentanone & 5.0 & $\mathrm{U}$ \\
\hline $108-88-3$ & Toluené & 0.50 & $\mathrm{U}$ \\
\hline $10061-02-6$ & trans-1,3-Dichloropropene & 0.50 & $\mathrm{U}$ \\
\hline $79-00-5$ & $1,1,2$-Trichloroethane & 0.50 & $\mathrm{U}$ \\
\hline $127-18-4$ & Tetrachloroethene & 0.50 & $\mathrm{U}$ \\
\hline $591-78-6$ & 2-Hexanone & 5.0 & $\mathrm{U}$ \\
\hline $124-48-1$ & Dibromochloromethane & 0.50 & $\mathrm{U}$ \\
\hline $106-93-4$ & 1,2-Dibromoethane & 0.50 & U \\
\hline $108-90-7$ & Chlorobenzene & 0.50 & $\mathrm{U}$ \\
\hline $100-41-4$ & Ethylbenzene & 0.50 & $\mathrm{U}$ \\
\hline $95-47-6$ & o-Xylene & 0.50 & $\mathrm{U}$ \\
\hline $179601-23-1$ & $\mathrm{~m}, \mathrm{p}-\mathrm{xy}$ lene & 0.50 & $\mathrm{U}$ \\
\hline $100-42-5$ & styrene & 0.50 & $\mathrm{U}$ \\
\hline $75-25-2$ & Bromoform & 0.50 & $\mathrm{U}$ \\
\hline $98-82-8$ & Isopropylbenzene & 0.50 & $\mathrm{U}$ \\
\hline $79-34-5$ & $1,1,2,2$-Tetrachloroethane & 0.50 & U \\
\hline $541-73-1$ & 1,3-Dichlorobenzene & 0.50 & U \\
\hline $106-46-7$ & 1,4-Dichlorobenzene & 0.50 & $\mathrm{U}$ \\
\hline $95-50-1$ & 1;2-Dichlorobenzene & 0.50 & U \\
\hline $96-12-8$ & 1,2-Dibromo-3-chloropropane & 0.50 & $\mathrm{U}$ \\
\hline $120-82-1$ & $1,2,4$-Trichlorobenzene & 0.50 & $\mathrm{U}$ \\
\hline $87-6 I-6$ & $1,2,3$-Trichlorobenzene & 0.50 & $\mathrm{U}$ \\
\hline
\end{tabular}


$1 A$ - FORM I VOA-I

VOLATILE ORGANICS ANALYSIS DATA SHEET
EPA SAMPLE NO.

CNMW04W26676

Lab Name: TestAmerica BURLINGTON

Contract : 21005

Lab Code: STLV Case No.: CENTRALIA Mod. Ref No.:

SDG NO.: 127561

Matrix: (SOIL/SED/WATER) Water

Sample wt/vol: $25.0 \quad(\mathrm{~g} / \mathrm{mL}) \mathrm{mL}$

Level: (TRACE/LOW/MED) TRACE

$\%$ Moisture: not dec.

GC Column: DB-624

ID $: 0.53$

Soil Extract Volume:

Purge Volume: 25.0
Lab Sample ID: 767258

Lab File ID: 767258

Date Received: 09/11/2008

Date Analyzed: 09/12/2008

(mm) Dilution Factor: 1.0

(uL) Soil Aliquot Volume:

(uL)

\begin{tabular}{|c|c|c|c|}
\hline CAS NO. & COMPOUND & $\begin{array}{l}\text { CONCENTRATION UNITS : } \\
(\mathrm{ug} / \mathrm{L} \text { or } \mathrm{ug} / \mathrm{kg}) \mathrm{ug} / \mathrm{L}\end{array}$ & $Q$ \\
\hline$===========\approx$ & 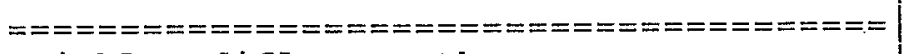 & 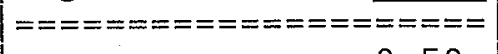 & $==z====$ \\
\hline $75-71-8$ & Dichlorodifluoromethane & 0.50 & $\mathrm{U}$ \\
\hline $74-87-3$ & Chloromethane & 0.50 & $\mathrm{U}$ \\
\hline $75-01-4$ & Vinyl chloride & 0.50 & $\mathrm{U}$ \\
\hline $74-83-9$ & Bromomethane & 0.50 & $\mathrm{U}$ \\
\hline $75-00-3$ & Chloroethane & 0.50 & U \\
\hline $75-69-4$ & Trichlorofluoromethane & 0.50 & $\mathrm{U}$ \\
\hline $75-35-4$ & 1,1-Dichloroethene & 0.50 & $\mathrm{U}$ \\
\hline $76-13-1$ & 1,1,2-Trichloro-1,2,2-trifluoroethane & 0.50 & $\mathrm{U}$ \\
\hline $67-64-1$ & Acetone & 1.6 & JB \\
\hline $75-15-0$ & Carbon disulfide & 0.50 & $\mathrm{U}$ \\
\hline $79-20-9$ & Methyl acetate & 0.50 & $\mathrm{U}$ \\
\hline $75-09-2$ & Methylene chloride & 0.50 & $\mathrm{U}$ \\
\hline $156-60-5$ & trans-1,2-Dichloroethene & 0.50 & $\mathrm{U}$ \\
\hline $1634-04-4$ & Methyl tert-butyl ether & 0.50 & $\mathrm{U}$ \\
\hline $75-34-3$ & 1,1-Dichloroethane & 0.50 & $\mathrm{U}$ \\
\hline $156-59-2$ & cis-1,2-Dichloroethene & 0.50 & U \\
\hline $78-93-3$ & 2-Butanone & 5.0 & $\mathrm{U}$ \\
\hline $74-97-5$ & Bromochloromethane & 0.50 & $\mathrm{U}$ \\
\hline $67-66-3$ & Chloroform & 0.50 & $\mathrm{U}$ \\
\hline $71-55-6$ & 1,1,1-Trichloroethane & 0.50 & $\mathrm{U}$ \\
\hline $110-82-7$ & Cyclohexane & 0.50 & $U$ \\
\hline $56-23-5$ & Carbon tetrachloride & 1.8 & \\
\hline $71-43-2$ & Benzene & 0.50 & $\overline{\mathrm{U}}$ \\
\hline $107-06-2$ & 1,2-Dichloroethane & 0.50 & $\mathrm{U}$ \\
\hline
\end{tabular}

Report 1,4-Dioxane for Low-Medium VOA analysis only 
$1 B$ - FORM I VOA-2

VOLATILE ORGANICS ANALYSIS DATA SHEET
EPA SAMPLE NO.
Lab Name: TestAmerica BURLINGTON

Contract: 21005

Lab Code: STLV Case No.: CENTRALIA Mod. Ref No.:

SDG NO.: 127561

Matrix: (SOIL/SED/WATER) Water

Sample wt/vol: 25.0

$(\mathrm{g} / \mathrm{mL}) \mathrm{mL}$

Level: (TRACE/LOW/MED) TRACE

$\%$ Moisture: not dec.

GC Column: $\mathrm{DB}-624$

ID: 0.53

Soil Extract Volume:

Purge Volume: 25.0
Lab Sample ID: 767258

Lab File ID: 767258

Date Received: 09/11/2008

Date Analyzed: 09/12/2008

Dilution Factor: 1.0

Soil Aliquot Volume:

(mL)

\begin{tabular}{|c|c|c|c|}
\hline CAS NO. & COMPOUND & $\begin{array}{l}\text { CONCENTRATION UNITS: } \\
(\mathrm{ug} / \mathrm{L} \text { or } \mathrm{ug} / \mathrm{kg}) \mathrm{ug} / \mathrm{L}\end{array}$ & $Q$ \\
\hline$===========$ & 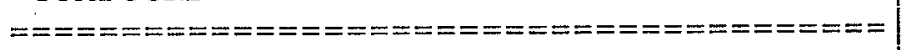 & 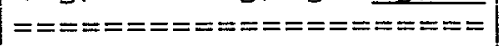 & $=======$ \\
\hline $79-01-6$ & Trichloroethene & 0.50 & $\mathrm{U}$ \\
\hline $108-87-2$ & Methylcyclohexane & 0.50 & $\mathrm{U}$ \\
\hline $78-87-5$ & 1,2-Dichloropropane & 0.50 & $\mathrm{U}$ \\
\hline $75-27-4$ & Bromodichloromethane & 0.50 & $\mathrm{U}$ \\
\hline $10061-01-5$ & cis-1,3-Dichloropropene & 0.50 & $\mathrm{U}$ \\
\hline $108-10-1$ & 4-Methy1-2-pentanone & 5.0 & $\mathrm{U}$ \\
\hline $108-88-3$ & Toluene & 0.50 & $\mathrm{U}$ \\
\hline $10061-02-6$ & trans-1,3-Dichloropropene & 0.50 & $\mathrm{U}$ \\
\hline $79-00-5$ & $1,1,2$-Trichloroethane & 0.50 & $\mathrm{U}$ \\
\hline $127-18-4$ & Tetrachloroethene & 0.50 & $\mathrm{U}$ \\
\hline $591-78-6$ & 2-Hexanone & 5.0 & $\mathrm{U}$ \\
\hline $124-48-1$ & Dibromochloromethane & 0.50 & $\mathrm{U}$ \\
\hline $106-93-4$ & 1,2-Dibromoethane & 0.50 & $\mathrm{U}$ \\
\hline $108-90-7$ & Chlorobenzene & 0.50 & U \\
\hline $100-41-4$ & Ethylbenzene & 0.50 & $\mathrm{U}$ \\
\hline $95-47-6$ & o-Xylene: & 0.50 & U \\
\hline $179601-23-1$ & $\mathrm{~m}, \mathrm{p}$-Xylene & 0.50 & U \\
\hline $100-42-5$ & Styrene & 0.50 & $\mathrm{U}$ \\
\hline $75-25-2$ & Bromoform & 0.50 & $\mathrm{U}$ \\
\hline $98-82-8$ & Isopropylbenzene & 0.50 & $\mathrm{U}$ \\
\hline $79-34-5$ & $1,1,2,2$-Tetrachloroethane & 0.50 & U \\
\hline $541-73-1$ & 1,3-Dichlorobenzene & 0.50 & $\mathrm{U}$ \\
\hline $106-46-7$ & 1,4-Dichlorobenzene & 0.50 & $\mathrm{U}$ \\
\hline $95-50-1$ & 1,2-Dichlorobenzene & 0.50 & $\mathrm{U}$ \\
\hline $96-12-8$ & 1,2-Dibromo-3-chloropropane & 0.50 & $\mathrm{U}$ \\
\hline $120-82-1$ & $1,2,4$-Trichlorobenzene & 0.50 & $\mathrm{U}$ \\
\hline $87-61-6$ & 1,2,3-Trichlorobenzene & 0.50 & $\mathrm{U}$ \\
\hline
\end{tabular}


IA - FORM I VOA- 1

VOLATILE ORGANICS ANALYSIS DATA SHEET
EPA SAMPLE NO.

CNQCTBW2 6702

Lab Name: TestAmerica BURLINGTON

Contract: 21005

Lab Code: STLV Case No.: CENTRALIA Mod. Ref No.:

SDG No.: 127561

Matrix: (SOIL/SED/WATER) Water

Sample wt/vol: 25.0

$(\mathrm{g} / \mathrm{mL}) \mathrm{mL}$

Level: (TRACE/LOW/MED) TRACE

\% Moisture: not dec.

GC Column: DB-624

Soil Extract Volume:

ID $: 0.53$

$(\mathrm{mm})$

(UL)

(mL)

Purge Volume: 25.0
Lab Sample ID: 767260

Lab File ID: 767260

Date Received: 09/11/2008

Date Analyzed: 09/12/2008

Dilution Factor: 1.0

Soil Aliquot Volume:

(UL)

\section{(m工)}

\begin{tabular}{|c|c|c|c|}
\hline CAS NO. & COMPOUND & $\begin{array}{l}\text { CONCENTRATION UNITS: } \\
(\mathrm{ug} / \mathrm{L} \text { or } \mathrm{ug} / \mathrm{kg}) \mathrm{ug} / \mathrm{L}\end{array}$ & Q \\
\hline 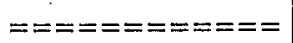 & 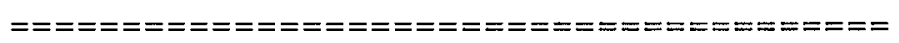 & 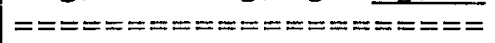 & $=======$ \\
\hline $75-71-8$ & Dichlorodifluoromethane & 0.50 & $\mathrm{U}$ \\
\hline $74-87-3$ & Chloromethane & 0.50 & $\mathrm{U}$ \\
\hline $75-01-4$ & Vinyl chloride & 0.50 & $\mathrm{U}$ \\
\hline $74-83-9$ & Bromomethane & 0.50 & U \\
\hline $75-00-3$ & Chloroethane & 0.50 & $\mathrm{U}$ \\
\hline $75-69-4$ & Trichlorofluoromethane & 0.50 & $\mathrm{U}$ \\
\hline $75-35-4$ & 1,1-Dichloroethene & 0.50 & $\mathrm{U}$ \\
\hline $76-13-1$ & 1,1,2-Trichloro-1,2,2-trifluoroethane & 0.50 & $\mathrm{U}$ \\
\hline $67-64-1$ & Acetone & 2.5 & JB \\
\hline $75-15-0$ & Carbon disulfide & 0.50 & $\mathrm{U}$ \\
\hline $79-20-9$ & Methy.l acetate & 0.50 & $\mathrm{U}$ \\
\hline $75-09-2$ & Methylene chloride & $0.50^{\circ}$ & $\mathrm{U}$ \\
\hline $156-60-5$ & trans-1,2-Dichloroethene & 0.50 & U \\
\hline $1634-04-4$ & Methyl tert-butyl ether & 0.50 & $\mathrm{U}$ \\
\hline $75-34-3$ & 1,1-Dichloroethane & 0.50 & $\mathrm{U}$ \\
\hline $156-59-2$ & cis-1,2-Dichloroethene & 0.50 & $\mathrm{U}$ \\
\hline $78-93-3$ & 2-Butanone & 5.0 & $\mathrm{U}$ \\
\hline $74-97-5$ & Bromochloromethane & 0.50 & $\mathrm{U}$ \\
\hline $67-66-3$ & Chloroform & 0.50 & $\mathrm{U}$ \\
\hline $71-55-6$ & I,I,1-Trichloroethane & 0.50 & $\mathrm{U}$ \\
\hline $110-82-7$ & Cyclohexane & 0.50 & $\mathrm{U}$ \\
\hline $56-23-5$ & Carbon tetrachloride & 0.50 & $\mathrm{U}$ \\
\hline $71-43-2$ & Benzene & 0.50 & $\mathrm{U}$ \\
\hline $107-06-2$ & 1,2-Dichloroethane & 0.50 & $\mathrm{U}$ \\
\hline
\end{tabular}

Report 1,4-Dioxane for Low-Medium VOA analysis only 
Lab Name: TestAmerica BURLINGTON

Contract: 21005

Lab Code: STLV Case No.: CENTRALIA Mod. Ref No.:

SDG NO.: 127561

Matrix: (SOIL/SED/WATER) Water

Sample wt/vol: 25.0

$(\mathrm{g} / \mathrm{mL}) \mathrm{mL}$

Level: (TRACE/LOW/MED) TRACE

\% Moisture: not dec.

GC Column: DB-624

Soil Extract Volume:

ID : 0.53

(mm)

(uL)

(mI)

Purge Volume: 25.0
Lab Sample ID: 767260

Lab File ID: 767260

Date Received: 09/11/2008

Date Analyzed: 09/12/2008

Dilution Factor: 1.0

Soil Aliquot Volume:

(UL)

(mis)

\begin{tabular}{|c|c|c|c|}
\hline CAS NO. & COMPOUND & $\begin{array}{l}\text { CONCENTRATION UNITS: } \\
(\mathrm{ug} / \mathrm{L} \text { or } \mathrm{ug} / \mathrm{kg}) \mathrm{ug} / \mathrm{L}\end{array}$ & $Q$ \\
\hline$===========$ & 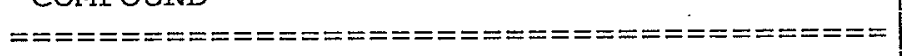 & $===================$ & $=======$ \\
\hline $79-01-6$ & Trichloroethene & 0.50 & $\mathrm{U}$ \\
\hline $108-87-2$ & Methylcyclohexane & 0.50 & $\mathrm{U}$ \\
\hline $78-87-5$ & 1,2-Dichloropropane & 0.50 & $\mathrm{U}$ \\
\hline $75-27-4$ & Bromodichloromethane & 0.50 & U \\
\hline $10061-01-5$ & cis-1,3-Dichloropropene & 0.50 & $\mathrm{U}$ \\
\hline $108-10-1$ & 4-Methyl-2-pentanone & 5.0 & $\mathrm{U}$ \\
\hline $108-88-3$ & Toluene & 0.50 & $\mathrm{U}$ \\
\hline $10061-02-6$ & trans-1,3-Dichloropropene & 0.50 & $\mathrm{U}$ \\
\hline $79-00-5$ & 1,1,2-Trichloxoethane & 0.50 & $\mathrm{U}$ \\
\hline $127-18-4$ & Tetrachloroethene & 0.50 & $\mathrm{U}$ \\
\hline $591-78-6$ & 2 -Hexanone & 5.0 & $\mathrm{U}$ \\
\hline $124-48-1$ & Dibromochloromethane & 0.50 & $\mathrm{U}$ \\
\hline $106-93-4$ & 1,2-Dibromoethane & 0.50 & $\mathrm{U}$ \\
\hline $108-90-7$ & Chlorobenzene & 0.50 & $\mathrm{U}$ \\
\hline $100-41-4$ & Ethylbenzène & 0.50 & U \\
\hline $95-47-6$ & o-Xylene & 0.50 & U \\
\hline $179601-23-1$ & $\mathrm{~m}, \mathrm{p}$-Xylene & 0.50 & U \\
\hline $100-42-5$ & styrene & 0.50 & $\mathrm{U}$ \\
\hline $75-25-2$ & Bromoform & 0.50 & $\mathrm{U}$ \\
\hline $98-82-8$ & Isopropylbenzene & 0.50 & U \\
\hline $79-34-5$ & $1,1,2,2$-Tetrachloroethane & 0.50 & $U$ \\
\hline $541-73-1$ & 1,3-Dichlorobenzene & 0.50 & U \\
\hline $106-46-7$ & 1,4-Dichlorobenzene & 0.50 & $\mathrm{U}$ \\
\hline $95-50-1$ & 1,2-Dichlorobenzene & 0.50 & $\mathrm{U}$ \\
\hline $96-12-8$ & 1,2-Dibromo-3-chloropropane & 0.50 & $\mathrm{U}$ \\
\hline $120-82-1$ & $1,2,4$-Trichlorobenzene & 0.50 & $\mathrm{U}$ \\
\hline $87-61-6$ & $1,2,3$-Trichlorobenzene & 0.50 & U \\
\hline
\end{tabular}


Lab Name: TestAmerica BURLINGTON

Lab Code: STLV Case No.: CENTRALIA Mod. Ref No.:
Contract: 21005
Matrix: (SOIL/SED/WATER) water

Sample wt/vol: 25.0

$(\mathrm{g} / \mathrm{mL}) \mathrm{mL}$

Level: (TRACE/LOW/MED) TRACE

\% Moisture: not dec.

GC Column: DB-624

Soil Extract Volume:

ID $: 0.53$

(mm)

(uL)

(mL)
Lab Sample ID: 767259

Lab File ID: 767259

Date Received: 09/11/2008

Date Analyzed: 09/12/2008

Dilution Factor: 1.0

Soil Aliquot Volume:

(uL)

\begin{tabular}{|c|c|c|c|}
\hline CAS NO. & COMPOUND & $\begin{array}{l}\text { CONCENTRATION UNITS: } \\
(\mathrm{ug} / \mathrm{L} \text { or } \mathrm{ug} / \mathrm{kg}) \mathrm{ug} / \mathrm{L}\end{array}$ & $Q$ \\
\hline$==========$ & 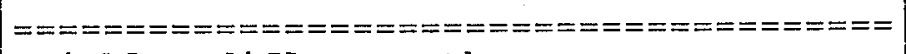 & 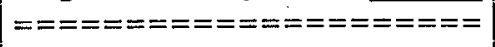 & $===== \pm==$ \\
\hline $75-71-8$ & Dichlorodifluoromethane & 0.50 & $\mathrm{U}$ \\
\hline $74-87-3$ & Chloromethane & 0.50 & $\mathrm{U}$ \\
\hline $75-01-4$ & Vinyl chloride & 0.50 & $\mathrm{U}$ \\
\hline $74-83-9$ & Bromomethane & 0.50 & $\mathrm{U}$ \\
\hline $75-00-3$ & Chloroethane & 0.50 & $\mathrm{U}$ \\
\hline $75-69-4$ & Trichlorofluoromethane & 0.50 & $\mathrm{U}$ \\
\hline $75-35-4$ & 1,1 -Dichloroethene & 0.50 & $\mathrm{U}$ \\
\hline $76-13-1$ & $1,1,2$-Trichloro-1,2,2-trifluoroethane & 0.50 & $\mathrm{U}$ \\
\hline $67-64-1$ & Acetone & 32 & B \\
\hline $75-15-0$ & Carbon disulfide & 0.26 & $\mathrm{~J}$ \\
\hline $79-20-9$ & Methyl acetate & 0.50 & $\mathrm{U}$ \\
\hline $75-09-2$ & Methylene chloride & 0.29 & $\mathrm{~J}$ \\
\hline $156-60-5$ & trans-1,2-Dichloroethene & 0.50 & $\mathrm{U}$ \\
\hline $1634-04-4$ & Methyl tert-butyl ether & 0.50 & $\mathrm{U}$ \\
\hline $75-34-3$ & 1,1-Dichloroethane & 0.50 & $\mathrm{u}$ \\
\hline $156-59-2$ & cis-1,2-Dichloroethene & 0.50 & $\mathrm{U}$ \\
\hline $78-93-3$ & 2-Butanone & 5.5 & \\
\hline $74-97-5$ & Bromochloromethane & 0.50 & $\mathrm{U}$ \\
\hline $67-66-3$ & Chloroform & 23 & $\mathrm{E}$ \\
\hline $71-55-6$ & $1,1,1$-Trichloroethane & 0.50 & U \\
\hline $110-82-7$ & Cyclohexane & 0.50 & $\mathrm{U}$ \\
\hline $56-23-5$ & Carbon tetrachloride & 87 & $\mathrm{E}$ \\
\hline $71-43-2$ & Benzene & 0.50 & $\mathrm{U}$ \\
\hline $107-06-2$ & 1,2-Dichloroethane & 0.50 & $\mathrm{U}$ \\
\hline
\end{tabular}

Report 1,4-Dioxane for Low-Medium VOA analysis only 
$1 B$ - FORM I VOA-2

VOLATILE ORGANICS ANALYSIS DATA SHEET
EPA SAMPLE NO.

PMP1W26689

Lab Name: TestAmerica BURLINGTON

Contract: 21005

Lab Code: STLV Case No.: CENTRALIA Mod. Ref No.:

SDG No.: 127561

Matrix: (SOIL/SED/WATER) Water

Sample wt/vol: 25.0

$(\mathrm{g} / \mathrm{mL}) \mathrm{mL}$

Level : (TRACE/LOW/MED) TRACE

$\%$ Moisture: not dec.

GC Column: DB-624

ID $: 0.53$

Soil Extract Volume:

Purge Volume: 25.0
Lab Sample ID: 767259

Lab File ID: 767259

Date Received: 09/11/2008

Date Analyzed: 09/12/2008

Dilution Factor: 1.0

Soil Aliquot Volume:

(mL)

\begin{tabular}{|c|c|c|c|}
\hline CAS NO. & COMPOUND & $\begin{array}{l}\text { CONCENTRATION UNITS: } \\
(\mathrm{ug} / \mathrm{L} \text { or } \mathrm{ug} / \mathrm{kg}) \mathrm{ug} / \mathrm{L}\end{array}$ & $Q$ \\
\hline $\begin{array}{c}=========== \\
79-01-6\end{array}$ & 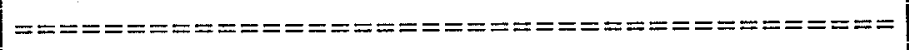 & 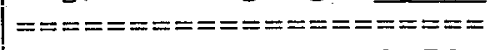 & $=======$ \\
\hline $\begin{array}{r}79-01-6 \\
108-87-2\end{array}$ & Trichloroethene & 0.50 & $\mathrm{U}$ \\
\hline $108-87-2$ & Methylcyclohexane & 0.50 & $\mathrm{U}$ \\
\hline $78-87-5$ & 1,2-Dichloropropane & 0.50 & $\mathrm{U}$ \\
\hline $75-27-4$ & Bromodichloromethane & 0.50 & $\mathrm{U}$ \\
\hline $10061-01-5$ & Cis-1,3-Dichloropropene & 0.50 & U \\
\hline $108-10-1$ & 4-Methyl-2-pentanone & 5.0 & $\mathrm{U}$ \\
\hline $108-88-3$ & Toluene & 33 & $\mathrm{E}$ \\
\hline $10061-02-6$ & trans-1,3-Dichloropropene & 0.50 & $\mathrm{U}$ \\
\hline $79-00-5$ & $1,1,2$-Trichloroethane & 0.50 & $\mathrm{U}$ \\
\hline $127-18-4$ & Tetrachloroethene & 0.50 & U \\
\hline $591-78-6$ & 2-Hexanone & 5.0 & $\mathrm{U}$ \\
\hline $124-48-1$ & Dibromochloromethane & 0.50 & $\mathrm{U}$ \\
\hline $106-93-4$ & 1,2-Dibromoethane & 0.50 & $\mathrm{U}$ \\
\hline $108-90-7$ & Chlorobenzene & 0.50 & $\mathrm{U}$ \\
\hline $100-41-4$ & Ethylbenzene & 0.33 & $\mathrm{~J}$ \\
\hline $95-47-6$ & o-xylene & 0.50 & U \\
\hline $179601-23-1$ & $\mathrm{~m}, \mathrm{p}$-Xylene & 0.50 & $\mathrm{U}$ \\
\hline $100-42-5$ & styrene & 0.50 & $\mathrm{U}$ \\
\hline $75-25-2$ & Bromoform & 0.50 & U \\
\hline $98-82-8$ & Isopropylbenzene & 0.50 & U \\
\hline $79-34-5$ & $1,1,2,2$-Tetrachloroethane & 0.50 & $\mathrm{U}$ \\
\hline $541-73-1$ & 1,3 -Dichlorobenzene & 0.50 & $\mathrm{U}$ \\
\hline $106-46-7$ & 1,4-Dichlorobenzene & 0.50 & $\mathrm{U}$ \\
\hline $95-50-1$ & 1,2-Dichlorobenzene & 0.50 & $\mathrm{U}$ \\
\hline $96-12-8$ & 1,2-Dibromo-3-chloropropane & 0.50 & $\mathrm{U}$ \\
\hline $120-82-1$ & $1,2,4$-Trichlorobenzene & 0.50 & $\mathrm{U}$ \\
\hline $87-61-6$ & $1,2,3$-Trichlorobenzene & 0.50 & U \\
\hline
\end{tabular}


IA - FORM I VOA-1

VOLATILE ORGANICS ANALYSIS DATA SHEET
EPA SAMPLE NO.

PMP1W26689DL

Lab Name: TestAmerica BURLINGTON

Contract: 21005

Lab Code: STLV Case No.: CENTRALIA Mod. Ref No.:

SDG No.: 127561

Matrix: (SOIL/SED/WATER) Water

Sample wt/vol: 25.0

$(\mathrm{g} / \mathrm{mL}) \mathrm{mL}$

Level: (TRACE/LOW/MED) TRACE

$\%$ Moisture: not dec.

GC Column: DB-624

ID $: 0.53$

Soil Extract Volume:

Purge Volume: 25.0
Lab Sample ID: 767259D1

Lab File ID: 767259 D

Date Received: 09/11/2008

Date Analyzed: 09/12/2008

Dilution Factor: 9.6

Soil Aliquot Volume:

(uL)

\begin{tabular}{|c|c|c|c|}
\hline CAS NO. & COMPOUND & $\begin{array}{l}\text { CONCENTRATION UNITS: } \\
(\mathrm{ug} / \mathrm{L} \text { or } \mathrm{ug} / \mathrm{kg}) \mathrm{ug} / \mathrm{L}\end{array}$ & $Q$ \\
\hline$===========$ & 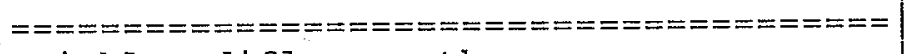 & 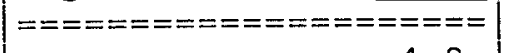 & $========$ \\
\hline $75-71-8$ & Dichlorodifluoromethane & 4.8 & $\mathrm{U}$ \\
\hline $74-87-3$ & Chloromethane & 4.8 & $\mathrm{U}$ \\
\hline $75-01-4$ & Vinyl chloride & 4.8 & $\mathrm{U}$ \\
\hline $74-83-9$ & Bromomethane & 4.8 & $\mathrm{U}$ \\
\hline $75-0.0-3$ & Chloroethane & 4.8 & U \\
\hline $75-69-4$ & Trichlorofluoromethane & 4.8 & $\mathrm{U}$ \\
\hline $75-35-4$ & 1,1-Dichloroethene & 4.8 & $\mathrm{U}$ \\
\hline $76-13-1$ & $1,1,2$-Trichloro-1,2,2-trifluoroethane & 4.8 & $\mathrm{U}$ \\
\hline $67-64-1$ & Acetone & 39 & DJB \\
\hline $75-15-0$ & Carbon disulfide & 4.8 & $\mathrm{U}$ \\
\hline $79-20-9$ & Methyl acetate & 4.8 & $U$ \\
\hline $75-09-2$ & Methylene chloride & 4.8 & $\mathrm{U}$ \\
\hline $156-60-5$ & trans-1,2-Dichloroethene & 4.8 & $\mathrm{U}$ \\
\hline $1634-04-4$ & Methyl tert-butyl ether & 4.8 & $\mathrm{U}$ \\
\hline $75-34-3$ & 1,1-Dichloroethane & 4.8 & $\mathrm{U}$ \\
\hline $156-59-2$ & cis-1,2-Dichloroethene & 4.8 & $\mathrm{U}$ \\
\hline $78-93-3$ & 2-Butanone & $5 \cdot 3$ & $\mathrm{DJ}$ \\
\hline $74-97-5$ & Bromochloromethane & 4.8 & $\mathrm{U}$ \\
\hline $67-66-3$ & Chloroform & 24 & $\mathrm{D}$ \\
\hline $71-55-6$ & $1,1,1$-Trichloroethane & 4.8 & $\mathrm{U}$ \\
\hline $110-82-7$ & Cyclohexane & 4.8 & $\mathrm{U}$ \\
\hline $56-23-5$ & Carbon tetrachloride & 120 & $\mathrm{D}$ \\
\hline $71-43-2$ & Benzene & 4.8 & $\mathrm{U}$ \\
\hline $107-06-2$ & 1,2-Dichloroethane & 4.8 & $\mathrm{U}$ \\
\hline
\end{tabular}

Report 1,4-Dioxane for Low-Medium VOA analysis only 
IB - FORM I VOA-2

VOLATILE ORGANICS ANALYSIS DATA SHEET
EPA SAMPLE NO.

Lab Name: TestAmerica BURLINGTON

Contract: 21005

Lab Code: STLV Case No.: CENTRALIA Mod. Ref No.:

SDG No.: 127561

Matrix: (SOIL/SED/WATER) Water

Sample wt/vol: 25.0

$(\mathrm{g} / \mathrm{mL}) \mathrm{mL}$

LeVel : (TRACE/LOW/MED) TRACE

\% Moisture: not dec.

GC Column: DB-624

Soil Extract Volume:

ID $: 0.53$

(mm)

(uL)

(mL)

Purge Volume: 25.0
Lab Sample ID: 767259D1

Lab File ID: 767259D

Date Received: 09/11/2008

Date Analyzed: 09/12/2008

Dilution Factor: 9.6

Soil Aliquot Volume:

(uL)

\begin{tabular}{|c|c|c|c|}
\hline CAS NO. & COMPOUND & $\begin{array}{l}\text { CONCENTRATION UNITS: } \\
(\mathrm{ug} / \mathrm{L} \text { or } \mathrm{ug} / \mathrm{kg}) \mathrm{ug} / \mathrm{L}\end{array}$ & Q \\
\hline 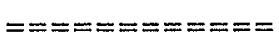 & 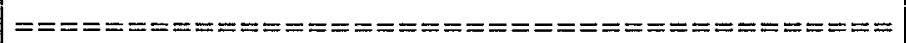 & 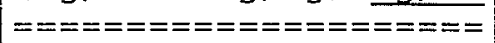 & $=== \pm====$ \\
\hline $79-01-6$ & Trichloroethene & 4.8 & $\mathrm{U}$ \\
\hline $108-87-2$ & Methylcyclohexane & 4.8 & $\mathrm{U}$ \\
\hline $78-87-5$ & 1,2-Dichloropropane & 4.8 & U \\
\hline $75-27-4$ & Bromodichloromethane & 4.8 & $\mathrm{U}$ \\
\hline $10061-01-5$ & Cis-1,3-Dichloropropene & 4.8 & $\mathrm{U}$ \\
\hline $108-10-1$ & 4-MethyI-2-pentanone & 48 & $\mathrm{U}$ \\
\hline $108-88-3$ & Toluene & 46 & $\mathrm{D}$ \\
\hline $10061-02-6$ & trans-1,3-Dichloropropene & 4.8 & $\mathrm{U}$ \\
\hline $79-00-5$ & $1,1,2$-Trichloroethane & 4.8 & $\mathrm{U}$ \\
\hline $127-18-4$ & Tetrachloroethene & 4.8 & $\mathrm{U}$ \\
\hline $591-78-6$ & 2-Hexanone & 48 & $\mathrm{U}$ \\
\hline $124-48-1$ & Dibromochloromethane & 4.8 & $\mathrm{U}$ \\
\hline $106-93-4$ & 1,2-Dibromoethane & 4.8 & $\mathrm{U}$ \\
\hline $108-90-7$ & Chlorobenzene & 4.8 & $\mathrm{U}$ \\
\hline $100-41-4$ & Ethylbenzene & 4.8 & $\mathrm{U}$ \\
\hline $95-47-6$ & o-Xylene & 4.8 & $\mathrm{U}$ \\
\hline $179601-23-1$ & $\mathrm{~m}, \mathrm{p}$-xylene & 4.8 & $\mathrm{U}$ \\
\hline $100-42-5$ & styrene & 4.8 & $\mathrm{U}$ \\
\hline $75-25-2$ & Bromoform & 4.8 & $\mathrm{U}$ \\
\hline $98-82-8$ & Isopropylbenzene & 4.8 & $\mathrm{U}$ \\
\hline $79-34-5$ & $1,1,2,2$-Tetrachloroethane & 4.8 & $\mathrm{U}$ \\
\hline $541-73-1$ & 1,3-Dichlorobenzene & 4.8 & $\mathrm{U}$ \\
\hline $106-46-7$ & 1,4-Dichlorobenzene & 4.8 & $\mathrm{U}$ \\
\hline $95-50-1$ & 1,2-Dichlorobenzene & 4.8 & $\mathrm{U}$ \\
\hline $96-12-8$ & 1,2-Dibromo-3-chloropropane & 4.8 & U \\
\hline $120-82-1$ & 1,2,4-Trichlorobenzene & 4.8 & $\mathrm{U}$ \\
\hline $87-61-6$ & 1,2,3-Trichlorobenzene & 4.8 & $\mathrm{U}$ \\
\hline
\end{tabular}


Argonne

Environmental Science Division

Argonne National Laboratory

9700 South Cass Avenue, Bldg. 203

Argonne, IL 60439-4843

www.anl.gov

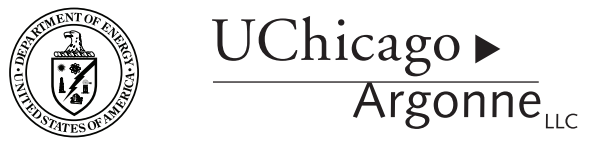

A U.S. Department of Energy laboratory

managed by UChicago Argonne, LLC 